\title{
RADIOLOGICAL FINAL STATUS SURVEY OF THE HAMMOND DEPOT HAMMOND, INDIANA
}

\section{T.J. VITKUS}

\section{Prepared for the}

Defense National Stockpile Center of the

Defense Logistics Agency

\section{O R I S E}

Oak Ridge Institute for Science and Education

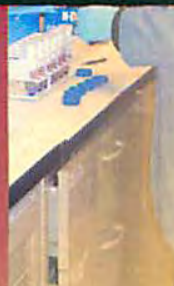

Approved for public release; further dissemination unlimited. 


\title{
RADIOLOGICAL FINAL STATUS SURVEY \\ OF THE \\ HAMMOND DEPOT \\ HAMMOND, INDIANA
}

\author{
Prepared by \\ T. J. Vitkus \\ Oak Ridge Institute for Science and Education \\ Oak Ridge, Tennessee 37831-0117 \\ Prepared for the \\ Defense National Stockpile Center \\ Defense Logistics Agency
}

FINAL REPORT

APRIL 2008 


\section{RADIOLOGICAL FINAL STATUS SURVEY OF THE \\ HAMMOND DEPOT HAMMOND, INDIANA}

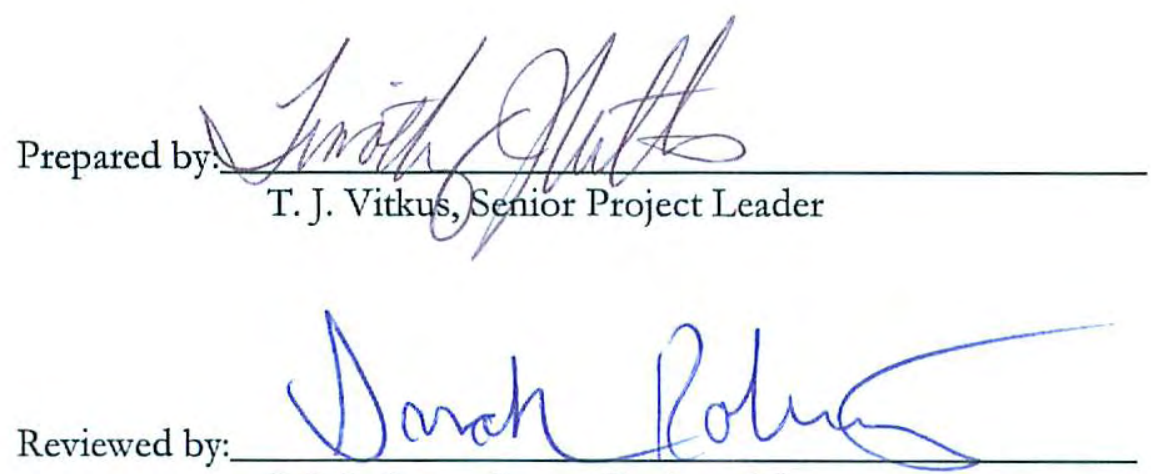

S. J. Roberts, Survey Projects Manager

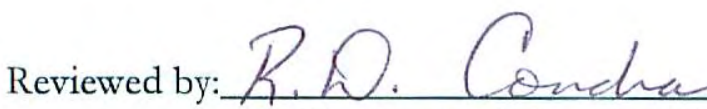

R. D. Condra, Laboratory Manager

Reviewed by: $\frac{\text { Omanc, Quality Manager }}{\text { A.T. Payne }}$

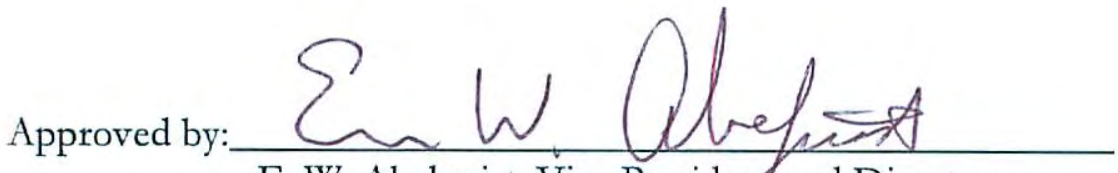

E. W. Abelquist, Vice Presideny and Director

Independent Environmental Assessment and Verification
Date: $4 / 1 / 2008$

Date: $4 / 108$

Date: $4 / 2 / 08$

Date: $4 / 2 / 08$

Date: $4 / 3 / 68$ 


\title{
ACKNOWLEDGMENTS
}

The author would like to acknowledge the significant contributions of the following staff members:

FIELD AND SUPPORT STAFF

E.N. Bailey

T.D. Herrera

E. Montalvo

\section{LABORATORY STAFF}

R.D. Condra

W.P. Ivey

J.S. Cox

W.F. Smith

\section{CLERICAL STAFF}

R.M. Fink

K.M. Moore

A. Ramsey

\section{ILLUSTRATORS}

\author{
T.L. Brown
}

J.A.Viars 


\section{ABBREVIATIONS AND ACRONYMS}

\begin{tabular}{|c|c|}
\hline$\varepsilon_{\mathrm{i}}$ & instrument efficiency \\
\hline$\varepsilon_{\mathrm{s}}$ & surface efficiency \\
\hline$\varepsilon_{\text {total }}$ & total efficiency \\
\hline$b_{i}$ & number of background counts in the interval \\
\hline AEC & Atomic Energy Commission \\
\hline ALARA & as low as reasonable achievable \\
\hline $\mathrm{AOC}$ & area of concern \\
\hline $\mathrm{BKG}$ & background \\
\hline COMPASS & $\begin{array}{l}\text { Computerization of MARSSIM for Planning and Assessing } \\
\text { Site Surveys }\end{array}$ \\
\hline $\mathrm{cm}$ & centimeter \\
\hline $\mathrm{cm}^{2}$ & square centimeter \\
\hline cpm & counts per minute \\
\hline $\mathrm{d}^{\prime}$ & index of sensitivity \\
\hline DCGL & derived concentration guideline level \\
\hline DLA & Defense Logistics Agency \\
\hline DNSC & Defense National Stockpile Center \\
\hline DOE & U.S. Department of Energy \\
\hline $\mathrm{dpm} / 100 \mathrm{~cm}^{2}$ & disintegrations per minute per 100 square centimeters \\
\hline DQA & data quality assessment \\
\hline DQO & data quality objective \\
\hline EMC & elevated measurement comparison \\
\hline FRS & final remediation survey \\
\hline FSS & final status survey \\
\hline GPS & global positioning system \\
\hline GSA & General Services Administration \\
\hline $\mathrm{H}_{0}$ & null hypothesis \\
\hline $\mathrm{H}_{\mathrm{A}}$ & alternative hypothesis \\
\hline HD & Hammond Depot \\
\hline HSA & historical site assessment \\
\hline IEAV & Independent Environmental Assessment and Verification \\
\hline ITP & Intercomparison Testing Program \\
\hline JHA & job hazard analysis \\
\hline MAPEP & Mixed Analyte Performance Evaluation Program \\
\hline MARSSIM & $\begin{array}{l}\text { Multi-Agency Radiation Survey and Site Investigation } \\
\text { Manual }\end{array}$ \\
\hline MDC & minimum detectable concentration \\
\hline MDCR & minimum detectable count rate \\
\hline $\mathrm{MeV}$ & million electron volts \\
\hline $\mathrm{m}$ & meters \\
\hline $\mathrm{m}^{2}$ & square meter \\
\hline $\mathrm{mg} / \mathrm{cm}^{2}$ & milligram per square centimeter \\
\hline $\min$ & minute \\
\hline $\mathrm{mm}$ & millimeter \\
\hline mrem $/ y$ & millirem per year \\
\hline
\end{tabular}




\section{ABBREVIATIONS AND ACRONYMS (Continued)}

$\begin{array}{ll}\text { NaI } & \text { sodium iodide } \\ \text { NIST } & \text { National Institute of Standards and Technology } \\ \text { NORM } & \text { naturally occurring radioactive material } \\ \text { NRC } & \text { U.S. Nuclear Regulatory Commission } \\ \text { NRIP } & \text { NIST Radiochemistry Intercomparison Program } \\ \text { ORISE } & \text { Oak Ridge Institute for Science and Education } \\ \text { ORNL } & \text { Oak Ridge National Laboratory } \\ \text { pCi/g } & \text { picocuries per gram } \\ \text { RESRAD } & \text { RESidual RADioactive material software } \\ \text { s } & \text { second } \\ \text { SOR } & \text { sum of ratios } \\ \text { TAP } & \text { total absorption peak } \\ \text { ThN } & \text { thorium nitrate } \\ \text { VSP } & \text { Visual Sampling Plan } \\ \text { WEI } & \text { World Environmental, Inc. } \\ \text { WRS } & \text { Wilcoxon Ranked Sum }\end{array}$




\section{TABLE OF CONTENTS}

1.0 INTRODUCTION..

PAGE

1.1 Objectives 1

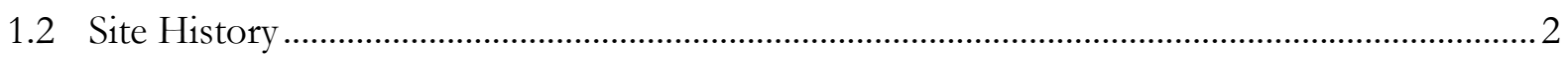

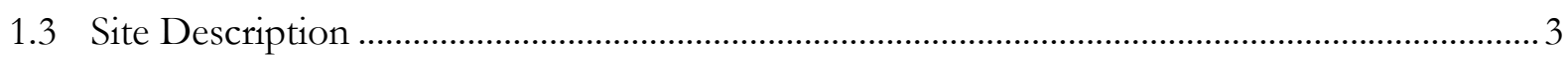

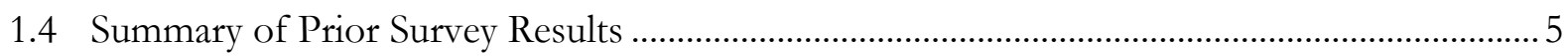

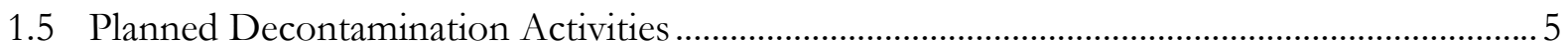

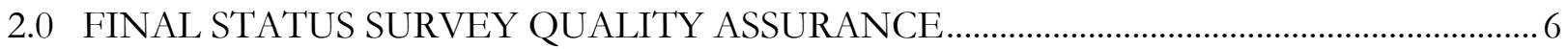

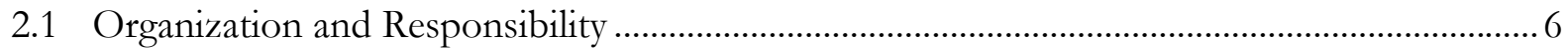

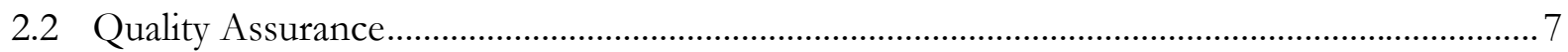

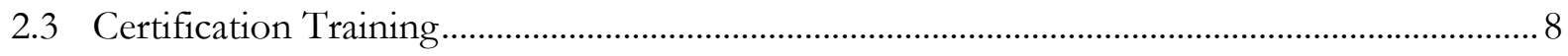

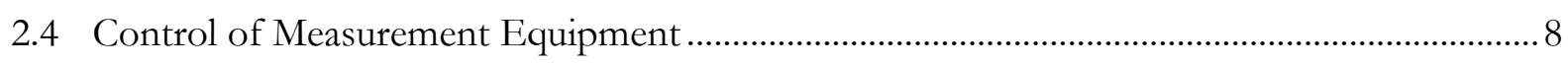

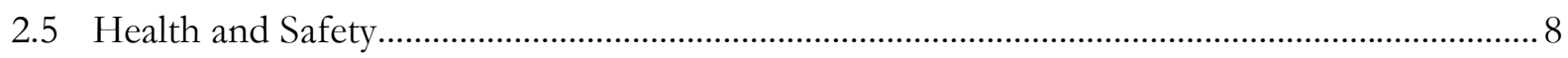

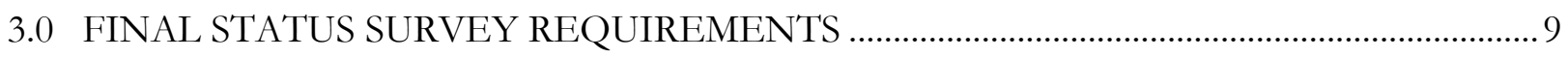

3.1 Derived Concentration Guideline Levels ...................................................................................... 9

3.2 Classification of Areas by Contamination Potential...................................................................10

3.3 Identification of Survey Units ..............................................................................................11

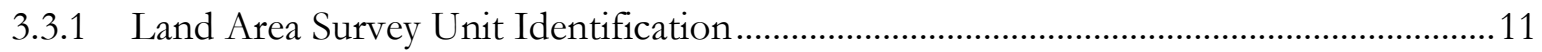

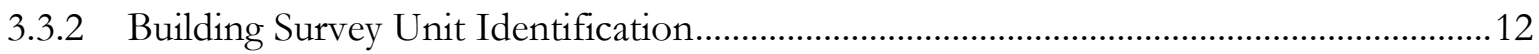

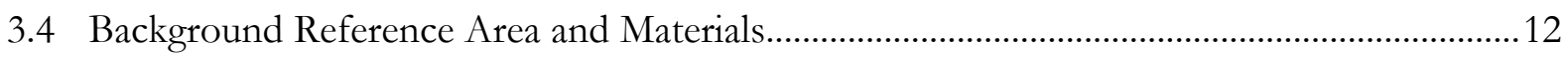

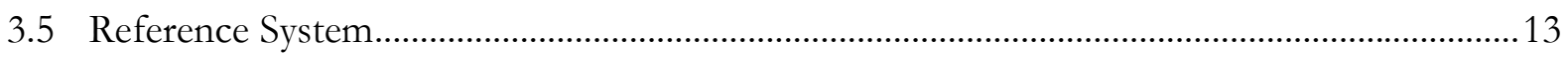

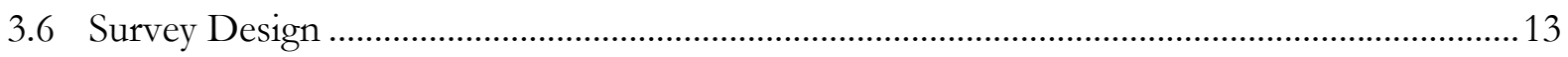

3.6.1 Calculation of Required Number of Measurements ...........................................................14

3.6.2 Determining Measurement/Sampling Locations ............................................................15

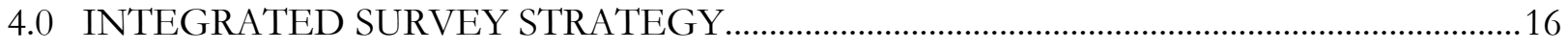

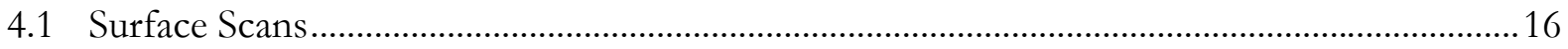

4.1.1 Class 1 Land Area Survey Units................................................................................... 17

4.1.2 Class 2 and 3 Land Area Survey Units...................................................................... 18

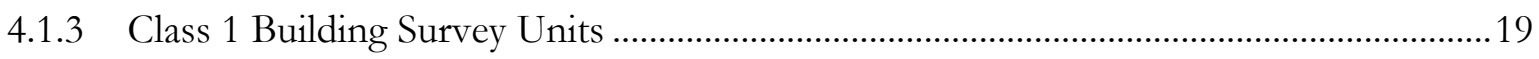

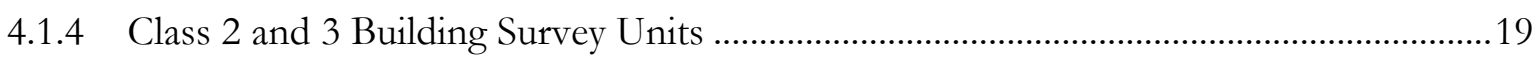

4.2 Soil Sampling and Surface Activity Measurements....................................................................20

4.2.1 Class 1 Land Area Survey Units.................................................................................21 


\section{TABLE OF CONTENTS (Continued)}

PAGE

4.2.2 Class 2 Land Area Survey Units........................................................................................22

4.2.3 Class 3 Land Area Survey Units.....................................................................................22

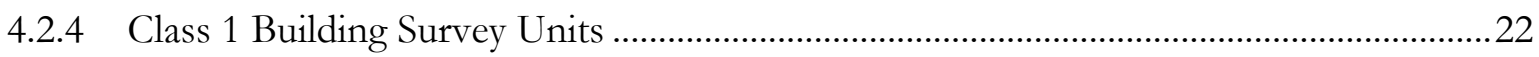

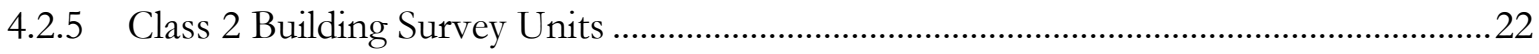

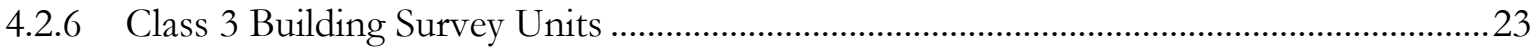

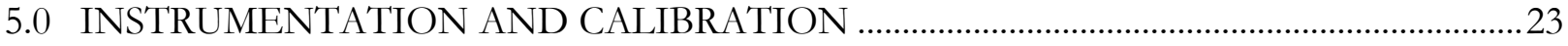

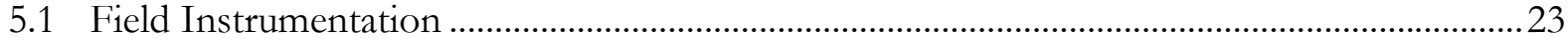

5.1.1 Scanning Instrument/Detector Combinations .................................................................2 24

5.1.2 Direct Measurement Instrument/Detector Combinations …...........................................25

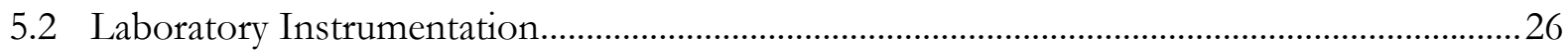

5.2.1 Gross Alpha/Beta (Removable Activity) ….................................................................27

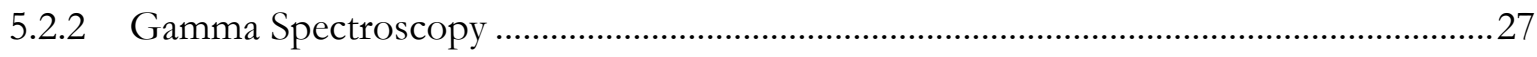

6.0 DATA REVIEW AND INVESTIGATION THRESHOLDS ..............................................28

7.0 DETERMINING COMPLIANCE WITH DCGLs ....................................................................28

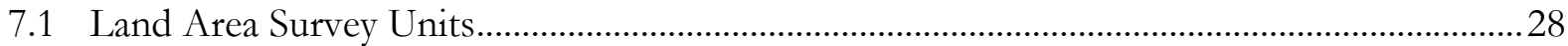

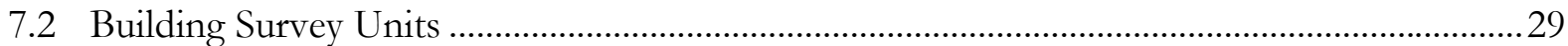

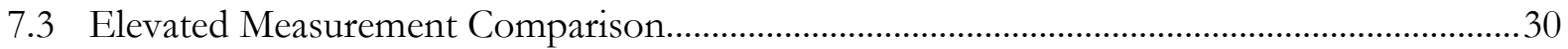

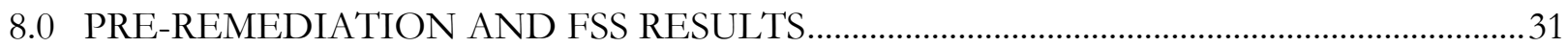

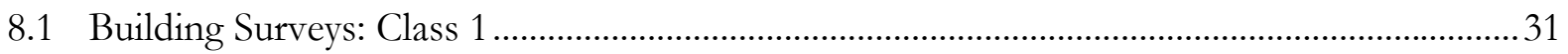

8.1.1 Class 1 Scoping/Characterization Surface Scans and Surface Activity Levels ...............31

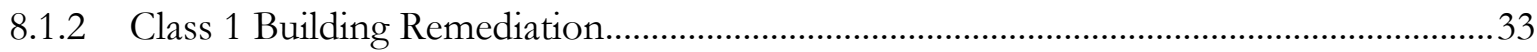

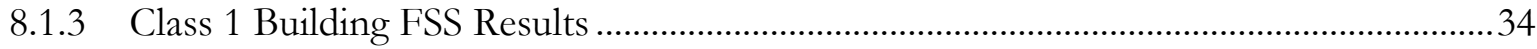

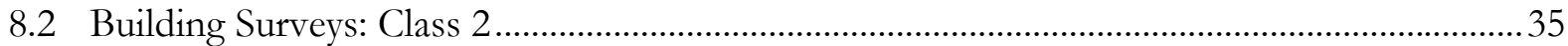

8.2.1 Class 2 Scoping/Characterization/FSS Surface Scans \& Surface Activity Levels ..........35

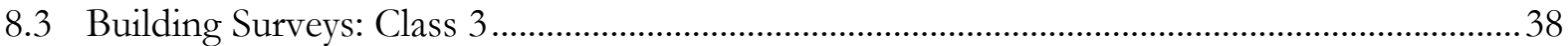

8.3.1 Class 3 Scoping/Characterization/FSS Surface Scans \& Surface Activity Levels ..........38

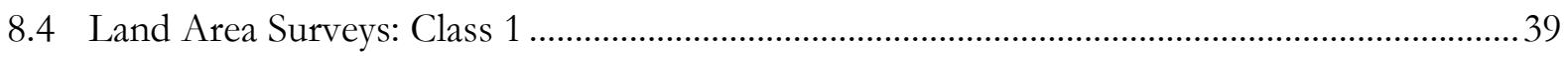

8.4.1 Class 1 Land Area Scoping/Characterization Surface Scans \& Sample Results.............39

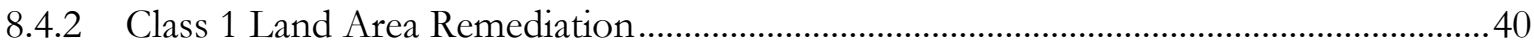

8.4.3 Class 1 Land Area FSS Surface Scans \& Sample Results ..................................................44

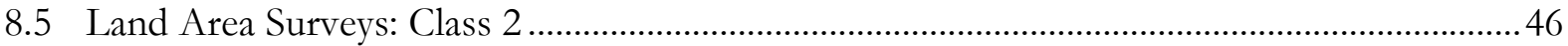




\section{TABLE OF CONTENTS (Continued)}

PAGE

8.5.1 Class 2 Land Area Scoping/Characterization/FSS Surface Scans \& Sample Results ...46

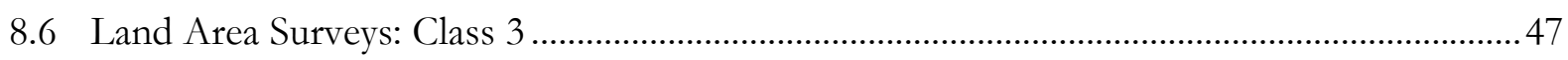

8.6.1 Class 3 Land Area Scoping/Characterization/FSS Surface Scans \& Sample Results ...47

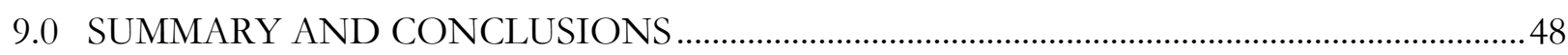

APPENDIX A: FIGURES

APPENDIX B: TABLES

APPENDIX C: REFERENCES

APPENDIX D: LAND SURVEY UNIT DQOs

APPENDIX E: STRUCTURE SURVEY UNIT DQOs

APPENDIX F: STRUCTURE SURVEY UNIT DQAs

APPENDIX G: LAND SURVEY UNIT DQAs 


\section{LIST OF FIGURES}

Figure 2-1: IEAV Organizational Chart

Figure A-1: Hammond Depot, Hammond, Indiana_-Plot Plan

Figure A-2: Hammond Depot_Contaminated Buildings and Soil AOCs...

Figure A-3: Hammond Depot—Background Reference Area and FSS Survey Units (\# ID) .........A-3

Figure A-4: Background Reference Area—FSS Soil Sampling Locations...........................................4

Figure A-5: Example Instrument Calibration Efficiency Determination ...........................................A-5

Figure A-6: Building 200E South, Floor-FSS Survey Units and Direct Measurement Locations ......

Figure A-7: Building 200E South, Walls (C1 SU22, 23, and C2 SU56 through 58)—FSS Survey Units and Direct Measurement Locations.

Figure A-8: Building 200E South, Columns 1 through 20 (C1 SU33)—FSS Survey Unit and Direct Measurement Locations

Figure A-9: Building 200E South, Columns 21 through 36 (C1 SU34)—FSS Survey Unit and Direct Measurement Locations

Figure A-10: Building 200E South, Ceiling (C1 SU24 through 31 and C2 SU35 and 36)—FSS Survey Unit and Direct Measurement Locations A-10

Figure A-11: Building 200E, NW Closet (C1 SU41)_FSS Survey Unit and Direct Measurement Locations

Figure A-12: Building 200E, North (C2 SU37 and 38 and C3 SU40)—FSS Survey Units and Direct Measurement Locations A-12

Figure A-13: Building 200E, North, Locker Room (C2 SU42)—FSS Survey Unit and Direct Measurement Locations....

Figure A-14: Building 100E, North-end Floor (C1 SU43, 44, and 59; C2 SU45, 60, and 61)—FSS Survey Units and Direct Measurement Locations.

Figure A-15: Building 100E, North-end Walls (C2 SU46)—FSS Survey Units and Direct Measurement Locations

Figure A-16: Building 100E, North-end Ceiling (C3 SU47)—FSS Survey Units and Direct Measurement Locations.....

Figure A-17: Building 100E, North-end Ceiling (C3 SU47)—FSS Survey Units and Direct Measurement Locations.

Figure A-18: Building 100W (C2 SU49 through 51 and C3 SU52)—FSS Survey Units and Direct Measurement Locations

Figure A-19: Garage Building (C3 SU53)—FSS Survey Units and Direct Measurement Locations..... 


\section{LIST OF FIGURES (Continued)}

Figure A-20: Workshop Building (C3 SU54)—FSS Survey Units and Direct Measurement Locations

Figure A-21: Building Exteriors (C3 SU55)_FSS Survey Units and Direct Measurement Locations A-21

Figure A-22: Survey Unit C1 SU1 — FSS Gamma Scan Results ................................................... A-22

Figure A-23: Survey Unit C1 SU1-FSS Soil Sampling Locations .................................................. A-23

Figure A-24: Survey Unit Survey Unit C1 SU2_FSS Gamma Scan Results ................................ A-24

Figure A-25: Survey Unit C1 SU2-FSS Soil Sampling Locations ................................................... A-25

Figure A-26: Survey Unit C1 SU3_FSS Gamma Scan Results ..................................................... A-26

Figure A-27: Survey Unit C1 SU3-FSS Soil Sampling Locations ................................................ A-27

Figure A-28: Survey Unit C1 SU4-FSS Gamma Scan Results ..................................................... A-28

Figure A-29: Survey Unit C1 SU4-FSS Soil Sampling Locations ................................................... A-29

Figure A-30: Survey Unit C1 SU5-FSS Gamma Scan Results ..................................................... A-30

Figure A-31: Survey Unit C1 SU5-FSS Soil Sampling Locations ................................................. A-31

Figure A-32: Survey Unit C1 SU6_-FSS Gamma Scan Results .................................................... A-32

Figure A-33: Survey Unit C1 SU6-FSS Soil Sampling Locations .................................................... A-33

Figure A-34: Survey Unit C1 SU7-FSS Gamma Scan Results ................................................... A-34

Figure A-35: Survey Unit C1 SU7-FSS Soil Sampling Locations .................................................. A-35

Figure A-36: Survey Units C1 SU2.1 through 5.8-FSS Gamma Scan Results ............................. A-36

Figure A-37: Survey Unit C1 SU2.1 through 5.8-FSS Direct Measurement Locations .............. A-37

Figure A-38: Survey Unit C2 SU1—FSS Gamma Scan Results ..................................................... A-38

Figure A-39: Survey Unit C2 SU1-FSS Soil Sampling Locations ................................................... A-39

Figure A-40: Survey Unit C2 SU2-FSS Gamma Scan Results ..................................................... A-40

Figure A-41: Survey Unit C2 SU2-FSS Soil Sampling Locations ................................................. A-41

Figure A-42: Survey Unit C2 SU3_-FSS Gamma Scan Results ................................................... A-42

Figure A-43: Survey Unit C2 SU3_-FSS Soil Sampling Locations ................................................. A-43

Figure A-44: Survey Unit C2 SU4—FSS Gamma Scan Results ..................................................... A-44

Figure A-45: Survey Unit C2 SU4-FSS Soil Sampling Locations .................................................. A-45

Figure A-46: Survey Unit C2 SU5-FSS Gamma Scan Results ...................................................... A-46

Figure A-47: Survey Unit C2 SU5-FSS Soil Sampling Locations ............................................... A-47 


\section{LIST OF FIGURES (Continued)}

Figure A-48: Survey Unit C2 SU6_-FSS Gamma Scan Results ................................................... A-48

Figure A-49: Survey Unit C2 SU6_-FSS Soil Sampling Locations ................................................... A-49

Figure A-50: Survey Unit C2 SU7-FSS Gamma Scan Results ....................................................... A-50

Figure A-51: Survey Unit C2 SU7-FSS Soil Sampling Locations ............................................... A-51

Figure A-52: Survey Unit C2 SU8-FSS Gamma Scan Results .................................................. A-52

Figure A-53: Survey Unit C2 SU8 —-FSS Soil Sampling Locations ................................................ A-53

Figure A-54: Survey Unit C2 SU9_FSS Gamma Scan Results ..................................................... A-54

Figure A-55: Survey Unit C2 SU9-FSS Soil Sampling Locations ................................................... A-55

Figure A-56: Survey Unit C2 SU10-FSS Gamma Scan Results ………….................................... A-56

Figure A-57: Survey Unit C2 SU10—FSS Soil Sampling Locations ................................................ A-57

Figure A-58: Survey Unit C2 SU11-FSS Gamma Scan Results .................................................. A-58

Figure A-59: Survey Unit C2 SU11—FSS Soil Sampling Locations ............................................... A-59

Figure A-60: Survey Unit C2 SU12-FSS Soil Sampling Locations ................................................... A-60

Figure A-61: Survey Unit C2 SU12, Debris Pile—FSS Direct Measurement Locations ............... A-61

Figure A-62: Survey Unit C3 SU1-FSS Gamma Scan Results .................................................. A-62

Figure A-63: Survey Unit C3 SU1-FSS Soil Sampling Locations ................................................. A-63

Figure A-64: Survey Unit C3 SU2-FSS Gamma Scan Results .................................................... A-64

Figure A-65: Survey Unit C3 SU2-FSS Soil Sampling Locations ................................................... A-65

Figure A-66: Survey Unit C3 SU1-FSS Gamma Scan Results ...................................................... A-66

Figure A-67: Survey Unit C3 SU3 —-FSS Soil Sampling Locations ................................................. A-67

Figure A-68: Survey Unit C3 SU4-FSS Gamma Scan Results .................................................... A-68

Figure A-69: Survey Unit C3 SU4-FSS Soil Sampling Locations ............................................... A-69

Figure A-70: Survey Unit C3 SU5-FSS Gamma Scan Results .................................................... A-70

Figure A-71: Survey Unit C3 SU5-FSS Soil Sampling Locations ................................................ A-71 


\section{LIST OF TABLES}

Table 3-1: MARSSIM-Recommended Survey Unit Sizes ...................................................................... 11

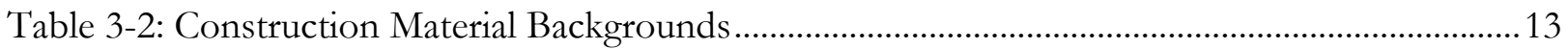

Table 4-1: MARSSIM-Recommended FSS Survey Scan Coverage .....................................................17

Table 8-1: Class 1 B-Line Building Characterization Surface Activity Levels......................................33

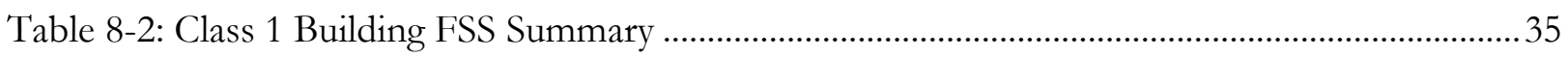

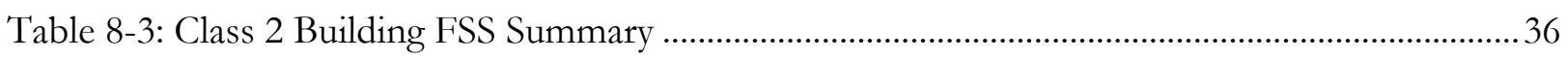

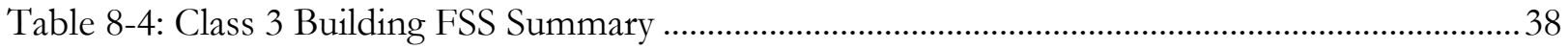

Table 8-5: Characterization Survey Radionuclide Concentrations ......................................................40

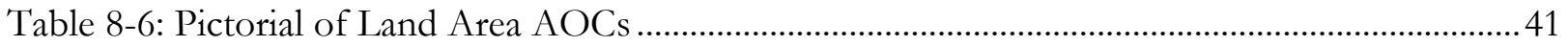

Table 8-7a: FSS Class 1 Radionuclide Concentrations in Soil Samples Summary Results..................45

Table 8-7b: FSS Class 1 Slag Beta Surface Activity...............................................................................45

Table 8-8a: FSS Class 2 Radionuclide Concentrations in Soil Samples Summary Results..................46

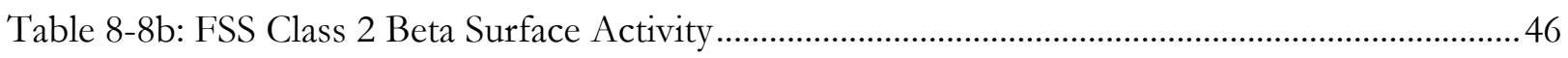

Table 8-9: FSS Class 3 Radionuclide Concentrations in Soil Samples Summary Results....................47

Table B-1: Survey Unit Descriptions and Results .............................................................................

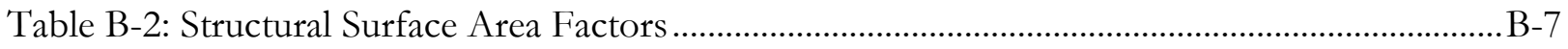

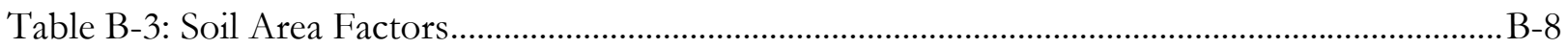

Table B-4: Investigation Results .....................................................................................................

Table B-5: FSS Structural Survey Units and Surface Activity Levels ............................................... B-11

Table B-6: FSS Survey Units and Radionuclide Concentration Levels ..............................................3-38

Table B-7: FSS Exterior Slag Survey Units and Surface Activity Levels ........................................... B-50 


\section{RADIOLOGICAL FINAL STATUS SURVEY \\ OF THE \\ HAMMOND DEPOT \\ HAMMOND, INDIANA}

\subsection{INTRODUCTION}

This report provides a detailed discussion of the radiological survey planning, survey implementation, remediation, and the results for these activities supporting the conclusion that radioactive contamination previously identified at the Hammond Depot (HD) has been reduced to levels such that the site may be released without radiological restrictions.

\subsection{OBJECTIVES}

The objective of the radiological final status survey (FSS) was to obtain the data necessary to demonstrate compliance with the U.S. Nuclear Regulatory Commission (NRC)-approved site-specific derived concentration guideline levels (DCGLs) for both structural surfaces and outdoor areas (ORISE 2006a and NRC 2007). The DCGLs were modeled such that any residual licensed material would not exceed the NRC's basic dose limit for license termination of 25 millirem per year (mrem/y). Specifically, when the DCGLs are applied to the FSS and the final survey results show that the DCGLs have been satisfied, the following 10 CFR 20.1402 requirements are met:

"Title 10 CFR 20.1402: Radiological criteria for unrestricted use. A site will be considered acceptable for unrestricted use if the residual radioactivity that is distinguishable from background radiation results in a total effective dose equivalent to an average member of the critical group that does not exceed 25 mrem (0.25 millisieverts) per year, including that from groundwater sources of drinking water, and that the residual radioactivity has been reduced to levels that are as low as reasonably achievable (ALARA). Determination of the levels which are ALARA must take into account consideration of any detriments, such as deaths from transportation accidents, expected to potentially result from decontamination and waste disposal." 
The FSS was conducted in accordance with the design inputs provided in the FSS plan (ORISE 2007a). These design inputs were obtained through the performance of a historical site assessment (HSA), scoping surveys, and a detailed characterization survey (ORISE 2005a, 2005b, and 2006b). Furthermore, the survey plans implemented for the scoping and characterization surveys were designed following the process detailed in the plan in order to satisfy the FSS data quality objectives (DQOs) for data quantity and quality, such that some or all of the data generated for those areas of the site with little potential for residual contamination could be used as FSS data (ORISE 2005c and 2006c).

\subsection{SITE HISTORY}

In 1946, the National Stockpile program began with the goal of mitigating dependence on foreign sources of vital materials during times of national emergencies. The Hammond Depot in Hammond, Indiana was established as part of this program in 1948. The land area for the Hammond Depot originally consisted of approximately 52.8 hectares (130.5 acres) of land leased on June 24, 1948 from the Indiana Harbor Belt Railroad Company. On June 27, 1969 the General Services Administration (GSA) purchased the entire site. The original site had eight warehouses and 80 above ground storage tanks. GSA sold portions of the property, including three warehouses, during the 1970s. The current site consists of 27.2 hectares.

The Defense National Stockpile Center (DNSC) used the Hammond Depot to store strategic materials (bulk ores, minerals, and metals). The materials stored in outdoor piles either on the ground or on pads were chrome, ferrochrome, ferromanganese, lead, tin, and others.

Beginning in approximately 1958, additional stored materials included monazite sand comprised of 2.4 to $3.4 \%$ thorium dioxide $\left(\mathrm{ThO}_{2}\right)$ and bastnesite with 0.01 to $0.11 \%$ of $\mathrm{ThO}_{2}$. Storage of thorium nitrate (reactor grade consisting of 46.0 to $47.15 \%$ by weight of $\mathrm{ThO}_{2}$ ) began in 1962 , followed by sodium sulfate, tantalum pentoxide, and columbium tantalum minerals in the 1980s. These latter materials contained from $<0.001$ to $0.053 \%$ by weight $\mathrm{ThO}_{2}$ and 0.012 to $0.156 \%$ by weight uranium oxide. All of these materials were contained in fiber and steel drums and stored in warehouses. Some materials contained radioactive material at concentrations that required a U.S. Atomic Energy Commission (AEC)—predecessor to the NRC—-source material license (License STC-133). 
The DNSC of the Defense Logistics Agency (DLA) is now in the process of closing out many of its depots across the country and seeking to terminate its NRC license for those facilities. In the early 1970s, warehouses (Warehouses 1, 2, and 3) where source or other materials were stored, were emptied and remediated and surveyed, if contaminated. These warehouses were then sold as excess property. All current site clean-up work at the HD is sponsored by the DNSC Thorium Nitrate Stewardship and Disposition Program - Phase 4 - Decontamination \& Decommissioning and is being supported under the Department of Energy (DOE)-Oak Ridge Operations Work for Others Program. The project is supported and coordinated by the Oak Ridge National Laboratory (ORNL), per DOE Proposal Number \# 1872-M171-A1. Removal of the thorium nitrate $(\mathrm{ThN})$ source material from the site, Phase 3 of the project, was completed in fiscal year 2005, which completed the initial phase of the current cleanup activities by removing the remaining source material that had been stored within two of the current site warehouses. In conjunction with site cleanup, at the request of ORNL, the Oak Ridge Institute for Science and Education (ORISE) performed an HSA of the Hammond Depot in order to plan for future site investigations and eventual remediation activities (ORISE 2005a). Additionally, ORISE was tasked to conduct scoping and characterization surveys of the site to validate the results of the HSA and to provide radiological information for the development of a decontamination scope of work for areas of the site identified with excess residual radioactivity levels (ORISE 2005b and 2006b, ORNL 2006). These surveys were designed in an integrated, graded approach following the radiological survey guidance and DQO process provided in the Multi-Agency Radiation Survey and Site Investigation Manual (MARSSIM) (NRC 2000).

\subsection{SiTE DESCRIPTION}

The Hammond Depot site is located on the west side of Hammond, Indiana on Sheffield Avenue-about 150 meters (500 feet) east of the Indiana-Illinois state line. The property currently consists of ten structures, mostly in good condition, including the three current warehouses used to store raw materials, and outdoor storage areas (Figure A-1). The depot is bounded on the east and southeast by the Indiana Harbor Belt railway, the Wolf Lake Industrial Center access road on the east, the Wolf Lake industrial/commercial complex on the north, Wolf Lake on the northern one-third of the western property boundary, and a drainage ditch on the west and southwest property boundary. A security fence encloses the facility. A number of 
road and railroad tracks provide access on the site. On site drainage ditches direct surface water runoff to Wolf Lake.

The three current warehouses are located in the central area of the site and are designated as Buildings 100W, 100E, and 200E. The dimensions of the three warehouses are each 38 meters by 122 meters (126 feet by 401 feet) and construction is cinder block walls on a concrete slab floor with steel beams, columns, and roof joists. Building 200E is divided by a cinder block wall into a northern and southern half. The southern half had been used for radioactive material storage and also had an asphalt overlayment covering the floor. The asphalt was laid down over the original floor surface to level it after extensive remediation in the 1980s. When the leaking drums of $\mathrm{ThN}$ were repackaged, they were moved into Building $100 \mathrm{~W}$ for interim storage. There was no history of any previous remedial activities or contamination events in Building 100W. Building 100E had no history of radioactive material storage. The interior of each warehouse was subdivided into 20 bay areas which correspond to the support column lines.

The warehouses continue to be used for storage of materials. These materials include tannin as well as tungsten and aluminum oxide. Both the tungsten and aluminum oxide contain radioactive materials, but at concentrations below licensable requirements. Although these materials do not require licensing, gamma radiation emitted from the natural thorium present in the material caused the local ambient background radiation levels to increase.

A unique site feature was the presence of both monolithic and unconsolidated slag throughout the site. The slag, originating from area steel mills, had historically been used as a backfill material over an approximately 20 square mile area, as much as seven meters or more in thickness. The physical-chemical slag properties were noted to vary somewhat based on general hardness and the concentration of naturally occurring radioactive material. The monolithic slag was typically beneath a surface soil layer of 0.15 to 0.30 meters in depth, although the slag could be encountered anywhere from the immediate surface to more than one meter below the overlying soil. 


\subsection{Summary OF Prior Survey Results}

The contaminant of concern for the Hammond Depot is primarily thorium with the potential for lesser quantities of uranium. All scoping and characterization survey results for the northern half of Building 200E, Building 100W, and the majority of the exterior areas satisfied the DCGL $_{\mathbb{W}}$ of $400 \mathrm{dpm} / 100 \mathrm{~cm}^{2}$ for Th-232 surface activity or the soil DCGL $\mathrm{W}$ of $2.9 \mathrm{pCi} / \mathrm{g}$ and $2.5 \mathrm{pCi} / \mathrm{g}$ for Th-232 and U-238, respectively, and supported the initial survey classifications. However, the scoping and characterization surveys identified residual contamination within the southern half and a closet area in the northwest corner of Building 200E, and a localized area within Building 100E. Several site soil areas of concern (AOCs) were determined to be present over a broad area near the former Burn Cage area and Ferrochrome Pile \#6 as well as several smaller AOCs; all of which were located on the western portion of the site. The locations of each of these AOCs, together with the previously discussed contaminated buildings, are shown on Figure A-2.

\subsection{Planned Decontamination Activities}

A detailed decontamination/remediation plan was prepared and submitted to NRC for review and approval (ORISE 2006d). The information provided in this plan was used to develop the scope of work requirements that were followed by the decontamination and remediation contractor. The requirements of the scope of work were the removal of contamination from structures using proven remedial technologies and the excavation of contaminated soils to levels below the DCGL $\mathrm{W}_{\mathrm{W}}$ (ORNL 2006).

The U.S. Army Joint Munitions Command contractor for the site remediation was World Environmental, Inc (WEI). WEI began remediation of contaminated soils and structures in August 2007 and completed remedial actions and final remediation support surveys in December 2007. There were two buildings and six soil AOCs that were identified as contaminated. Contaminated structural surfaces within Buildings 200E and 100E were remediated using a variety of techniques that involved scabbling, grinding, washing or complete structural removal and disposal. The contaminated soils were excavated and contaminated slag surfaces were scraped or broken out. Site remediation resulted in the removal and off-site shipment and disposal of over 4,000 tons of soil, concrete, slag, and debris. These activities are further discussed in Sections 8.1.2 and 8.4.2. WEI performed extensive post-remedial action 
scans, measurements, and sampling of each AOC, to ensure contamination levels were below the DCGLs prior to releasing an area for FSS. These activities were documented in certificates of completion and in the daily ORNL project reports.

\subsection{FINAL STATUS SURVEY QUALITY ASSURANCE}

The FSS project responsibilities, training requirements, and quality assurance are described below.

\subsection{ORGANIZATION AND RESPONSIBILITY}

ORISE conducts radiological survey activities as one of its core competencies through the Independent Environmental Assessment and Verification Program (IEAV). Figure 2-1 represents the generic organizational structure of the IEAV survey staff.

Detailed responsibilities for various staff positions are documented in Position Questionnaires, which have been developed for all employees. Additional detailed information regarding various staff position responsibilities is included in the IEAV Quality Program Manual (ORAU 2007). 


\section{Figure 2- 1: IEAV Organization Chart}

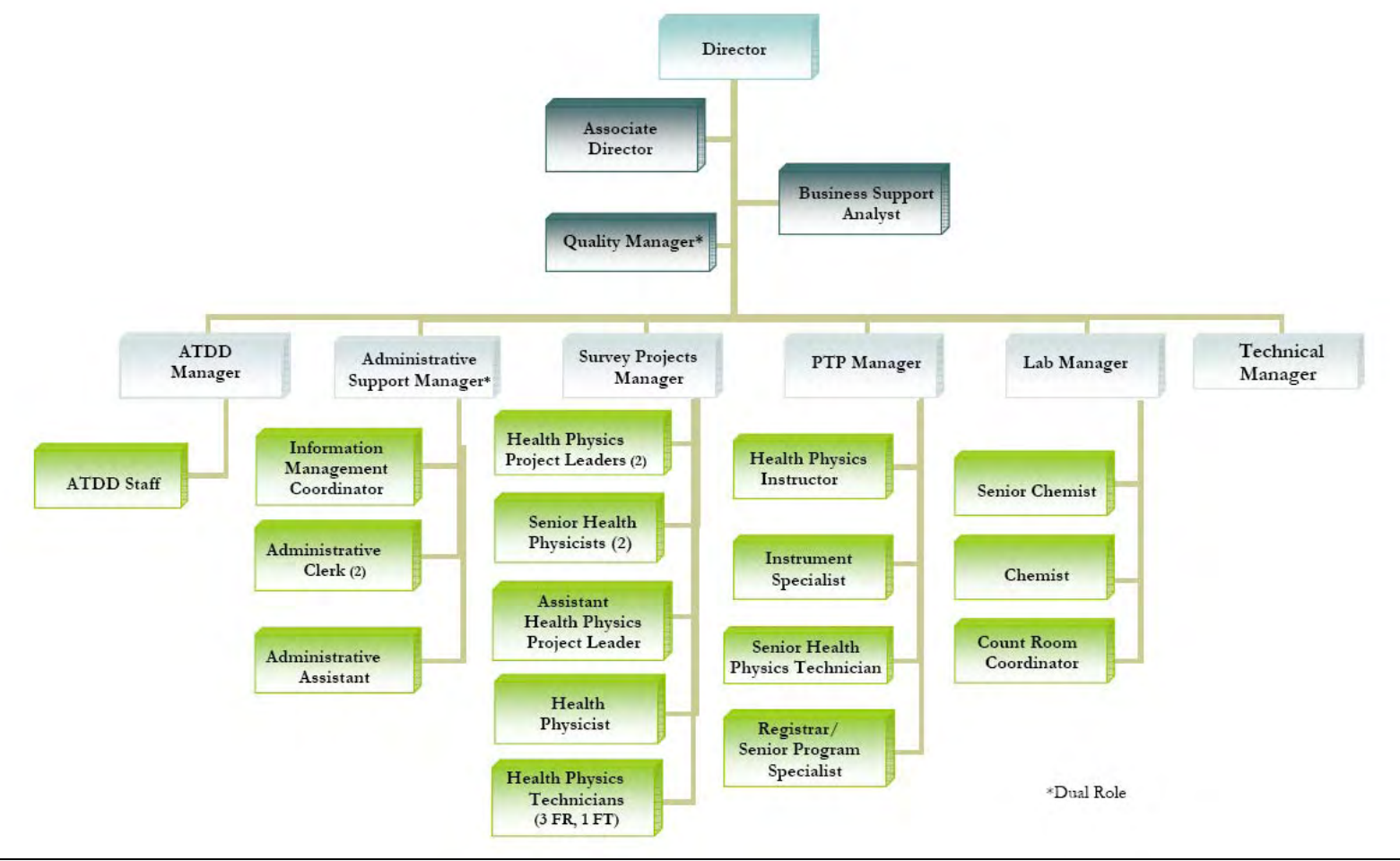

\subsection{QUALITY ASSURANCE}

ORISE conducted all field surveys in a manner that assured the quality and accuracy of resulting data and provided auditable documentation of activities. Details of the field quality assurance and quality control procedures are documented in the IEAV Quality Program Manual (ORAU 2007).

Quality control procedures included:

- Twice daily instrument background and check-source measurements to confirm that equipment operation was within acceptable response limits.

- Laboratory participation in the Mixed Analyte Performance Evaluation Program (MAPEP), National Institute of Standards and Technology (NIST) Radiochemistry Intercomparison Program (NRIP), and Intercomparison Testing Program (ITP) Laboratory Quality Assurance Programs. 
- $\quad$ Training and certification of all individuals performing procedures.

- $\quad$ Periodic internal and external audits.

\subsection{CERTIFICATION Training}

New employee indoctrination and orientation training is conducted to provide new survey staff with basic information about IEAV survey procedures. This initial training is followed by survey and quality assurance procedure training. The training consists of studying all applicable manual procedures, oral instruction, hands-on training, written testing, and demonstration of proficiency. The veteran staff members participate in annual procedure refresher training, and additional training when a procedure is revised or new procedure introduced.

In addition to survey and quality assurance procedure training, field personnel participate in training to satisfy regulatory requirements such as Occupational Safety and Health Administration HAZWOPER and DOE radiological worker, site-specific and generic safety, first aid and CPR, transportation, and other related requirements.

\subsection{CONTROL OF MEASUREMENT EQUiPMENT}

Radiological survey instruments were calibrated in accordance with IEAV Survey Procedures Manual requirements (ORISE 2007b). Procedures included electronic and NIST-traceable source calibration as well as twice daily operational check outs. Additional information on calibration and survey instrumentation is provided in Section 5.1.

\subsection{HEALTH AND SAFETY}

The proposed survey and sampling procedures were evaluated to ensure that any hazards inherent to the procedures themselves were addressed in current job hazard analyses (JHAs). The procedures entailed minimal potential hazards, all of which were addressed in current IEAV JHAs.

The project area was evaluated for potential health and safety issues. FSS survey activities were performed in accordance with the ORISE Radiation Protection Plan and radiation work permits as required (ORISE 2005d). Personnel also adhered to health and safety requirements discussed 
during the daily plan-of-the-day meetings which were held with all site personnel to address specific health and safety topics. Site remediation activities did result in the creation of additional hazards such as the excavations, protruding rebar, and other construction hazards. Identification of previously unaddressed hazards was consistently discussed during the daily meetings prior to performing work.

\subsection{FINAL STATUS SURVEY REQUIREMENTS}

A specific FSS was planned and conducted for each survey unit or group of similar survey units associated with each of the building and outdoor soil areas. The FSS plans were prepared in accordance with the guidance presented in MARSSIM. The plans followed the DQO process and ensured that all building and land areas were surveyed with the necessary rigor that corresponded with a given building or land area contamination potential. The DQO process included the following seven steps:

Step 1: State the problem

Step 2: Identify the decisions

Step 3: Identify inputs to the decisions

Step 4: Define the study boundaries

Step 5: Develop a decision rule

Step 6: Specify the decision errors

Step 7: Optimize the survey design

The following sections describe the requirements for the planning phase of the FSS including DCGLs, site classification and survey unit designations, survey planning parameters, instrumentation, measurement and sampling procedures, and the data quality assessments that were implemented.

\subsection{Derived ConCENTRATION GUIDELINE LEVELS}

Th-232 and its associated decay products and U-238 and its associated decay products were identified through process knowledge and characterization survey results as the contaminants of concern. Site-specific DCGL $\mathrm{W}_{\mathrm{W}}$ for both Th-232 and U-238 on building surfaces and within soils were developed using the RESRAD and RESRAD-BUILD computer codes and were approved 
by the NRC (ORISE 2006a and NRC 2007). These DCGL $\mathrm{W}_{\mathbb{W}}$ s accounted for all decay products found in secular equilibrium, including, the slight natural contribution from U-235 and its decay products. The above background DCGL $\mathrm{W}_{\mathrm{W}} \mathrm{s}$ for structural surfaces were $400 \mathrm{dpm} / 100 \mathrm{~cm}^{2}$ for Th-232 and its decay products and $800 \mathrm{dpm} / 100 \mathrm{~cm}^{2}$ for U-238 and decay products. These same DCGLs were also considered applicable to the monolithic slag underlying the site. It was expected that the slag surface would be the final surface encountered for the Building 200E south floor and for AOCs 1 and 2.

The structural/slag surface FSS planning and data quality assessment considered only the surface activity DCGL $\mathrm{L}_{\mathbb{W}}$ for Th-232. Use of only the more restrictive Th-232 surface activity DCGL rather than modifying the DCGL $\mathrm{W}_{\mathbb{W}}$ to also account for any small percentage of natural uranium activity that may have been present, allowed for simplification of the survey process yet provided an overall more conservative approach for determining compliance with remediation criteria. For soil area FSS planning, confirmation that U-238 was present in insignificant concentrations, relative to the Th-232, was achieved by evaluating the Th-232 to U-238 ratios in scoping and characterization survey soil samples. Only those samples containing greater than $5 \mathrm{pCi} / \mathrm{g}$ of Th-232 were selected for calculating the average net ratio which was approximately 11 to 1 . The above background DCGL $\mathrm{WW}_{\mathrm{W}}$ of $2.9 \mathrm{pCi} / \mathrm{g}$ for Th-232 and $2.5 \mathrm{pCi} / \mathrm{g}$ for U-238 were used for soil survey unit FSS planning and for the data quality assessments. Because of multiple contaminants, FSS planning and DQA for soils included an appropriate application of the unity rule in accordance with the equation:

$$
\frac{\text { Conc }_{\cdot T h-232}}{\text { DCGL }_{T h-232}}+\frac{\text { Conc }_{{ }_{U-238}}}{\text { DCGL }_{U-238}}<1
$$

Lastly, the potential for the concentration of Th-230 from the raw materials into the ThN product was evaluated and the determination made that there was no impact on the Th-232 or U-238 DCGLs, nor a need to develop a separate DCGL.

\subsection{Classification of Areas by Contamination Potential}

The HD site was subdivided into three classes, based on contamination potential, as either Class 1, 2, or 3 in accordance with MARSSIM. A description of each is as follows: 
Class 1: Buildings or land areas that have a significant potential for radioactive contamination (based on site operating history) or known contamination (based on previous radiological surveys) that exceeds the expected $\mathrm{DCGL}_{\mathrm{W}}$.

Class 2: $\quad$ Buildings or land areas, often contiguous to Class 1 areas, that have a potential for radioactive contamination but at levels less than the expected DCGL $L_{W}$.

Class 3: $\quad$ Remaining buildings and land areas that are expected to contain little or no residual contamination based on site operating history or previous radiological surveys.

Non-impacted: Areas that have no reasonable potential for residual contamination.

Furthermore, buildings and land areas were subdivided into survey units, which provided the fundamental unit for demonstrating compliance with the DCGLs. Survey unit size restrictions generally followed the recommended size limitations provided in MARSSIM.

\subsection{IDENTIFICATION OF SURVEY UNITS}

All impacted buildings and land areas were subdivided into Class 1, 2, or 3 survey units. Each survey unit represented a portion of the site with similar contamination potential. Table 3-1 provides the MARSSIM-recommended survey unit areas. The interiors of the Administration Building, Guard House, and Pump House were considered to be non-impacted.

\begin{tabular}{|c|c|c|}
\hline \multicolumn{3}{|c|}{ Table 3-1: MARSSIM-Recommended Survey Unit Sizes } \\
\hline Class & Structures & Land Areas \\
\hline 1 & Up to $100 \mathrm{~m}^{2}$ & Up to $2,000 \mathrm{~m}^{2}$ \\
\hline 2 & 100 to $1,000 \mathrm{~m}^{2}$ & 2,000 to $10,000 \mathrm{~m}^{2}$ \\
\hline 3 & No limit & No limit \\
\hline
\end{tabular}

\subsubsection{Land Area Survey Unit Identification}

Land area survey units for FSS are identified and illustrated on Figure A-3. There were five Class 3 soil area survey units, twelve Class 2 soil area survey units, and seven Class 1 soil/slag area survey units. In addition to the seven Class 1 soil survey units, once soil excavations were completed twenty-two slag surface Class 1 survey units were established within the original 
boundaries of AOCs 1, 2, and 3. The depot land area represented by each classification was 57.8\% Class 3, 35.7\% Class 2, and 6.5\% Class 1.

The DQOs implemented are provided in Sections 3.6 and 4.0. The scoping, characterization, and/or remedial support data collected were used for the DQO inputs, as appropriate, for each Class 1, 2, or 3 survey unit. In general, survey unit sizes followed the MARSSIM guidance. Table B-1 provides land area survey unit designations, classifications, and areas.

\subsubsection{Building Survey Unit Identification}

Building survey units for FSS are listed in Table B-1. Building areas originally classified as Class 2 or 3 where contamination was identified were reclassified as Class 1 . For the FSS phase, there were two buildings that contained Class 1 survey units. Among the remaining buildings, six survey units were surveyed during scoping/characterization as Class 2 and two as Class 3. The scoping surveys were designed and conducted in such a manner that the results for Class 2 and 3 building areas would meet FSS requirements. The FSS phase included forty Class 1, seven Class 2, and six Class 3 survey units. The DQOs implemented are provided in Sections 3.6 and 4.0. The characterization and remedial action support data collected from within building areas with Class 1 areas requiring remediation were used for the DQO inputs to design the FSS for remediated survey units.

\subsection{BaCkground Reference Area ANd MATERIALS}

A background soil reference area was selected and sampled/measured for comparing site soil sample data to and in evaluation of the FSS data in accordance with the planned non-parametric Wilcoxon Rank Sum (WRS) statistical test for land area survey units. The original background reference area selected, and discussed in the characterization survey report, did not appropriately represent the geo-physical properties of the site as the ubiquitous surface slag backfill deposits were not evident (ORISE 2006b). Following the characterization survey, a new background reference area was identified and sampled during the FSS (Figures A-3 and A-4). Random sampling locations were generated, and the soil sampled and analyzed. The analytical data were used for comparison with the site soil sample data and for the evaluation of the FSS data in accordance with the WRS test. 
Structural and monolithic slag survey units were evaluated using the non-parametric Sign test. Construction material-specific backgrounds were determined during scoping surveys and repeated during the FSS activities in areas of similar construction but without a history of radioactive material use (the Pump House and off-site slag surfaces). These construction material-specific measurements were used to correct direct measurements for background contributions, prior to converting data to the DCGL compliance unit of $\mathrm{dpm} / 100 \mathrm{~cm}^{2}$. Survey background count rates were as follows in Table 3-2:

\begin{tabular}{|c|c|c||}
\hline \multicolumn{3}{|c|}{ Table 3-2: Construction Material Backgrounds } \\
\hline Material & $\begin{array}{c}\text { Scoping/Characterization } \\
\text { (counts per minute) }\end{array}$ & $\begin{array}{c}\text { Final Status Survey } \\
\text { (counts per minute) }\end{array}$ \\
\hline \hline Concrete & 335 & 337 \\
\hline Cinder Block & 406 & 406 to 448 (painted/unpainted) \\
\hline Brick & 535 & 577 \\
\hline Slag & NA & 473 (average of two slag types) \\
\hline Metal & 216 & 178 \\
\hline
\end{tabular}

\subsection{REFERENCE SYSTEM}

FSS measurement and sampling locations were referenced as follows: direct measurements on structural surfaces were referenced to the X, Y metric distance from either the southwest corner of a floor/overhead structure or the lower left corner of a specific wall. Soil sampling locations were referenced to global positioning system (GPS) north/east coordinates obtained using hand-held GPS units. The GPS reference coordinate system used during scoping/characterization survey phases was WGS 84 latitude/longitude and WGS 84 UTM Zone 16 was used for referencing the exterior FSS sampling/measurement locations.

\subsection{SuRVEY DESIGN}

Structural surfaces were assessed by collecting the required number of gross beta surface activity measurements within each survey unit. The basis for assessing Th-232 surface activity levels via gross beta measurements is provided in Section 5.1.2. The Sign test was applied as the non-parametric statistical test for demonstrating compliance with the DCGL $\mathrm{L}_{\mathrm{W}}$. Land area 
compliance with the $\mathrm{DCGL}_{\mathbb{W}} \mathrm{s}$ was demonstrated through the application of the WRS test to soil sample results collected from each survey unit. Both Th-232 and U-238 activity concentrations in soil samples were measured by gamma spectroscopy. The Sign test was used to evaluate the surface activity measurements made for land area slag surface survey units as the slag shares the characteristics of a structural surface.

The applicable statistical test was performed if a survey unit measurement/sample exceeded the applicable DCGL $L_{\mathbb{W}}$ to evaluate the survey unit mean concentration relative to the null hypothesis $\left(\mathrm{H}_{0}\right)$. Simply stated, $\mathrm{H}_{0}$ is that the residual contamination in the survey unit exceeds the release criterion. Provided that the statistical test is satisfied at the desired confidence level, then $\mathrm{H}_{0}$ is rejected and the alternate hypothesis $\left(\mathrm{H}_{\mathrm{A}}\right)$, that residual contamination meets the release criterion, is accepted. The data needs for the statistical tests were determined in accordance with the following steps.

\subsubsection{Calculation of Required Number of Measurements}

The relative shift $(\Delta / \sigma)$ was calculated for each survey unit or group of similar units where:

$$
\Delta=\text { DCGLw - LBGR }
$$

DCGLw $=$ the gross or radionuclide specific guideline

LBGR = Lower Bound of the Gray Region; should be established as the estimated mean activity within the survey unit, but may be adjusted to maximize survey design

$\sigma=$ variability in concentration where:

1) The larger variability between the survey unit, $\sigma_{\mathrm{s}}$, and the background reference area, $\sigma_{\mathrm{r}}$, is selected for the WRS test and;

2) The survey unit and construction material-specific background count rate errors are propagated for the Sign test.

The DQOs were evaluated for each survey unit or survey area and the decision errors selected. The Type I decision error - the probability of incorrectly rejecting $\mathrm{H}_{0}$ when it is true-was 0.05 and the Type II error - the probability of incorrectly accepting $\mathrm{H}_{0}$ when it is false-was either 0.05 or 0.10 . Once the above parameters were established, the number of data points required 
by the statistical test were obtained either from Tables 5.3 (WRS test) or 5.5 (Sign test) in MARSSIM or otherwise generated using either COMPASS or Visual Sampling Plan (VSP) software. The FSS plan provided the tabulated estimated mean and standard deviation for Class 2 and 3 land area survey units (ORISE 2007a). These data were determined from scoping and characterization survey data. Remedial action support data supplemented by characterization data were required for determining the FSS data needs for Class 1 survey units. Appendix D contains the FSS planning DQO output for each group or individual land area survey unit.

For building structural survey units, the mean activity and variability was estimated for Class 2 and 3 structures prior to the implementation of the scoping survey. These tabulated estimates were also provided in the FSS plan and were used to determine the number of direct measurements required to satisfy FSS requirements (ORISE 2007a). Collection of remedial action support data, in conjunction with already acquired scoping/characterization survey data, was required for determining the data needs for Class 1 structural survey units. These data were also used to plan the FSS for those Class 2/3 survey units that were co-located with Class 1 remediated areas. Appendix E provides the FSS planning DQO output for each individual structural survey unit or survey unit group.

\subsubsection{Determining Measurement/Sampling Locations}

Measurement/sampling locations were established in either a random-start/systematic fashion for Class 1 and Class 2 survey units or at randomly generated locations for Class 3 survey units. Random start/systematic determinations followed the MARSSIM recommended guidance using a triangular measurement or sampling pattern to increase the probability of identifying small areas of residual activity. The spacing $(\mathrm{L})$ between data points on a triangular pattern was determined by:

$$
\mathrm{L}=[(\text { Survey Unit Area }) /(0.866 \times \text { number of data points })]^{1 / 2}
$$

The spacing between rows was calculated as: $0.866 \times \mathrm{L}$

For soil and exterior slag survey units, a unique GPS northing/easting coordinate location was generated for each sample location. For structural survey units a unique X, Y coordinate 
location was generated for each direct measurement location. The VSP software application, v.4.6, was used in plotting sampling coordinates for both structures and soil areas. When sample/measurement locations were either inaccessible or on a surface that had been removed during remediation, the sample was either not collected or replaced by a new randomly generated location. As the statistical sample size was already increased by $20 \%$, the decision as to whether to generate new locations was based upon the number of samples/measurements already collected and the anticipated resulting power of the statistical test. The anticipated power was based on data already collected and analyzed from similar survey units as the FSS progressed. In other words, in almost all cases the prospective survey unit mean concentration used for FSS planning was significantly greater than retrospective mean concentration which results in similar statistical power with fewer samples.

\subsection{INTEGRATED SURVEY STRATEGY}

FSS data collected for structural surfaces consisted of gamma and alpha plus beta or beta scans to identify locations of residual contamination as well as direct measurements of beta surface activity. Smear samples, although not used in the final data quality assessment, were collected during the scoping and characterization surveys from both judgmental and random-start/systematic locations to measure removable alpha and beta surface activity.

FSS of open land areas consisted of gamma scans to identify locations of residual contamination and samples of soil that were analyzed for Th-232 and U-238, or direct beta surface activity measurements on slag. Table B-1 provides survey unit nomenclature and information for land areas and buildings. Figure A-3 shows the FSS land area survey unit configuration. Figures A-6 through A-21 shows the structural survey units.

\subsection{SURFACE SCANS}

Land area gamma radiation surface scans were performed using $\mathrm{NaI}$ scintillation detectors. Structures were scanned for gamma radiation using $\mathrm{NaI}$ scintillation detectors and also for direct alpha plus beta or beta radiation using gas proportional detectors. Detectors were coupled to ratemeters or ratemeter-scalers with audible indicators. Characterization gamma surface scan data of land areas that did not require remediation were also used as FSS data, but were further augmented with additional scans during the FSS phase. The FSS gamma scan data were 
collected using a GPS system that enabled real time gamma count rate and position data capture. Table 4-1 shows the recommended surface scan coverage discussed in MARSSIM. Sections 4.1.1 and 4.1.2 discuss the actual scan coverage which met or exceeded the recommended coverages.

\begin{tabular}{|c|c|c|}
\hline \multicolumn{2}{|c|}{ Table 4-1: MARSSIM-Recommended FSS Survey Scan Coverage } \\
\hline Class & Structures & Land Areas \\
\hline 1 & $100 \%$ & $100 \%$ \\
\hline 2 & $\begin{array}{l}10 \text { to } 100 \% \text { floors and lower walls } \\
10 \text { to } 50 \% \text { upper walls and ceilings }\end{array}$ & 10 to $100 \%$ \\
\hline 3 & Judgmental & Judgmental \\
\hline
\end{tabular}

\subsubsection{Class 1 Land Area Survey Units}

All Class 1 land survey areas were gamma scanned 100\% during characterization using $\mathrm{NaI}$ detectors coupled to ratemeters with audible indicators. These areas were subdivided into survey units and scanned $100 \%$ following the completion of the remediation. A large debris pile, consisting of soil and structural debris, that overlaid the northwest corner of AOC 1 was removed to allow complete access to AOC 1, moved to the north and laid out in wind-rows for survey as a consolidated Class 2 survey unit, independent of the underlying land areas. The FSS gamma scans of the Class 1 survey units were performed using $\mathrm{NaI}$ detectors coupled to ratemeter-scalers with audible indicators. In addition, the gamma scanning of most survey units included the use of a GPS system coupled to the ratemeter-scalers enabling the resultant gamma count rate data to be logged and geo-referenced.

A special evaluation was required for the FSS assessment of the monolithic slag to address a deviation to the original survey plan for the exterior slag surfaces. The original plan required alpha plus beta or beta scans of the exposed slag surfaces (ORISE 2007a). However, once the slag layer was completely exposed, it was apparent that the surface undulations were too severe to permit scanning with a large area gas proportional detector. Therefore, an empirical evaluation was performed to determine the detection capability of the $\mathrm{NaI}$ scintillation detector for small areas $\left(100 \mathrm{~cm}^{2}\right)$ of residual contamination on the slag surface and establish the scan 
MDC capability relative to the $400 \mathrm{dpm} / 100 \mathrm{~cm}^{2}$ DCGL $_{\mathrm{W}}$. A large area of AOC 2 was gamma scanned using detectors with a crystal size of either $3.2 \mathrm{~cm} \times 3.8 \mathrm{~cm}$ or $5.1 \mathrm{~cm} \times 5.1 \mathrm{~cm}$. All locations considered distinguishable from background gamma radiation levels were marked for follow-up direct measurements with a hand-held gas proportional detector to quantify the beta activity. This evaluation determined that small areas of residual activity comparable to the DCGL $_{W}$ were readily detectable, therefore satisfying the required scan MDC, with the $5.1 \mathrm{~cm} \times 5.1 \mathrm{~cm} \mathrm{NaI}$ detector. Also, the approximate volumetric activity concentration equivalent to $400 \mathrm{dpm} / 100 \mathrm{~cm}^{2}$ within a thin layer of slag was calculated to be $3.8 \mathrm{pCi} / \mathrm{g}$. The scan MDC for the SPA-3 was approximately $1.8 \mathrm{pCi} / \mathrm{g}$, thereby confirming the field study result.

\subsubsection{Class 2 and 3 Land Area Survey Units}

As a result of the identification of AOC 2, both Class 2 and 3 land areas received high density gamma surface scans during the characterization survey. Characterization scans were performed using $\mathrm{NaI}$ detectors coupled to ratemeters with audible indicators. Class 2 and 3 scan coverage density began as 100\% coverage near roadways, railroads, and around buildings and then was gradually decreased to 50 to $75 \%$ coverage in outlying areas. These gamma scans identified three additional AOCs: AOCs 4, 5, and 6 on Figure 2.

FSS gamma surface scans were performed in all Class 2 and 3 survey units that met the minimum requirements in Table 4-1. The FSS scans were performed using $\mathrm{NaI}$ detectors coupled to the GPS-enabled ratemeter-scalers with audible indicators. Surface scan coverage was low to medium density (10 to $20 \%$ ) for Class 2 survey units and low density ( 1 to $5 \%$ ) for Class 3 survey units. Figure A-3 shows the Class 2 and 3 FSS survey units.

The Class 2 debris pile wind-rows survey unit (survey unit 12), discussed above in Section 4.1.1, was evaluated as two sub-units. The wind-rows, consisting of large quantities of soil intermixed with pieces of concrete, brick, re-bar, etc. were FSS gamma scanned using a NaI detector coupled to a ratemeter with audible indicator. The accessible solid debris surfaces were then scanned for beta activity using a hand-held gas proportional detector coupled to a ratemeter-scaler with audible indicator. 


\subsubsection{Class 1 Building Survey Units}

All Class 1 building surfaces received medium density (25 to 50\%) scans during the scoping survey and were then scanned 100\% during characterization. These areas were subdivided into survey units for the FSS and scanned 100\% for alpha plus beta or beta radiation following the completion of the remediation. Because of a similar slag surface condition to that discussed in Section 4.1.1, the slag surface in portions of Building 200E south (northeast corner, center, and southwest corner) required FSS gamma scans rather than the originally planned alpha plus beta scans. Figure A-1 highlights the Class 1 areas of the site buildings. The Class 1 FSS survey units included the floor, portions of the lower walls and overhead surfaces of Bays 1 through 10 of Building 200E south, the floor and lower wall of the northwest closet area of Building 200E, and three areas of the floor of Bays 13 through 20 of Building 100E.

\subsubsection{Class 2 and 3 Building Survey Units}

Twenty-five to $50 \%$ of accessible surfaces were systematically scanned during scoping surveys. Results of these scans as the survey progressed also resulted in the identification of additional areas for judgmental scanning. Upper walls, ceilings, and overhead structures were scanned with emphasis on horizontal surfaces where residual contamination may have settled and accumulated. Detailed discussions of the scanning processes are provided later in this section.

Characterization surface scans were performed over $100 \%$ of floor surfaces where anomalies were identified during the scoping surveys. Class 2 areas subject to expanded scan coverage included the eastern-most section of Bays 7 through 17 of Building $100 \mathrm{~W}$ and Bays 15 through 20 of Building 100E. The remaining Class 2 survey units addressed during the FSS included wall and overhead surfaces in Building 200E south, Bays 1 through 10.

Class 3 floors and lower walls were judgmentally scanned for alpha plus beta and gamma radiation during the scoping survey. Up to $25 \%$ of the accessible floor surfaces were scanned for direct gamma and alpha plus beta radiation. The remaining Class 3 areas assessed during the FSS were the lower walls of Building 200E north-Bays 11 through 20-the Workshop Building, Garage Building, and the collective building exteriors. Figure A-1 shows the locations of these other site buildings. 
The floors, lower walls, and upper surfaces of the northern section of Building 200E and all of Building 100W were scanned for alpha plus beta, beta, and/or gamma radiation during the scoping survey. Additionally, the central floor of Building $100 \mathrm{~W}$ was rescanned during the characterization survey. The rescanning was necessary due to identified ambient gamma radiation background interference that resulted from the drums of tungsten that were present during the scoping survey but removed during the characterization phase. These scoping/characterization scans were conducted such that FSS scanning requirements were satisfied in all cases. No additional scans of the survey units in these building areas were necessary during the FSS phase.

The surfaces of Building 100E—originally a Class 3 structure— were similarly scanned during the scoping survey. However, contamination was identified on numerous empty pallets that were stored in the northwest corner of the building. The floor of the northern-most five bays of Building 100E were therefore reclassified as Class 2 and scanned accordingly during the characterization survey. Additional contamination was identified in two expansion joints, and subsequently two Class 1 floor survey units were established for the FSS. An additional Class 1 and two Class 2 floor survey units were established during the FSS as a result of the identification of a small group of tannin pallets contaminated with radioactive material. The investigation determined that the material was likely residue from an old spill of monazite sand that may have occurred while moving a drum through the aisle way that these pallets abutted. To ensure this was an isolated incident, approximately 300 pallets of the stored tannin were individually moved and gamma scanned. Also, all accessible pallets of tannin within the entire warehouse were gamma scanned with no further anomalies identified.

Class 2 and 3 building survey units that were co-located with remediated Class 1 survey units were rescanned during the FSS using a graded approach. That is, 100\% high density scans were performed of the survey unit surface adjacent to any Class 1 survey unit. For example, a Class 2 wall was scanned $100 \%$ over the lower two meters while the upper wall was scanned 10 to $20 \%$.

\subsection{SoIl SAMPling ANd Surface ACtivity MEASUREMENTS}

FSS surface soil samples (0 to $0.15 \mathrm{~m}$ ) were collected from random-start/systematic or random locations, dependent upon the survey unit classification. Additional judgmental samples were 
obtained, as necessary, from locations where scans indicated potential residual contamination or from within remediated areas in those cases where systematic sampling locations did not fall within the remediated portion of a given survey unit. Soil samples were maintained under formal chain-of-custody procedures then analyzed in the IEAV laboratory by gamma spectroscopy and results reported in units of $\mathrm{pCi} / \mathrm{g}$. A number of the planned FSS soil samples could not be collected due to inaccessibility beneath concrete pads or similar overlying structures. In the event an excessive number of samples from a specific survey unit were inaccessible, additional random sample locations were generated and samples collected.

FSS direct measurements to quantify total beta activity levels were performed at pre-determined random start/systematic or random locations as applicable on building surfaces and interior and exterior slag surfaces. In cases where a measurement location was inaccessible or the structure had been physically removed, replacement locations were randomly generated as needed. Additional judgmental samples were obtained, as necessary, from locations where scans indicated potential residual contamination or from within remediated areas if a systematic measurement location did not fall within the remediated potion of a given survey unit. Measurements were made using gas proportional detectors coupled to ratemeter-scalers. Surface activity data were converted to units of $\mathrm{dpm} / 100 \mathrm{~cm}^{2}$.

The procedures used for calculating the number of and location for samples/measurements that are discussed below were determined in accordance with Sections 3.6.1 and 3.6.2.

\subsubsection{Class 1 Land Area Survey Units}

The specific DQO inputs for calculating the required FSS soil sample quantity in Class 1 land area survey units were derived from characterization samples and remedial action support survey results. The Class 1 survey units are shown on Figure A-3. There were three scenarios encountered during the FSS of Class 1 land area survey units. Each scenario required implementing a variation in the sampling/measurement FSS approach. Most of the Class 1 land area survey units in AOCs 1 and 2 were comprised of the monolithic slag surface as the overlying contaminated soil had been completely removed. For these survey units, direct measurements of residual beta surface activity were planned for and collected from random-start/systematic locations. Other survey units consisted of primarily soil, as either 
excavation was not required over large areas and/or the excavation did not expose the monolithic slag. Soil samples were collected for the FSS of these survey units. The final scenario encountered was a survey unit consisting of primarily soil, but where remediation exposed the slag surfaces over small areas of the survey unit. For this survey unit, the basis of the FSS was the collection of soil samples. However, additional judgmental direct measurements of the beta surface activity levels were made on the surface of the exposed slag. Appendix D provides the specific, detailed DQO reports for each Class 1 survey unit.

\subsubsection{Class 2 Land Area Survey Units}

The scoping survey sample results were used for generating the DQO inputs for calculating the required number of FSS soil samples for each Class 2 survey unit. The number of samples required and locations were generated in accordance with Sections 3.6.1 and 3.6.2. Survey units are shown on Figure A-3. Appendix D provides the detailed DQO report for Class 2 survey units.

\subsubsection{Class 3 Land Area Survey Units}

The number of FSS soil samples for each of the five Class 3 survey units was also calculated using the DQO inputs used for the Class 2s. Survey units are shown on Figure A-3 with the DQO report provided in Appendix D.

\subsubsection{Class 1 Building Survey Units}

There were forty Class 1 structural survey units established within Buildings 200E and 100E. The DQO inputs were derived from the remedial action support survey measurement results for Building 200E and characterization results (excluding data from the contaminated expansion joints) for the Class 1 areas of Building 100E.

\subsubsection{Class 2 Building Survey Units}

FSS direct measurements of six of the 15 Class 2 structural survey units associated with Buildings 100W and 200E (north end) were completed during the scoping survey phase. The DQO mean concentration and variability inputs for determining the number of direct 
measurements to satisfy FSS requirements were prospectively estimated. The actual data results were retrospectively reviewed to determine the adequacy of the estimated surface activity concentration. This planning followed the procedure described in Sections 3.6.1 and 3.6.2. For those Class 2 survey units that were co-located with Class 1 areas requiring remediation in Building 200E (south) and 100E, the characterization data provided most of the mean concentration and variability inputs for the FSS design or the original prospective parameters were used in the event that their use resulted in a more robust FSS.

\subsubsection{Class 3 Building Survey Units}

FSS direct measurements for three of the eight Class 3 structural survey units were completed during the scoping survey phase as no, or very limited, decontamination was required within the building. These Class 3 survey units were located in Buildings 100W, 100E, and 200E (north). The DQO mean concentration and variability inputs for determining the number of direct measurements to satisfy FSS requirements were prospectively estimated. These same parameters were also used in the DQO process for the remaining Class 3 units addressed during the FSS phase of the project. The actual data results for the completed Class 3 survey units were retrospectively reviewed to determine the adequacy of the estimated surface activity concentration. This planning followed the procedure described in Sections 3.6.1 and 3.6.2.

\subsection{INSTRUMENTATION AND CALIBRATION}

Calibration of all field and laboratory instrumentation was based on standards/sources, traceable to NIST. Specific field and laboratory instrumentation parameters are discussed below.

\subsection{FIELD INSTRUMENTATION}

The following, or similar, survey instrumentation were used during the FSS or for the collection of FSS data during the scoping survey. 


\title{
5.1.1 Scanning Instrument/Detector Combinations
}

\author{
Alpha plus Beta
}

Ludlum Floor Monitor Model 239-1 combined with Ludlum Ratemeter-Scaler Model 2221 coupled to Ludlum Gas Proportional Detector Model 43-37, Physical Area: $550 \mathrm{~cm}^{2}$ (Ludlum Measurements, Inc., Sweetwater, TX), Minimum Detectable Concentration (MDC) = $300 \mathrm{dpm} / 100 \mathrm{~cm}^{2} \mathrm{Th}-232$, based on a scanning total efficiency for the Th-232 decay series of approximately 1.50 .

Beta

Ludlum Ratemeter-Scaler Model 2221 coupled to Ludlum Gas Proportional Detector Model 43-68, Physical Area: $126 \mathrm{~cm}^{2}$ equipped with a $3.8 \mathrm{mg} / \mathrm{cm}^{2}$ Mylar window (Ludlum Measurements, Inc., Sweetwater, TX) MDC $=800 \mathrm{dpm} / 100 \mathrm{~cm}^{2}$ Th-232, based on a scanning total efficiency for the beta-only component of the Th-232 decay series of approximately 0.40 .

The actual scanning MDC for the instrumentation was compared with the required scanning MDC determined at the time of the Class 1 final status survey DQO development. Sample spacing adjustment was not necessary as the actual scan MDC was less than the required scan MDC for each Class 1 survey unit. A review of the area factors presented in Table B-2 demonstrates that a sample spacing of less than $100 \mathrm{~m}^{2}$ ensured that the required scan MDC was satisfied.

\section{Gamma}

Ludlum Pulse Ratemeter Model 12 or Ratemeter-Scaler Model 2221 enabled with RS-232 data collection capabilities (Ludlum Measurements, Inc., Sweetwater, TX) coupled to: Victoreen sodium iodide (NaI) Scintillation Detector Model 489-55, Crystal: $3.2 \mathrm{~cm}$ x $3.8 \mathrm{~cm}$ (Victoreen, Cleveland, $\mathrm{OH})$.

Th-232 $\mathrm{MDC}_{\text {Scan }}=2.8 \mathrm{pCi} / \mathrm{g}$ (assumes secular equilibrium with the decay series) $\mathrm{U}-238 \mathrm{MDC}=4.5 \mathrm{pCi} / \mathrm{g}$ (assumes secular equilibrium with the decay series)

or a: 
Ludlum SPA-3 NaI Scintillation Detector Model 44-10, Crystal: $5.1 \mathrm{~cm}$ x $5.1 \mathrm{~cm}$ (Ludlum Measurements, Inc., Sweetwater, TX).

Th-232 $\mathrm{MDC}_{\mathrm{Scan}}=1.8 \mathrm{pCi} / \mathrm{g}$ (assumes secular equilibrium with the decay series) $\mathrm{U}-238 \mathrm{MDC}=2.8 \mathrm{pCi} / \mathrm{g}$ (assumes secular equilibrium with the decay series)

Detector/ratemeter or ratemeter-scaler combinations were also coupled to a Trimble GeoXH Receiver and Data Logger (Trimble Navigation Limited, Sunnyvale, CA) when collecting scan data during the FSS. Gamma count rates (in cpm) and position were captured each second. Positional accuracy was typically within one to five meters.

Based on characterization data demonstrating that U-238 concentrations from licensed material contamination existed as a mixture dominated by Th-232, a combined scan MDC for the mixture was calculated from the observed fractional amounts. The observed Th-232:U-238 ratio averaged 11:1 in characterization samples. The calculated scan MDC for the 11:1 activity ratio was calculated to be $2.89 \mathrm{pCi} / \mathrm{g}$ total activity and can be compared with the similarly calculated total activity DCGL of $2.77 \mathrm{pCi} / \mathrm{g}$. The actual scanning MDC for the instrumentation was compared with the required scanning MDC for Class 1 survey units. Sample spacing adjustment was not necessary as the actual scan MDC was less than the required scan MDC for each Class 1 survey unit without the application of the area factors provided in Table B-3. Notes have been included in the DQO reports for Class 1 survey units indicating that although the scan MDC for $\mathrm{U}-238$ in equilibrium with the decay series is approximately $4 \mathrm{pCi} / \mathrm{g}$, the $\mathrm{U}-238$ scan $\mathrm{MDC}$ for the elevated measurement comparison was established as $2.9 \mathrm{pCi} / \mathrm{g}$ to reflect the gross activity scan MDC.

\subsubsection{Direct Measurement Instrument/Detector Combinations}

Beta

Ludlum Ratemeter-Scaler Model 2221 coupled to Ludlum Gas Proportional Detector Model 43-68, Physical Area: $126 \mathrm{~cm}^{2}$ (Ludlum Measurements, Inc., Sweetwater, TX).

MDC $=200 \mathrm{dpm} / 100 \mathrm{~cm}^{2}$ Th-232, based on the beta-only total efficiency of 0.40 to 0.42 . 
Use of only the more restrictive Th-232 surface activity DCGL $L_{\mathbb{W}}$, rather than modifying the DCGL $_{\mathbb{W}}$ to also account for any small percentage of natural uranium activity that could have been present, allowed for simplification of the survey process yet provided an overall more conservative approach for assessing surface activity levels. Therefore, the detectors used for assessing surface activity were calibrated only for the Th-232 decay series beta emissions by using $3.8 \mathrm{mg} / \mathrm{cm}^{2}$ Mylar windows to block alpha contributions. The calibration procedure was in accordance with ISO- $7503^{1}$ recommendations. Total beta efficiencies $\left(\boldsymbol{\varepsilon}_{\text {total }}\right)$ were determined for each instrument/detector combination and consisted of the product of the $2 \pi$ instrument efficiency $\left(\varepsilon_{\mathrm{i}}\right)$ and surface efficiency $\left(\varepsilon_{\mathrm{s}}\right): \varepsilon_{\mathrm{total}}=\varepsilon_{\mathrm{i}} \times \varepsilon_{\mathrm{s}}$. Beta total efficiencies were determined based on a beta energy multi-point calibration, development of instrument efficiency to beta energy calibration curves, and the calculation of the weighted efficiency representing the Th-232 decay series. Included in the weighted efficiency was an empirically determined correction for disequilibrium in the decay series that results from Rn-220 loss. A $3.8 \mathrm{mg} / \mathrm{cm}^{2}$ density thickness Mylar window was used on the beta detectors to block detector response contributions from alpha radiation.

ISO-7503 recommends an $\varepsilon_{\mathrm{s}}$ of 0.25 for beta emitters with a maximum energy of less than $0.4 \mathrm{MeV}$ and an $\boldsymbol{\varepsilon}_{\mathrm{s}}$ of 0.5 for maximum beta energies greater than $0.4 \mathrm{MeV}$. Figure A-5 illustrates an example multi-point calibration efficiency determination.

Direct measurement count rates were corrected for an appropriate construction material-specific background. The net count rates were then converted and reported in units of $\mathrm{dpm} / 100 \mathrm{~cm}^{2}$ by dividing by the detector total efficiency and geometry.

\subsection{LABORATORY INSTRUMENTATION}

Scoping/characterization and FSS samples were analyzed in accordance with the ORISE Laboratory Procedures Manual (ORISE 2007c).

${ }^{1}$ International Standard. ISO 7503-1, Evaluation of Surface Contamination - Part 1: Beta-emitters (maximum beta energy greater than $0.15 \mathrm{MeV}$ ) and alpha-emitters. August 1, 1988. 


\subsubsection{Gross Alpha/Beta (Removable Activity)}

Smear samples were analyzed using the following equipment and results were reported in units of $\mathrm{dpm} / 100 \mathrm{~cm}^{2}$.

Low Background Gas Proportional Counter Model LB-5100-W (Tennelec/Canberra, Meriden, CT). MDCs $=9 \mathrm{dpm} / 100 \mathrm{~cm}^{2}$ for alpha and $15 \mathrm{dpm} / 100 \mathrm{~cm}^{2}$ for beta with a two-minute count time.

\subsubsection{Gamma Spectroscopy}

Soil samples were analyzed by gamma spectroscopy using the following equipment and results reported in units of picocuries per gram $(\mathrm{pCi} / \mathrm{g})$ :

- High Purity Extended Range Intrinsic Detector CANBERRA/Tennelec Model No: ERVDS30-25195 (Canberra, Meriden, CT) used in conjunction with Lead Shield Model G-11 (Nuclear Lead, Oak Ridge, TN) and Multichannel Analyzer DEC ALPHA Workstation (Canberra, Meriden, CT).

- High Purity Extended Range Intrinsic Detector Model No. GMX-45200-5 (AMETEK/ORTEC, Oak Ridge, TN) used in conjunction with Lead Shield Model SPG-16-K8 (Nuclear Data) Multichannel Analyzer DEC ALPHA Workstation (Canberra, Meriden, CT).

- High-Purity Germanium Detector Model GMX-30-P4, 30\% Eff. (AMETEK/ORTEC, Oak Ridge, TN) used in conjunction with Lead Shield Model G-16 (Gamma Products, Palos Hills, IL) and Multichannel Analyzer DEC ALPHA Workstation (Canberra, Meriden, CT).

- Gamma Spectroscopy MDC $=0.11 \mathrm{pCi} / \mathrm{g}$ for Th-232 (based on the $0.911 \mathrm{MeV}$ photopeak from Ac-228) and $0.70 \mathrm{pCi} / \mathrm{g}$ for $\mathrm{U}-238$ (based on the $0.063 \mathrm{MeV}$ photopeak from Th-234) based on a 60-minute count time. 


\subsection{DATA REVIEW AND INVESTIGATION THRESHOLDS}

Data were reviewed to assure that the type, quantity, and quality were consistent with the survey plan and design assumptions. Data standard deviations were compared with the assumptions made in establishing the statistically-based number of direct measurement/sampling points. Individual and average data values were compared with guideline values and proper survey area classifications were confirmed. Individual measurements in excess of the guideline level for Class 1 and 2 areas were investigated. For Class 3 survey units, although less conservative than the recommendation provided in MARSSIM, measurements in excess of $75 \%$ of the guideline for Class 3 areas were investigated. The requirement for increasing the investigation threshold was due to the low DCGLs relative to background. For survey unit investigations, reclassifications, remediations, and/or resurveys, a determination of the cause was initiated and the data conversion and assessment process repeated. Additional information regarding the evaluation of measurement results in excess of the DCGLs is provided in Section 7.3 and specific occurrences are listed in Table B-4 and discussed in the appropriate sub-section of Section 8 of this report.

\subsection{DETERMINING COMPLIANCE WITH DCGLs}

As discussed in Section 3.1, both soil concentration and surface activity DCGLs were developed with which FSS data were compared. These DCGLs included both the mean concentrations $\left(D C G L_{W}\right)$ and also provided for small areas of elevated contamination in excess of the DCGL $\mathrm{L}_{\mathrm{W}}$,

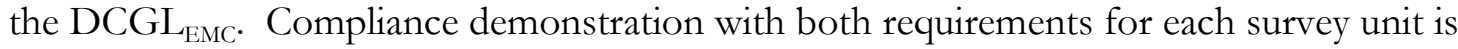
discussed below.

\subsection{LAND AREA SURVEY UNITS}

Land area survey units were evaluated using the WRS test. Survey unit and background reference area soil sample results collected from the random or random-start/systematic locations were converted to unity in accordance with the equation in Section 3.1. The DCGL in this case is also established as 1 . The reference area results were adjusted by adding the DCGL to the unity concentration value. The results for both data sets were then ranked as follows:

- Rank all (survey unit and reference area) measurements in order of increasing size from 1 to $\mathrm{N}$, where $\mathrm{N}$ is the total number of pooled measurements. 
- If several measurements have the same value, assign them the average ranking of the group of tied measurements.

- Sum the ranks of the adjusted reference area measurements; this value is the test statistic, $\mathrm{W}_{\mathrm{R}}$.

- Compare the value of $\mathrm{W}_{\mathrm{R}}$ to the critical value in MARSSIM Table I.4 for the appropriate sample size and decision level.

Prior to applying the test, if the difference between the largest survey unit result and the smallest reference area result was less than the $\mathrm{DCGL}_{\mathrm{W}}$, the survey unit would always pass a complete application of the WRS test. In this situation, no further evaluation was necessary and the null hypothesis was rejected. Otherwise, $\mathrm{W}_{\mathrm{R}}$ was calculated. If $\mathrm{W}_{\mathrm{R}}$ was greater than the critical value, $\mathrm{H}_{0}$ was rejected, and the survey unit met the established criteria. If $\mathrm{W}_{\mathrm{R}}$ was less than or equal to the critical value, $\mathrm{H}_{0}$ was not rejected, and the survey unit did not meet the established criteria; investigation, remediation, reclassification, and/or resurvey would have been performed as appropriate. However, application of the WRS resulted in the rejection of the null hypothesis for all survey units.

\subsection{BUILDING SURVEY UNITS}

Structural survey units were evaluated using the Sign test. Individual activity values and the average activity value were calculated.

If all values from the random or random-start/systematic locations for a survey unit were less than the guideline level, the survey unit satisfied the criterion and no further evaluation was necessary.

If the average activity value was greater than the guideline, the survey unit would not satisfy the criterion, and further investigation, possible reclassification, remediation, and/or resurvey would have been required. However, the average activity never exceeded the criterion.

If the average activity value was less than the guideline level, but some individual values were greater than the guideline, data evaluation by the Sign test proceeded, as follows: 
- List each of the survey unit measurements.

- Subtract each measurement from the guideline level.

- Discard all differences which are " 0 "; determine a revised sample size.

- Count the number of positive differences; this value is the test statistic $(\mathrm{S}+)$.

- Compare the value of S+ to the critical value in MARSSIM Table I.3 for the appropriate sample size and decision level.

When $\mathrm{S}+$ was greater than the critical value, $\mathrm{H}_{0}$ was rejected, and the survey unit met the established criteria. If $\mathrm{S}+$ had been less than or equal to the critical value, $\mathrm{H}_{0}$ would not have been rejected, and the survey unit would not have met the established criteria; investigation, remediation, reclassification, and/or resurvey would have been performed, as appropriate.

\subsection{Elevated Measurement Comparison}

Soil sample or direct measurement results that exceeded the DCGL were also evaluated for

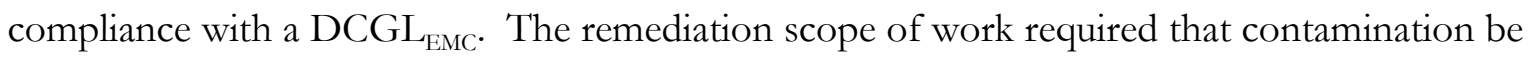
reduced to levels below the $\mathrm{DCGL}_{\mathbb{W}}$ and WEI performed remedial action support surveys as assurance that this requirement was met (ORNL 2006). However because contamination was present prior to remediation in Class 1 survey units, the potential existed that isolated locations of residual soil concentrations or surface activity could have been identified during the FSS that exceeded the DCGL $\mathrm{W}_{\mathrm{W}}$. The statistical tests for demonstrating compliance are such that some samples/measurements may exceed the $\mathrm{DCGL}_{\mathrm{W}}$, yet still reject the null hypothesis. Therefore, both the statistically-based and judgmental samples exceeding the DCGL $\mathrm{W}_{\mathbb{W}}$ by a predetermined threshold were compared with a DCGL $\mathrm{EMC}_{\mathrm{E}}$ that corresponded with the size of a given area of elevated activity—defined as the DCGL $\mathrm{DW}_{\mathrm{W}} \times$ Area Factor. The concentration threshold for soil samples from Class 1 survey units that required an EMC comparison was defined as either the Th-232 or U-238 DCGL W $_{\mathbb{W}}$ plus the sum of the respective mean background concentration and two standard deviations. For Class 1 surfaces, the corresponding threshold would be the surface activity DCGL $\mathrm{L}_{\mathrm{W}}$, in terms of counts per minute, plus the sum of the mean construction material-specific background count rate and two standard deviations. Tables B-2 and B-3 provide the area factors for both soil concentrations and surface activity. Area factors were developed using the identical inputs used in generating the site-specific DCGL $\mathrm{WW}_{\mathbb{W}}$ with only the size of the area of contamination changed and for soil, the length parallel to the aquifer flow also 
must be changed to match the hot spot size. When individual samples/measurements with elevated concentrations were less than the respective DCGL $\mathrm{EMC}_{\mathrm{EC}}$ the impact of multiple hot spots on the mean concentration in a survey unit would have been evaluated. This would have been performed using equation 8-2 in MARSSIM, but was not necessary for any survey unit. Any measurement that exceeded the DCGL $\mathrm{W}_{\mathrm{W}}$ within a Class 2 or 3 survey unit was investigated as discussed in Section 6.0 and in some cases required reclassification of portions of the survey unit.

\subsection{PRE-REMEDIATION AND FSS RESULTS}

The results of the FSS are provided in the following sections. The information below and in accompanying appendices provides all applicable data and documentation necessary to support the request for removal of the Hammond Depot from the DNSC's NRC license.

\subsection{Building Surveys: Class 1}

The results for the surveys of Buildings 200E and 100E are provided below.

\subsubsection{Class 1 Scoping/Characterization Surface Scans and Surface Activity Levels}

\section{Buildings 200E South, 200E NW Closet, and 100E}

The scoping and characterization surveys found extensive contamination on the floors of the south half of Building $200 \mathrm{E}$ where containers of $\mathrm{ThN}$ were stored and known to have leaked. The contamination was on the original concrete floor of the building, beneath the asphalt overlayment. Multiple small area and distributed locations of elevated gamma radiation were detected - indicating contamination beneath the asphalt - that ranged from 5,000 to 200,000 counts per minute (cpm). Investigations beneath the asphalt overlayment found that much of the contamination was associated with expansion joints and cracks in the original concrete floor slab. The contamination was also found to have migrated through these openings and spread to the monolithic slag layer that was immediately beneath the floor. Surveys confirmed the presence of contamination in excess of the DCGL $\mathrm{L}_{\mathrm{W}}$ on overhead structural steel and pipes, support columns and bases, and isolated lower wall areas. 
One area of contamination was identified in the closet area at the northwest end of Building 200E. Investigations identified jars of source material stored in the closet and one jar was noted to be leaking, causing localized floor and wall surface contamination.

Contaminated pallets were identified in Building 100E during the scoping survey within Bays 19 and 20. Once the pallets were surveyed and removed, the characterization survey identified three expansion joint locations with activity levels in excess of the DCGL $\mathrm{D}_{\mathrm{W}}$. Also, the pallets of tannin contaminated with monazite sand were present at this time but were not found until the FSS, after other materials had been moved out of the way. It is believed that the monazite sand was also the source of the contamination found within the expansion joints.

Table 8-1 summarizes the removable and total beta surface activity levels present within Class 1 areas following the characterization. 


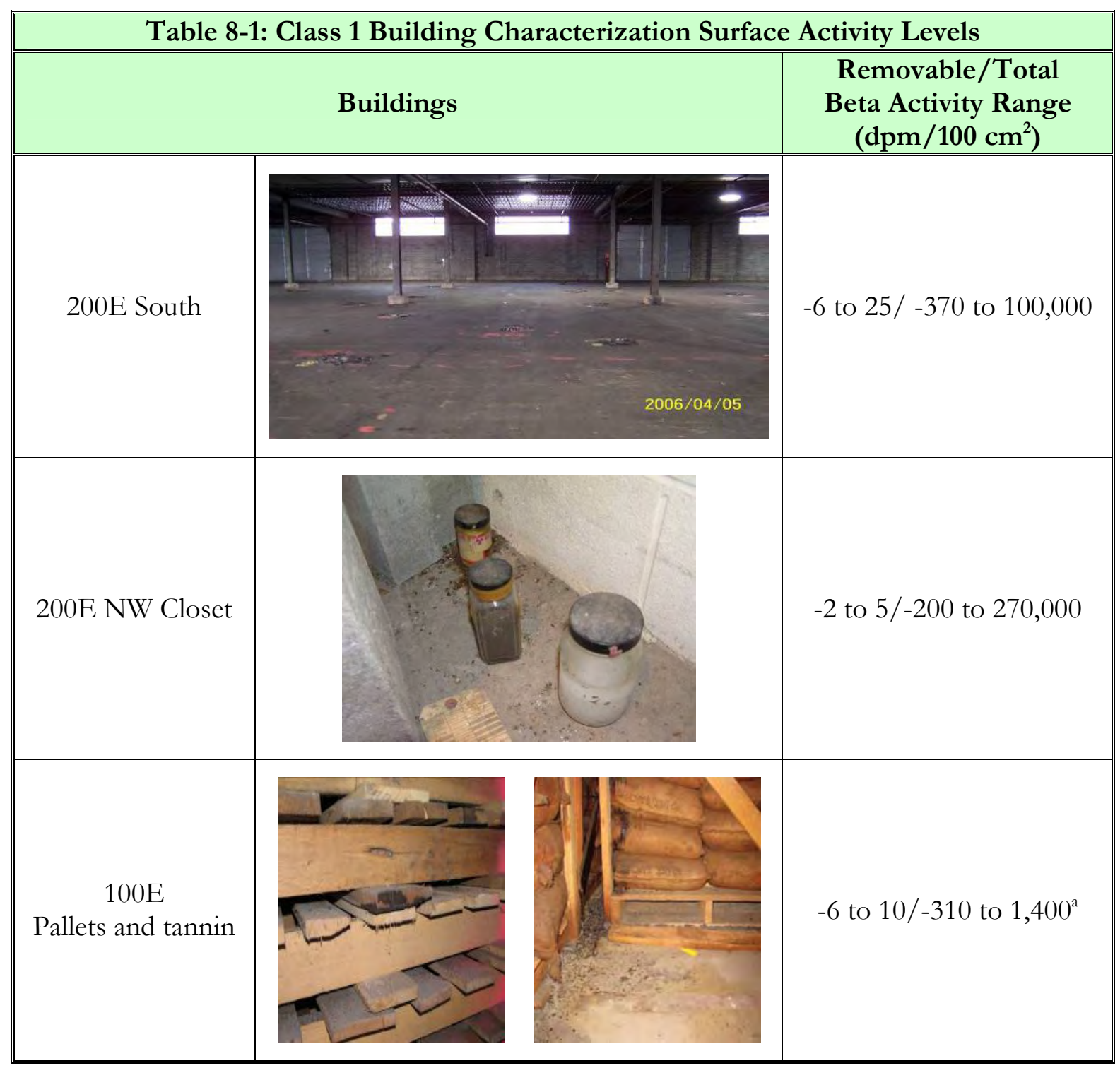

${ }^{a}$ Excludes the measurement results for the contaminated pallets.

\subsubsection{Class 1 Building Remediation}

The remedial actions that WEI conducted within the contaminated structures are summarized below.

\section{Building 200E South}

Aggressive decontamination efforts were necessary in Building 200E. The asphalt overlayment was removed to permit access to $100 \%$ of the original concrete floor. Surveys confirmed 
extensive contamination in the area referred to as the "spillway". The spillway began in southwest corner of the structure, continued through the central area, and extended up to the northeast corner. Surveys of the southeast and northwest corners of the floor did identify a number of isolated areas of contamination. Based on this, the decision was made to remove the entire floor slab for disposal and to expose the underlying monolithic slag layer to allow for thorough investigation of the slag contamination profile. The southeast and northwest corners did not require decontamination, with the exception of a few spots. The spillway observed on the floor was essentially mirrored on the slag and required extensive remediation by breaking, chipping, and scraping the contaminated slag. Contaminated lower walls and columns were spot remediated by scabbling, chipping, and grinding. The overhead structures were decontaminated by hydro-washing, wiping, and vacuuming.

\section{Building 200E Northwest Closet}

Remediation of this area included removal of the jars of $\mathrm{ThN}$ solution during an earlier interim cleanup action. Structural remediation involved scabbling a small area of the closet floor and the adjoining east lower wall.

\section{Building 100E}

Building 100E was remediated by first removing the contaminated pallets during the interim cleanup action. The contaminated expansion joints were chipped out. The pallets of tannin that were found contaminated with monazite sand during the FSS were wrapped and disposed of as waste. The surrounding floor was vacuumed and an associated expansion joint required removal of the mastic and concrete.

\subsubsection{Class 1 Building FSS Results}

As previously discussed, the FSS surveys of these Class 1 structures included 100\% alpha plus beta/beta and/or gamma surface scans of the Class 1 survey units associated with each structure. Class 2 survey units associated with these structures received FSS scan coverage generally ranging from 10 to 50\%, several Class 2 survey units, such as the Building 200E (south) lower walls, were scanned $100 \%$. These scans identified several additional areas of 
contamination requiring further remediation on the floor, columns, and overheads of Building $200 \mathrm{E}$ as well as the pallets of contaminated tannin in Building 100E. Most of the Building 200E locations would have readily met the $\mathrm{DCGL}_{\mathrm{EMC}}$. The additional remediation was performed as an ALARA practice. Of the approximately 600 FSS direct measurements made on these structural survey units, none exceeded the Th-232 surface activity DCGL $\mathrm{L}_{\mathbb{W}}$ of $400 \mathrm{dpm} / 100 \mathrm{~cm}^{2}$. Table 8-2 summarizes the FSS surface activity results for these Class 1 survey units. Complete data are provided in Table B-5.

\begin{tabular}{|c|c|c||}
\hline \multicolumn{3}{|c|}{ Table 8-2: Class 1 Building FSS Summary } \\
\hline Building & $\begin{array}{c}\text { Number of Survey Units/ } \\
\text { Measurements }\end{array}$ & $\begin{array}{c}\text { Total Beta Activity Range/Average } \\
\text { (dpm/100 cm } \mathbf{~}\end{array}$ \\
\hline $200 \mathrm{E}$ (south) & $34 / 534$ & -430 to 390/67 \\
\hline $200 \mathrm{E}$ NW closet & $1 / 19$ & -200 to $150 /-90$ \\
\hline $100 \mathrm{E}$ & $3 / 51$ & -130 to 230/22 \\
\hline
\end{tabular}

The DQA report for each survey unit for these structures is provided in Appendix F. The DQA reports include individual direct measurement results, both summary and graphical prospective and retrospective FSS planning information, summary statistics, and the Sign test results when applicable. Figures A-6 through A-11, and A-14 show the VSP-generated drawings for these structures with survey units shown and the location of direct measurements. $\mathrm{H}_{0}$ was rejected for all survey units and $\mathrm{H}_{\mathrm{A}}$ accepted, thus demonstrating that the release criteria have been met.

\subsection{Building Surveys: Class 2}

The results for the surveys of Class 2 survey units within Buildings 200E, 100E, and 100W are provided below.

\subsubsection{Class 2 Scoping/Characterization/FSS Surface Scans \& Surface Activity Levels}

Surface scans of Bays 11 through 20 in Building 200E (north) identified elevated gamma radiation associated with the northwest corner wall. Further investigation identified the presence of the three small jars of $\mathrm{ThN}$ within the locker room/closet area, previously discussed, that were located on the opposite side of the wall. Surface scans did not identify any additional areas 
of elevated direct radiation distinguishable from background in the remainder of the building. Surface scans of Building 100W Bays 1 through 7, those portions of Bays 8 through 18 outside of the former ThN storage area, and Bays 19 and 20 did not identify any elevated direct gamma or alpha plus beta radiation with the exception of two locations where the stored tungsten contributed to the background radiation. Therefore, DNSC moved the materials and the characterization surface scans of these suspect locations determined that the anomalies were false positives resulting from the increased ambient gamma radiation background levels. These scoping/characterization scan data were of sufficient quantity and quality to satisfy the FSS requirements.

The FSS beta surface scans of the Class 2 survey units in Buildings 200E (south) and 100E did not identify the any residual contamination discernable above the scan MDC.

The summary of the beta surface activity FSS results is provided below in Table 8-3. Complete data are provided in Table B-5.

\begin{tabular}{|c|c|c||}
\hline & \multicolumn{2}{|c|}{ Table 8-3: Class 2 Building FSS Summary } \\
\hline Building & $\begin{array}{c}\text { Number of Survey Units/ } \\
\text { Measurements }\end{array}$ & $\begin{array}{c}\text { Total Beta Activity Range/Average } \\
\text { (dpm/100 } \mathbf{c m}^{\mathbf{2}} \text { ) }\end{array}$ \\
\hline $200 \mathrm{E}$ (north) & $3 / 67$ & -200 to 200/-7 \\
\hline $200 \mathrm{E}$ (south) & $5 / 79$ & -380 to 520/61 \\
\hline $100 \mathrm{E}$ & $4 / 59$ & -310 to 230/37 \\
\hline $100 \mathrm{~W}$ & $3 / 50$ & -290 to 370/59 \\
\hline
\end{tabular}

The beta surface activity level at one direct measurement location in Building 200E (south) survey unit C2 SU57 exceeded the Th-232 DCGL $\mathrm{D}_{\mathbb{W}}$ of $400 \mathrm{dpm} / 100 \mathrm{~cm}^{2}$. The activity at this location was $520 \mathrm{dpm} / 100 \mathrm{~cm}^{2}$. This was a systematic measurement location on the lower west wall concrete footer at coordinates 35.6, 0.2. Identification of this single outlier occurred after site personnel had demobilized from the site. Therefore, a follow-up investigation was not possible to either confirm the anomaly as contamination or to otherwise disposition the anomaly if the cause was found to be unrelated to contamination such as a differing construction material background. Because this was a Class 2 survey unit, contamination above the DCGL was not expected. The observed beta count rate at this location was typical of the cinder block 
background count rates measured on the majority of the wall, but exceeded the background of the concrete footer by approximately $200 \mathrm{cpm}$. The overall opinion is that the anomaly is a false positive in view of the results of all other surface scans and direct measurements within Building 200E (south). However, the following sequence may have occurred had the anomaly been confirmed.

1.) A portion of the survey unit may have been reclassified. The measurement in question, was the first lower wall measurement location adjacent to Class 1 survey unit C1 SU23. The activity level was less than the scan MDC. Because of the proximity of the lower walls to the previously heavily contaminated floor and the adjoining Class 1 wall survey unit, this and all other lower walls had been scanned $100 \%$. No other contamination was noted or identified on any of the remaining lower walls.

2.) Therefore, had this area been either established as a new Class 1 survey unit or been included with existing Class 1 unit C1 SU23, Class 1 scanning requirements were met.

3.) The location would have either been remediated as an ALARA action or compared to the

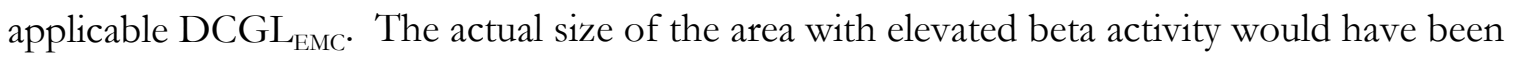
determined for the comparison. Without this information, the assumption can be made that the size corresponds to the sample spacing of the survey unit. The survey unit C2 SU57 sample spacing was $12.5 \mathrm{~m}^{2}$. As a conservative comparison, the area factor for $100 \mathrm{~m}^{2}$ is 1.96 and the corresponding DCGL EMC $_{1}$ is $784 \mathrm{dpm} / 100 \mathrm{~cm}^{2}$. The activity level of $520 \mathrm{dpm} / 100 \mathrm{~cm}^{2}$ is below this threshold.

The complete DQA report for each survey unit for these structures is provided in Appendix F. The DQA reports include individual direct measurement results, both summary and graphical prospective and retrospective FSS planning information, and summary statistics. Figures A-7, A-10, A-12 through A-15, and A-18 contain maps for these structures with direct measurement locations, some of which were previously presented in the characterization survey report (ORISE 2006b). $\mathrm{H}_{0}$ was rejected for all survey units and the $\mathrm{H}_{\mathrm{A}}$ accepted, thus demonstrating that the release criteria have been met. 


\subsection{Building Surveys: Class 3}

The results for the surveys of the Class 3 survey units associated with Buildings 100E, 200E (north), 100W, the Garage, the Workshop, and combined building exteriors are provided below.

\subsubsection{Class 3 Scoping/Characterization/FSS Surface Scans \& Surface Activity Levels}

Surface scans of Building 100E Bays 1 through 14 floors, walls, and overhead surfaces conducted at the time of the scoping survey did not identify any indications of elevated direct gamma or alpha plus beta radiation on surfaces other than an open drum noted to have elevated gamma radiation. The drum was stored near the central part of the building. The elevated radiation levels were the result of the presence of tungsten material within the drum. Additional gamma scans at the time of the FSS also did not identify any elevated direct gamma radiation other than that which was associated with stored drums of tungsten and previously discussed monazite sand-contaminated tannin.

Class 3 surface scans of Buildings 200E (north), 100E (overheads of Bays 14 through 20), 100W, the Garage, the Workshop, and building exteriors did not identify any gamma or beta radiation levels distinguishable from background.

The summary of the beta surface activity FSS results is provided below in Table 8-4. Complete data are provided in Tables B-5.

\begin{tabular}{|c|c|c||}
\hline \multicolumn{3}{|c|}{ Table 8-4: Class 3 Building FSS Summary } \\
\hline Building & $\begin{array}{c}\text { Number of Survey Units/ } \\
\text { Measurements }\end{array}$ & $\begin{array}{c}\text { Total Beta Activity Range/Average } \\
\text { (dpm/100 } \mathbf{~ c m}^{2} \text { ) }\end{array}$ \\
\hline \hline $200 \mathrm{E}$ (north) & $2 / 25$ & -440 to $150 /-21$ \\
\hline $100 \mathrm{E}$ & $2 / 21$ & -65 to $100 / 2$ \\
\hline $100 \mathrm{~W}$ & $1 / 21$ & -110 to $230 / 44$ \\
\hline Garage & $1 / 15$ & -120 to $110 / 19$ \\
\hline Workshop & $1 / 14$ & -210 to $140 / 7$ \\
\hline Building exteriors & $1 / 15$ & -150 to $68 /-8$ \\
\hline
\end{tabular}


The complete DQA report for each survey unit for these structures is provided in Appendix F. The DQA reports include individual direct measurement results, both summary and graphical prospective and retrospective FSS planning information, and summary statistics. Figures A-12 and A-16 through A-21 contain maps for these structures with direct measurement locations, some of which were previously presented in the characterization survey report (ORISE 2006b). $\mathrm{H}_{0}$ was rejected for all survey units and the $\mathrm{H}_{\mathrm{A}}$ accepted, thus demonstrating that the release criteria have been met.

\subsection{Land AREa Surveys: Class 1}

The survey results for the Class 1 land areas are described below. The FSS survey unit designations are shown on Figure A-3. Soil sample results, including the background reference area samples are provided in Table B-6.

\subsubsection{Class 1 Land Area Scoping/Characterization Surface Scans \& Sample Results}

There was one Class 1 designated land area at the time of the scoping survey, the Burn Cage area. Gamma radiation surface scans which covered 100\% of the Burn Cage area identified an approximately $50 \mathrm{~m}^{2}$ area of elevated direct radiation to the immediate east of a large debris pile and a small area with lower gamma radiation levels next to the actual Burn Cage. Scoping survey gamma scans of the remaining site land areas were low density overall as they focused on roadways, railroads, and building vicinities.

The characterization survey high density gamma scans and investigation soil sampling began in the Burn Cage area and considerably expanded due to the identification of seven new AOCs in the western sector of the site. Additional high density characterization gamma scans were performed over these new Class 1 areas to better delineate the gamma radiation isopleths.

The concentration ranges for Th-232 and U-238 for Class 1 area scoping and characterization survey investigation soil samples are summarized in Table 8-5. The Th-232 analytical results from samples collected from slag layer underlying the contaminated soil are also summarized. 


\begin{tabular}{|c|c|c|}
\hline \multicolumn{3}{|c|}{$\begin{array}{c}\text { Table 8-5: Characterization Survey } \\
\text { Radionuclide Concentrations }\end{array}$} \\
\hline \multirow{2}{*}{ Area } & \multicolumn{2}{|c|}{$\begin{array}{r}\text { Radionuclide Concentration } \\
\text { (pCi/g) }\end{array}$} \\
\cline { 2 - 3 } & Th-232 & U-238 \\
\hline Class 1 & 1.12 to 1630 & 1.44 to 57 \\
\hline Slag & 0.57 to 1.06 & --- $^{a}$ \\
\hline
\end{tabular}

${ }^{a}$ Analysis not performed.

The complete scoping/characterization survey results for Class 1 land areas were provided in the characterization survey report (ORISE 2006c).

\subsubsection{Class 1 Land Area Remediation}

The remedial actions that WEI conducted within the contaminated areas are summarized below. Table 8-6 provides a general pictorial representation of each land area AOC. 


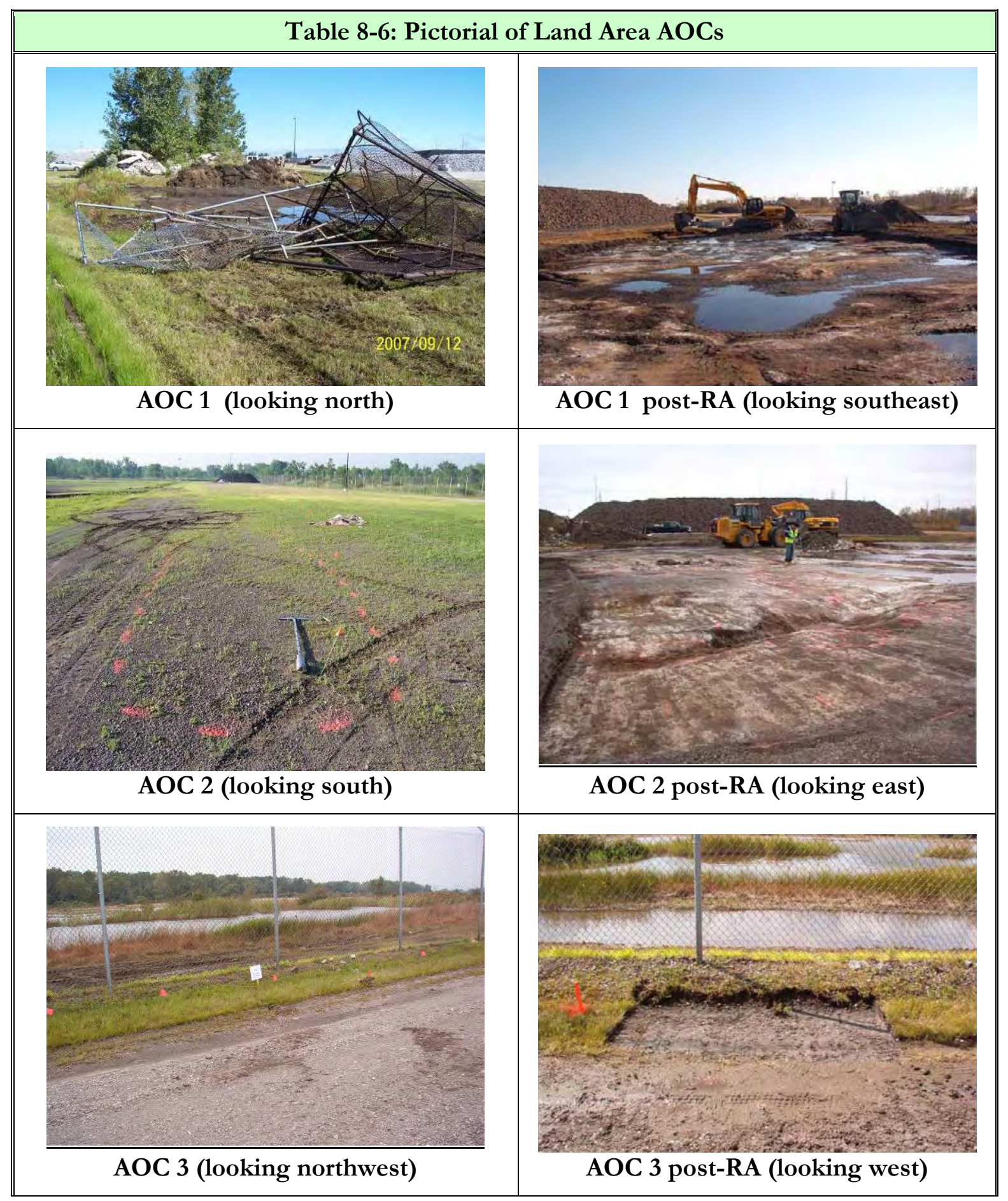




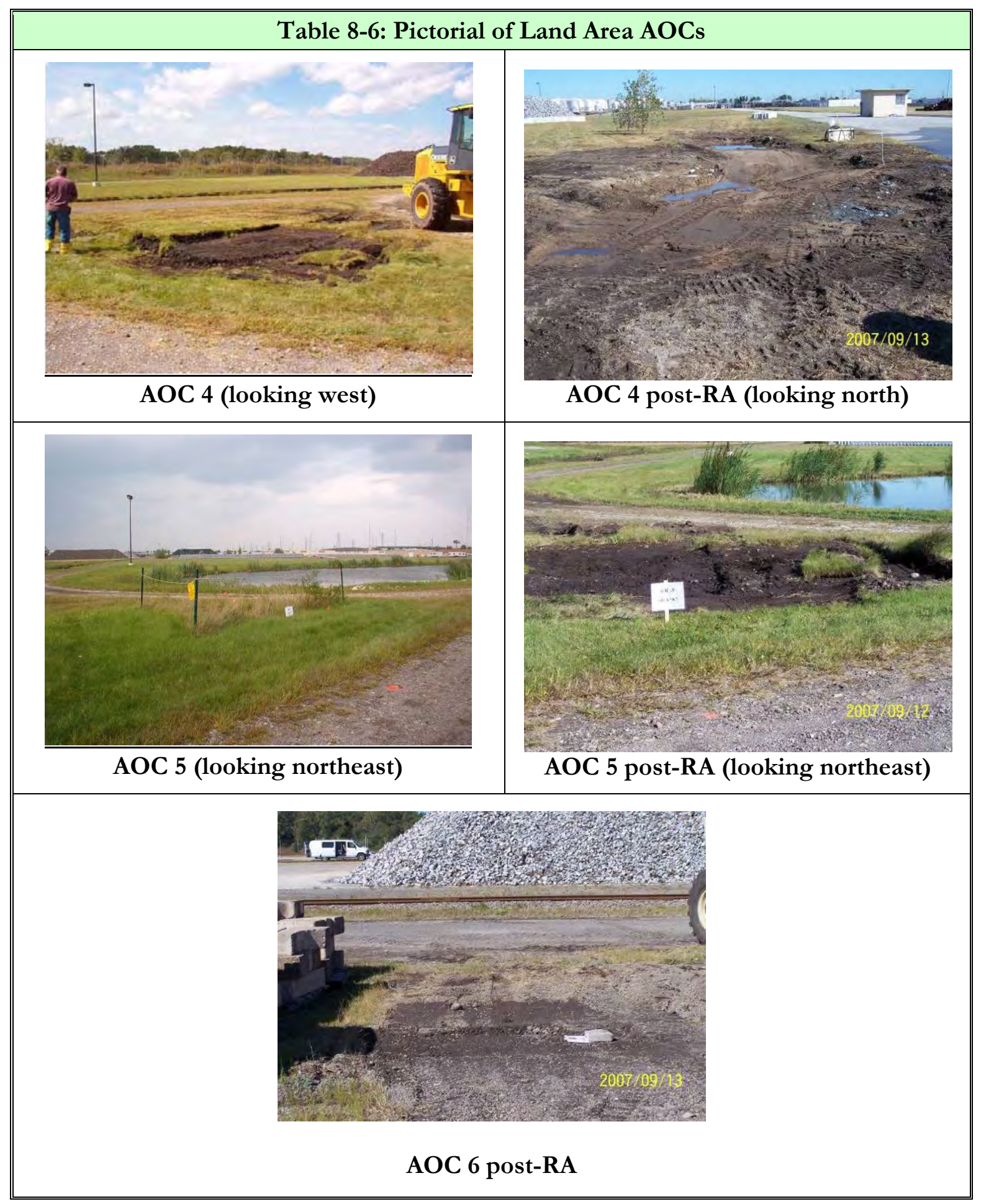




\section{$\underline{\mathrm{AOC} 1}$}

AOC 1 was associated with the former Burn Cage area in depot Area "V'. The excavation bounds covered approximately $1,050 \mathrm{~m}^{2}$ with the excavation depth to approximately 0.5 meters. The monolithic slag layer was completely exposed. Isolated hotspots identified on the slag surface required chipping followed by scraping of the entire surface.

\section{$\underline{\mathrm{AOC}} 2$}

AOC 2 was contiguous with AOC 1 to the southwest. The $2,800 \mathrm{~m}^{2}$ area was completely excavated of soil to the slag layer which varied in depth from a few centimeters to approximately one meter. As with AOC 1, a number of slag areas required chipping to remove surficial hot spots. The entire surface of the exposed slag was then scraped to remove residual contaminated soil. Contamination was also found to extend beneath the southern edge of the Ferrochrome \#6 pile. This finding required that the ore be moved to allow for remediation of the contamination that had migrated beneath the pad.

\section{$\underline{\mathrm{AOC} 3}$}

This AOC consisted of intermittent areas of contamination between the road edge and the western fence line totaling approximately $10 \mathrm{~m}^{2}$ and was directly across the perimeter road from AOC 2. Due to the proximity to the depot boundary, investigative gamma scans were performed outside the western property fence, between the fence line and Wolf Lake. There were no anomalies found. Also, a trench was excavated beneath the roadway separating AOCs 2 and 3. The trench provided the means to investigate whether AOCs 2 and 3 were connected. There was no contamination identified between the two AOCs. The localized soil contamination was excavated to the slag.

\section{$\underline{\mathrm{AOC}} 4$}

This $250 \mathrm{~m}^{2}$ area of contamination was identified near the Scale House in depot Area "V". Soils were excavated to approximately 0.30 meters in depth. Soils did not require complete removal to the slag layer in this AOC. 


\section{$\underline{\mathrm{AOC} 5}$}

AOC 5 was located beyond Area " $K$ " at the southern end of the site and was adjacent to where the railroad spur entered the site. Soils were excavated from an area of approximately $10 \mathrm{~m}^{2}$ to depths of 0.15 to 0.30 meters.

\section{$\underline{\mathrm{AOC} 6}$}

AOC 6 was located at the southwest edge of Area "D" where a contaminated pallet was identified. Shallow excavation was performed to remediate the small soil contaminated area of 1 to $2 \mathrm{~m}^{2}$.

\section{$\underline{\mathrm{AOC}} 7$}

AOC 7 was located adjacent to the railroad track that bounds the Burn Cage area and Ferrochrome Pile \#6 AOCs. The area measured approximately $2 \mathrm{~m}^{2}$ in size and was successfully remediated. The remediation included investigation to ensure that contamination had not migrated beneath the railroad track.

\subsubsection{Class 1 Land Area FSS Surface Scans \& Sample Results}

The final remediation support survey (FRS) and/or FSS high density gamma surface scans addressed each of the 29 Class 1 land area survey units. These survey units consisted of both soil and slag land area units. Where large areas of a proposed soil survey unit had been excavated to slag, sub-survey units were established. These sub-survey units consisted of the monolithic slag and were addressed for the FSS as a structural survey unit.

FRS and FSS scans identified several small areas of soil or surficial slag residual contamination that were investigated. Each location that exceeded the DCGLs was noted and additional remediation performed. The gamma radiation levels for most of the final FSS gamma surface scans are included within Figures A-22 through A-37 and are provided for informational purposes. Although $100 \%$ of the Class 1 units were scanned, the position correlated data were not captured for all areas due to signal loss between the GPS unit and the ratemeter-scaler. 
FSS scanning MDCs and decisions were based on the audible output from the detector/ratemeter-scaler combinations and documented accordingly.

The FSS systematic and judgmental sample results are provided in Table B-6. Summary data are provided below in Table 8-7a and 8-7b. The results presented in this table represent 123 soil and 308 beta activity FSS systematic and judgmental samples/measurements. Judgmental sample results from locations that required additional remediation are not included in the summary.

\begin{tabular}{|c|c|c|c|}
\hline \multicolumn{4}{|c|}{$\begin{array}{c}\text { Table 8-7a: FSS Class } 1 \text { Radionuclide Concentrations in Soil Samples } \\
\text { Summary Results }\end{array}$} \\
\hline Area & Th-232 (pCi/g) & $\mathrm{U}-238$ (pCi/g) & Net SOR/Average \\
\hline C1 SU1 - 7 & 0.32 to 3.02 & 0.48 to 4.09 & -0.93 to $1.13 /-0.14$ \\
\hline \multicolumn{4}{|c|}{ Table 8-7b: FSS Class 1 Slag Beta Surface Activity Summary Results } \\
\hline Area & \multicolumn{3}{|c|}{$\begin{array}{l}\text { Total Beta Activity Range/Average } \\
\qquad\left(\mathrm{dpm} / 100 \mathrm{~cm}^{2}\right)\end{array}$} \\
\hline C1 SU2.1 - 5.8 & \multicolumn{3}{|c|}{-300 to $350 /-27$} \\
\hline
\end{tabular}

The DQA reports for individual Class 1 land area survey units are provided in Appendix G. The DQA reports include individual analytical results, both summary and graphical prospective and retrospective FSS planning information, and summary statistics. Figures A-22 through A-37 included the maps for these survey units with soil sampling locations. $\mathrm{H}_{0}$ was rejected for all survey units and the $\mathrm{H}_{\mathrm{A}}$ accepted, thus demonstrating that the release criteria have been met. One sample from C1 SU4 contained residual soil concentrations exceeding an SOR of 1. The survey unit readily passed the WRS test. An EMC evaluation was not required as the primary cause for the sample exceeding unity was the result of the increased U-238 concentrations from the sample matrix. Samples within this excavation contained a large quantity of unconsolidated slag relative to soil. The Th-232 concentration in this sample was $2.47 \mathrm{pCi} / \mathrm{g}$. 


\subsection{LAND AREa Surveys: Class 2}

Surface scan and soil sampling results for the 13 Class 2 land area survey units (including the debris pile solid debris survey unit) are described below. The FSS Class 2 survey units are illustrated on Figure A-3. The debris pile maps are provided in Figures A-60 and A-61.

\subsubsection{Class 2 Land Area Scoping/Characterization/FSS Surface Scans \& Sample Results}

The scoping/characterization gamma surface scans of the Class 2 land areas did identify locations of residual contamination discussed in Sections 4.1.1 and 4.1.2 (AOCs 2, 5, and 7). Judgmental samples collected during the scoping survey of other investigated locations were provided in the characterization report (ORISE 2006b).

Surface scans of the land areas that were within the Class 2 configuration in place at the time of the FSS did not identify any locations of elevated direct radiation. Figures A-38 through A-59 include maps showing the FSS gamma radiation levels for the Class 2 survey units.

The FSS random-start/systematic sample results are provided in Table B-6. Summary data are provided below in Table 8-8a and 8-8b. The results presented in this table represent 175 FSS samples.

\begin{tabular}{|c|c|c|c|}
\hline \multicolumn{4}{|c|}{$\begin{array}{c}\text { Table 8-8a: FSS Class } 2 \text { Radionuclide Concentrations in Soil Samples } \\
\text { Summary Results }\end{array}$} \\
\hline Area & Th-232 (pCi/g) & $\mathrm{U}-238(\mathrm{pCi} / \mathrm{g})$ & $\begin{array}{l}\text { Net } \\
\text { SOR/Average }\end{array}$ \\
\hline C2 SU1 - 12 & 0.28 to 2.31 & 0.43 to 3.45 & -1.0 to $0.61 /-0.29$ \\
\hline \multicolumn{4}{|c|}{ Table 8-8b: FSS Class 2 Beta Surface Activity Summary Results } \\
\hline Area & \multicolumn{3}{|c|}{$\begin{array}{l}\text { Total Beta Activity Range/Average } \\
\qquad\left(\mathrm{dpm} / 100 \mathrm{~cm}^{2}\right)\end{array}$} \\
\hline C2 SU12 Debris & \multicolumn{3}{|c|}{-200 to $410^{a} /-57$} \\
\hline
\end{tabular}

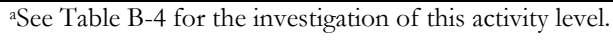

The DQA reports for the individual Class 2 land area survey units are provided in Appendix G. The DQA reports include individual analytical results, both summary and graphical prospective 
and retrospective FSS planning information, and summary statistics. Figures A-38 through A-58 contain the maps for these survey units with soil sampling/measurement locations. $\mathrm{H}_{0}$ was rejected for all survey units and the $\mathrm{H}_{\mathrm{A}}$ accepted, thus demonstrating that the release criteria have been met. The total beta activity of one direct measurement on the solid debris of survey unit C2 SU12 exceeded the DCGL $L_{\mathbb{W}}$ of $400 \mathrm{dpm} / 100 \mathrm{~cm}^{2}$. Further investigation determined the debris was a firebrick which naturally exhibits a higher background radiation level due to the thorium and uranium content. An EMC evaluation was not required nor was reclassification of the survey unit.

\subsection{LAND AREA Surveys: Class 3}

Surface scan and soil sampling results for the five Class 3 land area survey units are described below. The Class 3 survey units are shown collectively on Figure A-3.

\subsubsection{Class 3 Land Area Scoping/Characterization/FSS Surface Scans \& Sample Results}

The scoping survey gamma radiation surface scans of the site's Class 3 areas identified several locations distinguishable from background that were investigated by sampling in depot Areas "C", "D", and "M" and near the Pump House. The sample results showed that the Class 3 designation was appropriate for most of the areas. The high density characterization gamma scans identified two eventual AOCs; the contaminated pallet and soil in Area "D" (AOC 6) and the contaminated soil near the Scale House in Area "V" (AOC 4).

The FSS gamma scan illustrations and soil sampling location maps are included within Figures A-62 through A-71. All scan results were representative of area background levels. The results for the 81 FSS random samples collected from the five survey units are summarized below in Table 8-9 and individually in Table B-6.

\begin{tabular}{|c|c|c|c|}
\hline \multicolumn{4}{|c|}{ Table 8-9: FSS Class 3 Radionuclide Concentrations in Soil Samples } \\
Summary Results \\
\hline Area & Th-232 (pCi/g) & U-238 (pCi/g) & Net SOR/Average \\
\hline \hline C3 SU1 - 5 & 0.29 to 3.02 & 0.60 to 4.09 & -0.93 to $0.68 /-0.29$ \\
\hline
\end{tabular}




\subsection{SUMMARY AND CONCLUSIONS}

ORISE conducted extensive scoping, characterization, and final status surveys of land areas and structures at the DNSC's Hammond Depot located in Hammond, Indiana in multiple phases during 2005, 2006 and 2007. Surveys were designed and implemented in accordance with the guidance provided in MARSSIM for 108 land area and structural survey units.

World Environmental, Inc. successfully remediated contaminated soils from seven land areas of concern. Two of the depot's warehouses required remediation ranging from spot decontamination to removal of large volumes of contaminated structural materials and underlying slag.

The results of the FSS showed that all surface activity levels on structural survey units and residual Th-232 and U-238 concentrations, including the sum-of-ratios, were well below the NRC's basic dose limit of $25 \mathrm{mrem} / \mathrm{y}$ plus ALARA. The collective mean for the net Th-232 concentration was $0.05 \mathrm{pCi} / \mathrm{g}$ or approximately $2 \%$ of the dose limit, or $0.5 \mathrm{mrem}$, and the mean sum-of-ratio concentrations for all FSS soil samples was -0.24 . This slight negative bias was the result of the U-238 background variability. These results demonstrate that any residual licensable material would contribute a small fraction of the basic dose limit and is essentially indistinguishable from background. The average beta surface activity in Building 200E (south) was $67 \mathrm{dpm} / 100 \mathrm{~cm}^{2}$, or equates to $4 \mathrm{mrem} / \mathrm{y}$ above background based on the DCGL $\mathrm{w}_{\mathrm{W}}$. These results demonstrate that the Hammond Depot in Hammond, Indiana satisfies the requirements for unrestricted release from the DNSC's NRC license. 
APPENDIX A: FIGURES 


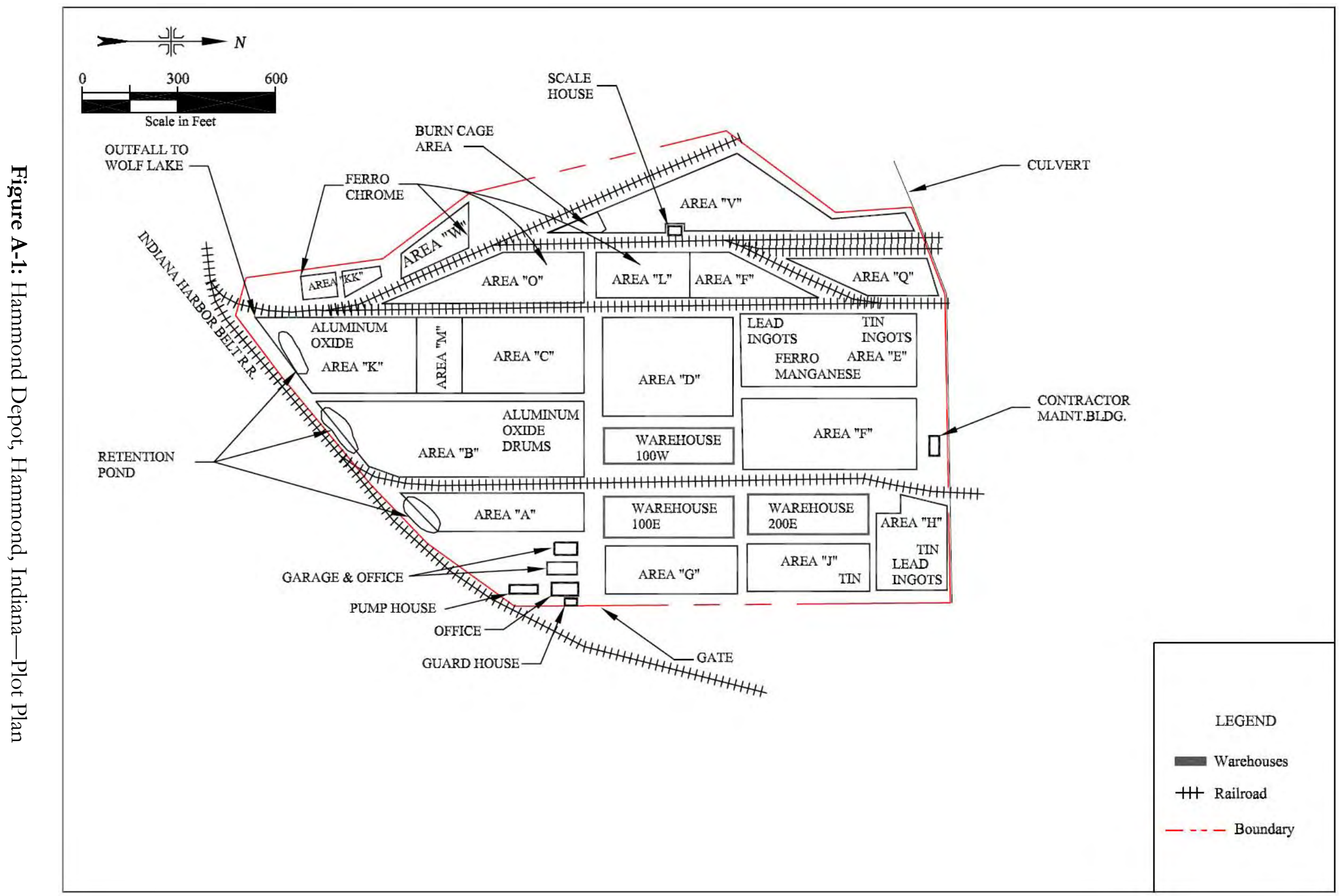




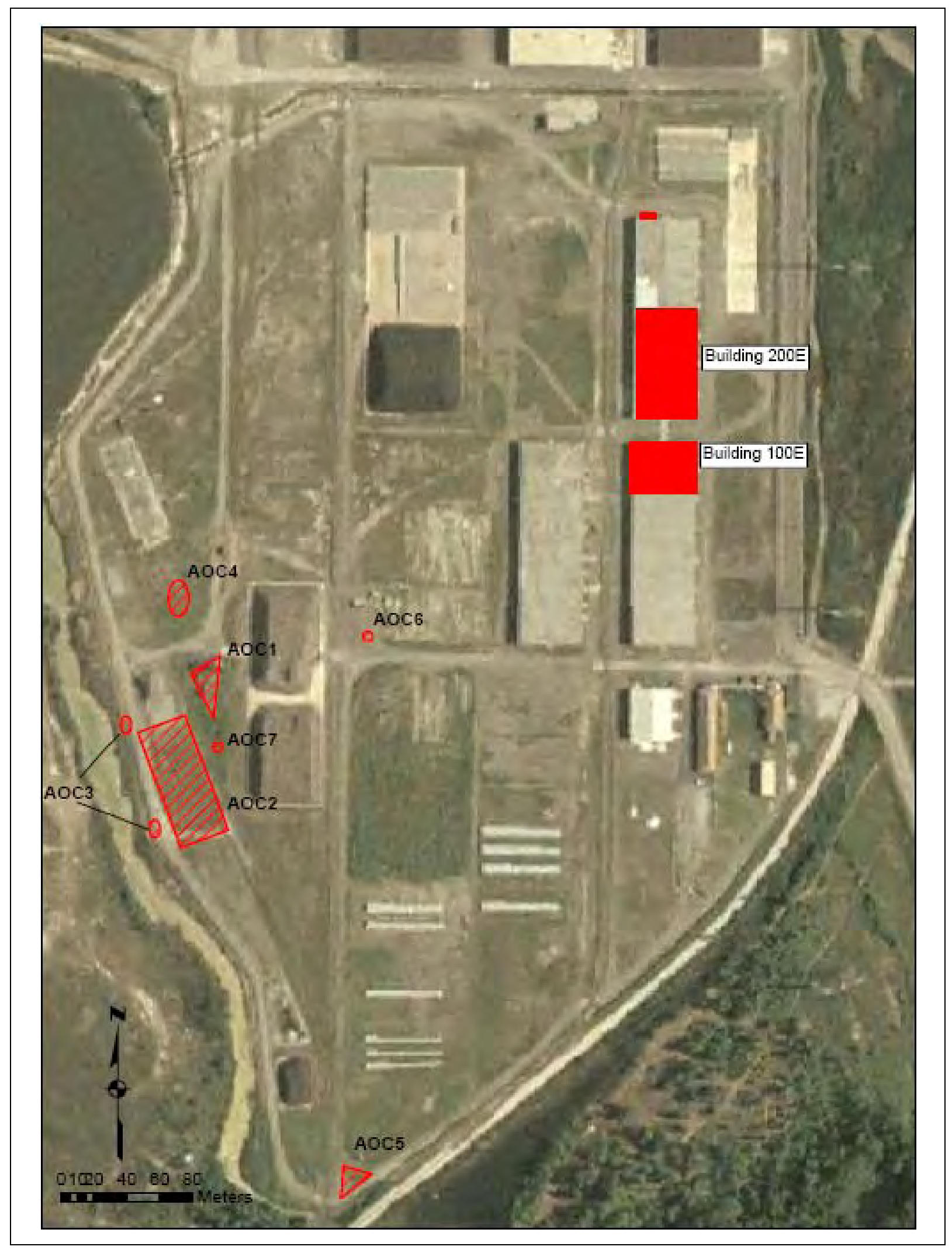

Figure A-2: Hammond Depot-Contaminated Buildings and Soil AOCs 


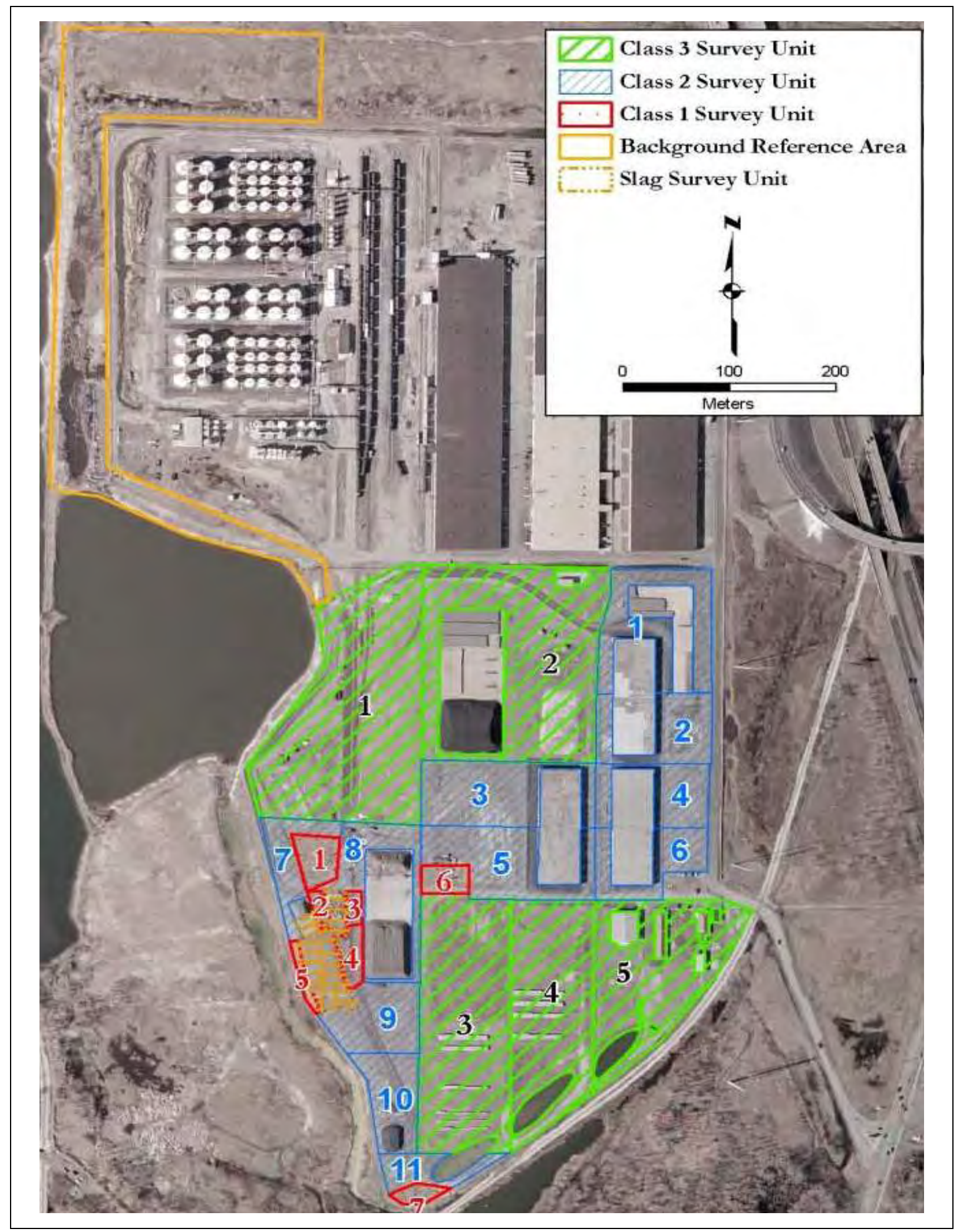

Figure A-3: Hammond Depot_-Background Reference Area and FSS Survey Units (\# ID) 


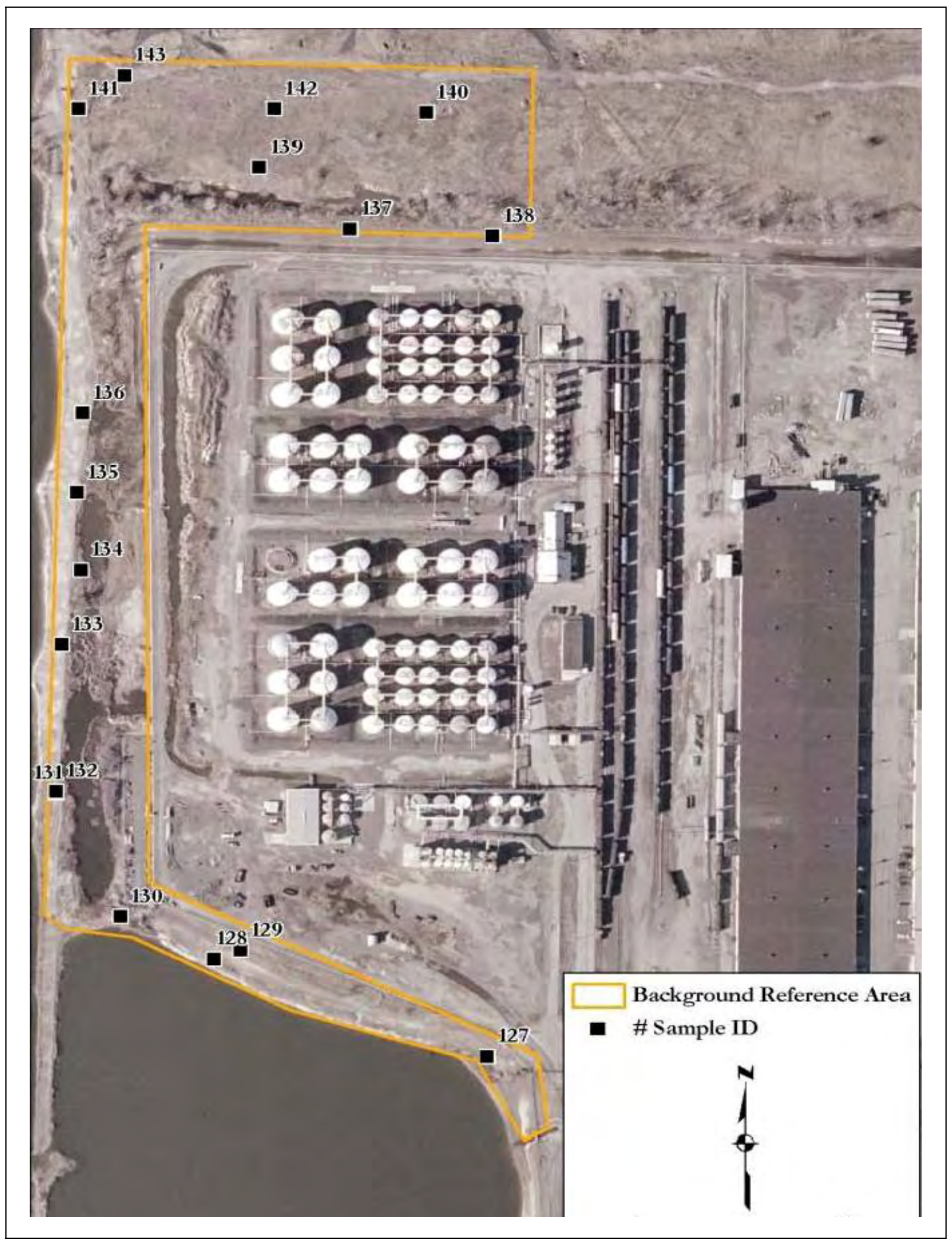

Figure A-4: Background Reference Area-FSS Soil Sampling Locations 


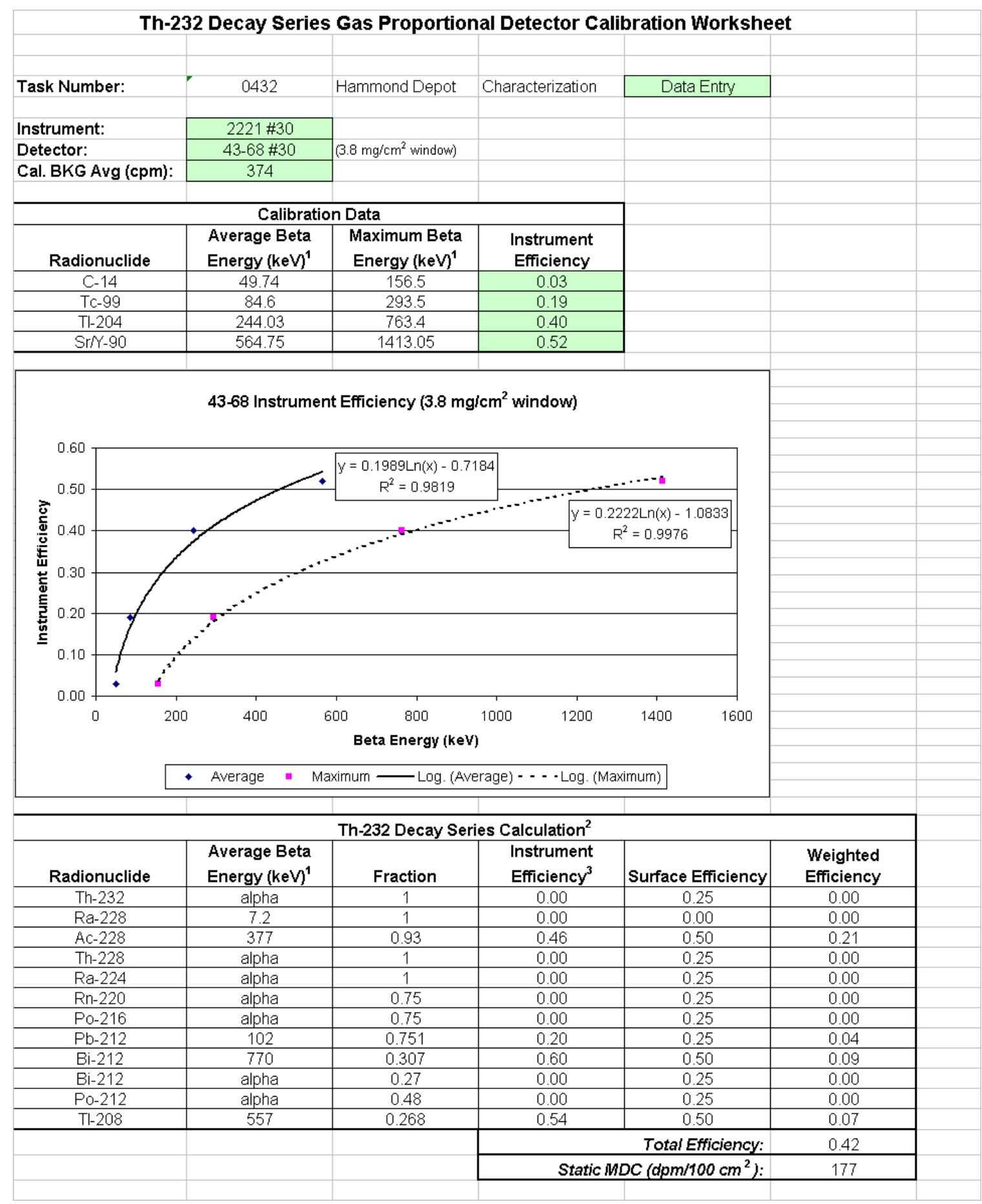

Figure A-5: Example Instrument Calibration Efficiency Determination 


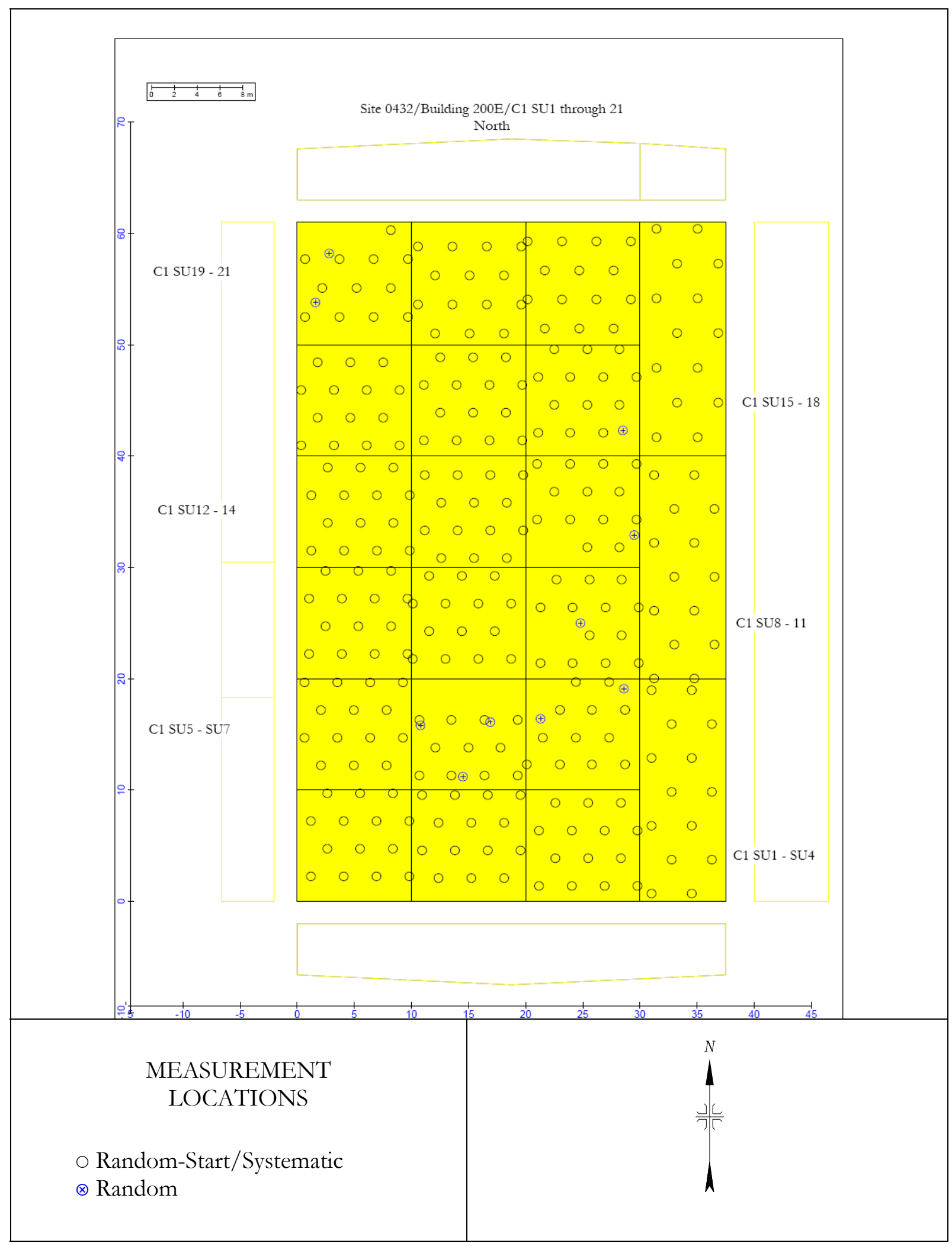

Figure A-6: Building 200E South, Floor—FSS Survey Units and Direct Measurement Locations 


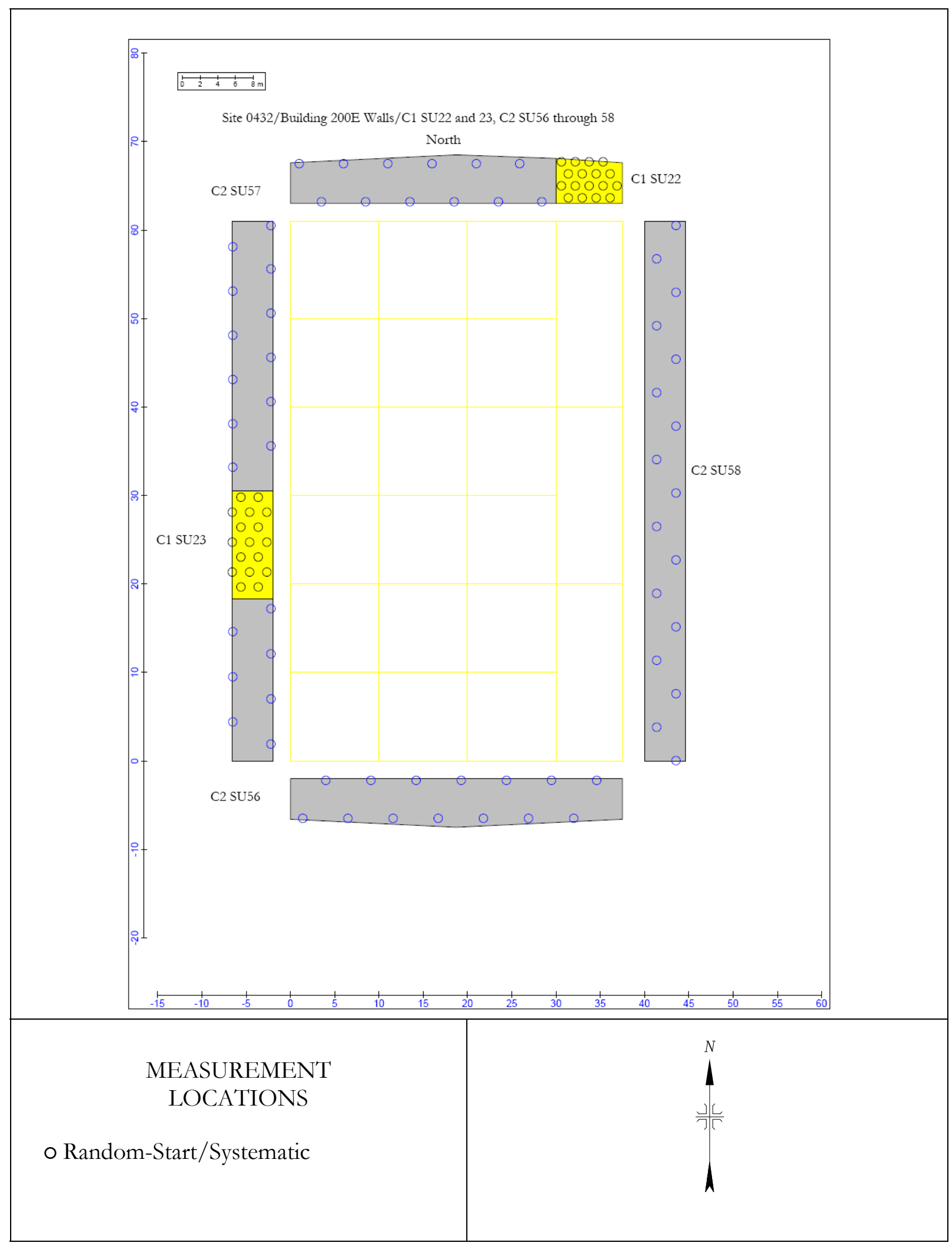

Figure A-7: Building 200E South, Walls (C1 SU22, 23, and C2 SU56 through 58)FSS Survey Units and Direct Measurement Locations 


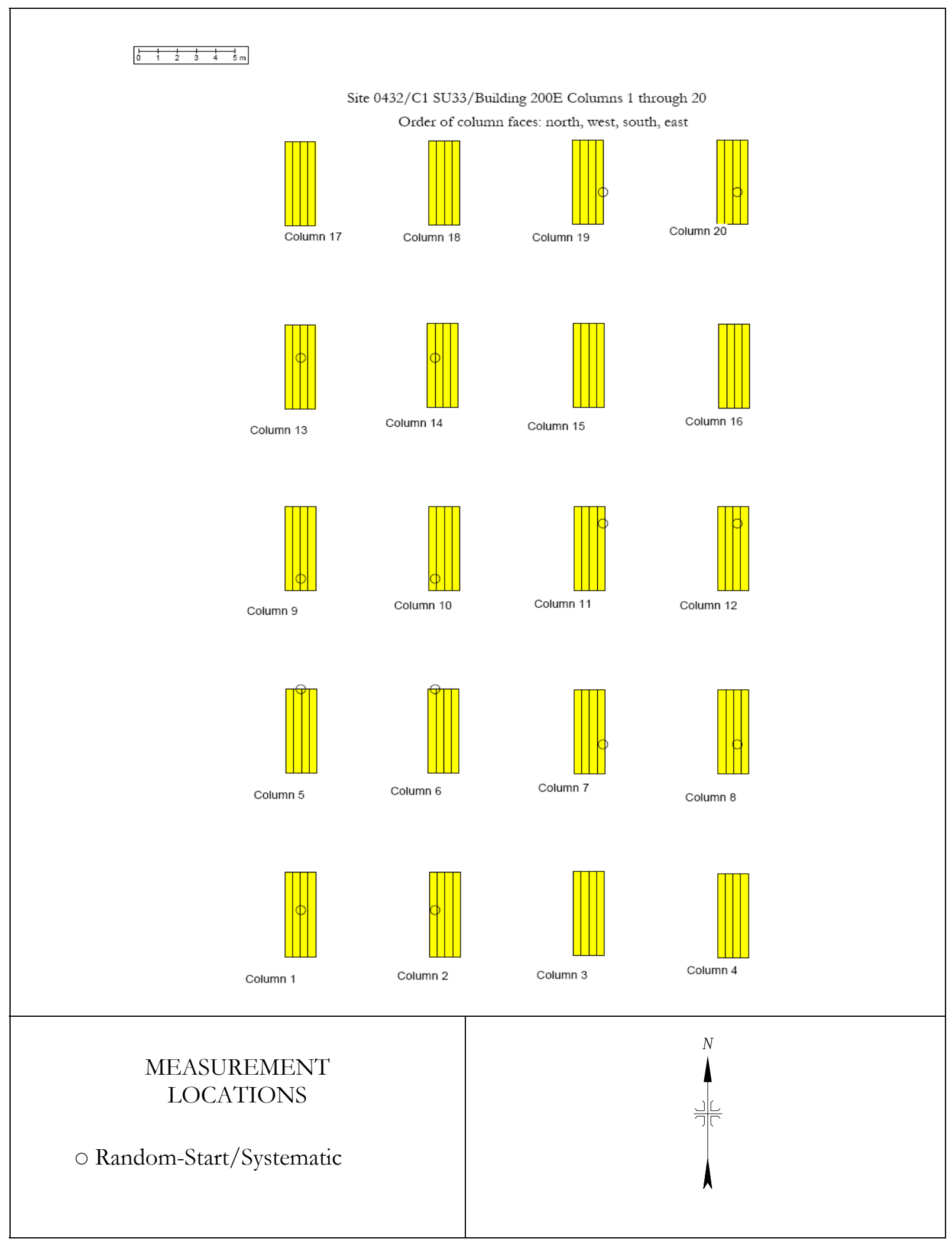

Figure A-8: Building 200E South, Columns 1 through 20 (C1 SU33)FSS Survey Unit and Direct Measurement Locations 


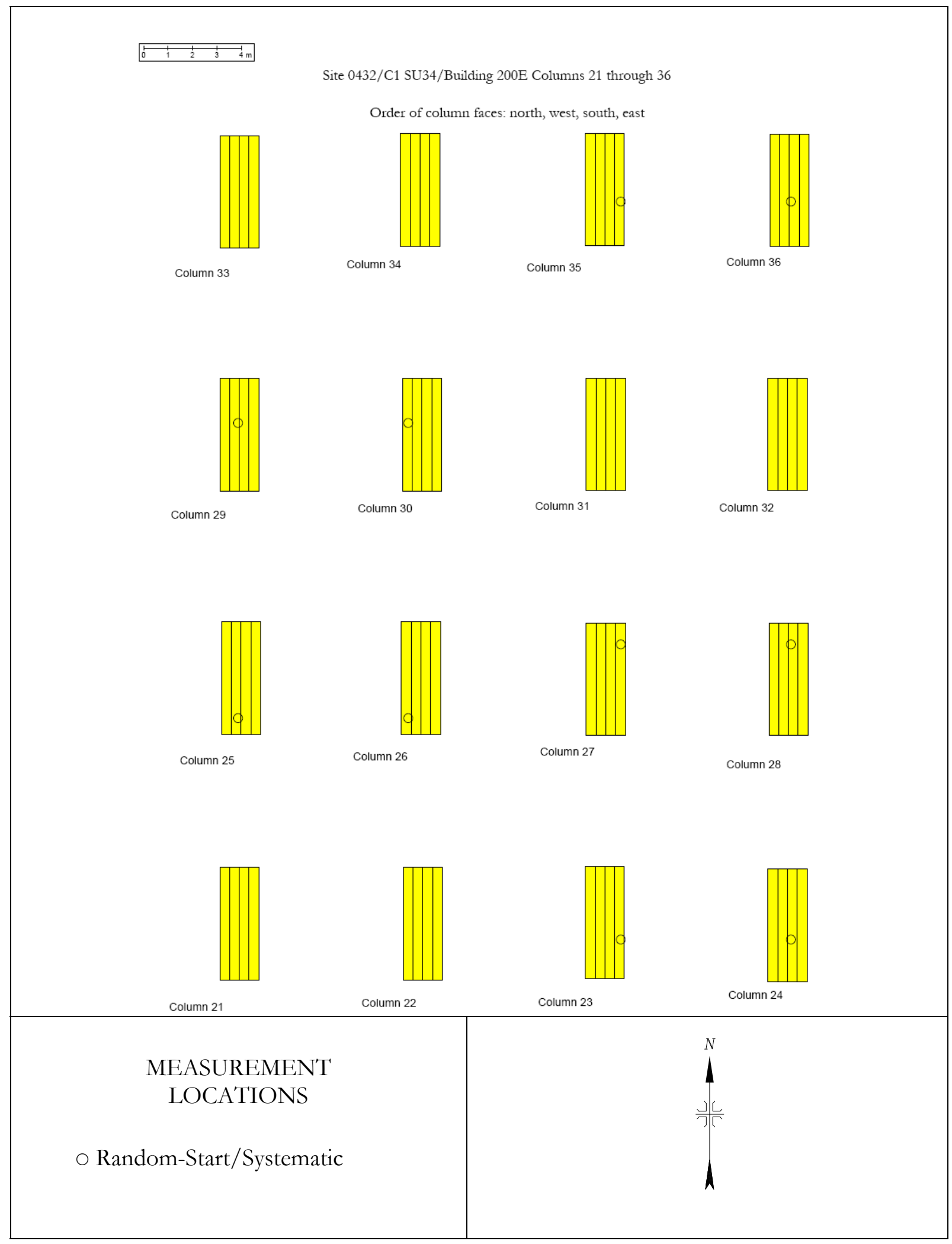

Figure A-9: Building 200E South, Columns 21 through 36 (C1 SU34)FSS Survey Unit and Direct Measurement Locations 


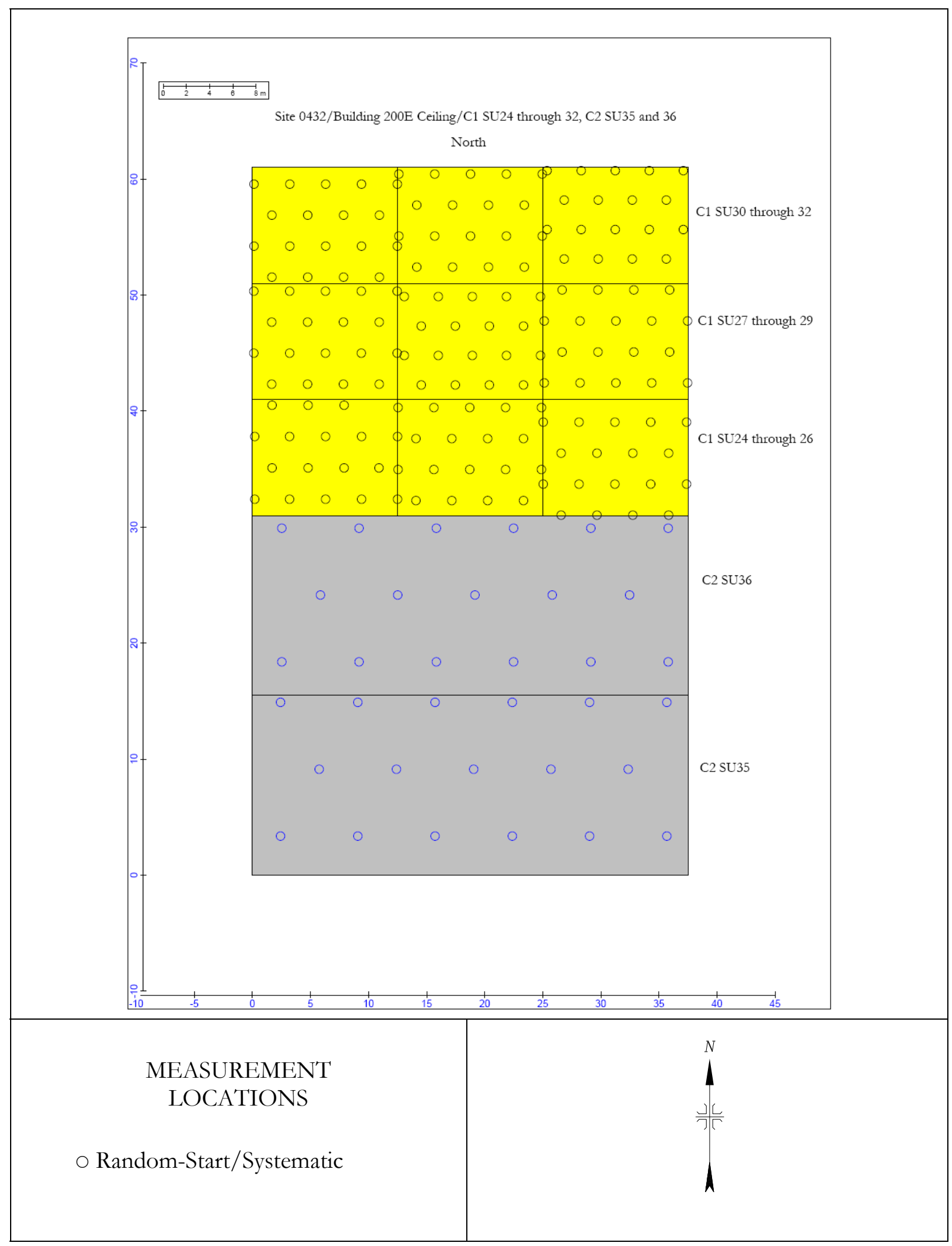

Figure A-10: Building 200E South, Ceiling (C1 SU24 through 31 and C2 SU35 and 36)_FSS Survey Units and Direct Measurement Locations 
Site 0432/Hammond Depot/Bldg 200E NW Closet/C1 SU41

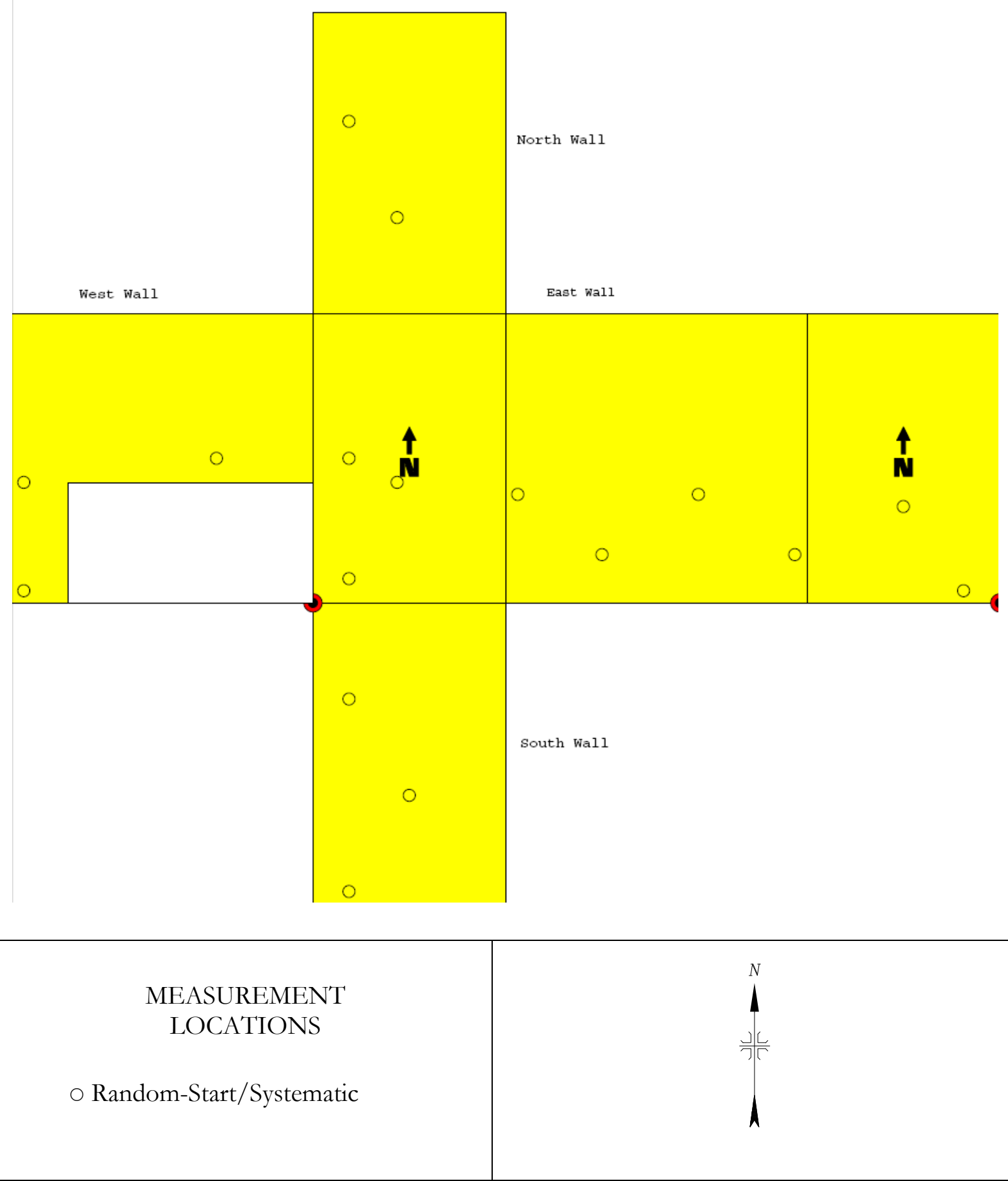

Figure A-11: Building 200E, NW Closet (C1 SU41)—FSS Survey Unit and Direct Measurement Locations 


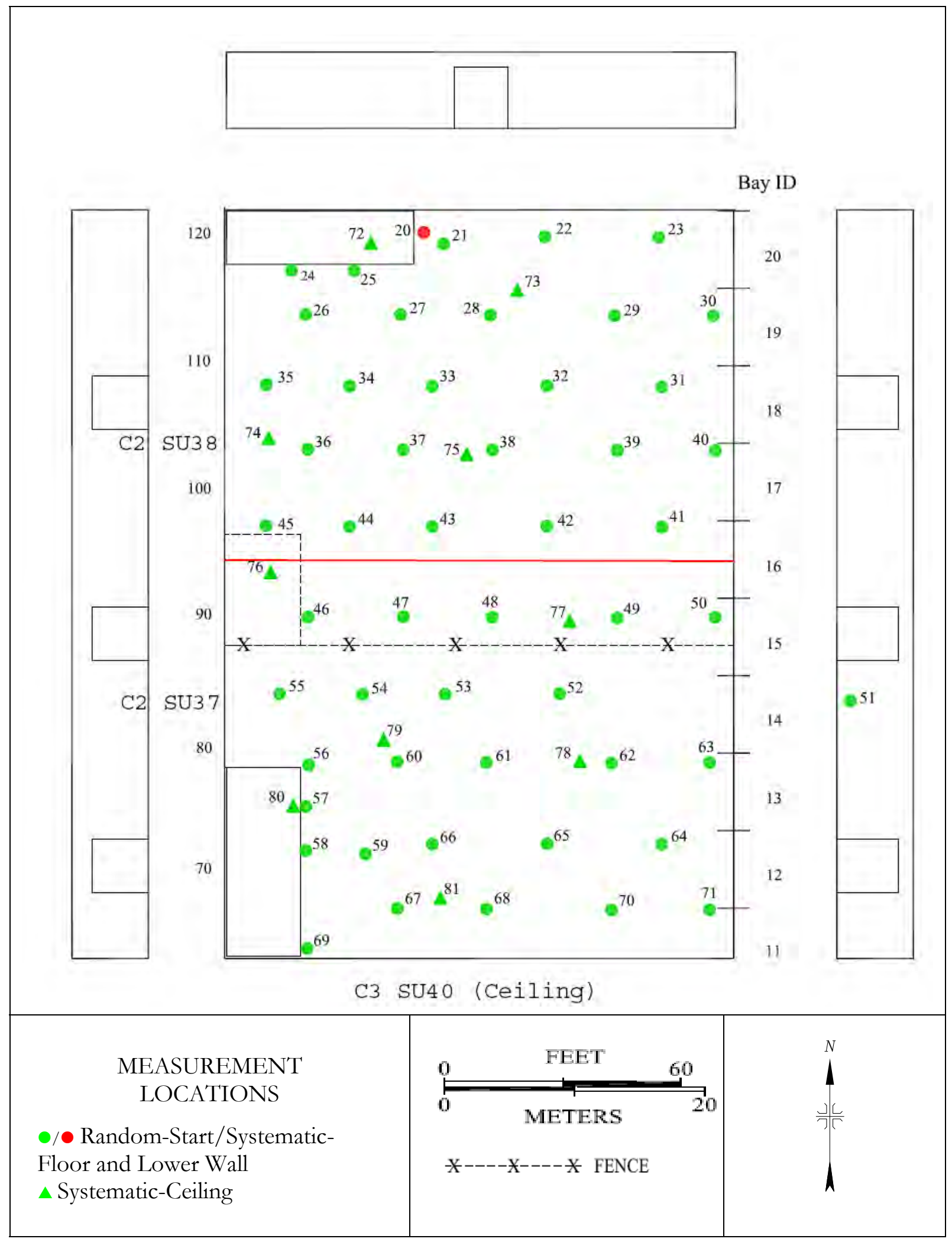

Figure A-12: Building 200E, North (C2 SU37 and 38 and C3 SU40)FSS Survey Units and Direct Measurement Locations 


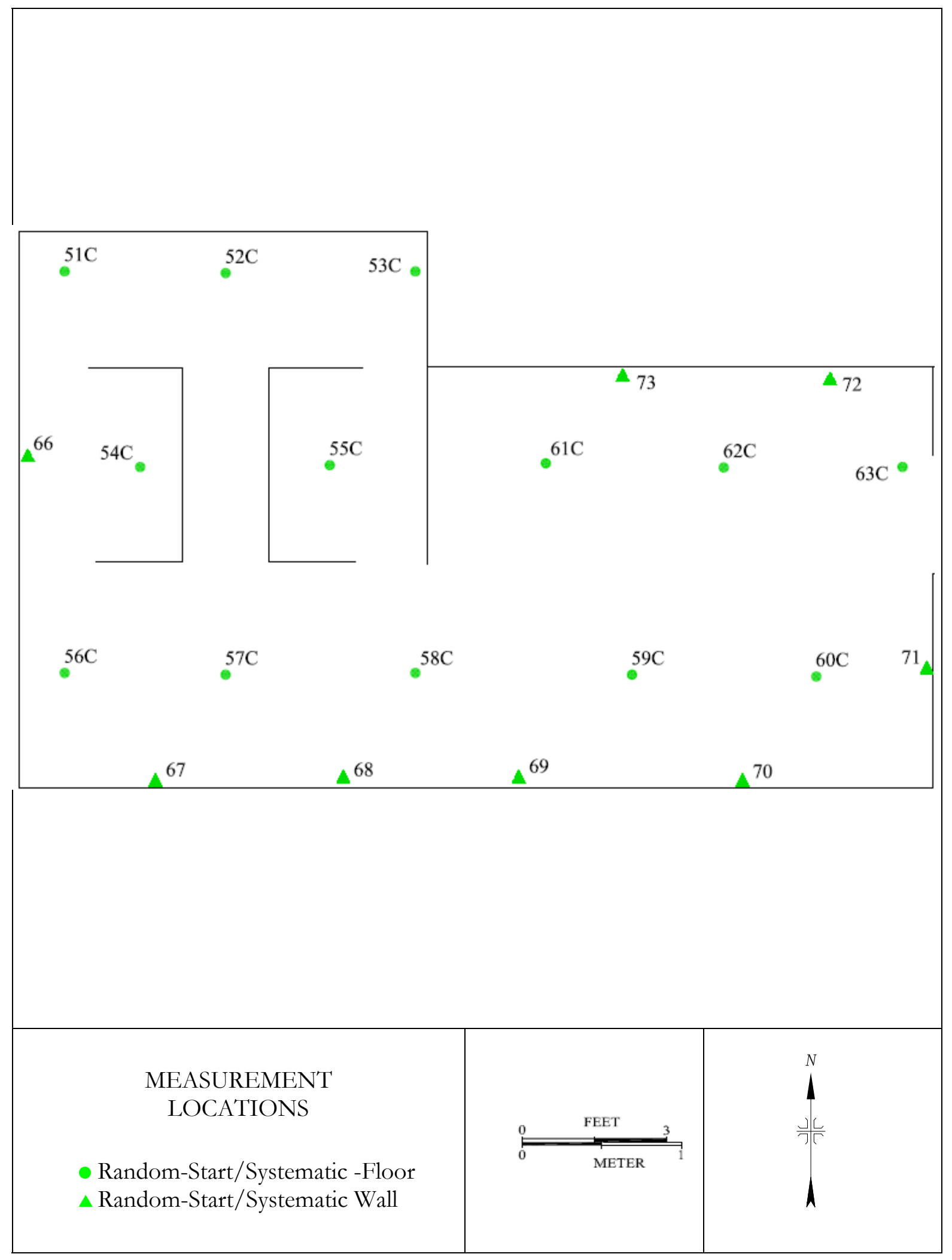

Figure A-13: Building 200E, North, Locker Room (C2 SU42)—FSS Survey Unit and Direct Measurement Locations 


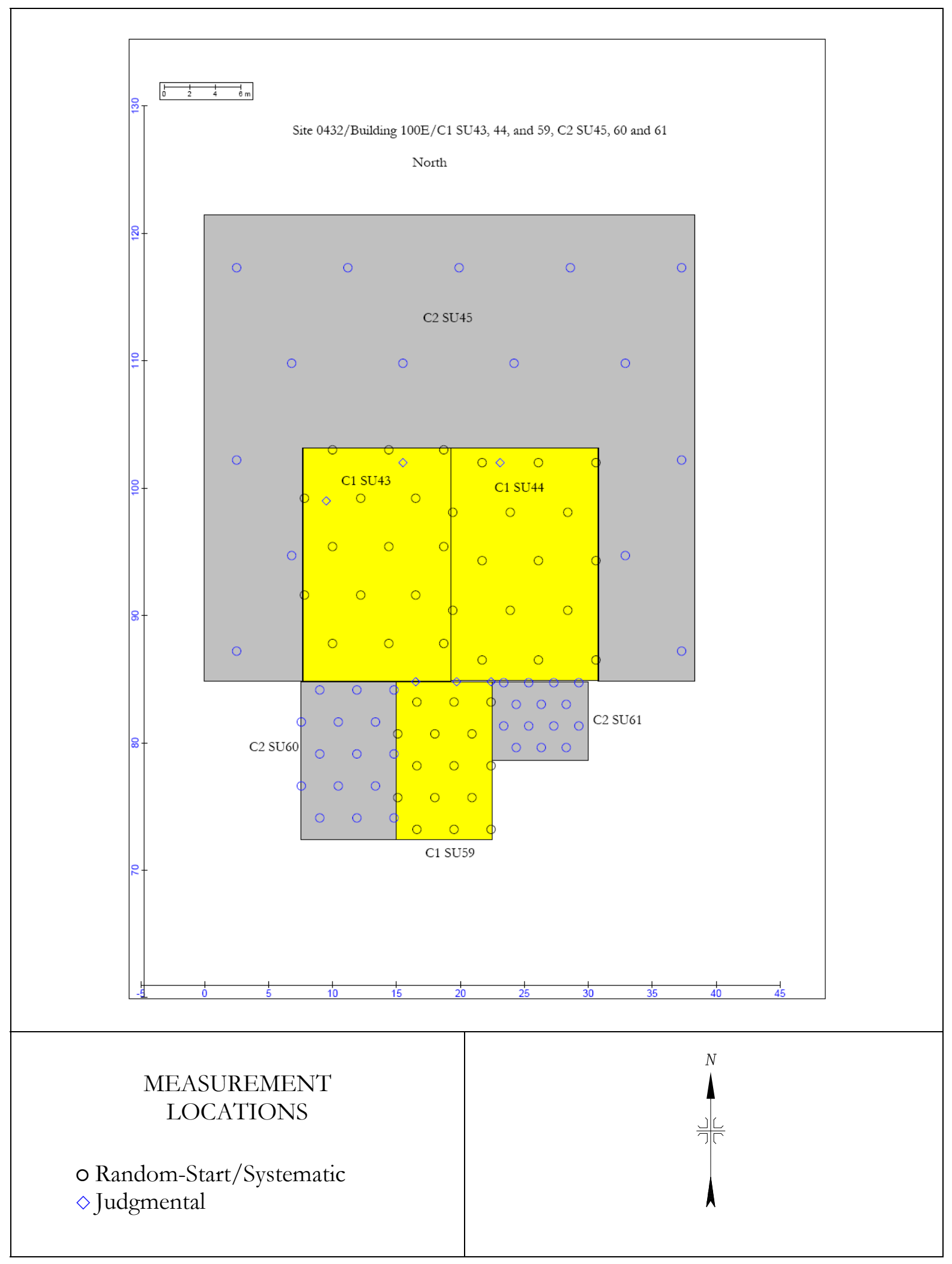

Figure A-14: Building 100E, North-end Floor (C1 SU43, 44, and 59; C2 SU45, 60, and 61)_FSS Survey Units and Direct Measurement Locations 


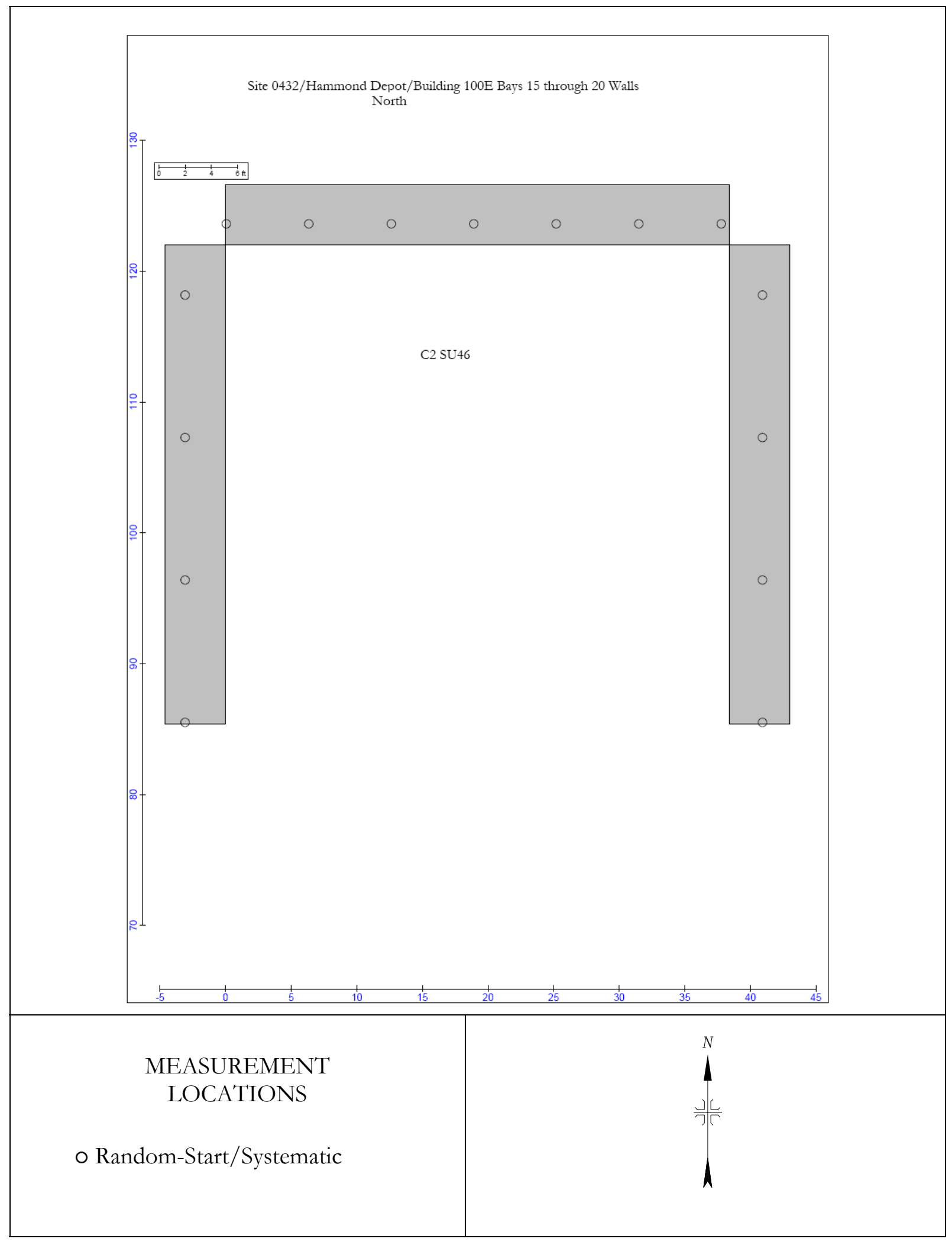

Figure A-15: Building 100E, North-end Walls (C2 SU46)—FSS Survey Units and Direct Measurement Locations 


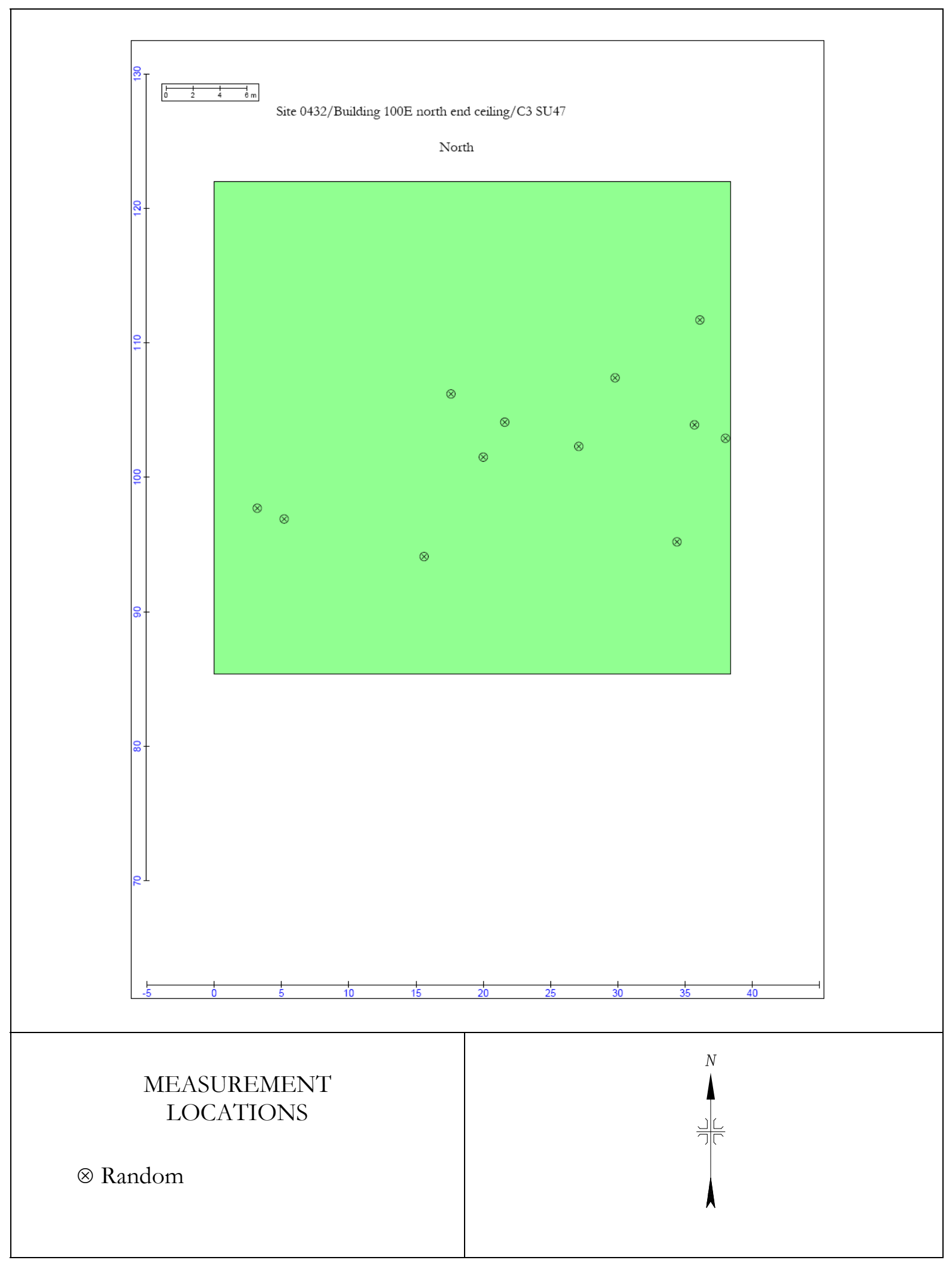

Figure A-16: Building 100E, North-end Ceiling (C3 SU47)—FSS Survey Units and Direct Measurement Locations 


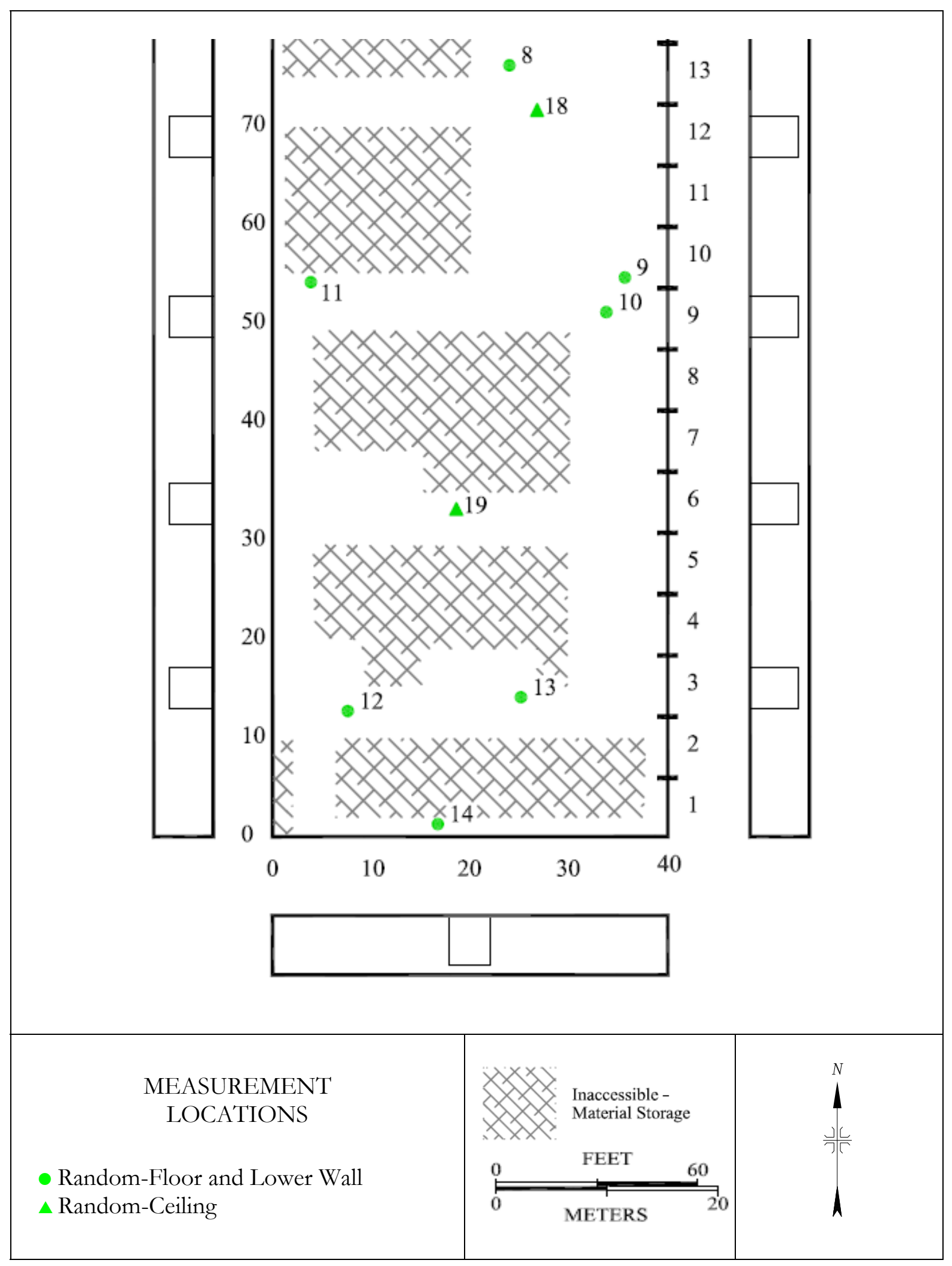

Figure A-17: Building 100E, North-end Ceiling (C3 SU47)—FSS Survey Units and Direct Measurement Locations 


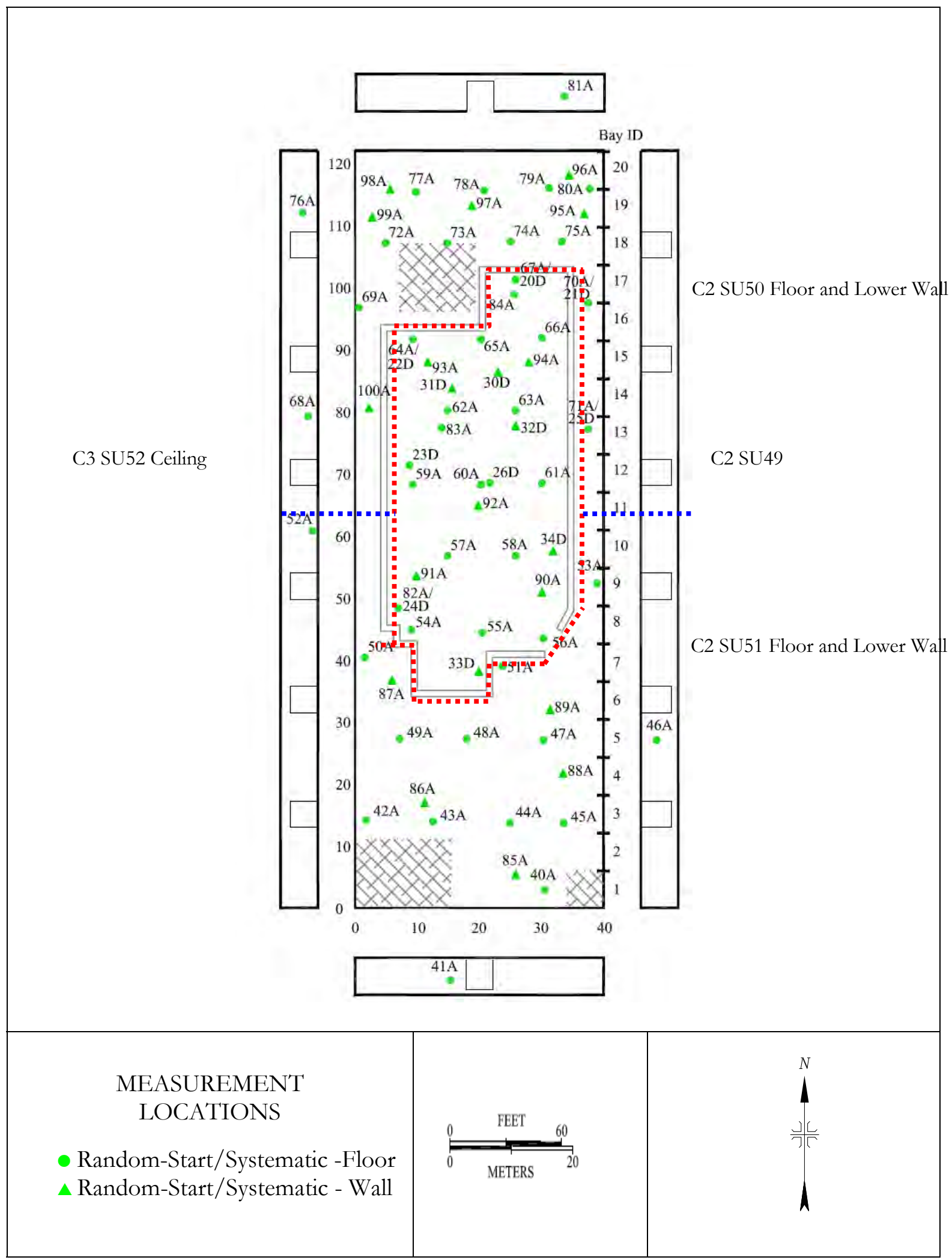

Figure A-18: Building 100W (C2 SU49 through 51 and C3 SU52)—FSS Survey Units and Direct Measurement Locations 


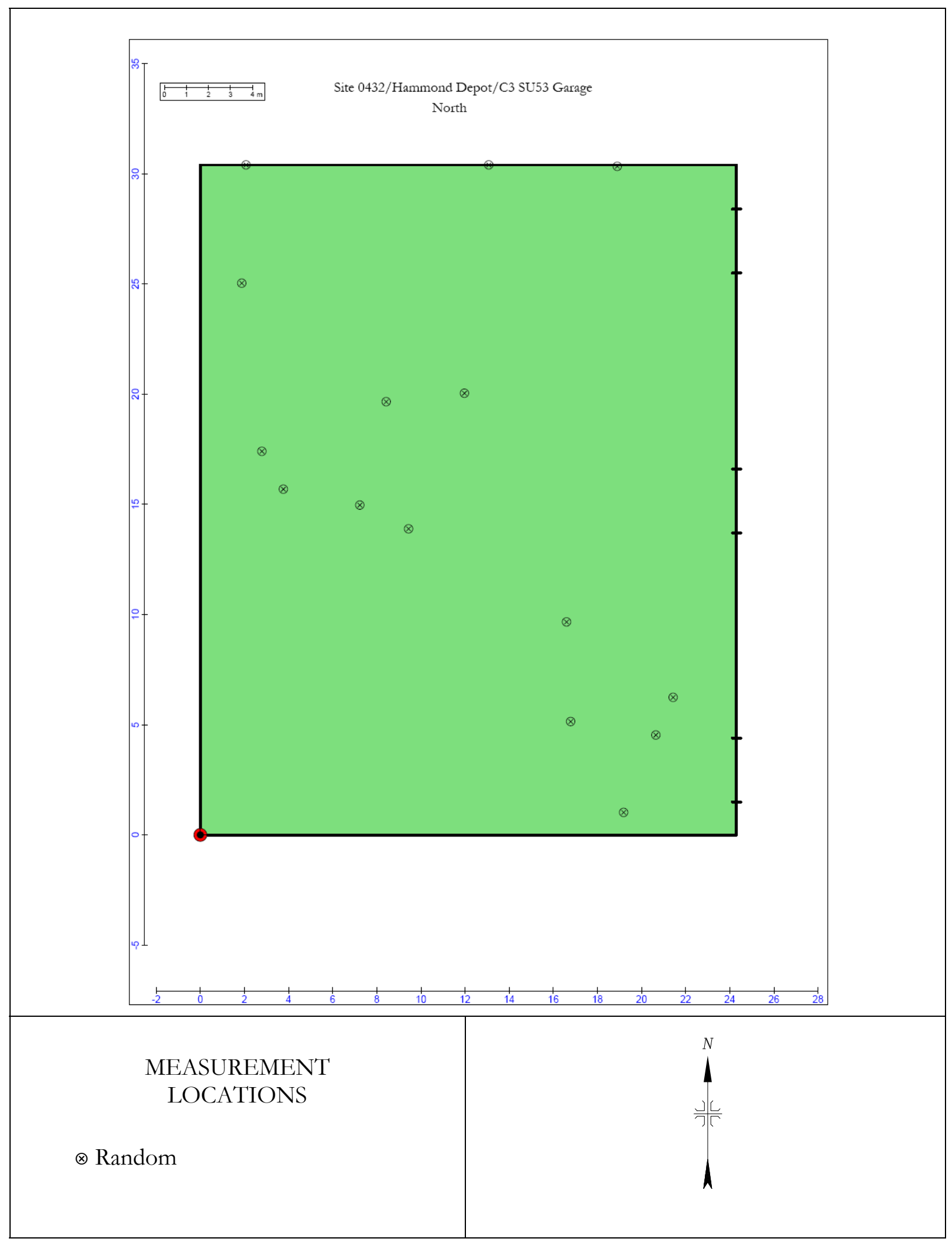

Figure A-19: Garage Building (C3 SU53)—FSS Survey Unit and Direct Measurement Locations 


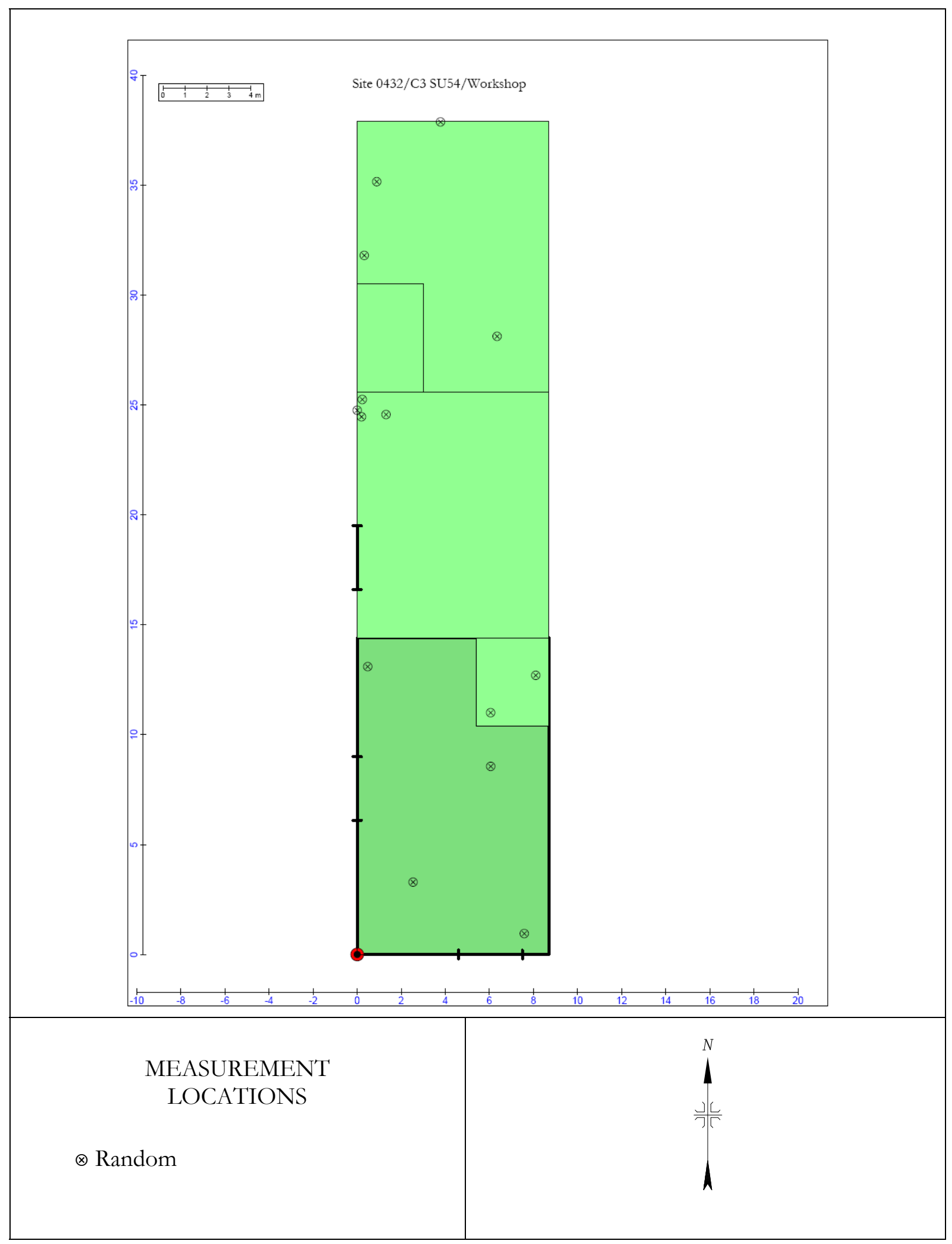

Figure A-20: Workshop Building (C3 SU54)—FSS Survey Unit and Direct Measurement Locations 


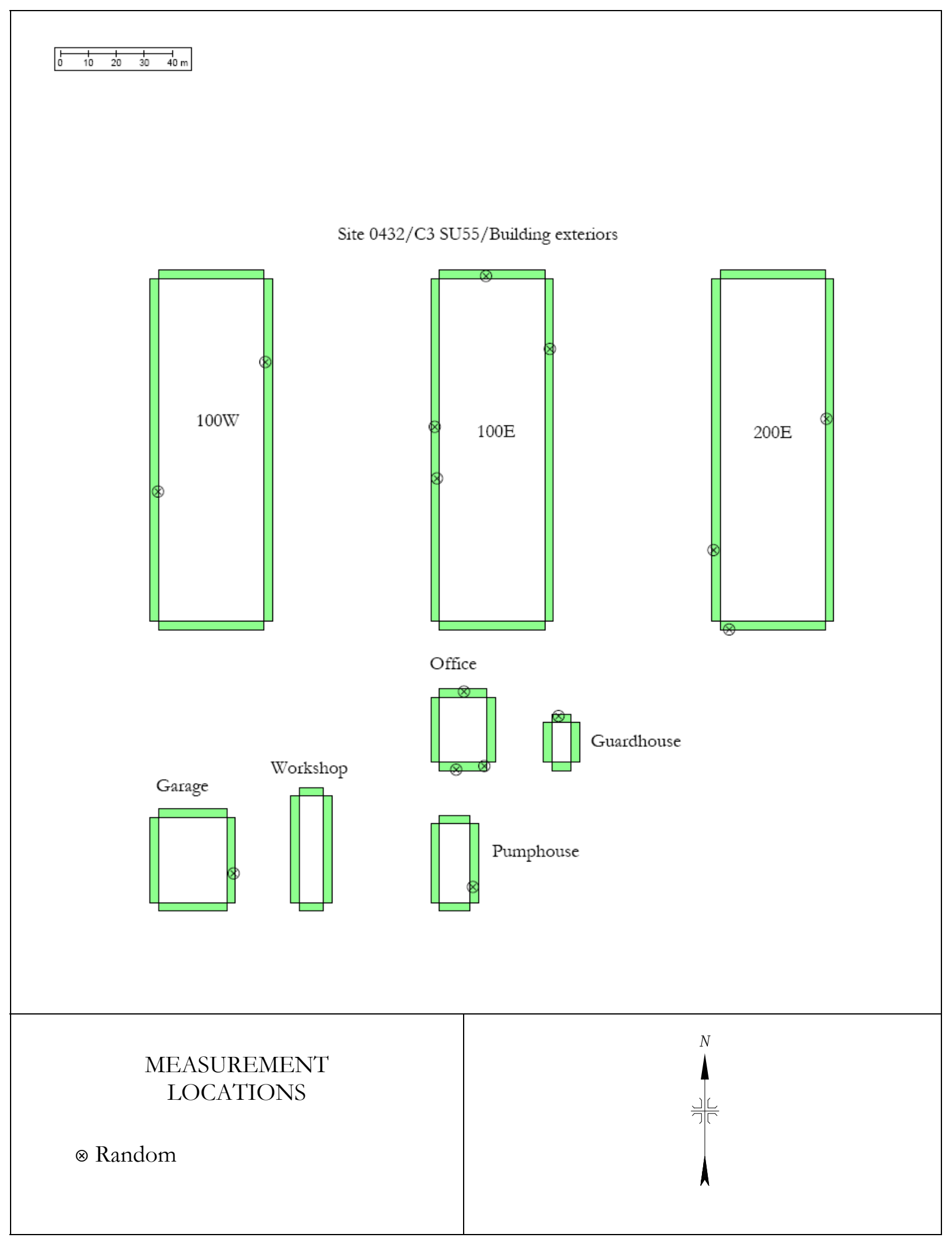

Figure A-21: Building Exteriors (C3 SU55)_FSS Survey Unit and Direct Measurement Locations 


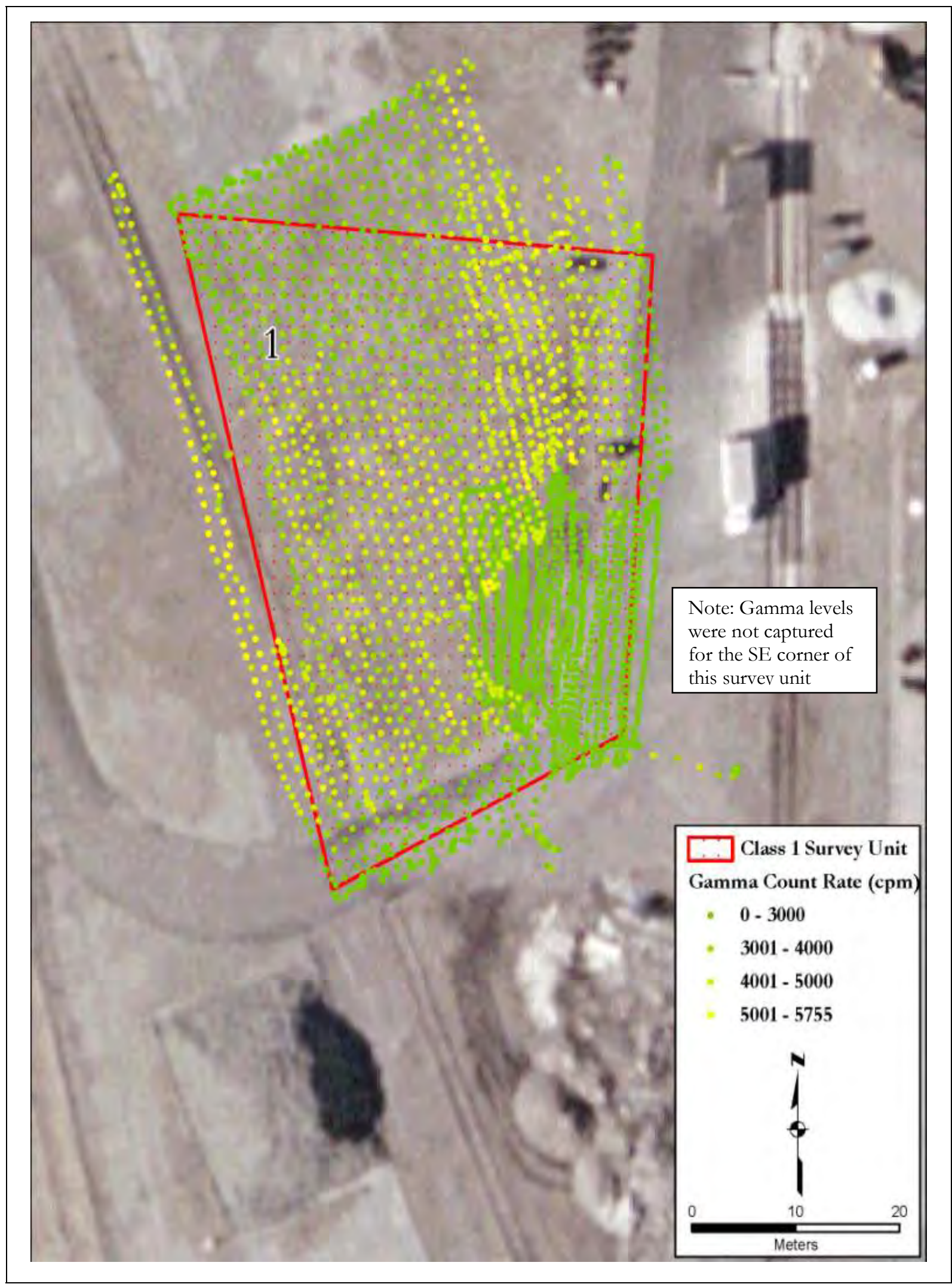

Figure A-22: Survey Unit C1 SU1-FSS Gamma Scan Results 


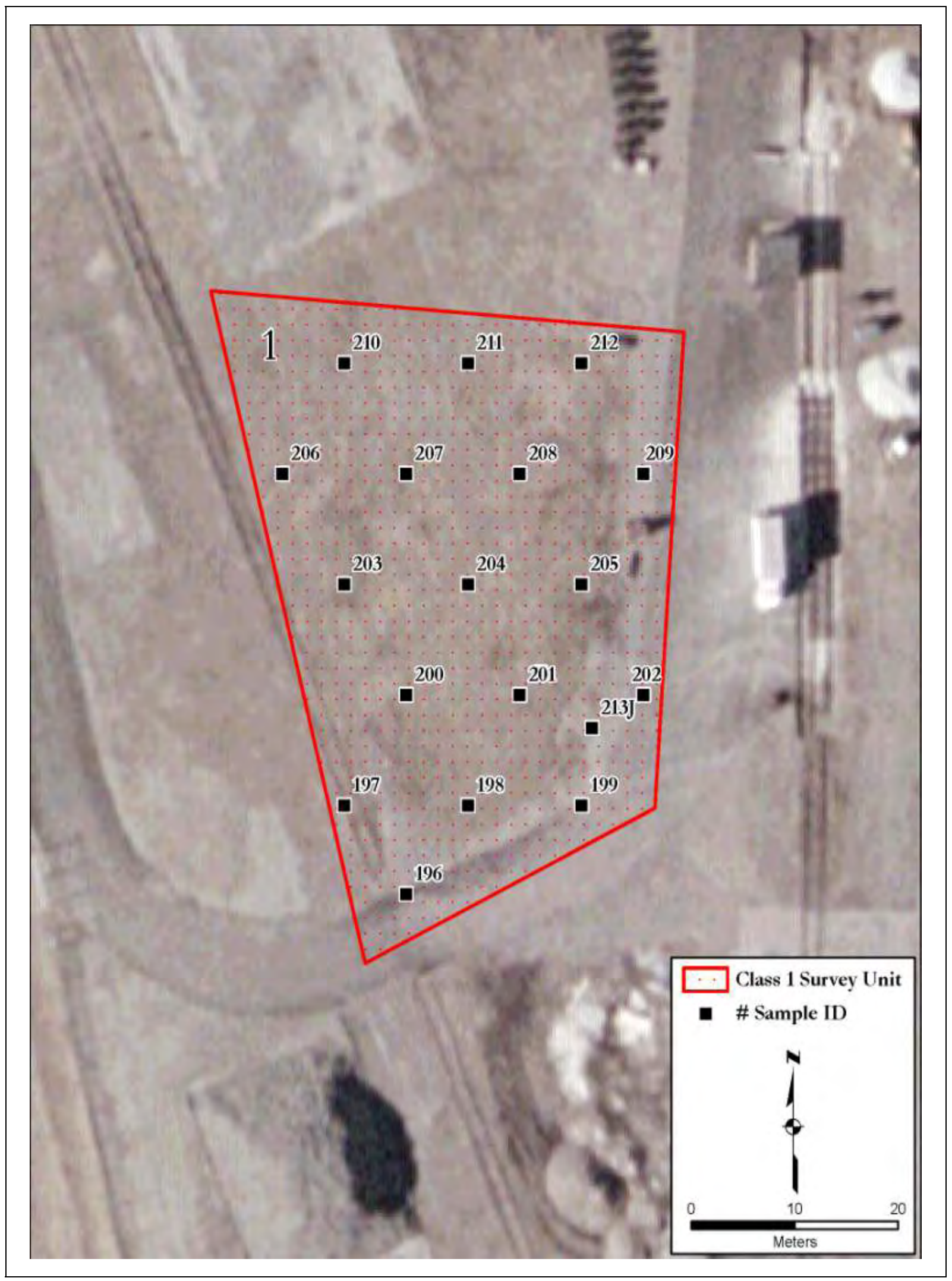

Figure A-23: Survey Unit C1 SU1-FSS Soil Sampling Locations 


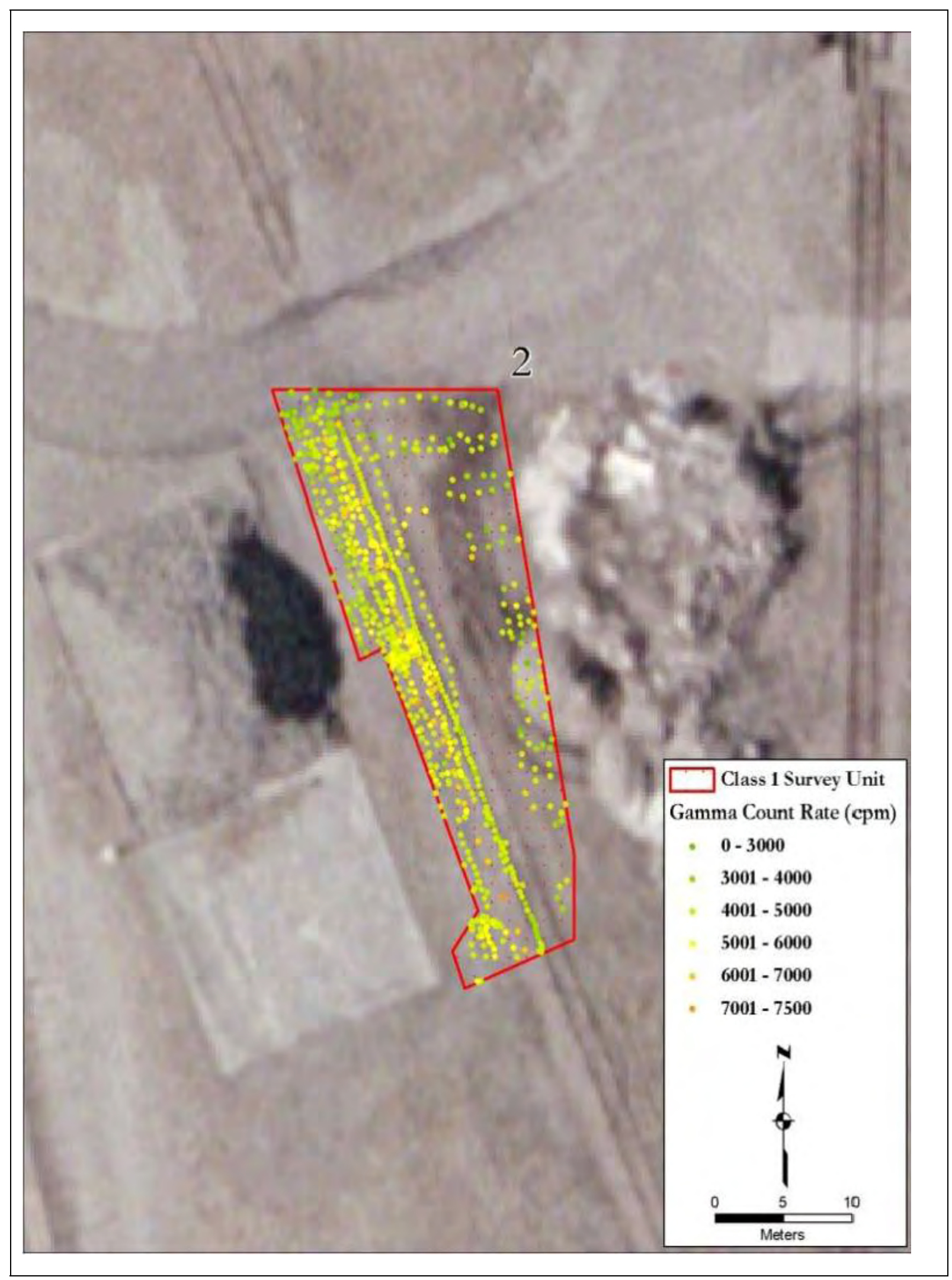

Figure A-24: Survey Unit Survey Unit C1 SU2-FSS Gamma Scan Results 


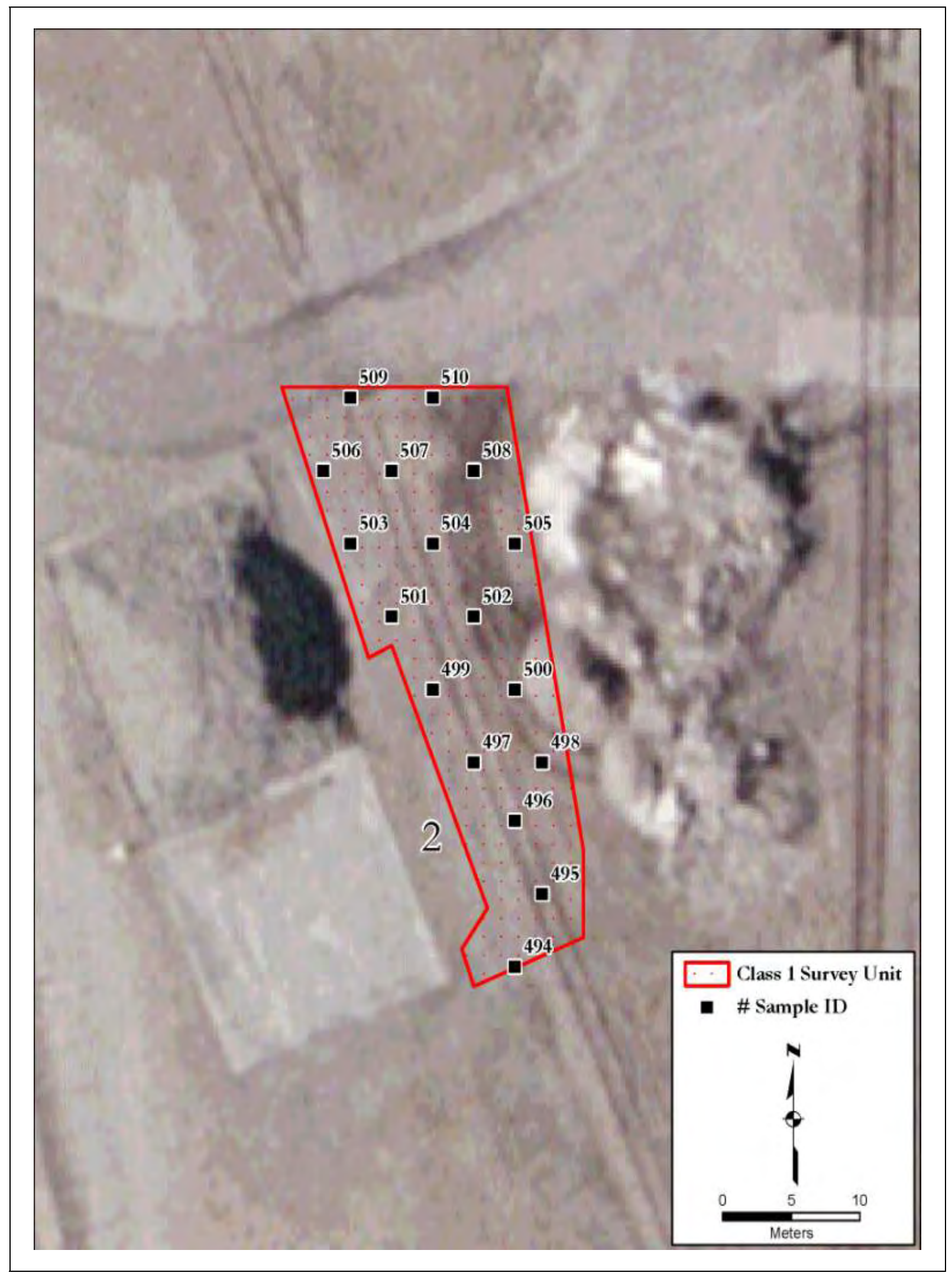

Figure A-25: Survey Unit C1 SU2-FSS Soil Sampling Locations 


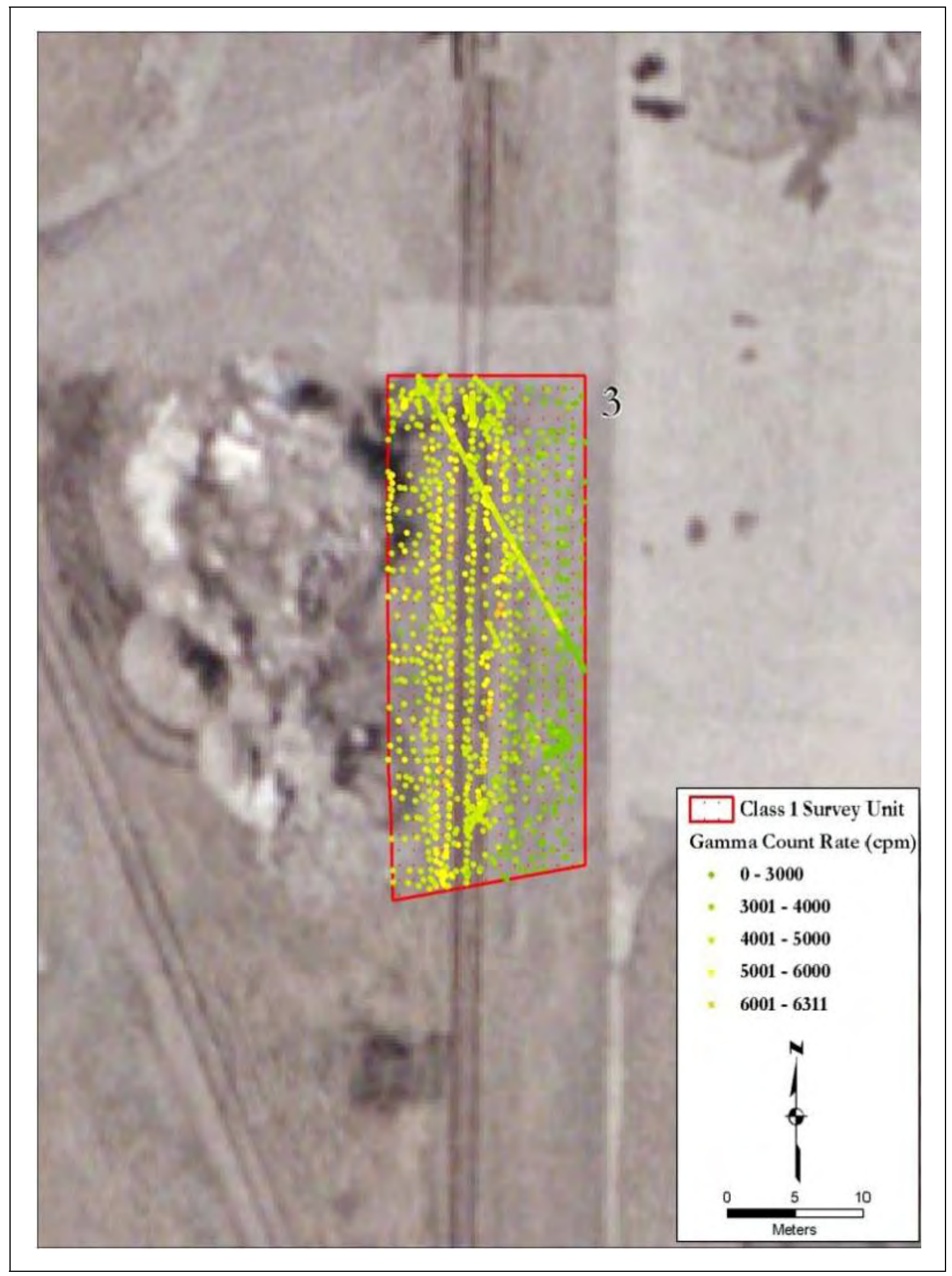

Figure A-26: Survey Unit C1 SU3-FSS Gamma Scan Results 


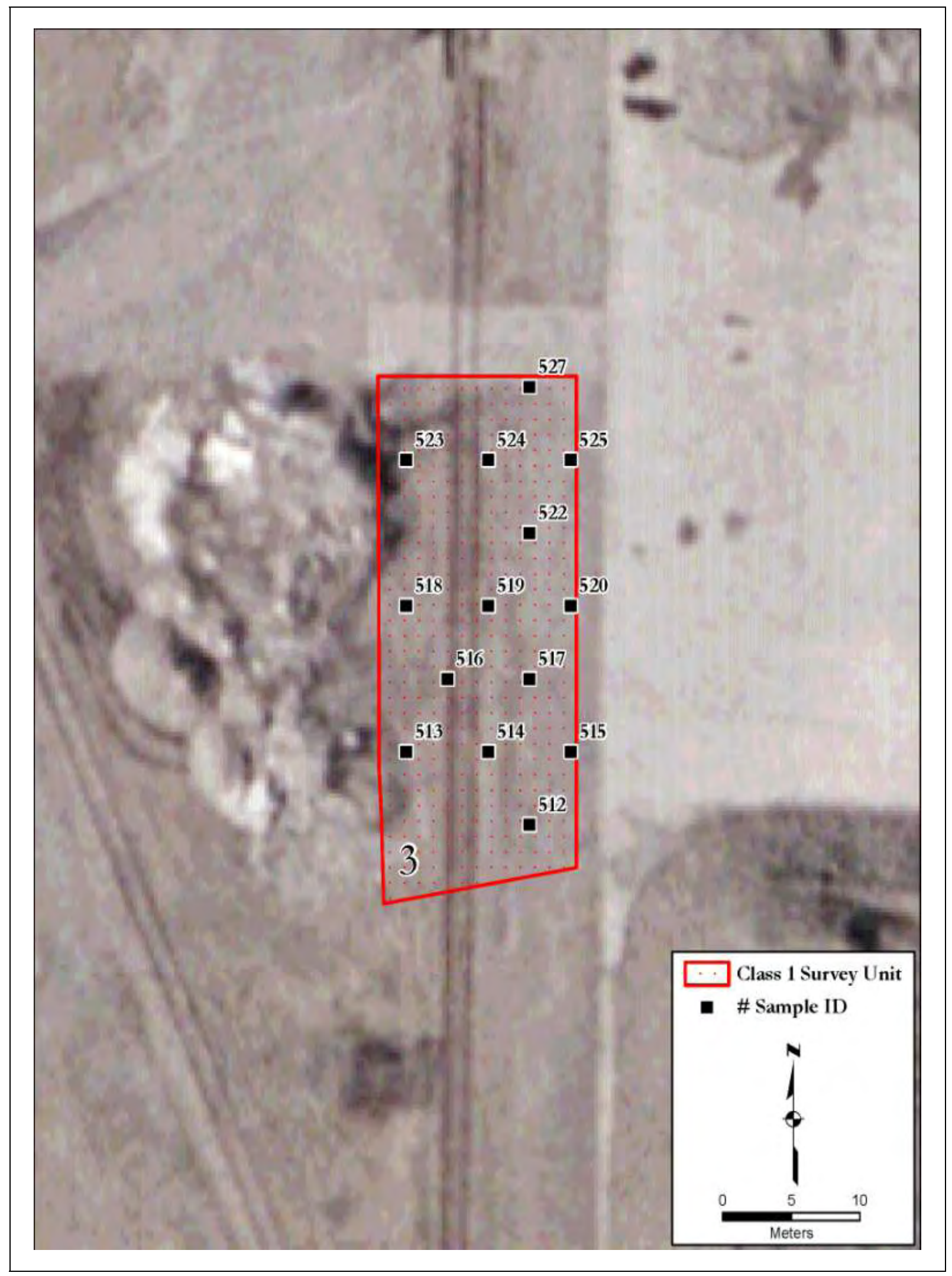

Figure A-27: Survey Unit C1 SU3-FSS Soil Sampling Locations 


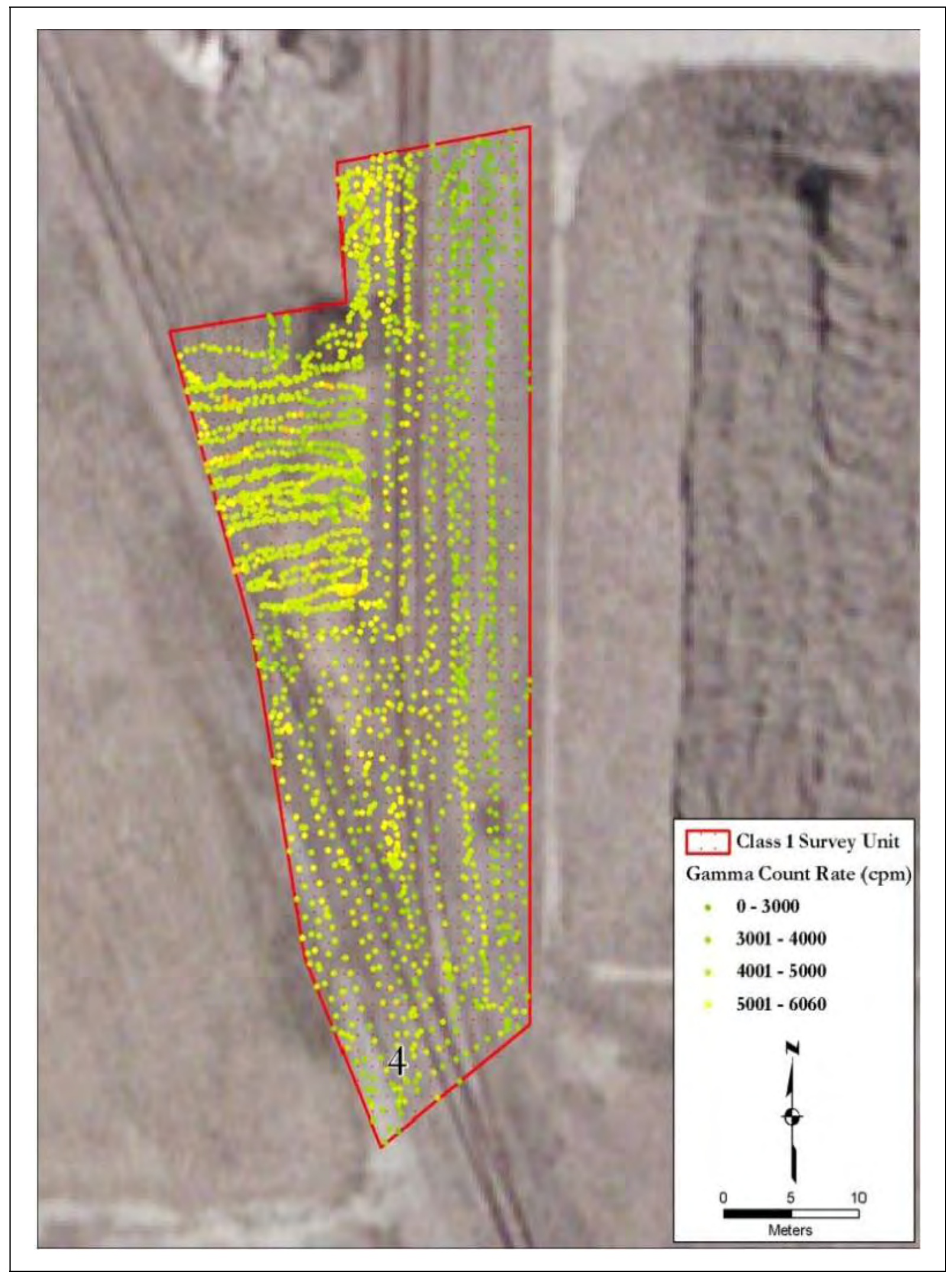

Figure A-28: Survey Unit C1 SU4-FSS Gamma Scan Results 


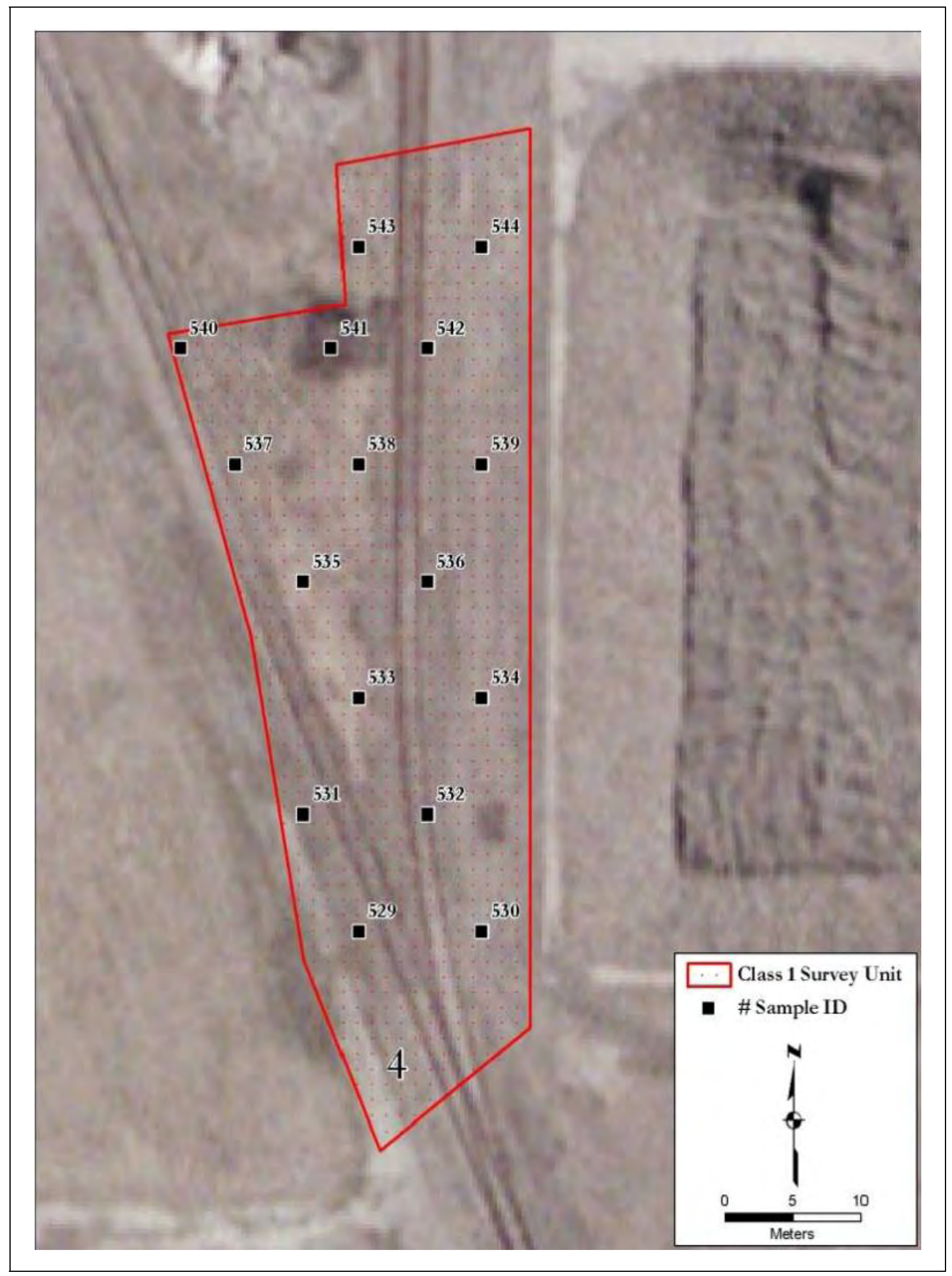

Figure A-29: Survey Unit C1 SU4-FSS Soil Sampling Locations 


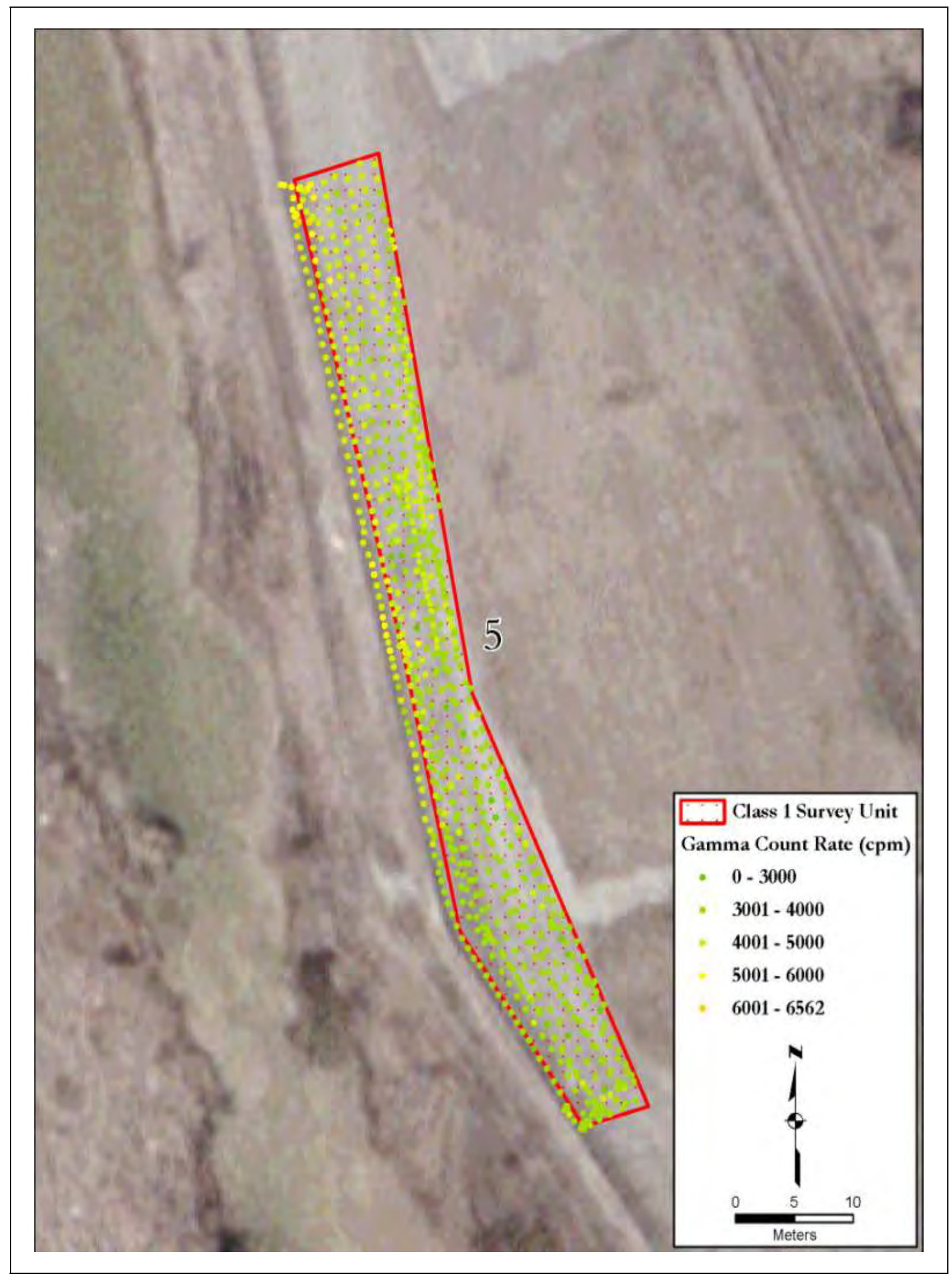

Figure A-30: Survey Unit C1 SU5-FSS Gamma Scan Results 


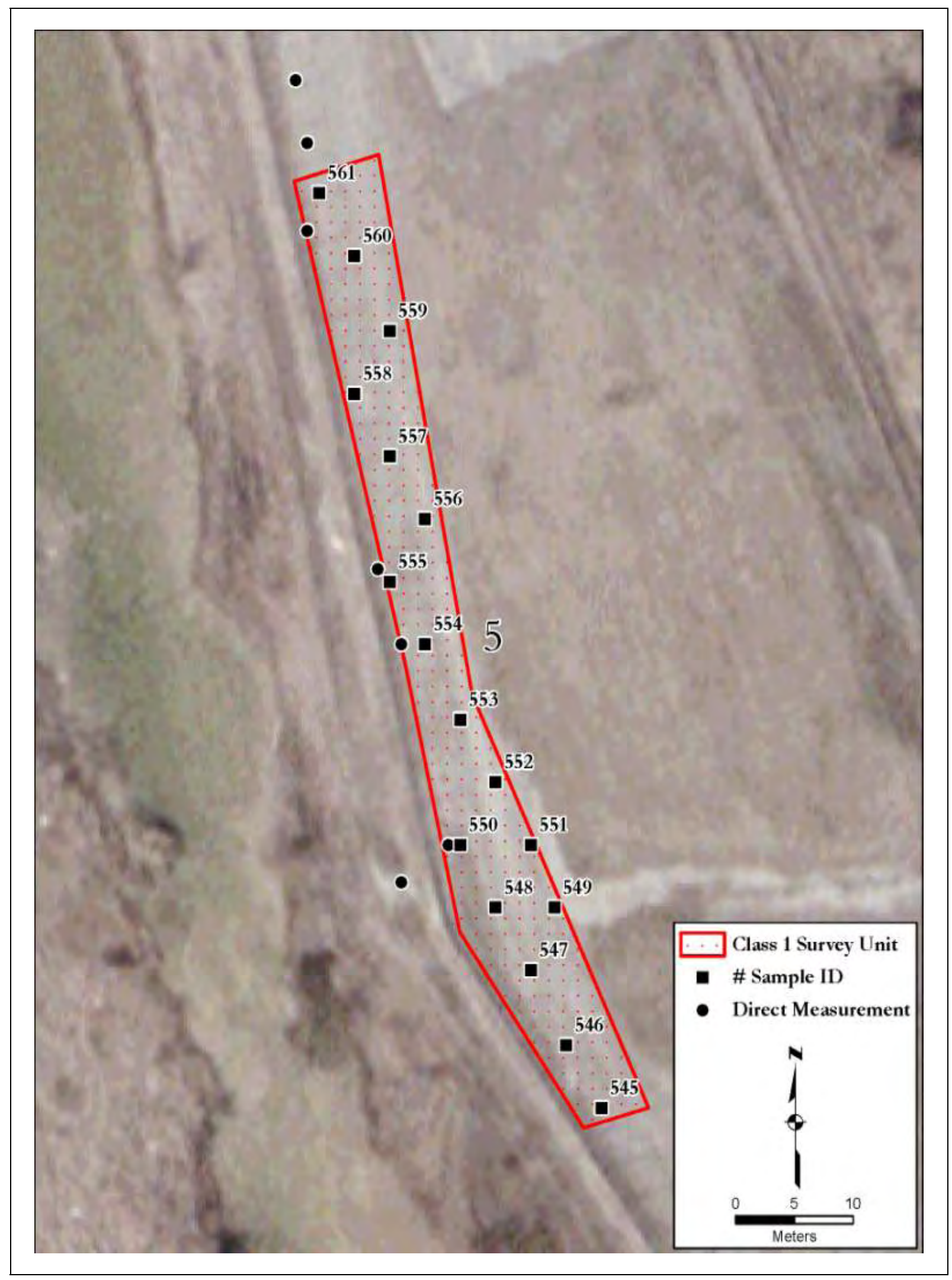

Figure A-31: Survey Unit C1 SU5-FSS Soil Sampling Locations 


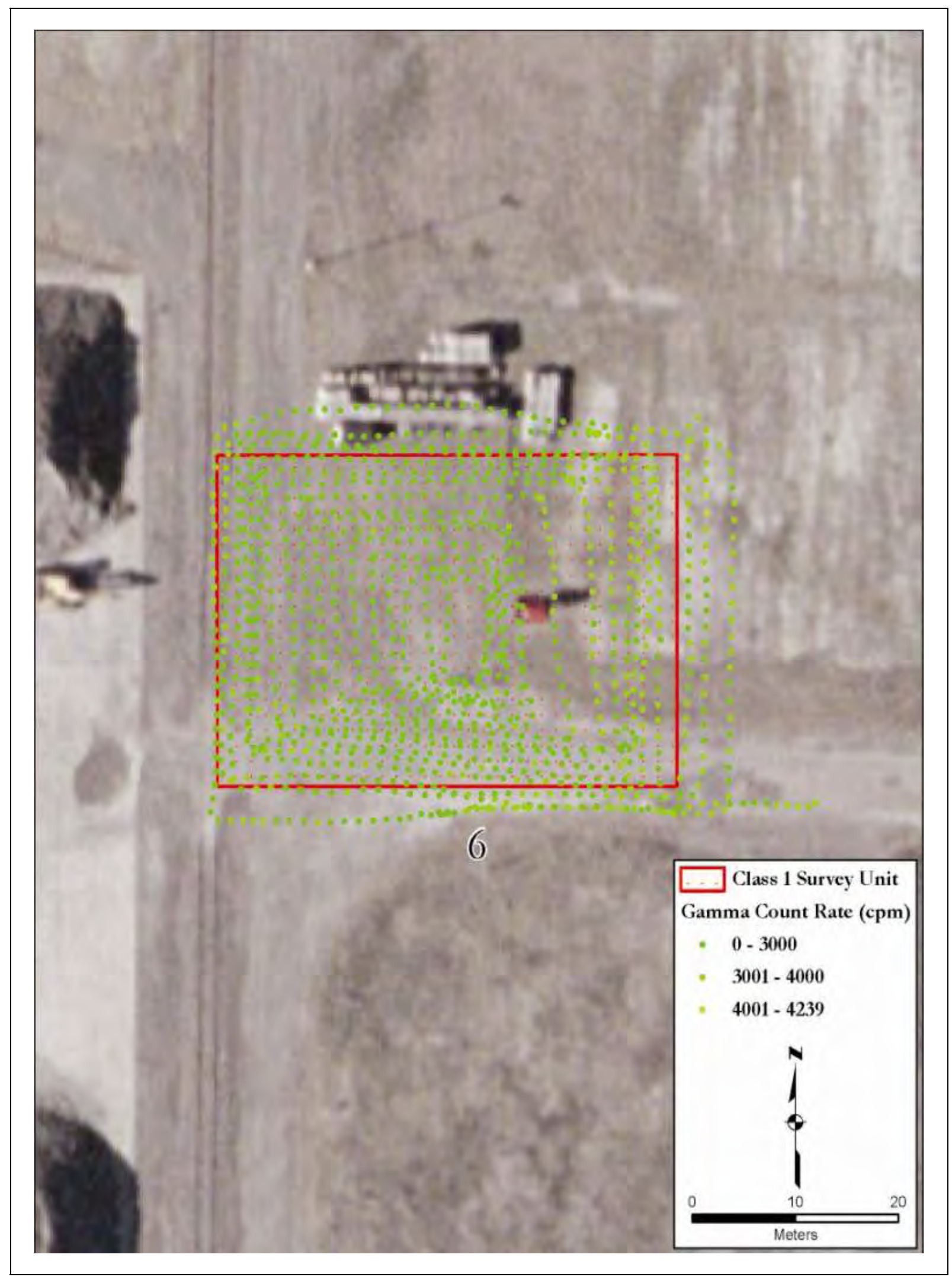

Figure A-32: Survey Unit C1 SU6-FSS Gamma Scan Results 


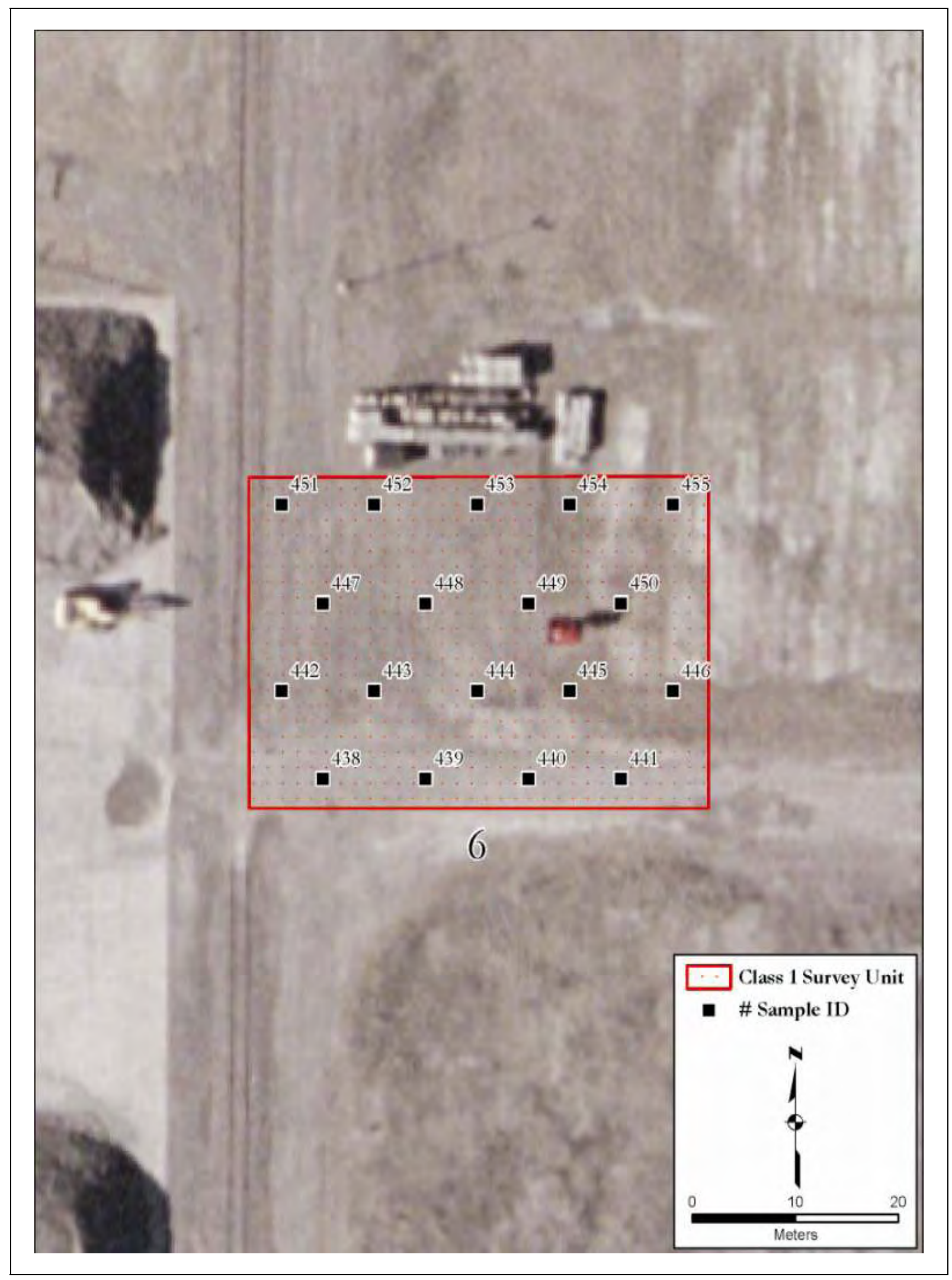

Figure A-33: Survey Unit C1 SU6_-FSS Soil Sampling Locations 


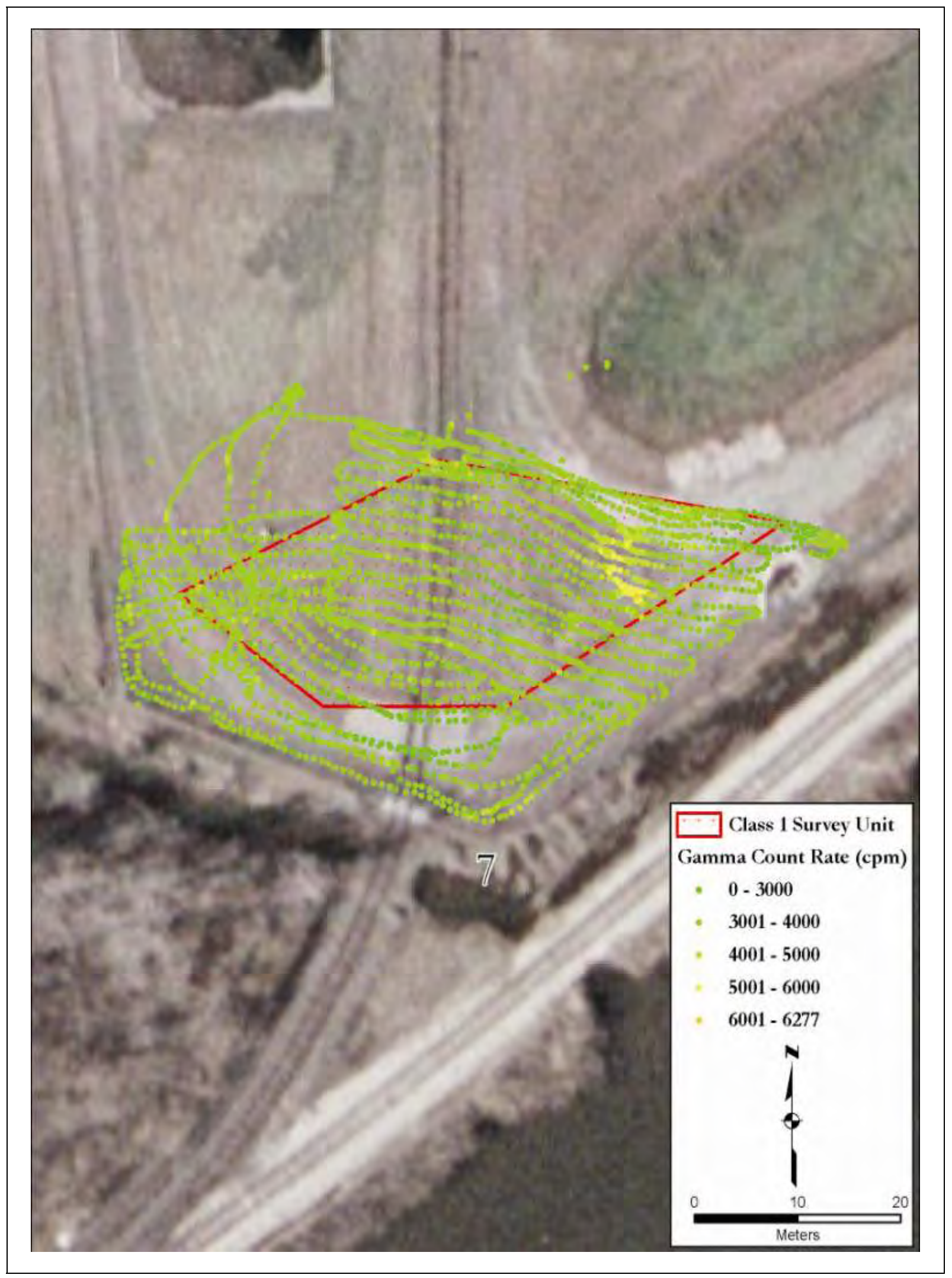

Figure A-34: Survey Unit C1 SU7-FSS Gamma Scan Results 


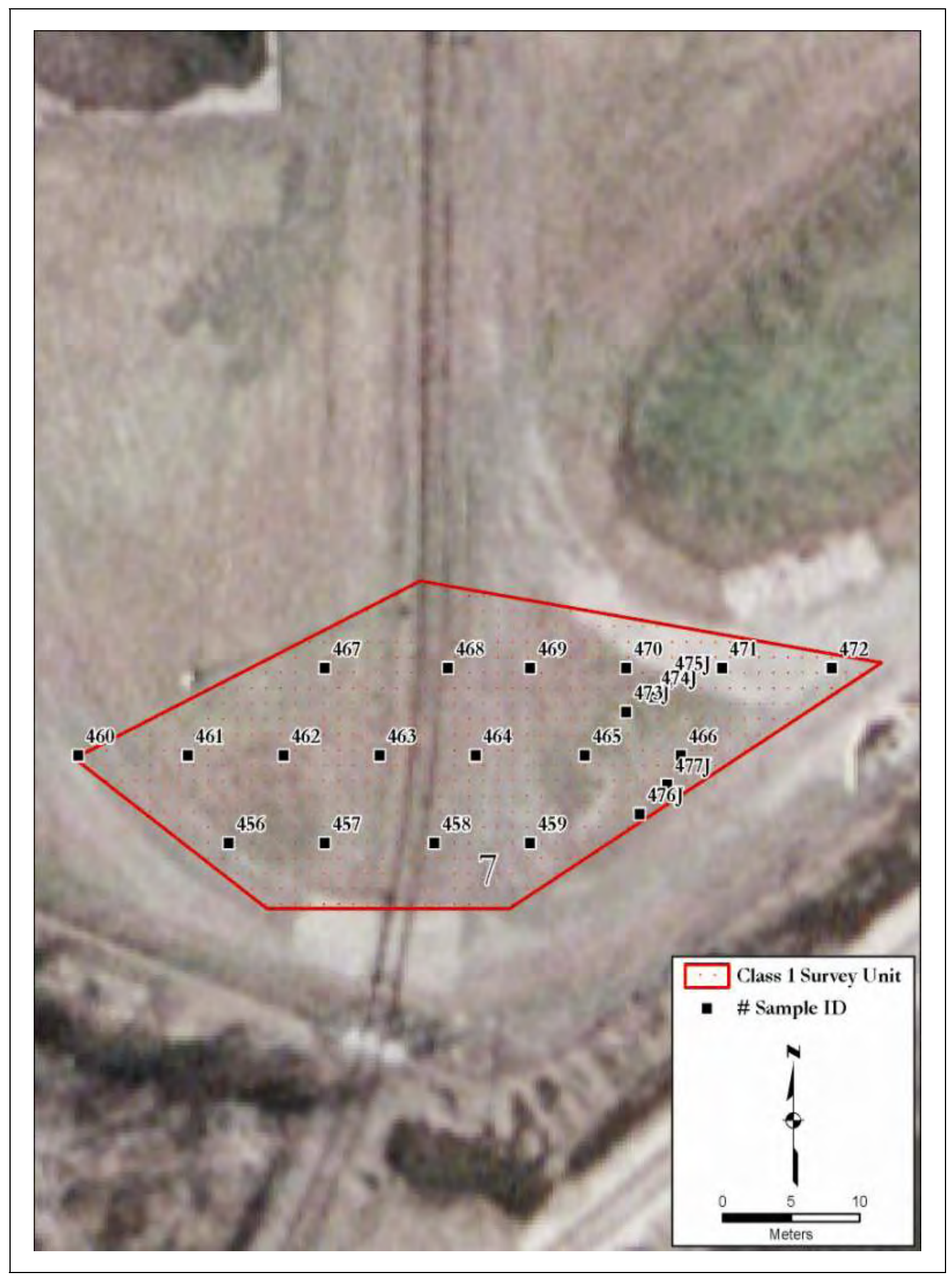

Figure A-35: Survey Unit C1 SU7-FSS Soil Sampling Locations 


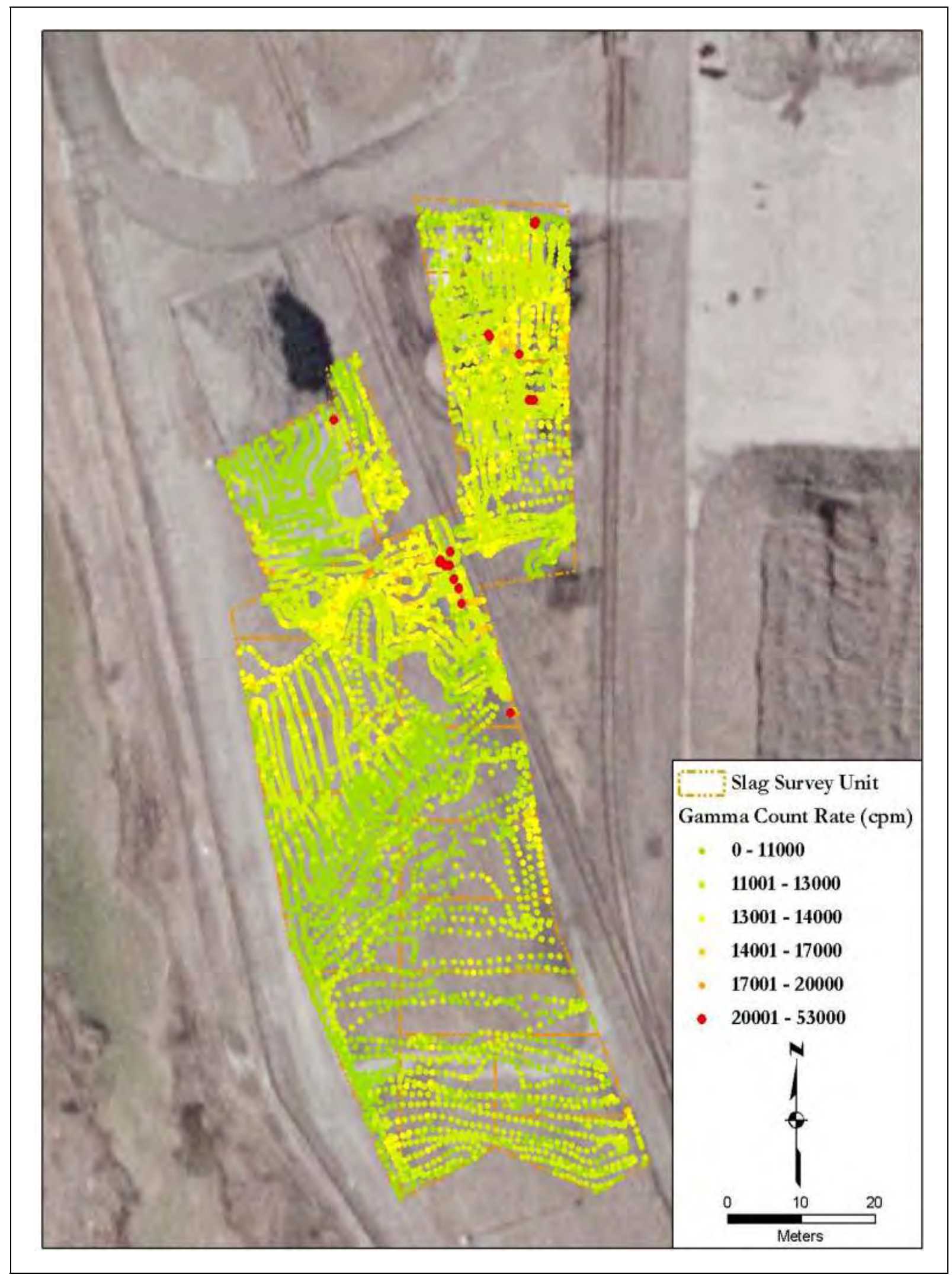

Figure A-36: Survey Units C1 SU2.1 through 5.8-FSS Gamma Scan Results 


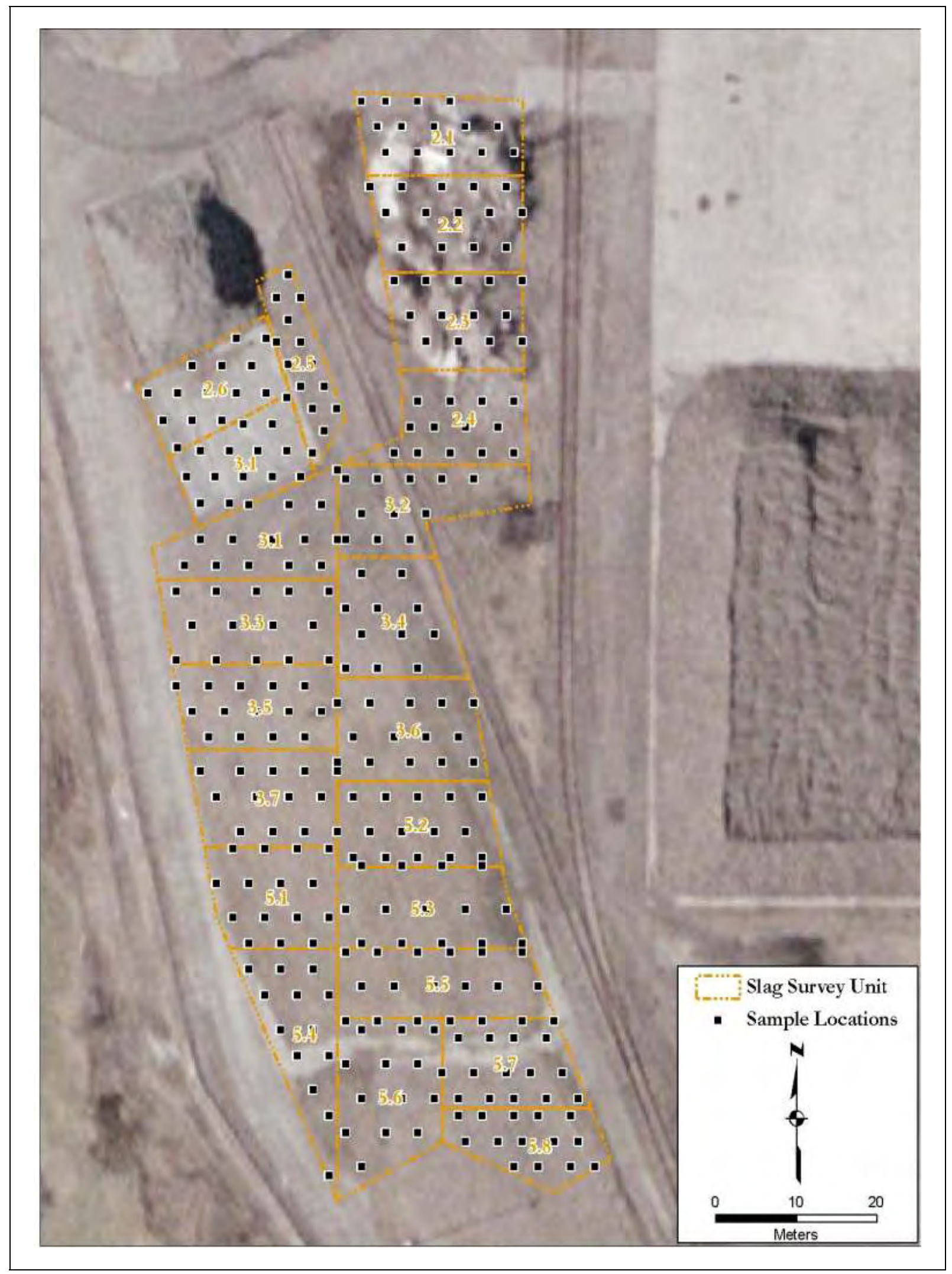

Figure A-37: Survey Unit C1 SU2.1 through 5.8-FSS Direct Measurement Locations 


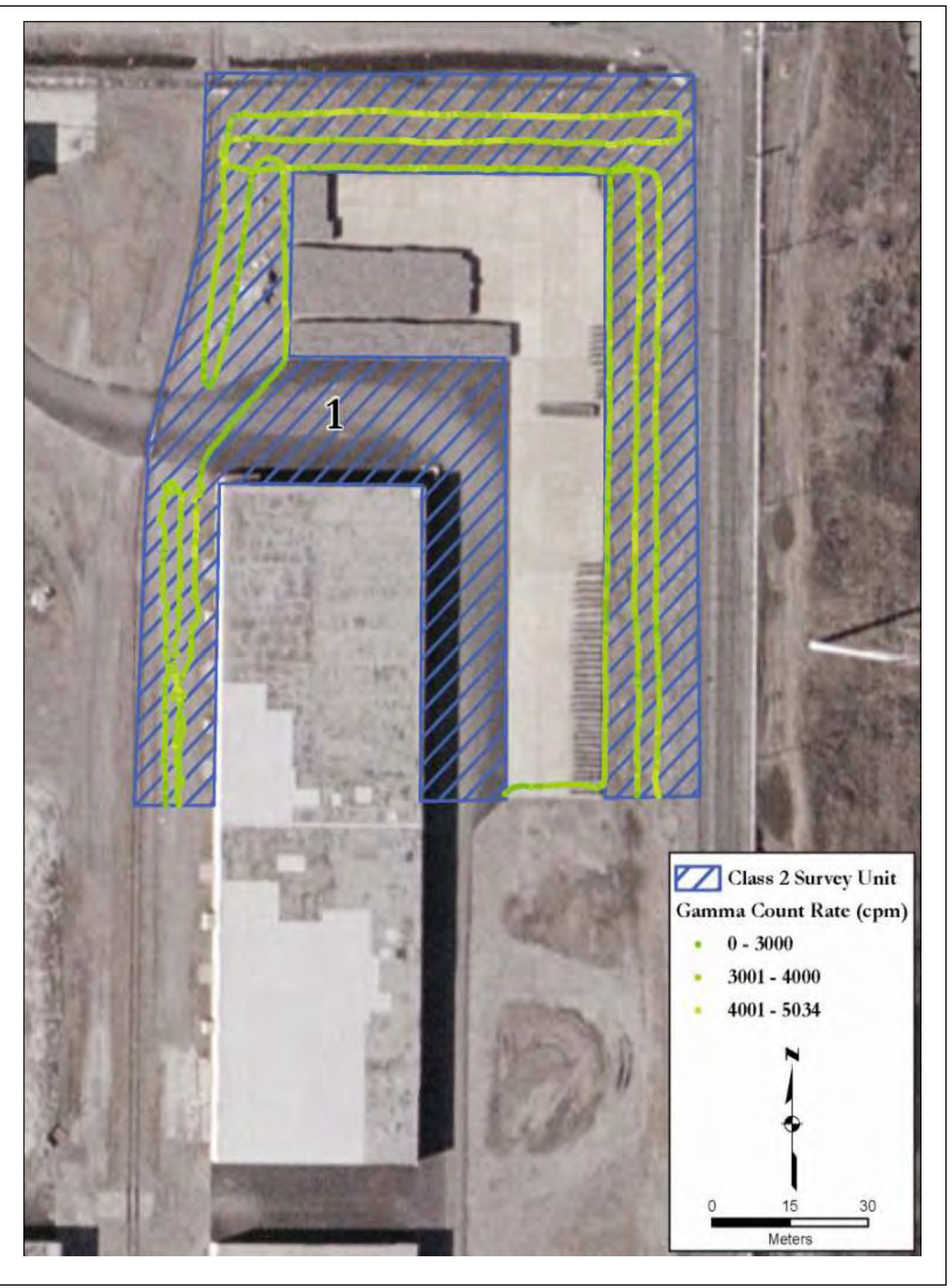

Figure A-38: Survey Unit C2 SU1—FSS Gamma Scan Results 


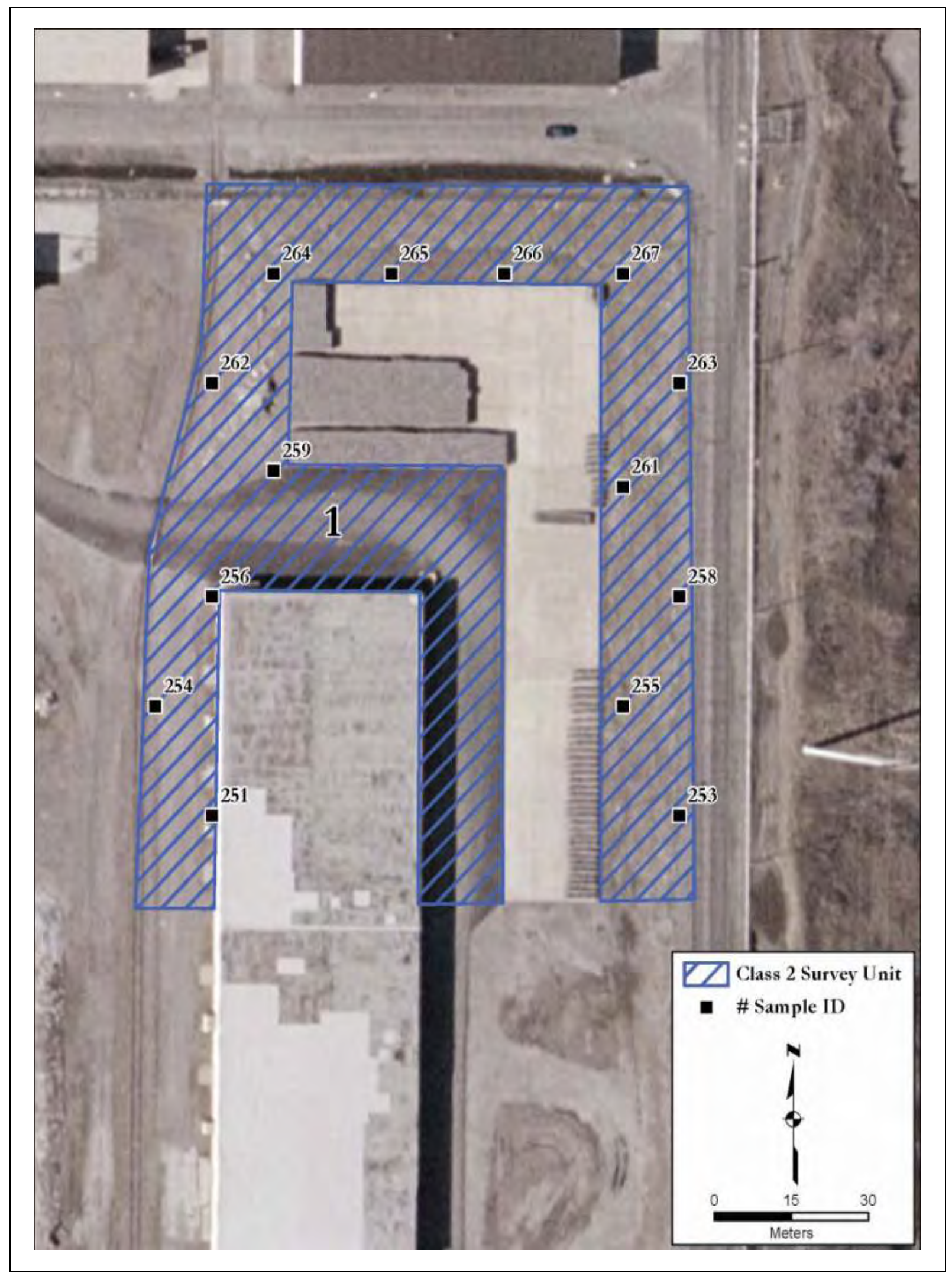

Figure A-39: Survey Unit C2 SU1-FSS Soil Sampling Locations 


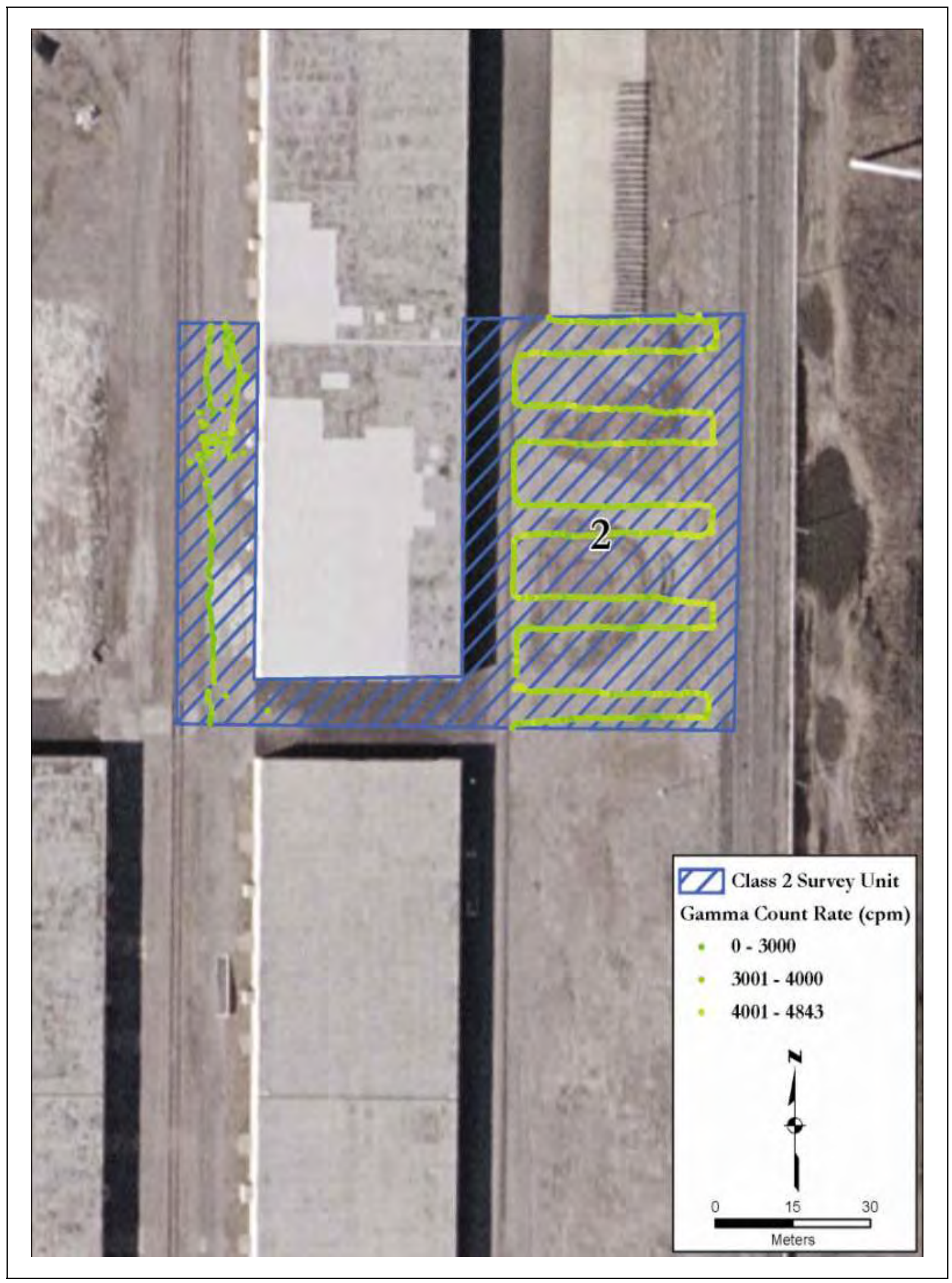

Figure A-40: Survey Unit C2 SU2-FSS Gamma Scan Results 


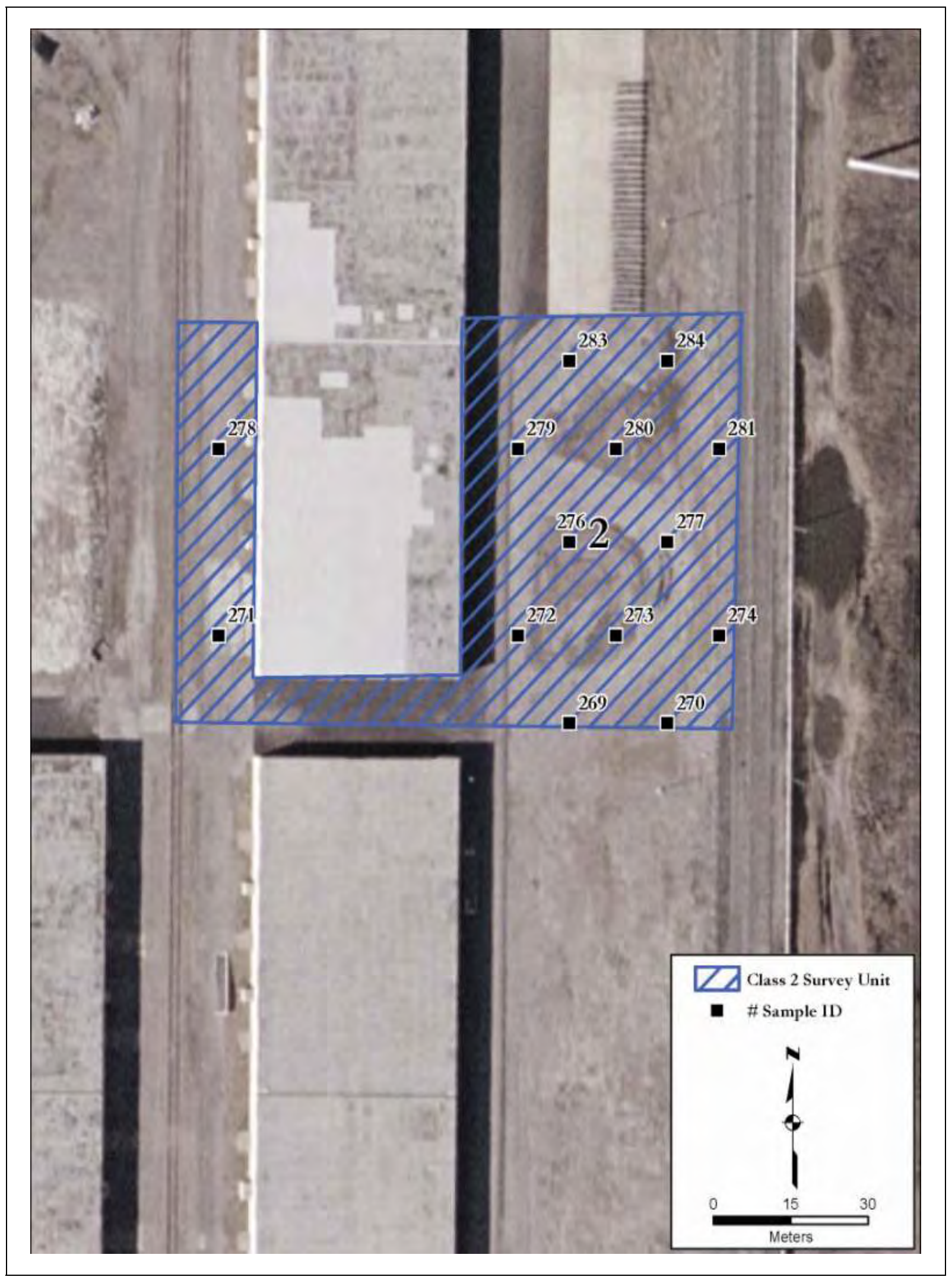

Figure A-41: Survey Unit C2 SU2-FSS Soil Sampling Locations 


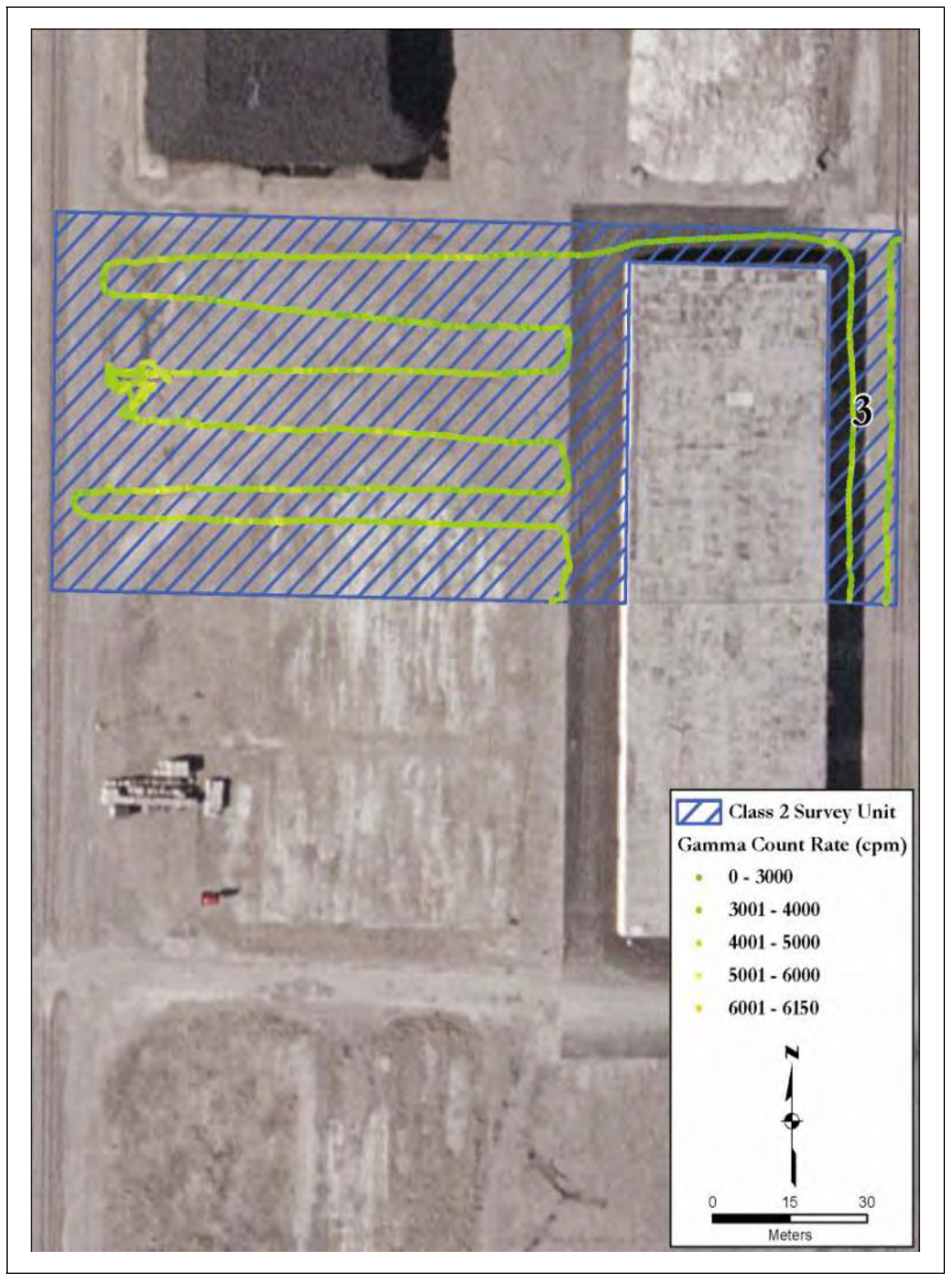

Figure A-42: Survey Unit C2 SU3-FSS Gamma Scan Results 


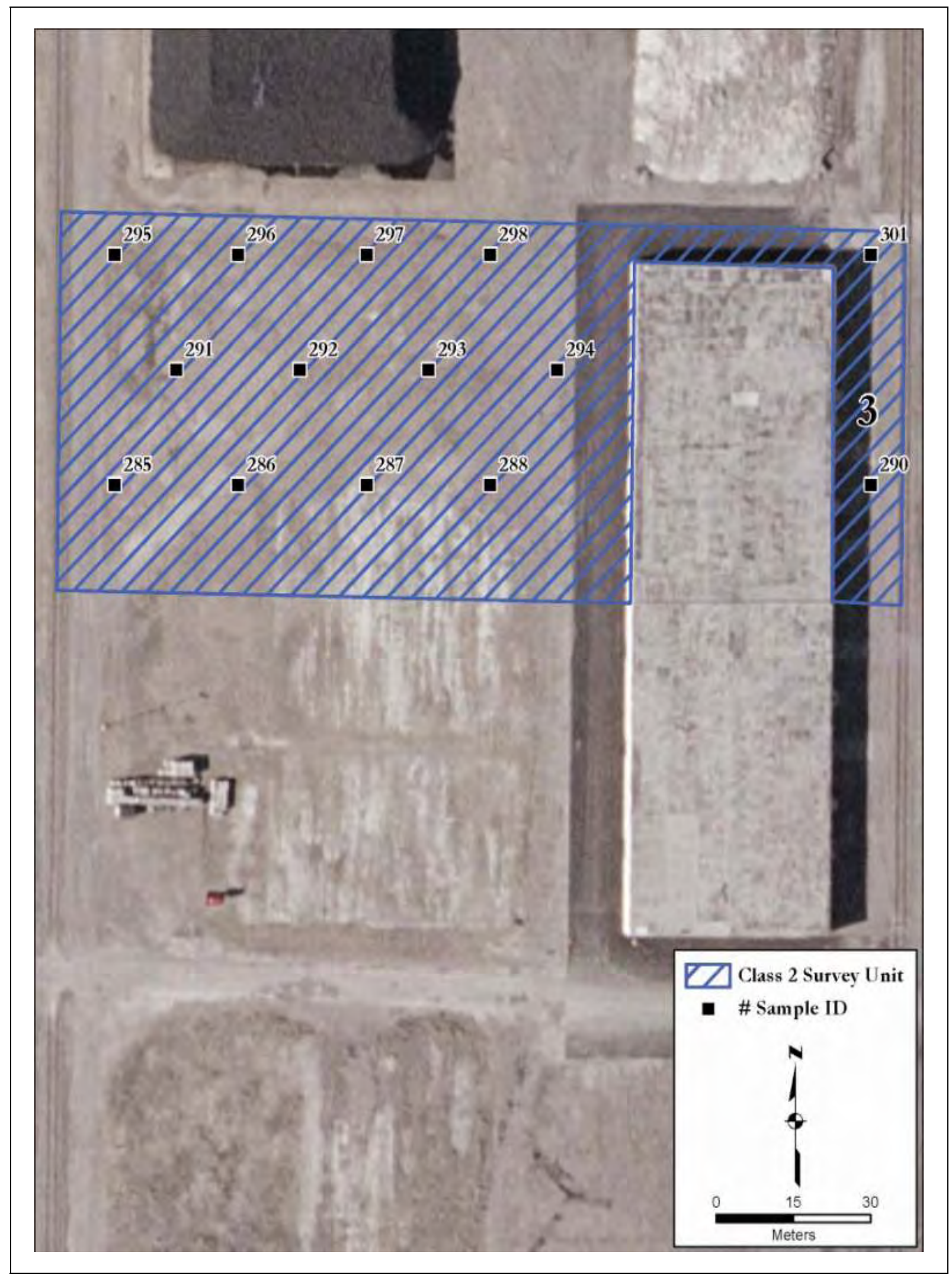

Figure A-43: Survey Unit C2 SU3-FSS Soil Sampling Locations 


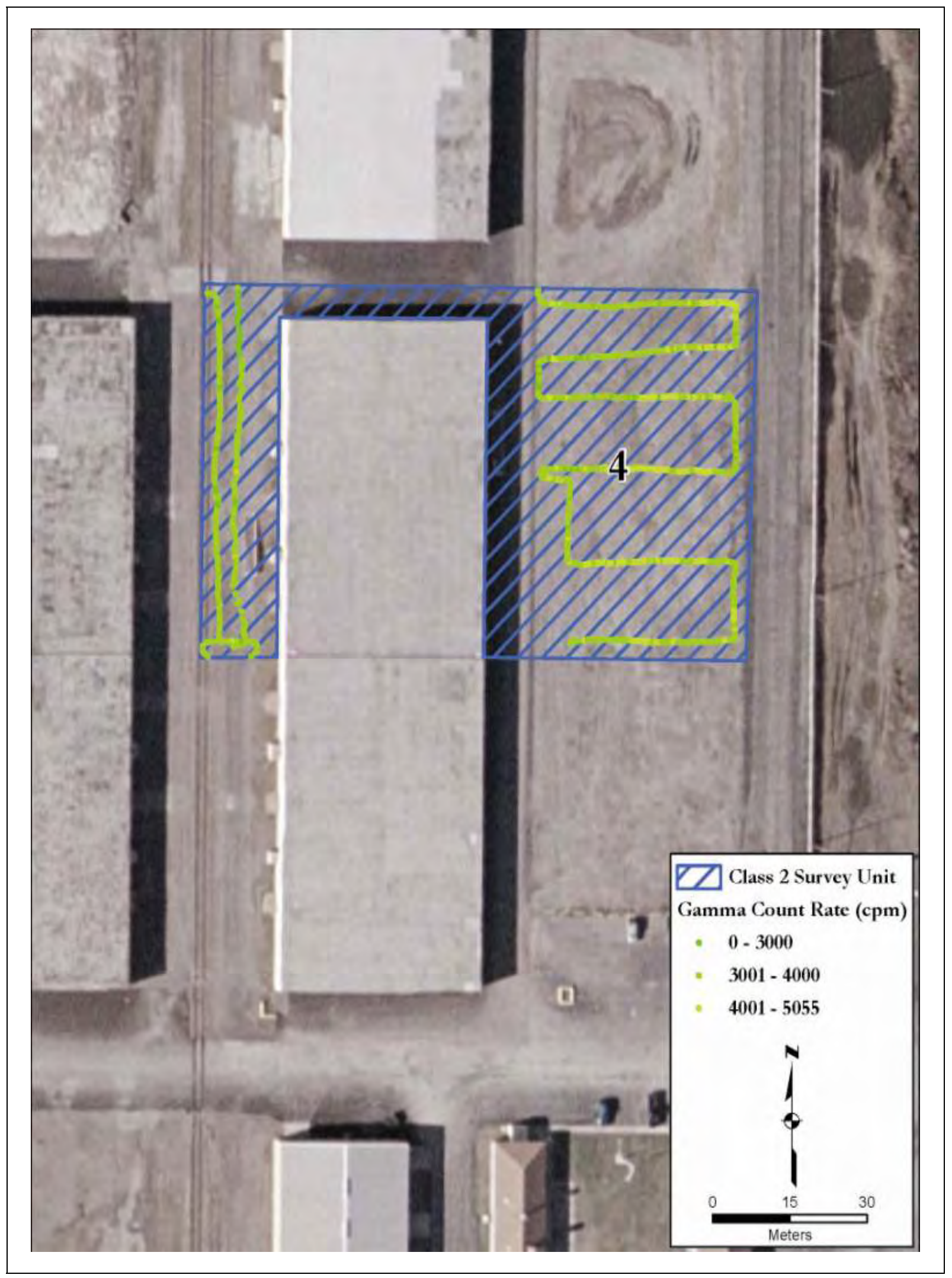

Figure A-44: Survey Unit C2 SU4—FSS Gamma Scan Results 


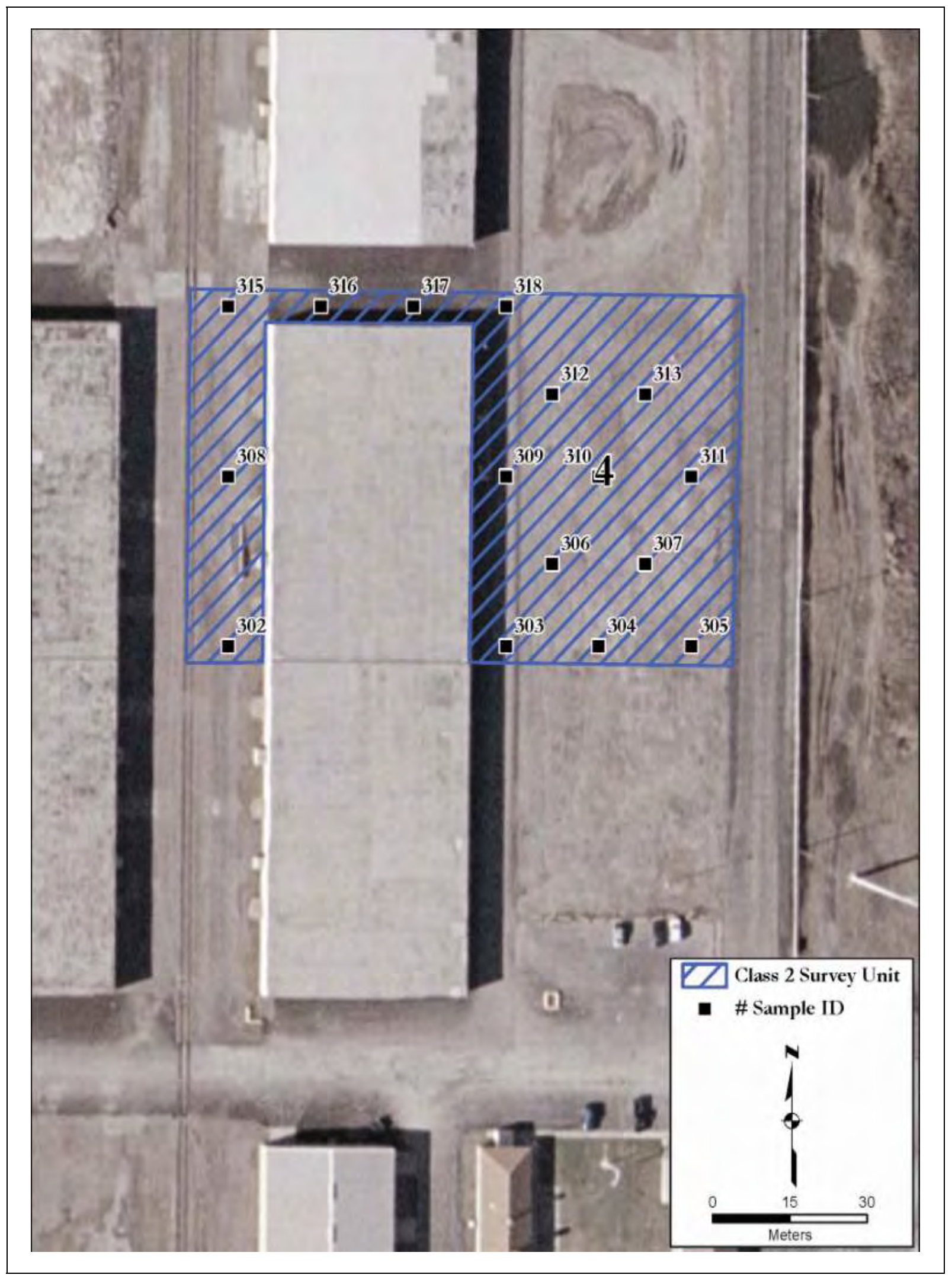

Figure A-45: Survey Unit C2 SU4-FSS Soil Sampling Locations 


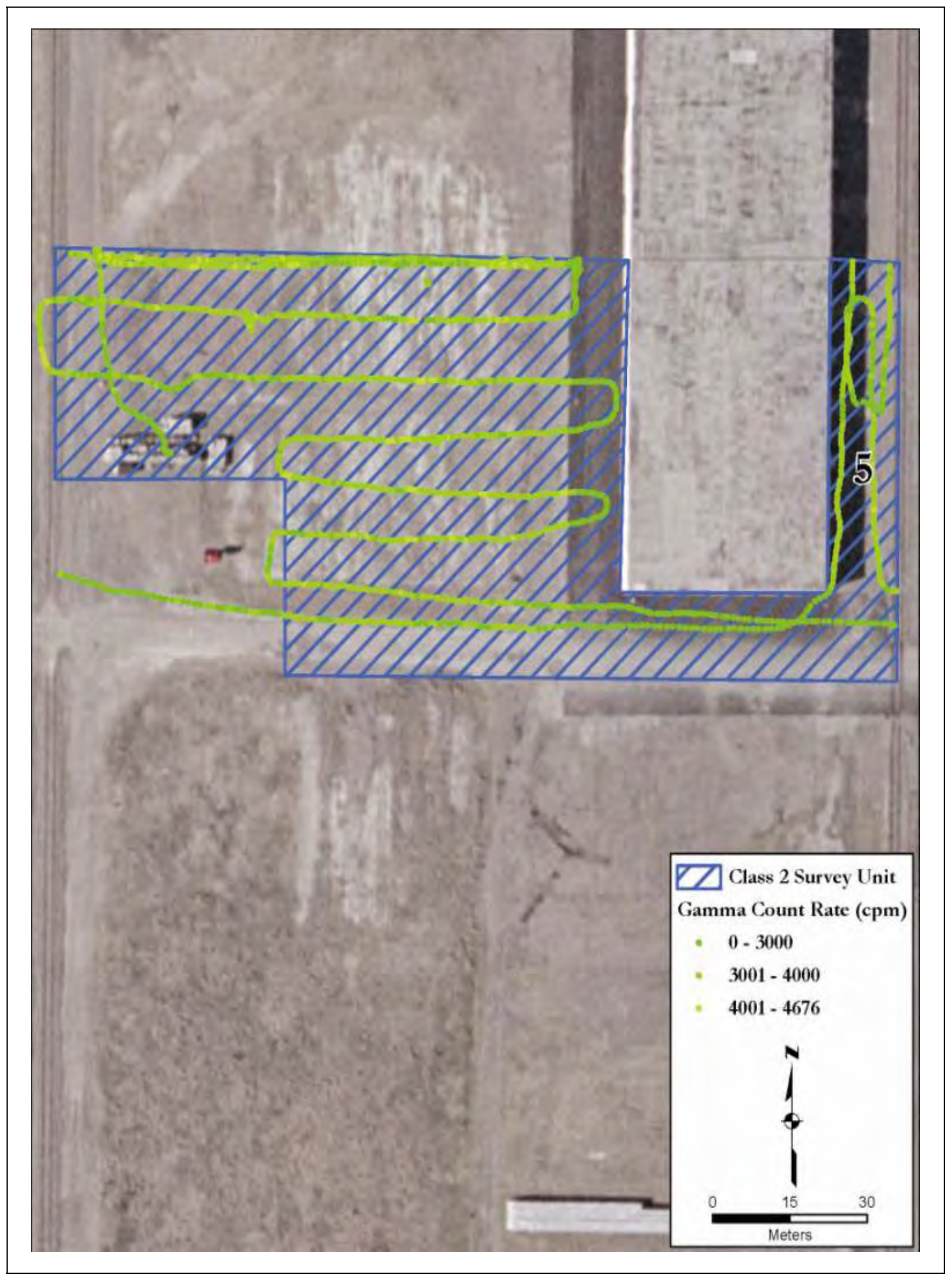

Figure A-46: Survey Unit C2 SU5-FSS Gamma Scan Results 


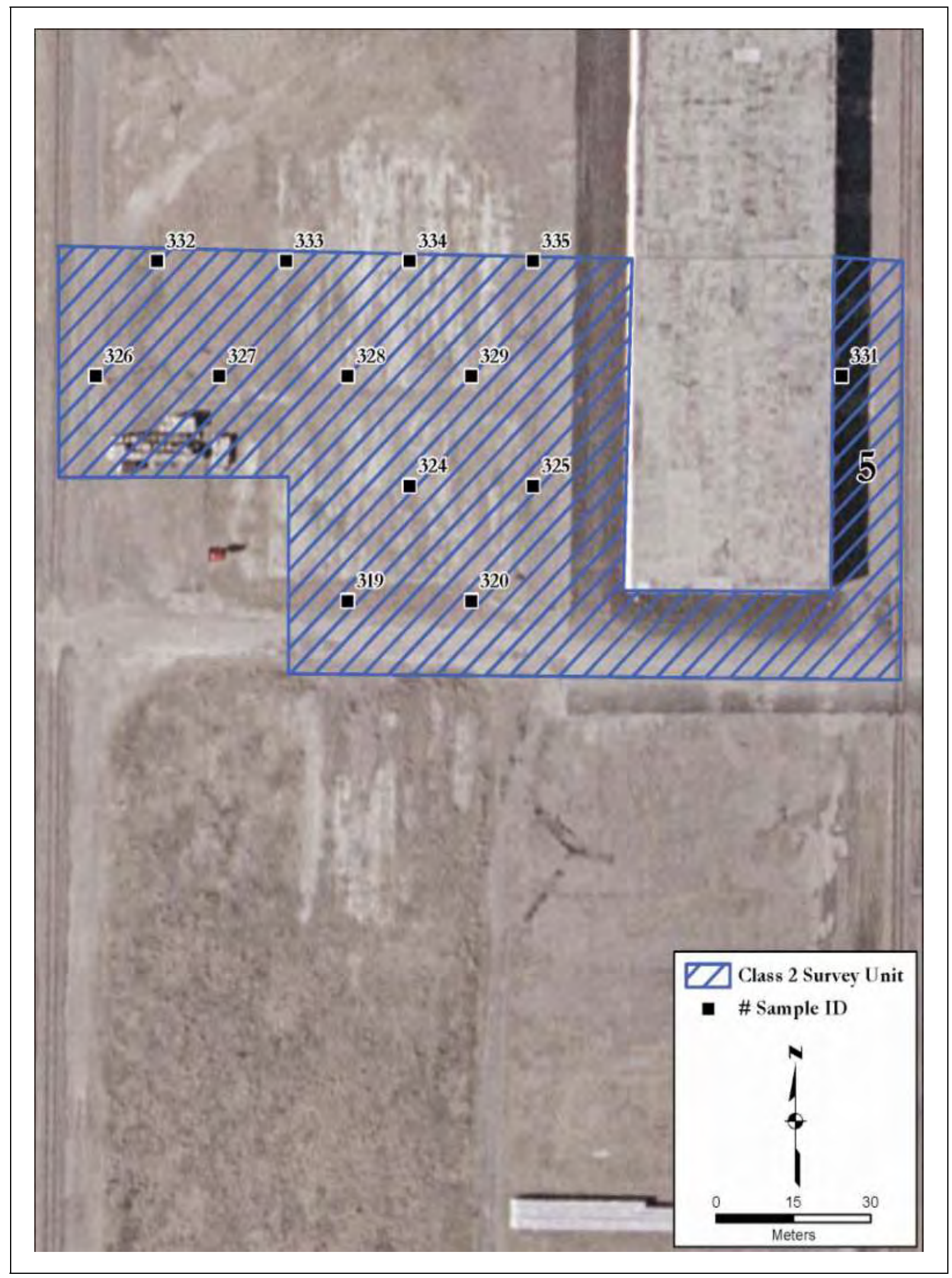

Figure A-47: Survey Unit C2 SU5-FSS Soil Sampling Locations 


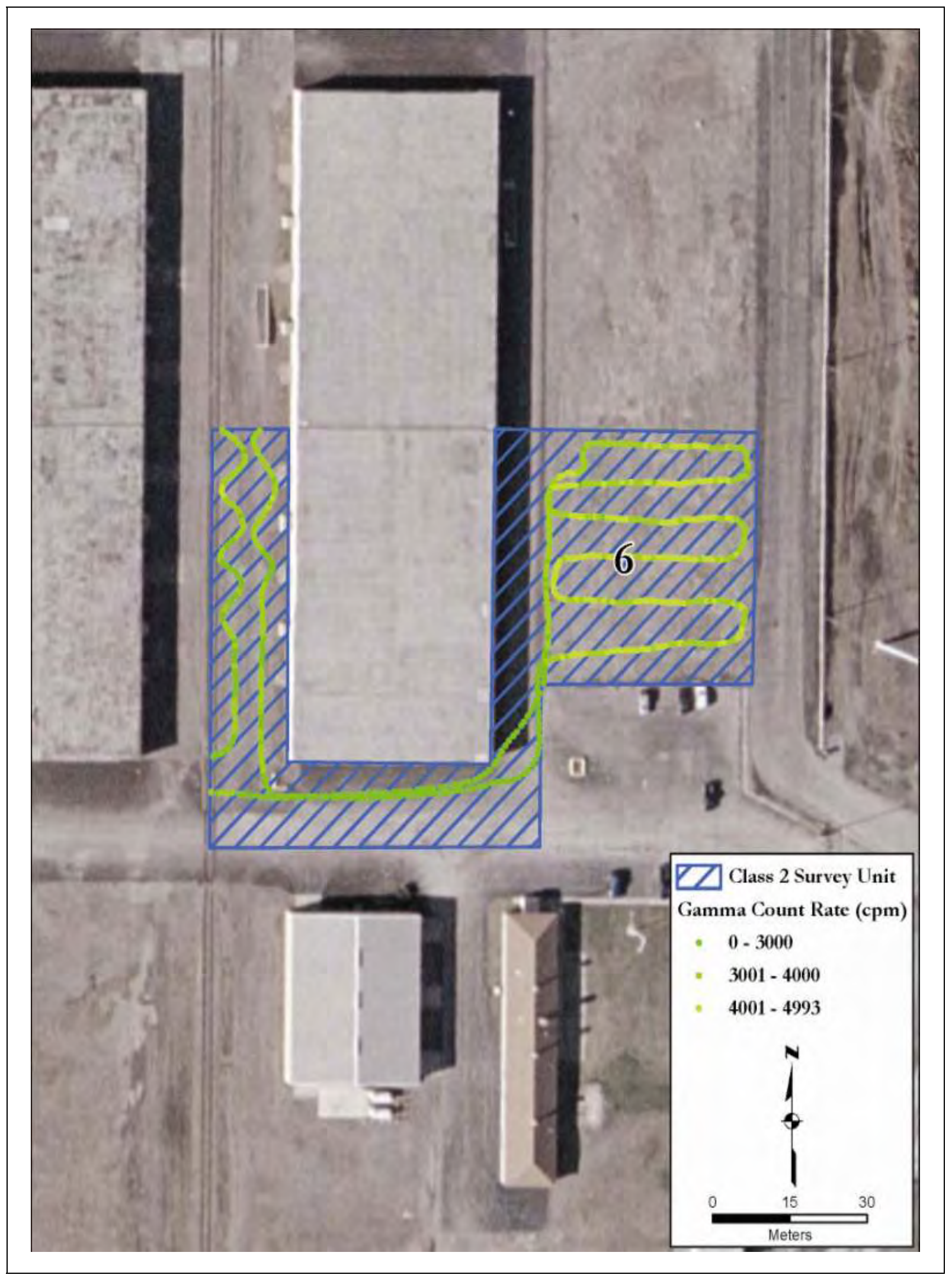

Figure A-48: Survey Unit C2 SU6-FSS Gamma Scan Results 


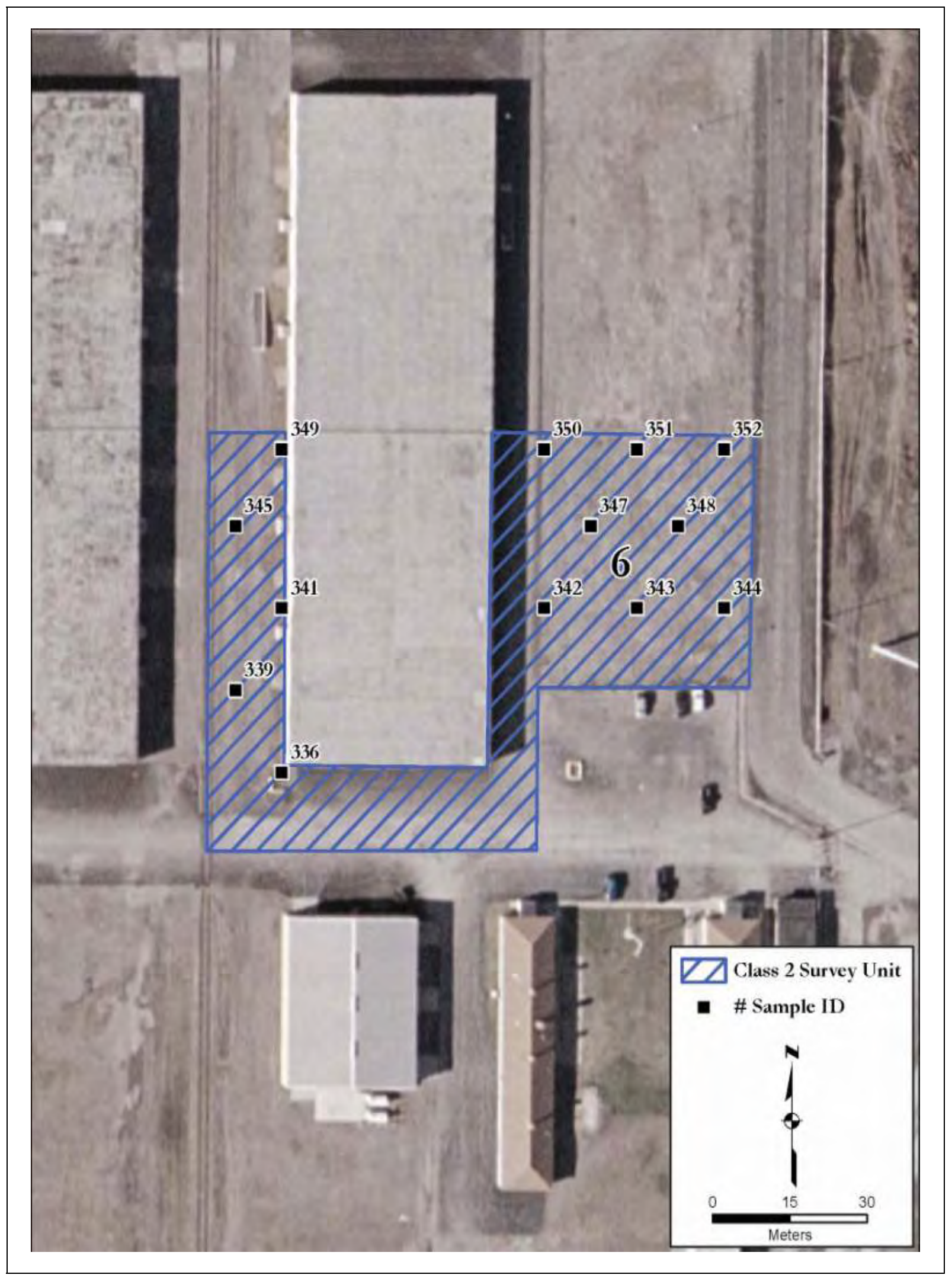

Figure A-49: Survey Unit C2 SU6_-FSS Soil Sampling Locations 


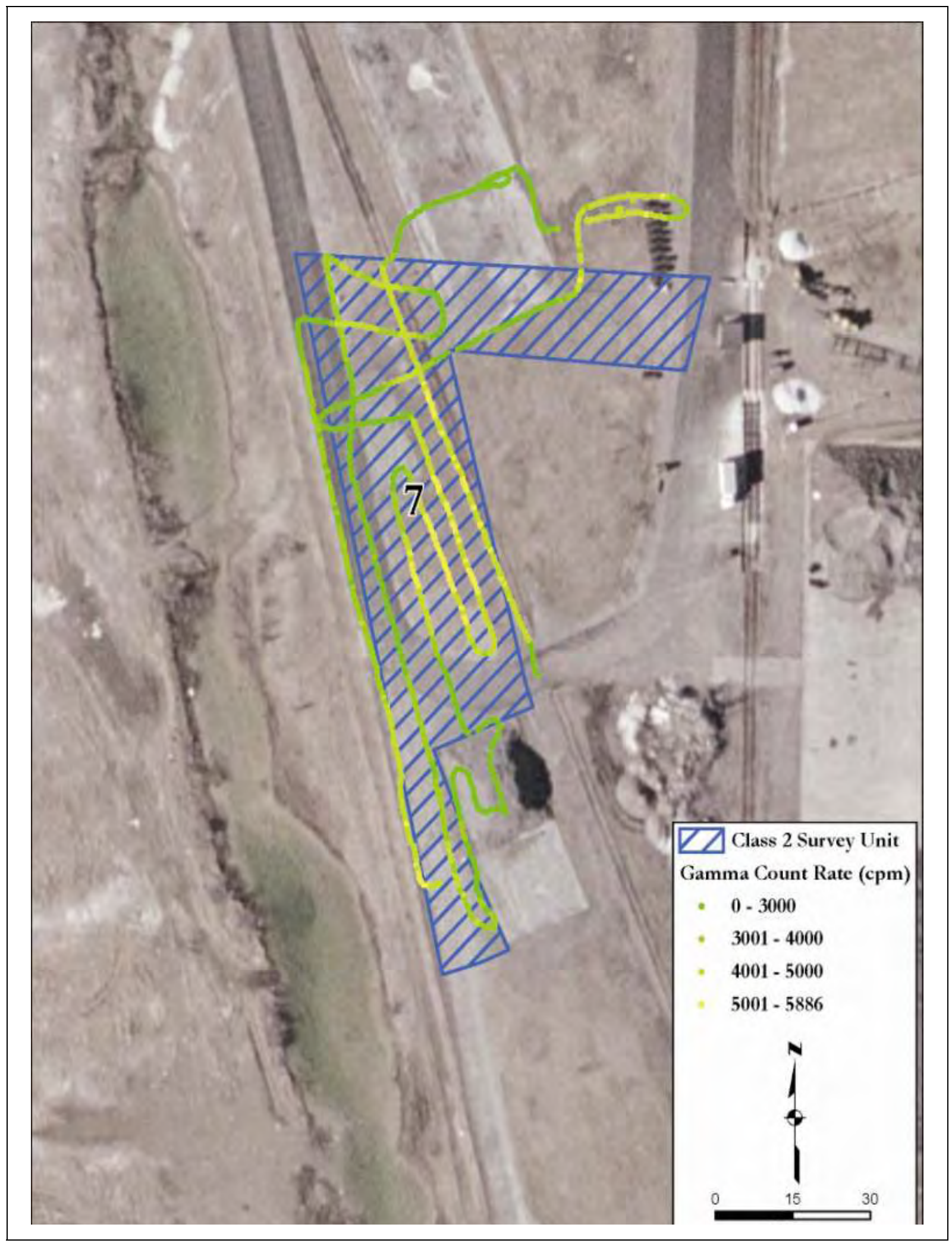

Figure A-50: Survey Unit C2 SU7-FSS Gamma Scan Results 


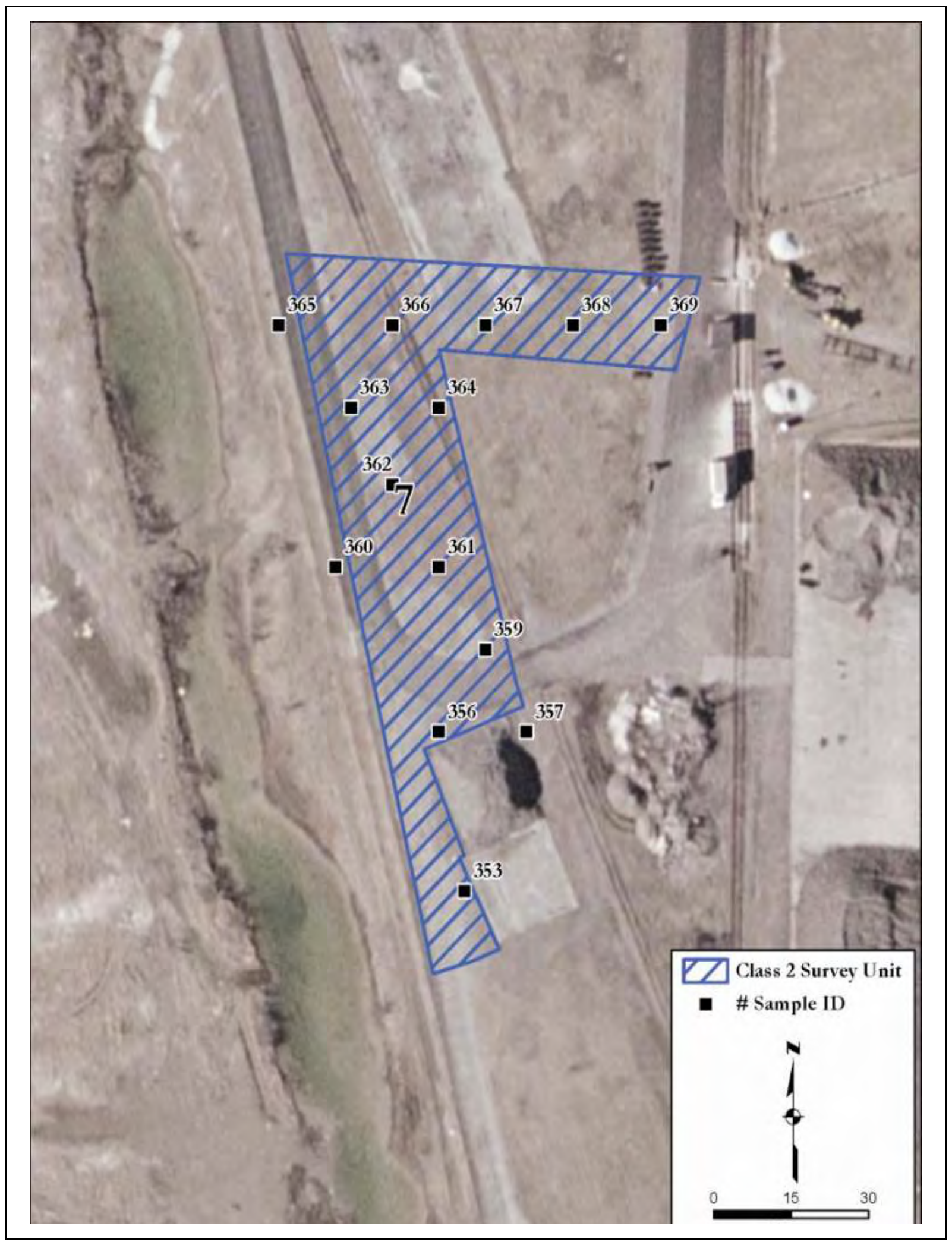

Figure A-51: Survey Unit C2 SU7-FSS Soil Sampling Locations 


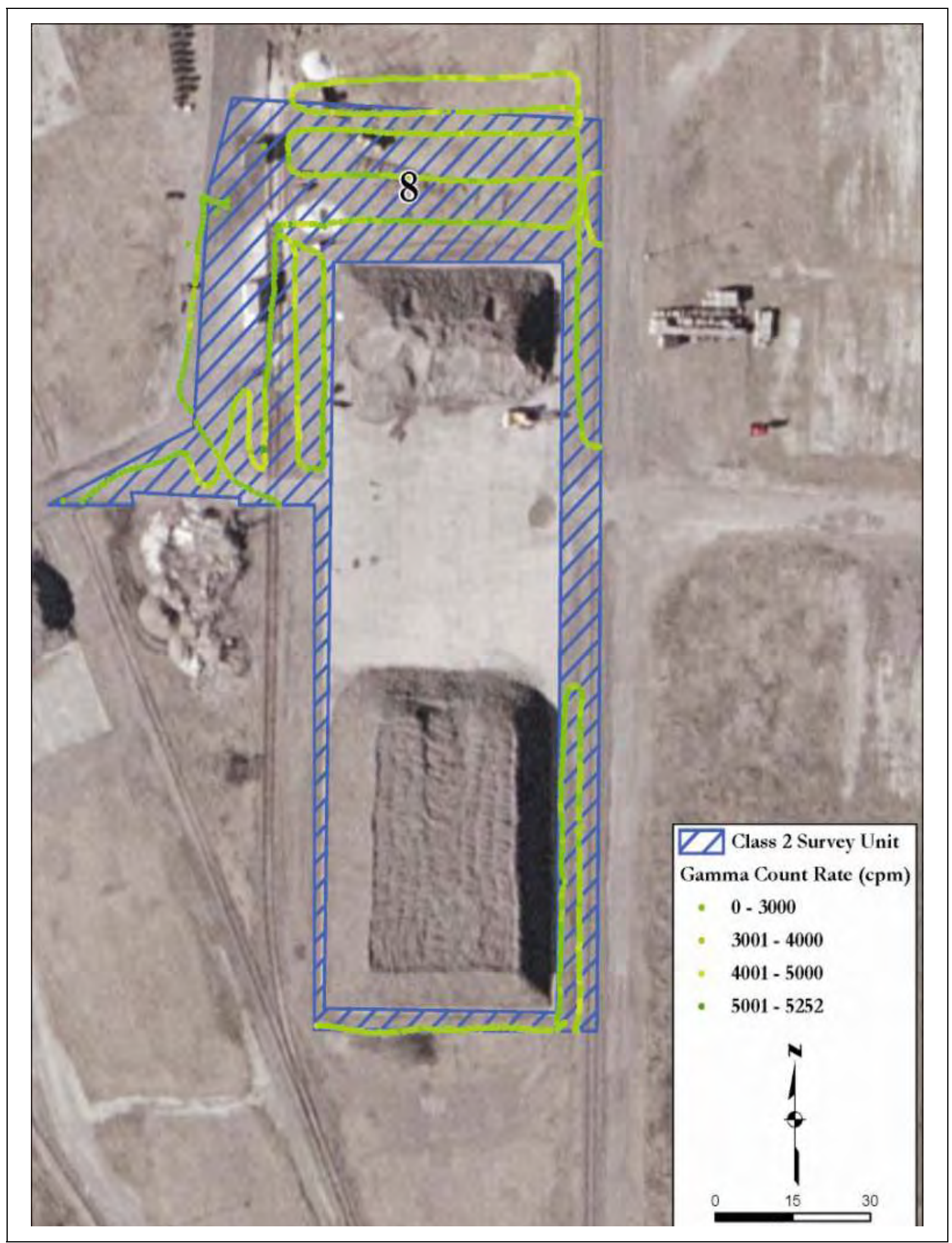

Figure A-52: Survey Unit C2 SU8-FSS Gamma Scan Results 


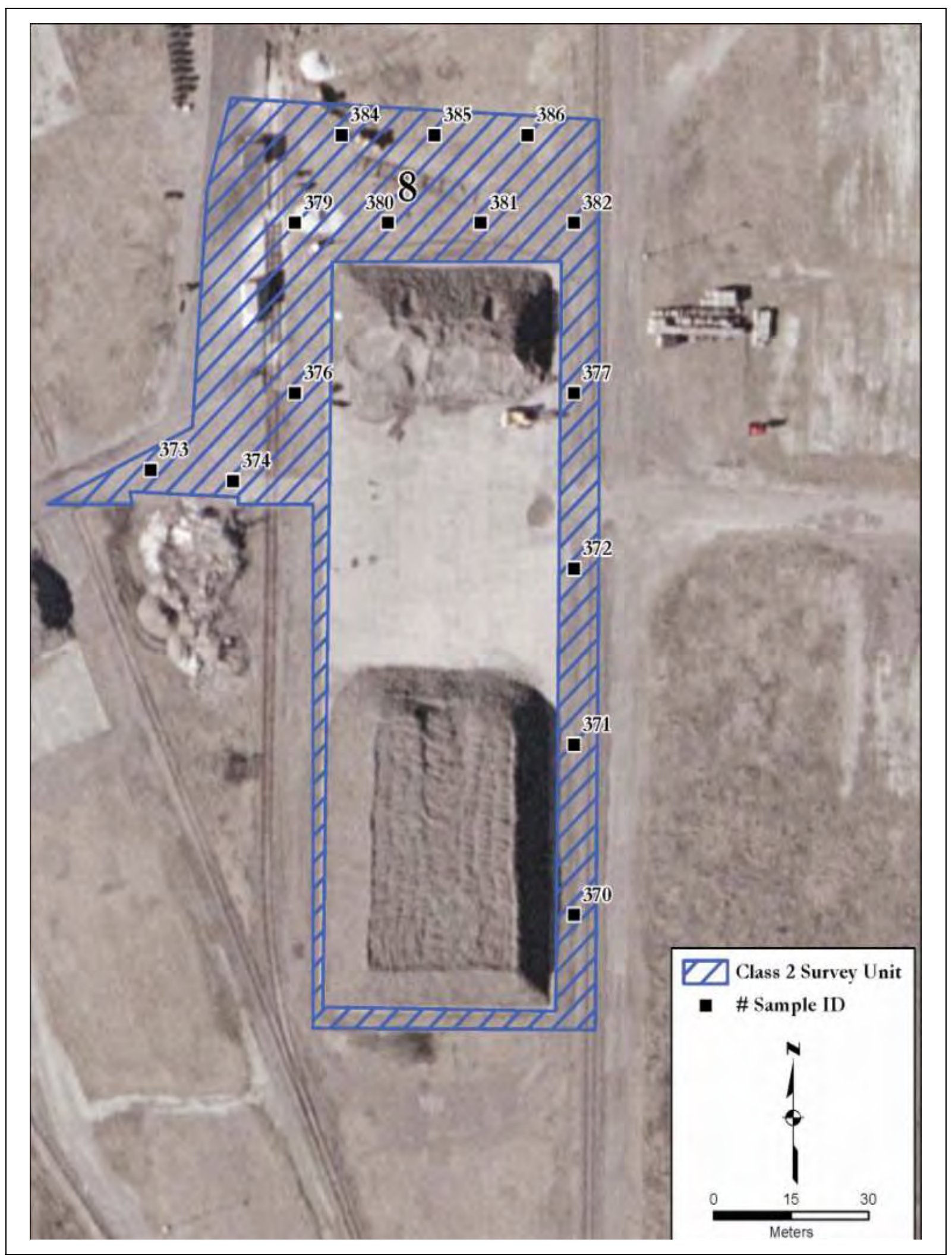

Figure A-53: Survey Unit C2 SU8-FSS Soil Sampling Locations 


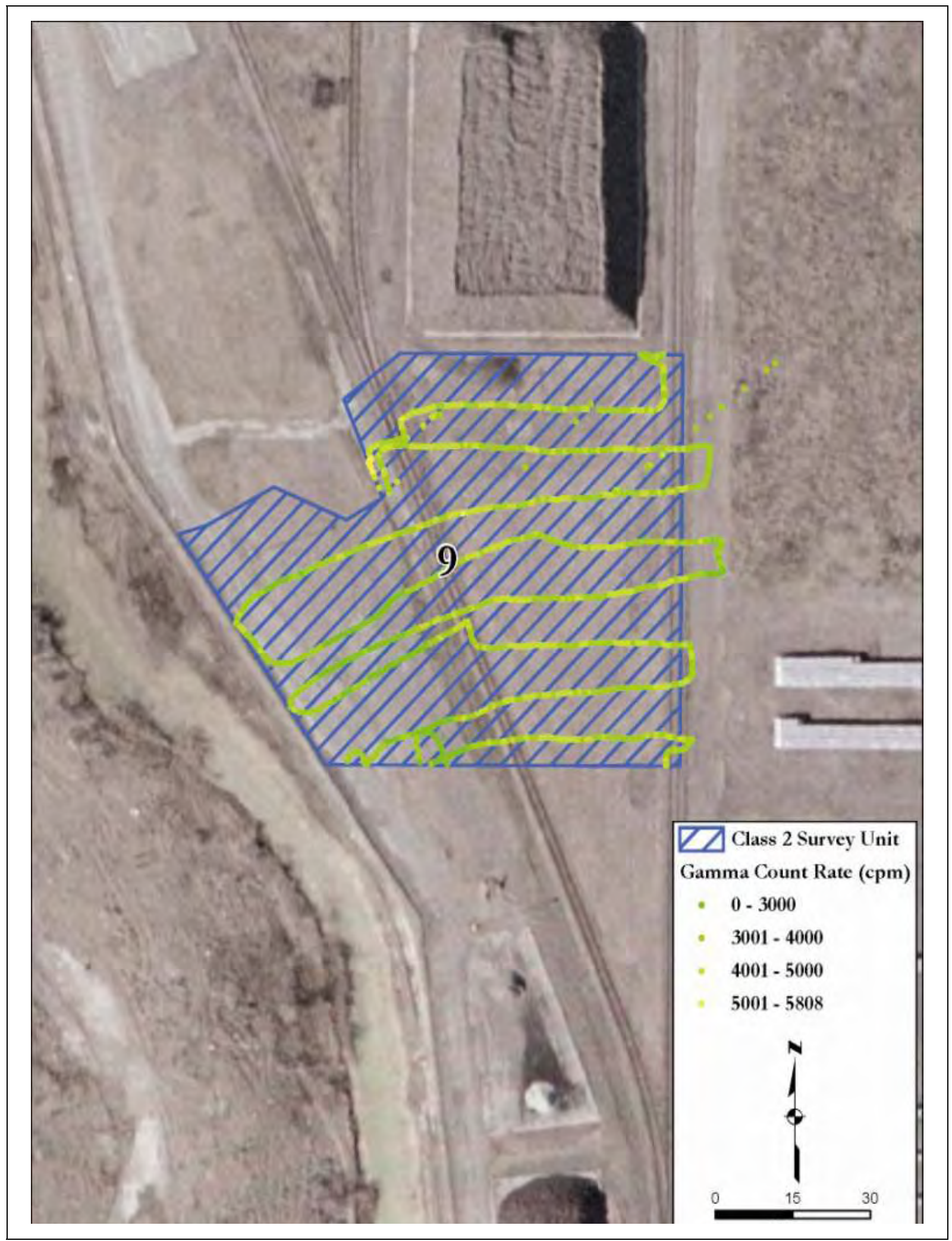

Figure A-54: Survey Unit C2 SU9-FSS Gamma Scan Results 


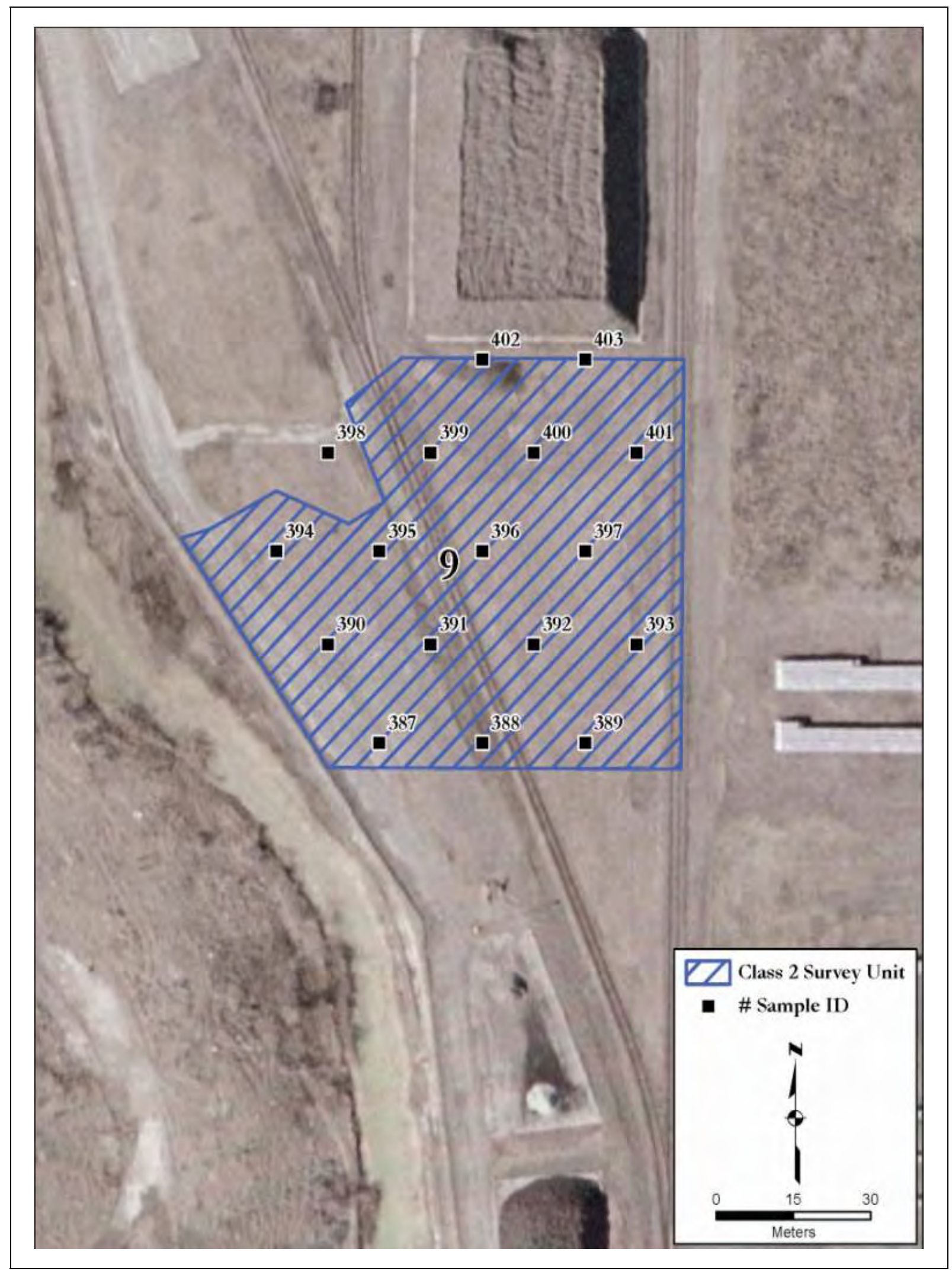

Figure A-55: Survey Unit C2 SU9-FSS Soil Sampling Locations 


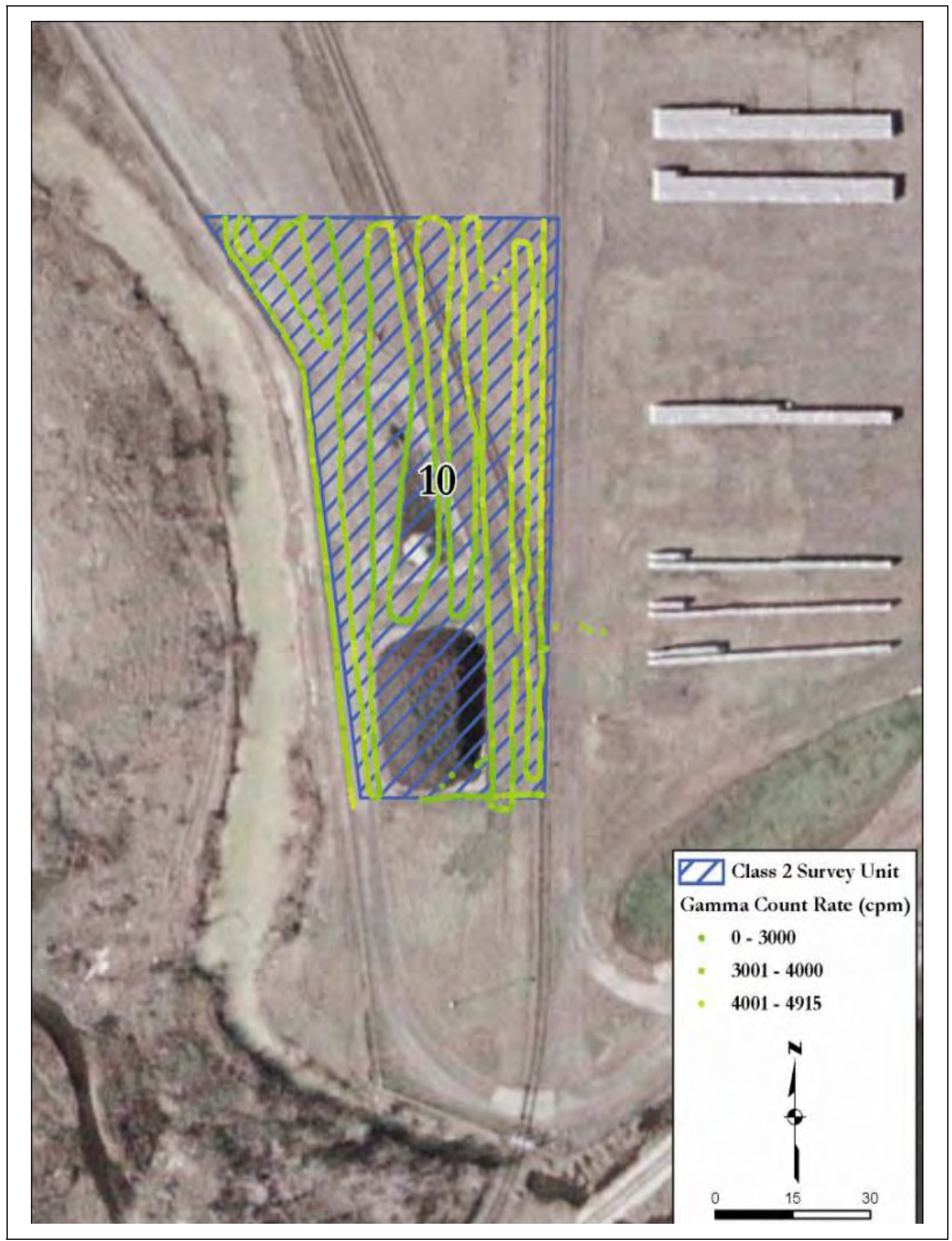

Figure A-56: Survey Unit C2 SU10_FSS Gamma Scan Results 


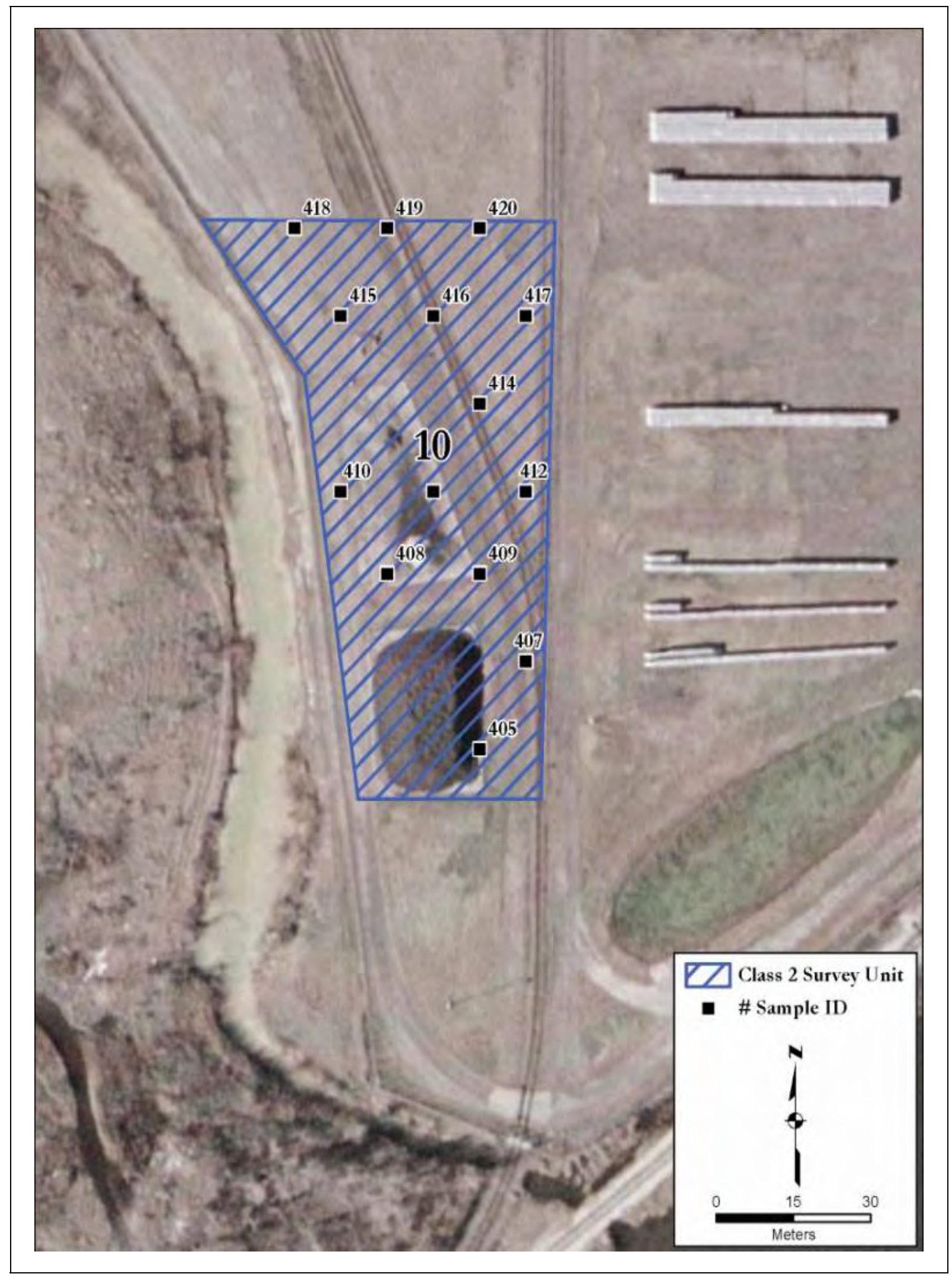

Figure A-57: Survey Unit C2 SU10—FSS Soil Sampling Locations 


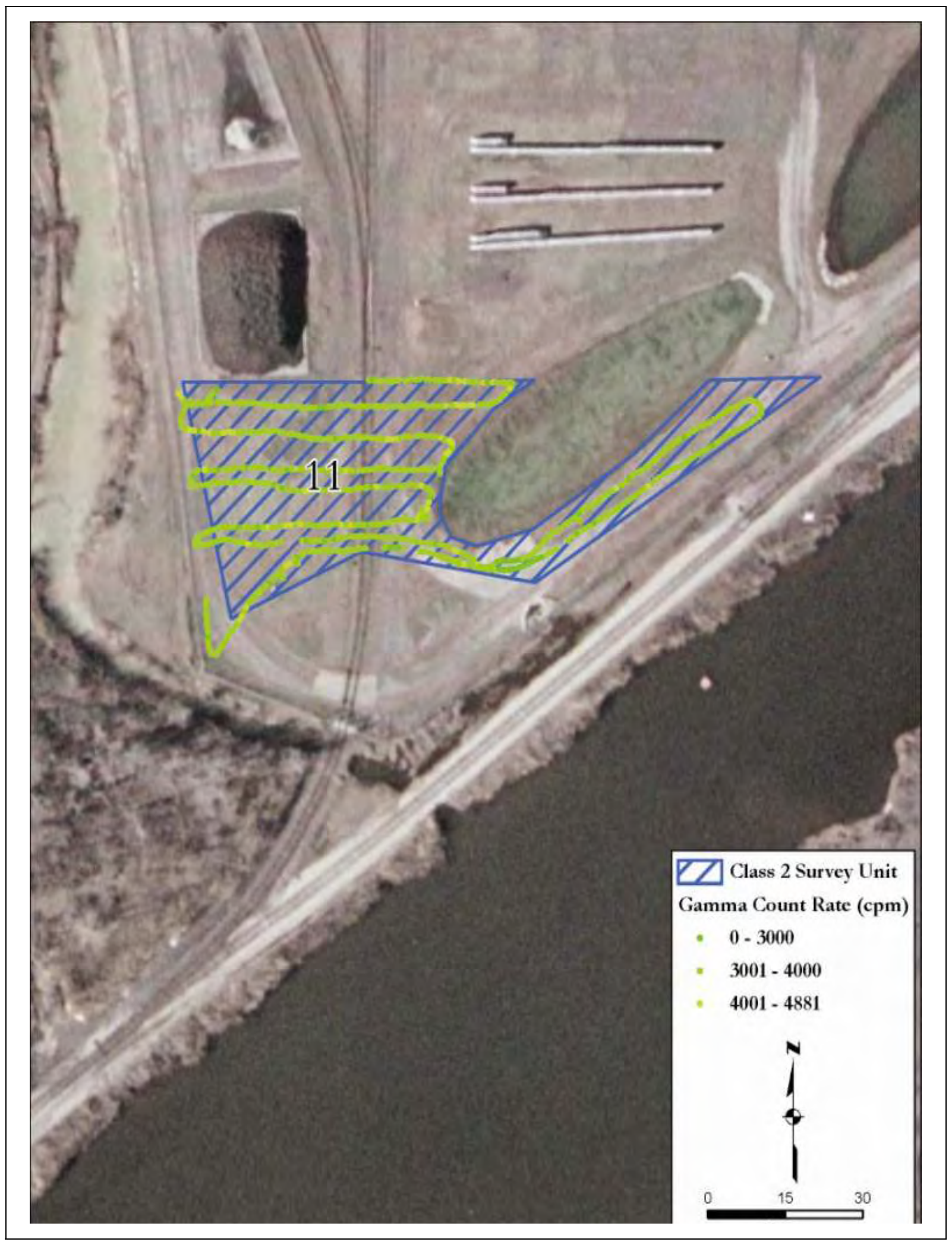

Figure A-58: Survey Unit C2 SU11-FSS Gamma Scan Results 


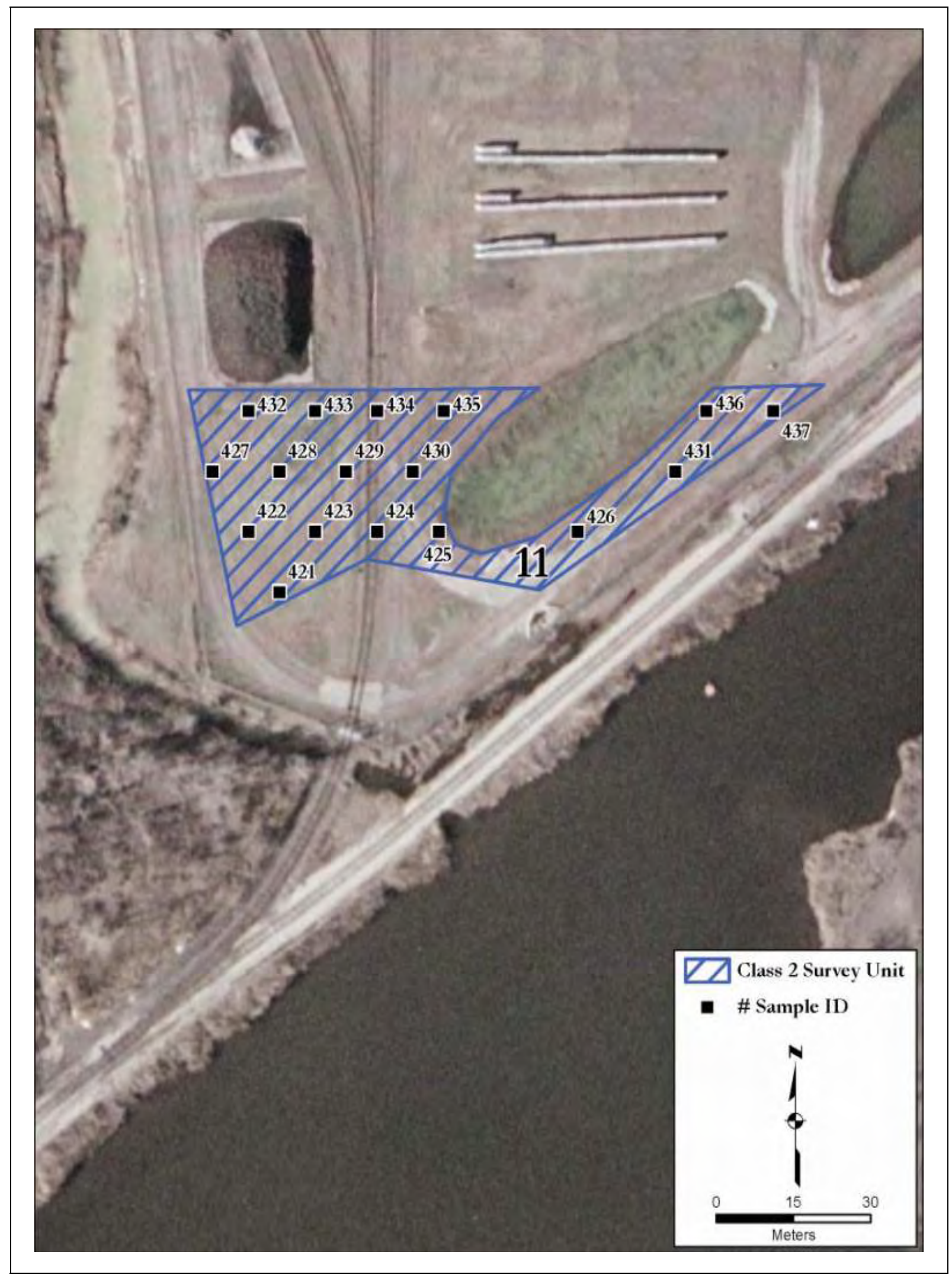

Figure A-59: Survey Unit C2 SU11—FSS Soil Sampling Locations 


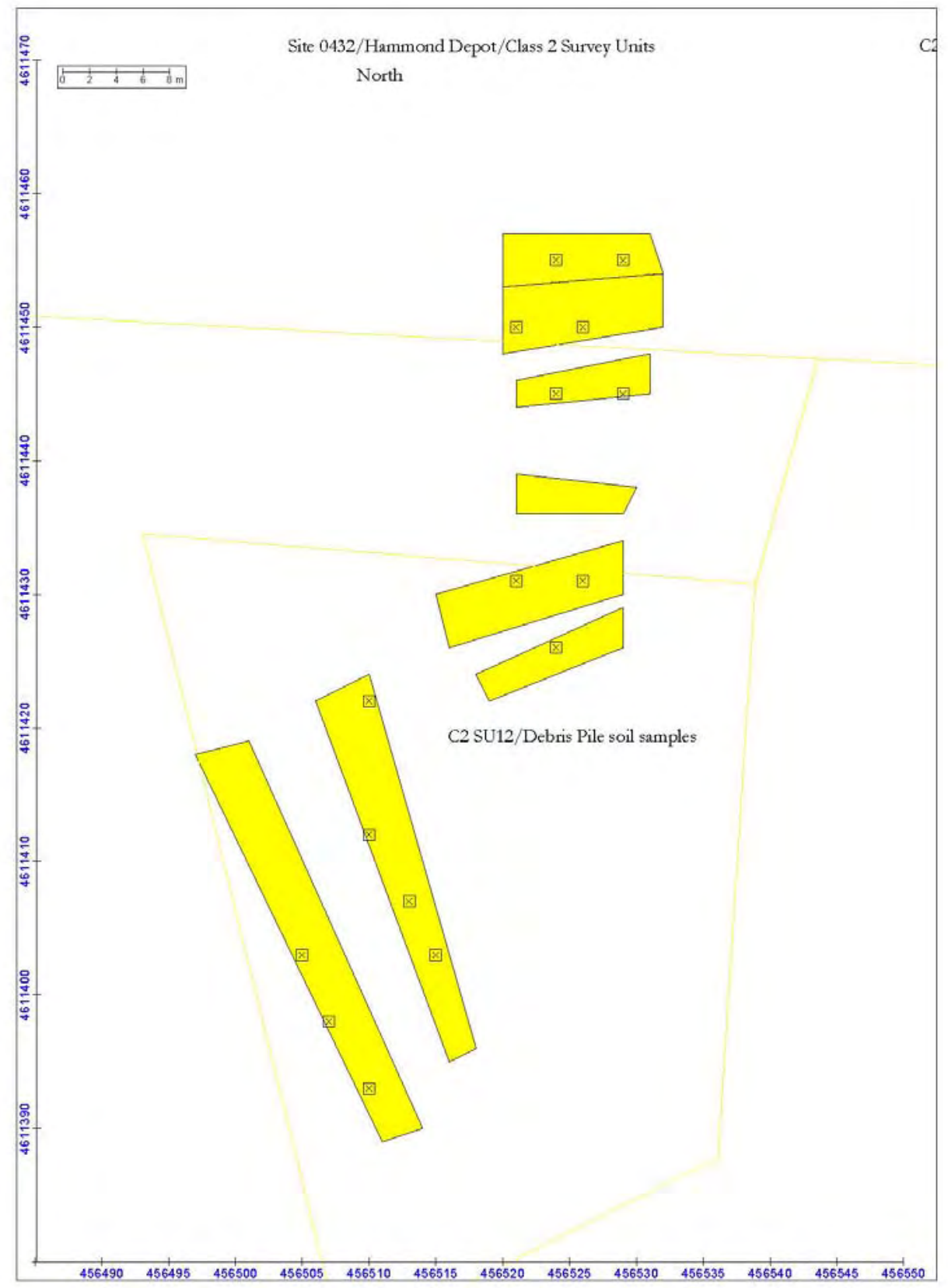

凶 Soil Sample Location

Figure A-60: Survey Unit C2 SU12-FSS Soil Sampling Locations 


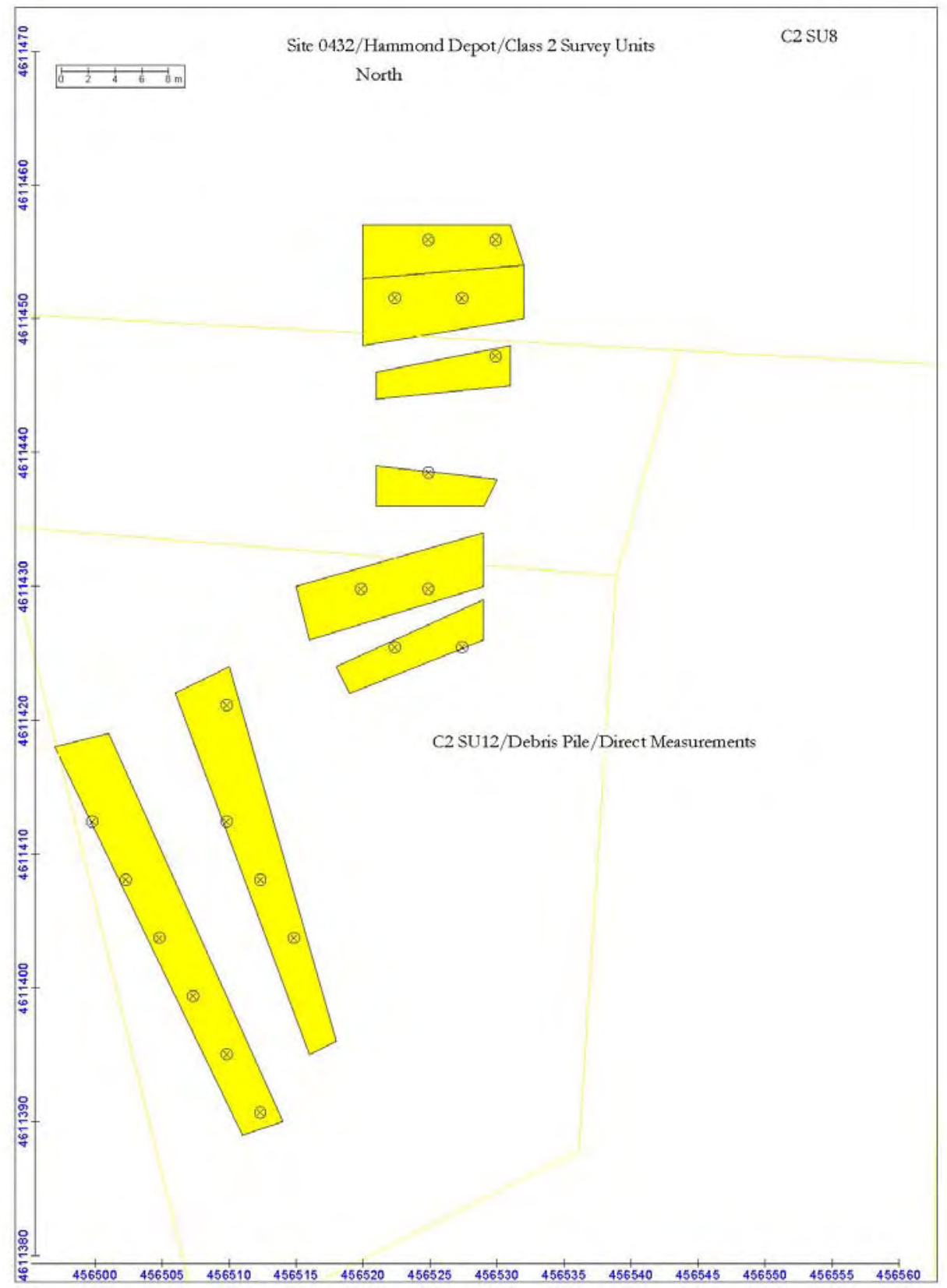

$\otimes$ Direct Measurement Location

Figure A-61: Survey Unit C2 SU12, Debris Pile—FSS Direct Measurement Locations 


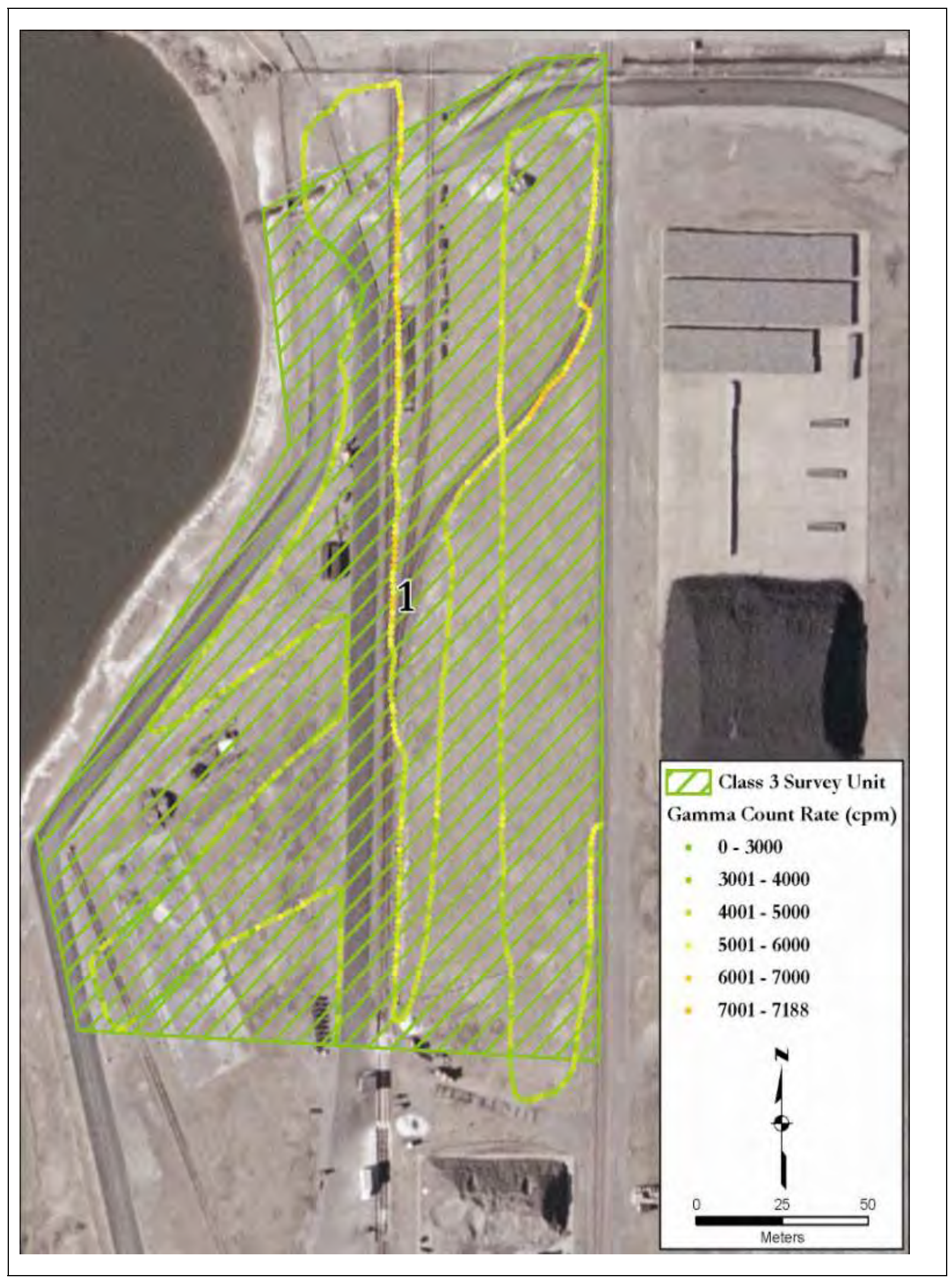

Figure A-62: Survey Unit C3 SU1—FSS Gamma Scan Results 


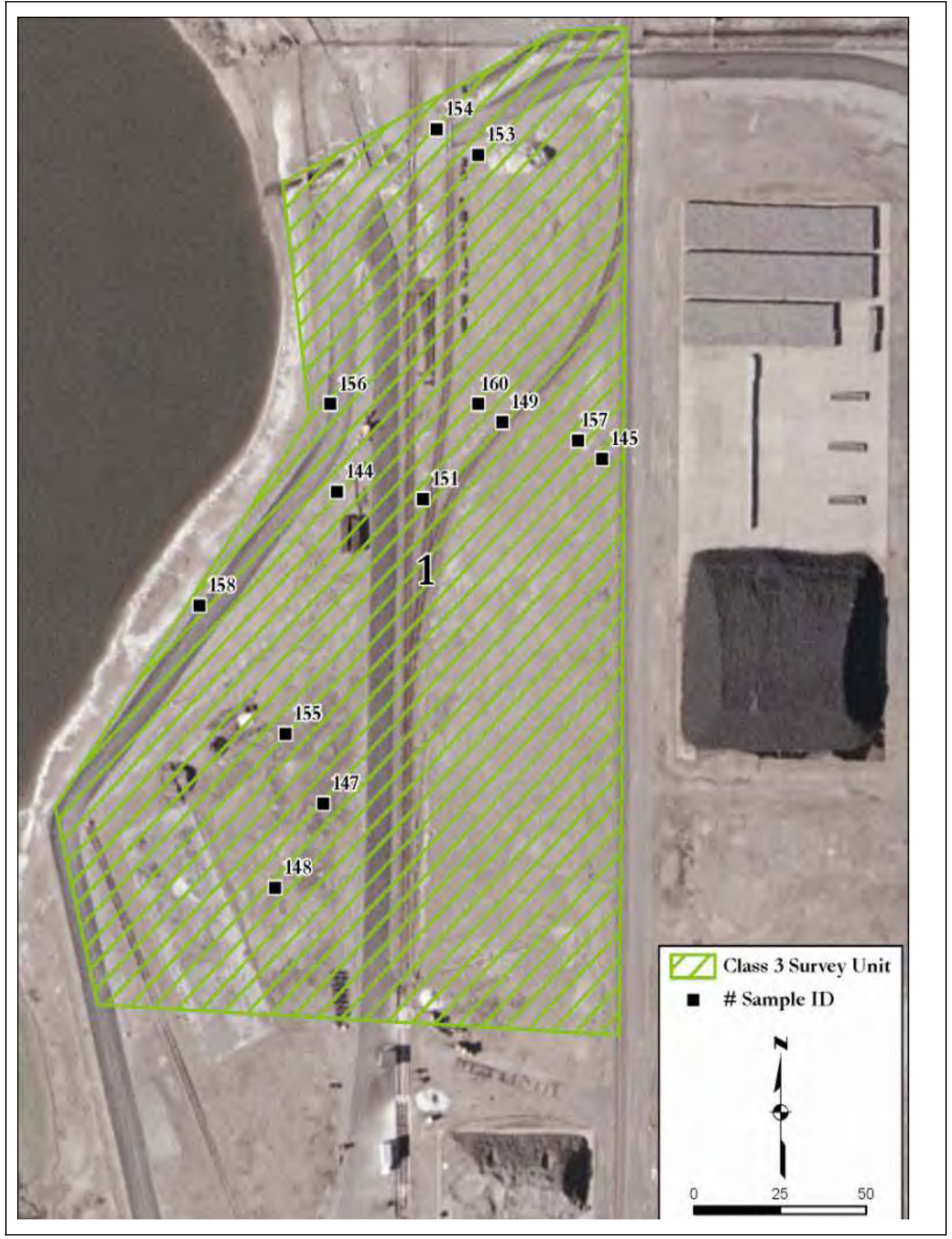

Figure A-63: Survey Unit C3 SU1-FSS Soil Sampling Locations 


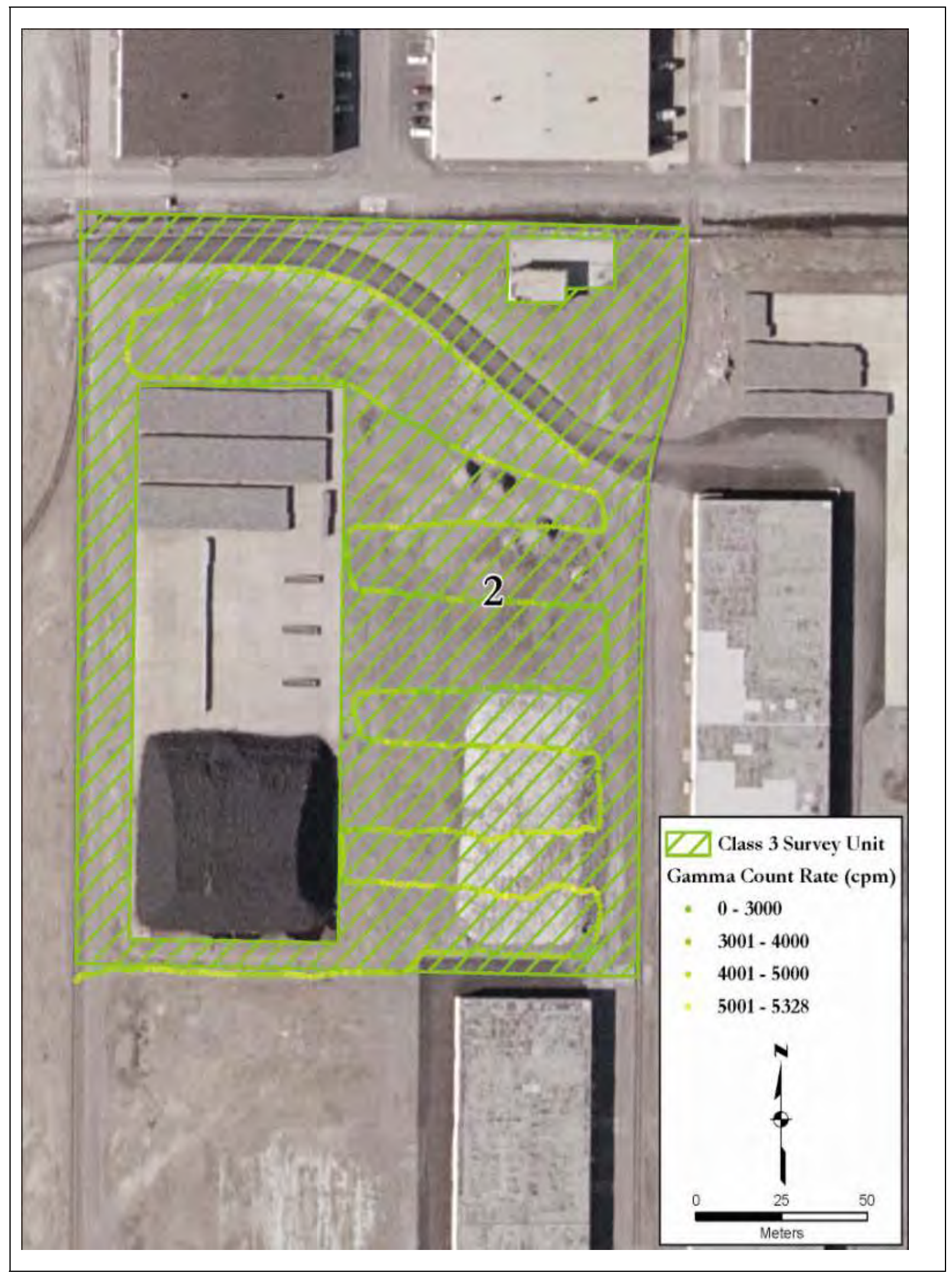

Figure A-64: Survey Unit C3 SU2-FSS Gamma Scan Results 


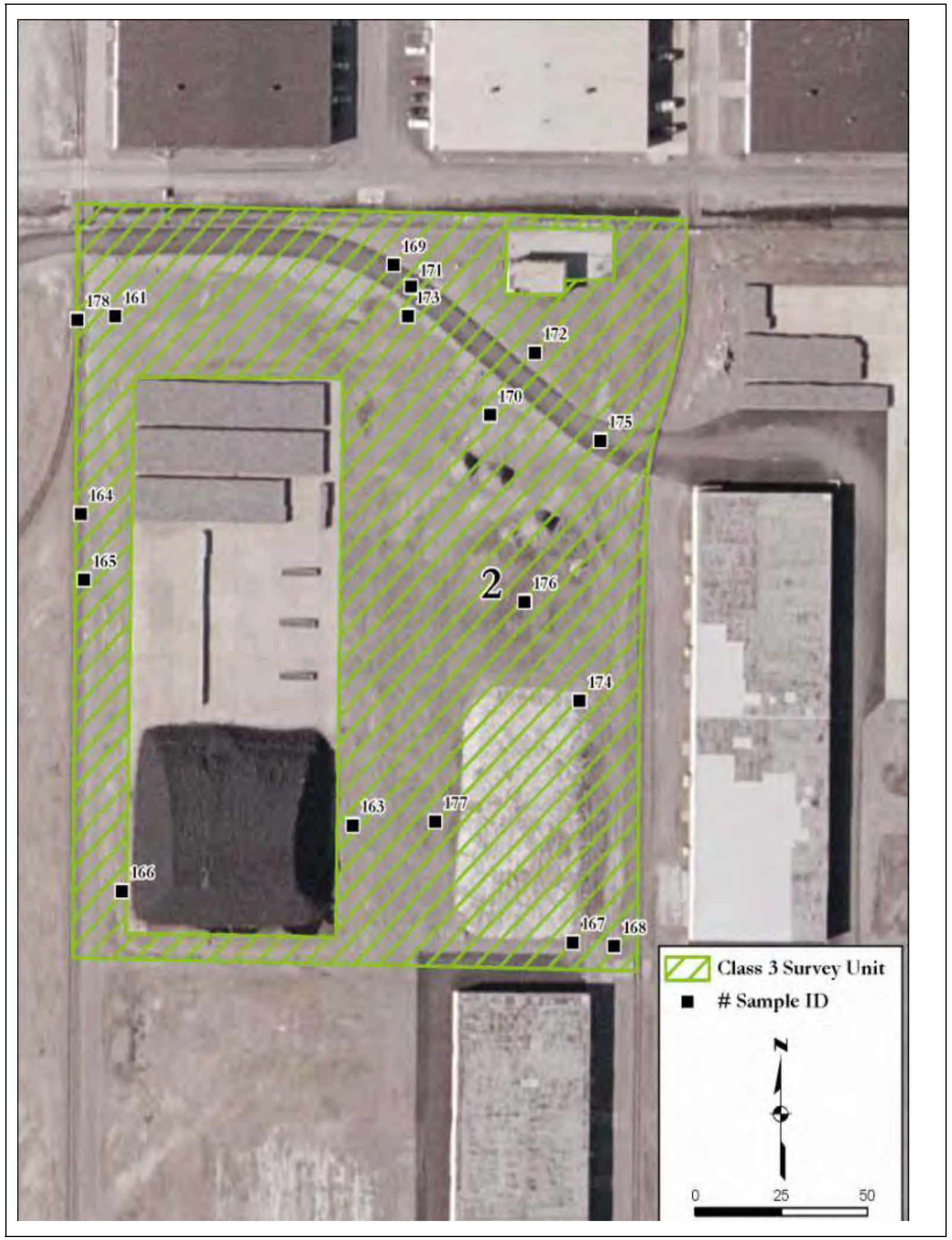

Figure A-65: Survey Unit C3 SU2-FSS Soil Sampling Locations 


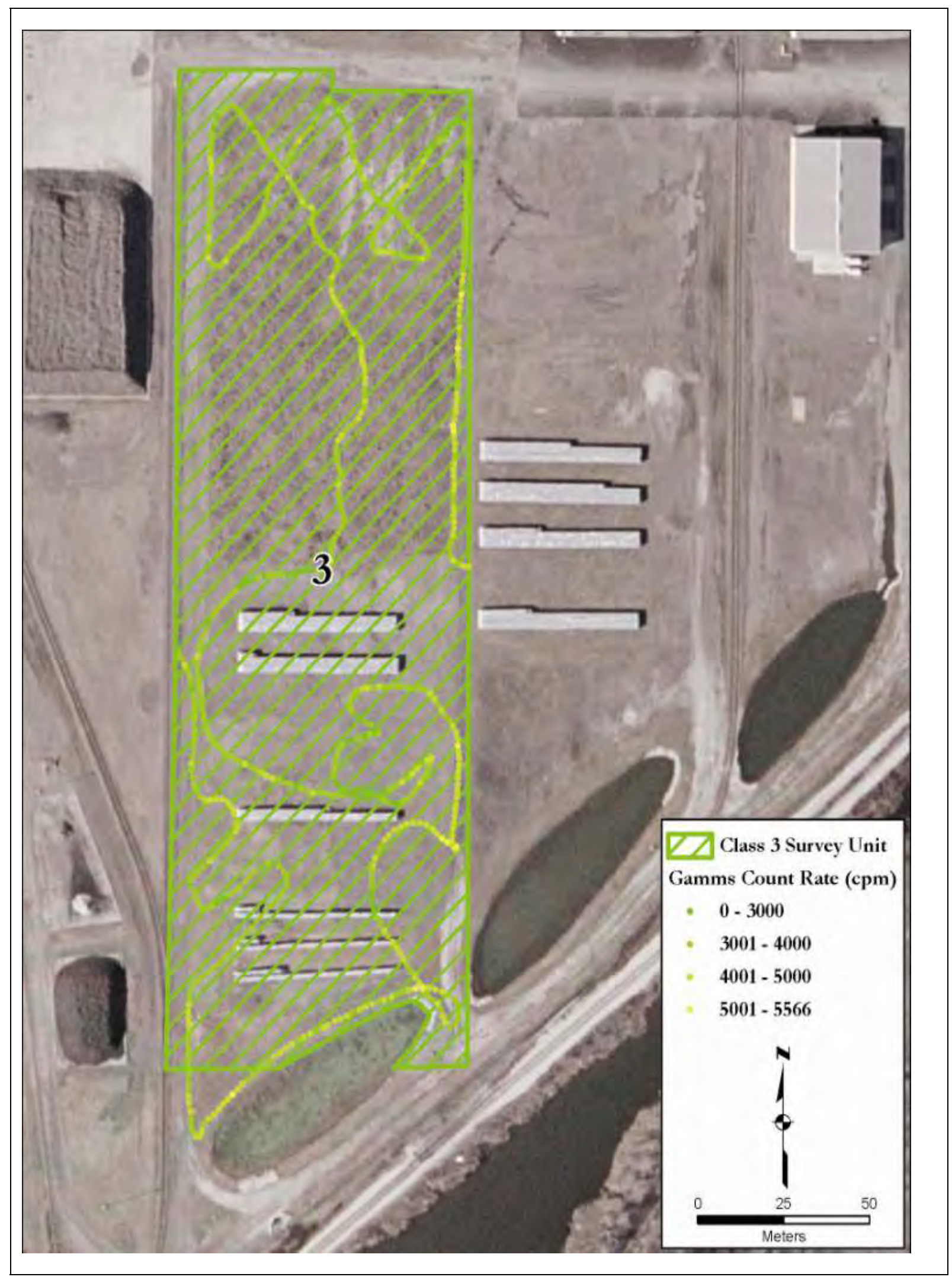

Figure A-66: Survey Unit C3 SU1-FSS Gamma Scan Results 


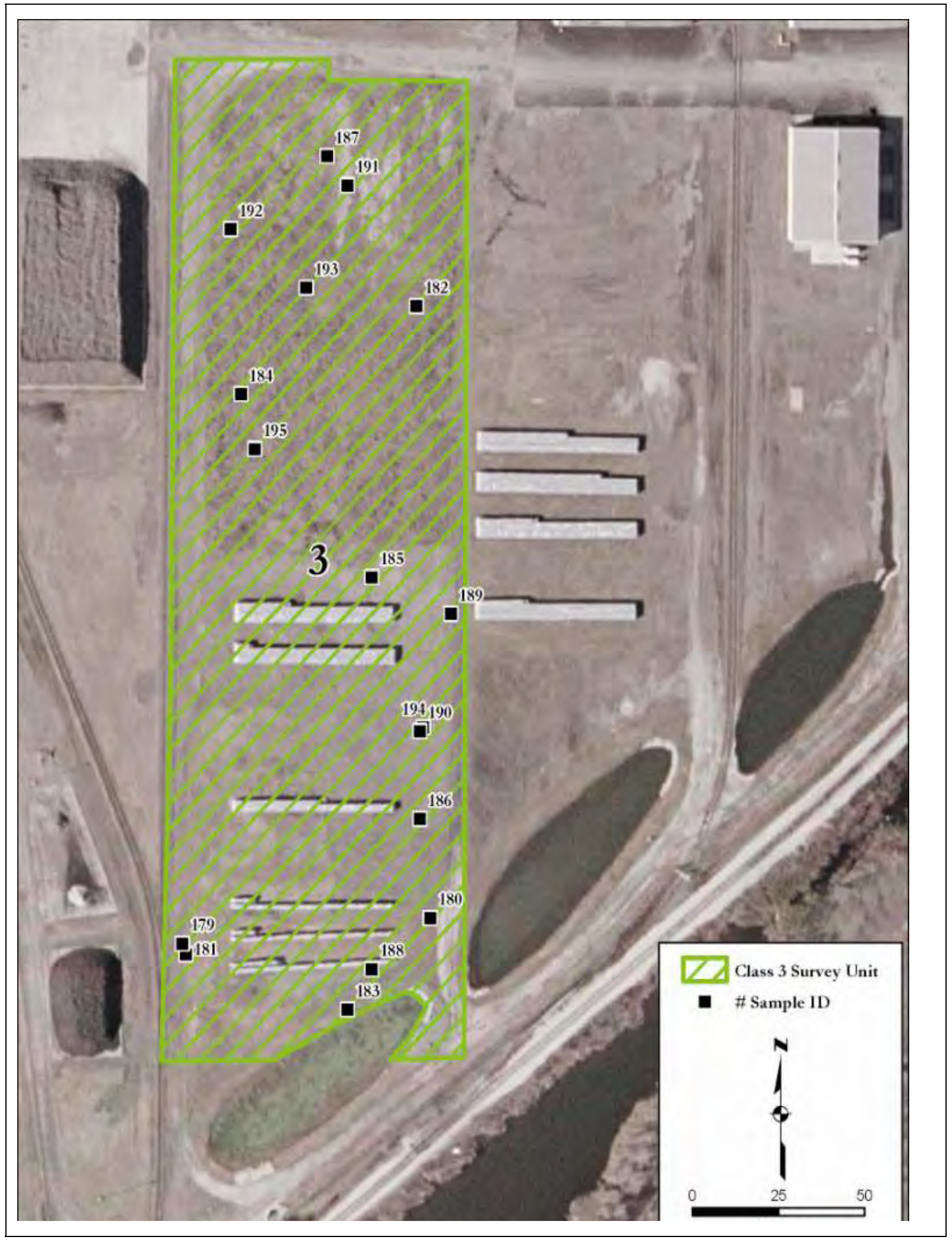

Figure A-67: Survey Unit C3 SU3-FSS Soil Sampling Locations 


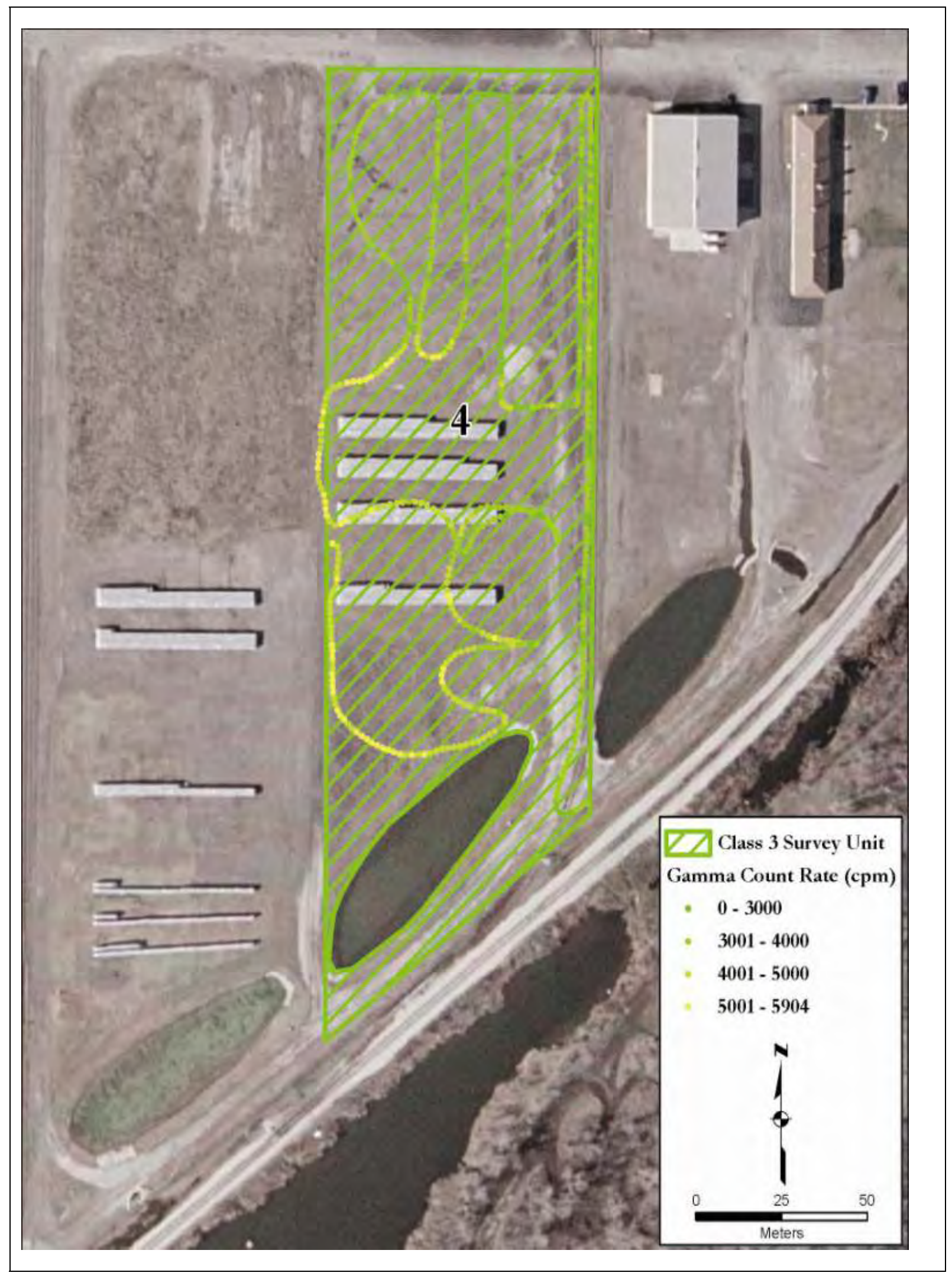

Figure A-68: Survey Unit C3 SU4-FSS Gamma Scan Results 


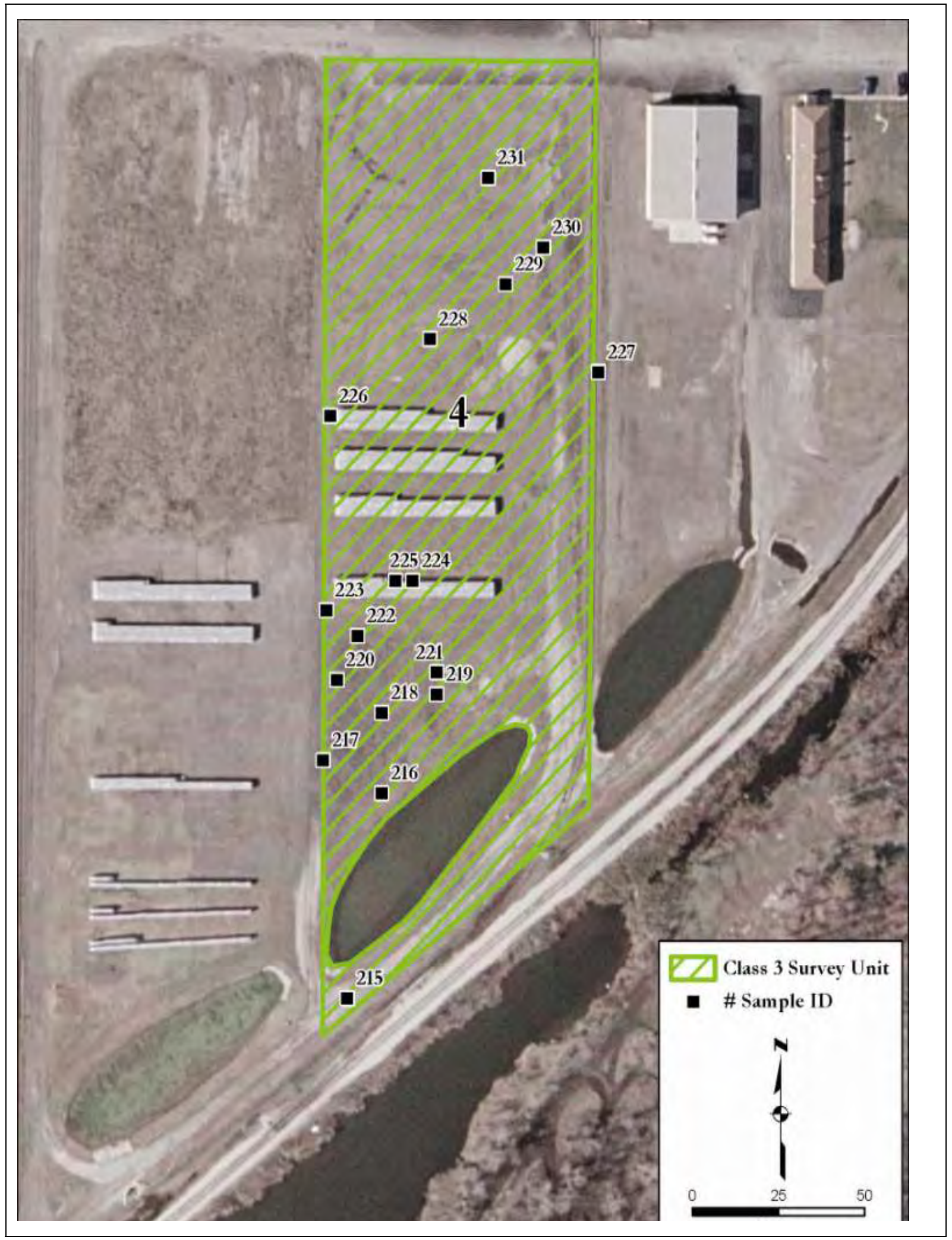

Figure A-69: Survey Unit C3 SU4-FSS Soil Sampling Locations 


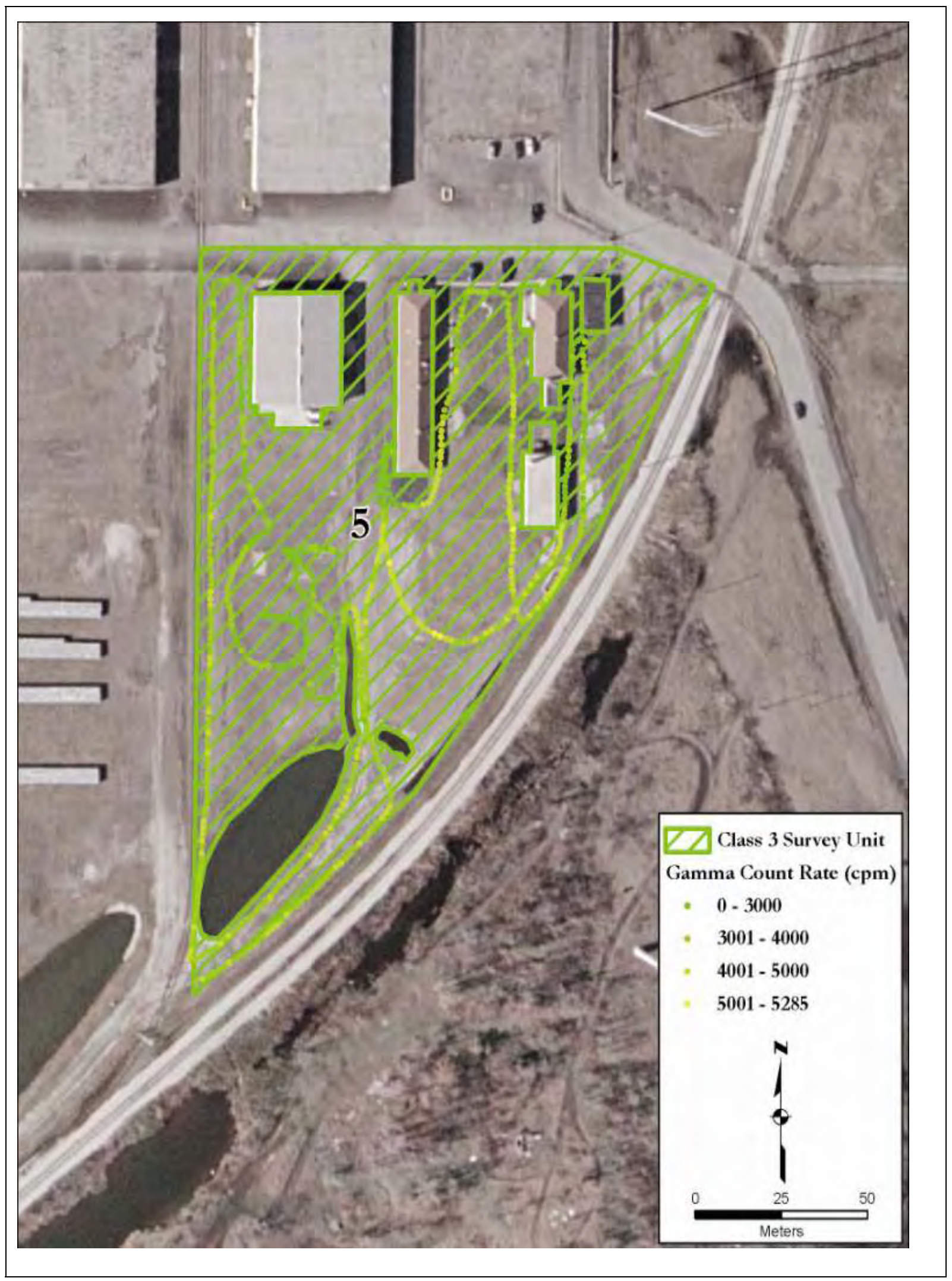

Figure A-70: Survey Unit C3 SU5-FSS Gamma Scan Results 


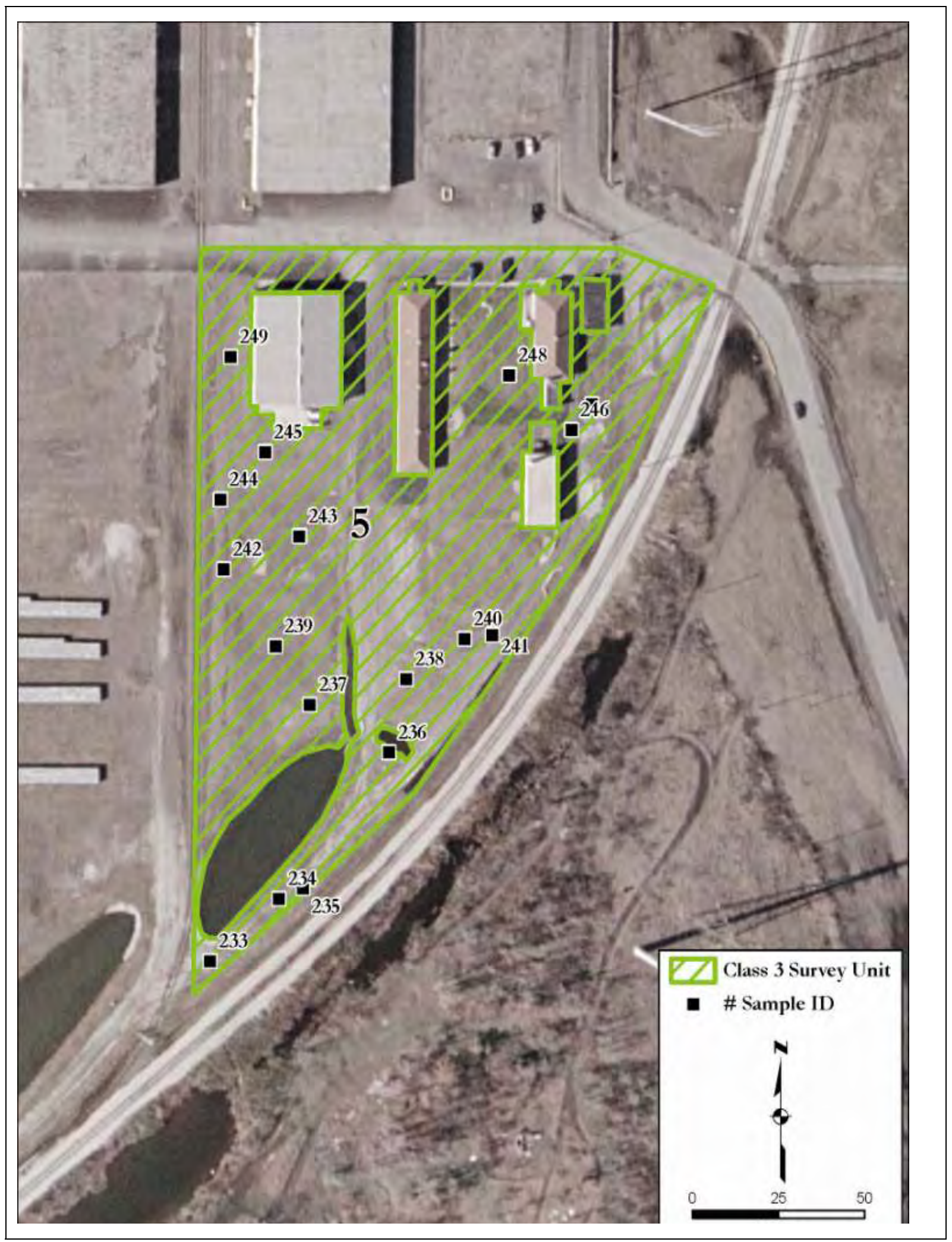

Figure A-71: Survey Unit C3 SU5-FSS Soil Sampling Locations 
APPENDIX B: TABLES 


\begin{tabular}{|c|c|c|c|c|c|c|c|}
\hline \multicolumn{8}{|c|}{$\begin{array}{c}\text { Table B-1: Survey Unit Descriptions and Results } \\
\text { Hammond Depot } \\
\text { Hammond, Indiana }\end{array}$} \\
\hline Class and Survey Unit & Class & Unit Area & Remarks & $\begin{array}{c}\text { DQO } \\
\text { complete }\end{array}$ & FSS complete & DQA Complete & Remarks \\
\hline \multicolumn{8}{|c|}{ Class 1 Survey Units (C1 SU\#) } \\
\hline C1 SU1 & 1 & $2,271 \mathrm{~m}^{2}$ & AOC 4 & Yes & FSS Completed & Yes & Pass \\
\hline C1 SU2 & 1 & $468 \mathrm{~m}^{2}$ & AOC 1 & Yes & FSS Completed & Yes & Pass \\
\hline C1 SU2.1 & 1 & $184 \mathrm{~m}^{2}$ & AOC 1 & Yes & FSS Completed & Yes & Pass \\
\hline C1 SU2.2 & 1 & $208 \mathrm{~m}^{2}$ & AOC 1 & Yes & FSS Completed & Yes & Pass \\
\hline C1 SU2.3 & 1 & $185 \mathrm{~m}^{2}$ & AOC 1 & Yes & FSS Completed & Yes & Pass \\
\hline C1 SU2.4 & 1 & $183 \mathrm{~m}^{2}$ & AOC 1 & Yes & FSS Completed & Yes & Pass \\
\hline C1 SU2.5 & 1 & $108 \mathrm{~m}^{2}$ & AOC 2 & Yes & FSS Completed & Yes & Pass \\
\hline C1 SU2.6 & 1 & $163 \mathrm{~m}^{2}$ & AOC 3 & Yes & FSS Completed & Yes & Pass \\
\hline C1 SU2.7 & 1 & $153 \mathrm{~m}^{2}$ & AOC 4 & Yes & FSS Completed & Yes & Pass \\
\hline C1 SU3 & 1 & $502 \mathrm{~m}^{2}$ & AOC 2 and 3 & Yes & FSS Completed & Yes & Pass \\
\hline C1 SU3.1 & 1 & $194 \mathrm{~m}^{2}$ & AOC 2 and 3 & Yes & FSS Completed & Yes & Pass \\
\hline C1 SU3.2 & 1 & $193 \mathrm{~m}^{2}$ & AOC 2 and 3 & Yes & FSS Completed & Yes & Pass \\
\hline C1 SU3.3 & 1 & $210 \mathrm{~m}^{2}$ & AOC 2 and 3 & Yes & FSS Completed & Yes & Pass \\
\hline C1 SU3.4 & 1 & $200 \mathrm{~m}^{2}$ & AOC 2 and 3 & Yes & FSS Completed & Yes & Pass \\
\hline C1 SU3.5 & 1 & $195 \mathrm{~m}^{2}$ & AOC 2 and 3 & Yes & FSS Completed & Yes & Pass \\
\hline C1 SU3.6 & 1 & $214 \mathrm{~m}^{2}$ & AOC 2 and 3 & Yes & FSS Completed & Yes & Pass \\
\hline C1 SU3.7 & 1 & $200 \mathrm{~m}^{2}$ & AOC 2 and 3 & Yes & FSS Completed & Yes & Pass \\
\hline C1 SU4 & 1 & $2,115 \mathrm{~m}^{2}$ & AOC 7 & Yes & FSS Completed & Yes & Pass \\
\hline C1 SU5 & 1 & $527 \mathrm{~m}^{2}$ & AOC 2 and 3 & Yes & FSS Completed & Yes & Pass \\
\hline C1 SU5.1 & 1 & $179 \mathrm{~m}^{2}$ & AOC 2 and 3 & Yes & FSS Completed & Yes & Pass \\
\hline
\end{tabular}




\begin{tabular}{|c|c|c|c|c|c|c|c|}
\hline \multicolumn{8}{|c|}{$\begin{array}{c}\text { Table B-1 (cont.): Survey Unit Descriptions and Results } \\
\text { Hammond Depot } \\
\text { Hammond, Indiana }\end{array}$} \\
\hline Class and Survey Unit & Class & Unit Area & Remarks & $\begin{array}{c}\text { DQO } \\
\text { complete }\end{array}$ & FSS complete & $\begin{array}{c}\text { DQA } \\
\text { Complete }\end{array}$ & Remarks \\
\hline C1 SU5.2 & 1 & $196 \mathrm{~m}^{2}$ & AOC 2 and 3 & Yes & FSS Completed & Yes & Pass \\
\hline C1 SU5.3 & 1 & $207 \mathrm{~m}^{2}$ & AOC 2 and 3 & Yes & FSS Completed & Yes & Pass \\
\hline C1 SU5.5 & 1 & $205 \mathrm{~m}^{2}$ & AOC 2 and 3 & Yes & FSS Completed & Yes & Pass \\
\hline C1 SU5.6 & 1 & $232 \mathrm{~m}^{2}$ & AOC 2 and 3 & Yes & FSS Completed & Yes & Pass \\
\hline C1 SU5.7 & 1 & $172 \mathrm{~m}^{2}$ & AOC 2 and 3 & Yes & FSS Completed & Yes & Pass \\
\hline C1 SU5.8 & 1 & $144 \mathrm{~m}^{2}$ & AOC 2 and 3 & Yes & FSS Completed & Yes & Pass \\
\hline C1 SU6 & 1 & $2,513 \mathrm{~m}^{2}$ & $\mathrm{AOC} 6$ & Yes & FSS Completed & Yes & Pass \\
\hline C1 SU7 & 1 & $1,575 \mathrm{~m}^{2}$ & $\mathrm{AOC} 5$ & Yes & FSS Completed & Yes & Pass \\
\hline C2 SU1 & 2 & $11,489 \mathrm{~m}^{2}$ & Land Area & Yes & FSS Completed & Yes & Pass \\
\hline C2 SU2 & 2 & $6,476 \mathrm{~m}^{2}$ & Land Area & Yes & FSS Completed & Yes & Pass \\
\hline C2 SU3 & 2 & $8,947 \mathrm{~m}^{2}$ & Land Area & Yes & FSS Completed & Yes & Pass \\
\hline C2 SU4 & 2 & $5,864 \mathrm{~m}^{2}$ & Land Area & Yes & FSS Completed & Yes & Pass \\
\hline C2 SU5 & 2 & $8,522 \mathrm{~m}^{2}$ & Land Area & Yes & FSS Completed & Yes & Pass \\
\hline C2 SU6 & 2 & $6,970 \mathrm{~m}^{2}$ & Land Area & Yes & FSS Completed & Yes & Pass \\
\hline C2 SU7 & 2 & $4,559 \mathrm{~m}^{2}$ & Land Area & Yes & FSS Completed & Yes & Pass \\
\hline C2 SU8 & 2 & $10,301 \mathrm{~m}^{2}$ & Land Area & Yes & FSS Completed & Yes & Pass \\
\hline C2 SU9 & 2 & $5,893 \mathrm{~m}^{2}$ & Land Area & Yes & FSS Completed & Yes & Pass \\
\hline C2 SU10 & 2 & $4,974 \mathrm{~m}^{2}$ & Land Area & Yes & FSS Completed & Yes & Pass \\
\hline C2 SU11 & 2 & $3,289 \mathrm{~m}^{2}$ & Land Area & Yes & FSS Completed & Yes & Pass \\
\hline C2 SU12 (soil) & 2 & $437 \mathrm{~m}^{2}$ & Debris Pile & Yes & FSS Completed & Yes & Pass \\
\hline
\end{tabular}




\begin{tabular}{|c|c|c|c|c|c|c|c|}
\hline \multicolumn{8}{|c|}{$\begin{array}{c}\text { Table B-1 (cont.): Survey Unit Descriptions and Results } \\
\text { Hammond Depot } \\
\text { Hammond, Indiana }\end{array}$} \\
\hline Class and Survey Unit & Class & Unit Area & Remarks & $\begin{array}{c}\text { DQO } \\
\text { complete }\end{array}$ & FSS complete & $\begin{array}{c}\text { DQA } \\
\text { Complete }\end{array}$ & Remarks \\
\hline C2 SU12 (solid debris) & 2 & $437 \mathrm{~m}^{2}$ & Debris Pile & Yes & FSS Completed & $\begin{array}{l}\text { Yes. } \\
\text { Investigation } \\
\text { Completed/See } \\
\text { Table B-4 }\end{array}$ & Pass \\
\hline \multicolumn{8}{|c|}{ Class 3 Survey Units (C3 SU\#) } \\
\hline C3 SU1 & 3 & $30,338 \mathrm{~m}^{2}$ & Land Area & Yes & FSS Completed & Yes & Pass \\
\hline C3 SU2 & 3 & $34,296 \mathrm{~m}^{2}$ & Land Area & Yes & FSS Completed & Yes & Pass \\
\hline C3 SU3 & 3 & $23,844 \mathrm{~m}^{2}$ & Land Area & Yes & FSS Completed & Yes & Pass \\
\hline C3 SU4 & 3 & $17,561 \mathrm{~m}^{2}$ & Land Area & Yes & FSS Completed & Yes & Pass \\
\hline C3 SU5 & 3 & $19,216 \mathrm{~m}^{2}$ & Land Area & Yes & FSS Completed & Yes & Pass \\
\hline \multicolumn{8}{|l|}{ Building 200E South } \\
\hline C1 SU1 & 1 & $100 \mathrm{~m}^{2}$ & Floor & Yes & FSS Completed & Yes & Pass \\
\hline C1 SU2 & 1 & $100 \mathrm{~m}^{2}$ & Floor & Yes & FSS Completed & Yes & Pass \\
\hline C1 SU3 & 1 & $100 \mathrm{~m}^{2}$ & Floor & Yes & FSS Completed & Yes & Pass \\
\hline C1 SU4 & 1 & $150 \mathrm{~m}^{2}$ & Floor & Yes & FSS Completed & Yes & Pass \\
\hline C1 SU5 & 1 & $100 \mathrm{~m}^{2}$ & Floor & Yes & FSS Completed & Yes & Pass \\
\hline C1 SU6 & 1 & $100 \mathrm{~m}^{2}$ & Floor & Yes & FSS Completed & Yes & Pass \\
\hline C1 SU7 & 1 & $100 \mathrm{~m}^{2}$ & Floor & Yes & FSS Completed & Yes & Pass \\
\hline C1 SU8 & 1 & $100 \mathrm{~m}^{2}$ & Floor & Yes & FSS Completed & Yes & Pass \\
\hline C1 SU9 & 1 & $100 \mathrm{~m}^{2}$ & Floor & Yes & FSS Completed & Yes & Pass \\
\hline C1 SU10 & 1 & $100 \mathrm{~m}^{2}$ & Floor & Yes & FSS Completed & Yes & Pass \\
\hline C1 SU11 & 1 & $150 \mathrm{~m}^{2}$ & Floor & Yes & FSS Completed & Yes & Pass \\
\hline C1 SU12 & 1 & $100 \mathrm{~m}^{2}$ & Floor & Yes & FSS Completed & Yes & Pass \\
\hline C1 SU13 & 1 & $100 \mathrm{~m}^{2}$ & Floor & Yes & FSS Completed & Yes & Pass \\
\hline
\end{tabular}




\begin{tabular}{|c|c|c|c|c|c|c|c|}
\hline \multicolumn{8}{|c|}{$\begin{array}{c}\text { Table B-1 (cont.): Survey Unit Descriptions and Results } \\
\text { Hammond Depot } \\
\text { Hammond, Indiana }\end{array}$} \\
\hline Class and Survey Unit & Class & Unit Area & Remarks & $\begin{array}{c}\text { DQO } \\
\text { complete }\end{array}$ & FSS complete & $\begin{array}{c}\text { DQA } \\
\text { Complete }\end{array}$ & Remarks \\
\hline C1 SU15 & 1 & $100 \mathrm{~m}^{2}$ & Floor & Yes & FSS Completed & Yes & Pass \\
\hline C1 SU16 & 1 & $100 \mathrm{~m}^{2}$ & Floor & Yes & FSS Completed & Yes & Pass \\
\hline C1 SU17 & 1 & $100 \mathrm{~m}^{2}$ & Floor & Yes & FSS Completed & Yes & Pass \\
\hline C1 SU18 & 1 & $150 \mathrm{~m}^{2}$ & Floor & Yes & FSS Completed & Yes & Pass \\
\hline C1 SU19 & 1 & $100 \mathrm{~m}^{2}$ & Floor & Yes & FSS Completed & Yes & Pass \\
\hline C1 SU20 & 1 & $100 \mathrm{~m}^{2}$ & Floor & Yes & FSS Completed & Yes & Pass \\
\hline C1 SU21 & 1 & $100 \mathrm{~m}^{2}$ & Floor & Yes & FSS Completed & Yes & Pass \\
\hline C1 SU22 & 1 & $33 \mathrm{~m}^{2}$ & Bay 10, Sect. 5 N. Wall & Yes & FSS Completed & Yes & Pass \\
\hline C1 SU23 & 1 & $50 \mathrm{~m}^{2}$ & Bay 4, $5 \mathrm{~W}$. Wall & Yes & FSS Completed & Yes & Pass \\
\hline C1 SU24 & 1 & $125 \mathrm{~m}^{2}$ & Overheads & Yes & FSS Completed & Yes & Pass \\
\hline C1 SU25 & 1 & $125 \mathrm{~m}^{2}$ & Overheads & Yes & FSS Completed & Yes & Pass \\
\hline C1 SU26 & 1 & $125 \mathrm{~m}^{2}$ & Overheads & Yes & FSS Completed & Yes & Pass \\
\hline C1 SU27 & 1 & $125 \mathrm{~m}^{2}$ & Overheads & Yes & FSS Completed & $\begin{array}{l}\text { Yes. } \\
\text { Investigation } \\
\text { Completed/See } \\
\text { Table B-4 } \\
\end{array}$ & Pass \\
\hline C1 SU28 & 1 & $125 \mathrm{~m}^{2}$ & Overheads & Yes & FSS Completed & \begin{tabular}{|l|} 
Yes. \\
Investigation \\
Completed/See \\
Table B-4 \\
\end{tabular} & Pass \\
\hline C1 SU29 & 1 & $125 \mathrm{~m}^{2}$ & Overheads & Yes & FSS Completed & \begin{tabular}{|l|} 
Yes. \\
Investigation \\
Completed/See \\
Table B-4 \\
\end{tabular} & Pass \\
\hline C1 SU30 & 1 & $125 \mathrm{~m}^{2}$ & Overheads & Yes & FSS Completed & $\begin{array}{l}\text { Yes. } \\
\text { Investigation } \\
\text { Completed/See } \\
\text { Table B-4 }\end{array}$ & Pass \\
\hline
\end{tabular}




\begin{tabular}{|c|c|c|c|c|c|c|c|}
\hline \multicolumn{8}{|c|}{$\begin{array}{c}\text { Table B-1 (cont.): Survey Unit Descriptions and Results } \\
\text { Hammond Depot } \\
\text { Hammond, Indiana }\end{array}$} \\
\hline Class and Survey Unit & Class & Unit Area & Remarks & $\begin{array}{c}\text { DQO } \\
\text { complete }\end{array}$ & FSS complete & $\begin{array}{c}\text { DQA } \\
\text { Complete }\end{array}$ & Remarks \\
\hline C1 SU31 & 1 & $125 \mathrm{~m}^{2}$ & Overheads & Yes & FSS Completed & $\begin{array}{l}\text { Yes. } \\
\text { Investigation } \\
\text { Completed/See } \\
\text { Table B-4 }\end{array}$ & Pass \\
\hline C1 SU32 & 1 & $125 \mathrm{~m}^{2}$ & Overheads & Yes & FSS Completed & $\begin{array}{l}\text { Yes. } \\
\text { Investigation } \\
\text { Completed/See } \\
\text { Table B-4 }\end{array}$ & Pass \\
\hline C1 SU33 & 1 & $72 \mathrm{~m}^{2}$ & Columns 1 through 20 & Yes & FSS Completed & Yes & Pass \\
\hline C1 SU34 & 1 & $72 \mathrm{~m}^{2}$ & Columns 21 through 40 & Yes & FSS Completed & Yes & Pass \\
\hline C1 SU35 & 2 & $545 \mathrm{~m}^{2}$ & Overheads, Bays 1-2.5 & Yes & FSS Completed & Yes & Pass \\
\hline C1 SU36 & 2 & $545 \mathrm{~m}^{2}$ & Overheads, Bays 2.5-5 & Yes & FSS Completed & Yes & Pass \\
\hline C2 SU56 & 2 & $251 \mathrm{~m}^{2}$ & S. Wall and W. Wall Bays 1-3 & Yes & FSS Completed & Yes & Pass \\
\hline C2 SU57 & 2 & $266 \mathrm{~m}^{2}$ & W. Wall Bay 6-10 and N. Wall Sections 1-4 & Yes & FSS Completed & Yes & Pass \\
\hline C2 SU58 & 2 & $281 \mathrm{~m}^{2}$ & East Wall & Yes & FSS Completed & Yes & Pass \\
\hline \multicolumn{8}{|l|}{ Building 200E North } \\
\hline C2 SU37 & 2 & $1,173 \mathrm{~m}^{2}$ & Floor & Yes & FSS Completed & Yes & Pass \\
\hline C2 SU38 & 2 & $1,173 \mathrm{~m}^{2}$ & Floor & Yes & FSS Completed & Yes & Pass \\
\hline C3 SU39 & 3 & $901 \mathrm{~m}^{2}$ & Walls & Yes & FSS Completed & Yes & Pass \\
\hline C3 SU40 & 3 & $2,350 \mathrm{~m}^{2}$ & Ceiling, & Yes & FSS Completed & Yes & Pass \\
\hline C1 SU41 & 1 & $12 \mathrm{~m}^{2}$ & Closet & Yes & FSS Completed & Yes & Pass \\
\hline C2 SU42 & 2 & $64 \mathrm{~m}^{2}$ & Locker Room F/LW & Yes & FSS Completed & Yes & Pass \\
\hline \multicolumn{8}{|l|}{ Building 100E } \\
\hline C1 SU43 & 1 & $94 \mathrm{~m}^{2}$ & Floor, Bay 16 & Yes & FSS Completed & $\begin{array}{l}\text { Yes. } \\
\text { Investigation } \\
\text { Completed/See } \\
\text { Table B-4 }\end{array}$ & Pass \\
\hline
\end{tabular}




\begin{tabular}{|c|c|c|c|c|c|c|c|}
\hline \multicolumn{8}{|c|}{$\begin{array}{c}\text { Table B-1 (cont.): Survey Unit Descriptions and Results } \\
\text { Hammond Depot } \\
\text { Hammond, Indiana }\end{array}$} \\
\hline Class and Survey Unit & Class & Unit Area & Remarks & $\begin{array}{c}\text { DQO } \\
\text { complete }\end{array}$ & FSS complete & $\begin{array}{c}\text { DQA } \\
\text { Complete }\end{array}$ & Remarks \\
\hline C1 SU44 & 1 & $94 \mathrm{~m}^{2}$ & Floor, Bay 17 & Yes & FSS Completed & \begin{tabular}{l|} 
Yes. \\
Investigation \\
Completed/See \\
Table B-4
\end{tabular} & Pass \\
\hline C1 SU59 & 1 & $93 \mathrm{~m}^{2}$ & Monazite floor & Yes & FSS Completed & Yes & Pass \\
\hline C2 SU45 & 2 & $1,090 \mathrm{~m}^{2}$ & Floor, Bays 15-20 & Yes & FSS Completed & Yes & Pass \\
\hline C2 SU46 & 2 & $663 \mathrm{~m}^{2}$ & Walls, Bays 15-20 & Yes & FSS Completed & Yes & Pass \\
\hline C3 SU47 & 3 & $1,278 \mathrm{~m} 2$ & Ceiling, Bays 15-20 & Yes & FSS Completed & Yes & Pass \\
\hline C3 SU48 & 3 & $7,400 \mathrm{~m}^{2}$ & All Surfaces, Bays 1-14 & Yes & FSS Completed & Yes & Pass \\
\hline C2 SU60 & 2 & $93 \mathrm{~m}^{2}$ & W. side of C1 SU59 floor & Yes & FSS Completed & Yes & Pass \\
\hline C2 SU61 & 2 & $46 \mathrm{~m}^{2}$ & E. Side of C1 SU59 floor & Yes & FSS Completed & Yes & Pass \\
\hline \multicolumn{8}{|l|}{ Building 100W } \\
\hline C2 SU 49 & 2 & $1,750 \mathrm{~m}^{2}$ & Floor, Bays 7-17 & Yes & FSS Completed & Yes & Pass \\
\hline C2 SU 50 & 2 & $2,100 \mathrm{~m}^{2}$ & Floor and Walls, Bays 1-10 & Yes & FSS Completed & Yes & Pass \\
\hline C2 SU 51 & 2 & $2,100 \mathrm{~m}^{2}$ & Floor and Walls, Bays 11-20 & Yes & FSS Completed & Yes & Pass \\
\hline C3 SU 52 & 3 & $4,514 \mathrm{~m}^{2}$ & Ceiling & Yes & FSS Completed & Yes & Pass \\
\hline \multicolumn{8}{|l|}{ Misc. Buildings } \\
\hline C3 SU53 & 3 & $739 \mathrm{~m}^{2}$ & Garage & Yes & FSS Completed & Yes & Pass \\
\hline C3 SU54 & 3 & $332 \mathrm{~m}^{2}$ & Workshop & Yes & FSS Completed & Yes & Pass \\
\hline C3 SU55 & 3 & $366 \mathrm{~m}^{2}$ & Building exteriors & Yes & FSS Completed & Yes & Pass \\
\hline
\end{tabular}




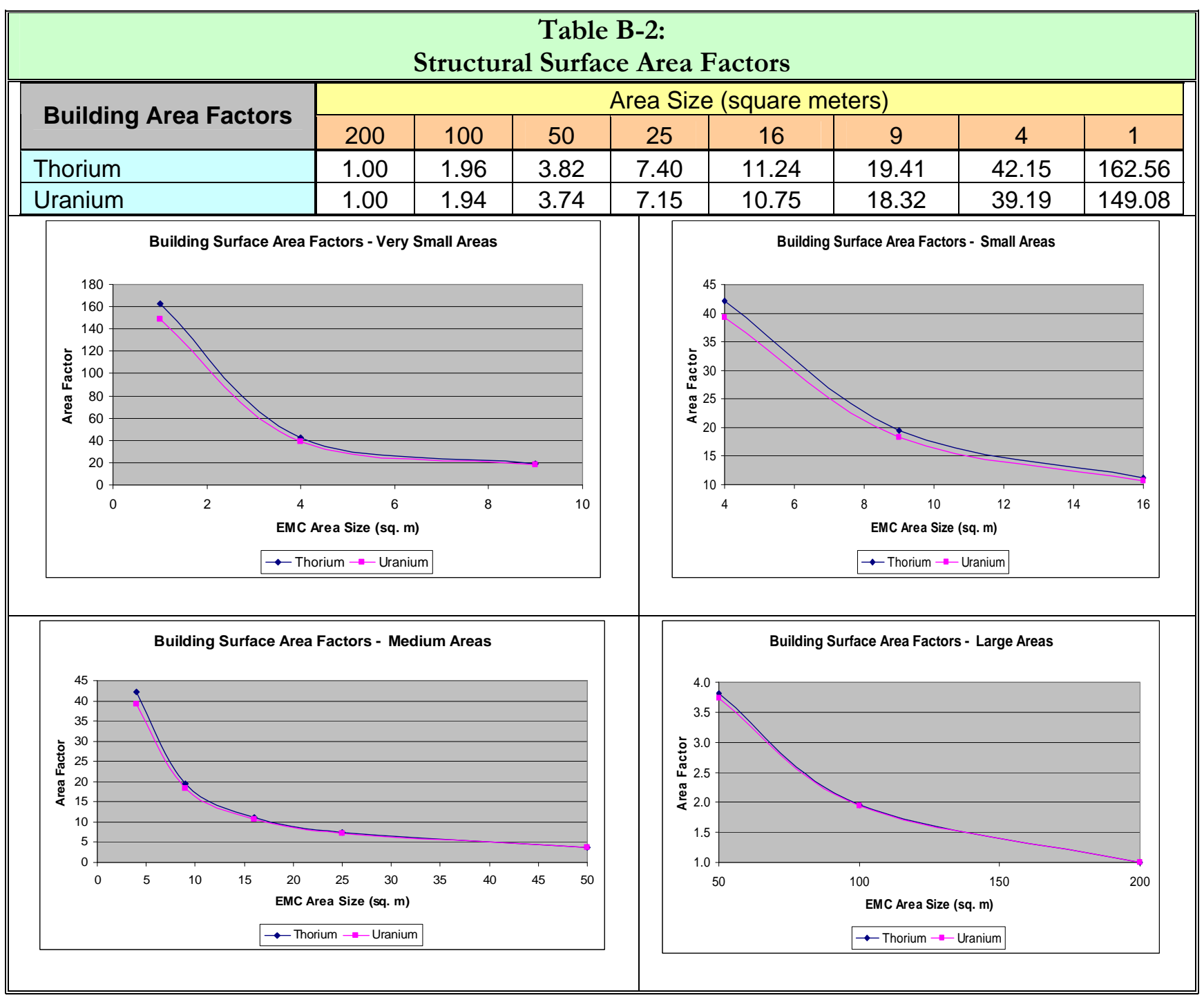




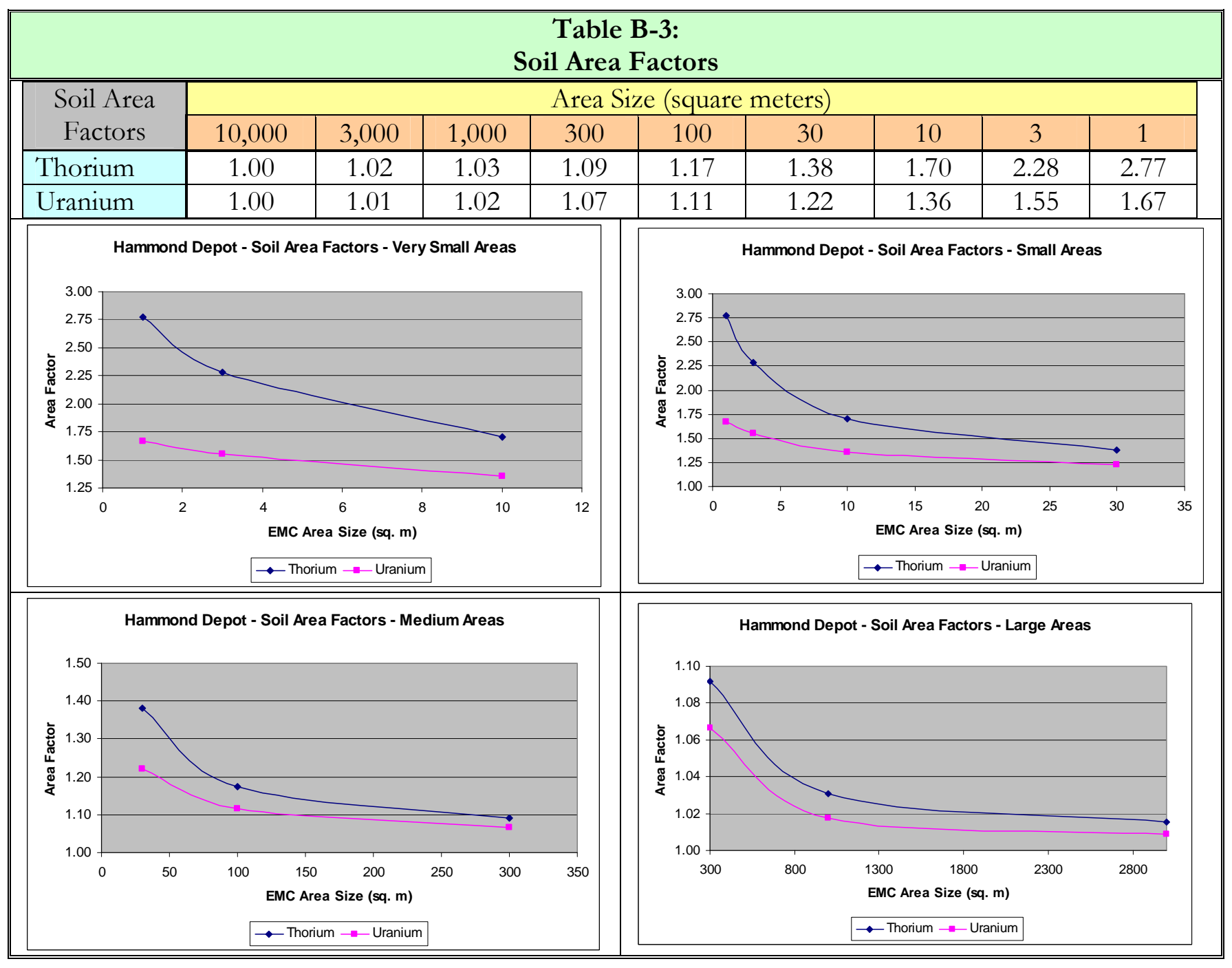




\begin{tabular}{|c|c|c|c|c|c|c|}
\hline \multicolumn{7}{|c|}{$\begin{array}{c}\text { Table B-4: Investigation Results } \\
\text { Hammond Depot } \\
\text { Hammond, Indiana }\end{array}$} \\
\hline $\begin{array}{c}\text { Survey } \\
\text { Unit }\end{array}$ & Class & Investigation Cause & $\begin{array}{l}\text { Sample } \\
\text { IDs }\end{array}$ & $\begin{array}{c}\text { Initial } \\
\text { Review }\end{array}$ & Further Investigation Required & $\begin{array}{l}\text { Open/Closed } \\
\text { Investigation }\end{array}$ \\
\hline $\begin{array}{l}\text { Bldg. 100E } \\
\text { C1 SU43 } \\
\text { and } 44\end{array}$ & 1 & $\begin{array}{l}\text { Elevated gamma activity } \\
\text { detected. Monazite sand } \\
\text { residue found on a pallet } \\
\text { of tannin }\end{array}$ & NA & NA & $\begin{array}{l}\text { Yes. Remediation of floor and expansion } \\
\text { required, three contaminated pallets of tannin } \\
\text { disposed of. Establishment of a new Class } 1 \\
\text { SU and two bounding Class } 2 \text { SUs required. } \\
\text { Surveys of tannin pallets required. } 100 \% \text { of } \\
\text { accessible pallets were gamma scanned. No } \\
\text { further contamination identified. } \\
\text { Approximately } 300 \text { individual pallets were } \\
\text { moved via fork lift and gamma scanned. No } \\
\text { further contaminated pallets identified. } \\
\text { Probable cause of contaminated pallets due } \\
\text { to spill of monazite drum while moving } \\
\text { between roll-up doors in Bay } 15 \text {. }\end{array}$ & Closed \\
\hline $\begin{array}{l}\text { Bldg 200E } \\
\text { SUs C2 } \\
\text { SU30 and } \\
31 \text { changed } \\
\text { to Class } 1\end{array}$ & 2 to 1 & $\begin{array}{l}\text { Prior to FSS, additional } \\
\text { characterization surveys } \\
\text { identified several small } \\
\text { areas of contamination on } \\
\text { concrete following } \\
\text { removal of asphalt } \\
\text { overlayment. }\end{array}$ & NA & NA & No. & Closed \\
\hline $\begin{array}{l}\text { C2 SU12 } \\
\text { Debris Piles } \\
\text { (solid } \\
\text { debris) }\end{array}$ & 2 & $\begin{array}{l}\text { One direct measurement } \\
\text { on a brick exceeded } \\
\text { DCGL of } 400 \mathrm{dpm} / 100 \\
\mathrm{~cm} 2\end{array}$ & NA & & $\begin{array}{l}\text { Yes. Brick was relocated and identified as a } \\
\text { common, yellow firebrick from furnaces used } \\
\text { in the steel industry. These firebricks contain } \\
\text { naturally occurring elevated levels of thorium } \\
\text { and uranium. A recount of the brick was } 693 \\
\text { cpm. }\end{array}$ & Closed \\
\hline
\end{tabular}




\begin{tabular}{|c|c|l|c|c|c|c||}
\hline \multicolumn{7}{|c|}{$\begin{array}{c}\text { Table B-4 (cont.): Investigation Results } \\
\text { Hammond Depot } \\
\text { Hammond, Indiana }\end{array}$} \\
\hline $\begin{array}{c}\text { Survey } \\
\text { Unit }\end{array}$ & Class & \multicolumn{1}{|c||}{ Investigation Cause } & $\begin{array}{c}\text { Sample } \\
\text { IDs }\end{array}$ & $\begin{array}{c}\text { Initial } \\
\text { Review }\end{array}$ & \multicolumn{1}{|c|}{ Further Investigation Required } & $\begin{array}{c}\text { Open/Closed } \\
\text { Investigation }\end{array}$ \\
\hline \hline C1 SU34 & 1 & $\begin{array}{l}\text { Contamination found on } \\
\text { column 22 during FSS }\end{array}$ & NA & NA & $\begin{array}{l}\text { Yes. World Environmental notified and } \\
\text { decon of two small spots performed. }\end{array}$ & Closed \\
\hline C1 SU14 & 1 & $\begin{array}{l}\text { Low-level contamination } \\
\text { found at systematic } \\
\text { measurement location }\end{array}$ & NA & NA & $\begin{array}{l}\text { Yes. World Environmental notified and } \\
\text { decontamination of one small spot } \\
\text { performed. }\end{array}$ & Closed \\
\hline C1 SU18 & 1 & $\begin{array}{l}\text { Low level contamination } \\
\text { found at systematic } \\
\text { measurement location }\end{array}$ & NA & NA & $\begin{array}{l}\text { Yes. World Environmental notified and } \\
\text { decontamination of one small spot } \\
\text { performed. }\end{array}$ & $\begin{array}{l}\text { Closed } \\
\text { truss supports in northern 1/3 were } \\
\text { decontaminated. }\end{array}$ \\
\hline C1 SU27-32 & 1 & $\begin{array}{l}\text { Low level contamination } \\
\text { found during FSS scans in } \\
\text { truss supports }\end{array}$ & NA & NA & $\begin{array}{l}\text { Yes. World Environmental notified and all } \\
\text { Closed }\end{array}$ \\
\hline \hline
\end{tabular}




\begin{tabular}{|c|c|c|c|}
\hline \multicolumn{4}{|c|}{$\begin{array}{c}\text { Table B-5: FSS Structural Survey Units and Surface Activity Levels } \\
\text { Hammond Depot } \\
\text { Hammond, Indiana }\end{array}$} \\
\hline $\begin{array}{l}\text { Building/ } \\
\text { Survey Unit ID/ } \\
\text { Surface }\end{array}$ & $\begin{array}{l}\text { Location }(X, Y /, Z \\
\quad \text { or Smear ID) }{ }^{\mathrm{a}}\end{array}$ & Counts per Minute & $\begin{array}{l}\text { Total Beta Activity } \\
\mathrm{dpm} / 100 \mathrm{~cm}^{2}\end{array}$ \\
\hline \multicolumn{4}{|c|}{ Building 200E (south) } \\
\hline \multicolumn{4}{|l|}{ C1 SU1 } \\
\hline Floor & $1.2,2.2$ & 530 & 110 \\
\hline Floor & $4.1,2.2$ & 453 & -40 \\
\hline Floor & $6.9,2.2$ & 517 & 87 \\
\hline Floor & $9.8,2.2$ & 481 & 16 \\
\hline Floor & $2.6,4.7$ & 509 & 71 \\
\hline Floor & $5.5,4.7$ & 476 & 6 \\
\hline Floor & $8.4,4.7$ & 458 & -30 \\
\hline Floor & $1.2,7.2$ & 477 & 8 \\
\hline Floor & $4.1,7.2$ & 470 & -6 \\
\hline Floor & $6.9,7.2$ & 481 & 16 \\
\hline Floor & $9.8,7.2$ & 461 & -24 \\
\hline Floor & $2.6,9.7$ & 491 & 36 \\
\hline Floor & $5.5,9.7$ & 476 & 6 \\
\hline Floor & $8.4,9.7$ & 487 & 28 \\
\hline \multicolumn{4}{|l|}{ C1 SU2 } \\
\hline Floor & $12.4,2.1$ & 458 & -30 \\
\hline Floor & $15.2,2.1$ & 418 & -110 \\
\hline Floor & $18.1,2.1$ & 462 & -22 \\
\hline Floor & $10.9,4.6$ & 493 & 40 \\
\hline Floor & $13.8,4.6$ & 476 & 6 \\
\hline Floor & $16.7,4.6$ & 468 & -10 \\
\hline Floor & 19.6, 4.6 & 493 & 40 \\
\hline Floor & $12.4,7.1$ & 508 & 69 \\
\hline Floor & $15.2,7.1$ & 475 & 4 \\
\hline Floor & $18.1,7.1$ & 491 & 36 \\
\hline Floor & $10.9,9.5$ & 461 & -24 \\
\hline Floor & $13.8,9.5$ & 482 & 18 \\
\hline Floor & $16.7,9.5$ & 525 & 100 \\
\hline Floor & $19.6,9.5$ & 474 & 2 \\
\hline \multicolumn{4}{|l|}{ C1 SU3 } \\
\hline Floor & $21.2,1.4$ & 429 & -87 \\
\hline Floor & $24.0,1.4$ & 442 & -62 \\
\hline Floor & $26.9,1.4$ & 450 & -46 \\
\hline Floor & $29.8,1.4$ & 465 & -16 \\
\hline Floor & $22.6,3.9$ & 485 & 24 \\
\hline Floor & $25.5,3.9$ & 459 & -28 \\
\hline Floor & $28.3,3.9$ & 449 & -48 \\
\hline
\end{tabular}




\begin{tabular}{|c|c|c|c|}
\hline \multicolumn{4}{|c|}{$\begin{array}{c}\text { Table B-5 (cont.): FSS Structural Survey Units and Surface Activity Levels } \\
\text { Hammond Depot } \\
\text { Hammond, Indiana }\end{array}$} \\
\hline $\begin{array}{l}\text { Building/ } \\
\text { Survey Unit ID/ } \\
\text { Surface }\end{array}$ & $\begin{array}{c}\text { Location }(\mathrm{X}, \mathrm{Y} /, \mathrm{Z} \\
\text { or Smear ID) }\end{array}$ & Counts per Minute & $\begin{array}{l}\text { Total Beta Activity } \\
\text { dpm } / 100 \mathrm{~cm}^{2}\end{array}$ \\
\hline \multicolumn{4}{|l|}{ C1 SU3 (cont.) } \\
\hline Floor & $21.2,6.4$ & 509 & 71 \\
\hline Floor & $24.0,6.4$ & 405 & -130 \\
\hline Floor & $26.9,6.4$ & 405 & -130 \\
\hline Floor & $29.8,6.4$ & 476 & 6 \\
\hline Floor & $22.6,8.8$ & 412 & -120 \\
\hline Floor & $25.5,8.8$ & 462 & -22 \\
\hline Floor & $28.3,8.8$ & 468 & -10 \\
\hline \multicolumn{4}{|l|}{ C1 SU4 } \\
\hline Floor & $31.0,0.7$ & 410 & -120 \\
\hline Floor & $34.5,0.7$ & 434 & -77 \\
\hline Floor & $32.8,3.7$ & 458 & -30 \\
\hline Floor & $36.3,3.7$ & 421 & -100 \\
\hline Floor & $31.0,6.8$ & 501 & 56 \\
\hline Floor & $34.5,6.8$ & 409 & -130 \\
\hline Floor & $32.8,9.8$ & 445 & -56 \\
\hline Floor & $36.3,9.8$ & 443 & -60 \\
\hline Floor & $31.0,12.9$ & 409 & -130 \\
\hline Floor & $34.5,12.9$ & 414 & -120 \\
\hline Floor & $32.8,15.9$ & 471 & -4 \\
\hline Floor & $36.3,15.9$ & 471 & -4 \\
\hline Floor & $31.0,19.0$ & 422 & -100 \\
\hline Floor & $34.5,19.0$ & 429 & -87 \\
\hline Driveway & $39.9,15.9$ & 302 & -69 \\
\hline \multicolumn{4}{|l|}{ C1 SU5 } \\
\hline Floor & $2.1,12.2$ & 464 & -18 \\
\hline Floor & $5.0,12.2$ & 451 & -44 \\
\hline Floor & $7.8,12.2$ & 480 & 14 \\
\hline Floor & $0.6,14.7$ & 461 & -24 \\
\hline Floor & $3.5,14.7$ & 485 & 24 \\
\hline Floor & $6.4,14.7$ & 489 & 32 \\
\hline Floor & $9.3,14.7$ & 439 & -67 \\
\hline Floor & $2.1,17.2$ & 537 & 130 \\
\hline Floor & $5.0,17.2$ & 465 & -16 \\
\hline Floor & $7.8,17.2$ & 444 & -58 \\
\hline Floor & $0.6,19.7$ & 559 & 170 \\
\hline Floor & $3.5,19.7$ & 466 & -14 \\
\hline Floor & $6.4,19.7$ & 496 & 46 \\
\hline Floor & $9.3,19.7$ & 517 & 87 \\
\hline Driveway & $-3.4,14.7$ & 378 & 81 \\
\hline
\end{tabular}




\begin{tabular}{|c|c|c|c|}
\hline \multicolumn{4}{|c|}{$\begin{array}{c}\text { Table B-5 (cont.): FSS Structural Survey Units and Surface Activity Levels } \\
\text { Hammond Depot } \\
\text { Hammond, Indiana }\end{array}$} \\
\hline $\begin{array}{l}\text { Building/ } \\
\text { Survey Unit ID/ } \\
\text { Surface }\end{array}$ & $\begin{array}{l}\text { Location (X, Y/, Z } \\
\text { or Smear ID) }^{\mathrm{a}}\end{array}$ & Counts per Minute & $\begin{array}{l}\text { Total Beta Activity } \\
\text { dpm } / 100 \mathrm{~cm}^{2}\end{array}$ \\
\hline \multicolumn{4}{|l|}{ C1 SU6 } \\
\hline Floor & $10.7,11.3$ & 486 & 26 \\
\hline Floor & $13.5,11.3$ & 469 & -8 \\
\hline Floor & $16.4,11.3$ & 508 & 69 \\
\hline Floor & $19.3,11.3$ & 494 & 42 \\
\hline Floor & $12.1,13.8$ & 483 & 20 \\
\hline Floor & $15.0,13.8$ & 475 & 4 \\
\hline Floor & $17.8,13.8$ & 503 & 60 \\
\hline Floor & $10.7,16.3$ & 483 & 20 \\
\hline Floor & $13.5,16.3$ & 493 & 40 \\
\hline Floor & $16.4,16.3$ & 492 & 38 \\
\hline Floor & $19.3,16.3$ & 488 & 30 \\
\hline Floor & $14.5,11.2$ & 448 & -50 \\
\hline Floor & $10.8,15.8$ & 428 & -89 \\
\hline Floor & $16.9,16.1$ & 407 & -130 \\
\hline \multicolumn{4}{|l|}{ C1 SU7 } \\
\hline Floor & $20.1,12.3$ & 449 & -48 \\
\hline Floor & $23.0,12.3$ & 430 & -85 \\
\hline Floor & $25.8,12.3$ & 493 & 40 \\
\hline Floor & $28.7,12.3$ & 497 & 48 \\
\hline Floor & $21.5,14.7$ & 475 & 4 \\
\hline Floor & $24.4,14.7$ & 446 & -54 \\
\hline Floor & $27.3,14.7$ & 485 & 24 \\
\hline Floor & $21.3,16.4$ & 426 & -93 \\
\hline Floor & $23.0,17.2$ & 457 & -32 \\
\hline Floor & $25.8,17.2$ & 479 & 12 \\
\hline Floor & $28.7,17.2$ & 488 & 30 \\
\hline Floor & $28.6,19.1$ & 493 & 40 \\
\hline Floor & $24.4,19.7$ & 508 & 69 \\
\hline Floor & $27.3,19.7$ & 510 & 73 \\
\hline \multicolumn{4}{|l|}{ C1 SU8 } \\
\hline Floor & $1.0,22.2$ & 480 & 14 \\
\hline Floor & $3.9,22.2$ & 444 & -58 \\
\hline Floor & $6.8,22.2$ & 478 & 10 \\
\hline Floor & $9.7,22.2$ & 466 & -14 \\
\hline Floor & $2.5,24.7$ & 256 & -430 \\
\hline Floor & $5.4,24.7$ & 595 & 240 \\
\hline Floor & $8.2,24.7$ & 384 & -180 \\
\hline Floor & $1.0,27.2$ & 437 & -71 \\
\hline Floor & $3.9,27.2$ & 538 & 130 \\
\hline
\end{tabular}




\begin{tabular}{|c|c|c|c|}
\hline \multicolumn{4}{|c|}{$\begin{array}{c}\text { Table B-5 (cont.): FSS Structural Survey Units and Surface Activity Levels } \\
\text { Hammond Depot } \\
\text { Hammond, Indiana }\end{array}$} \\
\hline $\begin{array}{l}\text { Building/ } \\
\text { Survey Unit ID/ } \\
\text { Surface }\end{array}$ & $\begin{array}{l}\text { Location }(\mathrm{X}, \mathrm{Y} /, \mathrm{Z} \\
\text { or Smear ID) }^{\mathrm{a}}\end{array}$ & Counts per Minute & $\begin{array}{l}\text { Total Beta Activity } \\
\text { dpm } / 100 \mathrm{~cm}^{2}\end{array}$ \\
\hline \multicolumn{4}{|l|}{ C1 SU8 (cont.) } \\
\hline Floor & $6.8,27.2$ & 433 & -79 \\
\hline Floor & $9.7,27.2$ & 454 & -38 \\
\hline Floor & $2.5,29.7$ & 376 & -190 \\
\hline Floor & $5.4,29.7$ & 356 & -230 \\
\hline Floor & $8.2,29.7$ & 583 & 220 \\
\hline \multicolumn{4}{|l|}{ C1 SU9 } \\
\hline Floor & $10.1,21.8$ & 509 & 71 \\
\hline Floor & $13.0,21.8$ & 535 & 120 \\
\hline Floor & $15.9,21.8$ & 500 & 54 \\
\hline Floor & $18.7,21.8$ & 511 & 75 \\
\hline Floor & $11.6,24.3$ & 510 & 73 \\
\hline Floor & $14.4,24.3$ & 476 & 6 \\
\hline Floor & $17.3,24.3$ & 580 & 210 \\
\hline Floor & $10.1,26.8$ & 534 & 120 \\
\hline Floor & $13.0,26.8$ & 499 & 52 \\
\hline Floor & $15.9,26.8$ & 534 & 120 \\
\hline Floor & $18.7,26.8$ & 484 & 22 \\
\hline Floor & $11.6,29.2$ & 534 & 120 \\
\hline Floor & $14.4,29.2$ & 485 & 24 \\
\hline Floor & $17.3,29.2$ & 485 & 24 \\
\hline \multicolumn{4}{|l|}{ C1 SU10 } \\
\hline Floor & $21.3,21.4$ & 493 & 40 \\
\hline Floor & $24.1,21.4$ & 578 & 210 \\
\hline Floor & $27.0,21.4$ & 479 & 12 \\
\hline Floor & $29.9,21.4$ & 456 & -34 \\
\hline Floor & $24.8,25.0$ & 526 & 110 \\
\hline Floor & $25.6,23.9$ & 470 & -6 \\
\hline Floor & $28.4,23.9$ & 485 & 24 \\
\hline Floor & $21.3,26.4$ & 489 & 32 \\
\hline Floor & $24.1,26.4$ & 485 & 24 \\
\hline Floor & $27.0,26.4$ & 486 & 26 \\
\hline Floor & $29.9,26.4$ & 467 & -12 \\
\hline Floor & $22.7,28.9$ & 466 & -14 \\
\hline Floor & $25.6,28.9$ & 491 & 36 \\
\hline Floor & $28.4,28.9$ & 451 & -44 \\
\hline \multicolumn{4}{|l|}{ C1 SU11 } \\
\hline Floor & $31.2,20.0$ & 406 & -130 \\
\hline Floor & $34.8,20.0$ & 478 & 10 \\
\hline Floor & $33.0,23.1$ & 475 & 4 \\
\hline
\end{tabular}




\begin{tabular}{|c|c|c|c|}
\hline \multicolumn{4}{|c|}{$\begin{array}{c}\text { Table B-5 (cont.): FSS Structural Survey Units and Surface Activity Levels } \\
\text { Hammond Depot } \\
\text { Hammond, Indiana }\end{array}$} \\
\hline $\begin{array}{l}\text { Building/ } \\
\text { Survey Unit ID/ } \\
\text { Surface }\end{array}$ & $\begin{array}{l}\text { Location (X, Y/, Z } \\
\text { or Smear ID) }^{\mathrm{a}}\end{array}$ & Counts per Minute & $\begin{array}{l}\text { Total Beta Activity } \\
\text { dpm } / 100 \mathrm{~cm}^{2}\end{array}$ \\
\hline \multicolumn{4}{|l|}{ C1 SU11 (cont.) } \\
\hline Floor & $36.5,23.1$ & 414 & -120 \\
\hline Floor & $31.2,26.1$ & 481 & 16 \\
\hline Floor & $34.8,26.1$ & 503 & 60 \\
\hline Floor & $33.0,29.2$ & 477 & 8 \\
\hline Floor & $36.5,29.2$ & 410 & -120 \\
\hline Floor & $31.2,32.2$ & 471 & -4 \\
\hline Floor & $34.8,32.2$ & 500 & 54 \\
\hline Floor & $33.0,35.3$ & 430 & -85 \\
\hline Floor & $36.5,35.3$ & 473 & 0 \\
\hline Floor & $31.2,38.3$ & 452 & -42 \\
\hline Floor & $34.8,38.3$ & 425 & -95 \\
\hline \multicolumn{4}{|l|}{ C1 SU12 } \\
\hline Floor & $1.2,31.5$ & 435 & -75 \\
\hline Floor & $4.1,31.5$ & 514 & 81 \\
\hline Floor & $7.0,31.5$ & 523 & 99 \\
\hline Floor & $9.9,31.5$ & 462 & -22 \\
\hline Floor & $2.7,34.0$ & 492 & 38 \\
\hline Floor & $5.5,34.0$ & 476 & 6 \\
\hline Floor & $8.4,34.0$ & 522 & 97 \\
\hline Floor & $1.2,36.5$ & 468 & -10 \\
\hline Floor & $4.1,36.5$ & 454 & -38 \\
\hline Floor & $7.0,36.5$ & 553 & 160 \\
\hline Floor & $9.9,36.5$ & 496 & 46 \\
\hline Floor & $2.7,39.0$ & 459 & -28 \\
\hline Floor & $5.5,39.0$ & 468 & -10 \\
\hline Floor & $8.4,39.0$ & 432 & -81 \\
\hline \multicolumn{4}{|l|}{ C1 SU13 } \\
\hline Floor & $12.6,30.8$ & 505 & 63 \\
\hline Floor & $15.5,30.8$ & 392 & -160 \\
\hline Floor & $18.4,30.8$ & 462 & -22 \\
\hline Floor & $11.2,33.3$ & 477 & 8 \\
\hline Floor & $14.0,33.3$ & 581 & 210 \\
\hline Floor & $16.9,33.3$ & 446 & -54 \\
\hline Floor & $19.8,33.3$ & 509 & 71 \\
\hline Floor & $12.6,35.8$ & 488 & 30 \\
\hline Floor & $15.5,35.8$ & 476 & 6 \\
\hline Floor & $18.4,35.8$ & 638 & 330 \\
\hline Floor & $11.2,38.3$ & 474 & 2 \\
\hline Floor & $14.0,38.3$ & 518 & 89 \\
\hline
\end{tabular}




\begin{tabular}{|c|c|c|c|}
\hline \multicolumn{4}{|c|}{$\begin{array}{c}\text { Table B-5 (cont.): FSS Structural Survey Units and Surface Activity Levels } \\
\text { Hammond Depot } \\
\text { Hammond, Indiana }\end{array}$} \\
\hline $\begin{array}{l}\text { Building/ } \\
\text { Survey Unit ID/ } \\
\text { Surface }\end{array}$ & $\begin{array}{l}\text { Location (X, Y/, Z } \\
\text { or Smear ID) }\end{array}$ & Counts per Minute & $\begin{array}{l}\text { Total Beta Activity } \\
\mathrm{dpm} / 100 \mathrm{~cm}^{2}\end{array}$ \\
\hline \multicolumn{4}{|l|}{ C1 SU13 (cont.) } \\
\hline Floor & $16.9,38.3$ & 500 & 54 \\
\hline Floor & $19.8,38.3$ & 472 & -2 \\
\hline \multicolumn{4}{|l|}{ C1 SU14 } \\
\hline Floor & $29.5,32.9$ & 484 & 22 \\
\hline Floor & $25.4,31.8$ & 502 & 58 \\
\hline Floor & $28.2,31.8$ & 479 & 12 \\
\hline Floor & $21.0,34.3$ & 495 & 44 \\
\hline Floor & $23.9,34.3$ & 489 & 32 \\
\hline Floor & $26.8,34.3$ & 501 & 56 \\
\hline Floor & $29.7,34.3$ & 530 & 110 \\
\hline Floor & $22.5,36.8$ & 407 & -130 \\
\hline Floor & $25.4,36.8$ & 520 & 93 \\
\hline Floor & $28.2,36.8$ & 506 & 65 \\
\hline Floor & $21.0,39.3$ & 582 & 220 \\
\hline Floor & $23.9,39.3$ & 513 & 79 \\
\hline Floor & $26.8,39.3$ & 537 & 130 \\
\hline Floor & $29.7,39.3$ & 747 & 540 \\
\hline Post-RA & $29.7,39.3$ & 589 & 230 \\
\hline \multicolumn{4}{|l|}{ C1 SU15 } \\
\hline Floor & $0.4,41.0$ & 457 & -32 \\
\hline Floor & $3.2,41.0$ & 505 & 63 \\
\hline Floor & $6.1,41.0$ & 503 & 60 \\
\hline Floor & $9.0,41.0$ & 469 & -8 \\
\hline Floor & $1.8,43.4$ & 508 & 69 \\
\hline Floor & $4.7,43.4$ & 456 & -34 \\
\hline Floor & $7.5,43.4$ & 509 & 71 \\
\hline Floor & $0.4,45.9$ & 493 & 40 \\
\hline Floor & $3.2,45.9$ & 465 & -16 \\
\hline Floor & $6.1,45.9$ & 480 & 14 \\
\hline Floor & $9.0,45.9$ & 512 & 77 \\
\hline Floor & $1.8,48.4$ & 471 & -4 \\
\hline Floor & $4.7,48.4$ & 522 & 97 \\
\hline Floor & $7.5,48.4$ & 428 & -89 \\
\hline \multicolumn{4}{|l|}{ C1 SU16 } \\
\hline Floor & $11.1,41.4$ & 494 & 42 \\
\hline Floor & $14.0,41.4$ & 520 & 93 \\
\hline Floor & $16.8,41.4$ & 501 & 56 \\
\hline Floor & $19.7,41.4$ & 565 & 180 \\
\hline Floor & $12.5,43.9$ & 539 & 130 \\
\hline
\end{tabular}




\begin{tabular}{|c|c|c|c|}
\hline \multicolumn{4}{|c|}{$\begin{array}{c}\text { Table B-5 (cont.): FSS Structural Survey Units and Surface Activity Levels } \\
\text { Hammond Depot } \\
\text { Hammond, Indiana }\end{array}$} \\
\hline $\begin{array}{l}\text { Building/ } \\
\text { Survey Unit ID/ } \\
\text { Surface }\end{array}$ & $\begin{array}{l}\text { Location (X, Y/, Z } \\
\text { or Smear ID) }^{\mathrm{a}}\end{array}$ & Counts per Minute & $\begin{array}{l}\text { Total Beta Activity } \\
\text { dpm } / 100 \mathrm{~cm}^{2}\end{array}$ \\
\hline \multicolumn{4}{|l|}{ C1 SU16 (cont.) } \\
\hline Floor & $15.4,43.9$ & 512 & 77 \\
\hline Floor & $18.3,43.9$ & 518 & 89 \\
\hline Floor & $11.1,46.4$ & 475 & 4 \\
\hline Floor & $14.0,46.4$ & 485 & 24 \\
\hline Floor & $16.8,46.4$ & 446 & -54 \\
\hline Floor & $19.7,46.4$ & 450 & -46 \\
\hline Floor & $12.5,48.9$ & 532 & 120 \\
\hline Floor & $15.4,48.9$ & 451 & -44 \\
\hline Floor & $18.3,48.9$ & 457 & -32 \\
\hline \multicolumn{4}{|l|}{ C1 SU17 } \\
\hline Floor & $21.1,42.1$ & 466 & -14 \\
\hline Floor & $23.9,42.1$ & 469 & -8 \\
\hline Floor & $26.8,42.1$ & 522 & 97 \\
\hline Floor & $28.5,42.3$ & 513 & 79 \\
\hline Floor & $22.5,44.6$ & 489 & 32 \\
\hline Floor & $25.4,44.6$ & 521 & 95 \\
\hline Floor & $28.2,44.6$ & 539 & 130 \\
\hline Floor & $21.1,47.1$ & 517 & 87 \\
\hline Floor & $23.9,47.1$ & 493 & 40 \\
\hline Floor & $26.8,47.1$ & 501 & 56 \\
\hline Floor & $29.7,47.1$ & 474 & 2 \\
\hline Floor & $22.5,49.6$ & 426 & -93 \\
\hline Floor & $25.4,49.6$ & 471 & -4 \\
\hline Floor & $28.2,49.6$ & 511 & 75 \\
\hline \multicolumn{4}{|l|}{ C1 SU18 } \\
\hline Floor & $31.4,41.7$ & 1234 & 1500 \\
\hline Post-RA & $31.4,41.7$ & 493 & 40 \\
\hline Floor & $35.1,41.7$ & 490 & 34 \\
\hline Floor & $33.3,44.8$ & 516 & 85 \\
\hline Floor & $36.9,44.8$ & 431 & -83 \\
\hline Floor & $31.4,47.9$ & 506 & 65 \\
\hline Floor & $35.1,47.9$ & 481 & 16 \\
\hline Floor & $33.3,51.0$ & 538 & 130 \\
\hline Floor & $36.9,51.0$ & 452 & -42 \\
\hline Floor & $31.4,54.2$ & 498 & 50 \\
\hline Floor & $35.1,54.2$ & 446 & -54 \\
\hline Floor & $33.3,57.3$ & 469 & -8 \\
\hline Floor & $36.9,57.3$ & 463 & -20 \\
\hline Floor & $31.4,60.4$ & 488 & 30 \\
\hline
\end{tabular}




\begin{tabular}{|c|c|c|c|}
\hline \multicolumn{4}{|c|}{$\begin{array}{c}\text { Table B-5 (cont.): FSS Structural Survey Units and Surface Activity Levels } \\
\text { Hammond Depot } \\
\text { Hammond, Indiana }\end{array}$} \\
\hline $\begin{array}{l}\text { Building/ } \\
\text { Survey Unit ID/ } \\
\text { Surface }\end{array}$ & $\begin{array}{l}\text { Location (X, Y/, Z } \\
\text { or Smear ID) }^{\mathrm{a}}\end{array}$ & Counts per Minute & $\begin{array}{l}\text { Total Beta Activity } \\
\text { dpm } / 100 \mathrm{~cm}^{2}\end{array}$ \\
\hline \multicolumn{4}{|l|}{ C1 SU18 (cont.) } \\
\hline Floor & $35.1,60.4$ & 521 & 95 \\
\hline \multicolumn{4}{|l|}{ C1 SU19 } \\
\hline Floor & $0.7,52.5$ & 455 & -36 \\
\hline Floor & $3.7,52.5$ & 467 & -12 \\
\hline Floor & $6.7,52.5$ & 454 & -38 \\
\hline Floor & $9.7,52.5$ & 490 & 34 \\
\hline Floor & $2.2,55.1$ & 464 & -18 \\
\hline Floor & $5.2,55.1$ & 435 & -75 \\
\hline Floor & $8.2,55.1$ & 470 & -6 \\
\hline Floor & $0.7,57.7$ & 455 & -36 \\
\hline Floor & $3.7,57.7$ & 490 & 34 \\
\hline Floor & $6.7,57.7$ & 487 & 28 \\
\hline Floor & $9.7,57.7$ & 477 & 8 \\
\hline Floor & $2.8,58.2$ & 596 & 240 \\
\hline Floor & $1.6,53.8$ & 478 & 10 \\
\hline Floor & $8.2,60.3$ & 499 & 52 \\
\hline \multicolumn{4}{|l|}{ C1 SU20 } \\
\hline Floor & $12.1,51.0$ & 524 & 100 \\
\hline Floor & $15.1,51.0$ & 509 & 71 \\
\hline Floor & $18.1,51.0$ & 473 & 0 \\
\hline Floor & $10.6,53.6$ & 503 & 60 \\
\hline Floor & $13.6,53.6$ & 514 & 81 \\
\hline Floor & $16.6,53.6$ & 511 & 75 \\
\hline Floor & $19.6,53.6$ & 521 & 95 \\
\hline Floor & $12.1,56.2$ & 471 & -4 \\
\hline Floor & $15.1,56.2$ & 491 & 36 \\
\hline Floor & $18.1,56.2$ & 474 & 2 \\
\hline Floor & $10.6,58.8$ & 526 & 110 \\
\hline Floor & $13.6,58.8$ & 496 & 46 \\
\hline Floor & $16.6,58.8$ & 549 & 150 \\
\hline Floor & $19.6,58.8$ & 448 & -50 \\
\hline \multicolumn{4}{|l|}{ C1 SU21 } \\
\hline Floor & $21.7,51.5$ & 511 & 75 \\
\hline Floor & $24.7,51.5$ & 407 & -130 \\
\hline Floor & $27.7,51.5$ & 438 & -69 \\
\hline Floor & $20.2,54.1$ & 477 & 8 \\
\hline Floor & $23.2,54.1$ & 440 & -65 \\
\hline Floor & $26.2,54.1$ & 486 & 26 \\
\hline Floor & $29.2,54.1$ & 396 & -150 \\
\hline
\end{tabular}




\begin{tabular}{|c|c|c|c|}
\hline \multicolumn{4}{|c|}{$\begin{array}{c}\text { Table B-5 (cont.): FSS Structural Survey Units and Surface Activity Levels } \\
\text { Hammond Depot } \\
\text { Hammond, Indiana }\end{array}$} \\
\hline $\begin{array}{l}\text { Building/ } \\
\text { Survey Unit ID/ } \\
\text { Surface }\end{array}$ & $\begin{array}{l}\text { Location }(\mathrm{X}, \mathrm{Y} /, \mathrm{Z} \\
\quad \text { or Smear ID) }\end{array}$ & Counts per Minute & $\begin{array}{l}\text { Total Beta Activity } \\
\mathrm{dpm} / 100 \mathrm{~cm}^{2}\end{array}$ \\
\hline \multicolumn{4}{|l|}{ C1 SU21 (cont.) } \\
\hline Floor & $21.7,56.7$ & 457 & -32 \\
\hline Floor & $24.7,56.7$ & 493 & 40 \\
\hline Floor & $27.7,56.7$ & 487 & 28 \\
\hline Floor & $20.2,59.3$ & 437 & -71 \\
\hline Floor & $23.2,59.3$ & 432 & -81 \\
\hline Floor & $26.2,59.3$ & 540 & 130 \\
\hline Floor & $29.2,59.3$ & 517 & 87 \\
\hline \multicolumn{4}{|l|}{ C1 SU22 } \\
\hline Wall & $1.4,0.6$ & 481 & 65 \\
\hline Wall & $3.0,0.6$ & 644 & 390 \\
\hline Wall & $4.5,0.6$ & 504 & 110 \\
\hline Wall & $6.1,0.6$ & 490 & 83 \\
\hline Wall & $0.6,2.0$ & 438 & -20 \\
\hline Wall & $2.2,2.0$ & 468 & 40 \\
\hline Wall & $3.8,2.0$ & 464 & 32 \\
\hline Wall & $5.3,2.0$ & 463 & 30 \\
\hline Wall & $6.9,2.0$ & 468 & 40 \\
\hline Wall & $1.4,3.4$ & 418 & -60 \\
\hline Wall & $3.0,3.4$ & 419 & -58 \\
\hline Wall & $4.5,3.4$ & 478 & 60 \\
\hline Wall & $6.1,3.4$ & 460 & 24 \\
\hline Wall & $0.6,4.7$ & 435 & -26 \\
\hline Wall & $2.2,4.7$ & 474 & 52 \\
\hline Wall & $3.8,4.7$ & 455 & 14 \\
\hline Wall & $5.3,4.7$ & 423 & -50 \\
\hline \multicolumn{4}{|l|}{ C1 SU23 } \\
\hline Wall & $19.7,3.6$ & 190 & 24 \\
\hline Wall & $19.7,1.6$ & 582 & 270 \\
\hline Wall & $21.3,4.6$ & 160 & -36 \\
\hline Wall & $21.3,2.6$ & 567 & 240 \\
\hline Wall & $21.3,0.7$ & 498 & 99 \\
\hline Wall & $23.0,3.6$ & 173 & -10 \\
\hline Wall & $23.0,1.6$ & 564 & 230 \\
\hline Wall & $24.7,4.6$ & 454 & 12 \\
\hline Wall & $24.7,2.6$ & 435 & -26 \\
\hline Wall & $24.7,0.7$ & 560 & 220 \\
\hline Wall & $26.4,3.6$ & 476 & 56 \\
\hline Wall & $26.4,1.6$ & 413 & -69 \\
\hline Wall & $28.1,4.6$ & 168 & -20 \\
\hline
\end{tabular}




\begin{tabular}{|c|c|c|c|}
\hline \multicolumn{4}{|c|}{$\begin{array}{c}\text { Table B-5 (cont.): FSS Structural Survey Units and Surface Activity Levels } \\
\text { Hammond Depot } \\
\text { Hammond, Indiana }\end{array}$} \\
\hline $\begin{array}{l}\text { Building/ } \\
\text { Survey Unit ID/ } \\
\text { Surface }\end{array}$ & $\begin{array}{l}\text { Location (X, Y/, Z } \\
\text { or Smear ID) }\end{array}$ & Counts per Minute & $\begin{array}{l}\text { Total Beta Activity } \\
\mathrm{dpm} / 100 \mathrm{~cm}^{2}\end{array}$ \\
\hline \multicolumn{4}{|l|}{ C1 SU23 (cont.) } \\
\hline Wall & $28.1,2.6$ & 442 & -12 \\
\hline Wall & $28.1,0.7$ & 238 & 120 \\
\hline Wall & $29.8,3.6$ & 473 & 50 \\
\hline Wall & $29.8,1.6$ & 447 & -2 \\
\hline \multicolumn{4}{|l|}{ C1 SU24 } \\
\hline Ceiling & $0.2,32.4$ & 233 & 110 \\
\hline Ceiling & $3.2,32.4$ & 232 & 110 \\
\hline Ceiling & $6.3,32.4$ & 238 & 120 \\
\hline Ceiling & $9.4,32.4$ & 250 & 140 \\
\hline Ceiling & $12.5,32.4$ & 256 & 150 \\
\hline Ceiling & $1.7,35.1$ & 241 & 120 \\
\hline Ceiling & $4.8,35.1$ & 235 & 110 \\
\hline Ceiling & $7.9,35.1$ & 234 & 110 \\
\hline Ceiling & $10.9,35.1$ & 259 & 160 \\
\hline Ceiling & $0.2,37.8$ & 238 & 120 \\
\hline Ceiling & $3.2,37.8$ & 240 & 120 \\
\hline Ceiling & $6.3,37.8$ & 268 & 180 \\
\hline Ceiling & $9.4,37.8$ & 262 & 170 \\
\hline Ceiling & $12.5,37.8$ & 245 & 130 \\
\hline Ceiling & $1.7,40.5$ & 242 & 130 \\
\hline Ceiling & $4.8,40.5$ & 239 & 120 \\
\hline Ceiling & $7.9,40.5$ & 268 & 180 \\
\hline \multicolumn{4}{|l|}{ C1 SU25 } \\
\hline Ceiling & $14.1,32.3$ & 244 & 130 \\
\hline Ceiling & $17.2,32.3$ & 271 & 180 \\
\hline Ceiling & $20.3,32.3$ & 261 & 160 \\
\hline Ceiling & $23.3,32.3$ & 289 & 220 \\
\hline Ceiling & $12.5,35.0$ & 372 & 380 \\
\hline Ceiling & $15.6,35.0$ & 200 & 44 \\
\hline Ceiling & $18.7,35.0$ & 292 & 230 \\
\hline Ceiling & $21.8,35.0$ & 282 & 210 \\
\hline Ceiling & $24.9,35.0$ & 275 & 190 \\
\hline Ceiling & $14.1,37.6$ & 290 & 220 \\
\hline Ceiling & $17.2,37.6$ & 253 & 150 \\
\hline Ceiling & $20.3,37.6$ & 280 & 200 \\
\hline Ceiling & $23.3,37.6$ & 293 & 230 \\
\hline Ceiling & $12.5,40.3$ & 269 & 180 \\
\hline Ceiling & $15.6,40.3$ & 301 & 240 \\
\hline Ceiling & $18.7,40.3$ & 279 & 200 \\
\hline
\end{tabular}




\begin{tabular}{|c|c|c|c|}
\hline \multicolumn{4}{|c|}{$\begin{array}{c}\text { Table B-5 (cont.): FSS Structural Survey Units and Surface Activity Levels } \\
\text { Hammond Depot } \\
\text { Hammond, Indiana }\end{array}$} \\
\hline $\begin{array}{l}\text { Building/ } \\
\text { Survey Unit ID/ } \\
\text { Surface }\end{array}$ & $\begin{array}{l}\text { Location (X, Y/, Z } \\
\text { or Smear ID) }\end{array}$ & Counts per Minute & $\begin{array}{l}\text { Total Beta Activity } \\
\mathrm{dpm} / 100 \mathrm{~cm}^{2}\end{array}$ \\
\hline \multicolumn{4}{|l|}{ C1 SU25 (cont.) } \\
\hline Ceiling & $21.8,40.3$ & 261 & 160 \\
\hline Ceiling & $24.9,40.3$ & 284 & 210 \\
\hline \multicolumn{4}{|l|}{ C1 SU26 } \\
\hline Ceiling & $26.6,31.0$ & 279 & 200 \\
\hline Ceiling & $29.7,31.0$ & 306 & 250 \\
\hline Ceiling & $32.8,31.0$ & 251 & 140 \\
\hline Ceiling & $35.8,31.0$ & 270 & 180 \\
\hline Ceiling & $25.0,33.7$ & 264 & 170 \\
\hline Ceiling & $28.1,33.7$ & 283 & 210 \\
\hline Ceiling & $31.2,33.7$ & 272 & 190 \\
\hline Ceiling & $34.3,33.7$ & 297 & 240 \\
\hline Ceiling & $37.4,33.7$ & 260 & 160 \\
\hline Ceiling & $26.6,36.4$ & 275 & 190 \\
\hline Ceiling & $29.7,36.4$ & 300 & 240 \\
\hline Ceiling & $32.8,36.4$ & 249 & 140 \\
\hline Ceiling & $35.8,36.4$ & 248 & 140 \\
\hline Ceiling & $25.0,39.0$ & 235 & 110 \\
\hline Ceiling & $28.1,39.0$ & 276 & 190 \\
\hline Ceiling & $31.2,39.0$ & 249 & 140 \\
\hline Ceiling & $34.3,39.0$ & 289 & 220 \\
\hline Ceiling & $37.4,39.0$ & 240 & 120 \\
\hline \multicolumn{4}{|l|}{ C1 SU27 } \\
\hline Ceiling & $1.7,42.3$ & 260 & 160 \\
\hline Ceiling & $4.8,42.3$ & 263 & 170 \\
\hline Ceiling & $7.8,42.3$ & 242 & 130 \\
\hline Ceiling & $10.9,42.3$ & 244 & 130 \\
\hline Ceiling & $0.1,45.0$ & 247 & 140 \\
\hline Ceiling & $3.2,45.0$ & 265 & 170 \\
\hline Ceiling & $6.3,45.0$ & 239 & 120 \\
\hline Ceiling & $9.4,45.0$ & 246 & 130 \\
\hline Ceiling & $12.5,45.0$ & 256 & 150 \\
\hline Ceiling & $1.7,47.7$ & 238 & 120 \\
\hline Ceiling & $4.8,47.7$ & 283 & 210 \\
\hline Ceiling & $7.8,47.7$ & 237 & 120 \\
\hline Ceiling & $10.9,47.7$ & 249 & 140 \\
\hline Ceiling & $0.1,50.3$ & 240 & 120 \\
\hline Ceiling & $3.2,50.3$ & 245 & 130 \\
\hline Ceiling & $6.3,50.3$ & 241 & 120 \\
\hline Ceiling & $9.4,50.3$ & 245 & 130 \\
\hline
\end{tabular}




\begin{tabular}{|c|c|c|c|}
\hline \multicolumn{4}{|c|}{$\begin{array}{c}\text { Table B-5 (cont.): FSS Structural Survey Units and Surface Activity Levels } \\
\text { Hammond Depot } \\
\text { Hammond, Indiana }\end{array}$} \\
\hline $\begin{array}{l}\text { Building/ } \\
\text { Survey Unit ID/ } \\
\text { Surface }\end{array}$ & $\begin{array}{l}\text { Location (X, Y/, Z } \\
\text { or Smear ID) }\end{array}$ & Counts per Minute & $\begin{array}{l}\text { Total Beta Activity } \\
\mathrm{dpm} / 100 \mathrm{~cm}^{2}\end{array}$ \\
\hline \multicolumn{4}{|l|}{ C1 SU27 (cont.) } \\
\hline Ceiling & $12.5,50.3$ & 247 & 140 \\
\hline \multicolumn{4}{|l|}{ C1 SU28 } \\
\hline Ceiling & $14.5,42.2$ & 249 & 140 \\
\hline Ceiling & $17.5,42.2$ & 250 & 140 \\
\hline Ceiling & $20.4,42.2$ & 248 & 140 \\
\hline Ceiling & $23.3,42.2$ & 252 & 150 \\
\hline Ceiling & $13.1,44.8$ & 288 & 220 \\
\hline Ceiling & $16.0,44.8$ & 239 & 120 \\
\hline Ceiling & $18.9,44.8$ & 264 & 170 \\
\hline Ceiling & $21.9,44.8$ & 238 & 120 \\
\hline Ceiling & $24.8,44.8$ & 277 & 200 \\
\hline Ceiling & $14.5,47.3$ & 265 & 170 \\
\hline Ceiling & $17.5,47.3$ & 313 & 270 \\
\hline Ceiling & $20.4,47.3$ & 281 & 200 \\
\hline Ceiling & $23.3,47.3$ & 280 & 200 \\
\hline Ceiling & $13.1,49.9$ & 267 & 180 \\
\hline Ceiling & $16.0,49.9$ & 264 & 170 \\
\hline Ceiling & $18.9,49.9$ & 286 & 210 \\
\hline Ceiling & $21.9,49.9$ & 247 & 140 \\
\hline Ceiling & $24.8,49.9$ & 279 & 200 \\
\hline \multicolumn{4}{|l|}{ C1 SU29 } \\
\hline Ceiling & $25.1,42.4$ & 268 & 180 \\
\hline Ceiling & $28.2,42.4$ & 294 & 230 \\
\hline Ceiling & $31.3,42.4$ & 272 & 190 \\
\hline Ceiling & $34.4,42.4$ & 275 & 190 \\
\hline Ceiling & $37.5,42.4$ & 290 & 220 \\
\hline Ceiling & $26.7,45.1$ & 260 & 160 \\
\hline Ceiling & $29.8,45.1$ & 365 & 370 \\
\hline Ceiling & $32.8,45.1$ & 350 & 340 \\
\hline Ceiling & $35.9,45.1$ & 216 & 75 \\
\hline Ceiling & $25.1,47.8$ & 321 & 280 \\
\hline Ceiling & $28.2,47.8$ & 247 & 140 \\
\hline Ceiling & $31.3,47.8$ & 266 & 170 \\
\hline Ceiling & $34.4,47.8$ & 242 & 130 \\
\hline Ceiling & $37.5,47.8$ & 287 & 220 \\
\hline Ceiling & $26.7,50.4$ & 266 & 170 \\
\hline Ceiling & $29.8,50.4$ & 283 & 210 \\
\hline Ceiling & $32.8,50.4$ & 255 & 150 \\
\hline Ceiling & $35.9,50.4$ & 246 & 130 \\
\hline
\end{tabular}




\begin{tabular}{|c|c|c|c|}
\hline \multicolumn{4}{|c|}{$\begin{array}{c}\text { Table B-5 (cont.): FSS Structural Survey Units and Surface Activity Levels } \\
\text { Hammond Depot } \\
\text { Hammond, Indiana }\end{array}$} \\
\hline $\begin{array}{l}\text { Building/ } \\
\text { Survey Unit ID/ } \\
\text { Surface }\end{array}$ & $\begin{array}{c}\text { Location }(\mathrm{X}, \mathrm{Y} /, \mathrm{Z} \\
\text { or Smear ID) }^{\mathrm{a}}\end{array}$ & Counts per Minute & $\begin{array}{l}\text { Total Beta Activity } \\
\mathrm{dpm} / 100 \mathrm{~cm}^{2}\end{array}$ \\
\hline \multicolumn{4}{|l|}{ C1 SU30 } \\
\hline Ceiling & $1.7,51.5$ & 236 & 120 \\
\hline Ceiling & $4.8,51.5$ & 229 & 100 \\
\hline Ceiling & $7.9,51.5$ & 242 & 130 \\
\hline Ceiling & $10.9,51.5$ & 253 & 150 \\
\hline Ceiling & $0.1,54.2$ & 262 & 170 \\
\hline Ceiling & $3.2,54.2$ & 274 & 190 \\
\hline Ceiling & $6.3,54.2$ & 293 & 230 \\
\hline Ceiling & $9.4,54.2$ & 290 & 220 \\
\hline Ceiling & $12.5,54.2$ & 236 & 120 \\
\hline Ceiling & $1.7,56.9$ & 275 & 190 \\
\hline Ceiling & $4.8,56.9$ & 261 & 160 \\
\hline Ceiling & $7.9,56.9$ & 242 & 130 \\
\hline Ceiling & $10.9,56.9$ & 257 & 160 \\
\hline Ceiling & $0.1,59.6$ & 262 & 170 \\
\hline Ceiling & $3.2,59.6$ & 263 & 170 \\
\hline Ceiling & $6.3,59.6$ & 280 & 200 \\
\hline Ceiling & $9.4,59.6$ & 263 & 170 \\
\hline Ceiling & $12.5,59.6$ & 249 & 140 \\
\hline \multicolumn{4}{|l|}{ C1 SU31 } \\
\hline Ceiling & $14.2,52.4$ & 301 & 240 \\
\hline Ceiling & $17.2,52.4$ & 269 & 180 \\
\hline Ceiling & $20.3,52.4$ & 294 & 230 \\
\hline Ceiling & $23.4,52.4$ & 283 & 210 \\
\hline Ceiling & $12.6,55.1$ & 265 & 170 \\
\hline Ceiling & $15.7,55.1$ & 269 & 180 \\
\hline Ceiling & $18.8,55.1$ & 264 & 170 \\
\hline Ceiling & $21.9,55.1$ & 272 & 190 \\
\hline Ceiling & $25.0,55.1$ & 268 & 180 \\
\hline Ceiling & $14.2,57.8$ & 283 & 210 \\
\hline Ceiling & $17.2,57.8$ & 231 & 110 \\
\hline Ceiling & $20.3,57.8$ & 278 & 200 \\
\hline Ceiling & $23.4,57.8$ & 286 & 210 \\
\hline Ceiling & $12.6,60.4$ & 250 & 140 \\
\hline Ceiling & $15.7,60.4$ & 271 & 180 \\
\hline Ceiling & $18.8,60.4$ & 276 & 190 \\
\hline Ceiling & $21.9,60.4$ & 281 & 200 \\
\hline Ceiling & $25.0,60.4$ & 253 & 150 \\
\hline \multicolumn{4}{|l|}{ C1 SU32 } \\
\hline Ceiling & $26.8,53.1$ & 261 & 160 \\
\hline
\end{tabular}




\begin{tabular}{|c|c|c|c|}
\hline \multicolumn{4}{|c|}{$\begin{array}{c}\text { Table B-5 (cont.): FSS Structural Survey Units and Surface Activity Levels } \\
\text { Hammond Depot } \\
\text { Hammond, Indiana }\end{array}$} \\
\hline $\begin{array}{l}\text { Building/ } \\
\text { Survey Unit ID/ } \\
\text { Surface }\end{array}$ & $\begin{array}{l}\text { Location (X, Y/, Z } \\
\text { or Smear ID) }\end{array}$ & Counts per Minute & $\begin{array}{l}\text { Total Beta Activity } \\
\mathrm{dpm} / 100 \mathrm{~cm}^{2}\end{array}$ \\
\hline \multicolumn{4}{|l|}{ C1 SU32 (cont.) } \\
\hline Ceiling & $29.8,53.1$ & 240 & 120 \\
\hline Ceiling & $32.7,53.1$ & 255 & 150 \\
\hline Ceiling & $35.6,53.1$ & 260 & 160 \\
\hline Ceiling & $25.4,55.6$ & 277 & 200 \\
\hline Ceiling & $28.3,55.6$ & 280 & 200 \\
\hline Ceiling & $31.2,55.6$ & 278 & 200 \\
\hline Ceiling & $34.2,55.6$ & 283 & 210 \\
\hline Ceiling & $37.1,55.6$ & 275 & 190 \\
\hline Ceiling & $26.8,58.2$ & 257 & 160 \\
\hline Ceiling & $29.8,58.2$ & 256 & 150 \\
\hline Ceiling & $32.7,58.2$ & 270 & 180 \\
\hline Ceiling & $35.6,58.2$ & 281 & 200 \\
\hline Ceiling & $25.4,60.7$ & 273 & 190 \\
\hline Ceiling & $28.3,60.7$ & 251 & 140 \\
\hline Ceiling & $31.2,60.7$ & 237 & 120 \\
\hline Ceiling & $34.2,60.7$ & 241 & 120 \\
\hline Ceiling & $37.1,60.7$ & 250 & 140 \\
\hline \multicolumn{4}{|l|}{ C1 SU33 } \\
\hline Column 1 & S. Face, 2.5 & 190 & 24 \\
\hline Column 2 & N. Face, 2.5 & 206 & 56 \\
\hline Column 5 & W. Face, 4.6 & 215 & 73 \\
\hline Column 6 & N. Face, 4.6 & 212 & 67 \\
\hline Column 7 & E. Face, 1.6 & 203 & 50 \\
\hline Column 8 & S. Face, 1.6 & 211 & 65 \\
\hline Column 9 & S. Face, 0.6 & 192 & 28 \\
\hline Column 10 & N. Face, 0.6 & 199 & 42 \\
\hline Column 11 & E. Face, 3.6 & 193 & 30 \\
\hline Column 12 & S. Face, 3.6 & 191 & 26 \\
\hline Column 13 & S. Face, 2.7 & 204 & 52 \\
\hline Column 14 & W. Face, 2.7 & 208 & 60 \\
\hline Column 19 & E. Face, 1.7 & 243 & 130 \\
\hline Column 20 & S. Face, 1.7 & 263 & 170 \\
\hline \multicolumn{4}{|l|}{ C1 SU34 } \\
\hline Column 23 & E. Face, 1.6 & 228 & 99 \\
\hline Column 24 & S. Face, 1.6 & 223 & 89 \\
\hline Column 25 & W. Face, 0.7 & 240 & 120 \\
\hline Column 26 & N. Face, 0.7 & 232 & 110 \\
\hline Column 27 & E. Face, 3.7 & 217 & 77 \\
\hline Column 28 & S. Face, 3.7 & 219 & 81 \\
\hline
\end{tabular}




\begin{tabular}{|c|c|c|c|}
\hline \multicolumn{4}{|c|}{$\begin{array}{c}\text { Table B-5 (cont.): FSS Structural Survey Units and Surface Activity Levels } \\
\text { Hammond Depot } \\
\text { Hammond, Indiana }\end{array}$} \\
\hline $\begin{array}{l}\text { Building/ } \\
\text { Survey Unit ID/ } \\
\text { Surface }\end{array}$ & $\begin{array}{l}\text { Location (X, Y/, Z } \\
\text { or Smear ID) }^{\mathrm{a}}\end{array}$ & Counts per Minute & $\begin{array}{l}\text { Total Beta Activity } \\
\mathrm{dpm} / 100 \mathrm{~cm}^{2}\end{array}$ \\
\hline \multicolumn{4}{|l|}{ C1 SU34 (cont.) } \\
\hline Column 29 & W. Face, 2.7 & 230 & 100 \\
\hline Column 30 & N. Face, 2.7 & 200 & 44 \\
\hline Column 35 & E. Face, 1.8 & 236 & 120 \\
\hline Column 36 & S. Face, 1.8 & 216 & 75 \\
\hline Column $22(\mathrm{~J})$ & E. Face, 0.4 & 907 & 1100 \\
\hline Column $22(\mathrm{~J})$ & S. Face, 0.2 & 1099 & 1500 \\
\hline Column 22/post $\mathrm{RA}$ & E. Face, 0.4 & 230 & -210 \\
\hline Column 22/ Post $\mathrm{R} A$ & S. Face, 0.2 & 206 & -260 \\
\hline \multicolumn{4}{|l|}{ C2 SU35 } \\
\hline Ceiling & $2.4,3.4$ & 245 & 130 \\
\hline Ceiling & $9.1,3.4$ & 281 & 200 \\
\hline Ceiling & $15.7,3.4$ & 276 & 190 \\
\hline Ceiling & $22.4,3.4$ & 250 & 140 \\
\hline Ceiling & $29.0,3.4$ & 244 & 130 \\
\hline Ceiling & $35.7,3.4$ & 252 & 150 \\
\hline Ceiling & $5.7,9.1$ & 248 & 140 \\
\hline Ceiling & $12.4,9.1$ & 237 & 120 \\
\hline Ceiling & $19.1,9.1$ & 278 & 200 \\
\hline Ceiling & $25.7,9.1$ & 237 & 120 \\
\hline Ceiling & $32.4,9.1$ & 252 & 150 \\
\hline Ceiling & $2.4,14.9$ & 229 & 100 \\
\hline Ceiling & $9.1,14.9$ & 268 & 180 \\
\hline Ceiling & $15.7,14.9$ & 254 & 150 \\
\hline Ceiling & $22.4,14.9$ & 270 & 180 \\
\hline Ceiling & $29.0,14.9$ & 267 & 180 \\
\hline Ceiling & $35.7,14.9$ & 264 & 170 \\
\hline \multicolumn{4}{|l|}{ C2 SU36 } \\
\hline Ceiling & $2.5,18.4$ & 296 & 230 \\
\hline Ceiling & $9.2,18.4$ & 291 & 220 \\
\hline Ceiling & $15.8,18.4$ & 301 & 240 \\
\hline Ceiling & $22.5,18.4$ & 298 & 240 \\
\hline Ceiling & $29.1,18.4$ & 297 & 240 \\
\hline Ceiling & $35.8,18.4$ & 276 & 190 \\
\hline Ceiling & $5.9,24.1$ & 263 & 170 \\
\hline Ceiling & $12.5,24.1$ & 312 & 270 \\
\hline Ceiling & $19.2,24.1$ & 290 & 220 \\
\hline Ceiling & $25.8,24.1$ & 263 & 170 \\
\hline Ceiling & $32.5,24.1$ & 289 & 220 \\
\hline Ceiling & $2.5,29.9$ & 269 & 180 \\
\hline
\end{tabular}




\begin{tabular}{|c|c|c|c|}
\hline \multicolumn{4}{|c|}{$\begin{array}{c}\text { Table B-5 (cont.): FSS Structural Survey Units and Surface Activity Levels } \\
\text { Hammond Depot } \\
\text { Hammond, Indiana }\end{array}$} \\
\hline $\begin{array}{l}\text { Building/ } \\
\text { Survey Unit ID/ } \\
\text { Surface }\end{array}$ & $\begin{array}{l}\text { Location }(X, Y /, Z \\
\text { or Smear ID) }\end{array}$ & Counts per Minute & $\begin{array}{l}\text { Total Beta Activity } \\
\mathrm{dpm} / 100 \mathrm{~cm}^{2}\end{array}$ \\
\hline \multicolumn{4}{|l|}{ C2 SU36 (cont.) } \\
\hline Ceiling & $9.2,29.9$ & 230 & 100 \\
\hline Ceiling & $15.8,29.9$ & 219 & 81 \\
\hline Ceiling & $22.5,29.9$ & 297 & 240 \\
\hline Ceiling & $29.1,29.9$ & 257 & 160 \\
\hline Ceiling & $35.8,29.9$ & 250 & 140 \\
\hline \multicolumn{4}{|l|}{ C2 SU56 } \\
\hline Wall & $1.9,0.2$ & 299 & -75 \\
\hline Wall & $4.4,4.5$ & 188 & 20 \\
\hline Wall & $7.0,0.2$ & 305 & -63 \\
\hline Wall & $9.5,4.5$ & 184 & 12 \\
\hline Wall & $12.1,0.2$ & 294 & -85 \\
\hline Wall & $14.6,4.5$ & 209 & 62 \\
\hline Wall & $17.2,0.2$ & 210 & 63 \\
\hline Wall & $36.1,4.5$ & 448 & 0 \\
\hline Wall & $33.5,0.2$ & 288 & -97 \\
\hline Wall & $31.0,4.5$ & 403 & -89 \\
\hline Wall & $28.4,0.2$ & 421 & -54 \\
\hline Wall & $25.9,4.5$ & 364 & -170 \\
\hline Wall & $23.3,0.2$ & 271 & -130 \\
\hline Wall & $20.8,4.5$ & 426 & -44 \\
\hline Wall & $18.2,0.2$ & 244 & 130 \\
\hline Wall & $15.7,4.5$ & 397 & -100 \\
\hline Wall & $13.1,0.2$ & 269 & -130 \\
\hline Wall & $10.6,4.5$ & 190 & 24 \\
\hline Wall & $8.0,0.2$ & 273 & -130 \\
\hline Wall & $5.5,4.5$ & 243 & -190 \\
\hline Wall & $2.9,0.2$ & 292 & -89 \\
\hline \multicolumn{4}{|l|}{ C2 SU57 } \\
\hline Wall & $33.2,4.5$ & 421 & -54 \\
\hline Wall & $35.6,0.2$ & 599 & 520 \\
\hline Wall & $38.1,4.5$ & 240 & 120 \\
\hline Wall & $40.6,0.2$ & 308 & -58 \\
\hline Wall & $43.1,4.5$ & 427 & -42 \\
\hline Wall & $45.6,0.2$ & 402 & 130 \\
\hline Wall & $48.1,4.5$ & 174 & -8 \\
\hline Wall & $50.6,0.2$ & 217 & 77 \\
\hline Wall & $53.1,4.5$ & 179 & 2 \\
\hline Wall & $55.6,0.2$ & 306 & -62 \\
\hline Wall & $58.1,4.5$ & 172 & -12 \\
\hline
\end{tabular}




\begin{tabular}{|c|c|c|c|}
\hline \multicolumn{4}{|c|}{$\begin{array}{c}\text { Table B-5 (cont.): FSS Structural Survey Units and Surface Activity Levels } \\
\text { Hammond Depot } \\
\text { Hammond, Indiana }\end{array}$} \\
\hline $\begin{array}{l}\text { Building/ } \\
\text { Survey Unit ID/ } \\
\text { Surface }\end{array}$ & $\begin{array}{l}\text { Location }(X, Y /, Z \\
\text { or Smear ID) }\end{array}$ & Counts per Minute & $\begin{array}{l}\text { Total Beta Activity } \\
\mathrm{dpm} / 100 \mathrm{~cm}^{2}\end{array}$ \\
\hline \multicolumn{4}{|l|}{ C2 SU57 (cont.) } \\
\hline Wall & $60.5,0.2$ & 289 & -95 \\
\hline Wall & $1.0,4.5$ & 254 & -380 \\
\hline Wall & $3.5,0.2$ & 294 & -310 \\
\hline Wall & $6.0,4.5$ & 349 & -200 \\
\hline Wall & $8.5,0.2$ & 481 & 65 \\
\hline Wall & $11.0,4.5$ & 434 & -28 \\
\hline Wall & $13.5,0.2$ & 477 & 58 \\
\hline Wall & $16.0,4.5$ & 445 & -6 \\
\hline Wall & $18.5,0.2$ & 527 & 160 \\
\hline Wall & $21.0,4.5$ & 464 & 32 \\
\hline Wall & $23.5,0.2$ & 431 & -34 \\
\hline Wall & $25.9,4.5$ & 461 & 26 \\
\hline Wall & $28.4,0.2$ & 519 & 140 \\
\hline \multicolumn{4}{|l|}{ C2 SU58 } \\
\hline Wall & $61.0,3.6$ & 453 & 10 \\
\hline Wall & $57.2,1.4$ & 539 & 180 \\
\hline Wall & $53.4,3.6$ & 415 & -65 \\
\hline Wall & $49.6,1.4$ & 482 & 67 \\
\hline Wall & $45.9,3.6$ & 519 & 140 \\
\hline Wall & $42.1,1.4$ & 515 & 130 \\
\hline Wall & $38.3,3.6$ & 436 & -24 \\
\hline Wall & $34.5,1.4$ & 200 & 44 \\
\hline Wall & $30.7,3.6$ & 476 & 56 \\
\hline Wall & $26.9,1.4$ & 511 & 120 \\
\hline Wall & $23.2,3.6$ & 190 & 24 \\
\hline Wall & $19.4,1.4$ & 564 & 230 \\
\hline Wall & $15.6,3.6$ & 197 & 38 \\
\hline Wall & $11.8,1.4$ & 532 & 170 \\
\hline Wall & $8.0,3.6$ & 411 & -73 \\
\hline Wall & $4.3,1.4$ & 465 & 34 \\
\hline Wall & $0.5,3.6$ & 461 & 26 \\
\hline \multicolumn{4}{|c|}{ Building 200E (north) } \\
\hline C2 SU37 & & & \\
\hline Floor & 46 & 296 & -81 \\
\hline Floor & 47 & 327 & -20 \\
\hline Floor & 48 & 304 & -65 \\
\hline Floor & 49 & 329 & -16 \\
\hline Floor & 50 & 337 & 0 \\
\hline Floor & 52 & 299 & -75 \\
\hline
\end{tabular}




\begin{tabular}{|c|c|c|c|}
\hline \multicolumn{4}{|c|}{$\begin{array}{c}\text { Table B-5 (cont.): FSS Structural Survey Units and Surface Activity Levels } \\
\text { Hammond Depot } \\
\text { Hammond, Indiana }\end{array}$} \\
\hline $\begin{array}{l}\text { Building/ } \\
\text { Survey Unit ID/ } \\
\text { Surface }\end{array}$ & $\begin{array}{l}\text { Location }(\mathrm{X}, \mathrm{Y} /, \mathrm{Z} \\
\text { or Smear ID) }^{\mathrm{a}}\end{array}$ & Counts per Minute & $\begin{array}{l}\text { Total Beta Activity } \\
\text { dpm } / 100 \mathrm{~cm}^{2}\end{array}$ \\
\hline \multicolumn{4}{|l|}{ C2 SU37 (cont.) } \\
\hline Floor & 53 & 324 & -26 \\
\hline Floor & 54 & 334 & -6 \\
\hline Floor & 55 & 336 & -2 \\
\hline Floor & 58 & 286 & -100 \\
\hline Floor & 59 & 347 & 20 \\
\hline Floor & 60 & 291 & -91 \\
\hline Floor & 61 & 307 & -60 \\
\hline Floor & 62 & 309 & -56 \\
\hline Floor & 63 & 369 & 63 \\
\hline Floor & 64 & 292 & -89 \\
\hline Floor & 65 & 289 & -95 \\
\hline Floor & 66 & 365 & 56 \\
\hline Floor & 67 & 434 & 190 \\
\hline Floor & 68 & 327 & -20 \\
\hline Floor & 70 & 332 & -10 \\
\hline Floor & 71 & 349 & 24 \\
\hline \multicolumn{4}{|l|}{ C2 SU38 } \\
\hline Floor & 21 & 348 & 22 \\
\hline Floor & 22 & 337 & 0 \\
\hline Floor & 23 & 329 & -16 \\
\hline Floor & 25 & 439 & 200 \\
\hline Floor & 26 & 346 & 18 \\
\hline Floor & 27 & 327 & -20 \\
\hline Floor & 28 & 312 & -50 \\
\hline Floor & 29 & 322 & -30 \\
\hline Floor & 30 & 319 & -36 \\
\hline Floor & 31 & 354 & 34 \\
\hline Floor & 32 & 351 & 28 \\
\hline Floor & 33 & 333 & -8 \\
\hline Floor & 34 & 356 & 38 \\
\hline Floor & 35 & 315 & -44 \\
\hline Floor & 36 & 294 & -85 \\
\hline Floor & 37 & 290 & -93 \\
\hline Floor & 38 & 371 & 67 \\
\hline Floor & 39 & 332 & -10 \\
\hline Floor & 40 & 325 & -24 \\
\hline Floor & 41 & 316 & -42 \\
\hline Floor & 42 & 311 & -52 \\
\hline Floor & 43 & 291 & -91 \\
\hline
\end{tabular}




\begin{tabular}{|c|c|c|c|}
\hline \multicolumn{4}{|c|}{$\begin{array}{c}\text { Table B-5 (cont.): FSS Structural Survey Units and Surface Activity Levels } \\
\text { Hammond Depot } \\
\text { Hammond, Indiana }\end{array}$} \\
\hline $\begin{array}{l}\text { Building/ } \\
\text { Survey Unit ID/ } \\
\text { Surface }\end{array}$ & $\begin{array}{l}\text { Location }(\mathrm{X}, \mathrm{Y} /, \mathrm{Z} \\
\quad \text { or Smear ID) }\end{array}$ & Counts per Minute & $\begin{array}{l}\text { Total Beta Activity } \\
\mathrm{dpm} / 100 \mathrm{~cm}^{2}\end{array}$ \\
\hline \multicolumn{4}{|l|}{ C2 SU38 (cont.) } \\
\hline Floor & 44 & 325 & -24 \\
\hline Floor & 45 & 323 & -28 \\
\hline \multicolumn{4}{|l|}{ C3 SU39 } \\
\hline W. Wall & $0,38.1,2.4$ & 523 & 140 \\
\hline W. Wall & $0,5.1,3.4$ & 217 & -440 \\
\hline E. Wall & $37.8,27.7,0.4$ & 198 & -34 \\
\hline E. Wall & $37.8,17.5,1.5$ & 472 & 45 \\
\hline W. Wall & $0,54.8,0.9$ & 461 & 25 \\
\hline E. Wall & $37.8,46.3,2$ & 165 & -96 \\
\hline E. Wall & $37.8,4.6,3.1$ & 424 & -45 \\
\hline N. Wall & $14.9,61,3.5$ & 364 & -160 \\
\hline E. Wall & $37.8,53.6,1.9$ & 453 & 9 \\
\hline W. Wall & $0,40.3,3.9$ & 530 & 150 \\
\hline N. Wall & $11.9,61,0.2$ & 287 & -300 \\
\hline W. Wall & $0,27.2,0.3$ & 210 & -11 \\
\hline E. Wall & $37.8,46.3,1.3$ & 181 & -66 \\
\hline S. Wall & $11.4,0,2.9$ & 504 & 110 \\
\hline S. Wall & $14,0,1.8$ & 482 & 64 \\
\hline \multicolumn{4}{|l|}{ C3 SU40 } \\
\hline US & 72 & 361 & 48 \\
\hline US & 73 & 199 & -34 \\
\hline US & 74 & 249 & 65 \\
\hline US & 75 & 218 & 4 \\
\hline US & 76 & 211 & -10 \\
\hline US & 77 & 110 & -210 \\
\hline US & 78 & 224 & 16 \\
\hline US & 79 & 230 & 28 \\
\hline US & 80 & 461 & 110 \\
\hline US & 81 & 246 & 60 \\
\hline \multicolumn{4}{|l|}{ C1 SU41 } \\
\hline Floor & $0.3,0.2$ & 259 & -150 \\
\hline Floor & $0.7,1.0$ & 328 & -18 \\
\hline US & $0.3,0.1,2.5$ & 235 & -200 \\
\hline US & $0.8,0.8,2.5$ & 269 & -130 \\
\hline S. Wall & $0.8,0.0,0.1$ & 234 & -200 \\
\hline E. Wall & $1.6,0.9,0.1$ & 250 & -170 \\
\hline N. Wall & $0.3,2.5,0.1$ & 411 & 150 \\
\hline S. Wall & $0.3,0.0,0.8$ & 234 & -200 \\
\hline E. Wall & $1.6,0.4,0.8$ & 236 & -200 \\
\hline
\end{tabular}




\begin{tabular}{|c|c|c|c|}
\hline \multicolumn{4}{|c|}{$\begin{array}{c}\text { Table B-5 (cont.): FSS Structural Survey Units and Surface Activity Levels } \\
\text { Hammond Depot } \\
\text { Hammond, Indiana }\end{array}$} \\
\hline $\begin{array}{l}\text { Building/ } \\
\text { Survey Unit ID/ } \\
\text { Surface }\end{array}$ & $\begin{array}{l}\text { Location }(X, Y /, Z \\
\text { or Smear ID) }\end{array}$ & Counts per Minute & $\begin{array}{l}\text { Total Beta Activity } \\
\mathrm{dpm} / 100 \mathrm{~cm}^{2}\end{array}$ \\
\hline \multicolumn{4}{|l|}{ C1 SU41 (cont.) } \\
\hline N. Wall & $0.7,2.6,0.8$ & 403 & 130 \\
\hline W. Wall & $0.0,1.0,0.8$ & 299 & -75 \\
\hline S. Wall & $0.8,0.0,1.6$ & 238 & -200 \\
\hline E. Wall & $1.6,0.9,1.6$ & 284 & -110 \\
\hline N. Wall & $0.3,2.5,1.6$ & 403 & 130 \\
\hline S. Wall & $0.3,0.0,2.4$ & 242 & -190 \\
\hline E. Wall & $1.6,0.4,2.2$ & 252 & -170 \\
\hline N. Wall & $0.7,2.6,2.2$ & 355 & 36 \\
\hline W. Wall & $0.0,1.0,2.0$ & 306 & -62 \\
\hline W. Wall & $0.0,0.7,1.5$ & 178 & -75 \\
\hline \multicolumn{4}{|l|}{ C2 SU42 } \\
\hline Floor & $51 \mathrm{C}$ & 362 & 50 \\
\hline Floor & $52 \mathrm{C}$ & 409 & 140 \\
\hline Floor & $53 \mathrm{C}$ & 376 & 77 \\
\hline Floor & $54 \mathrm{C}$ & 390 & 110 \\
\hline Floor & $55 \mathrm{C}$ & 367 & 60 \\
\hline Floor & $56 \mathrm{C}$ & 378 & 81 \\
\hline Floor & $57 \mathrm{C}$ & 360 & 46 \\
\hline Floor & $58 \mathrm{C}$ & 355 & 36 \\
\hline Floor & $59 \mathrm{C}$ & 371 & 67 \\
\hline Floor & $60 \mathrm{C}$ & 380 & 85 \\
\hline Floor & $61 \mathrm{C}$ & 403 & 130 \\
\hline Floor & $62 \mathrm{C}$ & 385 & 95 \\
\hline Floor & $63 \mathrm{C}$ & 396 & 120 \\
\hline Wall & $66 \mathrm{C}$ & 438 & 63 \\
\hline Wall & $67 \mathrm{C}$ & 331 & -150 \\
\hline Wall & $68 \mathrm{C}$ & 305 & -200 \\
\hline Wall & $69 \mathrm{C}$ & 322 & -170 \\
\hline Wall & $70 \mathrm{C}$ & 326 & -160 \\
\hline Wall & $71 \mathrm{C}$ & 330 & -150 \\
\hline Wall & $72 \mathrm{C}$ & 410 & 8 \\
\hline Wall & $73 \mathrm{C}$ & 340 & -130 \\
\hline \multicolumn{4}{|c|}{ Building 100E } \\
\hline C1 SU43 & & & \\
\hline Floor & $7.8,91.6$ & 346 & 22 \\
\hline Floor & $7.8,99.2$ & 383 & 95 \\
\hline Floor & $10.0,87.8$ & 341 & 12 \\
\hline Floor & $10.0,95.4$ & 376 & 81 \\
\hline Floor & $10.0,103.0$ & 372 & 73 \\
\hline
\end{tabular}




\begin{tabular}{|c|c|c|c|}
\hline \multicolumn{4}{|c|}{$\begin{array}{c}\text { Table B-5 (cont.): FSS Structural Survey Units and Surface Activity Levels } \\
\text { Hammond Depot } \\
\text { Hammond, Indiana }\end{array}$} \\
\hline $\begin{array}{l}\text { Building/ } \\
\text { Survey Unit ID/ } \\
\text { Surface }\end{array}$ & $\begin{array}{l}\text { Location (X, Y/, Z } \\
\text { or Smear ID) }\end{array}$ & Counts per Minute & $\begin{array}{l}\text { Total Beta Activity } \\
\mathrm{dpm} / 100 \mathrm{~cm}^{2}\end{array}$ \\
\hline \multicolumn{4}{|l|}{ C1 SU43 (cont.) } \\
\hline Floor & $12.2,91.6$ & 341 & 12 \\
\hline Floor & $12.2,99.2$ & 339 & 8 \\
\hline Floor & $14.4,87.8$ & 322 & -26 \\
\hline Floor & $14.4,95.4$ & 349 & 28 \\
\hline Floor & $14.4,103.0$ & 396 & 120 \\
\hline Floor & $16.5,91.6$ & 332 & -6 \\
\hline Floor & $16.5,99.2$ & 374 & 77 \\
\hline Floor & $18.7,87.8$ & 336 & 2 \\
\hline Floor & $18.7,95.4$ & 331 & -8 \\
\hline Floor & $18.7,103.0$ & 354 & 38 \\
\hline Floor $(\mathrm{J})$ & $9.5,99.0$ & 384 & 97 \\
\hline Floor (J) & $15.5,102.0$ & 337 & 4 \\
\hline \multicolumn{4}{|l|}{ C1 SU44 } \\
\hline Floor & $19.4,90.4$ & 368 & 65 \\
\hline Floor & $19.4,98.1$ & 340 & 10 \\
\hline Floor & $21.7,86.5$ & 324 & -22 \\
\hline Floor & $21.7,94.3$ & 334 & -2 \\
\hline Floor & $21.7,102.0$ & 372 & 73 \\
\hline Floor & $23.9,90.4$ & 359 & 48 \\
\hline Floor & $23.9,98.1$ & 365 & 60 \\
\hline Floor & $26.1,86.5$ & 432 & 190 \\
\hline Floor & $26.1,94.3$ & 341 & 12 \\
\hline Floor & $26.1,102.0$ & 449 & 230 \\
\hline Floor & $28.4,90.4$ & 369 & 67 \\
\hline Floor & $28.4,98.1$ & 408 & 140 \\
\hline Floor & $30.6,86.5$ & 389 & 110 \\
\hline Floor & $30.6,94.3$ & 350 & 30 \\
\hline Floor & $30.6,102.0$ & 331 & -8 \\
\hline Floor $(\mathrm{J})$ & $23.1,102.0$ & 339 & 8 \\
\hline \multicolumn{4}{|l|}{ C2 SU45 } \\
\hline Floor & $2.5,87.2$ & 350 & 30 \\
\hline Floor & $37.3,87.2$ & 370 & 69 \\
\hline Floor & $6.8,94.7$ & 376 & 81 \\
\hline Floor & $32.9,94.7$ & 358 & 46 \\
\hline Floor & $2.5,102.2$ & 356 & 42 \\
\hline Floor & $37.3,102.2$ & 348 & 26 \\
\hline Floor & $6.8,109.8$ & 327 & -16 \\
\hline Floor & $15.5,109.8$ & 339 & 8 \\
\hline Floor & $24.2,109.8$ & 348 & 26 \\
\hline
\end{tabular}




\begin{tabular}{|c|c|c|c|}
\hline \multicolumn{4}{|c|}{$\begin{array}{c}\text { Table B-5 (cont.): FSS Structural Survey Units and Surface Activity Levels } \\
\text { Hammond Depot } \\
\text { Hammond, Indiana }\end{array}$} \\
\hline $\begin{array}{l}\text { Building/ } \\
\text { Survey Unit ID/ } \\
\text { Surface }\end{array}$ & $\begin{array}{l}\text { Location (X, Y/, Z } \\
\text { or Smear ID) }\end{array}$ & Counts per Minute & $\begin{array}{l}\text { Total Beta Activity } \\
\mathrm{dpm} / 100 \mathrm{~cm}^{2}\end{array}$ \\
\hline \multicolumn{4}{|l|}{ C2 SU45 (cont.) } \\
\hline Floor & $32.9,109.8$ & 377 & 83 \\
\hline Floor & $2.5,117.3$ & 369 & 67 \\
\hline Floor & $12.3,117.3$ & 310 & -50 \\
\hline Floor & $19.9,117.3$ & 343 & 16 \\
\hline Floor & $28.6,117.3$ & 386 & 100 \\
\hline Floor & $36.0,117.3$ & 361 & 52 \\
\hline \multicolumn{4}{|l|}{ C2 SU46 } \\
\hline W. Wall & $85.5,3.1$ & 188 & 20 \\
\hline W. Wall & $96.4,3.1$ & 513 & 210 \\
\hline W. Wall & $107.3,3.1$ & 202 & 48 \\
\hline W. Wall & $118.2,3.1$ & 434 & 56 \\
\hline N. Wall & $0.1,1.6$ & 250 & -310 \\
\hline N. Wall & $6.3,1.6$ & 447 & 81 \\
\hline N. Wall & $12.6,1.6$ & 524 & 230 \\
\hline N. Wall & $18.9,1.6$ & 191 & 26 \\
\hline N. Wall & $25.2,1.6$ & 194 & 32 \\
\hline N. Wall & $31.5,1.6$ & 408 & 4 \\
\hline N. Wall & $37.8,1.6$ & 466 & 120 \\
\hline E. Wall & $36.5,2.5$ & 454 & 95 \\
\hline E. Wall & $25.6,2.5$ & 186 & 16 \\
\hline E. Wall & $14.7,2.5$ & 471 & 130 \\
\hline E. Wall & $3.8,2.5$ & 477 & 140 \\
\hline \multicolumn{4}{|l|}{ C3 SU47 } \\
\hline Ceiling & $20.0,101.5$ & 198 & 40 \\
\hline Ceiling & $34.4,95.2$ & 174 & -8 \\
\hline Ceiling & $5.2,96.9$ & 184 & 12 \\
\hline Ceiling & $38.0,102.9$ & 175 & -6 \\
\hline Ceiling & $29.8,107.4$ & 171 & -14 \\
\hline Ceiling & $27.1,102.3$ & 172 & -12 \\
\hline Ceiling & $3.2,97.7$ & 158 & -40 \\
\hline Ceiling & $36.1,111.7$ & 159 & -38 \\
\hline Ceiling & $35.7,103.9$ & 196 & 36 \\
\hline Ceiling & $21.6,104.1$ & 176 & -4 \\
\hline Ceiling & $17.6,106.2$ & 196 & 36 \\
\hline Ceiling & $15.6,94.1$ & 145 & -65 \\
\hline \multicolumn{4}{|l|}{ C3 SU48 } \\
\hline Floor & 8 & 366 & 62 \\
\hline Floor & 9 & 386 & 100 \\
\hline Floor & 10 & 313 & -44 \\
\hline
\end{tabular}




\begin{tabular}{|c|c|c|c|}
\hline \multicolumn{4}{|c|}{$\begin{array}{c}\text { Table B-5 (cont.): FSS Structural Survey Units and Surface Activity Levels } \\
\text { Hammond Depot } \\
\text { Hammond, Indiana }\end{array}$} \\
\hline $\begin{array}{l}\text { Building/ } \\
\text { Survey Unit ID/ } \\
\text { Surface }\end{array}$ & $\begin{array}{l}\text { Location }(\mathrm{X}, \mathrm{Y} /, \mathrm{Z} \\
\text { or Smear ID) }\end{array}$ & Counts per Minute & $\begin{array}{l}\text { Total Beta Activity } \\
\mathrm{dpm} / 100 \mathrm{~cm}^{2}\end{array}$ \\
\hline \multicolumn{4}{|l|}{ C3 SU48 (cont.) } \\
\hline Floor & 11 & 306 & -58 \\
\hline Floor & 12 & 341 & 12 \\
\hline Floor & 13 & 338 & 6 \\
\hline Floor & 14 & 320 & -30 \\
\hline Ceiling & 18 & 202 & 48 \\
\hline Ceiling & 19 & 183 & 10 \\
\hline \multicolumn{4}{|l|}{ C1 SU59 } \\
\hline Floor & $15.3,73.2$ & 341 & 12 \\
\hline Floor & $17.9,73.2$ & 323 & -24 \\
\hline Floor & $21.4,73.2$ & 271 & -130 \\
\hline Floor & $16.5,75.7$ & 315 & -40 \\
\hline Floor & $19.9,75.7$ & 357 & 44 \\
\hline Floor & $21.4,75.7$ & 324 & -22 \\
\hline Floor & $15.3,78.2$ & 326 & -18 \\
\hline Floor & $17.9,78.2$ & 320 & -30 \\
\hline Floor & $21.4,78.2$ & 334 & -2 \\
\hline Floor & $16.5,80.7$ & 303 & -63 \\
\hline Floor & $19.9,80.7$ & 310 & -50 \\
\hline Floor & $21.4,80.7$ & 329 & -12 \\
\hline Floor & $15.3,83.2$ & 343 & 16 \\
\hline Floor & $19.5,83.2$ & 365 & 60 \\
\hline Floor & $22.4,83.2$ & 329 & -12 \\
\hline Floor $(\mathrm{J})$ & $16.5,85.0$ & 286 & -97 \\
\hline Floor $(\mathrm{J})$ & $19.7,85.0$ & 292 & -85 \\
\hline Floor (J) & $22.6,85.0$ & 307 & -56 \\
\hline \multicolumn{4}{|l|}{ C2 SU60 } \\
\hline Floor & $9.0,74.1$ & 361 & 52 \\
\hline Floor & $11.9,74.1$ & 344 & 18 \\
\hline Floor & $14.8,74.1$ & 353 & 36 \\
\hline Floor & $7.6,76.6$ & 330 & -10 \\
\hline Floor & $10.5,76.6$ & 351 & 32 \\
\hline Floor & $13.4,76.6$ & 363 & 56 \\
\hline Floor & $9.0,79.1$ & 349 & 28 \\
\hline Floor & $11.9,79.1$ & 316 & -38 \\
\hline Floor & $14.8,79.1$ & 332 & -6 \\
\hline Floor & $7.6,81.6$ & 348 & 26 \\
\hline Floor & $10.5,81.6$ & 316 & -38 \\
\hline Floor & $13.4,81.6$ & 333 & -4 \\
\hline Floor & $9.0,84.1$ & 337 & 4 \\
\hline
\end{tabular}




\begin{tabular}{|c|c|c|c|}
\hline \multicolumn{4}{|c|}{$\begin{array}{c}\text { Table B-5 (cont.): FSS Structural Survey Units and Surface Activity Levels } \\
\text { Hammond Depot } \\
\text { Hammond, Indiana }\end{array}$} \\
\hline $\begin{array}{l}\text { Building/ } \\
\text { Survey Unit ID/ } \\
\text { Surface }\end{array}$ & $\begin{array}{l}\text { Location }(\mathrm{X}, \mathrm{Y} /, \mathrm{Z} \\
\text { or Smear ID) }^{\mathrm{a}}\end{array}$ & Counts per Minute & $\begin{array}{l}\text { Total Beta Activity } \\
\text { dpm } / 100 \mathrm{~cm}^{2}\end{array}$ \\
\hline \multicolumn{4}{|l|}{ C2 SU60 (cont.) } \\
\hline Floor & $11.9,84.1$ & 350 & 30 \\
\hline Floor & $14.8,84.1$ & 349 & 28 \\
\hline \multicolumn{4}{|l|}{ C2 SU61 } \\
\hline Floor & $24.4,79.6$ & 360 & 50 \\
\hline Floor & $26.3,79.6$ & 365 & 60 \\
\hline Floor & $28.3,79.6$ & 312 & -46 \\
\hline Floor & $23.4,81.3$ & 380 & 89 \\
\hline Floor & $25.3,81.3$ & 389 & 110 \\
\hline Floor & $27.3,81.3$ & 390 & 110 \\
\hline Floor & $29.3,81.3$ & 333 & -4 \\
\hline Floor & $24.4,83.0$ & 372 & 73 \\
\hline Floor & $26.3,83.0$ & 345 & 20 \\
\hline Floor & $28.3,83.0$ & 344 & 18 \\
\hline Floor & $23.4,84.7$ & 321 & -28 \\
\hline Floor & $25.3,84.7$ & 343 & 16 \\
\hline Floor & $27.3,84.7$ & 336 & 2 \\
\hline Floor & $29.3,84.7$ & 355 & 40 \\
\hline \multicolumn{4}{|c|}{ Building $100 \mathrm{~W}$} \\
\hline \multicolumn{4}{|l|}{ C2 SU49 } \\
\hline Floor Bay 7-17 & A54 & 488 & 300 \\
\hline Floor Bay 7-17 & A55 & 346 & 21 \\
\hline Floor Bay 7-17 & A56 & 489 & 300 \\
\hline Floor Bay 7-17 & A57 & 362 & 52 \\
\hline Floor Bay 7-17 & A58 & 373 & 74 \\
\hline Floor Bay 7-17 & A59 & 465 & 250 \\
\hline Floor Bay 7-17 & A60 & 350 & 29 \\
\hline Floor Bay 7-17 & A61 & 379 & 85 \\
\hline Floor Bay 7-17 & $\mathrm{A} 62$ & 409 & 140 \\
\hline Floor Bay 7-17 & A63 & 363 & 54 \\
\hline Floor Bay 7-17 & $\mathrm{A} 65$ & 410 & 150 \\
\hline Floor Bay 7-17 & A66 & 431 & 190 \\
\hline Floor Bay 7-17 & A83 & 524 & 370 \\
\hline Floor Bay 7-17 & A84 & 466 & 250 \\
\hline Floor Bay 7-17 & A90 & 243 & -180 \\
\hline Floor Bay 7-17 & A91 & 300 & -68 \\
\hline Floor Bay 7-17 & A92 & 250 & -160 \\
\hline Floor Bay 7-17 & A93 & 277 & -110 \\
\hline Floor Bay 7-17 & A94 & 297 & -74 \\
\hline Floor Bay 7-17 & $20 \mathrm{D}$ & 333 & -4 \\
\hline
\end{tabular}




\begin{tabular}{|c|c|c|c|}
\hline \multicolumn{4}{|c|}{$\begin{array}{c}\text { Table B-5 (cont.): FSS Structural Survey Units and Surface Activity Levels } \\
\text { Hammond Depot } \\
\text { Hammond, Indiana }\end{array}$} \\
\hline $\begin{array}{l}\text { Building/ } \\
\text { Survey Unit ID/ } \\
\text { Surface }\end{array}$ & $\begin{array}{c}\text { Location }(\mathrm{X}, \mathrm{Y} /, \mathrm{Z} \\
\text { or Smear ID) }\end{array}$ & Counts per Minute & $\begin{array}{l}\text { Total Beta Activity } \\
\text { dpm } / 100 \mathrm{~cm}^{2}\end{array}$ \\
\hline \multicolumn{4}{|l|}{ C2 SU49 (cont.) } \\
\hline Floor Bay 7-17 & $22 \mathrm{D}$ & 313 & -43 \\
\hline Floor Bay 7-17 & $24 \mathrm{D}$ & 319 & -31 \\
\hline \multicolumn{4}{|l|}{ C2 SU50 } \\
\hline F/LW Bay 1-10 & A40 & 284 & -99 \\
\hline F/LW Bay 1-10 & A41 & 451 & 87 \\
\hline F/LW Bay 1-10 & A42 & 334 & -2 \\
\hline F/LW Bay 1-10 & A43 & 298 & -72 \\
\hline F/LW Bay 1-10 & A44 & 338 & 6 \\
\hline F/LW Bay 1-10 & A45 & 342 & 14 \\
\hline F/LW Bay 1-10 & A46 & 505 & 190 \\
\hline F/LW Bay 1-10 & A47 & 353 & 35 \\
\hline F/LW Bay 1-10 & A48 & 362 & 52 \\
\hline F/LW Bay 1-10 & A49 & 345 & 19 \\
\hline F/LW Bay 1-10 & A50 & 390 & 110 \\
\hline F/LW Bay 1-10 & A51 & 391 & 110 \\
\hline F/LW Bay 1-10 & A52 & 509 & 200 \\
\hline F/LW Bay 1-10 & A53 & 338 & 6 \\
\hline \multicolumn{4}{|l|}{ C2 SU51 } \\
\hline F/LW Bay 11-20 & A68 & 533 & 250 \\
\hline F/LW Bay 11-20 & A69 & 398 & 120 \\
\hline F/LW Bay 11-20 & A70 & 307 & -54 \\
\hline F/LW Bay 11-20 & A71 & 329 & -12 \\
\hline F/LW Bay 11-20 & A72 & 351 & 31 \\
\hline F/LW Bay 11-20 & A73 & 373 & 74 \\
\hline F/LW Bay 11-20 & A74 & 425 & 170 \\
\hline F/LW Bay 11-20 & A75 & 395 & 120 \\
\hline F/LW Bay 11-20 & A76 & 484 & 150 \\
\hline F/LW Bay 11-20 & A77 & 362 & 52 \\
\hline F/LW Bay 11-20 & A78 & 339 & 8 \\
\hline F/LW Bay 11-20 & A79 & 367 & 62 \\
\hline F/LW Bay 11-20 & A80 & 344 & 17 \\
\hline F/LW Bay 11-20 & A81 & 256 & -290 \\
\hline \multicolumn{4}{|l|}{ C3 SU52 } \\
\hline Ceiling & A85 & 161 & -110 \\
\hline Ceiling & A86 & 235 & 37 \\
\hline Ceiling & A87 & 169 & -91 \\
\hline Ceiling & A88 & 241 & 48 \\
\hline Ceiling & A89 & 224 & 15 \\
\hline Ceiling & A90 & 243 & 52 \\
\hline
\end{tabular}




\begin{tabular}{|c|c|c|c|}
\hline \multicolumn{4}{|c|}{$\begin{array}{c}\text { Table B-5 (cont.): FSS Structural Survey Units an } \\
\text { Hammond Depot } \\
\text { Hammond, Indiana }\end{array}$} \\
\hline $\begin{array}{l}\text { Building/ } \\
\text { Survey Unit ID/ } \\
\text { Surface }\end{array}$ & $\begin{array}{l}\text { Location }(\mathrm{X}, \mathrm{Y} /, \mathrm{Z} \\
\quad \text { or Smear ID) }\end{array}$ & Counts per Minute & $\begin{array}{l}\text { Total Beta Activity } \\
\text { dpm/100 } \mathrm{cm}^{2}\end{array}$ \\
\hline \multicolumn{4}{|l|}{ C3 SU52 (cont.) } \\
\hline Ceiling & A91 & 300 & 160 \\
\hline Ceiling & A92 & 250 & 66 \\
\hline Ceiling & A93 & 277 & 120 \\
\hline Ceiling & A94 & 297 & 160 \\
\hline Ceiling & A95 & 282 & 130 \\
\hline Ceiling & A96 & 198 & -35 \\
\hline Ceiling & A97 & 187 & -56 \\
\hline Ceiling & A98 & 210 & -12 \\
\hline Ceiling & A99 & 251 & 68 \\
\hline Ceiling & A100 & 333 & 230 \\
\hline Ceiling & $30 \mathrm{D}$ & 246 & 58 \\
\hline Ceiling & $31 \mathrm{D}$ & 225 & 17 \\
\hline Ceiling & $32 \mathrm{D}$ & 217 & 2 \\
\hline Ceiling & $33 \mathrm{D}$ & 221 & 10 \\
\hline Ceiling & $34 \mathrm{D}$ & 244 & 54 \\
\hline \multicolumn{4}{|c|}{ Garage } \\
\hline \multicolumn{4}{|l|}{ C3 SU53 } \\
\hline Floor & $19.2,1$ & 332 & -6 \\
\hline Floor & $20.6,4.5$ & 395 & 110 \\
\hline Floor & $16.8,5.1$ & 376 & 77 \\
\hline Floor & $21.4,6.2$ & 369 & 64 \\
\hline Floor & $16.6,9.7$ & 343 & 15 \\
\hline Floor & $9.4,13.9$ & 328 & -13 \\
\hline Floor & $7.2,15$ & 381 & 87 \\
\hline Floor & $3.8,15.7$ & 372 & 70 \\
\hline Floor & $2.8,17.4$ & 355 & 38 \\
\hline Floor & $8.4,19.7$ & 323 & -23 \\
\hline Floor & 12,20 & 359 & 45 \\
\hline Floor & $1.9,25$ & 330 & -9 \\
\hline Floor & $18.9,30.3$ & 337 & 4 \\
\hline N. Wall & $2.1,30.4,1.5$ & 187 & -55 \\
\hline N. Wall & 13.1, 30.4. 0.9 & 155 & -120 \\
\hline \multicolumn{4}{|c|}{ Workshop } \\
\hline C3 SU54 & & & \\
\hline Floor & $6.1,8.6,0$ & 345 & 26 \\
\hline Floor & $7.6,0.9,0$ & 346 & 38 \\
\hline Floor & $0.5,13.1,0$ & 384 & 30 \\
\hline W. Wall & $0.8,3.3,3$ & 303 & -59 \\
\hline W. Wall & $8.6,7.3,3$ & 222 & -51 \\
\hline
\end{tabular}




\begin{tabular}{|c|c|c|c|}
\hline \multicolumn{4}{|c|}{$\begin{array}{c}\text { Table B-5 (cont.): FSS Structural Survey Units and Surface Activity Levels } \\
\text { Hammond Depot } \\
\text { Hammond, Indiana }\end{array}$} \\
\hline $\begin{array}{l}\text { Building/ } \\
\text { Survey Unit ID/ } \\
\text { Surface }\end{array}$ & $\begin{array}{l}\text { Location }(\mathrm{X}, \mathrm{Y} /, \mathrm{Z} \\
\text { or Smear ID) }^{\mathrm{a}}\end{array}$ & Counts per Minute & $\begin{array}{l}\text { Total Beta Activity } \\
\text { dpm } / 100 \mathrm{~cm}^{2}\end{array}$ \\
\hline \multicolumn{4}{|l|}{ C3 SU54 (cont.) } \\
\hline Ceiling & $6.1,11,3$ & 285 & -2 \\
\hline Floor & $0.2,24.5,0$ & 348 & -32 \\
\hline Floor & $0.2,25.2,0$ & 319 & 49 \\
\hline W. Wall & $0,24.8,0.9$ & 300 & 13 \\
\hline Floor & $1.3,24.6,0$ & 366 & -19 \\
\hline Ceiling & $0.3,31.8,3$ & 389 & 68 \\
\hline Ceiling & $0.9,35.2,3$ & 411 & -66 \\
\hline Floor & $3.8,37.9,0$ & 285 & -150 \\
\hline Floor & $6.4,28.1,0$ & 316 & 23 \\
\hline \multicolumn{4}{|c|}{ Building Exteriors } \\
\hline \multicolumn{4}{|l|}{ C3 SU55 } \\
\hline 100W West Wall & $75.8,2.7$ & 349 & 26 \\
\hline 100W East Wall & $92.2,2.5$ & 355 & 38 \\
\hline 100E West Wall & $52.8,1.4$ & 351 & 30 \\
\hline 100E West Wall & $71.1,2.2$ & 185 & -59 \\
\hline 100E North Wall & $20.9,2.1$ & 189 & -51 \\
\hline 100E East Wall & $96.9,1.9$ & 334 & -2 \\
\hline 200E West Wall & $96.6,0.7$ & 199 & -32 \\
\hline 200E East Wall & $72.1,2.6$ & 361 & 49 \\
\hline 200E South Wall & $3.2,0.1$ & 342 & 13 \\
\hline Office South Wall & $8.3,2$ & 206 & -19 \\
\hline Office South Wall & $6,0.3$ & 252 & 68 \\
\hline Office South Wall & $16,1.6$ & 181 & -66 \\
\hline $\begin{array}{c}\text { Guardhouse North } \\
\text { Wall }\end{array}$ & $4.5,0.6$ & 137 & -150 \\
\hline Pumphouse East Wall & $5.5,2.1$ & 347 & 23 \\
\hline Garage East Wall & $10.4,0.6$ & 226 & 19 \\
\hline
\end{tabular}

aRefer to Figures A-6 through A-21. 


\begin{tabular}{|c|c|c|c|c|c|}
\hline \multicolumn{6}{|c|}{$\begin{array}{c}\text { Table B-6: FSS Survey Units and Radionuclide Concentration Levels } \\
\text { Hammond Depot } \\
\text { Hammond, Indiana }\end{array}$} \\
\hline $\begin{array}{l}\text { Survey Unit/ } \\
\text { Sample ID } \\
\end{array}$ & $\begin{array}{c}\text { Coordinate } \mathbf{N}, \mathrm{E}^{\mathrm{a}} \\
(\mathrm{X}, \mathrm{Y})\end{array}$ & $\begin{array}{l}\text { Th-232 } \\
(\mathrm{pCi} / \mathrm{g}) \\
\end{array}$ & $\begin{array}{c}\mathrm{U}-238^{\mathrm{c}} \\
(\mathrm{pCi} / \mathrm{g})\end{array}$ & $\begin{array}{l}\text { SOR } \\
\text { Gross } \\
\end{array}$ & SOR Net \\
\hline \multicolumn{6}{|c|}{ Background Reference Area } \\
\hline 127 & $4611723 \mathrm{~N}, 456498 \mathrm{E}$ & $1.10 \pm 0.21$ & $2.54 \pm 0.73$ & 1.40 & 0.11 \\
\hline 128 & $4611778 \mathrm{~N}, 456355 \mathrm{E}$ & $0.85 \pm 0.19$ & $2.66 \pm 0.61$ & 1.36 & 0.07 \\
\hline 129 & $4611783 \mathrm{~N}, 456369 \mathrm{E}$ & $0.74 \pm 0.19$ & $2.44 \pm 0.79$ & 1.23 & -0.05 \\
\hline 130 & $4611802 \mathrm{~N}, 456306 \mathrm{E}$ & $1.09 \pm 0.22$ & $2.30 \pm 0.85$ & 1.30 & 0.01 \\
\hline 131 & $4611871 \mathrm{~N}, 456272 \mathrm{E}$ & $0.93 \pm 0.16$ & $2.50 \pm 0.79$ & 1.32 & 0.04 \\
\hline 132 & $4611872 \mathrm{~N}, 456272 \mathrm{E}$ & $0.79 \pm 0.12$ & $1.65 \pm 0.40$ & 0.93 & -0.35 \\
\hline 133 & $4611955 \mathrm{~N}, 456275 \mathrm{E}$ & $0.94 \pm 0.16$ & $2.30 \pm 0.52$ & 1.24 & -0.04 \\
\hline 134 & $4611997 \mathrm{~N}, 456285 \mathrm{E}$ & $0.98 \pm 0.18$ & $2.55 \pm 0.62$ & 1.36 & 0.08 \\
\hline 135 & $4612040 \mathrm{~N}, 456283 \mathrm{E}$ & $0.65 \pm 0.17$ & $2.06 \pm 0.63$ & 1.05 & -0.23 \\
\hline 136 & $4612085 \mathrm{~N}, 456286 \mathrm{E}$ & $0.73 \pm 0.21$ & $2.66 \pm 0.72$ & 1.32 & 0.03 \\
\hline 137 & $4612189 \mathrm{~N}, 456426 \mathrm{E}$ & $1.10 \pm 0.15$ & $2.44 \pm 0.56$ & 1.36 & 0.07 \\
\hline 138 & $4612185 \mathrm{~N}, 456501 \mathrm{E}$ & $1.03 \pm 0.21$ & $2.42 \pm 0.72$ & 1.32 & 0.04 \\
\hline 139 & $4612223 \mathrm{~N}, 456379 \mathrm{E}$ & $1.17 \pm 0.15$ & $2.72 \pm 0.49$ & 1.49 & 0.21 \\
\hline 140 & $4612254 \mathrm{~N}, 456466 \mathrm{E}$ & $1.36 \pm 0.28$ & $3.35 \pm 0.85$ & 1.81 & 0.53 \\
\hline 141 & $4612256 \mathrm{~N}, 456284 \mathrm{E}$ & $0.91 \pm 0.18$ & $1.44 \pm 0.82$ & 0.89 & -0.39 \\
\hline 142 & $4612256 \mathrm{~N}, 456387 \mathrm{E}$ & $1.71 \pm 0.26$ & $3.57 \pm 0.77$ & 2.02 & 0.74 \\
\hline 143 & $4612275 \mathrm{~N}, 456308 \mathrm{E}$ & $0.33 \pm 0.12$ & $0.63 \pm 0.50$ & 0.37 & -0.92 \\
\hline \multicolumn{6}{|l|}{ C1 SU1/AOC 4} \\
\hline 196 & $4611380 \mathrm{~N}, 456512 \mathrm{E}$ & $1.17 \pm 0.19$ & $2.16 \pm 0.61$ & 1.27 & -0.02 \\
\hline 197 & $4611388 \mathrm{~N}, 456506 \mathrm{E}$ & $1.15 \pm 0.16$ & $2.63 \pm 0.57$ & 1.45 & 0.17 \\
\hline 198 & $4611388 \mathrm{~N}, 456518 \mathrm{E}$ & $1.01 \pm 0.16$ & $2.42 \pm 0.45$ & 1.32 & 0.03 \\
\hline 199 & $4611388 \mathrm{~N}, 456529 \mathrm{E}$ & $1.15 \pm 0.18$ & $1.54 \pm 0.49$ & 1.01 & -0.27 \\
\hline 200 & $4611398 \mathrm{~N}, 456512 \mathrm{E}$ & $1.07 \pm 0.18$ & $2.82 \pm 0.84$ & 1.50 & 0.21 \\
\hline 201 & $4611398 \mathrm{~N}, 456523 \mathrm{E}$ & $1.13 \pm 0.16$ & $1.98 \pm 0.50$ & 1.18 & -0.10 \\
\hline 202 & $4611398 \mathrm{~N}, 456535 \mathrm{E}$ & $1.20 \pm 0.15$ & $1.37 \pm 0.43$ & 0.96 & -0.32 \\
\hline 203 & $4611408 \mathrm{~N}, 456506 \mathrm{E}$ & $0.51 \pm 0.11$ & $0.93 \pm 0.41$ & 0.55 & -0.73 \\
\hline 204 & $4611408 \mathrm{~N}, 456518 \mathrm{E}$ & $1.02 \pm 0.18$ & $2.72 \pm 0.67$ & 1.44 & 0.16 \\
\hline 205 & $4611408 \mathrm{~N}, 456529 \mathrm{E}$ & $1.22 \pm 0.20$ & $4.09 \pm 0.92$ & 2.06 & 0.77 \\
\hline 206 & $4611418 \mathrm{~N}, 456500 \mathrm{E}$ & $0.85 \pm 0.17$ & $2.58 \pm 0.72$ & 1.33 & 0.04 \\
\hline 207 & $4611418 \mathrm{~N}, 456512 \mathrm{E}$ & $1.05 \pm 0.16$ & $2.74 \pm 0.62$ & 1.46 & 0.18 \\
\hline 208 & $4611418 \mathrm{~N}, 456523 \mathrm{E}$ & $1.24 \pm 0.19$ & $2.80 \pm 0.69$ & 1.55 & 0.27 \\
\hline 209 & $4611418 \mathrm{~N}, 456535 \mathrm{E}$ & $1.25 \pm 0.16$ & $1.79 \pm 0.54$ & 1.15 & -0.14 \\
\hline 210 & $4611428 \mathrm{~N}, 456506 \mathrm{E}$ & $0.55 \pm 0.11$ & $1.08 \pm 0.41$ & 0.62 & -0.66 \\
\hline 211 & $4611428 \mathrm{~N}, 456518 \mathrm{E}$ & $0.88 \pm 0.21$ & $2.48 \pm 0.67$ & 1.30 & 0.01 \\
\hline 212 & $4611428 \mathrm{~N}, 456529 \mathrm{E}$ & $1.31 \pm 0.19$ & $3.10 \pm 0.60$ & 1.69 & 0.41 \\
\hline $213 \mathrm{~J}^{\mathrm{d}}$ & $4611395 \mathrm{~N}, 456530 \mathrm{E}$ & $1.51 \pm 0.24$ & $2.50 \pm 0.64$ & 1.52 & 0.24 \\
\hline $214 \mathrm{~J}$ & $4611400 \mathrm{~N}, 456525 \mathrm{E}$ & $1.43 \pm 0.21$ & $2.61 \pm 0.63$ & 1.54 & 0.25 \\
\hline \multicolumn{6}{|l|}{ C1 SU2/AOC 2} \\
\hline 494 & $4611334 \mathrm{~N}, 456525 \mathrm{E}$ & $1.19 \pm 0.20$ & $3.15 \pm 0.69$ & 1.67 & 0.39 \\
\hline
\end{tabular}




\begin{tabular}{|c|c|c|c|c|c|}
\hline \multicolumn{6}{|c|}{$\begin{array}{c}\text { Table B-6 (cont.): FSS Survey Units and Radionuclide Concentration Levels } \\
\text { Hammond Depot } \\
\text { Hammond, Indiana }\end{array}$} \\
\hline Sample ID & $\begin{array}{c}\text { Coordinate } \mathbf{N}, \mathrm{E}^{\mathrm{a}} \\
(\mathrm{X}, \mathrm{Y})\end{array}$ & $\begin{array}{l}\text { Th-232 } \\
\text { (pCi/g) }\end{array}$ & $\begin{array}{l}\mathrm{U}-238^{\mathrm{c}} \\
(\mathrm{pCi} / \mathrm{g})\end{array}$ & $\begin{array}{l}\text { SOR } \\
\text { Gross }\end{array}$ & SOR Net \\
\hline \multicolumn{6}{|c|}{ C1 SU2/AOC 2 (cont.) } \\
\hline 495 & $4611339 \mathrm{~N}, 456527 \mathrm{E}$ & $1.36 \pm 0.21$ & $2.95 \pm 0.74$ & 1.65 & 0.37 \\
\hline 496 & $4611344 \mathrm{~N}, 456525 \mathrm{E}$ & $1.19 \pm 0.21$ & $2.78 \pm 0.67$ & 1.52 & 0.24 \\
\hline 497 & $4611348 \mathrm{~N}, 456522 \mathrm{E}$ & $1.43 \pm 0.25$ & $3.14 \pm 0.98$ & 1.75 & 0.47 \\
\hline 498 & $4611348 \mathrm{~N}, 456527 \mathrm{E}$ & $0.87 \pm 0.13$ & $1.30 \pm 0.42$ & 0.82 & -0.46 \\
\hline 499 & $4611353 \mathrm{~N}, 456519 \mathrm{E}$ & $1.20 \pm 0.21$ & $2.89 \pm 0.69$ & 1.57 & 0.29 \\
\hline 500 & $4611353 \mathrm{~N}, 456525 \mathrm{E}$ & $0.80 \pm 0.15$ & $1.80 \pm 0.62$ & 1.00 & -0.29 \\
\hline 501 & $4611358 \mathrm{~N}, 456516 \mathrm{E}$ & $1.40 \pm 0.23$ & $3.81 \pm 0.95$ & 2.01 & 0.72 \\
\hline 502 & $4611358 \mathrm{~N}, 456522 \mathrm{E}$ & $1.33 \pm 0.18$ & $2.02 \pm 0.54$ & 1.27 & -0.02 \\
\hline 503 & $4611363 \mathrm{~N}, 456513 \mathrm{E}$ & $1.14 \pm 0.19$ & $2.59 \pm 0.73$ & 1.43 & 0.15 \\
\hline 504 & $4611363 \mathrm{~N}, 456519 \mathrm{E}$ & $1.32 \pm 0.21$ & $3.07 \pm 0.72$ & 1.68 & 0.40 \\
\hline 505 & $4611363 \mathrm{~N}, 456525 \mathrm{E}$ & $0.91 \pm 0.13$ & $1.82 \pm 0.49$ & 1.04 & -0.24 \\
\hline 506 & $4611368 \mathrm{~N}, 456511 \mathrm{E}$ & $1.13 \pm 0.14$ & $3.02 \pm 0.47$ & 1.60 & 0.32 \\
\hline 507 & $4611368 \mathrm{~N}, 456516 \mathrm{E}$ & $1.38 \pm 0.22$ & $3.72 \pm 0.67$ & 1.96 & 0.68 \\
\hline 508 & $4611368 \mathrm{~N}, 456522 \mathrm{E}$ & $1.12 \pm 0.20$ & $1.87 \pm 0.84$ & 1.13 & -0.15 \\
\hline 509 & $4611373 \mathrm{~N}, 456513 \mathrm{E}$ & $1.69 \pm 0.26$ & $2.96 \pm 0.98$ & 1.77 & 0.48 \\
\hline 510 & $4611373 \mathrm{~N}, 456519 \mathrm{E}$ & $1.03 \pm 0.17$ & $2.79 \pm 0.66$ & 1.47 & 0.19 \\
\hline \multicolumn{6}{|c|}{ C1 SU3/AOC 2 and 3} \\
\hline 511 & Sample not collected & & & & \\
\hline 512 & $4611343 \mathrm{~N}, 456556 \mathrm{E}$ & $0.44 \pm 0.10$ & $1.32 \pm 0.44$ & 0.68 & -0.60 \\
\hline 513 & $4611348 \mathrm{~N}, 456547 \mathrm{E}$ & $1.34 \pm 0.18$ & $2.94 \pm 0.65$ & 1.64 & 0.36 \\
\hline 514 & $4611348 \mathrm{~N}, 456553 \mathrm{E}$ & $0.76 \pm 0.17$ & $1.17 \pm 0.53$ & 0.73 & -0.55 \\
\hline 515 & $4611348 \mathrm{~N}, 456559 \mathrm{E}$ & $0.47 \pm 0.08$ & $0.91 \pm 0.29$ & 0.53 & -0.76 \\
\hline 516 & $4611353 \mathrm{~N}, 456550 \mathrm{E}$ & $1.34 \pm 0.23$ & $2.38 \pm 0.77$ & 1.41 & 0.13 \\
\hline 517 & $4611353 \mathrm{~N}, 456556 \mathrm{E}$ & $0.58 \pm 0.10$ & $1.08 \pm 0.47$ & 0.63 & -0.65 \\
\hline 518 & $4611358 \mathrm{~N}, 456547 \mathrm{E}$ & $1.48 \pm 0.24$ & $1.82 \pm 0.92$ & 1.24 & -0.04 \\
\hline 519 & $4611358 \mathrm{~N}, 456553 \mathrm{E}$ & $1.05 \pm 0.18$ & $2.74 \pm 0.81$ & 1.46 & 0.18 \\
\hline 520 & $4611358 \mathrm{~N}, 456559 \mathrm{E}$ & $0.70 \pm 0.11$ & $1.04 \pm 0.37$ & 0.66 & -0.63 \\
\hline 521 & Sample not collected & & & & \\
\hline 522 & $4611363 \mathrm{~N}, 456556 \mathrm{E}$ & $0.96 \pm 0.17$ & $1.72 \pm 0.59$ & 1.02 & -0.26 \\
\hline 523 & $4611368 \mathrm{~N}, 456547 \mathrm{E}$ & $1.62 \pm 0.21$ & $2.25 \pm 0.66$ & 1.46 & 0.18 \\
\hline 524 & $4611368 \mathrm{~N}, 456553 \mathrm{E}$ & $0.94 \pm 0.14$ & $1.67 \pm 0.51$ & 0.99 & -0.29 \\
\hline 525 & $4611368 \mathrm{~N}, 456559 \mathrm{E}$ & $0.47 \pm 0.10$ & $0.48 \pm 0.30$ & 0.35 & -0.93 \\
\hline 526 & Sample not collected & & & & \\
\hline 527 & $4611373 \mathrm{~N}, 456556 \mathrm{E}$ & $0.85 \pm 0.16$ & $1.35 \pm 0.68$ & 0.83 & -0.45 \\
\hline \multicolumn{6}{|c|}{ C1 SU4/AOC 7} \\
\hline 528 & Sample not collected & & & & \\
\hline 529 & $4611285 \mathrm{~N}, 456547 \mathrm{E}$ & $1.88 \pm 0.23$ & $3.33 \pm 0.79$ & 1.98 & 0.70 \\
\hline 530 & $4611285 \mathrm{~N}, 456556 \mathrm{E}$ & $1.11 \pm 0.17$ & $1.96 \pm 0.58$ & 1.17 & -0.12 \\
\hline 531 & $4611293 \mathrm{~N}, 456543 \mathrm{E}$ & $2.47 \pm 0.30$ & $3.9 \pm 1.0$ & 2.41 & 1.13 \\
\hline 532 & $4611293 \mathrm{~N}, 456552 \mathrm{E}$ & $1.08 \pm 0.15$ & $3.36 \pm 0.61$ & 1.72 & 0.43 \\
\hline
\end{tabular}




\begin{tabular}{|c|c|c|c|c|c|}
\hline \multicolumn{6}{|c|}{$\begin{array}{c}\text { Table B-6 (cont.): FSS Survey Units and Radionuclide Concentration Levels } \\
\text { Hammond Depot } \\
\text { Hammond, Indiana }\end{array}$} \\
\hline Sample ID & $\begin{array}{c}\text { Coordinate } \mathrm{N}, \mathrm{E}^{\mathrm{a}} \\
(\mathrm{X}, \mathrm{Y})\end{array}$ & $\begin{array}{l}\text { Th-232 } \\
\text { (pCi/g) }\end{array}$ & $\begin{array}{c}\mathrm{U}-238^{\mathrm{c}} \\
(\mathrm{pCi} / \mathrm{g})\end{array}$ & $\begin{array}{c}\text { SOR } \\
\text { Gross }\end{array}$ & SOR Net \\
\hline \multicolumn{6}{|c|}{ C1 SU4/AOC 7 (cont.) } \\
\hline 533 & $4611301 \mathrm{~N}, 456547 \mathrm{E}$ & $1.44 \pm 0.22$ & $3.55 \pm 0.80$ & 1.92 & 0.63 \\
\hline 534 & $4611301 \mathrm{~N}, 456556 \mathrm{E}$ & $0.99 \pm 0.14$ & $1.66 \pm 0.51$ & 1.01 & -0.28 \\
\hline 535 & $4611309 \mathrm{~N}, 456543 \mathrm{E}$ & $2.05 \pm 0.25$ & $2.29 \pm 0.78$ & 1.62 & 0.34 \\
\hline 536 & $4611309 \mathrm{~N}, 456552 \mathrm{E}$ & $0.85 \pm 0.16$ & $1.96 \pm 0.57$ & 1.08 & -0.21 \\
\hline 537 & $4611317 \mathrm{~N}, 456538 \mathrm{E}$ & $3.02 \pm 0.35$ & $2.56 \pm 0.85$ & 2.07 & 0.78 \\
\hline 538 & $4611317 \mathrm{~N}, 456547 \mathrm{E}$ & $1.97 \pm 0.27$ & $2.10 \pm 0.84$ & 1.52 & 0.24 \\
\hline 539 & $4611317 \mathrm{~N}, 456556 \mathrm{E}$ & $0.88 \pm 0.15$ & $1.54 \pm 0.53$ & 0.92 & -0.36 \\
\hline 540 & $4611325 \mathrm{~N}, 456534 \mathrm{E}$ & $1.26 \pm 0.18$ & $1.48 \pm 0.53$ & 1.03 & -0.26 \\
\hline 541 & $4611325 \mathrm{~N}, 456545 \mathrm{E}$ & $1.45 \pm 0.19$ & $2.27 \pm 0.68$ & 1.41 & 0.13 \\
\hline 542 & $4611325 \mathrm{~N}, 456552 \mathrm{E}$ & $0.64 \pm 0.14$ & $1.20 \pm 0.48$ & 0.70 & -0.58 \\
\hline 543 & $4611332 \mathrm{~N}, 456547 \mathrm{E}$ & $1.06 \pm 0.19$ & $2.76 \pm 0.45$ & 1.47 & 0.19 \\
\hline 544 & $4611332 \mathrm{~N}, 456556 \mathrm{E}$ & $0.41 \pm 0.08$ & $0.81 \pm 0.30$ & 0.47 & -0.82 \\
\hline \multicolumn{6}{|c|}{ C1 SU5/AOC 2 and 3} \\
\hline 545 & $4611247 \mathrm{~N}, 456518 \mathrm{E}$ & $1.06 \pm 0.16$ & $2.04 \pm 0.54$ & 1.18 & -0.10 \\
\hline 546 & $4611252 \mathrm{~N}, 456515 \mathrm{E}$ & $1.00 \pm 0.16$ & $1.56 \pm 0.61$ & 0.97 & -0.31 \\
\hline 547 & $4611258 \mathrm{~N}, 456512 \mathrm{E}$ & $1.06 \pm 0.18$ & $1.87 \pm 0.58$ & 1.11 & -0.17 \\
\hline 548 & $4611263 \mathrm{~N}, 456509 \mathrm{E}$ & $1.24 \pm 0.17$ & $1.46 \pm 0.58$ & 1.01 & -0.27 \\
\hline 549 & $4611263 \mathrm{~N}, 456514 \mathrm{E}$ & $1.01 \pm 0.15$ & $1.09 \pm 0.51$ & 0.78 & -0.50 \\
\hline 550 & $4611268 \mathrm{~N}, 456506 \mathrm{E}$ & $1.42 \pm 0.19$ & $2.06 \pm 0.71$ & 1.31 & 0.03 \\
\hline 551 & $4611268 \mathrm{~N}, 456512 \mathrm{E}$ & $1.12 \pm 0.16$ & $1.11 \pm 0.54$ & 0.83 & -0.45 \\
\hline 552 & $4611273 \mathrm{~N}, 456509 \mathrm{E}$ & $1.28 \pm 0.15$ & $1.48 \pm 0.49$ & 1.03 & -0.25 \\
\hline 553 & $4611278 \mathrm{~N}, 456506 \mathrm{E}$ & $1.42 \pm 0.19$ & $1.98 \pm 0.96$ & 1.28 & 0.00 \\
\hline 554 & $4611284 \mathrm{~N}, 456503 \mathrm{E}$ & $1.32 \pm 0.24$ & $1.61 \pm 0.67$ & 1.10 & -0.18 \\
\hline 555 & $4611289 \mathrm{~N}, 456500 \mathrm{E}$ & $1.48 \pm 0.20$ & $1.51 \pm 0.67$ & 1.11 & -0.17 \\
\hline 556 & $4611294 \mathrm{~N}, 456503 \mathrm{E}$ & $1.22 \pm 0.16$ & $1.60 \pm 0.43$ & 1.06 & -0.22 \\
\hline 557 & $4611299 \mathrm{~N}, 456500 \mathrm{E}$ & $1.47 \pm 0.19$ & $2.21 \pm 0.59$ & 1.39 & 0.11 \\
\hline 558 & $4611304 \mathrm{~N}, 456497 \mathrm{E}$ & $1.40 \pm 0.21$ & $1.58 \pm 0.67$ & 1.11 & -0.17 \\
\hline 559 & $4611309 \mathrm{~N}, 456500 \mathrm{E}$ & $1.13 \pm 0.14$ & $1.54 \pm 0.47$ & 1.01 & -0.28 \\
\hline 560 & $4611315 \mathrm{~N}, 456497 \mathrm{E}$ & $1.68 \pm 0.21$ & $1.85 \pm 0.66$ & 1.32 & 0.04 \\
\hline 561 & $4611320 \mathrm{~N}, 456494 \mathrm{E}$ & $1.52 \pm 0.20$ & $1.65 \pm 0.79$ & 1.18 & -0.10 \\
\hline \multicolumn{6}{|l|}{ C1 SU6/AOC 6} \\
\hline 438 & $4611374 \mathrm{~N}, 456622 \mathrm{E}$ & $0.56 \pm 0.11$ & $0.75 \pm 0.51$ & 0.49 & -0.79 \\
\hline 439 & $4611374 \mathrm{~N}, 456632 \mathrm{E}$ & $0.53 \pm 0.12$ & $0.64 \pm 0.32$ & 0.44 & -0.84 \\
\hline 440 & $4611374 \mathrm{~N}, 456642 \mathrm{E}$ & $0.65 \pm 0.13$ & $1.26 \pm 0.46$ & 0.73 & -0.55 \\
\hline 441 & $4611374 \mathrm{~N}, 456651 \mathrm{E}$ & $0.89 \pm 0.12$ & $1.42 \pm 0.44$ & 0.87 & -0.41 \\
\hline 442 & $4611382 \mathrm{~N}, 456618 \mathrm{E}$ & $0.38 \pm 0.12$ & $0.94 \pm 0.48$ & 0.51 & -0.78 \\
\hline 443 & $4611382 \mathrm{~N}, 456627 \mathrm{E}$ & $0.48 \pm 0.10$ & $0.81 \pm 0.33$ & 0.49 & -0.79 \\
\hline 444 & $4611382 \mathrm{~N}, 456637 \mathrm{E}$ & $0.65 \pm 0.13$ & $0.82 \pm 0.38$ & 0.55 & -0.73 \\
\hline 445 & $4611382 \mathrm{~N}, 456646 \mathrm{E}$ & $0.58 \pm 0.11$ & $1.40 \pm 0.47$ & 0.76 & -0.52 \\
\hline 446 & $4611382 \mathrm{~N}, 456656 \mathrm{E}$ & $0.57 \pm 0.12$ & $1.41 \pm 0.51$ & 0.76 & -0.52 \\
\hline
\end{tabular}




\begin{tabular}{|c|c|c|c|c|c|}
\hline \multicolumn{6}{|c|}{$\begin{array}{c}\text { Table B-6 (cont.): FSS Survey Units and Radionuclide Concentration Levels } \\
\text { Hammond Depot } \\
\text { Hammond, Indiana }\end{array}$} \\
\hline Sample ID & $\begin{array}{c}\text { Coordinate } \mathrm{N}, \mathrm{E}^{\mathrm{a}} \\
(\mathrm{X}, \mathrm{Y})\end{array}$ & $\begin{array}{l}\text { Th-232 } \\
\text { (pCi/g) }\end{array}$ & $\begin{array}{l}\mathrm{U}-238^{\mathrm{c}} \\
(\mathrm{pCi} / \mathrm{g})\end{array}$ & $\begin{array}{l}\text { SOR } \\
\text { Gross }\end{array}$ & SOR Net \\
\hline \multicolumn{6}{|c|}{ C1 SU6/AOC 6 (cont.) } \\
\hline 447 & $4611390 \mathrm{~N}, 456622 \mathrm{E}$ & $0.47 \pm 0.08$ & $0.75 \pm 0.26$ & 0.46 & -0.82 \\
\hline 448 & $4611390 \mathrm{~N}, 456632 \mathrm{E}$ & $0.48 \pm 0.10$ & $0.97 \pm 0.45$ & 0.55 & -0.73 \\
\hline 449 & $4611390 \mathrm{~N}, 456642 \mathrm{E}$ & $0.51 \pm 0.10$ & $0.77 \pm 0.36$ & 0.48 & -0.80 \\
\hline 450 & $4611390 \mathrm{~N}, 456651 \mathrm{E}$ & $0.77 \pm 0.15$ & $1.77 \pm 0.71$ & 0.97 & -0.31 \\
\hline 451 & $4611399 \mathrm{~N}, 456618 \mathrm{E}$ & $0.67 \pm 0.11$ & $0.92 \pm 0.43$ & 0.60 & -0.68 \\
\hline 452 & $4611399 \mathrm{~N}, 456627 \mathrm{E}$ & $0.64 \pm 0.13$ & $1.02 \pm 0.37$ & 0.63 & -0.65 \\
\hline 453 & $4611399 \mathrm{~N}, 456637 \mathrm{E}$ & $0.74 \pm 0.14$ & $0.67 \pm 0.50$ & 0.52 & -0.76 \\
\hline 454 & $4611399 \mathrm{~N}, 456646 \mathrm{E}$ & $0.49 \pm 0.13$ & $1.35 \pm 0.39$ & 0.71 & -0.57 \\
\hline 455 & $4611399 \mathrm{~N}, 456656 \mathrm{E}$ & $1.29 \pm 0.17$ & $2.29 \pm 0.59$ & 1.36 & 0.08 \\
\hline \multicolumn{6}{|l|}{ C1 SU7/AOC 5} \\
\hline 456 & $4611049 \mathrm{~N}, 456596 \mathrm{E}$ & $0.98 \pm 0.17$ & $1.90 \pm 0.52$ & 1.10 & -0.18 \\
\hline 457 & $4611049 \mathrm{~N}, 456603 \mathrm{E}$ & $0.83 \pm 0.19$ & $1.54 \pm 0.62$ & 0.90 & -0.38 \\
\hline 458 & $4611049 \mathrm{~N}, 456611 \mathrm{E}$ & $0.88 \pm 0.14$ & $1.82 \pm 0.58$ & 1.03 & -0.25 \\
\hline 459 & $4611049 \mathrm{~N}, 456618 \mathrm{E}$ & $0.72 \pm 0.14$ & $1.46 \pm 0.52$ & 0.83 & -0.45 \\
\hline 460 & $4611055 \mathrm{~N}, 456585 \mathrm{E}$ & $1.36 \pm 0.20$ & $1.84 \pm 0.77$ & 1.20 & -0.08 \\
\hline 461 & $4611055 \mathrm{~N}, 456593 \mathrm{E}$ & $0.75 \pm 0.14$ & $1.75 \pm 0.59$ & 0.96 & -0.32 \\
\hline 462 & $4611055 \mathrm{~N}, 456600 \mathrm{E}$ & $1.00 \pm 0.16$ & $1.84 \pm 0.58$ & 1.08 & -0.20 \\
\hline 463 & $4611055 \mathrm{~N}, 456607 \mathrm{E}$ & $0.57 \pm 0.12$ & $0.87 \pm 0.43$ & 0.54 & -0.74 \\
\hline 464 & $4611055 \mathrm{~N}, 456614 \mathrm{E}$ & $0.97 \pm 0.17$ & $1.77 \pm 0.57$ & 1.04 & -0.24 \\
\hline 465 & $4611055 \mathrm{~N}, 456622 \mathrm{E}$ & $0.73 \pm 0.13$ & $1.38 \pm 0.42$ & 0.80 & -0.48 \\
\hline 466 & $4611055 \mathrm{~N}, 456629 \mathrm{E}$ & $0.91 \pm 0.15$ & $2.19 \pm 0.43$ & 1.19 & -0.09 \\
\hline 467 & $4611061 \mathrm{~N}, 456603 \mathrm{E}$ & $0.95 \pm 0.21$ & $2.54 \pm 0.70$ & 1.34 & 0.06 \\
\hline 468 & $4611061 \mathrm{~N}, 456612 \mathrm{E}$ & $1.01 \pm 0.16$ & $1.50 \pm 0.45$ & 0.95 & -0.33 \\
\hline 469 & $4611061 \mathrm{~N}, 456618 \mathrm{E}$ & $0.76 \pm 0.13$ & $1.21 \pm 0.41$ & 0.75 & -0.54 \\
\hline 470 & $4611061 \mathrm{~N}, 456625 \mathrm{E}$ & $2.06 \pm 0.23$ & $2.42 \pm 0.55$ & 1.68 & 0.40 \\
\hline 471 & $4611061 \mathrm{~N}, 456632 \mathrm{E}$ & $1.32 \pm 0.20$ & $2.58 \pm 0.86$ & 1.49 & 0.20 \\
\hline 472 & $4611061 \mathrm{~N}, 456640 \mathrm{E}$ & $0.32 \pm 0.09$ & $0.72 \pm 0.51$ & 0.40 & -0.88 \\
\hline $473 \mathrm{~J}$ & $4611058 \mathrm{~N}, 456625 \mathrm{E}$ & $0.66 \pm 0.12$ & $1.24 \pm 0.47$ & 0.72 & -0.56 \\
\hline $474 \mathrm{~J}$ & $4611059 \mathrm{~N}, 456627 \mathrm{E}$ & $2.30 \pm 0.28$ & $2.44 \pm 0.70$ & 1.77 & 0.49 \\
\hline $475 \mathrm{~J}$ & $4611060 \mathrm{~N}, 456628 \mathrm{E}$ & $1.55 \pm 0.21$ & $2.30 \pm 0.69$ & 1.45 & 0.17 \\
\hline $476 \mathrm{~J}$ & $4611051 \mathrm{~N}, 456626 \mathrm{E}$ & $1.10 \pm 0.17$ & $2.04 \pm 0.60$ & 1.20 & -0.09 \\
\hline $477 \mathrm{~J}$ & $4611053 \mathrm{~N}, 456628 \mathrm{E}$ & $1.60 \pm 0.21$ & $1.39 \pm 0.57$ & 1.11 & -0.17 \\
\hline \multicolumn{6}{|l|}{ C2 SU1 } \\
\hline 0251 & $4611599 \mathrm{~N}, 456795 \mathrm{E}$ & $1.08 \pm 0.20$ & $2.40 \pm 0.81$ & 1.33 & 0.05 \\
\hline 0252 & Sample not collected & & & & \\
\hline 0253 & $4611599 \mathrm{~N}, 456886 \mathrm{E}$ & $0.75 \pm 0.20$ & $1.33 \pm 0.57$ & 0.79 & -0.49 \\
\hline 0254 & $4611619 \mathrm{~N}, 456784 \mathrm{E}$ & $1.19 \pm 0.18$ & $1.38 \pm 0.56$ & 0.96 & -0.32 \\
\hline 0255 & $4611619 \mathrm{~N}, 456875 \mathrm{E}$ & $1.15 \pm 0.20$ & $3.18 \pm 0.77$ & 1.67 & 0.39 \\
\hline 0256 & $4611639 \mathrm{~N}, 456795 \mathrm{E}$ & $0.60 \pm 0.14$ & $1.24 \pm 0.47$ & 0.70 & -0.58 \\
\hline 0257 & Sample not collected & & & & \\
\hline
\end{tabular}




\begin{tabular}{|c|c|c|c|c|c|}
\hline \multicolumn{6}{|c|}{$\begin{array}{c}\text { Table B-6 (cont.): FSS Survey Units and Radionuclide Concentration Levels } \\
\text { Hammond Depot } \\
\text { Hammond, Indiana }\end{array}$} \\
\hline Sample ID & $\begin{array}{c}\text { Coordinate } \mathrm{N}, \mathrm{E}^{\mathrm{a}} \\
(\mathrm{X}, \mathrm{Y})\end{array}$ & $\begin{array}{l}\text { Th-232 } \\
\text { (pCi/g) }\end{array}$ & $\begin{array}{c}\mathrm{U}-238^{\mathrm{c}} \\
(\mathrm{pCi} / \mathrm{g})\end{array}$ & $\begin{array}{c}\text { SOR } \\
\text { Gross }\end{array}$ & SOR Net \\
\hline \multicolumn{6}{|l|}{ C2 SU1 (cont.) } \\
\hline 0258 & $4611639 \mathrm{~N}, 456886 \mathrm{E}$ & $0.93 \pm 0.15$ & $1.95 \pm 0.55$ & 1.10 & -0.18 \\
\hline 0259 & $4611662 \mathrm{~N}, 456807 \mathrm{E}$ & $0.54 \pm 0.13$ & $0.94 \pm 0.54$ & 0.56 & -0.72 \\
\hline 0260 & Sample not collected & & & & \\
\hline 0261 & $4611659 \mathrm{~N}, 456875 \mathrm{E}$ & $0.98 \pm 0.21$ & $2.34 \pm 0.68$ & 1.27 & -0.01 \\
\hline 0262 & $4611678 \mathrm{~N}, 456795 \mathrm{E}$ & $0.77 \pm 0.14$ & $1.62 \pm 0.41$ & 0.91 & -0.37 \\
\hline 0263 & $4611678 \mathrm{~N}, 456886 \mathrm{E}$ & $0.93 \pm 0.17$ & $2.01 \pm 0.66$ & 1.12 & -0.16 \\
\hline 0264 & $4611698 \mathrm{~N}, 456807 \mathrm{E}$ & $0.80 \pm 0.17$ & $1.03 \pm 0.54$ & 0.69 & -0.59 \\
\hline 0265 & $4611698 \mathrm{~N}, 456830 \mathrm{E}$ & $1.03 \pm 0.18$ & $1.86 \pm 0.79$ & 1.10 & -0.18 \\
\hline 0266 & $4611698 \mathrm{~N}, 456852 \mathrm{E}$ & $0.59 \pm 0.11$ & $1.40 \pm 0.41$ & 0.76 & -0.52 \\
\hline 0267 & $4611698 \mathrm{~N}, 456875 \mathrm{E}$ & $1.17 \pm 0.18$ & $2.35 \pm 0.66$ & 1.34 & 0.06 \\
\hline \multicolumn{6}{|l|}{ C2 SU2 } \\
\hline 0268 & Sample not collected & & & & \\
\hline 0269 & $4611509 \mathrm{~N}, 456856 \mathrm{E}$ & $0.77 \pm 0.15$ & $1.47 \pm 0.66$ & 0.85 & -0.48 \\
\hline 0270 & $4611509 \mathrm{~N}, 456875 \mathrm{E}$ & $0.87 \pm 0.19$ & $1.81 \pm 0.65$ & 1.02 & -0.29 \\
\hline 0271 & $4611525 \mathrm{~N}, 456788 \mathrm{E}$ & $0.73 \pm 0.10$ & $1.01 \pm 0.34$ & 0.66 & -0.70 \\
\hline 0272 & $4611525 \mathrm{~N}, 456846 \mathrm{E}$ & $1.42 \pm 0.22$ & $1.75 \pm 0.73$ & 1.19 & -0.13 \\
\hline 0273 & $4611525 \mathrm{~N}, 456865 \mathrm{E}$ & $0.78 \pm 0.17$ & $1.55 \pm 0.52$ & 0.89 & -0.44 \\
\hline 0274 & $4611525 \mathrm{~N}, 456885 \mathrm{E}$ & $0.94 \pm 0.16$ & $2.10 \pm 0.38$ & 1.16 & -0.13 \\
\hline 0275 & Sample not collected & & & & \\
\hline 0276 & $4611542 \mathrm{~N}, 456856 \mathrm{E}$ & $0.96 \pm 0.20$ & $2.46 \pm 0.80$ & 1.32 & 0.04 \\
\hline 0277 & $4611542 \mathrm{~N}, 456875 \mathrm{E}$ & $1.30 \pm 0.18$ & $2.00 \pm 0.71$ & 1.25 & -0.05 \\
\hline 0278 & $4611559 \mathrm{~N}, 456788 \mathrm{E}$ & $0.57 \pm 0.10$ & $0.74 \pm 0.36$ & 0.49 & -0.88 \\
\hline 0279 & $4611559 \mathrm{~N}, 456846 \mathrm{E}$ & $1.05 \pm 0.14$ & $1.62 \pm 0.46$ & 1.01 & -0.31 \\
\hline 0280 & $4611559 \mathrm{~N}, 456865 \mathrm{E}$ & $1.19 \pm 0.19$ & $2.24 \pm 0.51$ & 1.31 & 0.02 \\
\hline 0281 & $4611559 \mathrm{~N}, 456885 \mathrm{E}$ & $0.81 \pm 0.17$ & $2.17 \pm 0.61$ & 1.15 & -0.15 \\
\hline 0282 & Sample not collected & & & & \\
\hline 0283 & $4611575 \mathrm{~N}, 456856 \mathrm{E}$ & $1.08 \pm 0.17$ & $1.58 \pm 0.43$ & 1.00 & -0.32 \\
\hline 0284 & $4611575 \mathrm{~N}, 456875 \mathrm{E}$ & $1.03 \pm 0.17$ & $2.04 \pm 0.82$ & 1.17 & -0.13 \\
\hline \multicolumn{6}{|l|}{ C2 SU3 } \\
\hline 0285 & $4611463 \mathrm{~N}, 456626 \mathrm{E}$ & $0.69 \pm 0.15$ & $2.06 \pm 0.59$ & 1.06 & -0.22 \\
\hline 0286 & $4611463 \mathrm{~N}, 456650 \mathrm{E}$ & $0.44 \pm 0.11$ & $1.12 \pm 0.45$ & 0.60 & -0.68 \\
\hline 0287 & $4611463 \mathrm{~N}, 456675 \mathrm{E}$ & $0.69 \pm 0.14$ & $1.80 \pm 0.49$ & 0.96 & -0.32 \\
\hline 0288 & $4611463 \mathrm{~N}, 456699 \mathrm{E}$ & $0.75 \pm 0.12$ & $1.33 \pm 0.48$ & 0.79 & -0.49 \\
\hline 0289 & Sample not collected & & & & \\
\hline 0290 & $4611463 \mathrm{~N}, 456773 \mathrm{E}$ & $0.99 \pm 0.18$ & $1.6 \pm 0.68$ & 0.98 & -0.30 \\
\hline 0291 & $4611484 \mathrm{~N}, 456638 \mathrm{E}$ & $1.16 \pm 0.19$ & $3.45 \pm 0.72$ & 1.78 & 0.50 \\
\hline 0292 & $4611484 \mathrm{~N}, 456662 \mathrm{E}$ & $0.62 \pm 0.13$ & $1.70 \pm 0.66$ & 0.89 & -0.39 \\
\hline 0293 & $4611484 \mathrm{~N}, 456687 \mathrm{E}$ & $0.65 \pm 0.15$ & $1.68 \pm 0.59$ & 0.90 & -0.39 \\
\hline 0294 & $4611484 \mathrm{~N}, 456712 \mathrm{E}$ & $0.75 \pm 0.12$ & $1.20 \pm 0.45$ & 0.74 & -0.54 \\
\hline 0295 & $4611505 \mathrm{~N}, 456626 \mathrm{E}$ & $0.58 \pm 0.14$ & $1.47 \pm 0.52$ & 0.79 & -0.49 \\
\hline
\end{tabular}




\begin{tabular}{|c|c|c|c|c|c|}
\hline \multicolumn{6}{|c|}{$\begin{array}{c}\text { Table B-6 (cont.): FSS Survey Units and Radionuclide Concentration Levels } \\
\text { Hammond Depot } \\
\text { Hammond, Indiana }\end{array}$} \\
\hline Sample ID & $\begin{array}{c}\text { Coordinate } \mathbf{N}, \mathrm{E}^{\mathrm{a}} \\
(\mathrm{X}, \mathrm{Y})\end{array}$ & $\begin{array}{l}\text { Th-232 } \\
\text { (pCi/g) }\end{array}$ & $\begin{array}{l}\mathrm{U}-238^{\mathrm{c}} \\
(\mathrm{pCi} / \mathrm{g})\end{array}$ & $\begin{array}{l}\text { SOR } \\
\text { Gross }\end{array}$ & SOR Net \\
\hline \multicolumn{6}{|l|}{ C2 SU3 (cont.) } \\
\hline 0296 & $4611505 \mathrm{~N}, 456650 \mathrm{E}$ & $0.84 \pm 0.17$ & $2.06 \pm 0.66$ & 1.11 & -0.17 \\
\hline 0297 & $4611505 \mathrm{~N}, 456675 \mathrm{E}$ & $0.48 \pm 0.15$ & $1.52 \pm 0.60$ & 0.77 & -0.51 \\
\hline 0298 & $4611505 \mathrm{~N}, 456699 \mathrm{E}$ & $0.96 \pm 0.13$ & $1.80 \pm 0.59$ & 1.05 & -0.23 \\
\hline 0299 & Sample not collected & & & & \\
\hline 0300 & Sample not collected & & & & \\
\hline 0301 & $4611505 \mathrm{~N}, 456773 \mathrm{E}$ & $0.77 \pm 0.13$ & $1.36 \pm 0.57$ & 0.81 & -0.47 \\
\hline \multicolumn{6}{|l|}{ C2 SU4 } \\
\hline 0302 & $4611444 \mathrm{~N}, 456787 \mathrm{E}$ & $0.86 \pm 0.15$ & $1.20 \pm 0.48$ & 0.78 & -0.51 \\
\hline 0303 & $4611444 \mathrm{~N}, 456841 \mathrm{E}$ & $0.94 \pm 0.17$ & $1.92 \pm 0.69$ & 1.09 & -0.19 \\
\hline 0304 & $4611444 \mathrm{~N}, 456859 \mathrm{E}$ & $0.88 \pm 0.19$ & $2.80 \pm 0.70$ & 1.42 & 0.14 \\
\hline 0305 & $4611444 \mathrm{~N}, 456877 \mathrm{E}$ & $0.89 \pm 0.15$ & $1.81 \pm 0.56$ & 1.03 & -0.25 \\
\hline 0306 & $4611459 \mathrm{~N}, 456850 \mathrm{E}$ & $0.84 \pm 0.15$ & $1.58 \pm 0.42$ & 0.92 & -0.36 \\
\hline 0307 & $4611459 \mathrm{~N}, 456868 \mathrm{E}$ & $0.68 \pm 0.19$ & $2.35 \pm 0.56$ & 1.17 & -0.11 \\
\hline 0308 & $4611475 \mathrm{~N}, 456787 \mathrm{E}$ & $1.18 \pm 0.16$ & $1.72 \pm 0.54$ & 1.09 & -0.19 \\
\hline 0309 & $4611475 \mathrm{~N}, 456841 \mathrm{E}$ & $0.96 \pm 0.14$ & $1.47 \pm 0.46$ & 0.92 & -0.36 \\
\hline 0310 & $4611475 \mathrm{~N}, 456859 \mathrm{E}$ & $0.78 \pm 0.16$ & $2.15 \pm 0.44$ & 1.13 & -0.15 \\
\hline 0311 & $4611475 \mathrm{~N}, 456877 \mathrm{E}$ & $0.88 \pm 0.15$ & $2.48 \pm 0.58$ & 1.30 & 0.01 \\
\hline 0312 & $4611490 \mathrm{~N}, 456850 \mathrm{E}$ & $0.83 \pm 0.17$ & $2.00 \pm 0.61$ & 1.09 & -0.20 \\
\hline 0313 & $4611490 \mathrm{~N}, 456868 \mathrm{E}$ & $0.81 \pm 0.14$ & $1.65 \pm 0.47$ & 0.94 & -0.34 \\
\hline 0314 & Sample not collected & & & & \\
\hline 0315 & $4611506 \mathrm{~N}, 456787 \mathrm{E}$ & $0.28 \pm 0.07$ & $0.57 \pm 0.3$ & 0.32 & -0.96 \\
\hline 0316 & $4611506 \mathrm{~N}, 456805 \mathrm{E}$ & $0.71 \pm 0.13$ & $1.79 \pm 0.46$ & 0.96 & -0.32 \\
\hline 0317 & $4611506 \mathrm{~N}, 456823 \mathrm{E}$ & $1.01 \pm 0.18$ & $1.53 \pm 0.61$ & 0.96 & -0.32 \\
\hline 0318 & $4611506 \mathrm{~N}, 456841 \mathrm{E}$ & $1.08 \pm 0.15$ & $1.35 \pm 0.49$ & 0.91 & -0.37 \\
\hline \multicolumn{6}{|l|}{ C2 SU5 } \\
\hline 0319 & $4611379 \mathrm{~N}, 456671 \mathrm{E}$ & $0.82 \pm 0.15$ & $2.00 \pm 0.52$ & 1.08 & -0.20 \\
\hline 0320 & $4611379 \mathrm{~N}, 456695 \mathrm{E}$ & $0.63 \pm 0.14$ & $1.31 \pm 0.44$ & 0.74 & -0.54 \\
\hline 0321 & Sample not collected & & & & \\
\hline 0322 & Sample not collected & & & & \\
\hline 0323 & Sample not collected & & & & \\
\hline 0324 & $4611400 \mathrm{~N}, 456683 \mathrm{E}$ & $0.55 \pm 0.11$ & $0.56 \pm 0.46$ & 0.41 & -0.87 \\
\hline 0325 & $4611400 \mathrm{~N}, 456707 \mathrm{E}$ & $0.99 \pm 0.18$ & $2.85 \pm 0.83$ & 1.48 & 0.20 \\
\hline 0326 & $4611420 \mathrm{~N}, 456622 \mathrm{E}$ & $0.64 \pm 0.11$ & $1.43 \pm 0.43$ & 0.79 & -0.49 \\
\hline 0327 & $4611420 \mathrm{~N}, 456646 \mathrm{E}$ & $0.85 \pm 0.16$ & $2.10 \pm 0.48$ & 1.13 & -0.15 \\
\hline 0328 & $4611420 \mathrm{~N}, 456671 \mathrm{E}$ & $0.42 \pm 0.09$ & $0.45 \pm 0.39$ & 0.32 & -0.96 \\
\hline 0329 & $4611420 \mathrm{~N}, 456695 \mathrm{E}$ & $0.96 \pm 0.15$ & $2.01 \pm 0.54$ & 1.14 & -0.15 \\
\hline 0330 & Sample not collected & & & & \\
\hline 0331 & $4611420 \mathrm{~N}, 456767 \mathrm{E}$ & $0.57 \pm 0.12$ & $0.78 \pm 0.54$ & 0.51 & -0.77 \\
\hline 0332 & $4611441 \mathrm{~N}, 456634 \mathrm{E}$ & $0.70 \pm 0.13$ & $1.37 \pm 0.68$ & 0.79 & -0.49 \\
\hline 0333 & $4611441 \mathrm{~N}, 456659 \mathrm{E}$ & $1.02 \pm 0.18$ & $2.43 \pm 0.43$ & 1.32 & 0.04 \\
\hline
\end{tabular}




\begin{tabular}{|c|c|c|c|c|c|}
\hline \multicolumn{6}{|c|}{$\begin{array}{c}\text { Table B-6 (cont.): FSS Survey Units and Radionuclide Concentration Levels } \\
\text { Hammond Depot } \\
\text { Hammond, Indiana }\end{array}$} \\
\hline Sample ID & $\begin{array}{c}\text { Coordinate } \mathrm{N}, \mathrm{E}^{\mathrm{a}} \\
(\mathrm{X}, \mathrm{Y})\end{array}$ & $\begin{array}{l}\text { Th-232 } \\
\text { (pCi/g) }\end{array}$ & $\begin{array}{c}\mathrm{U}-238^{\mathrm{c}} \\
(\mathrm{pCi} / \mathrm{g})\end{array}$ & $\begin{array}{l}\text { SOR } \\
\text { Gross }\end{array}$ & SOR Net \\
\hline \multicolumn{6}{|l|}{ C2 SU5 (cont.) } \\
\hline 0334 & $4611441 \mathrm{~N}, 456683 \mathrm{E}$ & $0.82 \pm 0.14$ & $2.05 \pm 0.53$ & 1.10 & -0.18 \\
\hline 0335 & $4611441 \mathrm{~N}, 456707 \mathrm{E}$ & $0.73 \pm 0.15$ & $1.97 \pm 0.67$ & 1.04 & -0.24 \\
\hline \multicolumn{6}{|l|}{ C2 SU6 } \\
\hline 0336 & $4611379 \mathrm{~N}, 456793 \mathrm{E}$ & $0.42 \pm 0.1$ & $0.77 \pm 0.37$ & 0.45 & -0.83 \\
\hline 0337 & Sample not collected & & & & \\
\hline 0338 & Sample not collected & & & & \\
\hline 0339 & $4611394 \mathrm{~N}, 456784 \mathrm{E}$ & $0.57 \pm 0.14$ & $1.34 \pm 0.59$ & 0.73 & -0.55 \\
\hline 0340 & Sample not collected & & & & \\
\hline 0341 & $4611409 \mathrm{~N}, 456793 \mathrm{E}$ & $0.58 \pm 0.12$ & $1.41 \pm 0.50$ & 0.76 & -0.52 \\
\hline 0342 & $4611409 \mathrm{~N}, 456844 \mathrm{E}$ & $1.42 \pm 0.19$ & $1.73 \pm 0.61$ & 1.18 & -0.10 \\
\hline 0343 & $4611409 \mathrm{~N}, 456862 \mathrm{E}$ & $0.87 \pm 0.17$ & $2.59 \pm 0.63$ & 1.34 & 0.05 \\
\hline 0344 & $4611409 \mathrm{~N}, 456879 \mathrm{E}$ & $0.86 \pm 0.16$ & $1.69 \pm 0.61$ & 0.97 & -0.31 \\
\hline 0345 & $4611424 \mathrm{~N}, 456784 \mathrm{E}$ & $0.65 \pm 0.14$ & $1.70 \pm 0.51$ & 0.90 & -0.38 \\
\hline 0346 & Sample not collected & & & & \\
\hline 0347 & $4611424 \mathrm{~N}, 456853 \mathrm{E}$ & $0.66 \pm 0.16$ & $1.35 \pm 0.62$ & 0.77 & -0.51 \\
\hline 0348 & $4611424 \mathrm{~N}, 456870 \mathrm{E}$ & $0.95 \pm 0.15$ & $2.58 \pm 0.51$ & 1.36 & 0.08 \\
\hline 0349 & $4611438 \mathrm{~N}, 456793 \mathrm{E}$ & $0.73 \pm 0.15$ & $1.64 \pm 0.50$ & 0.91 & -0.37 \\
\hline 0350 & $4611438 \mathrm{~N}, 456844 \mathrm{E}$ & $1.06 \pm 0.15$ & $1.51 \pm 0.44$ & 0.97 & -0.31 \\
\hline 0351 & $4611438 \mathrm{~N}, 456862 \mathrm{E}$ & $0.9 \pm 0.17$ & $2.16 \pm 0.54$ & 1.17 & -0.11 \\
\hline 0352 & $4611438 \mathrm{~N}, 456879 \mathrm{E}$ & $0.99 \pm 0.18$ & $1.90 \pm 0.69$ & 1.10 & -0.18 \\
\hline \multicolumn{6}{|l|}{ C2 SU7 } \\
\hline 0353 & $4611336 \mathrm{~N}, 456498$ & $0.67 \pm 0.13$ & $1.58 \pm 0.42$ & 0.86 & -0.42 \\
\hline 0354 & Sample not collected & & & & \\
\hline 0355 & Sample not collected & & & & \\
\hline 0356 & $4611365 \mathrm{~N}, 456493 \mathrm{E}$ & $0.84 \pm 0.12$ & $1.14 \pm 0.41$ & 0.75 & -0.54 \\
\hline 0357 & $4611365 \mathrm{~N}, 456510 \mathrm{E}$ & $1.23 \pm 0.18$ & $2.16 \pm 0.51$ & 1.29 & 0.01 \\
\hline 0358 & Sample not collected & & & & \\
\hline 0359 & $4611380 \mathrm{~N}, 456502 \mathrm{E}$ & $1.00 \pm 0.14$ & $1.78 \pm 0.38$ & 1.06 & -0.23 \\
\hline 0360 & $4611395 \mathrm{~N}, 456473 \mathrm{E}$ & $1.05 \pm 0.18$ & $1.96 \pm 0.60$ & 1.15 & -0.14 \\
\hline 0361 & $4611395 \mathrm{~N}, 456493 \mathrm{E}$ & $1.11 \pm 0.16$ & $3.07 \pm 0.62$ & 1.61 & 0.33 \\
\hline 0362 & $4611410 \mathrm{~N}, 456484 \mathrm{E}$ & $0.45 \pm 0.09$ & $0.93 \pm 0.25$ & 0.53 & -0.76 \\
\hline 0363 & $4611424 \mathrm{~N}, 456476 \mathrm{E}$ & $1.09 \pm 0.16$ & $2.28 \pm 0.54$ & 1.29 & 0.01 \\
\hline 0364 & $4611424 \mathrm{~N}, 456493 \mathrm{E}$ & $0.98 \pm 0.17$ & $2.26 \pm 0.74$ & 1.24 & -0.04 \\
\hline 0365 & $4611439 \mathrm{~N}, 456462 \mathrm{E}$ & $0.94 \pm 0.15$ & $1.89 \pm 0.54$ & 1.08 & -0.20 \\
\hline 0366 & $4611439 \mathrm{~N}, 456484 \mathrm{E}$ & $0.96 \pm 0.21$ & $3.35 \pm 0.90$ & 1.67 & 0.39 \\
\hline 0367 & $4611439 \mathrm{~N}, 456502 \mathrm{E}$ & $0.78 \pm 0.13$ & $1.28 \pm 0.49$ & 0.78 & -0.50 \\
\hline 0368 & $4611439 \mathrm{~N}, 456519 \mathrm{E}$ & $0.75 \pm 0.12$ & $1.75 \pm 0.48$ & 0.96 & -0.32 \\
\hline 0369 & $4611439 \mathrm{~N}, 456536 \mathrm{E}$ & $1.03 \pm 0.18$ & $1.72 \pm 0.64$ & 1.04 & -0.24 \\
\hline \multicolumn{6}{|l|}{ C2 SU8 } \\
\hline 370 & $4611299 \mathrm{~N}, 456610 \mathrm{E}$ & $0.76 \pm 0.12$ & $1.54 \pm 0.47$ & 0.88 & -0.40 \\
\hline
\end{tabular}




\begin{tabular}{|c|c|c|c|c|c|}
\hline \multicolumn{6}{|c|}{$\begin{array}{c}\text { Table B-6 (cont.): FSS Survey Units and Radionuclide Concentration Levels } \\
\text { Hammond Depot } \\
\text { Hammond, Indiana }\end{array}$} \\
\hline Sample ID & $\begin{array}{c}\text { Coordinate } \mathrm{N}, \mathrm{E}^{\mathrm{a}} \\
(\mathrm{X}, \mathrm{Y})\end{array}$ & $\begin{array}{l}\text { Th-232 } \\
\text { (pCi/g) }\end{array}$ & $\begin{array}{l}\mathrm{U}-238^{\mathrm{c}} \\
(\mathrm{pCi} / \mathrm{g})\end{array}$ & $\begin{array}{l}\text { SOR } \\
\text { Gross }\end{array}$ & SOR Net \\
\hline \multicolumn{6}{|l|}{ C2 SU8 (cont.) } \\
\hline 371 & $4611330 \mathrm{~N}, 456610 \mathrm{E}$ & $0.53 \pm 0.10$ & $0.98 \pm 0.41$ & 0.57 & -0.71 \\
\hline 372 & $4611362 \mathrm{~N}, 456610 \mathrm{E}$ & $0.45 \pm 0.10$ & $0.78 \pm 0.42$ & 0.47 & -0.82 \\
\hline 373 & $4611380 \mathrm{~N}, 456528 \mathrm{E}$ & $0.98 \pm 0.16$ & $1.50 \pm 0.62$ & 0.94 & -0.34 \\
\hline 374 & $4611378 \mathrm{~N}, 456544 \mathrm{E}$ & $0.88 \pm 0.11$ & $1.27 \pm 0.37$ & 0.81 & -0.47 \\
\hline 375 & Sample not collected & & & & \\
\hline 376 & $4611394 \mathrm{~N}, 456556 \mathrm{E}$ & $0.86 \pm 0.13$ & $2.13 \pm 0.53$ & 1.15 & -0.13 \\
\hline 377 & $4611394 \mathrm{~N}, 456610 \mathrm{E}$ & $0.48 \pm 0.08$ & $0.49 \pm 0.27$ & 0.36 & -0.92 \\
\hline 378 & Sample not collected & & & & \\
\hline 379 & $4611425 \mathrm{~N}, 456556 \mathrm{E}$ & $0.98 \pm 0.19$ & $1.52 \pm 0.62$ & 0.95 & -0.34 \\
\hline 380 & $4611425 \mathrm{~N}, 456574 \mathrm{E}$ & $0.69 \pm 0.15$ & $1.31 \pm 0.49$ & 0.76 & -0.52 \\
\hline 381 & $4611425 \mathrm{~N}, 456592 \mathrm{E}$ & $0.56 \pm 0.10$ & $1.17 \pm 0.36$ & 0.66 & -0.62 \\
\hline 382 & $4611425 \mathrm{~N}, 456610 \mathrm{E}$ & $1.31 \pm 0.17$ & $1.39 \pm 0.48$ & 1.01 & -0.27 \\
\hline 383 & Sample not collected & & & & \\
\hline 384 & $4611441 \mathrm{~N}, 456565 \mathrm{E}$ & $0.69 \pm 0.12$ & $1.24 \pm 0.43$ & 0.73 & -0.55 \\
\hline 385 & $4611441 \mathrm{~N}, 456583 \mathrm{E}$ & $0.79 \pm 0.14$ & $1.85 \pm 0.59$ & 1.01 & -0.27 \\
\hline 386 & $4611441 \mathrm{~N}, 456601 \mathrm{E}$ & $0.58 \pm 0.12$ & $1.64 \pm 0.48$ & 0.86 & -0.43 \\
\hline \multicolumn{6}{|l|}{ C2 SU9 } \\
\hline 387 & $4611208 \mathrm{~N}, 456555 \mathrm{E}$ & $0.79 \pm 0.15$ & $1.61 \pm 0.55$ & 0.92 & -0.37 \\
\hline 388 & $4611208 \mathrm{~N}, 456575 \mathrm{E}$ & $1.02 \pm 0.17$ & $1.93 \pm 0.75$ & 1.12 & -0.16 \\
\hline 389 & $4611208 \mathrm{~N}, 456595 \mathrm{E}$ & $0.80 \pm 0.13$ & $1.54 \pm 0.49$ & 0.89 & -0.39 \\
\hline 390 & $4611226 \mathrm{~N}, 456545 \mathrm{E}$ & $0.56 \pm 0.11$ & $0.84 \pm 0.34$ & 0.53 & -0.75 \\
\hline 391 & $4611226 \mathrm{~N}, 456565 \mathrm{E}$ & $1.02 \pm 0.16$ & $2.10 \pm 0.52$ & 1.19 & -0.09 \\
\hline 392 & $4611226 \mathrm{~N}, 456585 \mathrm{E}$ & $0.97 \pm 0.15$ & $1.14 \pm 0.56$ & 0.79 & -0.49 \\
\hline 393 & $4611226 \mathrm{~N}, 456605 \mathrm{E}$ & $0.91 \pm 0.16$ & $2.55 \pm 0.72$ & 1.33 & 0.05 \\
\hline 394 & $4611243 \mathrm{~N}, 456535 \mathrm{E}$ & $0.63 \pm 0.10$ & $1.08 \pm 0.30$ & 0.65 & -0.63 \\
\hline 395 & $4611243 \mathrm{~N}, 456555 \mathrm{E}$ & $0.99 \pm 0.17$ & $2.17 \pm 0.74$ & 1.21 & -0.07 \\
\hline 396 & $4611243 \mathrm{~N}, 456575 \mathrm{E}$ & $0.80 \pm 0.17$ & $1.90 \pm 0.56$ & 1.04 & -0.25 \\
\hline 397 & $4611243 \mathrm{~N}, 456595 \mathrm{E}$ & $0.94 \pm 0.20$ & $2.34 \pm 0.63$ & 1.26 & -0.02 \\
\hline 398 & \multicolumn{5}{|c|}{ Sample location within C1 SU2, soil remediated } \\
\hline 399 & $4611261 \mathrm{~N}, 456565 \mathrm{E}$ & $1.00 \pm 0.14$ & $1.76 \pm 0.55$ & 1.05 & -0.23 \\
\hline 400 & $4611261 \mathrm{~N}, 456585 \mathrm{E}$ & $0.97 \pm 0.12$ & $1.39 \pm 0.47$ & 0.89 & -0.39 \\
\hline 401 & $4611261 \mathrm{~N}, 456605 \mathrm{E}$ & $0.86 \pm 0.13$ & $1.93 \pm 0.53$ & 1.07 & -0.21 \\
\hline 402 & $4611278 \mathrm{~N}, 456575 \mathrm{E}$ & $0.82 \pm 0.14$ & $1.78 \pm 0.61$ & 0.99 & -0.29 \\
\hline 403 & $4611278 \mathrm{~N}, 456595 \mathrm{E}$ & $0.65 \pm 0.12$ & $0.98 \pm 0.40$ & 0.62 & -0.67 \\
\hline \multicolumn{6}{|l|}{ C2 SU10 } \\
\hline 0404 & Sample not collected & & & & \\
\hline 0405 & $4611107 \mathrm{~N}, 456599 \mathrm{E}$ & $0.33 \pm 0.10$ & $0.87 \pm 0.46$ & 0.46 & -0.82 \\
\hline 0406 & Sample not collected & & & & \\
\hline 0407 & $4611123 \mathrm{~N}, 456608 \mathrm{E}$ & $1.09 \pm 0.19$ & $2.22 \pm 0.60$ & 1.26 & -0.02 \\
\hline 0408 & $4611139 \mathrm{~N}, 456581 \mathrm{E}$ & $0.56 \pm 0.15$ & $1.14 \pm 0.45$ & 0.65 & -0.63 \\
\hline
\end{tabular}




\begin{tabular}{|c|c|c|c|c|c|}
\hline \multicolumn{6}{|c|}{$\begin{array}{c}\text { Table B-6 (cont.): FSS Survey Units and Radionuclide Concentration Levels } \\
\text { Hammond Depot } \\
\text { Hammond, Indiana }\end{array}$} \\
\hline Sample ID & $\begin{array}{c}\text { Coordinate } \mathrm{N}, \mathrm{E}^{\mathrm{a}} \\
(\mathrm{X}, \mathrm{Y})\end{array}$ & $\begin{array}{l}\text { Th-232 } \\
\text { (pCi/g) }\end{array}$ & $\begin{array}{c}\mathrm{U}-238^{\mathrm{c}} \\
(\mathrm{pCi} / \mathrm{g})\end{array}$ & $\begin{array}{c}\text { SOR } \\
\text { Gross }\end{array}$ & SOR Net \\
\hline \multicolumn{6}{|l|}{ C2 SU10 (cont.) } \\
\hline 0409 & $4611139 \mathrm{~N}, 456599 \mathrm{E}$ & $0.49 \pm 0.08$ & $0.82 \pm 0.23$ & 0.50 & -0.79 \\
\hline 0410 & $4611154 \mathrm{~N}, 456572 \mathrm{E}$ & $0.59 \pm 0.12$ & $1.10 \pm 0.48$ & 0.64 & -0.64 \\
\hline 0411 & $4611154 \mathrm{~N}, 456590 \mathrm{E}$ & $0.33 \pm 0.08$ & $0.43 \pm 0.26$ & 0.29 & -1.00 \\
\hline 0412 & $4611154 \mathrm{~N}, 456608 \mathrm{E}$ & $1.13 \pm 0.22$ & $2.62 \pm 0.81$ & 1.44 & 0.16 \\
\hline 0413 & Sample not collected & & & & \\
\hline 0414 & $4611170 \mathrm{~N}, 456599 \mathrm{E}$ & $0.97 \pm 0.18$ & $2.35 \pm 0.91$ & 1.27 & -0.01 \\
\hline 0415 & $4611186 \mathrm{~N}, 456572 \mathrm{E}$ & $0.71 \pm 0.12$ & $1.13 \pm 0.40$ & 0.70 & -0.59 \\
\hline 0416 & $4611186 \mathrm{~N}, 456590 \mathrm{E}$ & $0.99 \pm 0.15$ & $1.92 \pm 0.55$ & 1.11 & -0.17 \\
\hline 0417 & $4611186 \mathrm{~N}, 456608 \mathrm{E}$ & $0.81 \pm 0.19$ & $2.68 \pm 0.82$ & 1.35 & 0.07 \\
\hline 0418 & $4611202 \mathrm{~N}, 456563 \mathrm{E}$ & $0.61 \pm 0.11$ & $1.11 \pm 0.47$ & 0.65 & -0.63 \\
\hline 0419 & $4611202 \mathrm{~N}, 456581 \mathrm{E}$ & $1.01 \pm 0.17$ & $2.63 \pm 0.59$ & 1.40 & 0.12 \\
\hline 0420 & $4611202 \mathrm{~N}, 456599 \mathrm{E}$ & $0.74 \pm 0.18$ & $2.29 \pm 0.62$ & 1.17 & -0.11 \\
\hline \multicolumn{6}{|l|}{ C2 SU11 } \\
\hline 0421 & $4611061 \mathrm{~N}, 456593 \mathrm{E}$ & $0.98 \pm 0.19$ & $2.15 \pm 0.73$ & 1.20 & -0.08 \\
\hline 0422 & $4611072 \mathrm{~N}, 456587 \mathrm{E}$ & $1.09 \pm 0.16$ & $2.28 \pm 0.61$ & 1.29 & 0.01 \\
\hline 0423 & $4611072 \mathrm{~N}, 456600 \mathrm{E}$ & $0.95 \pm 0.17$ & $1.73 \pm 0.67$ & 1.02 & -0.26 \\
\hline 0424 & $4611072 \mathrm{~N}, 456612 \mathrm{E}$ & $0.68 \pm 0.12$ & $1.72 \pm 0.49$ & 0.92 & -0.36 \\
\hline 0425 & $4611072 \mathrm{~N}, 456624 \mathrm{E}$ & $1.31 \pm 0.23$ & $1.74 \pm 0.88$ & 1.15 & -0.13 \\
\hline 0426 & $4611072 \mathrm{~N}, 456651 \mathrm{E}$ & $1.26 \pm 0.17$ & $1.61 \pm 0.62$ & 1.08 & -0.20 \\
\hline 0427 & $4611083 \mathrm{~N}, 456580 \mathrm{E}$ & $1.14 \pm 0.20$ & $2.01 \pm 0.89$ & 1.20 & -0.09 \\
\hline 0428 & $4611083 \mathrm{~N}, 456593 \mathrm{E}$ & $0.62 \pm 0.15$ & $1.63 \pm 0.58$ & 0.87 & -0.42 \\
\hline 0429 & $4611083 \mathrm{~N}, 456606 \mathrm{E}$ & $0.75 \pm 0.18$ & $2.29 \pm 0.59$ & 1.17 & -0.11 \\
\hline 0430 & $4611083 \mathrm{~N}, 456619 \mathrm{E}$ & $0.82 \pm 0.14$ & $1.67 \pm 0.56$ & 0.95 & -0.33 \\
\hline 0431 & $4611083 \mathrm{~N}, 456670 \mathrm{E}$ & $1.05 \pm 0.17$ & $1.83 \pm 0.63$ & 1.09 & -0.19 \\
\hline 0432 & $4611094 \mathrm{~N}, 456587 \mathrm{E}$ & $0.77 \pm 0.13$ & $2.24 \pm 0.54$ & 1.16 & -0.12 \\
\hline 0433 & $4611094 \mathrm{~N}, 456600 \mathrm{E}$ & $1.01 \pm 0.16$ & $1.76 \pm 0.54$ & 1.05 & -0.23 \\
\hline 0434 & $4611094 \mathrm{~N}, 456612 \mathrm{E}$ & $0.96 \pm 0.17$ & $2.82 \pm 0.91$ & 1.46 & 0.18 \\
\hline 0435 & $4611094 \mathrm{~N}, 456625 \mathrm{E}$ & $1.21 \pm 0.18$ & $2.66 \pm 0.86$ & 1.48 & 0.20 \\
\hline 0436 & $4611094 \mathrm{~N}, 456676 \mathrm{E}$ & $1.02 \pm 0.21$ & $1.33 \pm 0.73$ & 0.88 & -0.40 \\
\hline 0437 & $4611094 \mathrm{~N}, 456689 \mathrm{E}$ & $0.87 \pm 0.14$ & $1.39 \pm 0.58$ & 0.86 & -0.43 \\
\hline \multicolumn{6}{|l|}{ C2 SU12 } \\
\hline 0478 & $4611393 \mathrm{~N}, 456510 \mathrm{E}$ & $0.71 \pm 0.14$ & $2.06 \pm 0.67$ & 1.07 & -0.21 \\
\hline 0479 & $4611398 \mathrm{~N}, 456507 \mathrm{E}$ & $0.77 \pm 0.13$ & $2.01 \pm 0.53$ & 1.07 & -0.21 \\
\hline 0480 & $4611403 \mathrm{~N}, 456505 \mathrm{E}$ & $0.67 \pm 0.15$ & $1.04 \pm 0.59$ & 0.65 & -0.64 \\
\hline 0481 & $4611403 \mathrm{~N}, 456515 \mathrm{E}$ & $0.91 \pm 0.17$ & $1.54 \pm 0.61$ & 0.93 & -0.35 \\
\hline 0482 & $4611407 \mathrm{~N}, 456513 \mathrm{E}$ & $0.78 \pm 0.12$ & $1.81 \pm 0.53$ & 0.99 & -0.29 \\
\hline 0483 & $4611412 \mathrm{~N}, 456510 \mathrm{E}$ & $0.56 \pm 0.13$ & $1.15 \pm 0.51$ & 0.65 & -0.63 \\
\hline 0484 & $4611422 \mathrm{~N}, 456510 \mathrm{E}$ & $0.76 \pm 0.14$ & $1.40 \pm 0.57$ & 0.82 & -0.46 \\
\hline 0485 & $4611426 \mathrm{~N}, 456524 \mathrm{E}$ & $0.75 \pm 0.14$ & $1.80 \pm 0.46$ & 0.98 & -0.30 \\
\hline 0486 & $4611431 \mathrm{~N}, 456521 \mathrm{E}$ & $0.77 \pm 0.14$ & $1.56 \pm 0.61$ & 0.89 & -0.39 \\
\hline
\end{tabular}




\begin{tabular}{|c|c|c|c|c|c|}
\hline \multicolumn{6}{|c|}{$\begin{array}{c}\text { Table B-6 (cont.): FSS Survey Units and Radionuclide Concentration Levels } \\
\text { Hammond Depot } \\
\text { Hammond, Indiana }\end{array}$} \\
\hline Sample ID & $\begin{array}{c}\text { Coordinate } \mathrm{N}, \mathrm{E}^{\mathrm{a}} \\
(\mathrm{X}, \mathrm{Y})\end{array}$ & $\begin{array}{l}\text { Th-232 } \\
\text { (pCi/g) }\end{array}$ & $\begin{array}{c}\mathrm{U}-238^{\mathrm{c}} \\
(\mathrm{pCi} / \mathrm{g})\end{array}$ & $\begin{array}{c}\text { SOR } \\
\text { Gross }\end{array}$ & SOR Net \\
\hline \multicolumn{6}{|l|}{ C2 SU12 (cont.) } \\
\hline 0487 & $4611431 \mathrm{~N}, 456526 \mathrm{E}$ & $0.90 \pm 0.13$ & $1.93 \pm 0.51$ & 1.08 & -0.20 \\
\hline 0488 & $4611445 \mathrm{~N}, 456524 \mathrm{E}$ & $0.77 \pm 0.17$ & $1.79 \pm 0.63$ & 0.98 & -0.30 \\
\hline 0489 & $4611445 \mathrm{~N}, 456529 \mathrm{E}$ & $1.36 \pm 0.18$ & $2.11 \pm 0.61$ & 1.31 & 0.03 \\
\hline 0490 & $4611450 \mathrm{~N}, 456521 \mathrm{E}$ & $2.31 \pm 0.27$ & $2.74 \pm 0.68$ & 1.89 & 0.61 \\
\hline 0491 & $4611450 \mathrm{~N}, 456526 \mathrm{E}$ & $1.43 \pm 0.23$ & $2.56 \pm 0.67$ & 1.52 & 0.23 \\
\hline 0492 & $4611455 \mathrm{~N}, 456524 \mathrm{E}$ & $0.87 \pm 0.16$ & $1.59 \pm 0.57$ & 0.94 & -0.35 \\
\hline 0493 & $4611455 \mathrm{~N}, 456529 \mathrm{E}$ & $1.5 \pm 0.3$ & $2.34 \pm 0.75$ & 1.45 & 0.17 \\
\hline \multicolumn{6}{|l|}{ C3 SU1 } \\
\hline 0144 & $4611592 \mathrm{~N}, 456533 \mathrm{E}$ & $0.77 \pm 0.19$ & $2.30 \pm 0.67$ & 1.31 & -0.10 \\
\hline 0145 & $4611601 \mathrm{~N}, 456610 \mathrm{E}$ & $0.95 \pm 0.19$ & $2.60 \pm 0.75$ & 1.51 & 0.09 \\
\hline 0146 & Sample not collected & & & & \\
\hline 0147 & $4611507 \mathrm{~N}, 456529 \mathrm{E}$ & $0.94 \pm 0.17$ & $2.46 \pm 0.64$ & 1.44 & 0.03 \\
\hline 0148 & $4611484 \mathrm{~N}, 456515 \mathrm{E}$ & $1.10 \pm 0.19$ & $2.90 \pm 0.71$ & 1.70 & 0.26 \\
\hline 0149 & $4611611 \mathrm{~N}, 456581 \mathrm{E}$ & $0.90 \pm 0.19$ & $2.40 \pm 0.66$ & 1.40 & -0.01 \\
\hline 0150 & Sample not collected & & & & \\
\hline 0151 & $4611590 \mathrm{~N}, 456558 \mathrm{E}$ & $0.67 \pm 0.18$ & $2.68 \pm 0.72$ & 1.45 & 0.02 \\
\hline 0152 & Sample not collected & & & & \\
\hline 0153 & $4611684 \mathrm{~N}, 456574 \mathrm{E}$ & $1.02 \pm 0.19$ & $2.66 \pm 0.69$ & 1.56 & 0.13 \\
\hline 0154 & $4611691 \mathrm{~N}, 456562 \mathrm{E}$ & $1.10 \pm 0.21$ & $2.81 \pm 0.96$ & 1.66 & 0.22 \\
\hline 0155 & $4611526 \mathrm{~N}, 456518 \mathrm{E}$ & $0.80 \pm 0.17$ & $1.96 \pm 0.62$ & 1.17 & -0.22 \\
\hline 0156 & $4611616 \mathrm{~N}, 456531 \mathrm{E}$ & $1.43 \pm 0.17$ & $1.84 \pm 0.53$ & 1.33 & -0.05 \\
\hline 0157 & $4611606 \mathrm{~N}, 456603 \mathrm{E}$ & $0.88 \pm 0.18$ & $2.85 \pm 0.79$ & 1.60 & 0.16 \\
\hline 0158 & $4611561 \mathrm{~N}, 456493 \mathrm{E}$ & $0.89 \pm 0.16$ & $1.40 \pm 0.58$ & 0.94 & -0.42 \\
\hline 0159 & Sample not collected & & & & \\
\hline 0160 & $4611616 \mathrm{~N}, 456574 \mathrm{E}$ & $1.13 \pm 0.21$ & $3.31 \pm 0.88$ & 1.89 & 0.43 \\
\hline \multicolumn{6}{|l|}{ C3 SU2 } \\
\hline 0161 & $4611688 \mathrm{~N}, 456628 \mathrm{E}$ & $0.82 \pm 0.16$ & $1.60 \pm 0.57$ & 1.01 & 0.35 \\
\hline 0162 & Sample not collected & & & & \\
\hline 0163 & $4611549 \mathrm{~N}, 456697 \mathrm{E}$ & $0.47 \pm 0.14$ & $1.34 \pm 0.69$ & 0.70 & -0.58 \\
\hline 0164 & $4611634 \mathrm{~N}, 456618 \mathrm{E}$ & $0.61 \pm 0.12$ & $1.20 \pm 0.50$ & 0.69 & -0.59 \\
\hline 0165 & $4611616 \mathrm{~N}, 456619 \mathrm{E}$ & $0.64 \pm 0.11$ & $1.39 \pm 0.51$ & 0.78 & -0.51 \\
\hline 0166 & $4611531 \mathrm{~N}, 456630 \mathrm{E}$ & $0.68 \pm 0.11$ & $1.60 \pm 0.42$ & 0.87 & -0.41 \\
\hline 0167 & $4611517 \mathrm{~N}, 456761 \mathrm{E}$ & $0.7 \pm 0.15$ & $1.08 \pm 0.40$ & 0.67 & -0.61 \\
\hline 0168 & $4611516 \mathrm{~N}, 456773 \mathrm{E}$ & $0.50 \pm 0.11$ & $0.66 \pm 0.33$ & 0.44 & -0.85 \\
\hline 0169 & $4611702 \mathrm{~N}, 456709 \mathrm{E}$ & $0.81 \pm 0.16$ & $1.19 \pm 0.68$ & 0.76 & -0.53 \\
\hline 0170 & $4611661 \mathrm{~N}, 456737 \mathrm{E}$ & $0.72 \pm 0.14$ & $1.83 \pm 0.40$ & 0.98 & -0.30 \\
\hline 0171 & $4611696 \mathrm{~N}, 456714 \mathrm{E}$ & $0.77 \pm 0.14$ & $1.06 \pm 0.51$ & 0.69 & -0.59 \\
\hline 0172 & $4611678 \mathrm{~N}, 456750 \mathrm{E}$ & $0.58 \pm 0.12$ & $0.99 \pm 0.51$ & 0.60 & -0.69 \\
\hline 0173 & $4611688 \mathrm{~N}, 456713 \mathrm{E}$ & $0.77 \pm 0.11$ & $1.19 \pm 0.29$ & 0.74 & -0.54 \\
\hline 0174 & $4611583 \mathrm{~N}, 456763 \mathrm{E}$ & $0.70 \pm 0.12$ & $1.46 \pm 0.45$ & 0.83 & -0.46 \\
\hline
\end{tabular}




\begin{tabular}{|c|c|c|c|c|c|}
\hline \multicolumn{6}{|c|}{$\begin{array}{c}\text { Table B-6 (cont.): FSS Survey Units and Radionuclide Concentration Levels } \\
\text { Hammond Depot } \\
\text { Hammond, Indiana }\end{array}$} \\
\hline Sample ID & $\begin{array}{c}\text { Coordinate } \mathrm{N}, \mathrm{E}^{\mathrm{a}} \\
(\mathrm{X}, \mathrm{Y})\end{array}$ & $\begin{array}{l}\text { Th-232 } \\
\text { (pCi/g) }\end{array}$ & $\begin{array}{c}\mathrm{U}-238^{\mathrm{c}} \\
(\mathrm{pCi} / \mathrm{g})\end{array}$ & $\begin{array}{l}\text { SOR } \\
\text { Gross }\end{array}$ & SOR Net \\
\hline \multicolumn{6}{|l|}{ C3 SU2 (cont.) } \\
\hline 0175 & $4611654 \mathrm{~N}, 456769 \mathrm{E}$ & $0.60 \pm 0.14$ & $0.86 \pm 0.42$ & 0.55 & -0.73 \\
\hline 0176 & $4611610 \mathrm{~N}, 456747 \mathrm{E}$ & $0.29 \pm 0.07$ & $0.62 \pm 0.22$ & 0.35 & -0.93 \\
\hline 0177 & $4611550 \mathrm{~N}, 456721 \mathrm{E}$ & $0.86 \pm 0.16$ & $1.54 \pm 0.66$ & 0.91 & -0.37 \\
\hline 0178 & $4611687 \mathrm{~N}, 456617 \mathrm{E}$ & $0.99 \pm 0.17$ & $2.16 \pm 0.62$ & 1.21 & -0.08 \\
\hline \multicolumn{6}{|l|}{ C3 SU3 } \\
\hline 0179 & $4611130 \mathrm{~N}, 456617 \mathrm{E}$ & $0.88 \pm 0.17$ & $1.74 \pm 0.70$ & 1.00 & -0.28 \\
\hline 0180 & $4611137 \mathrm{~N}, 456689 \mathrm{E}$ & $0.44 \pm 0.12$ & $1.37 \pm 0.57$ & 0.70 & -0.58 \\
\hline 0181 & $4611127 \mathrm{~N}, 456618 \mathrm{E}$ & $0.89 \pm 0.15$ & $1.53 \pm 0.68$ & 0.92 & -0.36 \\
\hline 0182 & $4611304 \mathrm{~N}, 456685 \mathrm{E}$ & $1.06 \pm 0.18$ & $2.78 \pm 0.66$ & 1.48 & 0.20 \\
\hline 0183 & $4611112 \mathrm{~N}, 456665 \mathrm{E}$ & $1.05 \pm 0.16$ & $1.60 \pm 0.54$ & 1.00 & -0.28 \\
\hline 0184 & $4611280 \mathrm{~N}, 456634 \mathrm{E}$ & $0.66 \pm 0.15$ & $1.59 \pm 0.52$ & 0.86 & -0.42 \\
\hline 0185 & $4611230 \mathrm{~N}, 456672 \mathrm{E}$ & $0.36 \pm 0.07$ & $0.69 \pm 0.25$ & 0.40 & -0.88 \\
\hline 0186 & $4611164 \mathrm{~N}, 456686 \mathrm{E}$ & $0.87 \pm 0.16$ & $1.37 \pm 0.58$ & 0.85 & -0.43 \\
\hline 0187 & $4611345 \mathrm{~N}, 456659 \mathrm{E}$ & $0.82 \pm 0.18$ & $1.82 \pm 0.66$ & 1.01 & -0.27 \\
\hline 0188 & $4611123 \mathrm{~N}, 456672 \mathrm{E}$ & $1.15 \pm 0.19$ & $2.43 \pm 0.67$ & 1.37 & 0.09 \\
\hline 0189 & $4611220 \mathrm{~N}, 456695 \mathrm{E}$ & $0.88 \pm 0.13$ & $1.18 \pm 0.38$ & 0.78 & -0.51 \\
\hline 0190 & $4611188 \mathrm{~N}, 456686 \mathrm{E}$ & $1.20 \pm 0.19$ & $2.24 \pm 0.66$ & 1.31 & 0.03 \\
\hline 0191 & $4611337 \mathrm{~N}, 456665 \mathrm{E}$ & $0.76 \pm 0.18$ & $2.22 \pm 0.64$ & 1.15 & -0.13 \\
\hline 0192 & $4611325 \mathrm{~N}, 456631 \mathrm{E}$ & $0.86 \pm 0.19$ & $2.31 \pm 0.57$ & 1.22 & -0.06 \\
\hline 0193 & $4611309 \mathrm{~N}, 456653 \mathrm{E}$ & $0.74 \pm 0.17$ & $2.39 \pm 0.46$ & 1.21 & -0.07 \\
\hline 0194 & $4611189 \mathrm{~N}, 456687 \mathrm{E}$ & $1.02 \pm 0.16$ & $1.82 \pm 0.53$ & 1.08 & -0.20 \\
\hline 0195 & $4611265 \mathrm{~N}, 456638 \mathrm{E}$ & $0.39 \pm 0.11$ & $0.97 \pm 0.40$ & 0.52 & -0.76 \\
\hline \multicolumn{6}{|l|}{ C3 SU4 } \\
\hline 0215 & $4611109 \mathrm{~N}, 456706 \mathrm{E}$ & $1.40 \pm 0.26$ & $3.7 \pm 1.0$ & 1.96 & 0.68 \\
\hline 0216 & $4611165 \mathrm{~N}, 456716 \mathrm{E}$ & $1.66 \pm 0.25$ & $2.63 \pm 0.83$ & 1.62 & 0.34 \\
\hline 0217 & $4611174 \mathrm{~N}, 456699 \mathrm{E}$ & $1.19 \pm 0.17$ & $1.88 \pm 0.55$ & 1.16 & -0.12 \\
\hline 0218 & $4611187 \mathrm{~N}, 456716 \mathrm{E}$ & $1.01 \pm 0.12$ & $1.24 \pm 0.46$ & 0.84 & -0.44 \\
\hline 0219 & $4611192 \mathrm{~N}, 456732 \mathrm{E}$ & $1.53 \pm 0.25$ & $2.30 \pm 0.86$ & 1.45 & 0.17 \\
\hline 0220 & $4611196 \mathrm{~N}, 456703 \mathrm{E}$ & $1.55 \pm 0.23$ & $2.16 \pm 0.86$ & 1.40 & 0.12 \\
\hline 0221 & $4611198 \mathrm{~N}, 456732 \mathrm{E}$ & $1.38 \pm 0.20$ & $2.34 \pm 0.65$ & 1.41 & 0.13 \\
\hline 0222 & $4611208 \mathrm{~N}, 456709 \mathrm{E}$ & $1.50 \pm 0.20$ & $2.31 \pm 0.56$ & 1.44 & 0.16 \\
\hline 0223 & $4611215 \mathrm{~N}, 456700 \mathrm{E}$ & $0.90 \pm 0.14$ & $2.01 \pm 0.69$ & 1.11 & -0.17 \\
\hline 0224 & $4611223 \mathrm{~N}, 456725 \mathrm{E}$ & $1.04 \pm 0.15$ & $1.72 \pm 0.51$ & 1.05 & -0.24 \\
\hline 0225 & $4611223 \mathrm{~N}, 456720 \mathrm{E}$ & $1.44 \pm 0.19$ & $2.30 \pm 0.61$ & 1.42 & 0.13 \\
\hline 0226 & $4611268 \mathrm{~N}, 456701 \mathrm{E}$ & $1.24 \pm 0.17$ & $1.65 \pm 0.51$ & 1.09 & -0.19 \\
\hline 0227 & $4611280 \mathrm{~N}, 456779 \mathrm{E}$ & $1.09 \pm 0.16$ & $1.39 \pm 0.52$ & 0.93 & -0.35 \\
\hline 0228 & $4611289 \mathrm{~N}, 456730 \mathrm{E}$ & $0.96 \pm 0.16$ & $1.94 \pm 0.59$ & 1.11 & -0.18 \\
\hline 0229 & $4611304 \mathrm{~N}, 456752 \mathrm{E}$ & $0.47 \pm 0.09$ & $0.60 \pm 0.29$ & 0.40 & -0.88 \\
\hline 0230 & $4611314 \mathrm{~N}, 456763 \mathrm{E}$ & $0.41 \pm 0.09$ & $0.74 \pm 0.29$ & 0.44 & -0.85 \\
\hline 0231 & $4611333 \mathrm{~N}, 456747 \mathrm{E}$ & $0.53 \pm 0.11$ & $0.85 \pm 0.56$ & 0.52 & -0.76 \\
\hline
\end{tabular}




\begin{tabular}{|c|c|c|c|c|c|}
\hline \multicolumn{6}{|c|}{$\begin{array}{c}\text { Table B-6 (cont.): FSS Survey Units and Radionuclide Concentration Levels } \\
\text { Hammond Depot } \\
\text { Hammond, Indiana }\end{array}$} \\
\hline Sample ID & $\begin{array}{c}\text { Coordinate } \mathrm{N}, \mathrm{E}^{\mathrm{a}} \\
(\mathrm{X}, \mathrm{Y})\end{array}$ & $\begin{array}{l}\text { Th-232 } \\
(\mathrm{pCi} / \mathrm{g})\end{array}$ & $\begin{array}{c}\mathrm{U}-238^{\mathrm{c}} \\
(\mathrm{pCi} / \mathrm{g})\end{array}$ & $\begin{array}{c}\text { SOR } \\
\text { Gross }\end{array}$ & SOR Net \\
\hline \multicolumn{6}{|l|}{ C3 SU4 (cont.) } \\
\hline 0232 & Sample not collected & & & & \\
\hline \multicolumn{6}{|l|}{ C3 SU5 } \\
\hline 0233 & $4611170 \mathrm{~N}, 456781 \mathrm{E}$ & $1.21 \pm 0.19$ & $1.15 \pm 0.61$ & 0.88 & -0.41 \\
\hline 0234 & $4611187 \mathrm{~N}, 456801 \mathrm{E}$ & $1.18 \pm 0.17$ & $1.72 \pm 0.42$ & 1.09 & -0.19 \\
\hline 0235 & $4611190 \mathrm{~N}, 456808 \mathrm{E}$ & $0.99 \pm 0.16$ & $1.60 \pm 0.67$ & 0.98 & -0.30 \\
\hline 0236 & $4611227 \mathrm{~N}, 456833 \mathrm{E}$ & $1.45 \pm 0.20$ & $1.94 \pm 0.60$ & 1.28 & -0.01 \\
\hline 0237 & $4611240 \mathrm{~N}, 456810 \mathrm{E}$ & $0.38 \pm 0.08$ & $0.60 \pm 0.26$ & 0.37 & -0.91 \\
\hline 0238 & $4611247 \mathrm{~N}, 456838 \mathrm{E}$ & $0.99 \pm 0.14$ & $1.67 \pm 0.44$ & 1.01 & -0.27 \\
\hline 0239 & $4611256 \mathrm{~N}, 456800 \mathrm{E}$ & $0.36 \pm 0.10$ & $0.63 \pm 0.31$ & 0.38 & -0.91 \\
\hline 0240 & $4611258 \mathrm{~N}, 456855 \mathrm{E}$ & $0.87 \pm 0.15$ & $1.55 \pm 0.55$ & 0.92 & -0.36 \\
\hline 0241 & $4611259 \mathrm{~N}, 456863 \mathrm{E}$ & $1.35 \pm 0.20$ & $2.42 \pm 0.93$ & 1.43 & 0.15 \\
\hline 0242 & $4611277 \mathrm{~N}, 456785 \mathrm{E}$ & $0.63 \pm 0.09$ & $0.84 \pm 0.33$ & 0.55 & -0.73 \\
\hline 0243 & $4611286 \mathrm{~N}, 456807 \mathrm{E}$ & $0.88 \pm 0.13$ & $1.43 \pm 0.45$ & 0.88 & -0.41 \\
\hline 0244 & $4611296 \mathrm{~N}, 456784 \mathrm{E}$ & $0.75 \pm 0.12$ & $0.91 \pm 0.35$ & 0.62 & -0.66 \\
\hline 0245 & $4611309 \mathrm{~N}, 456797 \mathrm{E}$ & $0.59 \pm 0.12$ & $1.14 \pm 0.51$ & 0.66 & -0.62 \\
\hline 0246 & $4611315 \mathrm{~N}, 456886 \mathrm{E}$ & $0.76 \pm 0.13$ & $1.62 \pm 0.52$ & 0.91 & -0.37 \\
\hline 0247 & $4611322 \mathrm{~N}, 456892 \mathrm{E}$ & $0.38 \pm 0.11$ & $0.65 \pm 0.41$ & 0.39 & -0.89 \\
\hline 0248 & $4611330 \mathrm{~N}, 456868 \mathrm{E}$ & $0.64 \pm 0.19$ & $1.48 \pm 0.65$ & 0.81 & -0.47 \\
\hline 0249 & $4611335 \mathrm{~N}, 456787 \mathrm{E}$ & $0.58 \pm 0.12$ & $1.25 \pm 0.36$ & 0.70 & -0.58 \\
\hline 0250 & Sample not collected & & & & \\
\hline
\end{tabular}




\begin{tabular}{|c|c|c|c|}
\hline \multicolumn{4}{|c|}{$\begin{array}{c}\text { Table B-7: FSS Exterior Slag Survey Units and Surface Activity Levels } \\
\text { Hammond Depot } \\
\text { Hammond, Indiana }\end{array}$} \\
\hline $\begin{array}{c}\text { Survey Unit ID/ } \\
\text { Surface }\end{array}$ & Location $(\mathrm{N}, \mathrm{E})^{\mathrm{a}}$ & Counts per Minute & $\begin{array}{l}\text { Total Beta Activity } \\
\mathrm{dpm} / 100 \mathrm{~cm}^{2}\end{array}$ \\
\hline \multicolumn{4}{|c|}{ C1 SU2 } \\
\hline \multicolumn{4}{|l|}{ C1 SU2.1 } \\
\hline Slag & 4611369,456528 & 479 & 11 \\
\hline Slag & 4611369,456532 & 541 & 130 \\
\hline Slag & 4611369,456536 & 569 & 180 \\
\hline Slag & 4611369,456540 & 481 & 15 \\
\hline Slag & 4611369,456544 & 462 & -21 \\
\hline Slag & 4611372,456527 & 466 & -13 \\
\hline Slag & 4611372,456530 & 498 & 47 \\
\hline Slag & 4611372,456534 & 404 & -130 \\
\hline Slag & 4611372,456538 & 451 & -42 \\
\hline Slag & 4611372,456542 & 408 & -120 \\
\hline Slag & 4611375,456525 & 398 & -140 \\
\hline Slag & 4611375,456528 & 347 & -240 \\
\hline Slag & 4611375,456532 & 405 & -130 \\
\hline Slag & 4611375,456536 & 498 & 47 \\
\hline \multicolumn{4}{|l|}{ C1 SU2.2 } \\
\hline Slag & 4611358,456530 & 525 & 98 \\
\hline Slag & 4611358,456535 & 503 & 57 \\
\hline Slag & 4611358,456539 & 530 & 110 \\
\hline Slag & 4611358,456543 & 552 & 150 \\
\hline Slag & 4611362,456528 & 434 & -74 \\
\hline Slag & 4611362,456533 & 506 & 62 \\
\hline Slag & 4611362,456537 & 487 & 26 \\
\hline Slag & 4611362,456541 & 503 & 57 \\
\hline Slag & 4611362,456545 & 570 & 180 \\
\hline Slag & 4611365,456526 & 459 & -26 \\
\hline Slag & 4611365,456530 & 495 & 42 \\
\hline Slag & 4611365,456535 & 476 & 6 \\
\hline Slag & 4611365,456539 & 485 & 23 \\
\hline Slag & 4611365,456543 & 513 & 76 \\
\hline \multicolumn{4}{|l|}{ C1 SU2.3 } \\
\hline Slag & 4611347,456529 & 539 & 120 \\
\hline Slag & 4611347,456533 & 486 & 25 \\
\hline Slag & 4611347,456537 & 480 & 13 \\
\hline Slag & 4611347,456541 & 478 & 9 \\
\hline Slag & 4611347,456545 & 484 & 21 \\
\hline Slag & 4611350,456531 & 507 & 64 \\
\hline Slag & 4611350,456535 & 534 & 120 \\
\hline
\end{tabular}




\begin{tabular}{|c|c|c|c|}
\hline \multicolumn{4}{|c|}{$\begin{array}{c}\text { Table B-7 (cont.): FSS Exterior Slag Survey Units and Surface Activity Levels } \\
\text { Hammond Depot } \\
\text { Hammond, Indiana }\end{array}$} \\
\hline $\begin{array}{l}\text { Survey Unit ID/ } \\
\text { Surface }\end{array}$ & Location $(\mathrm{N}, \mathrm{E})^{\mathrm{a}}$ & Counts per Minute & $\begin{array}{l}\text { Total Beta Activity } \\
\mathrm{dpm} / 100 \mathrm{~cm}^{2}\end{array}$ \\
\hline \multicolumn{4}{|l|}{ C1 SU2.3 (cont.) } \\
\hline Slag & 4611350,456539 & 481 & 15 \\
\hline Slag & 4611350,456543 & 509 & 68 \\
\hline Slag & 4611354,456529 & 505 & 60 \\
\hline Slag & 4611354,456533 & 549 & 140 \\
\hline Slag & 4611354,456537 & 529 & 110 \\
\hline Slag & 4611354,456541 & 518 & 85 \\
\hline Slag & 4611354,456545 & 548 & 140 \\
\hline \multicolumn{4}{|l|}{ C1 SU2.4 } \\
\hline Slag & 4611334,456525 & 498 & 47 \\
\hline Slag & 4611334,456529 & 559 & 160 \\
\hline Slag & 4611334,456532 & 475 & 4 \\
\hline Slag & 4611334,456536 & 431 & -79 \\
\hline Slag & 4611334,456540 & 455 & -34 \\
\hline Slag & 4611334,456544 & 448 & -47 \\
\hline Slag & 4611337,456531 & 589 & 220 \\
\hline Slag & 4611337,456534 & 533 & 110 \\
\hline Slag & 4611337,456538 & 506 & 62 \\
\hline Slag & 4611337,456542 & 480 & 13 \\
\hline Slag & 4611340,456532 & 524 & 96 \\
\hline Slag & 4611340,456536 & 477 & 8 \\
\hline Slag & 4611340,456540 & 457 & -30 \\
\hline Slag & 4611340,456544 & 525 & 98 \\
\hline \multicolumn{4}{|l|}{ C1 SU2.5 } \\
\hline Slag & 4611334,456519 & 658 & 350 \\
\hline Slag & 4611337,456520 & 550 & 150 \\
\hline Slag & 4611339,456519 & 475 & 4 \\
\hline Slag & 4611339,456522 & 541 & 130 \\
\hline Slag & 4611342,456517 & 513 & 76 \\
\hline Slag & 4611342,456520 & 442 & -59 \\
\hline Slag & 4611344,456516 & 447 & -49 \\
\hline Slag & 4611344,456519 & 530 & 110 \\
\hline Slag & 4611347,456514 & 564 & 170 \\
\hline Slag & 4611347,456517 & 482 & 17 \\
\hline Slag & 4611349,456516 & 539 & 120 \\
\hline Slag & 4611352,456514 & 490 & 32 \\
\hline Slag & 4611352,456517 & 450 & -43 \\
\hline Slag & 4611355,456516 & 494 & 40 \\
\hline \multicolumn{4}{|l|}{ C1 SU2.6 } \\
\hline Slag & 4611335,456502 & 428 & -85 \\
\hline Slag & 4611338,456500 & 413 & -110 \\
\hline
\end{tabular}




\begin{tabular}{|c|c|c|c|}
\hline \multicolumn{4}{|c|}{$\begin{array}{c}\text { Table B-7 (cont.): FSS Exterior Slag Survey Units and Surface Activity Levels } \\
\text { Hammond Depot } \\
\text { Hammond, Indiana }\end{array}$} \\
\hline $\begin{array}{c}\text { Survey Unit ID/ } \\
\text { Surface }\end{array}$ & Location $(\mathrm{N}, \mathrm{E})^{\mathrm{a}}$ & Counts per Minute & $\begin{array}{l}\text { Total Beta Activity } \\
\text { dpm } / 100 \mathrm{~cm}^{2}\end{array}$ \\
\hline \multicolumn{4}{|l|}{ C1 SU2.6 (cont.) } \\
\hline Slag & 4611338,456504 & 381 & -170 \\
\hline Slag & 4611338,456508 & 338 & -260 \\
\hline Slag & 4611341,456499 & 320 & -290 \\
\hline Slag & 4611341,456502 & 361 & -210 \\
\hline Slag & 4611341,456506 & 389 & -160 \\
\hline Slag & 4611341,456510 & 314 & -300 \\
\hline Slag & 4611341,456513 & 352 & -230 \\
\hline Slag & 4611344,456504 & 358 & -220 \\
\hline Slag & 4611344,456508 & 325 & -280 \\
\hline Slag & 4611344,456511 & 363 & -210 \\
\hline Slag & 4611347,456510 & 529 & 110 \\
\hline Slag & 4611347,456513 & 459 & -26 \\
\hline \multicolumn{4}{|l|}{ C1 SU2.7 } \\
\hline Slag & 4611328,456505 & 409 & -120 \\
\hline Slag & 4611328,456509 & 424 & -93 \\
\hline Slag & 4611331,456503 & 426 & -89 \\
\hline Slag & 4611331,456507 & 543 & 130 \\
\hline Slag & 4611331,456510 & 358 & -220 \\
\hline Slag & 4611331,456514 & 430 & -81 \\
\hline Slag & 4611331,456517 & 445 & -53 \\
\hline Slag & 4611334,456505 & 362 & -210 \\
\hline Slag & 4611334,456509 & 365 & -200 \\
\hline Slag & 4611334,456512 & 390 & -160 \\
\hline Slag & 4611334,456516 & 554 & 150 \\
\hline Slag & 4611337,456510 & 343 & -250 \\
\hline Slag & 4611337,456514 & 408 & -120 \\
\hline Slag & 4611340,456516 & 464 & -17 \\
\hline \multicolumn{4}{|c|}{ C1 SU3 } \\
\hline C1 SU3.1 & & & \\
\hline Slag & 4611321,456503 & 450 & -43 \\
\hline Slag & 4611321,456507 & 589 & 220 \\
\hline Slag & 4611321,456511 & 511 & 72 \\
\hline Slag & 4611321,456513 & 565 & 170 \\
\hline Slag & 4611321,456520 & 463 & -19 \\
\hline Slag & 4611324,456505 & 518 & 85 \\
\hline Slag & 4611324,456509 & 511 & 72 \\
\hline Slag & 4611324,456514 & 509 & 68 \\
\hline Slag & 4611324,456518 & 534 & 120 \\
\hline Slag & 4611324,456522 & 539 & 120 \\
\hline Slag & 4611328,456511 & 507 & 64 \\
\hline
\end{tabular}




\begin{tabular}{|c|c|c|c|}
\hline \multicolumn{4}{|c|}{$\begin{array}{c}\text { Table B-7 (cont.): FSS Exterior Slag Survey Units and Surface Activity Levels } \\
\text { Hammond Depot } \\
\text { Hammond, Indiana }\end{array}$} \\
\hline $\begin{array}{l}\text { Survey Unit ID/ } \\
\text { Surface }\end{array}$ & Location $(\mathrm{N}, \mathrm{E})^{\mathrm{a}}$ & Counts per Minute & $\begin{array}{l}\text { Total Beta Activity } \\
\mathrm{dpm} / 100 \mathrm{~cm}^{2}\end{array}$ \\
\hline \multicolumn{4}{|l|}{ C1 SU3.1 (cont.) } \\
\hline Slag & 4611328,456516 & 479 & 11 \\
\hline Slag & 4611328,456520 & 549 & 140 \\
\hline Slag & 4611332,456522 & 513 & 76 \\
\hline \multicolumn{4}{|l|}{ C1 SU3.2 } \\
\hline Slag & 4611324,456523 & 500 & 51 \\
\hline Slag & 4611324,456527 & 588 & 220 \\
\hline Slag & 4611324,456531 & 570 & 180 \\
\hline Slag & 4611327,456525 & 610 & 260 \\
\hline Slag & 4611327,456529 & 504 & 59 \\
\hline Slag & 4611327,456533 & 512 & 74 \\
\hline Slag & No Measurement & & \\
\hline Slag & 4611327,456541 & 490 & 32 \\
\hline Slag & 4611331,456523 & 518 & 85 \\
\hline Slag & 4611331,456527 & 491 & 34 \\
\hline Slag & 4611331,456531 & 540 & 130 \\
\hline Slag & 4611331,456535 & 469 & -8 \\
\hline Slag & 4611331,456539 & 432 & -77 \\
\hline Slag & No Measurement & & \\
\hline \multicolumn{4}{|l|}{ C1 SU3.3 } \\
\hline Slag & 4611310,456502 & 585 & 210 \\
\hline Slag & 4611310,456507 & 481 & 15 \\
\hline Slag & 4611310,456512 & 489 & 30 \\
\hline Slag & 4611310,456516 & 385 & -170 \\
\hline Slag & 4611310,456521 & 498 & 47 \\
\hline Slag & 4611314,456504 & 563 & 170 \\
\hline Slag & 4611314,456509 & 393 & -150 \\
\hline Slag & 4611314,456514 & 476 & 6 \\
\hline Slag & 4611314,456519 & 368 & -200 \\
\hline Slag & 4611318,456502 & 633 & 300 \\
\hline Slag & 4611318,456507 & 547 & 140 \\
\hline Slag & 4611318,456512 & 466 & -13 \\
\hline Slag & 4611318,456516 & 447 & -49 \\
\hline Slag & 4611318,456521 & 542 & 130 \\
\hline \multicolumn{4}{|l|}{ C1 SU3.4 } \\
\hline Slag & 4611309,456523 & 440 & -62 \\
\hline Slag & 4611309,456527 & 368 & -200 \\
\hline Slag & 4611313,456532 & 519 & 87 \\
\hline Slag & No Measurement & & \\
\hline Slag & 4611313,456527 & 497 & 45 \\
\hline Slag & 4611313,456530 & 480 & 13 \\
\hline
\end{tabular}




\begin{tabular}{|c|c|c|c|}
\hline \multicolumn{4}{|c|}{$\begin{array}{c}\text { Table B-7 (cont.): FSS Exterior Slag Survey Units and Surface Activity Levels } \\
\text { Hammond Depot } \\
\text { Hammond, Indiana }\end{array}$} \\
\hline $\begin{array}{c}\text { Survey Unit ID/ } \\
\text { Surface }\end{array}$ & Location $(\mathrm{N}, \mathrm{E})^{\mathrm{a}}$ & Counts per Minute & $\begin{array}{l}\text { Total Beta Activity } \\
\mathrm{dpm} / 100 \mathrm{~cm}^{2}\end{array}$ \\
\hline \multicolumn{4}{|l|}{ C1 SU3.4 (cont.) } \\
\hline Slag & 4611313,456534 & 514 & 77 \\
\hline Slag & 4611316,456525 & 390 & -160 \\
\hline Slag & 4611316,456527 & 489 & 30 \\
\hline Slag & 4611316,456532 & 513 & 76 \\
\hline Slag & No Measurement & & \\
\hline Slag & 4611320,456525 & 541 & 130 \\
\hline Slag & 4611320,456530 & 568 & 180 \\
\hline Slag & No Measurement & & \\
\hline \multicolumn{4}{|l|}{ C1 SU3.5 } \\
\hline Slag & 4611301,456506 & 479 & 11 \\
\hline Slag & 4611301,456510 & 517 & 83 \\
\hline Slag & 4611301,456514 & 424 & -93 \\
\hline Slag & 4611301,456518 & 409 & -120 \\
\hline Slag & 4611304,456504 & 501 & 53 \\
\hline Slag & 4611304,456508 & 441 & -60 \\
\hline Slag & 4611304,456512 & 458 & -28 \\
\hline Slag & 4611304,456516 & 466 & -13 \\
\hline Slag & 4611304,456520 & 423 & -94 \\
\hline Slag & 4611307,456502 & 523 & 94 \\
\hline Slag & 4611307,456506 & 506 & 62 \\
\hline Slag & 4611307,456510 & 480 & 13 \\
\hline Slag & 4611307,456514 & 464 & -17 \\
\hline Slag & 4611307,456518 & 502 & 55 \\
\hline \multicolumn{4}{|l|}{ C1 SU3.6 } \\
\hline Slag & 4611298,456522 & 375 & -190 \\
\hline Slag & 4611298,456526 & 391 & -150 \\
\hline Slag & 4611298,456531 & 392 & -150 \\
\hline Slag & 4611298,456535 & 495 & 42 \\
\hline Slag & 4611298,456539 & 654 & 340 \\
\hline Slag & 4611301,456524 & 375 & -190 \\
\hline Slag & 4611301,456529 & 398 & -140 \\
\hline Slag & 4611301,456533 & 618 & 270 \\
\hline Slag & 4611301,456537 & 457 & -30 \\
\hline Slag & 4611305,456522 & 373 & -190 \\
\hline Slag & 4611305,456526 & 368 & -200 \\
\hline Slag & 4611305,456531 & 376 & -180 \\
\hline Slag & 4611305,456535 & 421 & -98 \\
\hline Slag & 4611305,456539 & 411 & -120 \\
\hline \multicolumn{4}{|l|}{ C1 SU3.7 } \\
\hline Slag & 4611290,456505 & 376 & -180 \\
\hline
\end{tabular}




\begin{tabular}{|c|c|c|c|}
\hline \multicolumn{4}{|c|}{$\begin{array}{c}\text { Table B-7 (cont.): FSS Exterior Slag Survey Units and Surface Activity Levels } \\
\text { Hammond Depot } \\
\text { Hammond, Indiana }\end{array}$} \\
\hline $\begin{array}{c}\text { Survey Unit ID/ } \\
\text { Surface }\end{array}$ & Location $(\mathrm{N}, \mathrm{E})^{\mathrm{a}}$ & Counts per Minute & $\begin{array}{l}\text { Total Beta Activity } \\
\text { dpm } / 100 \mathrm{~cm}^{2}\end{array}$ \\
\hline \multicolumn{4}{|l|}{ C1 SU3.7 (cont.) } \\
\hline Slag & 4611290,456510 & 367 & -200 \\
\hline Slag & 4611290,456514 & 361 & -210 \\
\hline Slag & 4611290,456518 & 417 & -110 \\
\hline Slag & 4611290,456522 & 376 & -180 \\
\hline Slag & 4611294,456507 & 432 & -77 \\
\hline Slag & 4611294,456512 & 385 & -170 \\
\hline Slag & 4611294,456516 & 474 & 2 \\
\hline Slag & 4611294,456520 & 454 & -36 \\
\hline Slag & 4611297,456505 & 420 & -100 \\
\hline Slag & 4611297,456510 & 399 & -140 \\
\hline Slag & 4611297,456514 & 374 & -190 \\
\hline Slag & 4611297,456518 & 359 & -220 \\
\hline Slag & 4611297,456522 & 409 & -120 \\
\hline \multicolumn{4}{|l|}{ C1 SU5.1 } \\
\hline Slag & 4611277,456511 & 386 & -160 \\
\hline Slag & 4611277,456515 & 465 & -15 \\
\hline Slag & 4611277,456519 & 387 & -160 \\
\hline Slag & 4611280,456509 & 414 & -110 \\
\hline Slag & 4611280,456513 & 392 & -150 \\
\hline Slag & 4611280,456517 & 487 & 26 \\
\hline Slag & 4611280,456521 & 428 & -85 \\
\hline Slag & 4611284,456507 & 383 & -170 \\
\hline Slag & 4611284,456511 & 515 & 79 \\
\hline Slag & 4611284,456515 & 450 & -43 \\
\hline Slag & 4611284,456519 & 453 & -38 \\
\hline Slag & 4611288,456509 & 346 & -240 \\
\hline Slag & 4611288,456513 & 366 & -200 \\
\hline Slag & 4611288,456517 & 425 & -91 \\
\hline \multicolumn{4}{|c|}{ C1 SU5 } \\
\hline C1 SU5.2 & & & \\
\hline Slag & 4611287,456524 & 373 & -190 \\
\hline Slag & 4611287,456528 & 350 & -230 \\
\hline Slag & 4611287,456532 & 366 & -200 \\
\hline Slag & 4611287,456536 & 503 & 57 \\
\hline Slag & 4611287,456540 & 464 & -17 \\
\hline Slag & 4611290,456526 & 436 & -70 \\
\hline Slag & 4611290,456530 & 357 & -220 \\
\hline Slag & 4611290,456534 & 402 & -130 \\
\hline Slag & 4611290,456538 & 551 & 150 \\
\hline Slag & 4611294,456524 & 344 & -240 \\
\hline
\end{tabular}




\begin{tabular}{|c|c|c|c|}
\hline \multicolumn{4}{|c|}{$\begin{array}{c}\text { Table B-7 (cont.): FSS Exterior Slag Survey Units and Surface Activity Levels } \\
\text { Hammond Depot } \\
\text { Hammond, Indiana }\end{array}$} \\
\hline $\begin{array}{c}\text { Survey Unit ID/ } \\
\text { Surface }\end{array}$ & Location $(\mathrm{N}, \mathrm{E})^{\mathrm{a}}$ & Counts per Minute & $\begin{array}{l}\text { Total Beta Activity } \\
\text { dpm } / 100 \mathrm{~cm}^{2}\end{array}$ \\
\hline \multicolumn{4}{|l|}{ C1 SU5.2 (cont.) } \\
\hline Slag & 4611294,456528 & 348 & -240 \\
\hline Slag & 4611294,456532 & 380 & -180 \\
\hline Slag & 4611294,456536 & 437 & -68 \\
\hline Slag & 4611294,456540 & 524 & 96 \\
\hline \multicolumn{4}{|l|}{ C1 SU5.3 } \\
\hline Slag & 4611277,456525 & 344 & -240 \\
\hline Slag & 4611277,456530 & 523 & 94 \\
\hline Slag & 4611277,456535 & 489 & 30 \\
\hline Slag & 4611277,456540 & 386 & -160 \\
\hline Slag & 4611277,456545 & 455 & -34 \\
\hline Slag & 4611281,456523 & 391 & -150 \\
\hline Slag & 4611281,456528 & 408 & -120 \\
\hline Slag & 4611281,456533 & 502 & 55 \\
\hline Slag & 4611281,456538 & 439 & -64 \\
\hline Slag & 4611281,456543 & 511 & 72 \\
\hline Slag & 4611286,456525 & 362 & -210 \\
\hline Slag & 4611286,456530 & 399 & -140 \\
\hline Slag & 4611286,456535 & 449 & -45 \\
\hline Slag & 4611286,456540 & 443 & -57 \\
\hline \multicolumn{4}{|l|}{ C1 SU5.4 } \\
\hline Slag & 4611250,456521 & 359 & -220 \\
\hline Slag & 4611253,456519 & 592 & 220 \\
\hline Slag & 4611257,456521 & 520 & 89 \\
\hline Slag & 4611260,456519 & 415 & -110 \\
\hline Slag & 4611264,456517 & 393 & -150 \\
\hline Slag & 4611264,456521 & 418 & -100 \\
\hline Slag & 4611267,456515 & 402 & -130 \\
\hline Slag & 4611267,456519 & 444 & -55 \\
\hline Slag & 4611271,456513 & 447 & -49 \\
\hline Slag & 4611271,456517 & 370 & -190 \\
\hline Slag & 4611271,456521 & 330 & -270 \\
\hline Slag & 4611274,456511 & 406 & -130 \\
\hline Slag & 4611274,456515 & 391 & -150 \\
\hline Slag & 4611274,456519 & 463 & -19 \\
\hline \multicolumn{4}{|l|}{ C1 SU5.5 } \\
\hline Slag & 4611268,456523 & 388 & -160 \\
\hline Slag & 4611268,456527 & 434 & -74 \\
\hline Slag & 4611268,456532 & 458 & -28 \\
\hline Slag & 4611268,456536 & 563 & 170 \\
\hline Slag & 4611268,456540 & 447 & -49 \\
\hline
\end{tabular}




\begin{tabular}{|c|c|c|c|}
\hline \multicolumn{4}{|c|}{$\begin{array}{c}\text { Table B-7 (cont.): FSS Exterior Slag Survey Units and Surface Activity Levels } \\
\text { Hammond Depot } \\
\text { Hammond, Indiana }\end{array}$} \\
\hline $\begin{array}{c}\text { Survey Unit ID/ } \\
\text { Surface }\end{array}$ & Location $(\mathrm{N}, \mathrm{E})^{\mathrm{a}}$ & Counts per Minute & $\begin{array}{l}\text { Total Beta Activity } \\
\mathrm{dpm} / 100 \mathrm{~cm}^{2}\end{array}$ \\
\hline \multicolumn{4}{|l|}{ C1 SU5.5 (cont.) } \\
\hline Slag & 4611268,456545 & 396 & -150 \\
\hline Slag & 4611268,456549 & 380 & -180 \\
\hline Slag & 4611272,456525 & 358 & -220 \\
\hline Slag & 4611272,456529 & 440 & -62 \\
\hline Slag & 4611272,456534 & 524 & 96 \\
\hline Slag & 4611272,456538 & 427 & -87 \\
\hline Slag & 4611272,456542 & 477 & 8 \\
\hline Slag & 4611272,456547 & 462 & -21 \\
\hline Slag & 4611276,456523 & 412 & -120 \\
\hline Slag & 4611276,456527 & 387 & -160 \\
\hline Slag & 4611276,456532 & 509 & 68 \\
\hline Slag & 4611276,456536 & 558 & 160 \\
\hline Slag & 4611276,456540 & 440 & -62 \\
\hline Slag & 4611276,456545 & 471 & -4 \\
\hline \multicolumn{4}{|l|}{ C1 SU5.6 } \\
\hline Slag & 4611251,456525 & 448 & -47 \\
\hline Slag & 4611251,456530 & 352 & -230 \\
\hline Slag & 4611255,456523 & 449 & -45 \\
\hline Slag & 4611255,456528 & 458 & -28 \\
\hline Slag & 4611255,456532 & 471 & -4 \\
\hline Slag & 4611259,456525 & 476 & 6 \\
\hline Slag & 4611259,456530 & 461 & -23 \\
\hline Slag & 4611259,456534 & 463 & -19 \\
\hline Slag & 4611263,456523 & 517 & 83 \\
\hline Slag & 4611263,456528 & 389 & -160 \\
\hline Slag & 4611263,456531 & 399 & -140 \\
\hline Slag & 4611267,456525 & 479 & 11 \\
\hline Slag & 4611267,456530 & 447 & -49 \\
\hline Slag & 4611267,456534 & 505 & 60 \\
\hline \multicolumn{4}{|l|}{ C1 SU5.7 } \\
\hline Slag & 4611259,456537 & 481 & 15 \\
\hline Slag & 4611259,456541 & 480 & 13 \\
\hline Slag & 4611259,456544 & 455 & -34 \\
\hline Slag & 4611259,456548 & 438 & -66 \\
\hline Slag & 4611259,456552 & 471 & -4 \\
\hline Slag & 4611262,456535 & 461 & -23 \\
\hline Slag & 4611262,456539 & 377 & -180 \\
\hline Slag & 4611262,456543 & 376 & -180 \\
\hline Slag & 4611262,456546 & 411 & -120 \\
\hline Slag & 4611262,456550 & 460 & -25 \\
\hline
\end{tabular}




\begin{tabular}{|c|c|c|c|}
\hline \multicolumn{4}{|c|}{$\begin{array}{c}\text { Table B-7 (cont.): FSS Exterior Slag Survey Units and Surface Activity Levels } \\
\text { Hammond Depot } \\
\text { Hammond, Indiana }\end{array}$} \\
\hline $\begin{array}{c}\text { Survey Unit ID/ } \\
\text { Surface }\end{array}$ & 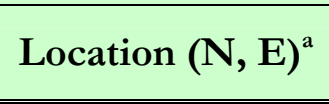 & Counts per Minute & $\begin{array}{l}\text { Total Beta Activity } \\
\mathrm{dpm} / 100 \mathrm{~cm}^{2}\end{array}$ \\
\hline \multicolumn{4}{|l|}{ C1 SU5.7 (cont.) } \\
\hline Slag & 4611266,456537 & 538 & 120 \\
\hline Slag & 4611266,456541 & 381 & -170 \\
\hline Slag & 4611266,456544 & 368 & -200 \\
\hline Slag & 4611266,456548 & 464 & -17 \\
\hline \multicolumn{4}{|l|}{ C1 SU5.8 } \\
\hline Slag & 4611251,456544 & 446 & -51 \\
\hline Slag & 4611251,456547 & 472 & -2 \\
\hline Slag & 4611251,456551 & 436 & -70 \\
\hline Slag & 4611251,456553 & 513 & 76 \\
\hline Slag & 4611254,456538 & 517 & 83 \\
\hline Slag & 4611254,456542 & 438 & -66 \\
\hline Slag & 4611254,456545 & 459 & -26 \\
\hline Slag & 4611254,456549 & 452 & -40 \\
\hline Slag & 4611254,456552 & 448 & -47 \\
\hline Slag & 4611257,456537 & 472 & -2 \\
\hline Slag & 4611257,456540 & 482 & 17 \\
\hline Slag & 4611257,456544 & 470 & -6 \\
\hline Slag & 4611257,456547 & 399 & -140 \\
\hline Slag & 4611257,456551 & 517 & 83 \\
\hline \multicolumn{4}{|l|}{ C1 SU5 } \\
\hline $\operatorname{Slag}(J)^{b}$ & 4611268,456505 & 534 & 120 \\
\hline Slag (J) & 4611265,456501 & 585 & 210 \\
\hline Slag (J) & 4611283,456501 & 660 & 350 \\
\hline Slag $(J)$ & 4611290,456499 & 461 & -23 \\
\hline Slag $(J)$ & 4611317,456492 & 559 & 160 \\
\hline Slag (J) & 4611323,456493 & 512 & 74 \\
\hline Slag $(J)$ & 4611329,456492 & 534 & 120 \\
\hline
\end{tabular}

aGlobal positioning coordinates are NAD 83 UTM Zone 16.

$\mathrm{b}=$ judgmental measurement. 
APPENDIX C: REFERENCES 


\section{REFERENCES}

Oak Ridge Associated Universities (ORAU). Quality Program for the Independent

Environmental Assessment and Verification Program. Oak Ridge, TN; November 1, 2007.

Oak Ridge Institute for Science and Education (ORISE) . Final Report-Historical Site

Assessment of the Hammond Depot, Hammond, Indiana. Oak Ridge, TN; August 2005a.

Oak Ridge Institute for Science and Education. Radiological Scoping Survey of the Hammond Depot, Hammond, Indiana. Oak Ridge, TN; December 2005b.

Oak Ridge Institute for Science and Education. Phase 2 Radiological Scoping Survey Plan for the Hammond Depot, Hammond, Indiana. Oak Ridge, TN; August 25, 2005c.

Oak Ridge Institute for Science and Education. Radiation Protection Plan. Oak Ridge, TN; September 10, 2005d.

Oak Ridge Institute for Science and Education. Preliminary Site-Specific Derived Concentration Guideline Levels for the Hammond Depot, Hammond, Indiana. Oak Ridge, TN;

January, 2006a.

Oak Ridge Institute for Science and Education. Radiological Characterization Survey of the Hammond Depot, Hammond, Indiana. Oak Ridge, TN; August 2006b.

Oak Ridge Institute for Science and Education. Final Radiological Characterization Survey Plan for the Hammond Depot, Hammond, Indiana. Oak Ridge, TN; March 15, 2006c.

Oak Ridge Institute for Science and Education. Final Report-Decommissioning/Remediation Plan for Hammond Depot, Hammond, Indiana and Curtis Bay Depot, Curtis Bay, Maryland. Oak Ridge, TN; September 25, 2006 d.

Oak Ridge Institute for Science and Education. Radiological Final Status Survey Plan for the Hammond Depot, Hammond, Indiana. Oak Ridge, TN; July 12, 2007a.

Oak Ridge Institute for Science and Education. Survey Procedures Manual for the Independent Environmental Assessment and Verification Program. Oak Ridge, TN; August 3, 2007b.

Oak Ridge Institute for Science and Education. Laboratory Procedures Manual for the Independent Environmental Assessment and Verification Program. Oak Ridge, TN; June 15, 2007c. 


\section{REFERENCES (continued)}

Oak Ridge National Laboratory (ORNL). Thorium Nitrate Project Phase 4-Decontamination and Decommissioning Statement of Work. Decontamination and Packaging for Solid Low-Level Waste Disposal at the Defense National Stockpile Center's Curtis Bay and Hammond Depots. Oak Ridge, TN; August 18, 2006.

U.S. Nuclear Regulatory Commission (NRC). Multi-Agency Radiation Survey and Site Investigation Manual (MARSSIM), NUREG-1575; Revision 1. Washington, DC; August 2000.

U.S. Nuclear Regulatory Commission. Defense Logistics Agency, License Amendment, Control No. 138087. King of Prussia, Pennsylvania; December 5, 2007. 


\section{APPENDIX D: LAND SURVEY UNIT DQOs}




\section{Surface Soil Survey Plan}

\section{Survey Plan Summary}

Site:

Hammond Depot FSS Planner

Planner(s):

Vitkus

Survey Unit Name:

C1 SU1

Comments:

Area $\left(m^{2}\right)$ :

1,949

Classification:

1

Selected Test:

WRS

Estimated Sigma (SOR):

0.39

DCGL (SOR):

1

Sample Size (N/2):

LBGR (SOR):

0.39

Estimated Conc. (SOR):

0.39

Alpha:

0.050

Estimated Power:

1

Beta:

0.050

EMC Sample Size (N):

Scanning Instrumentation:

Nal

\section{Prospective Power Curve}

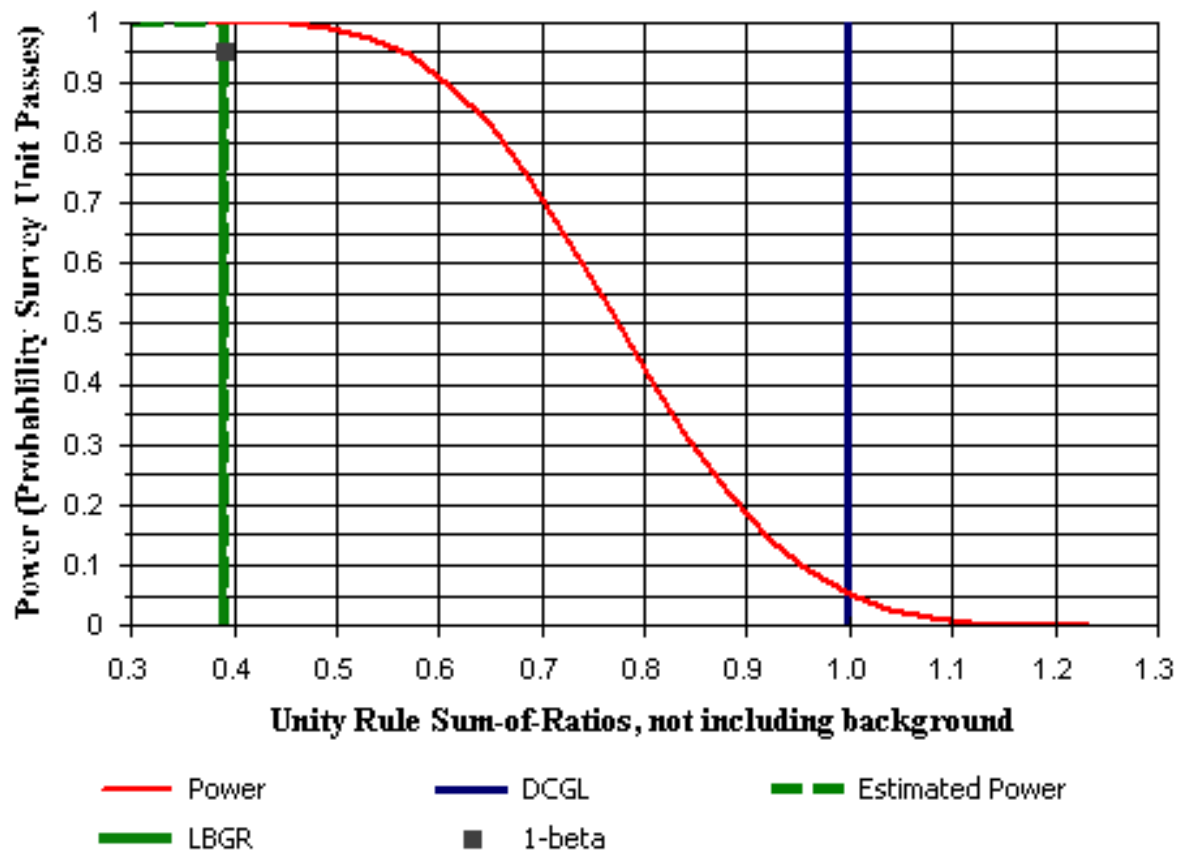




\section{Surface Soil Survey Plan}

\section{Contaminant Summary}

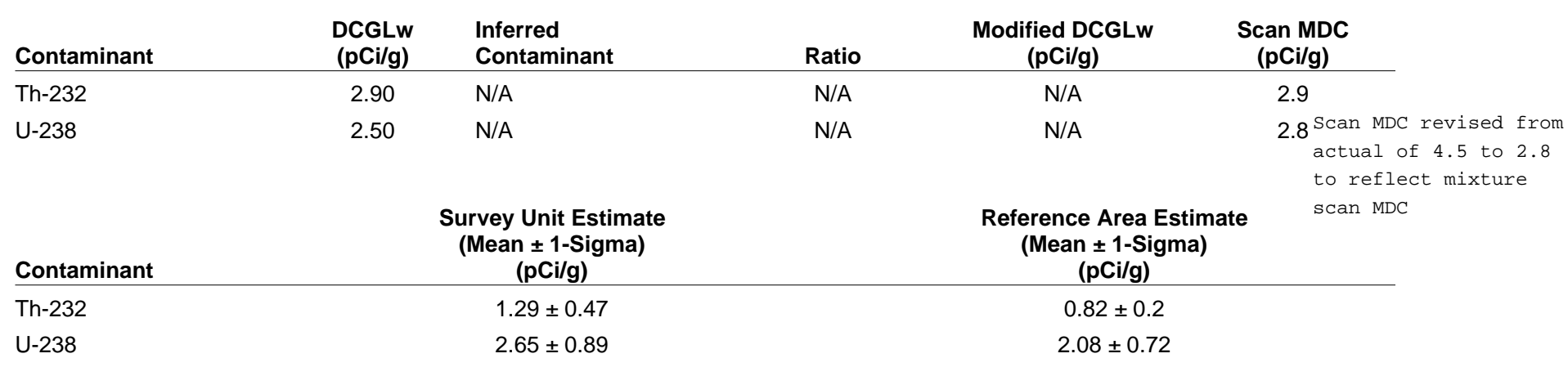




\section{Surface Soil Survey Plan}

\section{Survey Plan Summary}

Site:

Planner(s):

Survey Unit Name:

Comments:

Area $\left(m^{2}\right)$ :

Selected Test:

DCGL (SOR):

LBGR (SOR):

Alpha:

Beta:

Scanning Instrumentation:
Hammond Depot FSS Planner

Vitkus

C1 SU2, 3, 4, and 5 (AOC 1 and 2)

Survey unit area largest of the group

1,218

Classification:

1

WRS

Estimated Sigma (SOR):

0.39

Sample Size (N/2):

18

Estimated Conc. (SOR):

0.39

Estimated Power:

1

EMC Sample Size (N):

\section{Prospective Power Curve}

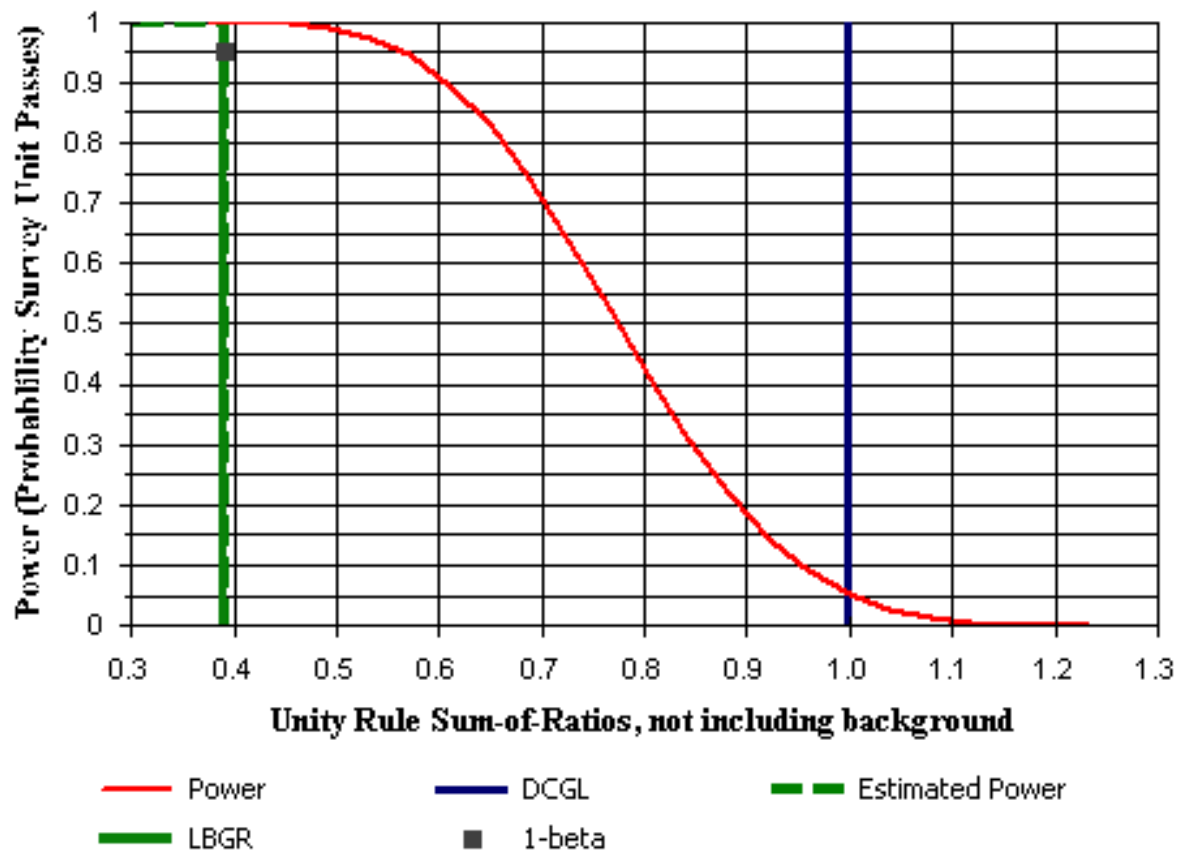




\section{Surface Soil Survey Plan}

\section{Contaminant Summary}

\begin{tabular}{|c|c|c|c|c|c|}
\hline Contaminant & $\begin{array}{l}\text { DCGLw } \\
\text { (pCi/g) }\end{array}$ & $\begin{array}{l}\text { Inferred } \\
\text { Contaminant }\end{array}$ & Ratio & $\begin{array}{c}\text { Modified DCGLw } \\
(\mathrm{pCi} / \mathrm{g})\end{array}$ & $\begin{array}{l}\text { Scan MDC } \\
(p C i / g)\end{array}$ \\
\hline Th-232 & 2.90 & $\mathrm{~N} / \mathrm{A}$ & N/A & $\mathrm{N} / \mathrm{A}$ & 2.8 \\
\hline \multirow[t]{2}{*}{$\mathrm{U}-238$} & 2.50 & $N / A$ & $\mathrm{~N} / \mathrm{A}$ & $\mathrm{N} / \mathrm{A}$ & $\begin{array}{l}2.9 \text { Scan } \\
\text { gross } \\
\text { vers? }\end{array}$ \\
\hline & & $\begin{array}{c}\text { Survey Unit Estimate } \\
\text { (Mean } \pm \text { 1-Sigma) } \\
\text { (pCi/g) }\end{array}$ & & $\begin{array}{r}\text { Reference Area } \\
\text { (Mean } \pm 1-S i \\
(\mathrm{pCi} / \mathrm{g})\end{array}$ & \\
\hline Th-232 & & $1.29 \pm 0.47$ & & $0.82 \pm 0.2$ & \\
\hline U-238 & & $2.65 \pm 0.89$ & & $2.08 \pm 0.7$ & \\
\hline
\end{tabular}




\section{Surface Soil Survey Plan}

\section{Survey Plan Summary}

Site:

Planner(s):

Survey Unit Name:

Comments:

Area $\left(m^{2}\right)$ :

Selected Test:

DCGL (SOR):

LBGR (SOR):

Alpha:

0.050

Beta:

0.050

2,513
Hammond Depot FSS Planner

Vitkus

C1 SU6 and 7

Survey unit area is the largest

Classification:

1

Estimated Sigma (SOR): $\quad 0.39$

Sample Size (N/2): $\quad 18$

Estimated Conc. (SOR): $\quad 0.39$

Estimated Power: $\quad 1$

EMC Sample Size (N): $\quad 18$

Scanning Instrumentation:

Nal

\section{Prospective Power Curve}

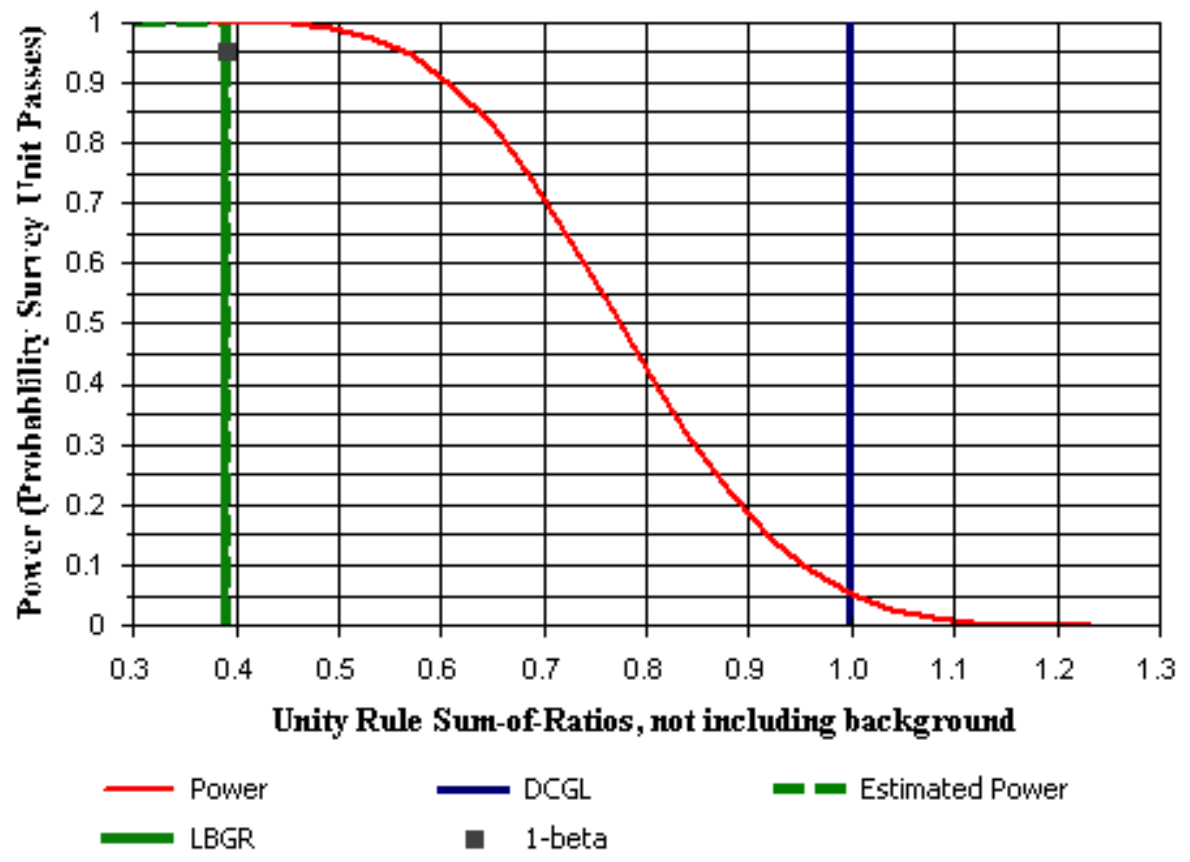




\section{Surface Soil Survey Plan}

\section{Contaminant Summary}

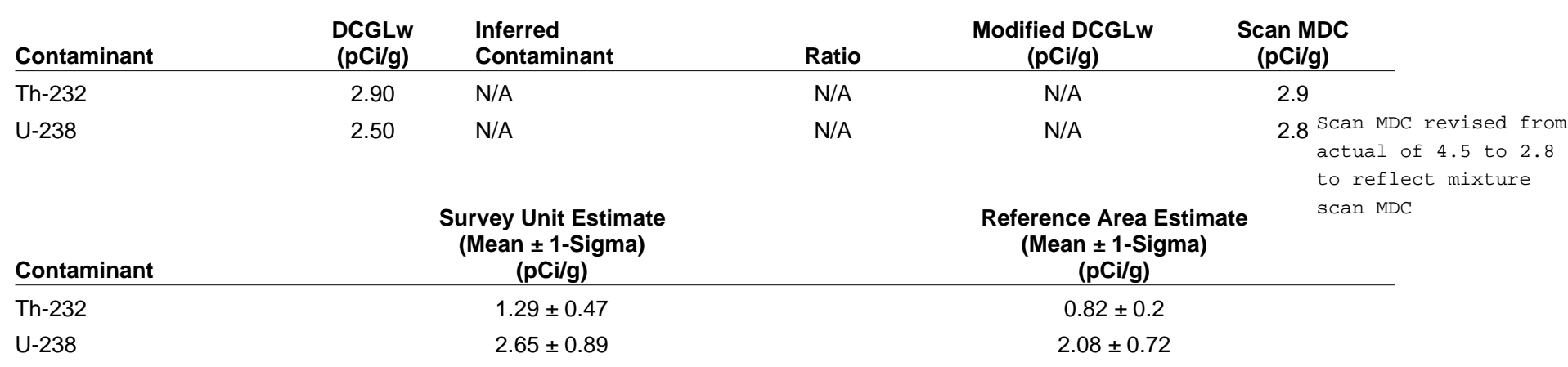




\section{Building Surface Survey Plan}

\section{Survey Plan Summary}

Site:

Planner(s):

Survey Unit Name:

Comments:

Area $\left(m^{2}\right)$ :

Selected Test:

DCGL (cpm):

LBGR (cpm):

Alpha:

0.050

Beta:

210

Sign

212
Hammond Depot FSS Planner

Vitkus

C1 SU2.1 through 5.8 AOC 2 and 3 slag

Survey unit is area is the largest of the units

Classification:

1

Estimated Sigma (cpm):

48.3

Sample Size (N):

Estimated Conc. (cpm):

120

Estimated Power:

1.00

EMC Sample Size (N):

\section{Prospective Power Curve}

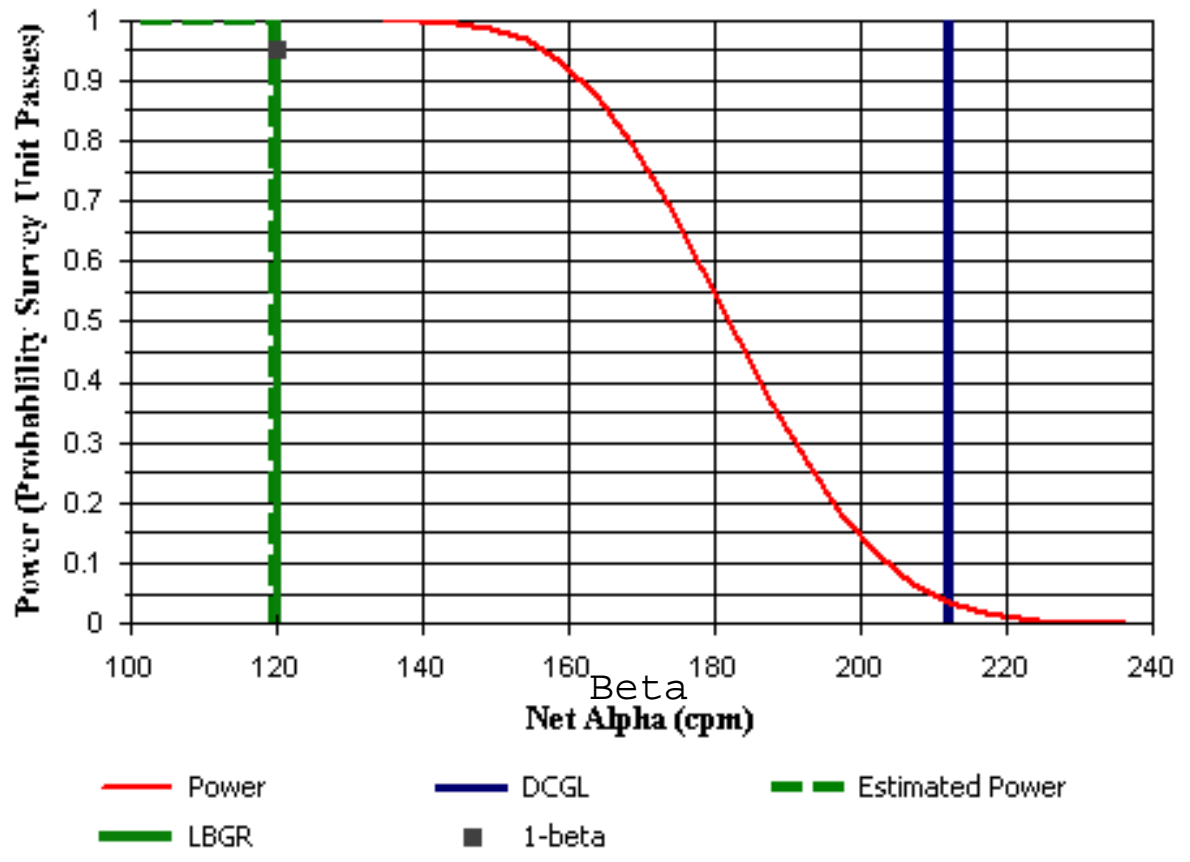




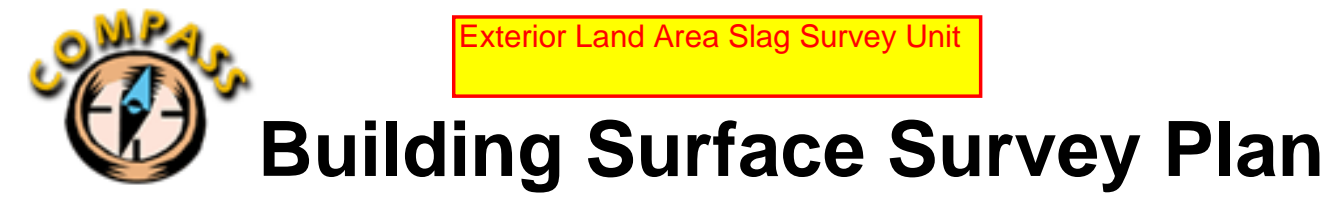

\section{Contaminant Summary}

\begin{tabular}{lc} 
Contaminant & $\begin{array}{c}\text { DCGLw } \\
\text { (dpm/100 cm } \mathbf{c m}^{2}\end{array}$ \\
\hline Th-232 & 400
\end{tabular}

Beta measurements performed see

calibration worksheets

\section{Alpha Instrumentation Summary}

Gross Alpha DCGLw (dpm/100 cm²):

Total Efficiency:

Gross Alpha DCGLw (cpm):

ID Type

$14 \quad 43-68$ \#30

Contaminant

Th-232
400

0.42

212

${ }^{1}$ Average beta energy (keV) [N/A indicates alpha emission]

2 Activity fraction

Gross Survey Unit Mean (cpm): $559 \pm 27$ (1-sigma)

Count Time (min): 1

\begin{tabular}{lcccc} 
Material & $\begin{array}{c}\text { Number of } \\
\text { BKG Counts }\end{array}$ & $\begin{array}{c}\text { Average } \\
\text { (cpm) }\end{array}$ & $\begin{array}{c}\text { Standard } \\
\text { Deviation (cpm) }\end{array}$ & $\begin{array}{c}\text { MDC } \\
\left.\text { (dpm/100 } \mathbf{~ c m}^{2}\right)\end{array}$ \\
\hline slag & 20 & 473.4 & 40.1 & 197 \\
Slag 1 & 10 & 507.2 & 22 & 204 \\
Slag 2 & 10 & 439.5 & 19.1 & 190
\end{tabular}




\section{Surface Soil Survey Plan}

\section{Survey Plan Summary}

Site:

Planner(s):

Survey Unit Name:

Comments:

Area $\left(m^{2}\right)$ :

Selected Test:

DCGL (SOR):

LBGR (SOR):

Alpha:

Beta:
Hammond Depot FSS Planner

Vitkus

C2 SU1 through 12

Survey unit sizes range from 3,289 to $11,489 \mathrm{~m} 2$

11,489

Classification:

WRS

Estimated Sigma (SOR):

0.39

Sample Size (N/2):

Estimated Conc. (SOR):

0.39

Estimated Power:

\section{Prospective Power Curve}

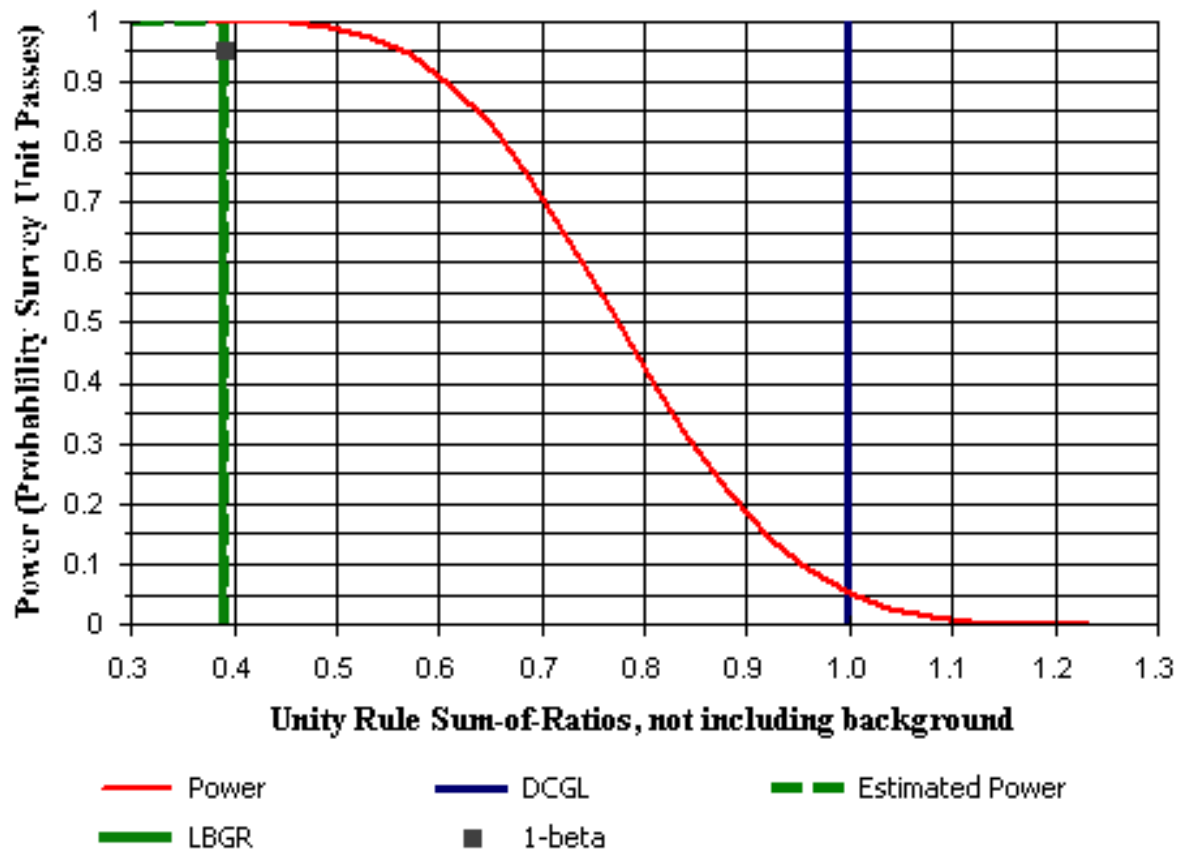




\section{Surface Soil Survey Plan}

\section{Contaminant Summary}

\begin{tabular}{|c|c|c|c|c|c|}
\hline Contaminant & $\begin{array}{l}\text { DCGLw } \\
\text { (pCi/g) }\end{array}$ & $\begin{array}{l}\text { Inferred } \\
\text { Contaminant }\end{array}$ & Ratio & $\begin{array}{l}\text { Modified DCGLw } \\
(\mathrm{pCi} / \mathrm{g})\end{array}$ & $\begin{array}{c}\text { Scan MDC } \\
(p C i / g)\end{array}$ \\
\hline Th-232 & 2.90 & $\mathrm{~N} / \mathrm{A}$ & $\mathrm{N} / \mathrm{A}$ & $\mathrm{N} / \mathrm{A}$ & $\mathrm{N} / \mathrm{A}$ \\
\hline U-238 & 2.50 & $\mathrm{~N} / \mathrm{A}$ & N/A & N/A & $\mathrm{N} / \mathrm{A}$ \\
\hline
\end{tabular}

\begin{tabular}{lcc} 
Contaminant & $\begin{array}{c}\text { Survey Unit Estimate } \\
\text { (Mean } \pm \text { 1-Sigma) } \\
(\mathbf{p C i} / \mathbf{g})\end{array}$ & $\begin{array}{c}\text { Reference Area Estimate } \\
\text { (Mean } \pm \text { 1-Sigma) } \\
(\text { pCi/g) }\end{array}$ \\
\hline Th-232 & $1.29 \pm 0.47$ & $0.82 \pm 0.2$ \\
U-238 & $2.65 \pm 0.89$ & $2.08 \pm 0.72$
\end{tabular}




\section{Survey Plan Summary}

Site:

Planner(s):

Survey Unit Name:

Comments:

Area $\left(m^{2}\right)$ :

Selected Test:

DCGL (cpm):

LBGR (cpm):

Alpha:

Beta:
Hammond Depot FSS Planner

Vitkus

C2 SU12 Debris Pile DM Rev. 1
Classification:

Estimated Sigma (cpm):

Sample Size (N):

Estimated Conc. (cpm):

Estimated Power:
2

67.3

20

120

1.00

\section{Prospective Power Curve}

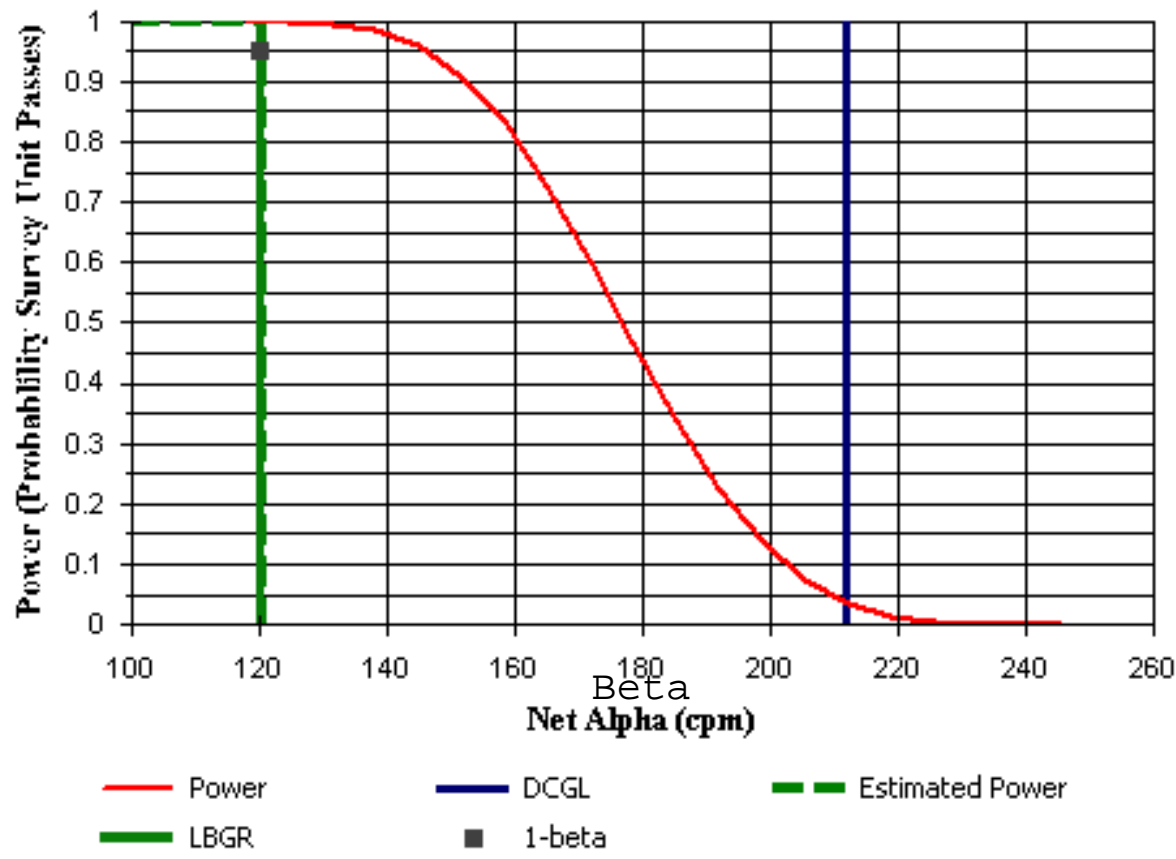




\section{Building Surface Survey Plan}

\section{Contaminant Summary}

\begin{tabular}{lc} 
Contaminant & $\begin{array}{c}\text { DCGLw } \\
\text { (dpm/100 cm²) }\end{array}$ \\
\hline Th-232 & 400
\end{tabular}

Beta measurements performed see

calibration worksheets

\section{Alpha Instrumentation Summary}

Gross Alpha DCGLw (dpm/100 $\left.\mathrm{cm}^{2}\right)$

Total Efficiency:

Gross Alpha DCGLw (cpm):

ID Type

$14 \quad 43-68 \# 30$

Contaminant

Th-232

${ }^{1}$ Average beta energy (keV) [N/A indicates alpha emission]

${ }^{2}$ Activity fraction

Gross Survey Unit Mean (cpm): $457 \pm 54$ (1-sigma)

Count Time (min): 1

\section{Material}

Concrete

slag

Slag 1

Slag 2

Small Brick

Unpainted Cinder Block
400

0.42

212

\begin{tabular}{cc} 
Mode & Area $\left(\mathbf{c m}^{2}\right)$ \\
\hline Alpha & 126
\end{tabular}

\begin{tabular}{cccc} 
Fraction $^{2}$ & Inst. Eff. & Surf. Eff. & Total Eff. \\
\hline 1.0000 & 0.42 & 1.00 & 0.4200
\end{tabular}




\section{Surface Soil Survey Plan}

\section{Survey Plan Summary}

Site:

Planner(s):

Survey Unit Name:

Comments:

Area $\left(m^{2}\right)$ :

Selected Test:

DCGL (SOR):

LBGR (SOR):

Alpha:

Beta:
Hammond Depot FSS Planner

Vitkus

C3 SU1 through 5

Survey unit sizes range from 17,561 to $34,296 \mathrm{~m} 2$

34,296

Classification:

WRS

Estimated Sigma (SOR):

0.39

Sample Size (N/2):

Estimated Conc. (SOR):

0.39

Estimated Power:

\section{Prospective Power Curve}

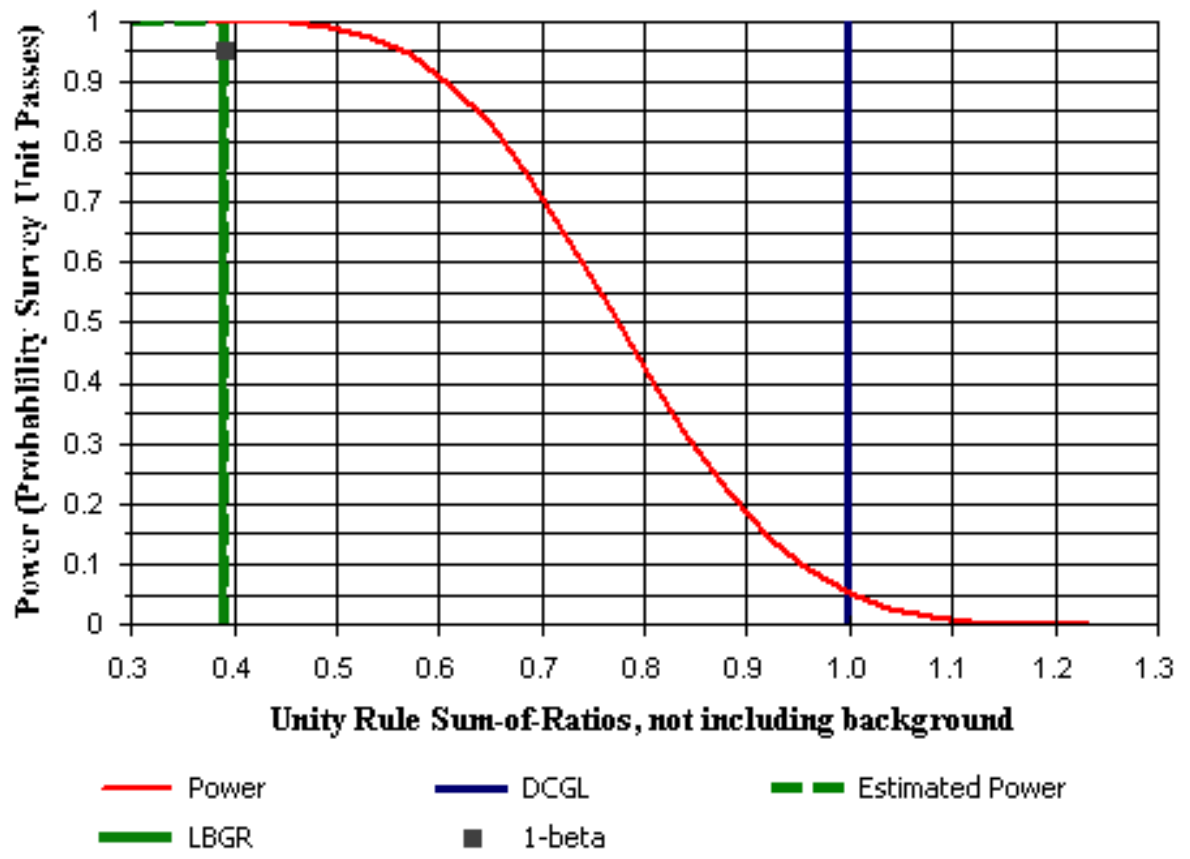




\section{Surface Soil Survey Plan}

\section{Contaminant Summary}

\begin{tabular}{|c|c|c|c|c|c|}
\hline Contaminant & $\begin{array}{l}\text { DCGLw } \\
\text { (pCi/g) }\end{array}$ & $\begin{array}{l}\text { Inferred } \\
\text { Contaminant }\end{array}$ & Ratio & $\begin{array}{l}\text { Modified DCGLw } \\
(\mathrm{pCi} / \mathrm{g})\end{array}$ & $\begin{array}{c}\text { Scan MDC } \\
(p C i / g)\end{array}$ \\
\hline Th-232 & 2.90 & $\mathrm{~N} / \mathrm{A}$ & $\mathrm{N} / \mathrm{A}$ & $\mathrm{N} / \mathrm{A}$ & $\mathrm{N} / \mathrm{A}$ \\
\hline U-238 & 2.50 & $\mathrm{~N} / \mathrm{A}$ & N/A & N/A & $\mathrm{N} / \mathrm{A}$ \\
\hline
\end{tabular}

\begin{tabular}{lcc} 
Contaminant & $\begin{array}{c}\text { Survey Unit Estimate } \\
\text { (Mean } \pm \text { 1-Sigma) } \\
(\mathbf{p C i} / \mathbf{g})\end{array}$ & $\begin{array}{c}\text { Reference Area Estimate } \\
\text { (Mean } \pm \text { 1-Sigma) } \\
(\text { pCi/g) }\end{array}$ \\
\hline Th-232 & $1.29 \pm 0.47$ & $0.82 \pm 0.2$ \\
U-238 & $2.65 \pm 0.89$ & $2.08 \pm 0.72$
\end{tabular}


APPENDIX E: STRUCTURE SURVEY UNIT DQOs 


\section{Building Surface Survey Plan}

\section{Survey Plan Summary}

Site:

Planner(s):

Survey Unit Name:

Comments:

Area $\left(m^{2}\right)$ :

Selected Test:

DCGL (cpm):

LBGR (cpm):

Alpha:

Beta:
Hammond Depot FSS Planner

Vitkus

C1 SU1 through 21 Bldg 200E slag Rev. 1

Survey unit area is largest of the SUs Rev. 1 to enter average slag BKG for DQA reporting 157

Classification:

1

Sign

Estimated Sigma (cpm):

48.3

212

Sample Size $(\mathrm{N})$ :

14

Estimated Conc. (cpm):

27.6

Estimated Power:

1.00

EMC Sample Size (N):

\section{Prospective Power Curve}

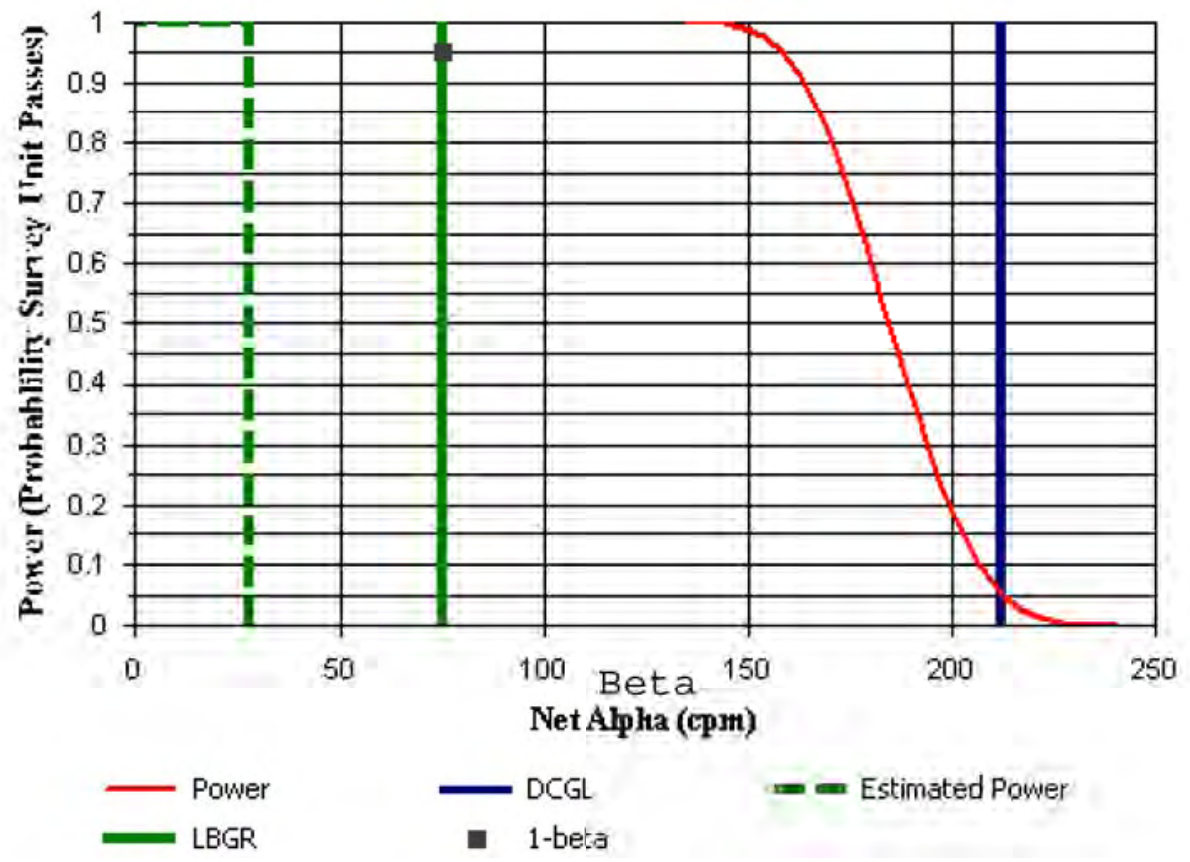




\section{Building Surface Survey Plan}

\section{Contaminant Summary}

\begin{tabular}{lc} 
Contaminant & $\begin{array}{c}\text { DCGLw } \\
\left(\mathrm{dpm} / 100 \mathrm{~cm}^{2}\right)\end{array}$ \\
\hline Th-232 & 400
\end{tabular}

Beta measurements performed see

calibration worksheets

\section{Alpha Instrumentation Summary}

Gross Alpha DCGLw (dpm/100 $\left.\mathrm{cm}^{2}\right)$

Total Efficiency:

Gross Alpha DCGLw (cpm):

ID Type

$14 \quad 43-68$ \#30

Contaminant

Th-232

\section{0}

0.42

212

${ }^{1}$ Average beta energy (keV) [N/A indicates alpha emission]

${ }^{2}$ Activity fraction

Gross Survey Unit Mean (cpm): $501 \pm 27$ (1-sigma)

Count Time (min): 1

\begin{tabular}{lcccc} 
Material & $\begin{array}{c}\text { Number of } \\
\text { BKG Counts }\end{array}$ & $\begin{array}{c}\text { Average } \\
(\mathbf{c p m})\end{array}$ & $\begin{array}{c}\text { Standard } \\
\text { Deviation }(\mathbf{c p m})\end{array}$ & $\begin{array}{c}\text { MDC } \\
\left(\mathbf{d p m} / 100 \mathrm{~cm}^{2}\right)\end{array}$ \\
\hline slag & 20 & 473.4 & 40.1 & 197
\end{tabular}

$\begin{array}{cc}\text { Mode } & \text { Area }\left(\mathbf{c m}^{2}\right) \\ \text { Alpha } & 126\end{array}$

Inst. Eff.

Surf. Eff. 1.00

Total Eff. 1.0000 0.4200
Material (cpm)
197 


\section{Building Surface Survey Plan}

\section{Survey Plan Summary}

Site: $\quad$ Hammond Depot FSS Planner

Planner(s): $\quad$ Vitkus

Survey Unit Name: $\quad$ C1 SU22 and 23 Building 200E west and north wall

Comments:

Area $\left(m^{2}\right):$

Classification: $\quad 1$

Selected Test: $\quad$ Sign

Estimated Sigma (cpm): $\quad 96.4$

DCGL (cpm): $\quad 212$

Sample Size $(\mathrm{N}):$

$\begin{array}{llll}\text { LBGR }(\mathrm{cpm}): & 50 & \text { Estimated Conc. }(\mathrm{cpm}): & 50.1\end{array}$

$\begin{array}{llll}\text { Alpha: } & 0.050 & \text { Estimated Power: } & 1.00\end{array}$

$\begin{array}{llll}\text { Beta: } & 0.050 & \text { EMC Sample Size (N): } & 17\end{array}$

\section{Prospective Power Curve}

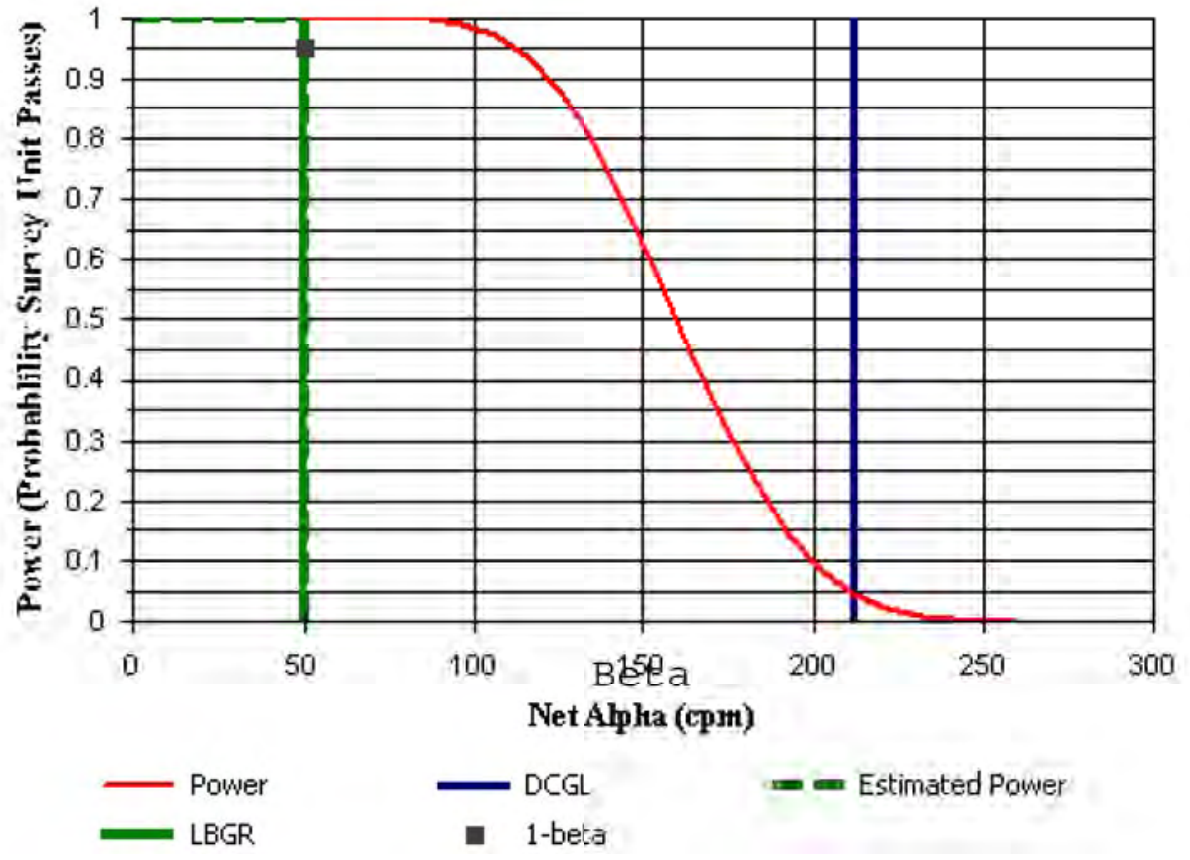




\section{Building Surface Survey Plan}

\section{Contaminant Summary}

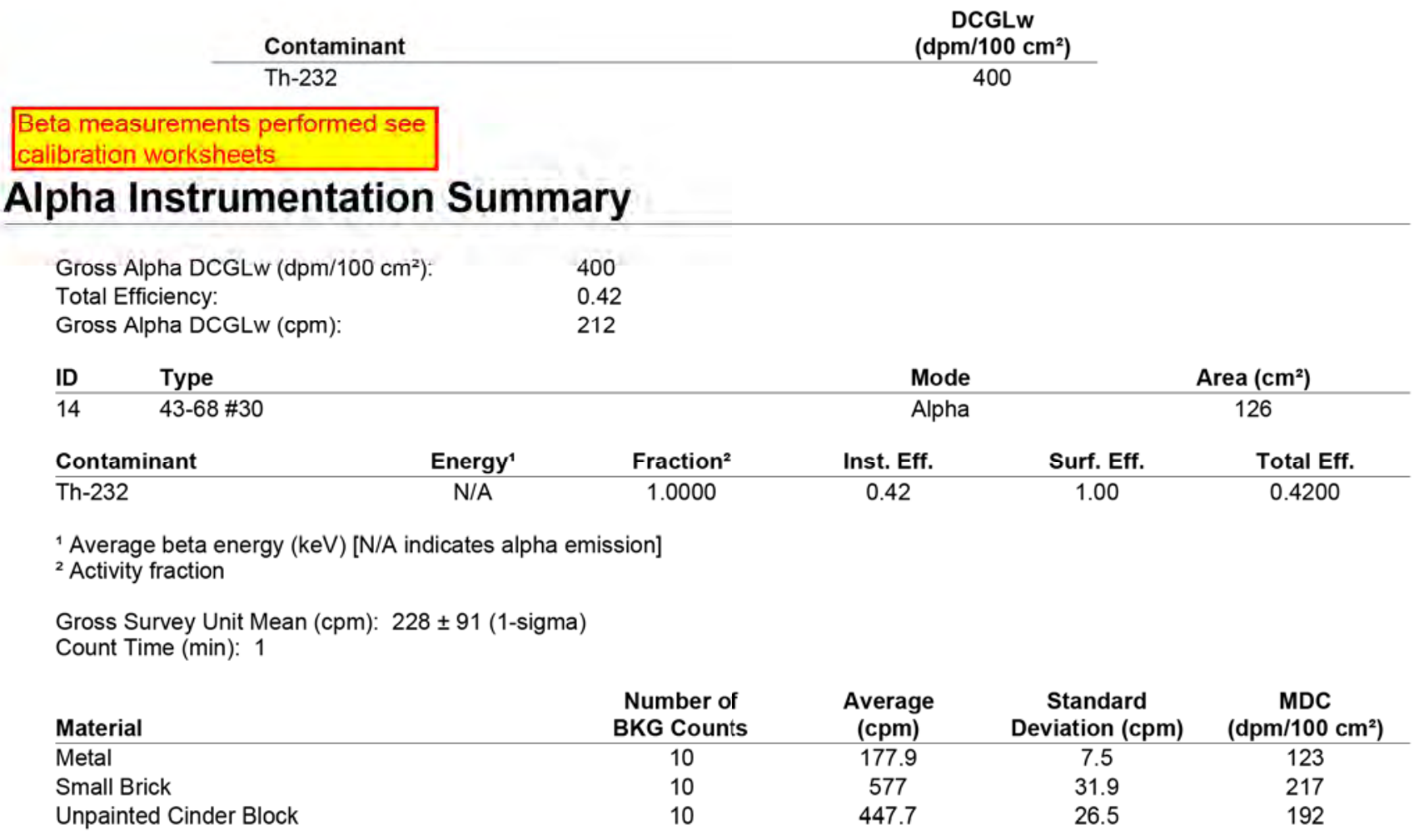




\section{Building Surface Survey Plan}

\section{Survey Plan Summary}

Site:

Planner(s):

Survey Unit Name:

Comments:

Area $\left(m^{2}\right)$ :

Selected Test:

DCGL (cpm):

LBGR (cpm):

Alpha:

Beta:
Hammond Depot FSS Planner

Vitkus

C1 SU24 through 32 Bldg 200E Ceiling

125

Classification:

1

Sign

Estimated Sigma (cpm):

61.9

Sample Size (N):

18

Estimated Conc. (cpm):

134

Estimated Power:

1.00

EMC Sample Size (N):

\section{Prospective Power Curve}

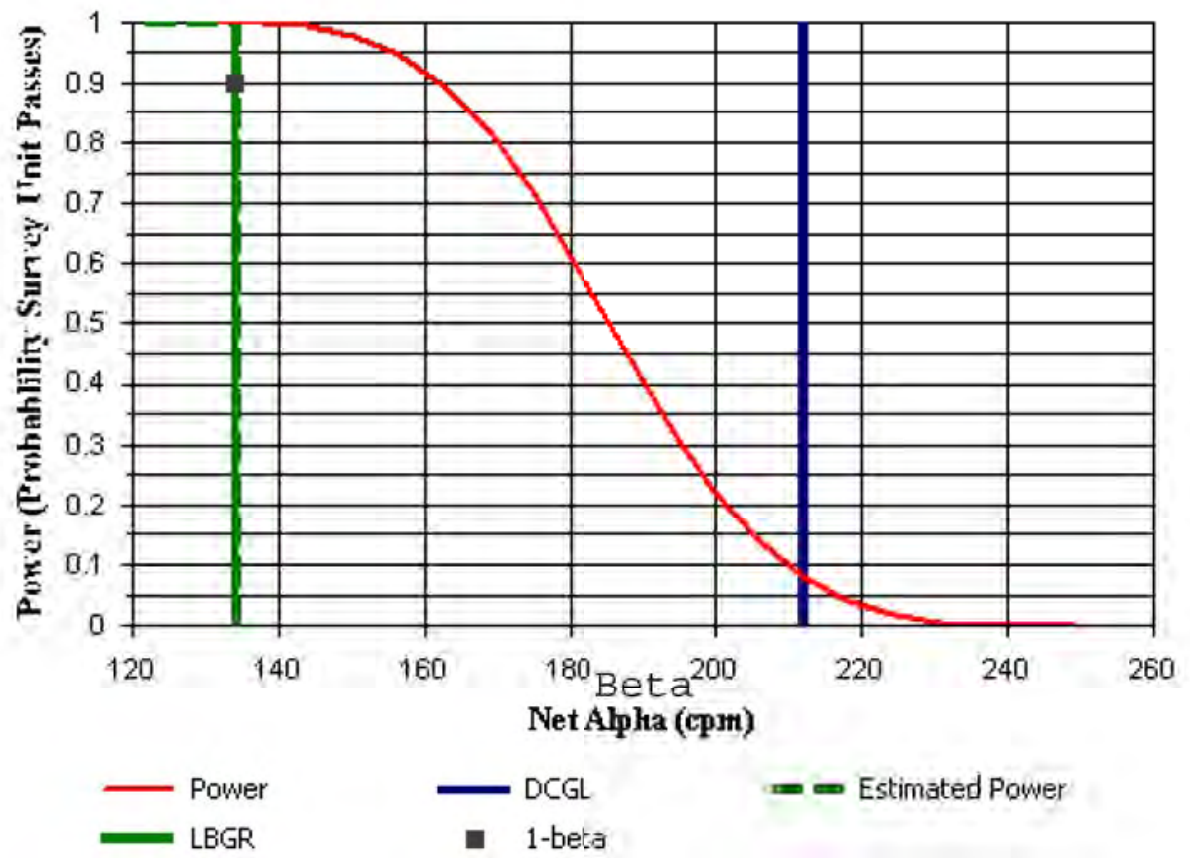




\section{Building Surface Survey Plan}

\section{Contaminant Summary}

\begin{tabular}{lc} 
Contaminant & $\begin{array}{c}\text { DCGLw } \\
\left(\mathrm{dpm} / 100 \mathrm{~cm}^{2}\right)\end{array}$ \\
\hline Th-232 & 400
\end{tabular}

Beta measurements performed see calibration worksheets

\section{Alpha Instrumentation Summary}

Gross Alpha DCGLw (dpm/100 $\left.\mathrm{cm}^{2}\right)$

Total Efficiency:

Gross Alpha DCGLw (cpm):
400

0.42

212
ID Type

14 43-68 \#30 nant

Th-232

Energy ${ }^{1}$

N/A
Mode

Alpha
Area $\left(\mathrm{cm}^{2}\right)$ 126

${ }^{1}$ Average beta energy (keV) [N/A indicates alpha emission]

${ }^{2}$ Activity fraction

Gross Survey Unit Mean (cpm): $312 \pm 53$ (1-sigma)

Count Time (min): 1

\section{Material}

Concrete

Metal

Small Brick

Unpainted Cinder Block

\begin{tabular}{cccc}
$\begin{array}{c}\text { Number of } \\
\text { BKG Counts }\end{array}$ & $\begin{array}{c}\text { Average } \\
(\mathbf{c p m})\end{array}$ & $\begin{array}{c}\text { Standard } \\
\text { Deviation }(\mathbf{c p m})\end{array}$ & $\begin{array}{c}\text { MDC } \\
\left(\mathbf{d p m} / \mathbf{1 0 0} \mathbf{~ c m}^{\mathbf{2}}\right)\end{array}$ \\
\hline 10 & 336.7 & 19.1 & 167 \\
10 & 177.9 & 7.5 & 123 \\
10 & 577 & 31.9 & 217 \\
10 & 447.7 & 26.5 & 192
\end{tabular}

Inst. Eff. 0.42
Surf. Eff. 1.00
Total Eff. 0.4200 


\section{Building Surface Survey Plan}

\section{Survey Plan Summary}

Site:

Planner(s):

Survey Unit Name:

Comments:

Area $\left(m^{2}\right)$ :

Selected Test:

DCGL (cpm):

LBGR (cpm):

Alpha:

Beta:
Hammond Depot FSS Planner

Vitkus

C1 SU33 and 34 Bldg 200E columns

72

Classification:

1

Sign

Estimated Sigma (cpm):

32.3

Sample Size (N):

14

Estimated Conc. (cpm):

64.1

Estimated Power:

1.00

EMC Sample Size (N):

\section{Prospective Power Curve}

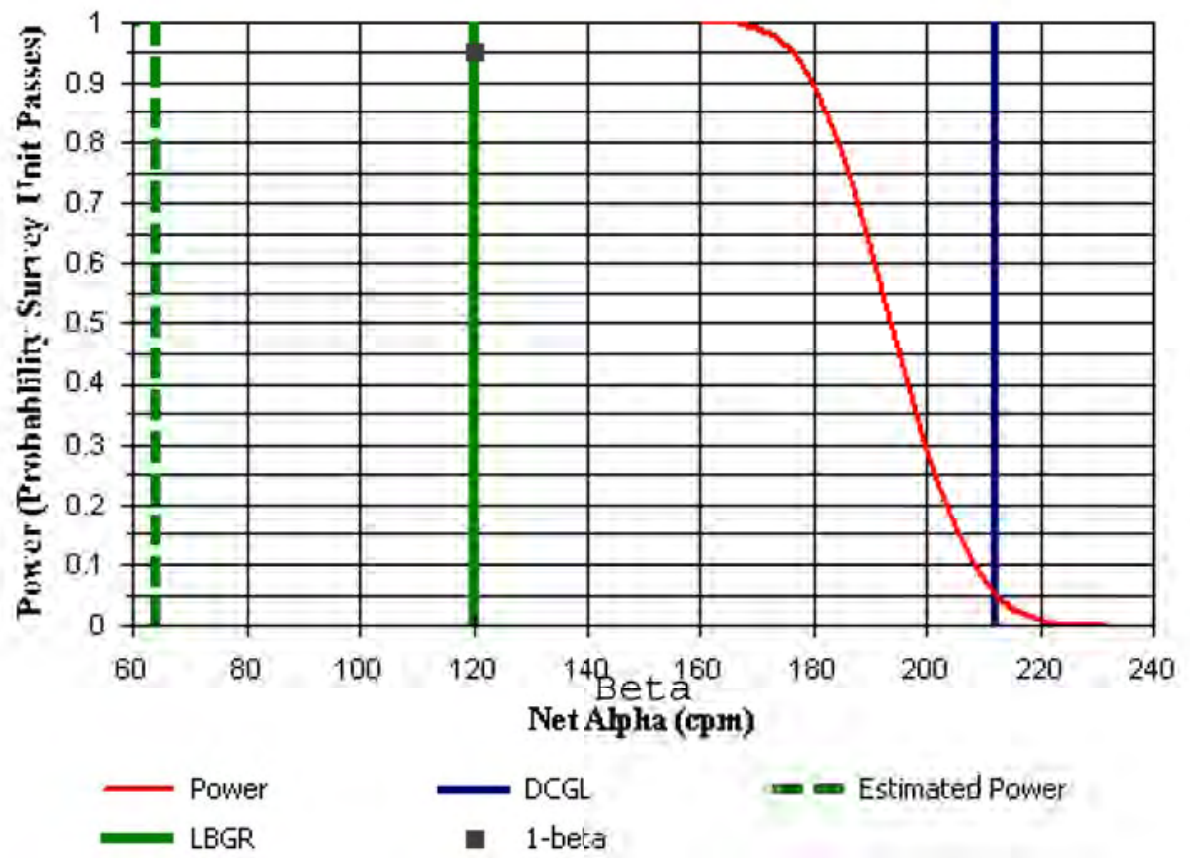




\section{Building Surface Survey Plan}

\section{Contaminant Summary}

\begin{tabular}{lc} 
Contaminant & $\begin{array}{c}\text { DCGLw } \\
\left(\mathrm{dpm} / 100 \mathrm{~cm}^{2}\right)\end{array}$ \\
\hline Th-232 & 400
\end{tabular}

Beta measurements performed see

calibration worksheets

\section{Alpha Instrumentation Summary}

Gross Alpha DCGLw (dpm/100 $\left.\mathrm{cm}^{2}\right)$

Total Efficiency:

Gross Alpha DCGLw (cpm):
400

0.42

212
ID Type

$14 \quad 43-68$ \#30
Energy ${ }^{1}$

N/A
Mode

Alpha
Area $\left(\mathrm{cm}^{2}\right)$ 126

Th-232

Fraction $^{2}$

Inst. Eff. 0.42

Surf. Eff. 1.00

Total Eff. 0.4200

${ }^{1}$ Average beta energy (keV) [N/A indicates alpha emission]

${ }^{2}$ Activity fraction

Gross Survey Unit Mean (cpm): $242 \pm 26$ (1-sigma)

Count Time (min): 1

\begin{tabular}{|c|c|c|c|c|}
\hline Material & $\begin{array}{c}\text { Number of } \\
\text { BKG Counts }\end{array}$ & $\begin{array}{l}\text { Average } \\
\text { (cpm) }\end{array}$ & $\begin{array}{c}\text { Standard } \\
\text { Deviation }(\mathrm{cpm})\end{array}$ & $\begin{array}{c}\text { MDC } \\
\left(\mathrm{dpm} / 100 \mathrm{~cm}^{2}\right)\end{array}$ \\
\hline Concrete & 10 & 336.7 & 19.1 & 167 \\
\hline Metal & 10 & 177.9 & 7.5 & 123 \\
\hline
\end{tabular}




\section{Building Surface Survey Plan}

\section{Survey Plan Summary}

Site:

Planner(s):

Survey Unit Name:

Comments:

Area $\left(m^{2}\right)$ :

Selected Test:

DCGL (cpm):

LBGR (cpm):

Alpha:

Beta:
Hammond Depot FSS Planner

Vitkus

C2 SU35 and 36 Bldg 200E Ceiling Rev. 1

545

Classification:

Sign

Estimated Sigma (cpm):

61.9

Sample Size (N):

18

Estimated Conc. (cpm):

134

Estimated Power:

1.00

\section{Prospective Power Curve}

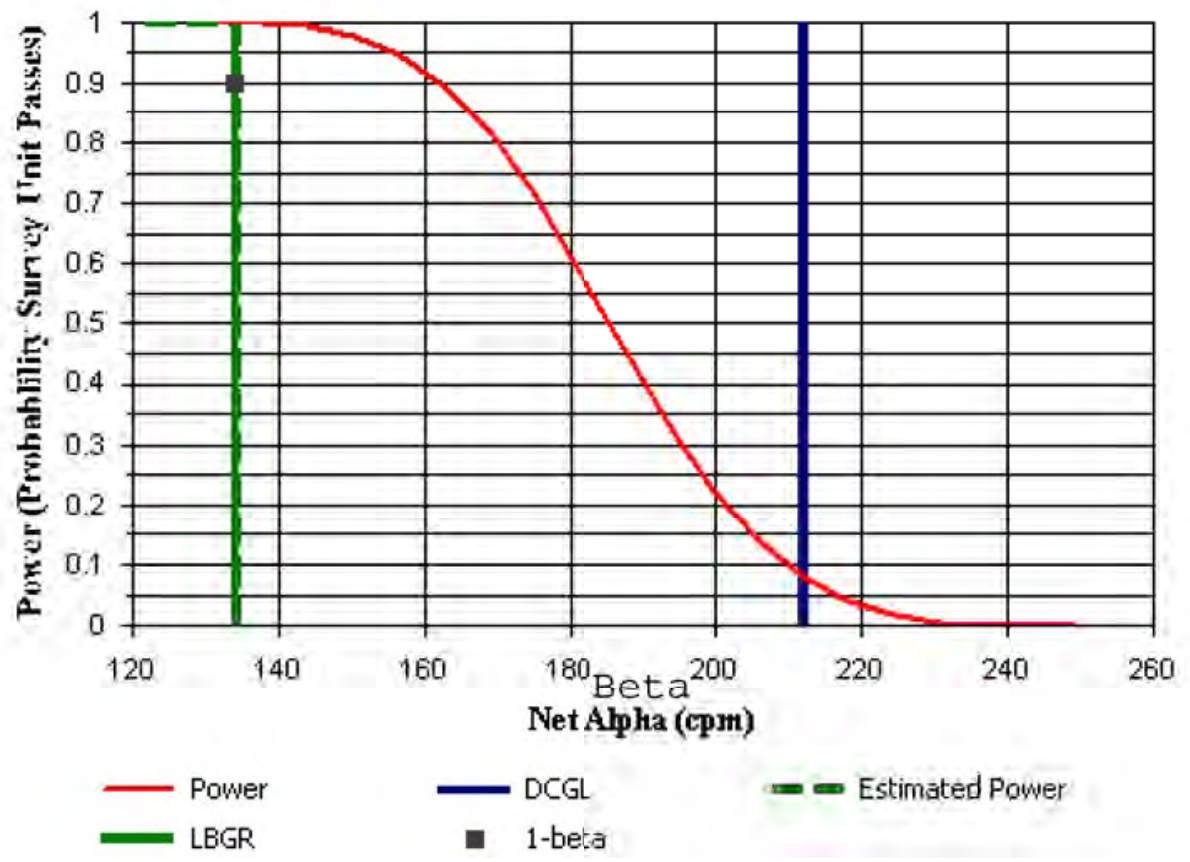




\section{Building Surface Survey Plan}

\section{Contaminant Summary}

\begin{tabular}{lc} 
Contaminant & $\begin{array}{c}\text { DCGLw } \\
\left(\mathrm{dpm} / 100 \mathrm{~cm}^{2}\right)\end{array}$ \\
\hline Th-232 & 400
\end{tabular}

Beta measurements performed see calibration worksheets

\section{Alpha Instrumentation Summary}

Gross Alpha DCGLw $\left(\mathrm{dpm} / 100 \mathrm{~cm}^{2}\right)$

Total Efficiency:

Gross Alpha DCGLw (cpm):
400

0.42

212
ID Type

14 43-68 \#30
Energy ${ }^{1}$

N/A
Mode

Alpha
Area $\left(\mathrm{cm}^{2}\right)$ 126

Fraction $^{2}$ Inst. Eff. 0.42 Surf. Eff. 1.00 Total Eff. 0.4200

${ }^{1}$ Average beta energy (keV) [N/A indicates alpha emission]

${ }^{2}$ Activity fraction

Gross Survey Unit Mean (cpm): $312 \pm 53$ (1-sigma)

Count Time (min): 1

\section{Material}

Concrete

Metal

Painted Cinder block

Small Brick

Unpainted Cinder Block

\begin{tabular}{cccc}
$\begin{array}{c}\text { Number of } \\
\text { BKG Counts }\end{array}$ & $\begin{array}{c}\text { Average } \\
(\mathbf{c p m})\end{array}$ & $\begin{array}{c}\text { Standard } \\
\text { Deviation }(\mathbf{c p m})\end{array}$ & $\begin{array}{c}\text { MDC } \\
\left(\mathbf{d p m} / \mathbf{1 0 0} \mathbf{~ c m}^{2}\right)\end{array}$ \\
\hline 10 & 336.7 & 19.1 & 167 \\
10 & 177.9 & 7.5 & 123 \\
10 & 395.7 & 20.9 & 180 \\
10 & 577 & 31.9 & 217 \\
10 & 447.7 & 26.5 & 192
\end{tabular}




\section{Building Surface Survey Plan}

\section{Survey Plan Summary}

Site:

Planner(s):

Survey Unit Name:

Comments:

Area $\left(m^{2}\right)$ :

Selected Test:

DCGL (cpm):

LBGR (cpm):

Alpha:

Beta:
Hammond Depot FSS Planner

Vitkus

C2 SU56 through 58 Bldg 200E walls

Survey unit area is largest or the survey units

281

Classification:

Sign

Estimated Sigma (cpm):

96.4

Sample Size (N):

17

Estimated Conc. (cpm):

50.1

Estimated Power:

1.00

\section{Prospective Power Curve}

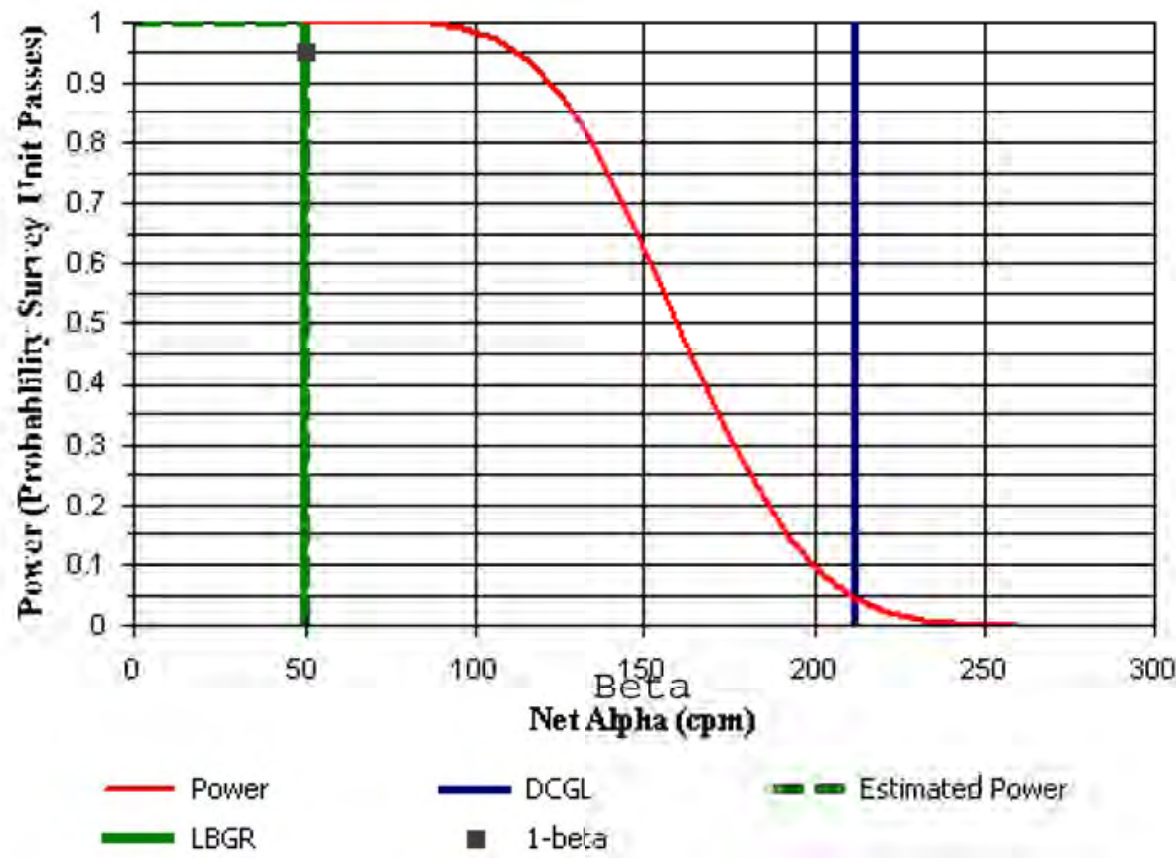




\section{Building Surface Survey Plan}

\section{Contaminant Summary}

\begin{tabular}{lc} 
Contaminant & $\begin{array}{c}\text { DCGLw } \\
\left(\mathrm{dpm} / 100 \mathrm{~cm}^{2}\right)\end{array}$ \\
\hline Th-232 & 400
\end{tabular}

Beta measurements performed see calibration worksheets

\section{Alpha Instrumentation Summary}

Gross Alpha DCGLw $\left(\mathrm{dpm} / 100 \mathrm{~cm}^{2}\right)$

Total Efficiency:

Gross Alpha DCGLw (cpm)
400

0.42

212
ID Type

$14 \quad 43-68$ \#30

Contaminant

Th-232

${ }^{1}$ Average beta energy (keV) [N/A indicates alpha emission]

${ }^{2}$ Activity fraction

Gross Survey Unit Mean (cpm): $228 \pm 91$ (1-sigma)

Count Time (min): 1

\section{Material}

Concrete

Metal

Painted Cinder block

Small Brick

Unpainted Cinder Block

\begin{tabular}{cccc}
$\begin{array}{c}\text { Number of } \\
\text { BKG Counts }\end{array}$ & $\begin{array}{c}\text { Average } \\
\text { (cpm) }\end{array}$ & $\begin{array}{c}\text { Standard } \\
\text { Deviation (cpm) }\end{array}$ & $\begin{array}{c}\text { MDC } \\
\text { (dpm/100 } \mathbf{c m}^{2} \text { ) }\end{array}$ \\
\hline 10 & 336.7 & 19.1 & 167 \\
10 & 177.9 & 7.5 & 123 \\
10 & 395.7 & 20.9 & 180 \\
10 & 577 & 31.9 & 217 \\
10 & 447.7 & 26.5 & 192
\end{tabular}

\begin{tabular}{cc} 
Mode & Area $\left(\mathbf{c m}^{2}\right)$ \\
\hline Alpha & 126
\end{tabular}

Inst. Eff.

Surf. Eff.

Total Eff.

1.0000

0.42

1.00

0.4200 


\section{Building Surface Survey Plan}

\section{Survey Plan Summary}

Site:

Planner(s):

Survey Unit Name:

Comments:

Area $\left(m^{2}\right)$ :

Selected Test:

DCGL (cpm):

LBGR (cpm):

Alpha:

Beta:
Hammond Depot FSS Planner

Vitkus

C1 SU41 Bldg 200E NW closet Rev 1

revised to add metal background

20

Classification:

1

Sign

Estimated Sigma (cpm):

98.2

212

Sample Size $(\mathrm{N})$ :

18

Estimated Conc. (cpm):

57.1

Estimated Power:

1.00

EMC Sample Size (N):

\section{Prospective Power Curve}

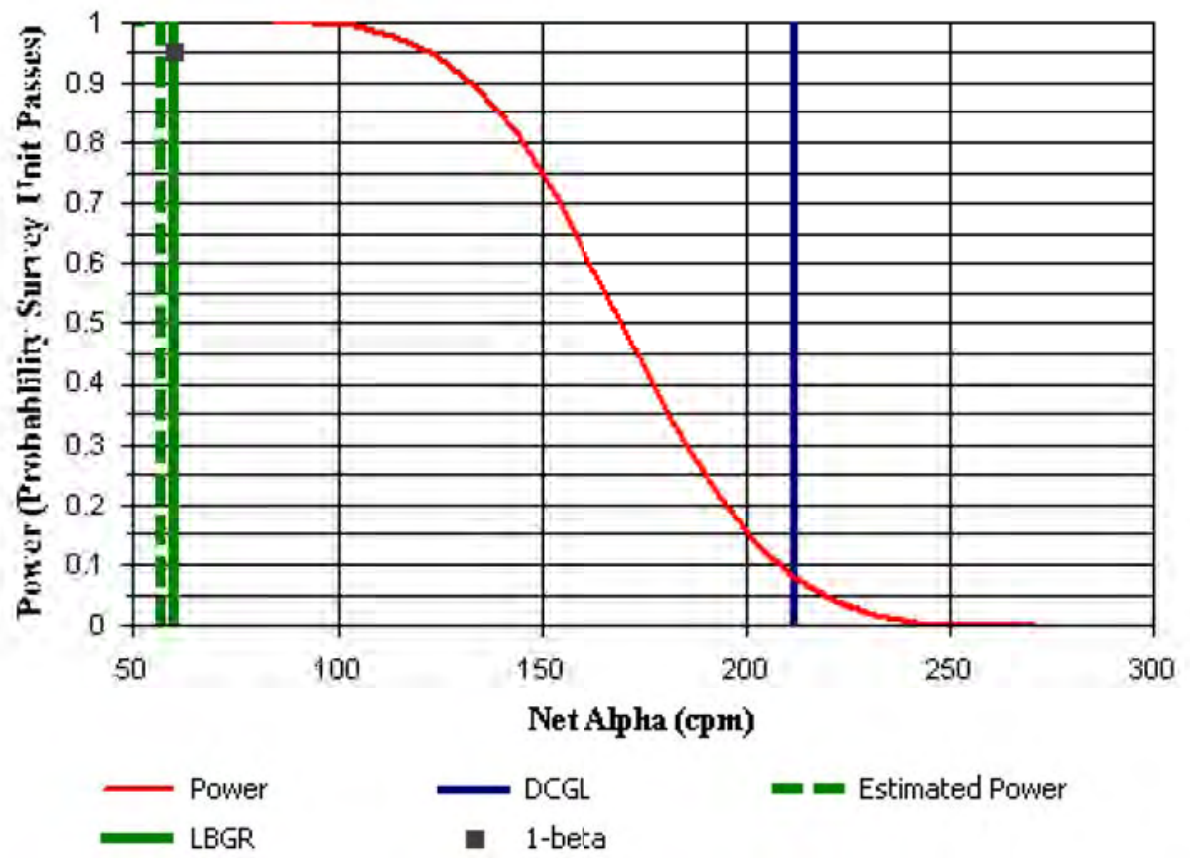




\section{Building Surface Survey Plan}

\section{Contaminant Summary}

\begin{tabular}{lc} 
Contaminant & $\begin{array}{c}\text { DCGLw } \\
\left(\mathrm{dpm} / 100 \mathrm{~cm}^{2}\right)\end{array}$ \\
\hline Th-232 & 400
\end{tabular}

Beta measurements performed see calibration worksheets

\section{Alpha Instrumentation Summary}

Gross Alpha DCGLw (dpm/100 $\left.\mathrm{cm}^{2}\right)$

Total Efficiency:

Gross Alpha DCGLw (cpm):
400

0.42

212

ID Typ

$14 \quad 43-68$ \#30

Mode

Alpha

Area $\left(\mathrm{cm}^{2}\right)$ 126

Contaminant

Energy ${ }^{1}$

Fraction $^{2}$

Inst. Eff.

N/A

1.0000

0.42

Surf. Eff. 1.00

Total Eff.

${ }^{1}$ Average beta energy (keV) [N/A indicates alpha emission]

${ }^{2}$ Activity fraction

Gross Survey Unit Mean (cpm): $235 \pm 96$ (1-sigma)

Count Time (min): 1

\begin{tabular}{|c|c|c|c|c|}
\hline Material & $\begin{array}{l}\text { Number of } \\
\text { BKG Counts }\end{array}$ & $\begin{array}{l}\text { Average } \\
\text { (cpm) }\end{array}$ & $\begin{array}{c}\text { Standard } \\
\text { Deviation }(\mathrm{cpm})\end{array}$ & $\begin{array}{c}\text { MDC } \\
\left(\mathrm{dpm} / 100 \mathrm{~cm}^{2}\right)\end{array}$ \\
\hline Concrete & 10 & 336.7 & 19.1 & 167 \\
\hline Metal & 10 & 177.9 & 7.5 & 123 \\
\hline Painted Cinder block & 10 & 395.7 & 20.9 & 180 \\
\hline
\end{tabular}




\section{Building Surface Survey Plan}

\section{Survey Plan Summary}

Site:

Planner(s):

Survey Unit Name:

Comments:

Area $\left(m^{2}\right)$ :

Selected Test:

DCGL (cpm):

LBGR (cpm):

Alpha:

Beta:
Hammond Depot FSS Planner

Vitkus

C1 SU43 and 44 Building 100E , C1 SU59

200

Classification:

1

Sign

Estimated Sigma (cpm):

27.7

212

Sample Size (N):

13

Estimated Conc. (cpm):

$-17.7$

Estimated Power:

1.00

EMC Sample Size (N):

\section{Prospective Power Curve}

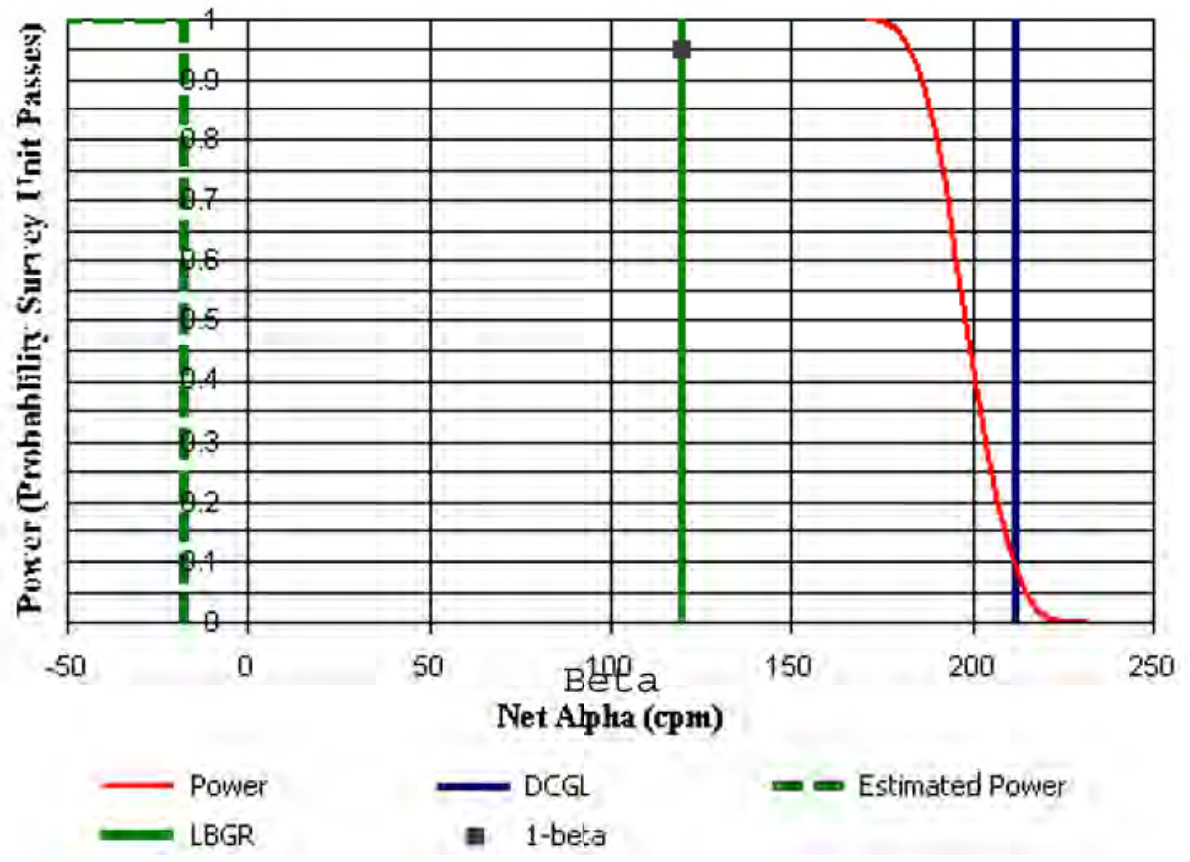




\section{Building Surface Survey Plan}

\section{Contaminant Summary}

\begin{tabular}{lc} 
Contaminant & $\begin{array}{c}\text { DCGLw } \\
\left(\mathrm{dpm} / 100 \mathrm{~cm}^{2}\right)\end{array}$ \\
\hline Th-232 & 400
\end{tabular}

Beta measurements performed see calibration worksheets

\section{Alpha Instrumentation Summary}

Gross Alpha DCGLw (dpm/100 $\left.\mathrm{cm}^{2}\right)$

Total Efficiency:

Gross Alpha DCGLw (cpm):
400

0.42

212
ID Type

$14 \quad 43-68$ \#30 nant

Th-232
Energy 1

N/A
Mode

Alpha Beta
Area $\left(\mathrm{cm}^{2}\right)$ 126

${ }^{1}$ Average beta energy (keV) [N/A indicates alpha emission]

${ }^{2}$ Activity fraction

Gross Survey Unit Mean (cpm): $319 \pm 20$ (1-sigma)

Count Time (min): 1

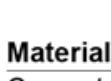

Number of BKG Counts 10

\begin{tabular}{c}
$\begin{array}{c}\text { Average } \\
\text { (cpm) }\end{array}$ \\
\hline 336.7
\end{tabular}

Standard Deviation (cpm)

19.1

336.7
MDC (dpm/100 cm ${ }^{2}$ )

167 


\section{Building Surface Survey Plan}

\section{Survey Plan Summary}

Site:

Planner(s):

Survey Unit Name:

Comments:

Area $\left(m^{2}\right)$ :

Selected Test:

DCGL (cpm):

LBGR (cpm):

Alpha:

Beta:
Hammond Depot FSS Planner

Vitkus

Class 2 SUs Scoping Rev. 1

Survey area is maximum SU size

2,100

Classification:

Sign

Estimated Sigma (cpm):

62.6

Sample Size $(\mathrm{N})$ :

20

Estimated Conc. (cpm):

120

Estimated Power:

1.00

\section{Prospective Power Curve}

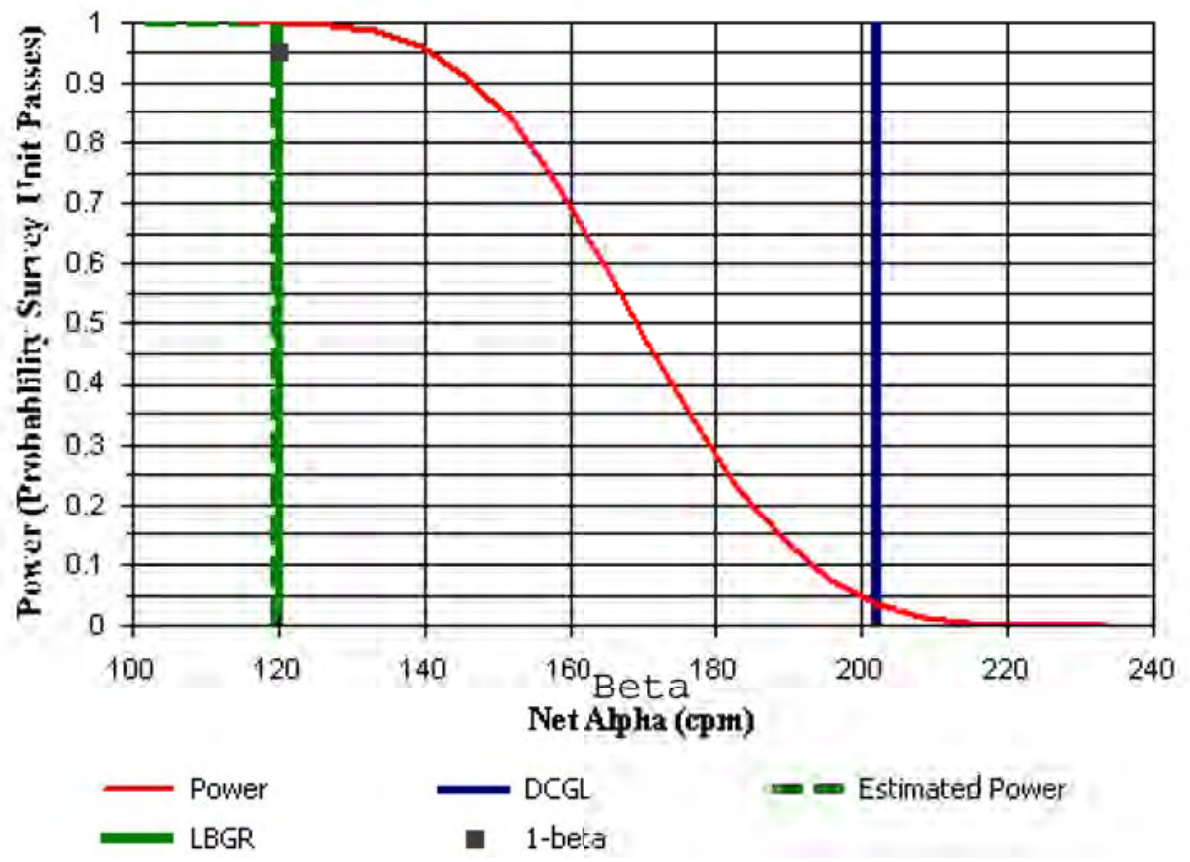




\section{Building Surface Survey Plan}

\section{Contaminant Summary}

\begin{tabular}{lc} 
Contaminant & $\begin{array}{c}\text { DCGLw } \\
\left(\mathrm{dpm} / 100 \mathrm{~cm}^{2}\right)\end{array}$ \\
\hline Th-232 & 400
\end{tabular}

Beta measurements performed see

calibration worksheets

\section{Alpha Instrumentation Summary}

Gross Alpha DCGLw (dpm/100 $\left.\mathrm{cm}^{2}\right)$

Total Efficiency:

Gross Alpha DCGLw (cpm):
400

0.40

202
ID Type

$15 \quad 43-68$ \#20
Energy ${ }^{1}$

N/A
Mode

Alpha
Area $\left(\mathrm{cm}^{2}\right)$ 126

Th-232

Fraction $^{2}$ Inst. Eff. 0.40

Surf. Eff. 1.00 Total Eff. 0.4000

${ }_{1}^{1}$ Average beta energy (keV) [N/A indicates alpha emission]

${ }^{2}$ Activity fraction

Gross Survey Unit Mean (cpm): $281 \pm 55$ (1-sigma)

Count Time (min): 1

\section{Material}

Concrete

Metal

Small Brick

Unpainted Cinder Block

\begin{tabular}{cccc}
$\begin{array}{c}\text { Number of } \\
\text { BKG Counts }\end{array}$ & $\begin{array}{c}\text { Average } \\
\text { (cpm) }\end{array}$ & $\begin{array}{c}\text { Standard } \\
\text { Deviation }(\mathbf{c p m})\end{array}$ & $\begin{array}{c}\text { MDC } \\
\left(\mathbf{d p m} / \mathbf{1 0 0} \mathbf{c m}^{2}\right)\end{array}$ \\
\hline 10 & 334.7 & 18 & 175 \\
10 & 161.3 & 6.4 & 123 \\
10 & 535.4 & 15.9 & 219 \\
10 & 405.8 & 29.9 & 192
\end{tabular}




\section{Building Surface Survey Plan}

\section{Survey Plan Summary}

Site:

Planner(s):

Survey Unit Name:

Comments:

Area $\left(m^{2}\right)$ :

Selected Test:

DCGL (cpm):

LBGR (cpm):

Alpha:

Beta:
Hammond Depot FSS Planner

Vitkus

Class 2 SUs

Survey unit area is maximum

2,100

Classification:

Sign

Estimated Sigma (cpm):

47.4

Sample Size (N):

15

Estimated Conc. (cpm):

$-57.9$

Estimated Power:

1.00

\section{Prospective Power Curve}

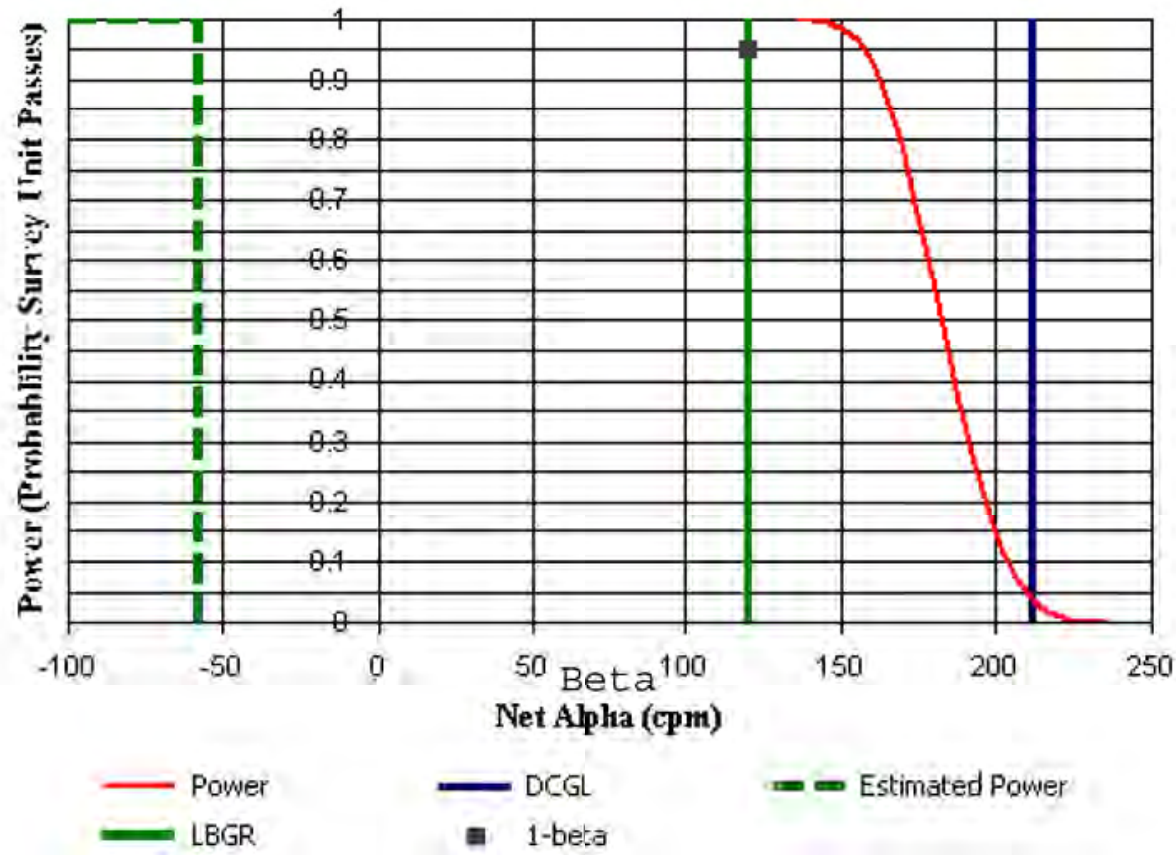




\section{Building Surface Survey Plan}

\section{Contaminant Summary}

Contaminant

Th-232
DCGLW

(dpm/100 cm²)

Beta measurements performed

see calibration worksheet

\section{Alpha Instrumentation Summary}

Gross Alpha DCGLw (dpm/100 cm²):

Total Efficiency:

Gross Alpha DCGLw (cpm):
400

0.42

212

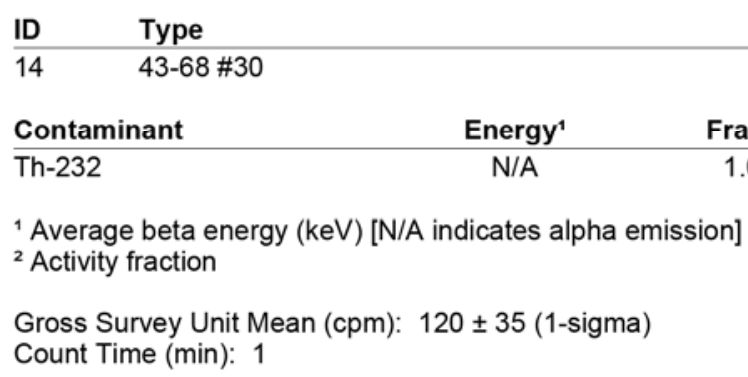

\begin{tabular}{|c|c|c|c|c|}
\hline Material & $\begin{array}{l}\text { Number of } \\
\text { BKG Counts }\end{array}$ & $\begin{array}{c}\text { Average } \\
\text { (cpm) }\end{array}$ & $\begin{array}{c}\text { Standard } \\
\text { Deviation }(\mathrm{cpm})\end{array}$ & $\begin{array}{c}\text { MDC } \\
\left(\mathrm{dpm} / 100 \mathrm{~cm}^{2}\right)\end{array}$ \\
\hline Concrete & 10 & 336.7 & 19.1 & 167 \\
\hline Metal & 10 & 177.9 & 7.5 & 123 \\
\hline Painted Cinder block & 10 & 395.7 & 20.9 & 180 \\
\hline Small Brick & 10 & 577 & 31.9 & 217 \\
\hline Unpainted Cinder Block & 10 & 447.7 & 26.5 & 192 \\
\hline
\end{tabular}

$\begin{array}{cc}\text { Mode } & \text { Area }\left(\mathrm{cm}^{2}\right) \\ \text { Alpha } & 126\end{array}$

Inst. Eff. 0.42

Surf. Eff. 1.00 Total Eff. 0.4200 


\section{Building Surface Survey Plan}

\section{Survey Plan Summary}

Site:

Planner(s):

Survey Unit Name:

Comments:

Area $\left(m^{2}\right)$ :

Selected Test:

DCGL (cpm):

LBGR (cpm):

Alpha:

Beta:
Hammond Depot FSS Planner

Vitkus

Class 3 SUs scoping

Survey unit area is maximum

7,400

Classification:

Sign

Estimated Sigma (cpm):

42.4

Sample Size (N):

15

Estimated Conc. (cpm):

120

Estimated Power:

1.00

\section{Prospective Power Curve}

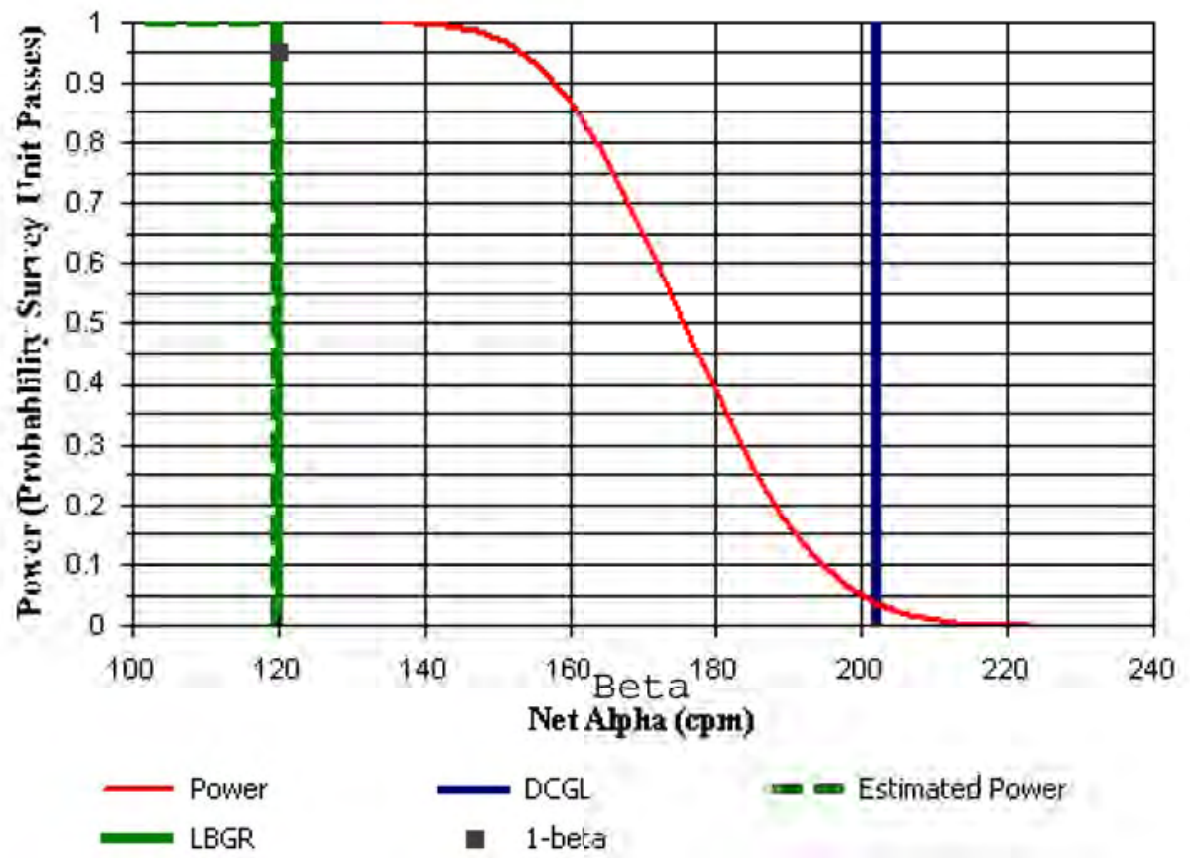




\section{Building Surface Survey Plan}

\section{Contaminant Summary}

\begin{tabular}{lc} 
Contaminant & $\begin{array}{c}\text { DCGLw } \\
\left(\mathrm{dpm} / 100 \mathrm{~cm}^{2}\right)\end{array}$ \\
\hline Th-232 & 400
\end{tabular}

Beta measurements performed see

calibration worksheets

\section{Alpha Instrumentation Summary}

Gross Alpha DCGLw $\left(\mathrm{dpm} / 100 \mathrm{~cm}^{2}\right)$

Total Efficiency:

Gross Alpha DCGLw (cpm):
400

0.40

202
ID Type

$15 \quad 43-68$ \#20
Energy 1

N/A
Mode

Alpha
Area $\left(\mathrm{cm}^{2}\right)$ 126

Th-232

Fraction $^{2}$ Inst. Eff. 0.40

Surf. Eff. 1.00 Total Eff. 0.4000

${ }^{1}$ Average beta energy (keV) [N/A indicates alpha emission]

${ }^{2}$ Activity fraction

Gross Survey Unit Mean (cpm): $281 \pm 30$ (1-sigma)

Count Time (min): 1

\section{Material}

Concrete

Metal

Painted Cinder block

Small Brick

Unpainted Cinder Block

\begin{tabular}{|c|c|c|c|}
\hline $\begin{array}{l}\text { Number of } \\
\text { BKG Counts }\end{array}$ & $\begin{array}{c}\text { Average } \\
\text { (cpm) }\end{array}$ & $\begin{array}{c}\text { Standard } \\
\text { Deviation (cpm) }\end{array}$ & $\begin{array}{c}\text { MDC } \\
\left(\mathrm{dpm} / 100 \mathrm{~cm}^{2}\right)\end{array}$ \\
\hline 10 & 334.7 & 18 & 175 \\
\hline 10 & 161.3 & 6.4 & 123 \\
\hline 10 & 334.7 & 18 & 175 \\
\hline 10 & 535.4 & 15.9 & 219 \\
\hline 10 & 405.8 & 29.9 & 192 \\
\hline
\end{tabular}




\section{Building Surface Survey Plan}

\section{Survey Plan Summary}

Site:

Planner(s):

Survey Unit Name:

Comments:

Area $\left(m^{2}\right)$ :

Selected Test:

DCGL (cpm):

LBGR (cpm):

Alpha:

Beta:
Hammond Depot FSS Planner

Vitkus

Class 3 SUs

Survey Unit area is maximum

7,400

Classification:

3

Sign

Estimated Sigma (cpm):

45.2

Sample Size (N):

15

Estimated Conc. (cpm):

$-57.9$

Estimated Power:

1.00

\section{Prospective Power Curve}

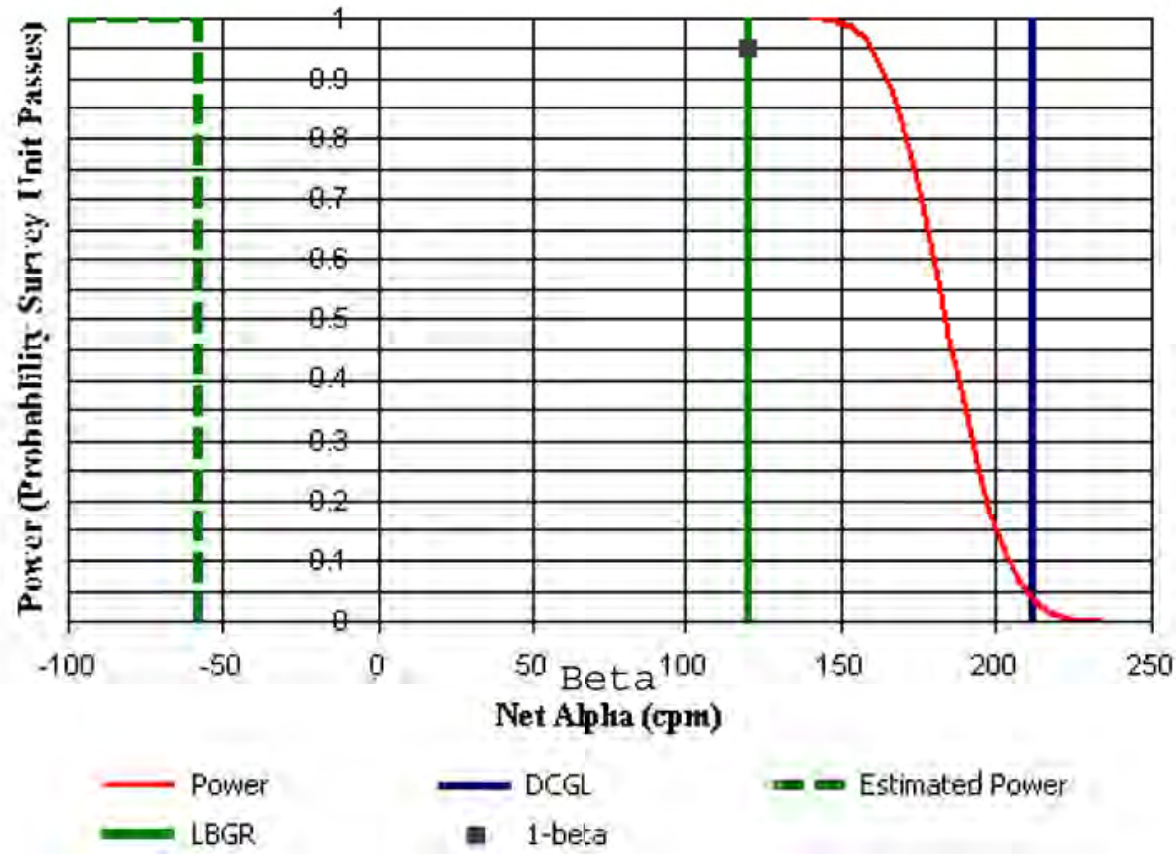




\section{Building Surface Survey Plan}

\section{Contaminant Summary}

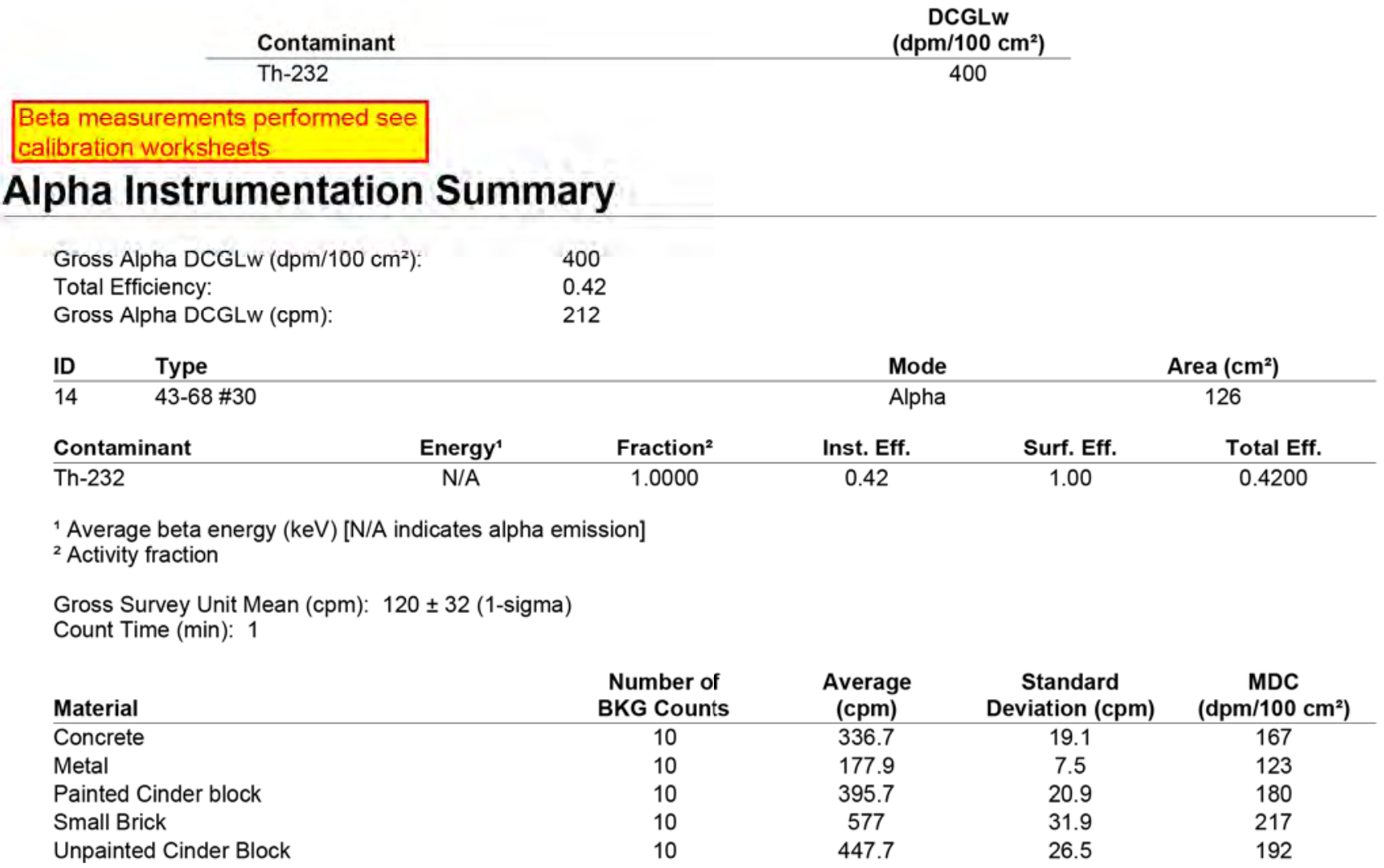


APPENDIX F: STRUCTURE SURVEY UNIT DQAs 


\section{DQA Building Surface Report}

\section{Assessment Summary}

Site:

Planner(s):

Survey Unit Name:

Report Number:

Survey Unit Measurements:

Reference Area Measurements:

Test Performed:

Judgmental Areas:

Assessment Conclusion:
Hammond Depot FSS Planner

Vitkus

C1 SU1 through 21 Bldg 200E slag Rev. 1 C1 SU1

1

14

0

Sign

Test Result:

Not Performed

0

EMC Result:

Not Performed

Reject Null Hypothesis (Survey Unit PASSES)

\section{Retrospective Power Curve}

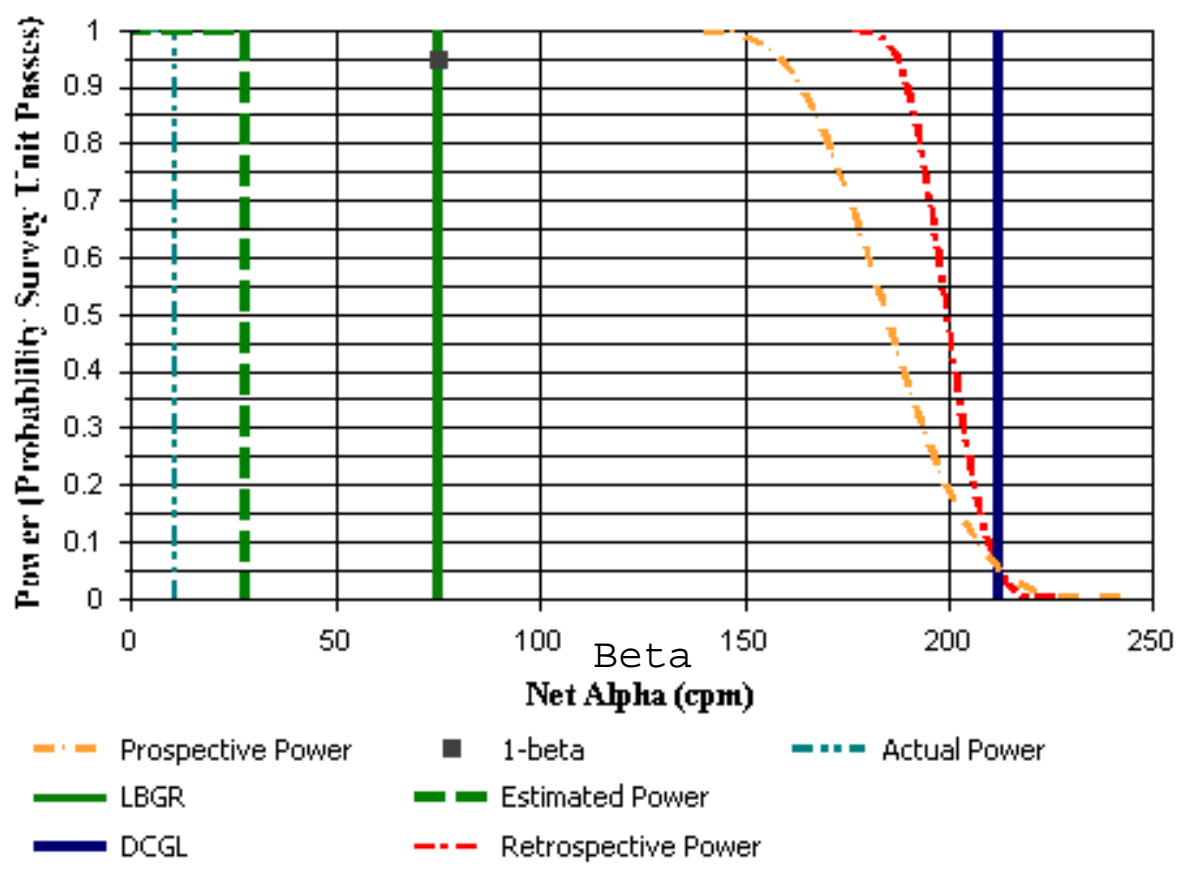




\section{DQA Building Surface Report}

\section{Survey Unit Data}

NOTE: $\quad$ Type $=$ "S" indicates survey measurement.

Type $=$ "R" indicates reference measurement.

\begin{tabular}{lccc} 
Measurement & Material & Type & Gross Alpha (cpm) \\
\hline $1.2,2.2$ & Slag & S & 530 \\
$4.1,2.2$ & Slag & S & 453 \\
$6.9,2.2$ & Slag & S & 517 \\
$9.8,2.2$ & Slag & S & 481 \\
$2.6,4.7$ & Slag & S & 509 \\
$5.5,4.7$ & Slag & S & 476 \\
$8.4,4.7$ & Slag & S & 458 \\
$1.2,7.2$ & Slag & S & 477 \\
$4.1,7.2$ & Slag & S & 470 \\
$6.9,7.2$ & Slag & S & 481 \\
$9.8,7.2$ & Slag & S & 461 \\
$2.6,9.7$ & Slag & S & 491 \\
$5.5,9.7$ & Slag & S & 476 \\
$8.4,9.7$ & Slag & S & 487
\end{tabular}

\section{Basic Statistical Quantities Summary}

Statistic

Sample Number

Mean $\left(\mathrm{dpm} / 100 \mathrm{~cm}{ }^{2}\right)$

Median (dpm/100 cm²)

Std Dev (dpm/100 $\left.\mathrm{cm}^{2}\right)$

High Value $\left(\mathrm{dpm} / 100 \mathrm{~cm}^{2}\right)$

Low Value $\left(\mathrm{dpm} / 100 \mathrm{~cm}^{2}\right)$
Survey Unit

14

18.91

10.68

42.06

107.05

$-38.45$
Background

N/A

N/A

N/A

N/A

N/A

N/A

N/A
DQO Results

$\mathrm{N}=14$

27.6

N/A

48.3

N/A 


\section{DQA Building Surface Report}

\section{Assessment Summary}

Site:

Planner(s):

Survey Unit Name:

Report Number:

Survey Unit Measurements:

Reference Area Measurements:

Test Performed:

Judgmental Areas:

Assessment Conclusion:
Hammond Depot FSS Planner

Vitkus

C1 SU1 through 21 Bldg 200E slag Rev. 1 C1 SU2

2

14

0

Sign

Test Result:

Not Performed

0

EMC Result:

Not Performed

Reject Null Hypothesis (Survey Unit PASSES)

\section{Retrospective Power Curve}

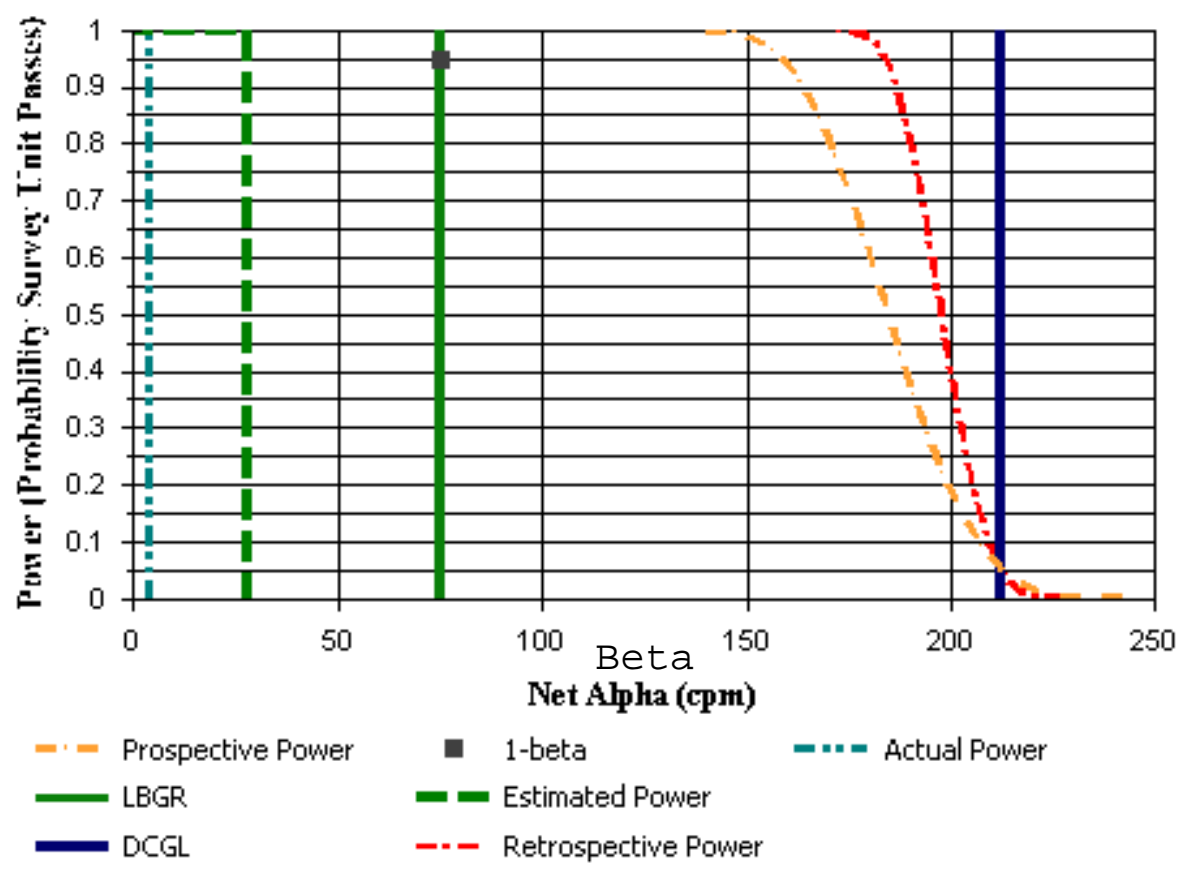




\section{DQA Building Surface Report}

\section{Survey Unit Data}

NOTE: $\quad$ Type $=$ "S" indicates survey measurement.

Type $=$ "R" indicates reference measurement.

\begin{tabular}{lccc} 
Measurement & Material & Type & Gross Alpha (cpm) \\
\hline $12.4,2.1$ & Slag & S & 458 \\
$15.2,2.1$ & Slag & S & 418 \\
$18.1,2.1$ & Slag & S & 462 \\
$10.9,4.6$ & Slag & S & 493 \\
$13.8,4.6$ & Slag & S & 476 \\
$16.7,4.6$ & Slag & S & 468 \\
$19.6,4.6$ & Slag & S & 493 \\
$12.4,7.1$ & Slag & S & 508 \\
$15.2,7.1$ & Slag & S & 475 \\
$18.1,7.1$ & Slag & S & 491 \\
$10.9,9.5$ & Slag & S & 461 \\
$13.8,9.5$ & Slag & S & 482 \\
$16.7,9.5$ & Slag & S & 525 \\
$19.6,9.5$ & Slag & S & 474
\end{tabular}

\section{Basic Statistical Quantities Summary}

Statistic

Sample Number

Mean $\left(\mathrm{dpm} / 100 \mathrm{~cm}^{2}\right)$

Median (dpm/100 cm²)

Std Dev (dpm/100 $\left.\mathrm{cm}^{2}\right)$

High Value $\left(\mathrm{dpm} / 100 \mathrm{~cm}^{2}\right)$

Low Value $\left(\mathrm{dpm} / 100 \mathrm{~cm}^{2}\right)$
Survey Unit

14

7.71

4.06

48.07

97.60

$-104.59$
Background

DQO Results

$\begin{array}{ll}\text { N/A } & \mathrm{N}=14 \\ \text { N/A } & 27.6 \\ \text { N/A } & \text { N/A } \\ \text { N/A } & 48.3 \\ \text { N/A } & \text { N/A } \\ \text { N/A } & \text { N/A }\end{array}$




\section{DQA Building Surface Report}

\section{Assessment Summary}

Site:

Planner(s):

Survey Unit Name:

Report Number:

Survey Unit Measurements:

Reference Area Measurements:

Test Performed:

Judgmental Areas:

Assessment Conclusion:
Hammond Depot FSS Planner

Vitkus

C1 SU1 through 21 Bldg 200E slag Rev. 1 C1 SU3

3

14

0

Sign

Test Result:

Not Performed

0

EMC Result:

Not Performed

\section{Retrospective Power Curve}

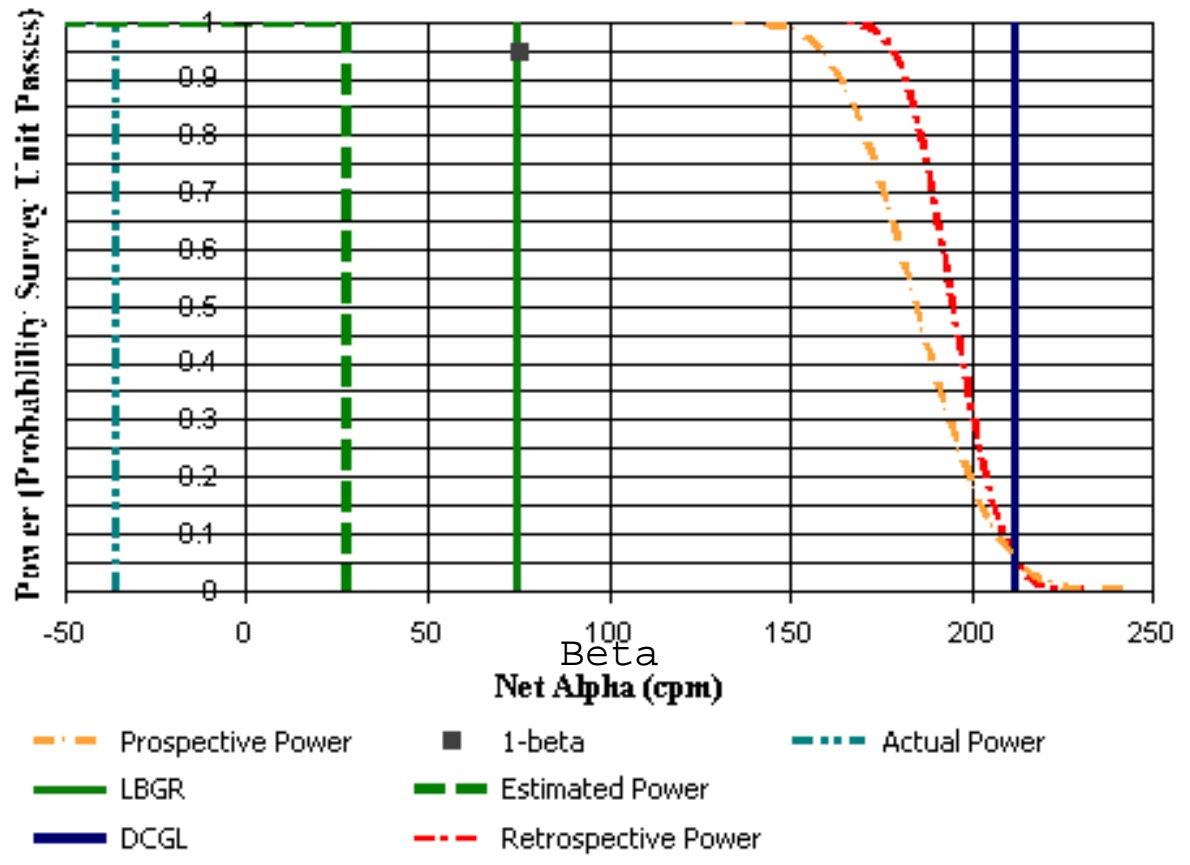




\section{DQA Building Surface Report}

\section{Survey Unit Data}

NOTE: $\quad$ Type = "S" indicates survey measurement.

Type $=$ "R" indicates reference measurement.

\begin{tabular}{lccc} 
Measurement & Material & Type & Gross Alpha (cpm) \\
\hline $21.2,1.4$ & Slag & S & 429 \\
$24.0,1.4$ & Slag & S & 442 \\
$26.9,1.4$ & Slag & S & 450 \\
$29.8,1.4$ & Slag & S & 465 \\
$22.6,3.9$ & Slag & S & 485 \\
$25.5,3.9$ & Slag & S & 459 \\
$28.3,3.9$ & Slag & S & 449 \\
$21.2,6.4$ & Slag & S & 509 \\
$24.0,6.4$ & Slag & S & 405 \\
$26.9,6.4$ & Slag & S & 405 \\
$29.8,6.4$ & Slag & S & 476 \\
$22.6,8.8$ & Slag & S & 412 \\
$25.5,8.8$ & Slag & S & 462 \\
$28.3,8.8$ & Slag & S & 468
\end{tabular}

\section{Basic Statistical Quantities Summary}

Statistic

Sample Number

Mean $\left(\mathrm{dpm} / 100 \mathrm{~cm}^{2}\right)$

Median (dpm/100 cm²)

Std Dev (dpm/100 $\left.\mathrm{cm}^{2}\right)$

High Value $\left(\mathrm{dpm} / 100 \mathrm{~cm}^{2}\right)$

Low Value $\left(\mathrm{dpm} / 100 \mathrm{~cm}^{2}\right)$
Survey Unit

14

$-41.96$

$-35.62$

57.74

67.37

$-129.16$
Background

DQO Results

$\begin{array}{ll}\text { N/A } & \mathrm{N}=14 \\ \text { N/A } & 27.6 \\ \text { N/A } & \text { N/A } \\ \text { N/A } & 48.3 \\ \text { N/A } & \text { N/A } \\ \text { N/A } & \text { N/A }\end{array}$




\section{DQA Building Surface Report}

\section{Assessment Summary}

Site:

Planner(s):

Survey Unit Name:

Report Number:

Survey Unit Measurements:

Reference Area Measurements:

Test Performed:

Judgmental Areas:

Assessment Conclusion:
Hammond Depot FSS Planner

Vitkus

C1 SU1 through 21 Bldg 200E slag Rev. 1 C1 SU4

4

14

0

Sign

Test Result:

Not Performed

0

EMC Result:

Not Performed

Reject Null Hypothesis (Survey Unit PASSES)

\section{Retrospective Power Curve}

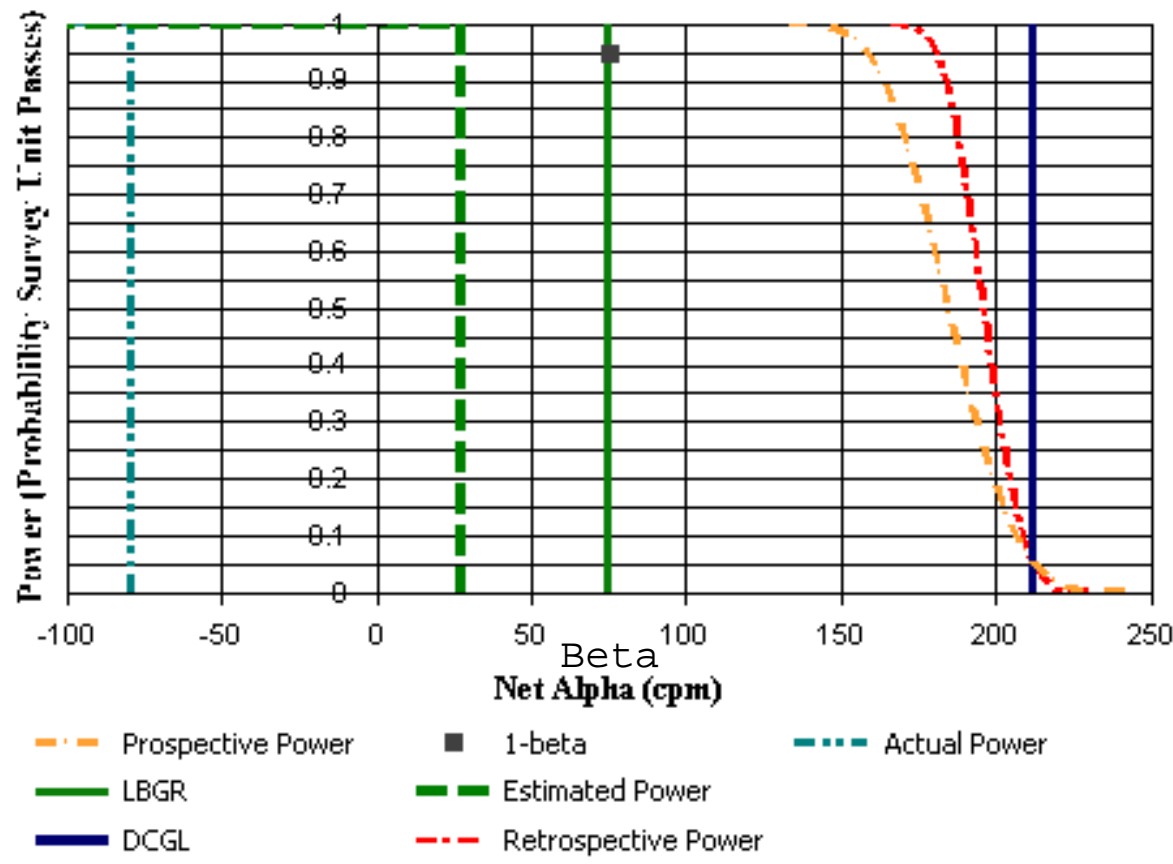




\section{DQA Building Surface Report}

\section{Survey Unit Data}

NOTE: $\quad$ Type $=$ "S" indicates survey measurement.

Type $=$ "R" indicates reference measurement.

\begin{tabular}{lccc} 
Measurement & Material & Type & Gross Alpha (cpm) \\
\hline $31.0,0.7$ & Slag & S & 410 \\
$34.5,0.7$ & Slag & S & 434 \\
$32.8,3.7$ & Slag & S & 458 \\
$36.3,3.7$ & Slag & S & 421 \\
$31.0,6.8$ & Slag & S & 501 \\
$34.5,6.8$ & Slag & S & 409 \\
$32.8,9.8$ & Slag & S & 445 \\
$36.3,9.8$ & Slag & S & 443 \\
$31.0,12.9$ & Slag & S & 409 \\
$34.5,12.9$ & Slag & S & 414 \\
$32.8,15.9$ & Slag & S & 471 \\
$36.3,15.9$ & Slag & S & 471 \\
$31.0,19.0$ & Slag & S & 422 \\
$34.5,19.0$ & Slag & S & 429
\end{tabular}

\section{Basic Statistical Quantities Summary}

Statistic

Sample Number

Mean $\left(\mathrm{dpm} / 100 \mathrm{~cm}^{2}\right)$

Median (dpm/100 cm²)

Std Dev (dpm/100 $\left.\mathrm{cm}^{2}\right)$

High Value $\left(\mathrm{dpm} / 100 \mathrm{~cm}^{2}\right)$

Low Value $\left(\mathrm{dpm} / 100 \mathrm{~cm}^{2}\right)$
Survey Unit

14
-66.12
-79.08
53.20
52.25
-121.60

14

$-121.60$
Background

DQO Results

$\begin{array}{ll}\text { N/A } & \mathrm{N}=14 \\ \text { N/A } & 27.6 \\ \text { N/A } & \text { N/A } \\ \text { N/A } & 48.3 \\ \text { N/A } & \text { N/A } \\ \text { N/A } & \text { N/A }\end{array}$




\section{DQA Building Surface Report}

\section{Assessment Summary}

Site:

Planner(s):

Survey Unit Name:

Report Number:

Survey Unit Measurements:

Reference Area Measurements:

Test Performed:

Judgmental Areas:

Assessment Conclusion:
Hammond Depot FSS Planner

Vitkus

C1 SU1 through 21 Bldg 200E slag Rev. 1 C1 SU5

5

14

0

Sign

Test Result:

Not Performed

0

EMC Result:

Not Performed

\section{Retrospective Power Curve}

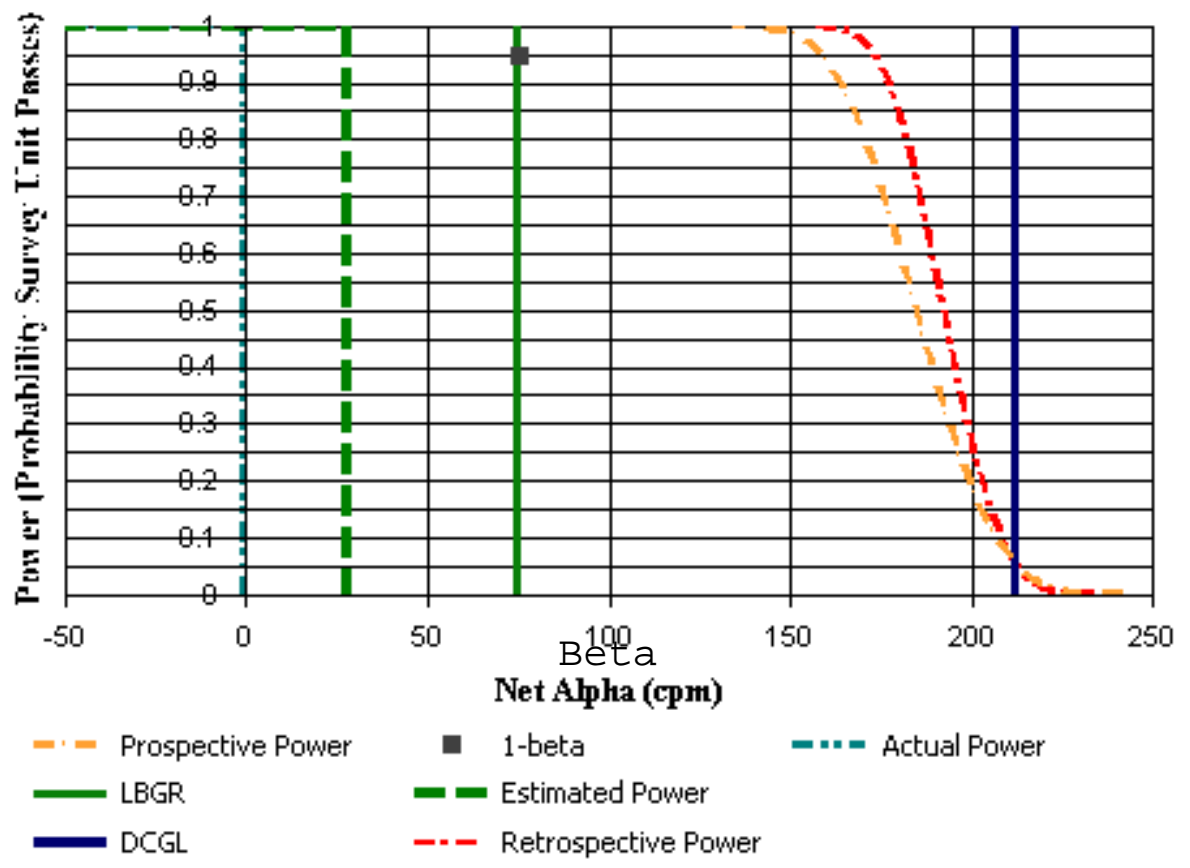




\section{DQA Building Surface Report}

\section{Survey Unit Data}

NOTE: $\quad$ Type = "S" indicates survey measurement.

Type $=$ "R" indicates reference measurement.

\begin{tabular}{lccc} 
Measurement & Material & Type & Gross Alpha (cpm) \\
\hline $2.1,12.2$ & Slag & S & 464 \\
$5.0,12.2$ & Slag & S & 451 \\
$7.8,12.2$ & Slag & S & 480 \\
$0.6,14.7$ & Slag & S & 461 \\
$3.5,14.7$ & Slag & S & 485 \\
$6.4,14.7$ & Slag & S & 489 \\
$9.3,14.7$ & Slag & S & 439 \\
$2.1,17.2$ & Slag & S & 537 \\
$5.0,17.2$ & Slag & S & 465 \\
$7.8,17.2$ & Slag & S & 444 \\
$0.6,19.7$ & Slag & S & 559 \\
$3.5,19.7$ & Slag & S & 466 \\
$6.4,19.7$ & Slag & S & 496 \\
$9.3,19.7$ & Slag & S & 517
\end{tabular}

\section{Basic Statistical Quantities Summary}

Statistic

Sample Number

Mean $\left(\mathrm{dpm} / 100 \mathrm{~cm}^{2}\right)$

Median (dpm/100 cm²)

Std Dev (dpm/100 $\left.\mathrm{cm}^{2}\right)$

High Value $\left(\mathrm{dpm} / 100 \mathrm{~cm}^{2}\right)$

Low Value $\left(\mathrm{dpm} / 100 \mathrm{~cm}^{2}\right)$
Survey Unit

14
17.02
-0.66
66.29
161.85
-64.91

Background

N/A

N/A

N/A

N/A

N/A

N/A
DQO Results

$\mathrm{N}=14$

27.6

N/A

48.3

N/A

N/A 


\section{DQA Building Surface Report}

\section{Assessment Summary}

Site:

Planner(s):

Survey Unit Name:

Report Number:

Survey Unit Measurements:

Reference Area Measurements:

Test Performed:

Judgmental Areas:

Assessment Conclusion:
Hammond Depot FSS Planner

Vitkus

C1 SU1 through 21 Bldg 200E slag Rev. 1 C1 SU6

6

14

0

Sign

Test Result:

Not Performed

0

EMC Result:

Not Performed

Reject Null Hypothesis (Survey Unit PASSES)

\section{Retrospective Power Curve}

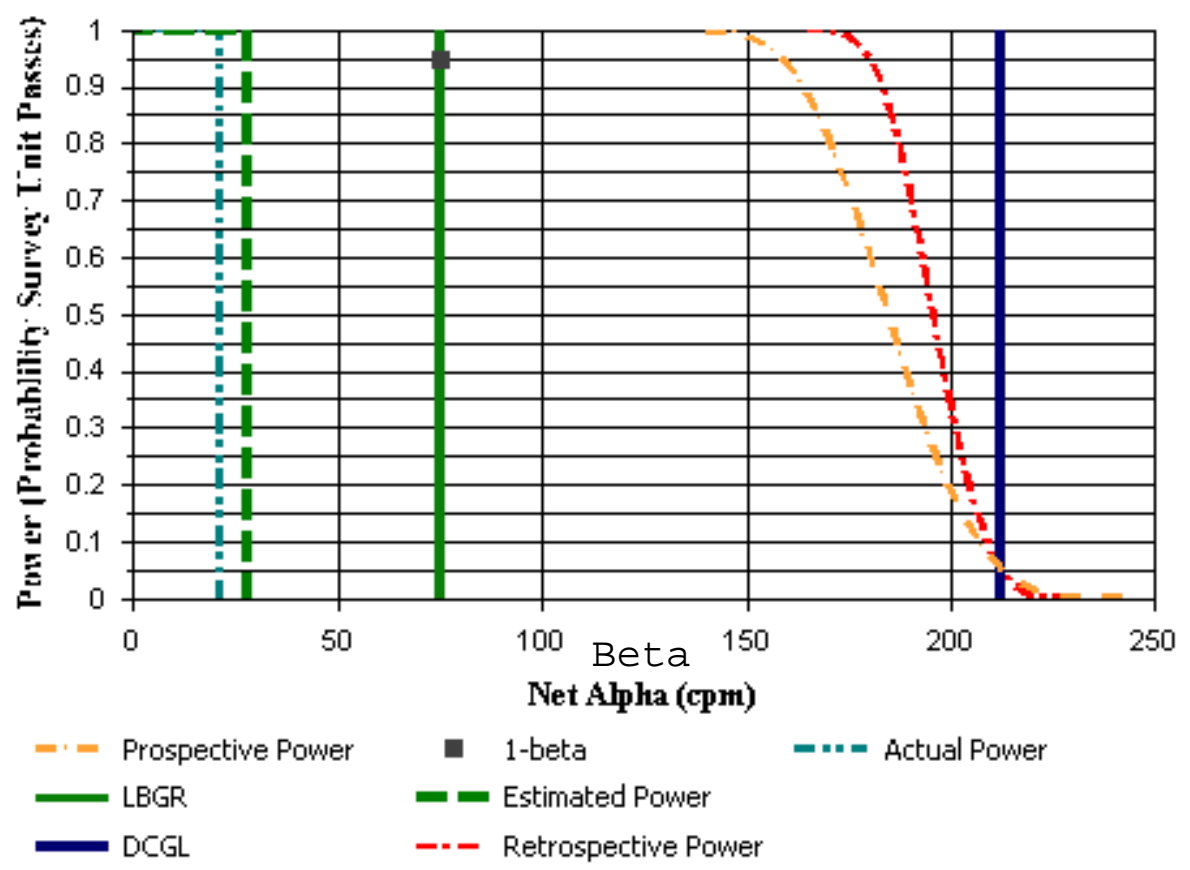




\section{DQA Building Surface Report}

\section{Survey Unit Data}

NOTE: $\quad$ Type $=$ "S" indicates survey measurement.

Type $=$ "R" indicates reference measurement.

\begin{tabular}{lccc} 
Measurement & Material & Type & Gross Alpha (cpm) \\
\hline $10.7,11.3$ & Slag & S & 486 \\
$13.5,11.3$ & Slag & S & 469 \\
$16.4,11.3$ & Slag & S & 508 \\
$19.3,11.3$ & Slag & S & 494 \\
$12.1,13.8$ & Slag & S & 483 \\
$15.0,13.8$ & Slag & S & 475 \\
$17.8,13.8$ & Slag & S & 503 \\
$10.7,16.3$ & Slag & S & 483 \\
$13.5,16.3$ & Slag & S & 493 \\
$16.4,16.3$ & Slag & S & 492 \\
$19.3,16.3$ & Slag & S & 488 \\
$14.5,11.2$ & Slag & S & 448 \\
$10.8,15.8$ & Slag & S & 428 \\
$16.9,16.1$ & Slag & S & 407
\end{tabular}

\section{Basic Statistical Quantities Summary}

Statistic

Sample Number

Mean $\left(\mathrm{dpm} / 100 \mathrm{~cm}^{2}\right)$

Median (dpm/100 cm²)

Std Dev (dpm/100 $\left.\mathrm{cm}^{2}\right)$

High Value $\left(\mathrm{dpm} / 100 \mathrm{~cm}^{2}\right)$

Low Value $\left(\mathrm{dpm} / 100 \mathrm{~cm}^{2}\right)$
Survey Unit

14

4.06

21.07

54.62

65.48

$-125.38$
Background

DQO Results

$\begin{array}{ll}\text { N/A } & \mathrm{N}=14 \\ \text { N/A } & 27.6 \\ \text { N/A } & \text { N/A } \\ \text { N/A } & 48.3 \\ \text { N/A } & \text { N/A } \\ \text { N/A } & \text { N/A }\end{array}$




\section{DQA Building Surface Report}

\section{Assessment Summary}

Site:

Planner(s):

Survey Unit Name:

Report Number:

Survey Unit Measurements:

Reference Area Measurements:

Test Performed:

Judgmental Areas:

Assessment Conclusion:
Hammond Depot FSS Planner

Vitkus

C1 SU1 through 21 Bldg 200E slag Rev. 1 C1 SU7

7

14

0

Sign

Test Result:

Not Performed

0

EMC Result:

Not Performed

Reject Null Hypothesis (Survey Unit PASSES)

\section{Retrospective Power Curve}

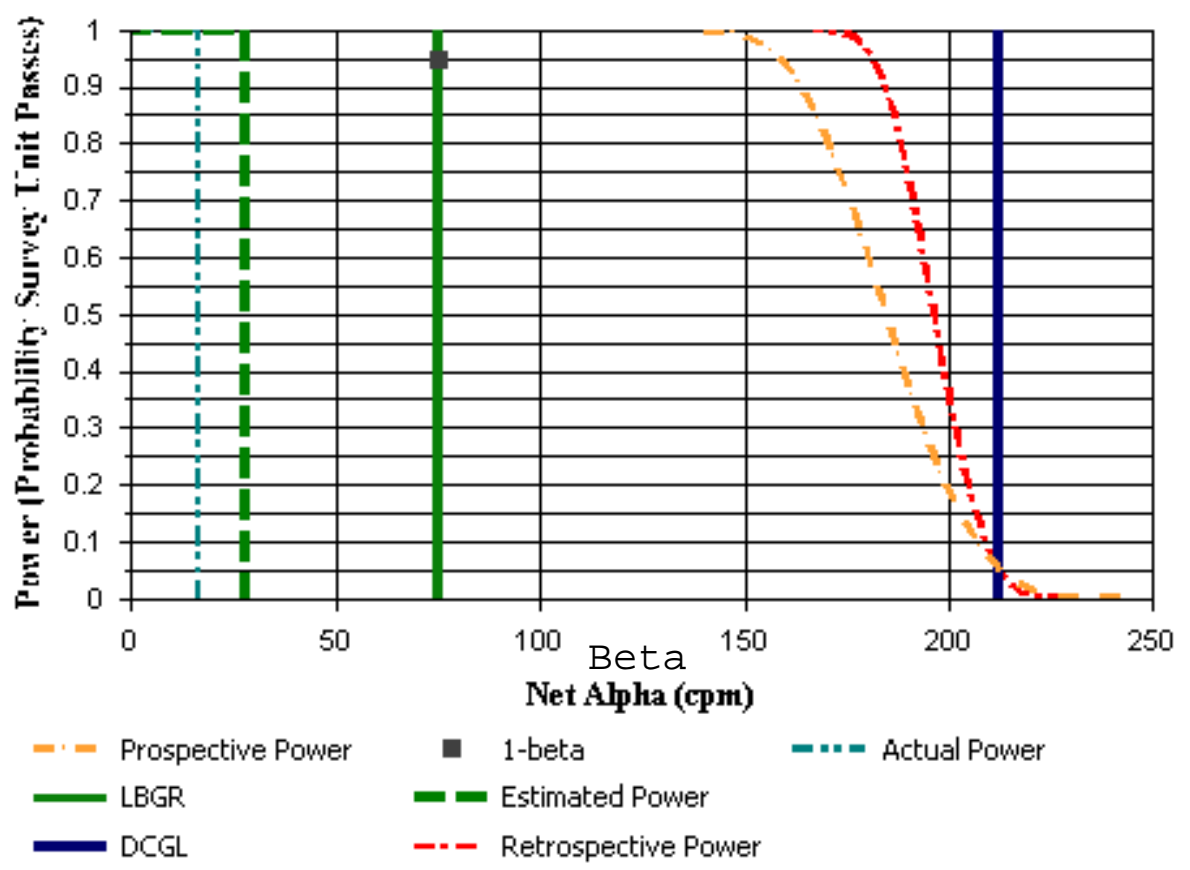




\section{DQA Building Surface Report}

\section{Survey Unit Data}

NOTE: $\quad$ Type = "S" indicates survey measurement.

Type $=$ "R" indicates reference measurement.

\begin{tabular}{lccc} 
Measurement & Material & Type & Gross Alpha (cpm) \\
\hline $20.1,12.3$ & Slag & S & 449 \\
$23.0,12.3$ & Slag & S & 430 \\
$25.8,12.3$ & Slag & S & 493 \\
$28.7,12.3$ & Slag & S & 497 \\
$21.5,14.7$ & Slag & S & 475 \\
$24.4,14.7$ & Slag & S & 446 \\
$27.3,14.7$ & Slag & S & 485 \\
$21.3,16.4$ & Slag & S & 426 \\
$23.0,17.2$ & Slag & S & 457 \\
$25.8,17.2$ & Slag & S & 479 \\
$28.7,17.2$ & Slag & S & 488 \\
$28.6,19.1$ & Slag & S & 493 \\
$24.4,19.7$ & Slag & S & 508 \\
$27.3,19.7$ & Slag & S & 510
\end{tabular}

\section{Basic Statistical Quantities Summary}

Statistic

Sample Number

Mean $\left(\mathrm{dpm} / 100 \mathrm{~cm}^{2}\right)$

Median (dpm/100 cm²)

Std Dev (dpm/100 $\left.\mathrm{cm}^{2}\right)$

High Value $\left(\mathrm{dpm} / 100 \mathrm{~cm}^{2}\right)$

Low Value $\left(\mathrm{dpm} / 100 \mathrm{~cm}^{2}\right)$
Survey Unit

14
1.23
16.35
52.37
69.26
-89.47

14

$-89.47$
Background

DQO Results

$\begin{array}{ll}\text { N/A } & \mathrm{N}=14 \\ \text { N/A } & 27.6 \\ \text { N/A } & \text { N/A } \\ \text { N/A } & 48.3 \\ \text { N/A } & \text { N/A } \\ \text { N/A } & \text { N/A }\end{array}$




\section{DQA Building Surface Report}

\section{Assessment Summary}

Site:

Planner(s):

Survey Unit Name:

Report Number:

Survey Unit Measurements:

Reference Area Measurements:

Test Performed:

Judgmental Areas:

Assessment Conclusion:
Hammond Depot FSS Planner

Vitkus

C1 SU1 through 21 Bldg 200E slag Rev. 1 C1 SU8

8

14

0

Sign

Test Result:

Not Performed

0

EMC Result:

Not Performed

Reject Null Hypothesis (Survey Unit PASSES)

\section{Retrospective Power Curve}

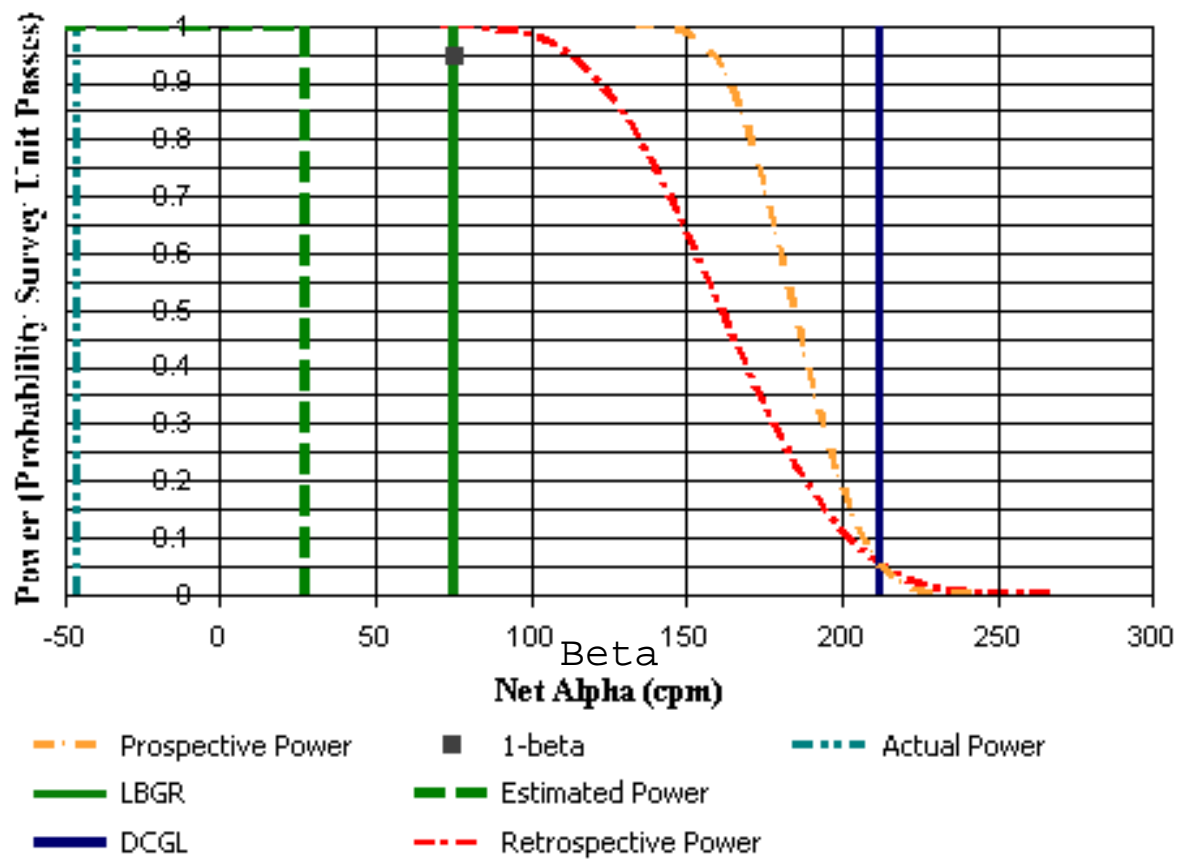




\section{DQA Building Surface Report}

\section{Survey Unit Data}

NOTE: $\quad$ Type = "S" indicates survey measurement.

Type $=$ "R" indicates reference measurement.

\begin{tabular}{lccc} 
Measurement & Material & Type & Gross Alpha (cpm) \\
\hline $1.0,22.2$ & Slag & S & 480 \\
$3.9,22.2$ & Slag & S & 444 \\
$6.8,22.2$ & Slag & S & 478 \\
$9.7,22.2$ & Slag & S & 466 \\
$2.5,24.7$ & Slag & S & 256 \\
$5.4,24.7$ & Slag & S & 595 \\
$8.2,24.7$ & Slag & S & 384 \\
$1.0,27.2$ & Slag & S & 437 \\
$3.9,27.2$ & Slag & S & 538 \\
$6.8,27.2$ & Slag & S & 433 \\
$9.7,27.2$ & Slag & S & 454 \\
$2.5,29.7$ & Slag & S & 376 \\
$5.4,29.7$ & Slag & S & 356 \\
$8.2,29.7$ & Slag & S & 583
\end{tabular}

\section{Basic Statistical Quantities Summary}

Statistic

Sample Number

Mean $\left(\mathrm{dpm} / 100 \mathrm{~cm}^{2}\right)$

Median (dpm/100 cm²)

Std Dev (dpm/100 $\left.\mathrm{cm}^{2}\right)$

High Value $\left(\mathrm{dpm} / 100 \mathrm{~cm}^{2}\right)$

Low Value $\left(\mathrm{dpm} / 100 \mathrm{~cm}^{2}\right)$
Survey Unit

14

$-46.82$

$-46.01$

169.97

229.88

$-410.71$
Background

DQO Results

$\begin{array}{ll}\text { N/A } & \mathrm{N}=14 \\ \text { N/A } & 27.6 \\ \text { N/A } & \text { N/A } \\ \text { N/A } & 48.3 \\ \text { N/A } & \text { N/A } \\ \text { N/A } & \text { N/A }\end{array}$




\section{DQA Building Surface Report}

\section{Assessment Summary}

Site:

Planner(s):

Survey Unit Name:

Report Number:

Survey Unit Measurements:

Reference Area Measurements:

Test Performed:

Judgmental Areas:

Assessment Conclusion:
Hammond Depot FSS Planner

Vitkus

C1 SU1 through 21 Bldg 200E slag Rev. 1 C1 SU9

9

14

0

Sign

Test Result:

Not Performed

0

EMC Result:

Not Performed

Reject Null Hypothesis (Survey Unit PASSES)

\section{Retrospective Power Curve}

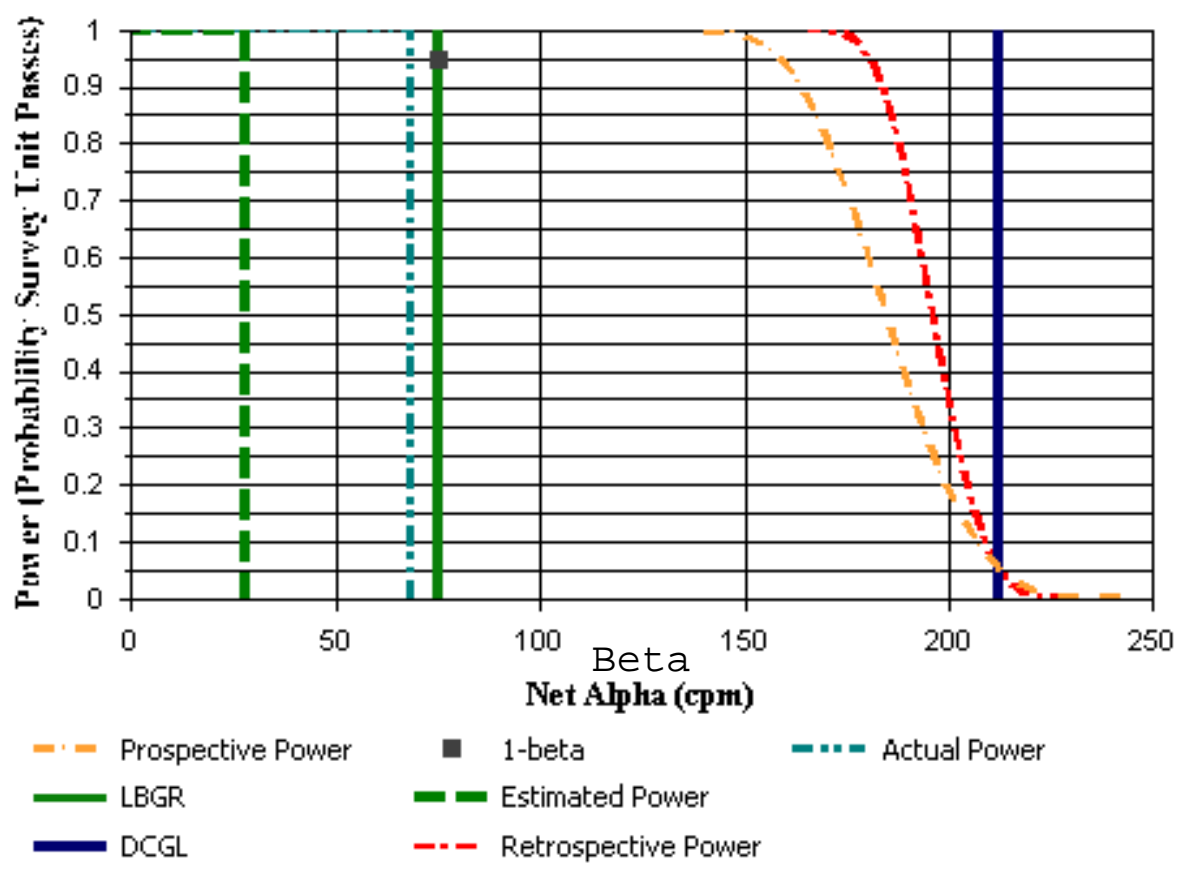




\section{DQA Building Surface Report}

\section{Survey Unit Data}

NOTE: $\quad$ Type = "S" indicates survey measurement.

Type $=$ "R" indicates reference measurement.

Measurement

$10.1,21.8$

$13.0,21.8$

$15.9,21.8$

$18.7,21.8$

$11.6,24.3$

$14.4,24.3$

$17.3,24.3$

$10.1,26.8$

$13.0,26.8$

$15.9,26.8$

$18.7,26.8$

$11.6,29.2$

$14.4,29.2$

$17.3,29.2$

Material

Slag

Slag

Slag

Slag

Slag

Slag

Slag

Slag

Slag

Slag

Slag

Slag

Slag

Slag
Beta

Type Gross Alpha (cpm)

509

535

500

511

510

476

580

534

499

534

484

534

485

485

\section{Basic Statistical Quantities Summary}

Statistic

Sample Number

Mean $\left(\mathrm{dpm} / 100 \mathrm{~cm}^{2}\right)$

Median (dpm/100 cm²)

Std Dev (dpm/100 $\left.\mathrm{cm}^{2}\right)$

High Value $\left(\mathrm{dpm} / 100 \mathrm{~cm}^{2}\right)$

Low Value $\left(\mathrm{dpm} / 100 \mathrm{~cm}^{2}\right)$
Survey Unit

14
74.11
68.31
53.62
201.53
5.01

5.01
Background

DQO Results

$\begin{array}{ll}\text { N/A } & \mathrm{N}=14 \\ \text { N/A } & 27.6 \\ \text { N/A } & \text { N/A } \\ \text { N/A } & 48.3 \\ \text { N/A } & \text { N/A } \\ \text { N/A } & \text { N/A }\end{array}$




\section{DQA Building Surface Report}

\section{Assessment Summary}

Site:

Planner(s):

Survey Unit Name:

Report Number:

Survey Unit Measurements:

Reference Area Measurements:

Test Performed:

Judgmental Areas:

Assessment Conclusion:
Hammond Depot FSS Planner

Vitkus

C1 SU1 through 21 Bldg 200E slag Rev. 1 C1 SU10

10

14

0

Sign

Test Result:

Not Performed

0

EMC Result:

Not Performed

Reject Null Hypothesis (Survey Unit PASSES)

\section{Retrospective Power Curve}

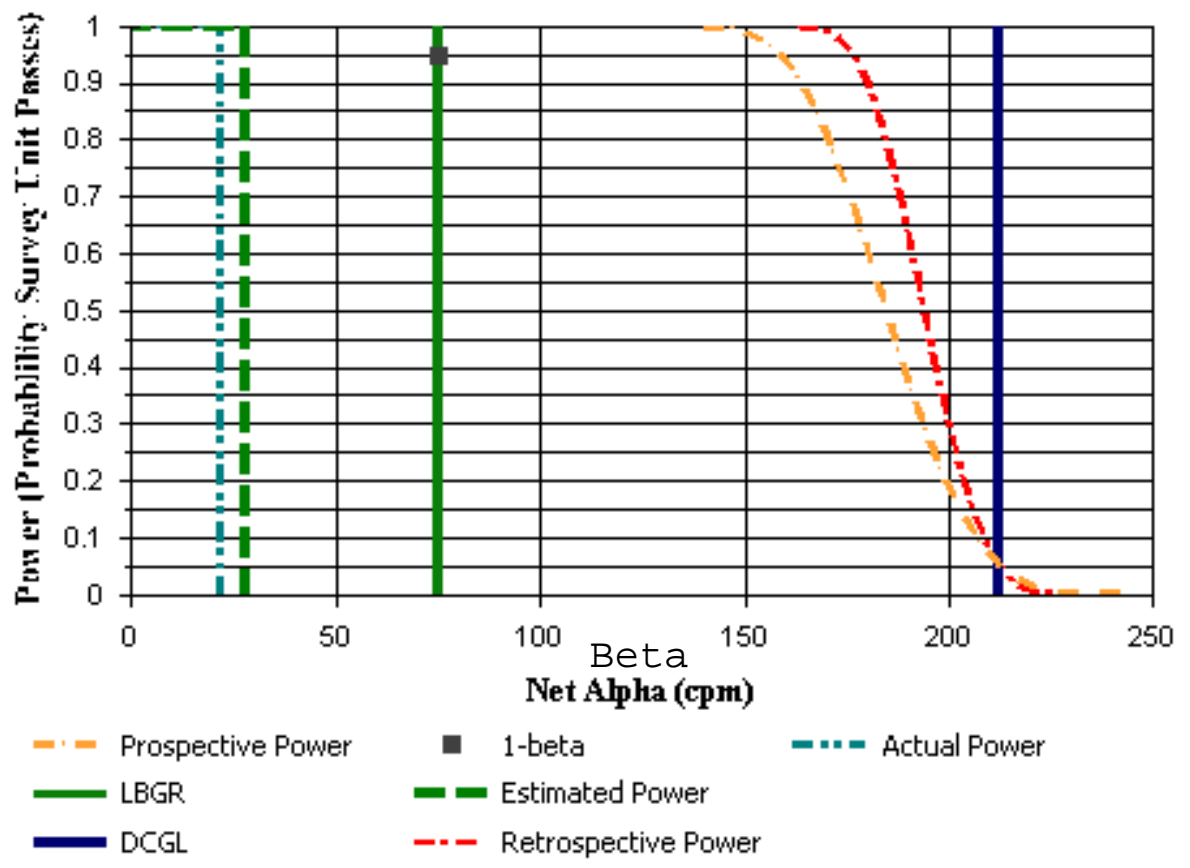




\section{DQA Building Surface Report}

\section{Survey Unit Data}

NOTE: $\quad$ Type $=$ "S" indicates survey measurement.

Type $=$ "R" indicates reference measurement.

\begin{tabular}{lccc} 
Measurement & Material & Type & Gross Alpha (cpm) \\
\hline $21.3,21.4$ & Slag & S & 493 \\
$24.1,21.4$ & Slag & S & 578 \\
$27.0,21.4$ & Slag & S & 479 \\
$29.9,21.4$ & Slag & S & 456 \\
$24.8,25.0$ & Slag & S & 526 \\
$25.6,23.9$ & Slag & S & 470 \\
$28.4,23.9$ & Slag & S & 485 \\
$21.3,26.4$ & Slag & S & 489 \\
$24.1,26.4$ & Slag & S & 485 \\
$27.0,26.4$ & Slag & S & 486 \\
$29.9,26.4$ & Slag & S & 467 \\
$22.7,28.9$ & Slag & S & 466 \\
$25.6,28.9$ & Slag & S & 491 \\
$28.4,28.9$ & Slag & S & 451
\end{tabular}

\section{Basic Statistical Quantities Summary}

Statistic

Sample Number

Mean $\left(\mathrm{dpm} / 100 \mathrm{~cm}^{2}\right)$

Median (dpm/100 cm²)

Std Dev (dpm/100 $\left.\mathrm{cm}^{2}\right)$

High Value $\left(\mathrm{dpm} / 100 \mathrm{~cm}^{2}\right)$

Low Value $\left(\mathrm{dpm} / 100 \mathrm{~cm}^{2}\right)$
Survey Unit

14
26.33
22.01
60.48
197.75
-42.23

Background

N/A

N/A

N/A

N/A

N/A

N/A
DQO Results

$\mathrm{N}=14$

27.6

N/A

48.3

N/A

N/A 


\section{DQA Building Surface Report}

\section{Assessment Summary}

Site:

Planner(s):

Survey Unit Name:

Report Number:

Survey Unit Measurements:

Reference Area Measurements:

Test Performed:

Judgmental Areas:

Assessment Conclusion:
Hammond Depot FSS Planner

Vitkus

C1 SU1 through 21 Bldg 200E slag Rev. 1 C1 SU11

11

14

0

Sign

Test Result:

Not Performed

0

EMC Result:

Not Performed

\section{Reject Null Hypothesis (Survey Unit PASSES)}

\section{Retrospective Power Curve}

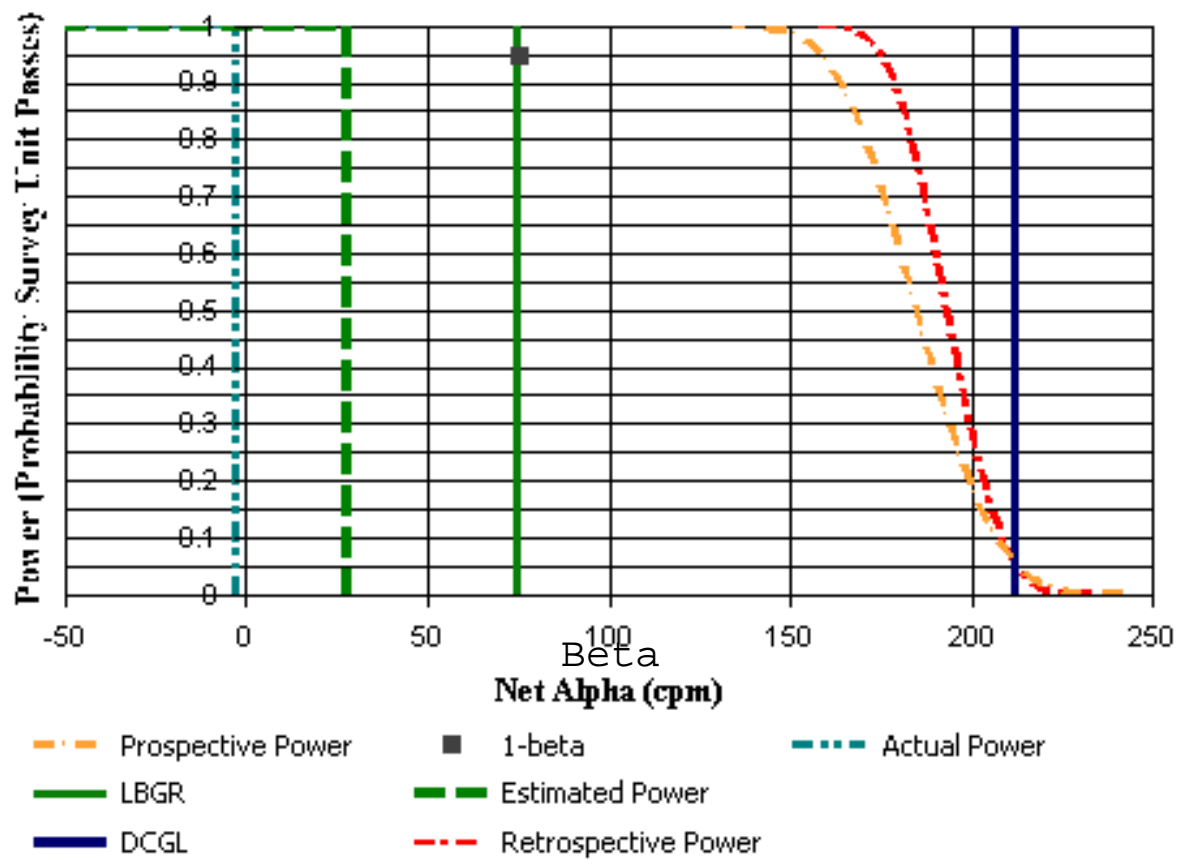




\section{DQA Building Surface Report}

\section{Survey Unit Data}

NOTE: $\quad$ Type $=$ "S" indicates survey measurement.

Type $=$ "R" indicates reference measurement.

Measurement

$31.2,20.0$

$34.8,20.0$

$33.0,23.1$

$36.5,23.1$

$31.2,26.1$

$34.8,26.1$

$33.0,29.2$

$36.5,29.2$

$31.2,32.2$

$34.8,32.2$

$33.0,35.3$

$36.5,35.3$

$31.2,38.3$

$34.8,38.3$

\section{Material}

Slag

Slag

Slag

Slag

Slag

Slag

Slag

Slag

Slag

Slag

Slag

Slag

Slag

Slag
Beta

Type Gross Alpha (cpm)

406

478

475

414

481

503

477

410

471

500

430

473

452

425

\section{Basic Statistical Quantities Summary}

Statistic

Sample Number

Mean $\left(\mathrm{dpm} / 100 \mathrm{~cm}^{2}\right)$

Median (dpm/100 cm²)

Std Dev (dpm/100 $\left.\mathrm{cm}^{2}\right)$

High Value $\left(\mathrm{dpm} / 100 \mathrm{~cm}^{2}\right)$

Low Value $\left(\mathrm{dpm} / 100 \mathrm{~cm}^{2}\right)$
Survey Unit

14
-31.30
-2.55
63.31
56.03
-127.27

Background

N/A

N/A

N/A

N/A

N/A

N/A
DQO Results

$\mathrm{N}=14$

27.6

N/A

48.3

N/A

N/A 


\section{DQA Building Surface Report}

\section{Assessment Summary}

Site:

Planner(s):

Survey Unit Name:

Report Number:

Survey Unit Measurements:

Reference Area Measurements:

Test Performed:

Judgmental Areas:

Assessment Conclusion:
Hammond Depot FSS Planner

Vitkus

C1 SU1 through 21 Bldg 200E slag Rev. 1 C1 SU12

12

14

0

Sign

Test Result:

Not Performed

0

EMC Result:

Not Performed

Reject Null Hypothesis (Survey Unit PASSES)

\section{Retrospective Power Curve}

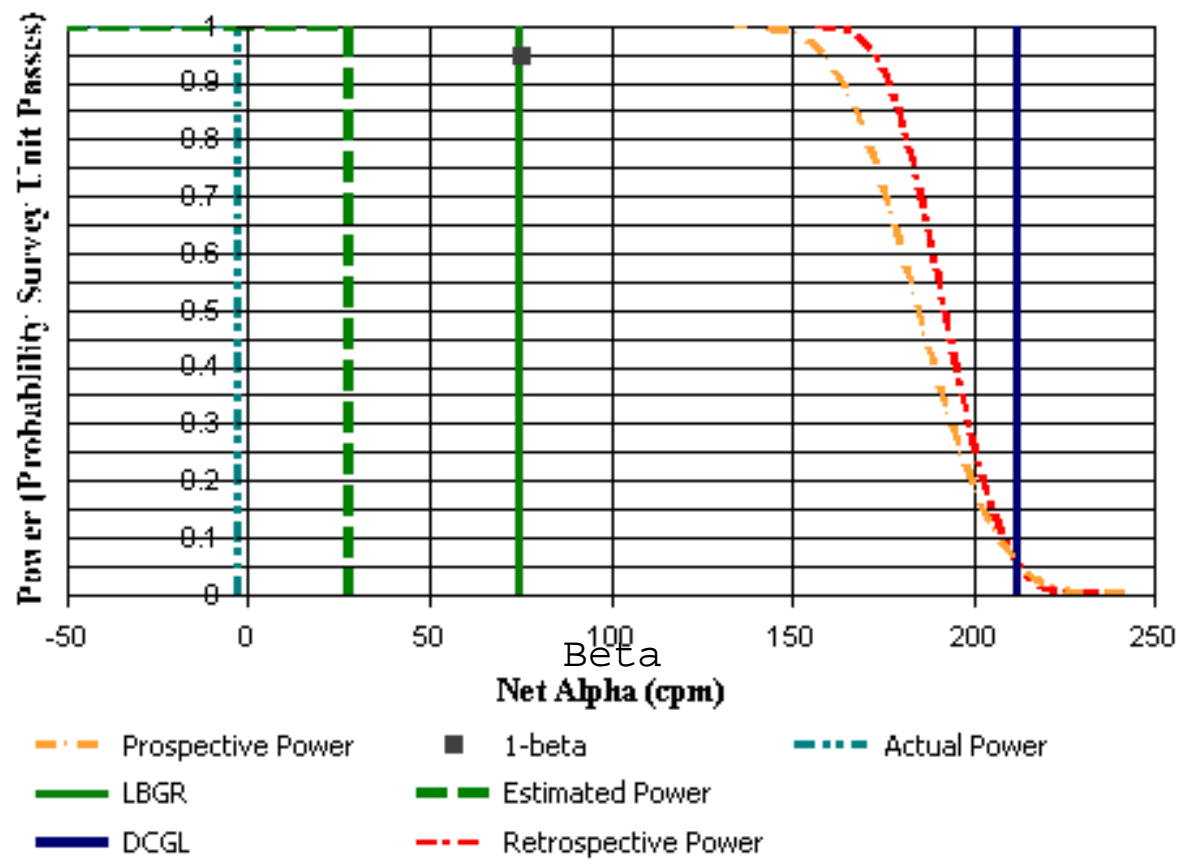




\section{DQA Building Surface Report}

\section{Survey Unit Data}

NOTE: $\quad$ Type = "S" indicates survey measurement.

Type $=$ "R" indicates reference measurement.

\begin{tabular}{lccc} 
Measurement & Material & Type & Gross Alpha (cpm) \\
\hline $1.2,31.5$ & Slag & S & 435 \\
$4.1,31.5$ & Slag & S & 514 \\
$7.0,31.5$ & Slag & S & 523 \\
$9.9,31.5$ & Slag & S & 462 \\
$2.7,34.0$ & Slag & S & 492 \\
$5.5,34.0$ & Slag & S & 476 \\
$8.4,34.0$ & Slag & S & 522 \\
$1.2,36.5$ & Slag & S & 468 \\
$4.1,36.5$ & Slag & S & 454 \\
$7.0,36.5$ & Slag & S & 553 \\
$9.9,36.5$ & Slag & S & 496 \\
$2.7,39.0$ & Slag & S & 459 \\
$5.5,39.0$ & Slag & S & 468 \\
$8.4,39.0$ & Slag & S & 432
\end{tabular}

\section{Basic Statistical Quantities Summary}

Statistic

Sample Number

Mean $\left(\mathrm{dpm} / 100 \mathrm{~cm}^{2}\right)$

Median (dpm/100 cm²)

Std Dev (dpm/100 $\left.\mathrm{cm}^{2}\right)$

High Value $\left(\mathrm{dpm} / 100 \mathrm{~cm}^{2}\right)$

Low Value $\left(\mathrm{dpm} / 100 \mathrm{~cm}^{2}\right)$
Survey Unit

14

17.16

$-2.55$

67.30

150.51

$-78.14$
Background

DQO Results

$\begin{array}{ll}\text { N/A } & \mathrm{N}=14 \\ \text { N/A } & 27.6 \\ \text { N/A } & \text { N/A } \\ \text { N/A } & 48.3 \\ \text { N/A } & \text { N/A } \\ \text { N/A } & \text { N/A }\end{array}$




\section{DQA Building Surface Report}

\section{Assessment Summary}

Site:

Planner(s):

Survey Unit Name:

Report Number:

Survey Unit Measurements:

Reference Area Measurements:

Test Performed:

Judgmental Areas:

Assessment Conclusion:
Hammond Depot FSS Planner

Vitkus

C1 SU1 through 21 Bldg 200E slag Rev. 1 C1 SU13

13

14

0

Sign

Test Result:

Not Performed

0

EMC Result:

Not Performed

Reject Null Hypothesis (Survey Unit PASSES)

\section{Retrospective Power Curve}

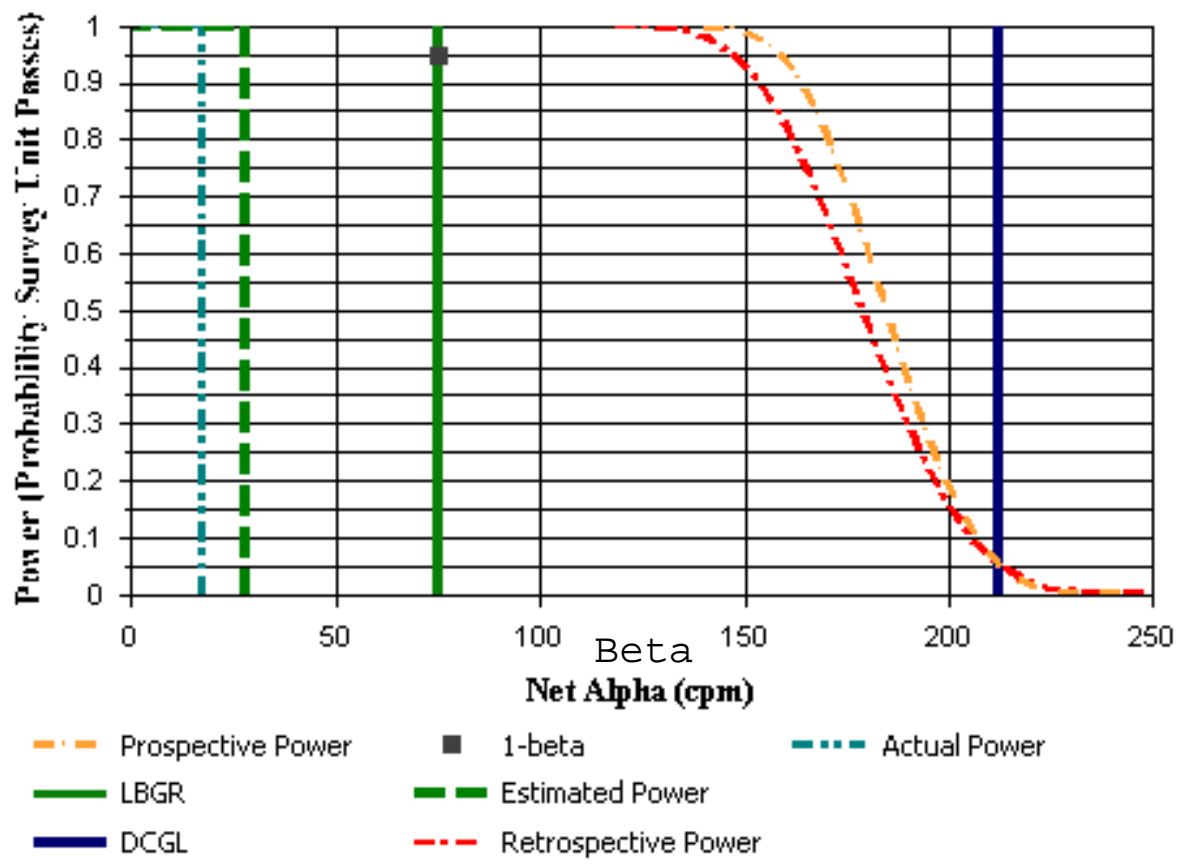




\section{DQA Building Surface Report}

\section{Survey Unit Data}

NOTE: $\quad$ Type = "S" indicates survey measurement.

Type $=$ "R" indicates reference measurement.

\begin{tabular}{lccc} 
Measurement & Material & Type & Gross Alpha (cpm) \\
\hline $12.6,30.8$ & Slag & S & 505 \\
$15.5,30.8$ & Slag & S & 392 \\
$18.4,30.8$ & Slag & S & 462 \\
$11.2,33.3$ & Slag & S & 477 \\
$14.0,33.3$ & Slag & S & 581 \\
$16.9,33.3$ & Slag & S & 446 \\
$19.8,33.3$ & Slag & S & 509 \\
$12.6,35.8$ & Slag & S & 488 \\
$15.5,35.8$ & Slag & S & 476 \\
$18.4,35.8$ & Slag & S & 638 \\
$11.2,38.3$ & Slag & S & 474 \\
$14.0,38.3$ & Slag & S & 518 \\
$16.9,38.3$ & Slag & $\mathrm{S}$ & 500 \\
$19.8,38.3$ & Slag & S & 472
\end{tabular}

\section{Basic Statistical Quantities Summary}

Statistic

Sample Number

Mean $\left(\mathrm{dpm} / 100 \mathrm{~cm}^{2}\right)$

Median (dpm/100 cm²)

Std Dev (dpm/100 $\left.\mathrm{cm}^{2}\right)$

High Value $\left(\mathrm{dpm} / 100 \mathrm{~cm}^{2}\right)$

Low Value $\left(\mathrm{dpm} / 100 \mathrm{~cm}^{2}\right)$
Survey Unit

14

41.99

17.29

110.55

311.13

$-153.72$
Background

DQO Results

$\begin{array}{ll}\text { N/A } & \mathrm{N}=14 \\ \text { N/A } & 27.6 \\ \text { N/A } & \text { N/A } \\ \text { N/A } & 48.3 \\ \text { N/A } & \text { N/A } \\ \text { N/A } & \text { N/A }\end{array}$




\section{DQA Building Surface Report}

\section{Assessment Summary}

Site:

Planner(s):

Survey Unit Name:

Report Number:

Survey Unit Measurements:

Reference Area Measurements:

Test Performed:

Judgmental Areas:

Assessment Conclusion:
Hammond Depot FSS Planner

Vitkus

C1 SU1 through 21 Bldg 200E slag Rev. 1 C1 SU14

14

14

0

Sign

Test Result:

Not Performed

0

EMC Result:

Not Performed

Reject Null Hypothesis (Survey Unit PASSES)

\section{Retrospective Power Curve}

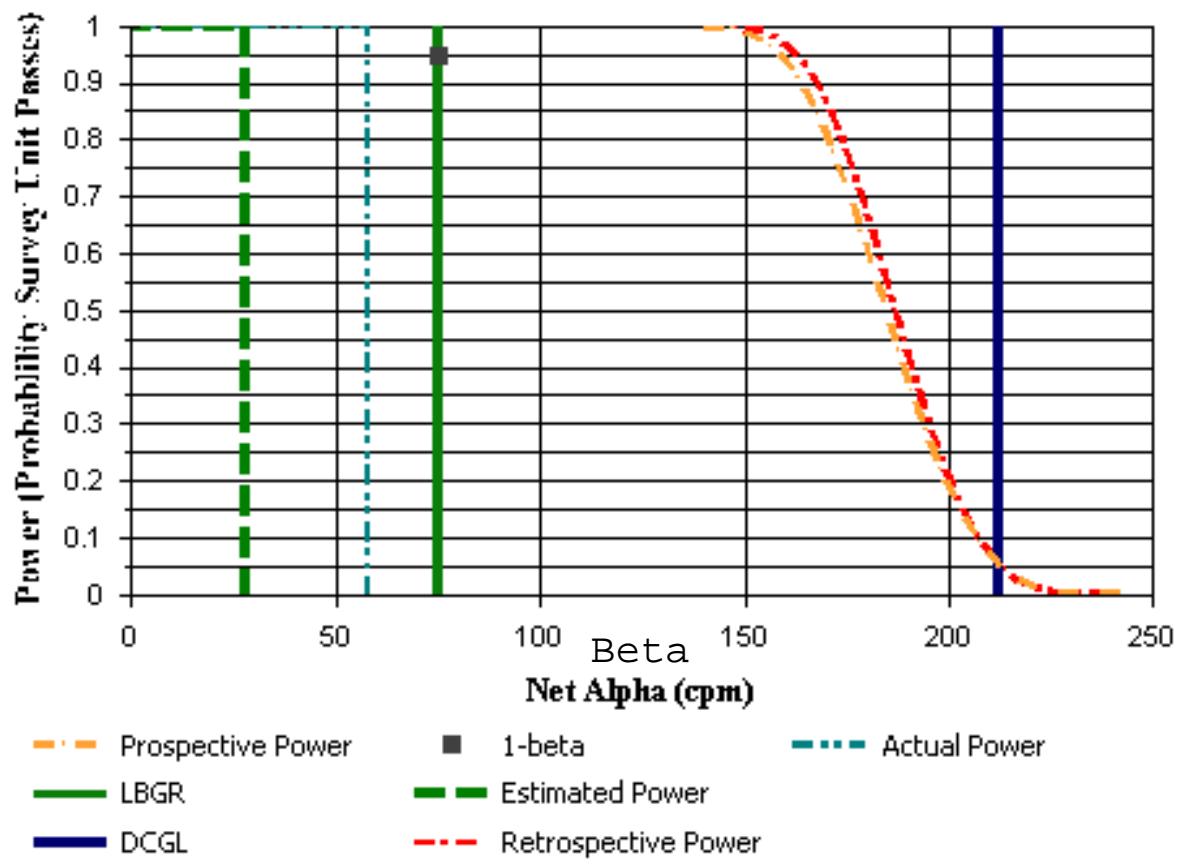




\section{DQA Building Surface Report}

\section{Survey Unit Data}

NOTE: $\quad$ Type $=$ "S" indicates survey measurement.

Type = "R" indicates reference measurement.

Beta

Measurement

$22.5,31.8$

$25.4,31.8$

$28.2,31.8$

$21.0,34.3$

$23.9,34.3$

$26.8,34.3$

$29.7,34.3$

$22.5,36.8$

$25.4,36.8$

$28.2,36.8$

$21.0,39.3$

$23.9,39.3$

$26.8,39.3$

$29.7,39.3$

Materia
Slag
Slag
Slag
Slag
Slag
Slag
Slag
Slag
Slag
Slag
Slag
Slag
Slag
Slag

Type Gross Alpha (cpm)

Slag $\quad$ S 484

$\begin{array}{lll}\text { Slag } & S & 502\end{array}$

Slag

Slag

Slag

Slag

Slag

Slag

Slag

Slag

Slag

Slag

479

495

489

501

530

407

520

506

582

513

537

589

\section{Basic Statistical Quantities Summary}

Statistic

Sample Number

Mean $\left(\mathrm{dpm} / 100 \mathrm{~cm}^{2}\right)$

Median (dpm/100 cm²)

Std Dev (dpm/100 $\left.\mathrm{cm}^{2}\right)$

High Value $\left(\mathrm{dpm} / 100 \mathrm{~cm}^{2}\right)$

Low Value $\left(\mathrm{dpm} / 100 \mathrm{~cm}^{2}\right)$
Survey Unit

14
68.45
57.92
84.07
218.54
-125.38

14

$-125.38$
Background

DQO Results

$\begin{array}{ll}\text { N/A } & \mathrm{N}=14 \\ \text { N/A } & 27.6 \\ \text { N/A } & \text { N/A } \\ \text { N/A } & 48.3 \\ \text { N/A } & \text { N/A } \\ \text { N/A } & \text { N/A }\end{array}$




\section{DQA Building Surface Report}

\section{Assessment Summary}

Site:

Planner(s):

Survey Unit Name:

Report Number:

Survey Unit Measurements:

Reference Area Measurements:

Test Performed:

Judgmental Areas:

Assessment Conclusion:
Hammond Depot FSS Planner

Vitkus

C1 SU1 through 21 Bldg 200E slag Rev. 1 C1 SU15

15

14

0

Sign

Test Result:

Not Performed

0

EMC Result:

Not Performed

Reject Null Hypothesis (Survey Unit PASSES)

\section{Retrospective Power Curve}

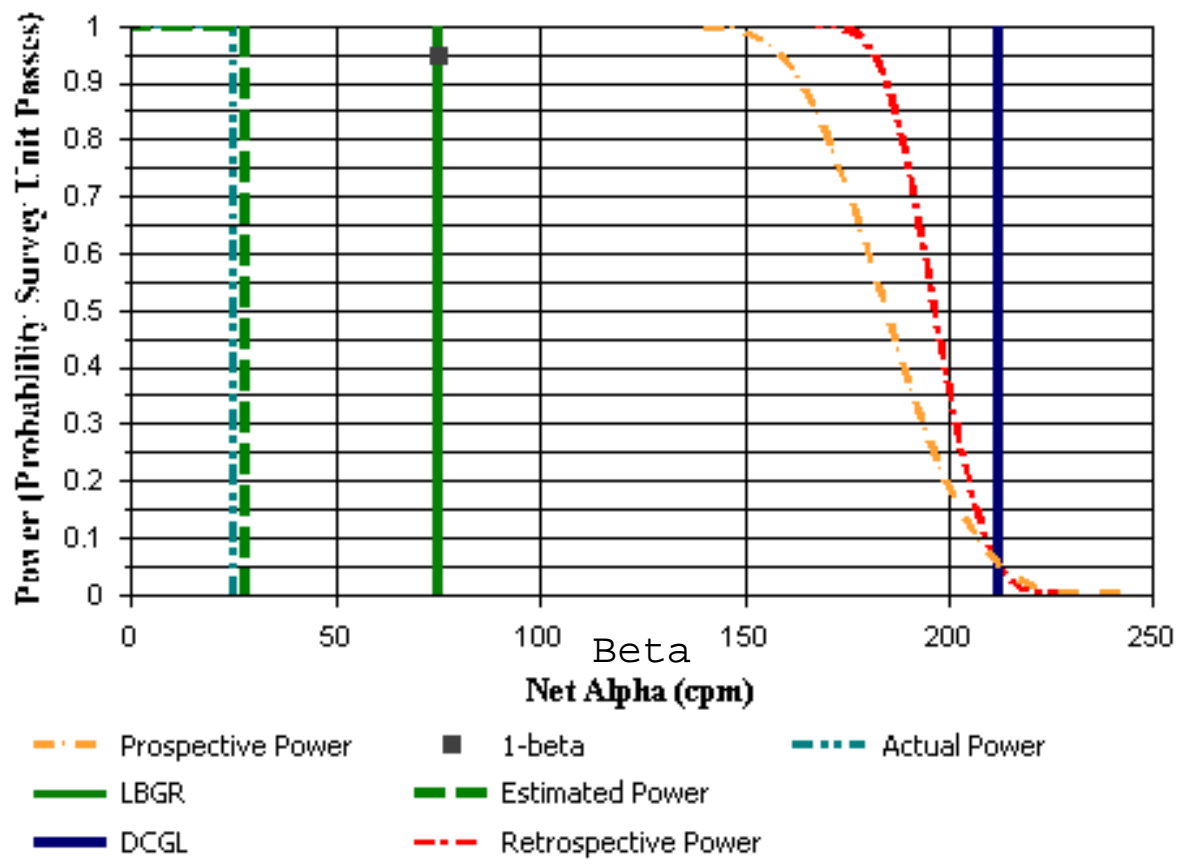




\section{DQA Building Surface Report}

\section{Survey Unit Data}

NOTE: $\quad$ Type = "S" indicates survey measurement.

Type $=$ "R" indicates reference measurement.

\begin{tabular}{lccc} 
Measurement & Material & Type & Gross Alpha (cpm) \\
\hline $0.4,41.0$ & Slag & S & 457 \\
$3.2,41.0$ & Slag & S & 505 \\
$6.1,41.0$ & Slag & S & 503 \\
$9.0,41.0$ & Slag & S & 469 \\
$1.8,43.4$ & Slag & S & 508 \\
$4.7,43.4$ & Slag & S & 456 \\
$7.5,43.4$ & Slag & S & 509 \\
$0.4,45.9$ & Slag & S & 493 \\
$3.2,45.9$ & Slag & S & 465 \\
$6.1,45.9$ & Slag & S & 480 \\
$9.0,45.9$ & Slag & S & 512 \\
$1.8,48.4$ & Slag & S & 471 \\
$4.7,48.4$ & Slag & S & 522 \\
$7.5,48.4$ & Slag & S & 428
\end{tabular}

\section{Basic Statistical Quantities Summary}

Statistic

Sample Number

Mean $\left(\mathrm{dpm} / 100 \mathrm{~cm}^{2}\right)$

Median (dpm/100 cm²)

Std Dev (dpm/100 $\left.\mathrm{cm}^{2}\right)$

High Value $\left(\mathrm{dpm} / 100 \mathrm{~cm}^{2}\right)$

Low Value $\left(\mathrm{dpm} / 100 \mathrm{~cm}^{2}\right)$
Survey Unit

14
20.39
24.85
51.70
91.93
-85.70

Background

N/A

N/A

N/A

N/A

N/A

N/A
DQO Results

$\mathrm{N}=14$

27.6

N/A

48.3

N/A

N/A 


\section{DQA Building Surface Report}

\section{Assessment Summary}

Site:

Planner(s):

Survey Unit Name:

Report Number:

Survey Unit Measurements:

Reference Area Measurements:

Test Performed:

Judgmental Areas:

Assessment Conclusion:
Hammond Depot FSS Planner

Vitkus

C1 SU1 through 21 Bldg 200E slag Rev. 1 C1 SU16

16

14

0

Sign

Test Result:

Not Performed

0

EMC Result:

Not Performed

\section{Retrospective Power Curve}

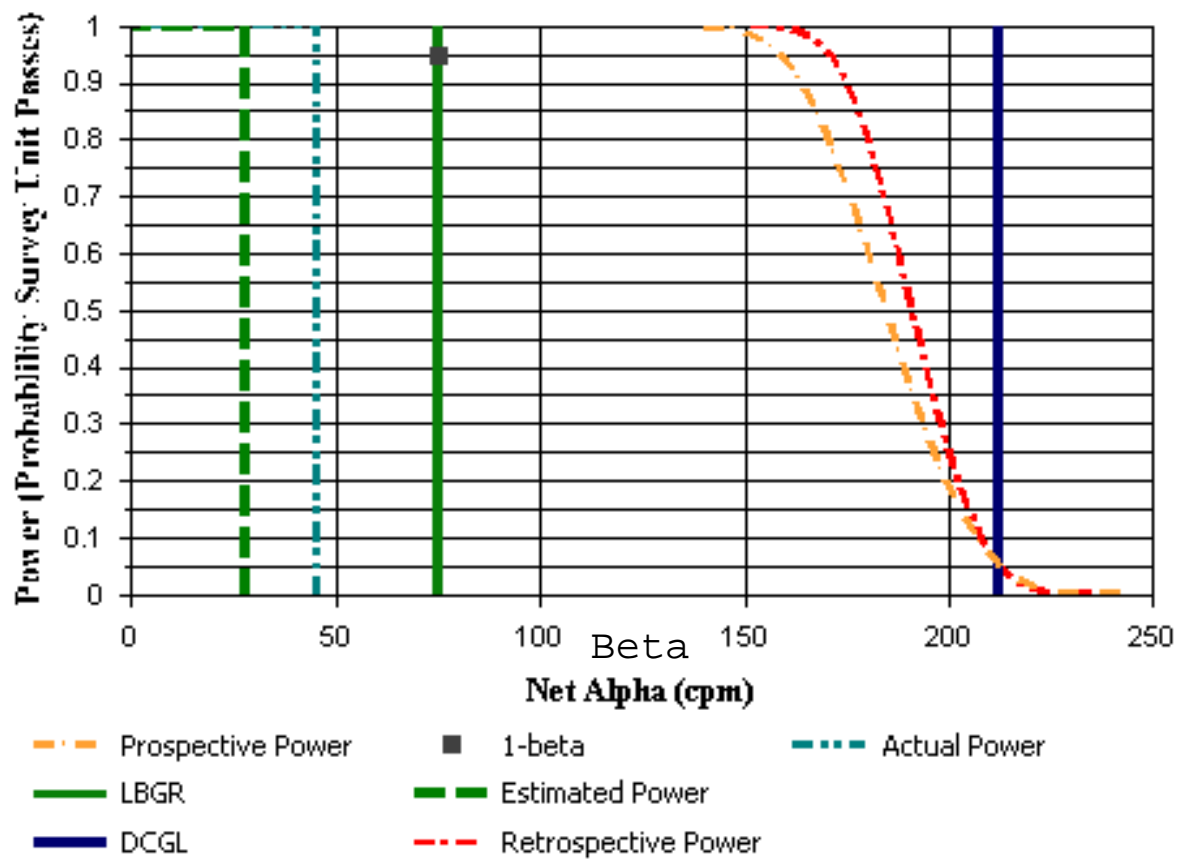




\section{DQA Building Surface Report}

\section{Survey Unit Data}

NOTE: $\quad$ Type $=$ "S" indicates survey measurement.

Type $=$ "R" indicates reference measurement.

Measurement

$11.1,41.4$

$14.0,41.4$

$16.8,41.4$

$19.7,41.4$

$12.5,43.9$

$15.4,43.9$

$18.3,43.9$

$11.1,46.4$

$14.0,46.4$

$16.8,46.4$

$19.7,46.4$

$12.5,48.9$

$15.4,48.9$

$18.3,48.9$
Material

Slag

Slag

Slag

Slag

Slag

Slag

Slag

Slag

Slag

Slag

Slag

Slag

Slag

Slag
Beta

Type Gross Alpha (cpm)

494

520

501

565

539

512

518

475

485

446

450

532

451

457

\section{Basic Statistical Quantities Summary}

Statistic

Sample Number

Mean $\left(\mathrm{dpm} / 100 \mathrm{~cm}^{2}\right)$

Median (dpm/100 cm²)

Std Dev (dpm/100 $\left.\mathrm{cm}^{2}\right)$

High Value $\left(\mathrm{dpm} / 100 \mathrm{~cm}^{2}\right)$

Low Value $\left(\mathrm{dpm} / 100 \mathrm{~cm}^{2}\right)$
Survey Unit

14

42.94

45.63

70.27

173.19

$-51.68$
Background

DQO Results

$\begin{array}{ll}\text { N/A } & \mathrm{N}=14 \\ \text { N/A } & 27.6 \\ \text { N/A } & \text { N/A } \\ \text { N/A } & 48.3 \\ \text { N/A } & \text { N/A } \\ \text { N/A } & \text { N/A }\end{array}$




\section{DQA Building Surface Report}

\section{Assessment Summary}

Site:

Planner(s):

Survey Unit Name:

Report Number:

Survey Unit Measurements:

Reference Area Measurements:

Test Performed:

Judgmental Areas:

Assessment Conclusion:
Hammond Depot FSS Planner

Vitkus

C1 SU1 through 21 Bldg 200E slag Rev. 1 C1 SU17

17

14

0

Sign

Test Result:

Not Performed

0

EMC Result:

Not Performed

\section{Retrospective Power Curve}

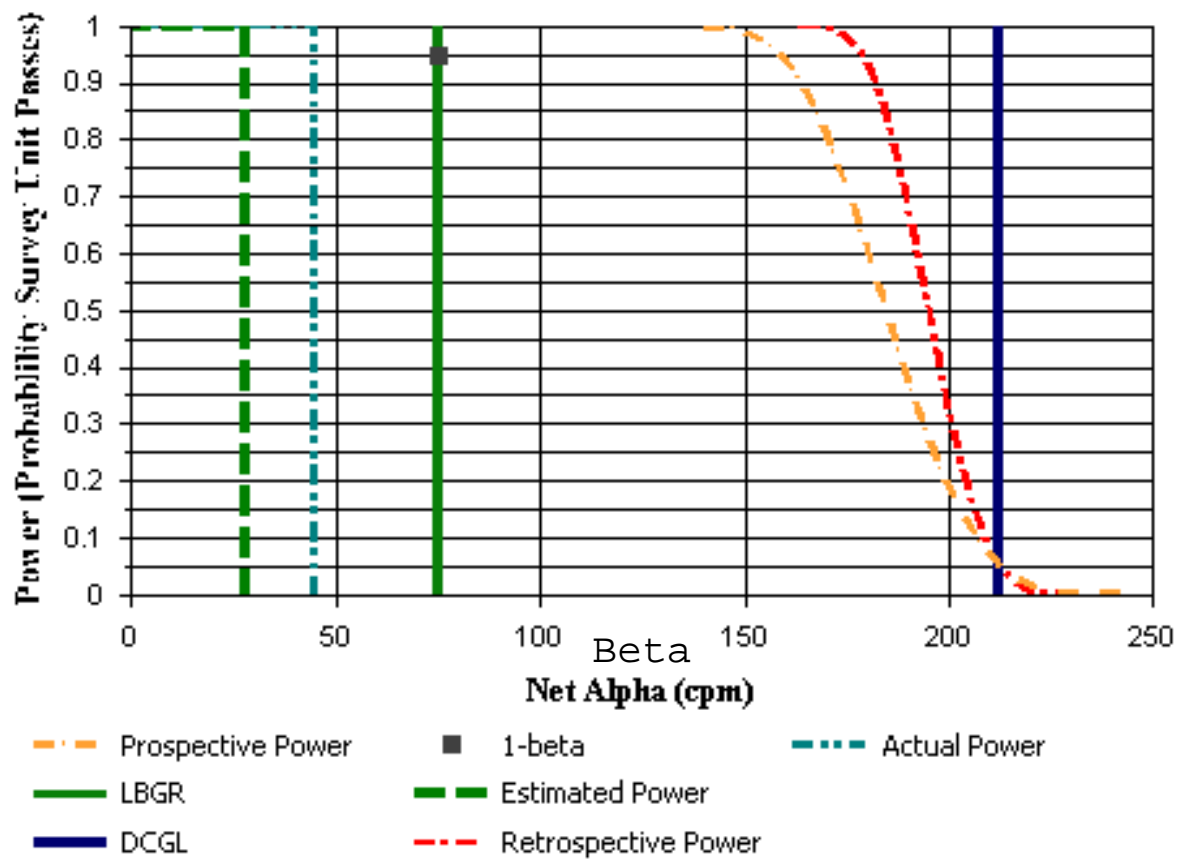




\section{DQA Building Surface Report}

\section{Survey Unit Data}

NOTE: $\quad$ Type = "S" indicates survey measurement.

Type $=$ "R" indicates reference measurement.

\begin{tabular}{lccc} 
Measurement & Material & Type & Gross Alpha (cpm) \\
\hline $21.1,42.1$ & Slag & S & 466 \\
$23.9,42.1$ & Slag & S & 469 \\
$26.8,42.1$ & Slag & S & 522 \\
$28.5,42.3$ & Slag & S & 513 \\
$22.5,44.6$ & Slag & S & 489 \\
$25.4,44.6$ & Slag & S & 521 \\
$28.2,44.6$ & Slag & S & 539 \\
$21.1,47.1$ & Slag & S & 517 \\
$23.9,47.1$ & Slag & S & 493 \\
$26.8,47.1$ & Slag & S & 501 \\
$29.7,47.1$ & Slag & S & 474 \\
$22.5,49.6$ & Slag & S & 426 \\
$25.4,49.6$ & Slag & S & 471 \\
$28.2,49.6$ & Slag & S & 511
\end{tabular}

\section{Basic Statistical Quantities Summary}

Statistic

Sample Number

Mean $\left(\mathrm{dpm} / 100 \mathrm{~cm}^{2}\right)$

Median (dpm/100 cm²)

Std Dev (dpm/100 $\left.\mathrm{cm}^{2}\right)$

High Value $\left(\mathrm{dpm} / 100 \mathrm{~cm}^{2}\right)$

Low Value $\left(\mathrm{dpm} / 100 \mathrm{~cm}^{2}\right)$
Survey Unit

14

38.48

44.69

56.82

124.06

$-89.47$
Background

DQO Results

$\begin{array}{ll}\text { N/A } & \mathrm{N}=14 \\ \text { N/A } & 27.6 \\ \text { N/A } & \text { N/A } \\ \text { N/A } & 48.3 \\ \text { N/A } & \text { N/A } \\ \text { N/A } & \text { N/A }\end{array}$




\section{DQA Building Surface Report}

\section{Assessment Summary}

Site:

Planner(s):

Survey Unit Name:

Report Number:

Survey Unit Measurements:

Reference Area Measurements:

Test Performed:

Judgmental Areas:

Assessment Conclusion:
Hammond Depot FSS Planner

Vitkus

C1 SU1 through 21 Bldg 200E slag Rev. 1 C1 SU18

18

14

0

Sign

Test Result:

Not Performed

0

EMC Result:

Not Performed

\section{Retrospective Power Curve}

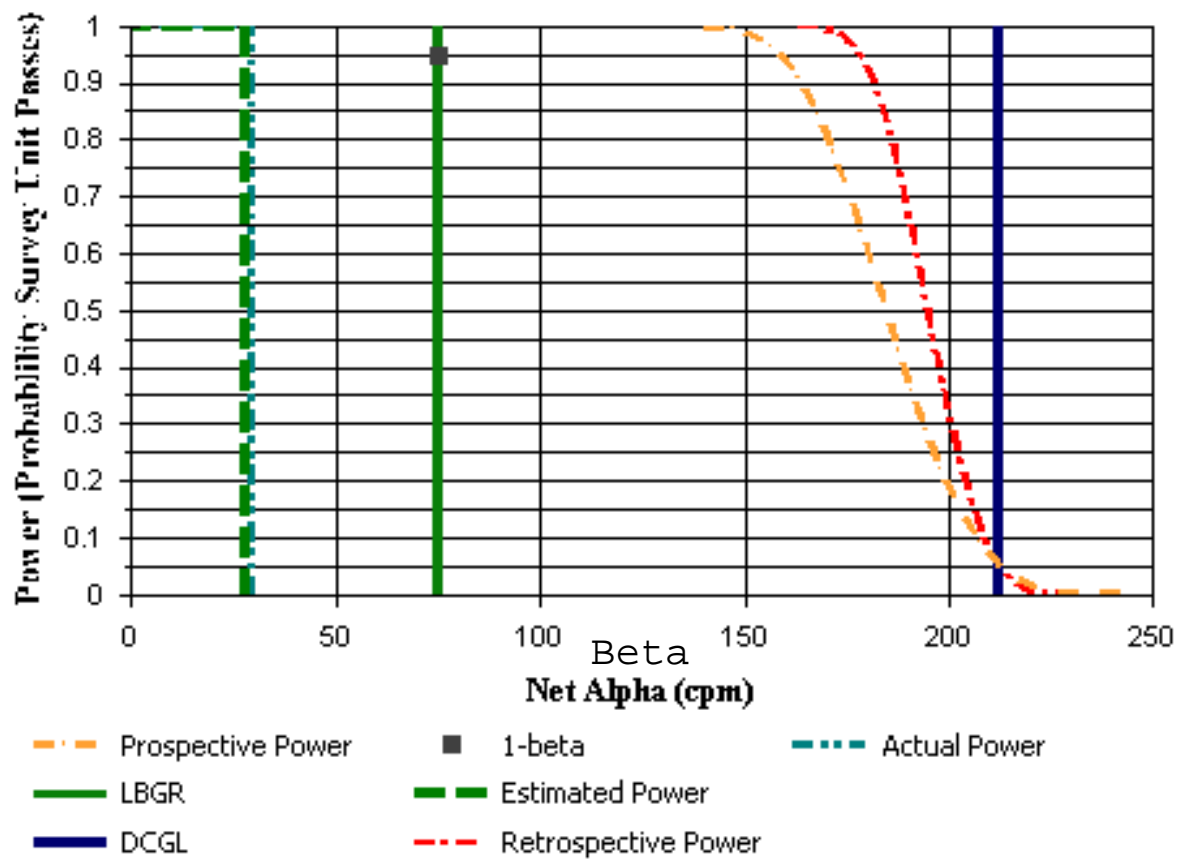




\section{DQA Building Surface Report}

\section{Survey Unit Data}

NOTE: $\quad$ Type $=$ "S" indicates survey measurement.

Type $=$ "R" indicates reference measurement.

\begin{tabular}{lccc} 
Measurement & Material & Type & Gross Alpha (cpm) \\
\hline $31.4,41.7$ & Slag & S & 493 \\
$35.1,41.7$ & Slag & S & 490 \\
$33.3,44.8$ & Slag & S & 516 \\
$36.9,44.8$ & Slag & S & 431 \\
$31.4,47.9$ & Slag & S & 506 \\
$35.1,47.9$ & Slag & S & 481 \\
$33.3,51.0$ & Slag & S & 538 \\
$36.9,51.0$ & Slag & S & 452 \\
$31.4,54.2$ & Slag & S & 498 \\
$35.1,54.2$ & Slag & S & 446 \\
$33.3,57.3$ & Slag & S & 469 \\
$36.9,57.3$ & Slag & S & 463 \\
$31.4,60.4$ & Slag & $S$ & 488 \\
$35.1,60.4$ & Slag & $S$ & 521
\end{tabular}

\section{Basic Statistical Quantities Summary}

Statistic

Sample Number

Mean $\left(\mathrm{dpm} / 100 \mathrm{~cm}{ }^{2}\right)$

Median (dpm/100 cm²)

Std Dev (dpm/100 $\left.\mathrm{cm}^{2}\right)$

High Value $\left(\mathrm{dpm} / 100 \mathrm{~cm}^{2}\right)$

Low Value $\left(\mathrm{dpm} / 100 \mathrm{~cm}^{2}\right)$
Survey Unit

14
22.28
29.57
57.54
122.17
-80.03

Background

N/A

N/A

N/A

N/A

N/A

N/A
DQO Results

$\mathrm{N}=14$

27.6

N/A

48.3

N/A

N/A 


\section{DQA Building Surface Report}

\section{Assessment Summary}

Site:

Planner(s):

Survey Unit Name:

Report Number:

Survey Unit Measurements:

Reference Area Measurements:

Test Performed:

Judgmental Areas:

Assessment Conclusion:
Hammond Depot FSS Planner

Vitkus

C1 SU1 through 21 Bldg 200E slag Rev. 1 C1 SU19

19

14

0

Sign

Test Result:

Not Performed

0

EMC Result:

Not Performed

Reject Null Hypothesis (Survey Unit PASSES)

\section{Retrospective Power Curve}

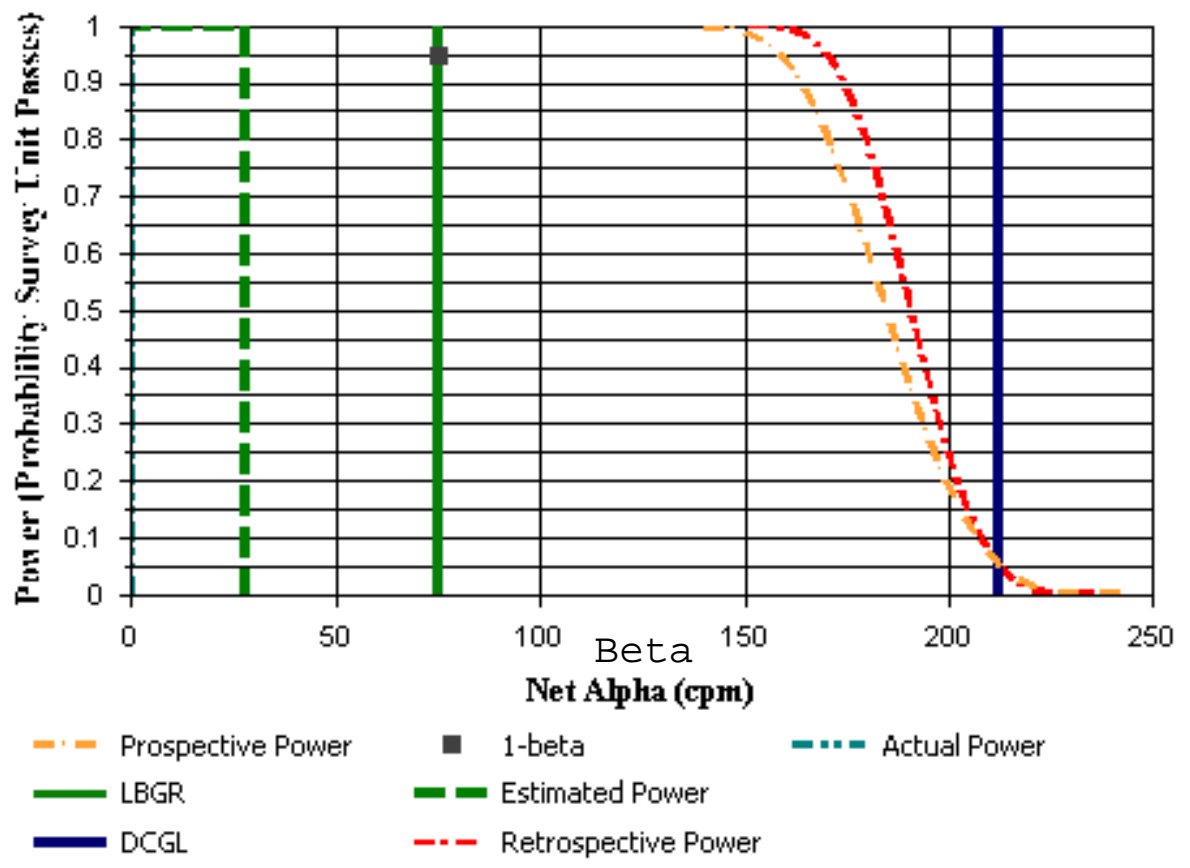




\section{DQA Building Surface Report}

\section{Survey Unit Data}

NOTE: $\quad$ Type $=$ "S" indicates survey measurement.

Type $=$ "R" indicates reference measurement.

\begin{tabular}{lccc} 
Measurement & Material & Type & Gross Alpha (cpm) \\
\hline $0.7,52.5$ & Slag & S & 455 \\
$3.7,52.5$ & Slag & S & 467 \\
$6.7,52.5$ & Slag & S & 454 \\
$9.7,52.5$ & Slag & S & 490 \\
$2.2,55.1$ & Slag & S & 464 \\
$5.2,55.1$ & Slag & S & 435 \\
$8.2,55.1$ & Slag & S & 470 \\
$0.7,57.7$ & Slag & S & 455 \\
$3.7,57.7$ & Slag & S & 490 \\
$6.7,57.7$ & Slag & S & 487 \\
$9.7,57.7$ & Slag & S & 477 \\
$2.8,58.2$ & Slag & S & 596 \\
$1.6,53.8$ & Slag & S & 478 \\
$8.2,60.3$ & Slag & S & 499
\end{tabular}

\section{Basic Statistical Quantities Summary}

Statistic

Sample Number

Mean $\left(\mathrm{dpm} / 100 \mathrm{~cm}^{2}\right)$

Median (dpm/100 cm²)

Std Dev (dpm/100 $\left.\mathrm{cm}^{2}\right)$

High Value $\left(\mathrm{dpm} / 100 \mathrm{~cm}^{2}\right)$

Low Value $\left(\mathrm{dpm} / 100 \mathrm{~cm}^{2}\right)$
Survey Unit

14
12.16
0.28
71.39
231.76
-72.47

Background

N/A

N/A

N/A

N/A

N/A

N/A
DQO Results

$\mathrm{N}=14$

27.6

N/A

48.3

N/A

N/A 


\section{DQA Building Surface Report}

\section{Assessment Summary}

Site:

Planner(s):

Survey Unit Name:

Report Number:

Survey Unit Measurements:

Reference Area Measurements:

Test Performed:

Judgmental Areas:

Assessment Conclusion:
Hammond Depot FSS Planner

Vitkus

C1 SU1 through 21 Bldg 200E slag Rev. 1 C1 SU20

20

14

0

Sign

Test Result:

Not Performed

0

EMC Result:

Not Performed

Reject Null Hypothesis (Survey Unit PASSES)

\section{Retrospective Power Curve}

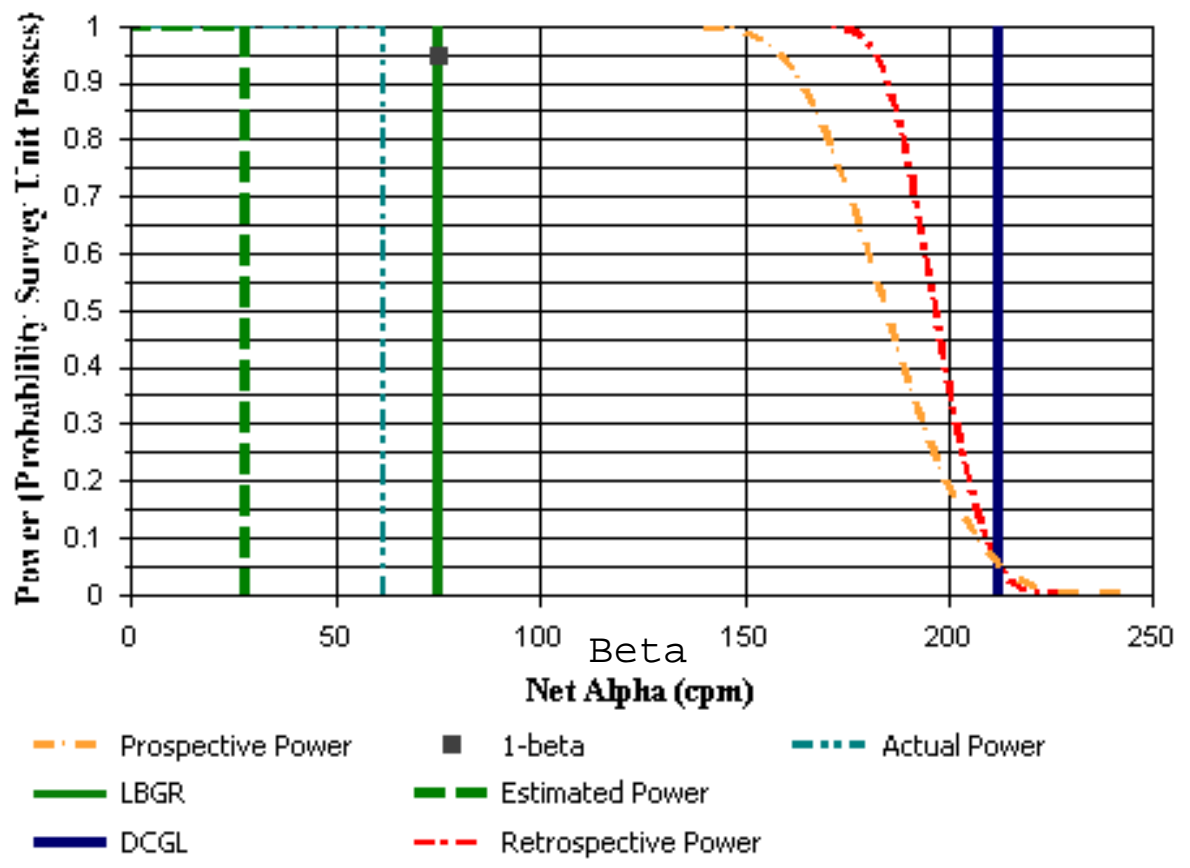




\section{DQA Building Surface Report}

\section{Survey Unit Data}

NOTE: $\quad$ Type = "S" indicates survey measurement.

Type $=$ "R" indicates reference measurement.

\begin{tabular}{lccc} 
Measurement & Material & Type & Gross Alpha (cpm) \\
\hline $12.1,51.0$ & Slag & S & 524 \\
$15.1,51.0$ & Slag & S & 509 \\
$18.1,51.0$ & Slag & S & 473 \\
$10.6,53.6$ & Slag & S & 503 \\
$13.6,53.6$ & Slag & S & 514 \\
$16.6,53.6$ & Slag & S & 511 \\
$19.6,53.6$ & Slag & S & 521 \\
$12.1,56.2$ & Slag & S & 471 \\
$15.1,56.2$ & Slag & S & 491 \\
$18.1,56.2$ & Slag & S & 474 \\
$10.6,58.8$ & Slag & S & 526 \\
$13.6,58.8$ & Slag & S & 496 \\
$16.6,58.8$ & Slag & S & 549 \\
$19.6,58.8$ & Slag & S & 448
\end{tabular}

\section{Basic Statistical Quantities Summary}

Statistic

Sample Number

Mean $\left(\mathrm{dpm} / 100 \mathrm{~cm}^{2}\right)$

Median (dpm/100 cm²)

Std Dev (dpm/100 $\left.\mathrm{cm}^{2}\right)$

High Value $\left(\mathrm{dpm} / 100 \mathrm{~cm}^{2}\right)$

Low Value $\left(\mathrm{dpm} / 100 \mathrm{~cm}^{2}\right)$
Survey Unit

14
51.71
61.70
51.19
142.95
-47.90

Background

N/A

N/A

N/A

N/A

N/A

N/A
DQO Results

$\mathrm{N}=14$

27.6

N/A

48.3

N/A

N/A 


\section{DQA Building Surface Report}

\section{Assessment Summary}

Site:

Planner(s):

Survey Unit Name:

Report Number:

Survey Unit Measurements:

Reference Area Measurements:

Test Performed:

Judgmental Areas:

Assessment Conclusion:
Hammond Depot FSS Planner

Vitkus

C1 SU1 through 21 Bldg 200E slag Rev. 1 C1 SU21

21

14

0

Sign

Test Result:

Not Performed

0

EMC Result:

Not Performed

Reject Null Hypothesis (Survey Unit PASSES)

\section{Retrospective Power Curve}

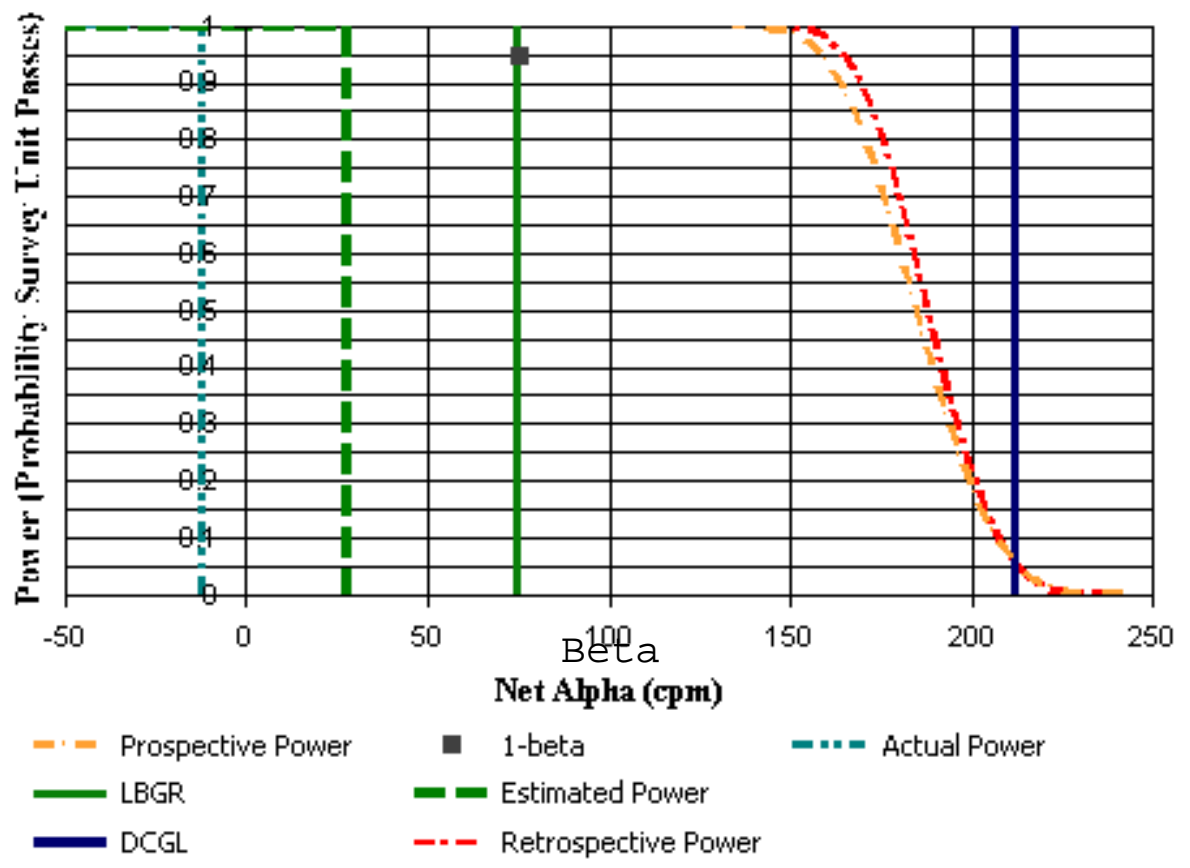




\section{DQA Building Surface Report}

\section{Survey Unit Data}

NOTE: $\quad$ Type = "S" indicates survey measurement.

Type $=$ "R" indicates reference measurement.

\begin{tabular}{lccc} 
Measurement & Material & Type & Gross Alpha (cpm) \\
\hline $21.7,51.5$ & Slag & S & 511 \\
$24.7,51.5$ & Slag & S & 407 \\
$27.7,51.5$ & Slag & S & 438 \\
$20.2,54.1$ & Slag & S & 477 \\
$23.2,54.1$ & Slag & S & 440 \\
$26.2,54.1$ & Slag & S & 486 \\
$29.2,54.1$ & Slag & S & 396 \\
$21.7,56.7$ & Slag & S & 457 \\
$24.7,56.7$ & Slag & S & 493 \\
$27.7,56.7$ & Slag & S & 487 \\
$20.2,59.3$ & Slag & S & 437 \\
$23.2,59.3$ & Slag & S & 432 \\
$26.2,59.3$ & Slag & S & 540 \\
$29.2,59.3$ & Slag & S & 517
\end{tabular}

\section{Basic Statistical Quantities Summary}

Statistic

Sample Number

Mean $\left(\mathrm{dpm} / 100 \mathrm{~cm}^{2}\right)$

Median (dpm/100 cm²)

Std Dev (dpm/100 $\left.\mathrm{cm}^{2}\right)$

High Value $\left(\mathrm{dpm} / 100 \mathrm{~cm}^{2}\right)$

Low Value $\left(\mathrm{dpm} / 100 \mathrm{~cm}^{2}\right)$
Survey Unit

14
-14.70
-12.00
80.70
125.94
-146.16

Background

N/A

N/A

N/A

N/A

N/A

N/A

N/A
N/A
N/A
N/A
N/A
N/A

DQO Results

$\mathrm{N}=14$

27.6

N/A

48.3

N/A

N/A 


\section{DQA Building Surface Report}

\section{Assessment Summary}

Site:

Planner(s):

Survey Unit Name:

Report Number:

Survey Unit Measurements:

Reference Area Measurements:

Test Performed:

Judgmental Areas:

Assessment Conclusion:
Hammond Depot FSS Planner

Vitkus

C1 SU22 and 23 Building 200E west and north wall C1 SU22 NE corner wall

17

0

Sign

Test Result:

Not Performed

0

EMC Result:

Not Performed

Reject Null Hypothesis (Survey Unit PASSES)

\section{Retrospective Power Curve}

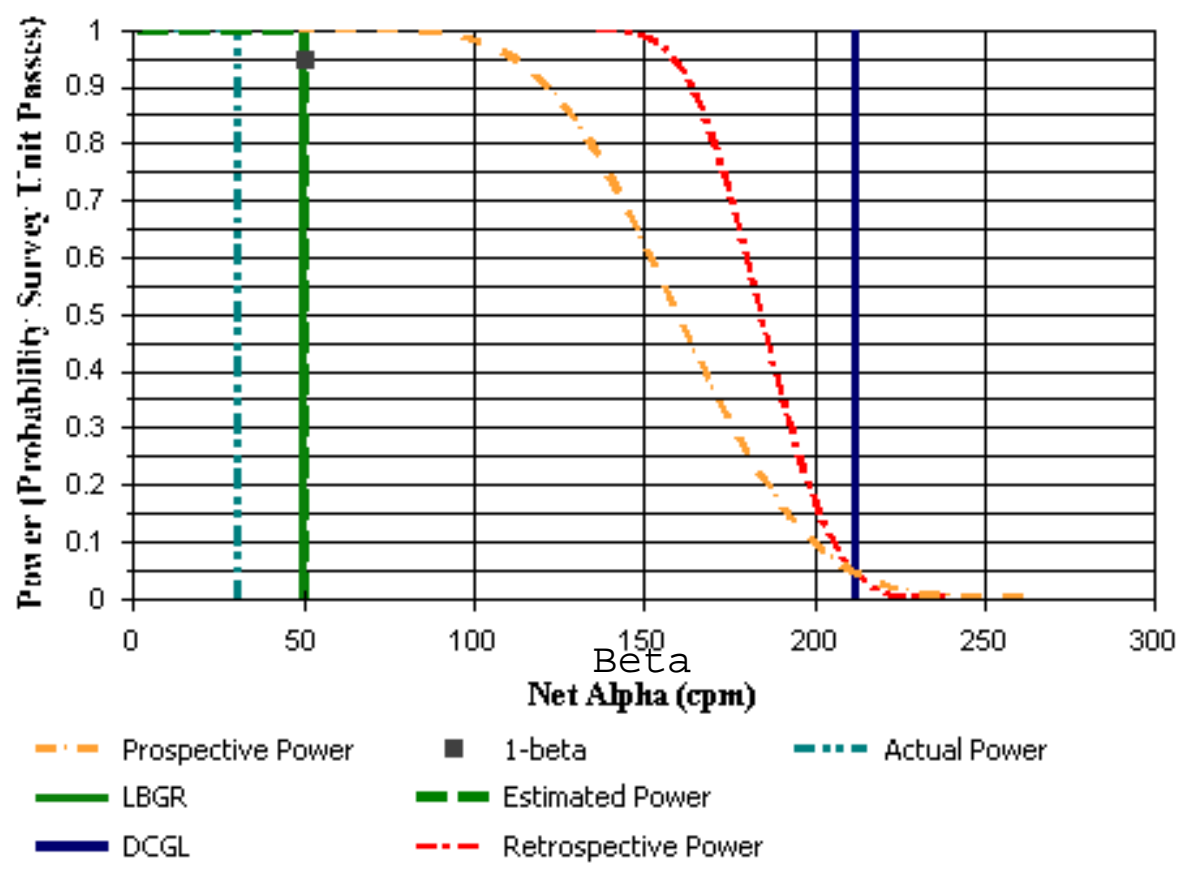




\section{DQA Building Surface Report}

\section{Survey Unit Data}

NOTE: Type $=$ "S" indicates survey measurement.

Type = "R" indicates reference measurement.

Measurement

$1.4,0.6$

$3.0,0.6$

$4.5,0.6$

$6.1,0.6$

$0.6,2.0$

2.2, 2.0

$3.8,2.0$

$5.3,2.0$

$6.9,2.0$

$1.4,3.4$

$3.0,3.4$

$4.5,3.4$

$6.1,3.4$

$0.6,4.7$

$2.2,4.7$

3.8, 4.7

$5.3,4.7$
Material

Unpainted Cinder Block

Unpainted Cinder Block

Unpainted Cinder Block

Unpainted Cinder Block

Unpainted Cinder Block

Unpainted Cinder Block

Unpainted Cinder Block

Unpainted Cinder Block

Unpainted Cinder Block

Unpainted Cinder Block

Unpainted Cinder Block

Unpainted Cinder Block

Unpainted Cinder Block

Unpainted Cinder Block

Unpainted Cinder Block

Unpainted Cinder Block

Unpainted Cinder Block
Beta

Type Gross Alpha (cpm)

481

644

504

490

438

468

464

463

468

418

419

478

460

435

474

455

423

\section{Basic Statistical Quantities Summary}

\section{Statistic}

Sample Number

Mean $\left(\mathrm{dpm} / 100 \mathrm{~cm}^{2}\right)$

Median (dpm/100 $\left.\mathrm{cm}^{2}\right)$

Std Dev (dpm/100 cm²)

High Value $\left(\mathrm{dpm} / 100 \mathrm{~cm}^{2}\right)$

Low Value $\left(\mathrm{dpm} / 100 \mathrm{~cm}^{2}\right)$
Survey Unit

17
41.25
30.80
97.06
370.94
-56.12

Background

N/A

N/A

N/A

N/A

N/A

N/A
DQO Results

$\mathrm{N}=17$

50.1

N/A

96.4

N/A

N/A 


\section{DQA Building Surface Report}

\section{Assessment Summary}

Site:

Planner(s):

Survey Unit Name:

Report Number:

Survey Unit Measurements:

Reference Area Measurements:

Test Performed:

Judgmental Areas:

Assessment Conclusion:
Hammond Depot FSS Planner

Vitkus

C1 SU24 through 32 Bldg 200E Ceiling C1 SU24

1

17

0

Sign

Test Result:

Not Performed

0

EMC Result:

Not Performed

Reject Null Hypothesis (Survey Unit PASSES)

\section{Retrospective Power Curve}

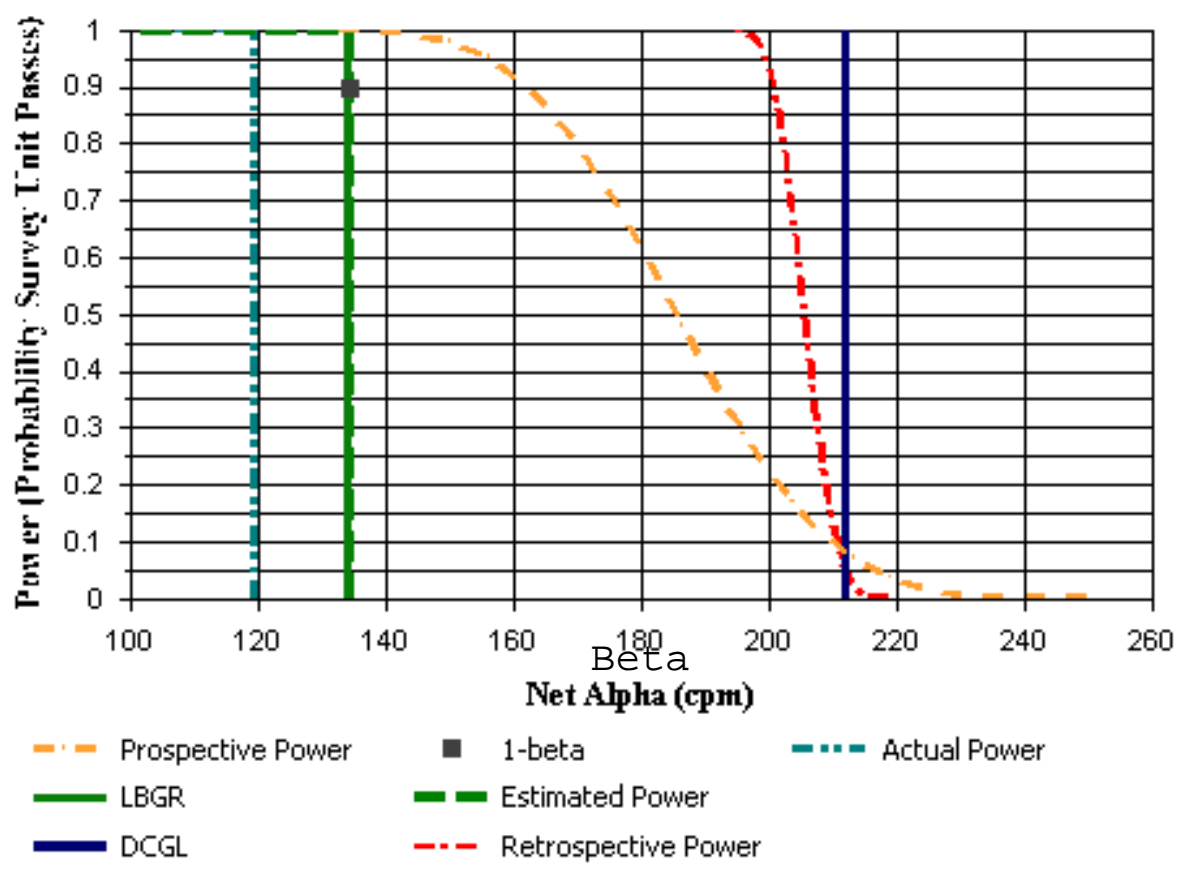




\section{DQA Building Surface Report}

\section{Survey Unit Data}

NOTE: $\quad$ Type $=$ "S" indicates survey measurement.

Type $=$ "R" indicates reference measurement.

\begin{tabular}{lccc} 
Measurement & Material & Type & Gross Alpha (cpm) \\
\hline $0.2,32.4$ & Metal & $\mathrm{S}$ & 233 \\
$3.2,32.4$ & Metal & $\mathrm{S}$ & 232 \\
$6.3,32.4$ & Metal & $\mathrm{S}$ & 238 \\
$9.4,32.4$ & Metal & $\mathrm{S}$ & 250 \\
$12.5,32.4$ & Metal & $\mathrm{S}$ & 256 \\
$1.7,35.1$ & Metal & $\mathrm{S}$ & 241 \\
$4.8,35.1$ & Metal & $\mathrm{S}$ & 235 \\
$7.9,35.1$ & Metal & $\mathrm{S}$ & 234 \\
$10.9,35.1$ & Metal & $\mathrm{S}$ & 259 \\
$0.2,37.8$ & Metal & $\mathrm{S}$ & 238 \\
$3.2,37.8$ & Metal & $\mathrm{S}$ & 240 \\
$6.3,37.8$ & Metal & $\mathrm{S}$ & 268 \\
$9.4,37.8$ & Metal & $\mathrm{S}$ & 262 \\
$12.5,37.8$ & Metal & $\mathrm{S}$ & 245 \\
$1.7,40.5$ & Metal & $\mathrm{S}$ & 242 \\
$4.8,40.5$ & Metal & $\mathrm{S}$ & 239 \\
$7.9,40.5$ & Metal & $\mathrm{S}$ & 268
\end{tabular}

\section{Basic Statistical Quantities Summary}

Statistic

Sample Number

Mean $\left(\mathrm{dpm} / 100 \mathrm{~cm}^{2}\right)$

Median (dpm/100 $\left.\mathrm{cm}^{2}\right)$

Std Dev (dpm/100 $\left.\mathrm{cm}^{2}\right)$

High Value $\left(\mathrm{dpm} / 100 \mathrm{~cm}^{2}\right)$

Low Value $\left(\mathrm{dpm} / 100 \mathrm{~cm}^{2}\right)$
Survey Unit

17
128.46
119.24
23.11
170.26
102.23

Background

N/A

N/A

N/A

N/A

N/A

N/A
DQO Results

$\mathrm{N}=18$

134

N/A

61.9

N/A

N/A 


\section{DQA Building Surface Report}

\section{Assessment Summary}

Site:

Planner(s):

Survey Unit Name:

Report Number:

Survey Unit Measurements:

Reference Area Measurements:

Test Performed:

Judgmental Areas:

Assessment Conclusion:
Hammond Depot FSS Planner

Vitkus

C1 SU22 and 23 Building 200E west and north wall C1 SU23 west central wall

2

17

0

Sign

Test Result:

Not Performed

0

EMC Result:

Not Performed

\section{Reject Null Hypothesis (Survey Unit PASSES)}

\section{Retrospective Power Curve}

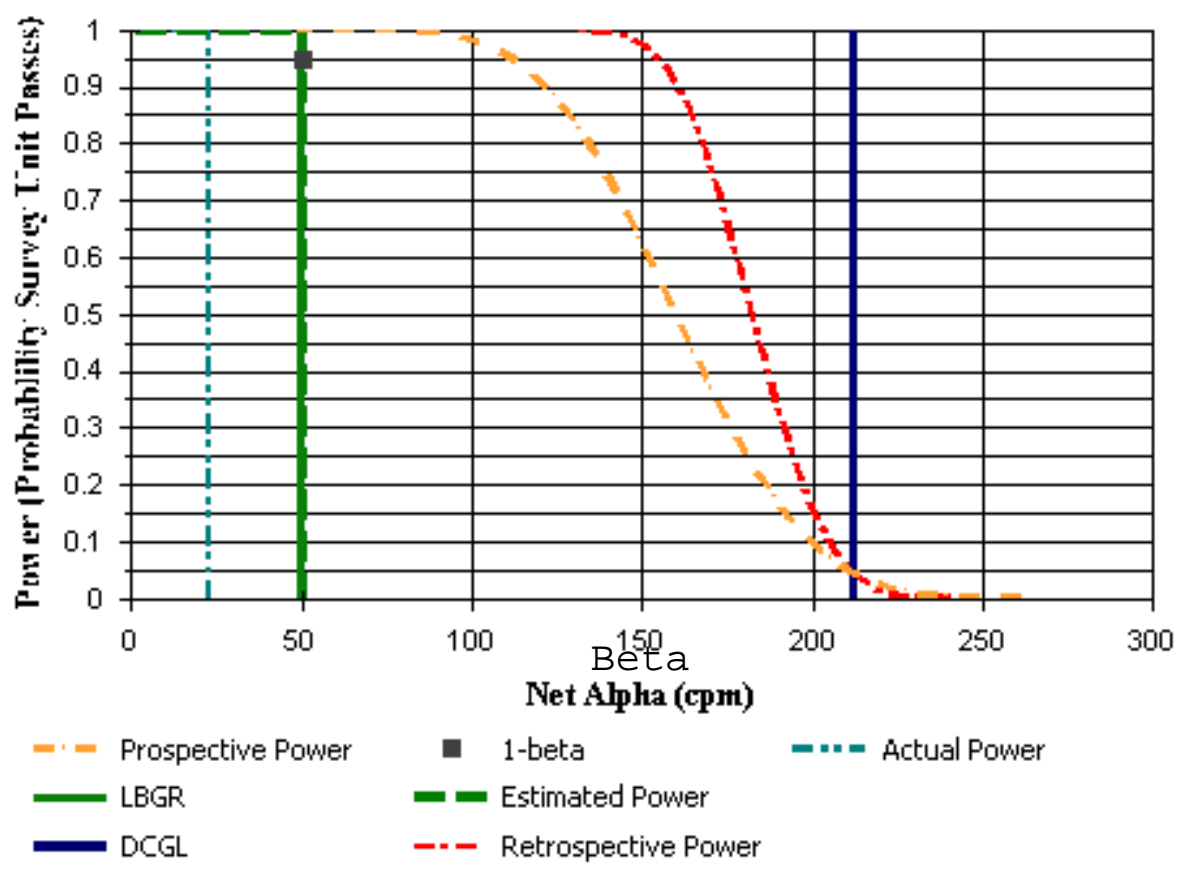




\section{DQA Building Surface Report}

\section{Survey Unit Data}

NOTE: $\quad$ Type $=$ "S" indicates survey measurement.

Type = "R" indicates reference measurement.

\begin{tabular}{|c|c|c|c|}
\hline Measurement & Material & Type & Gross Alpha (cpm) \\
\hline $19.7,3.6$ & Metal & S & 190 \\
\hline $19.7,1.6$ & Unpainted Cinder Block & S & 582 \\
\hline $21.3,4.6$ & Metal & S & 160 \\
\hline $21.3,2.6$ & Unpainted Cinder Block & S & 567 \\
\hline $21.3,0.7$ & Unpainted Cinder Block & $\mathrm{s}$ & 498 \\
\hline $23.0,3.6$ & Metal & $\mathrm{s}$ & 173 \\
\hline $23.0,1.6$ & Unpainted Cinder Block & S & 564 \\
\hline $24.7,4.6$ & Unpainted Cinder Block & S & 454 \\
\hline $24.7,2.6$ & Unpainted Cinder Block & S & 435 \\
\hline $24.7,0.7$ & Unpainted Cinder Block & S & 560 \\
\hline $26.4,3.6$ & Unpainted Cinder Block & $\mathrm{S}$ & 476 \\
\hline $26.4,1.6$ & Unpainted Cinder Block & $\mathrm{s}$ & 413 \\
\hline $28.1,4.6$ & Metal & $\mathrm{s}$ & 168 \\
\hline $28.1,2.6$ & Unpainted Cinder Block & $\mathrm{S}$ & 442 \\
\hline 28.1, 0.7 actual material concrete & e, Metal & $\mathrm{s}$ & 238 \\
\hline $29.8,3.6$ Metal BKG used (178 vs. & Unj) & S & 473 \\
\hline $29.8,1.6$ & Unpainted Cinder Block & $\mathrm{s}$ & 447 \\
\hline
\end{tabular}

\section{Basic Statistical Quantities Summary}

Statistic

Sample Number

Mean $\left(\mathrm{dpm} / 100 \mathrm{~cm}^{2}\right)$

Median (dpm/100 $\left.\mathrm{cm}^{2}\right)$

Std Dev (dpm/100 $\left.\mathrm{cm}^{2}\right)$

High Value $\left(\mathrm{dpm} / 100 \mathrm{~cm}^{2}\right)$

Low Value $\left(\mathrm{dpm} / 100 \mathrm{~cm}^{2}\right)$
Survey Unit

17
64.26
22.86
103.88
253.78
-65.57

Background

$\begin{array}{ll}\text { N/A } & \mathrm{N}=17 \\ \text { N/A } & 50.1 \\ \text { N/A } & \text { N/A } \\ \text { N/A } & 96.4 \\ \text { N/A } & \text { N/A } \\ \text { N/A } & \text { N/A }\end{array}$




\section{DQA Building Surface Report}

\section{Assessment Summary}

Site:

Planner(s):

Survey Unit Name:

Report Number:

Survey Unit Measurements:

Reference Area Measurements:

Test Performed:

Judgmental Areas:

Assessment Conclusion:
Hammond Depot FSS Planner

Vitkus

C1 SU24 through 32 Bldg 200E Ceiling C1 SU25

3

18

0

Sign

Test Result:

Not Performed

0

EMC Result:

Not Performed

Reject Null Hypothesis (Survey Unit PASSES)

\section{Retrospective Power Curve}

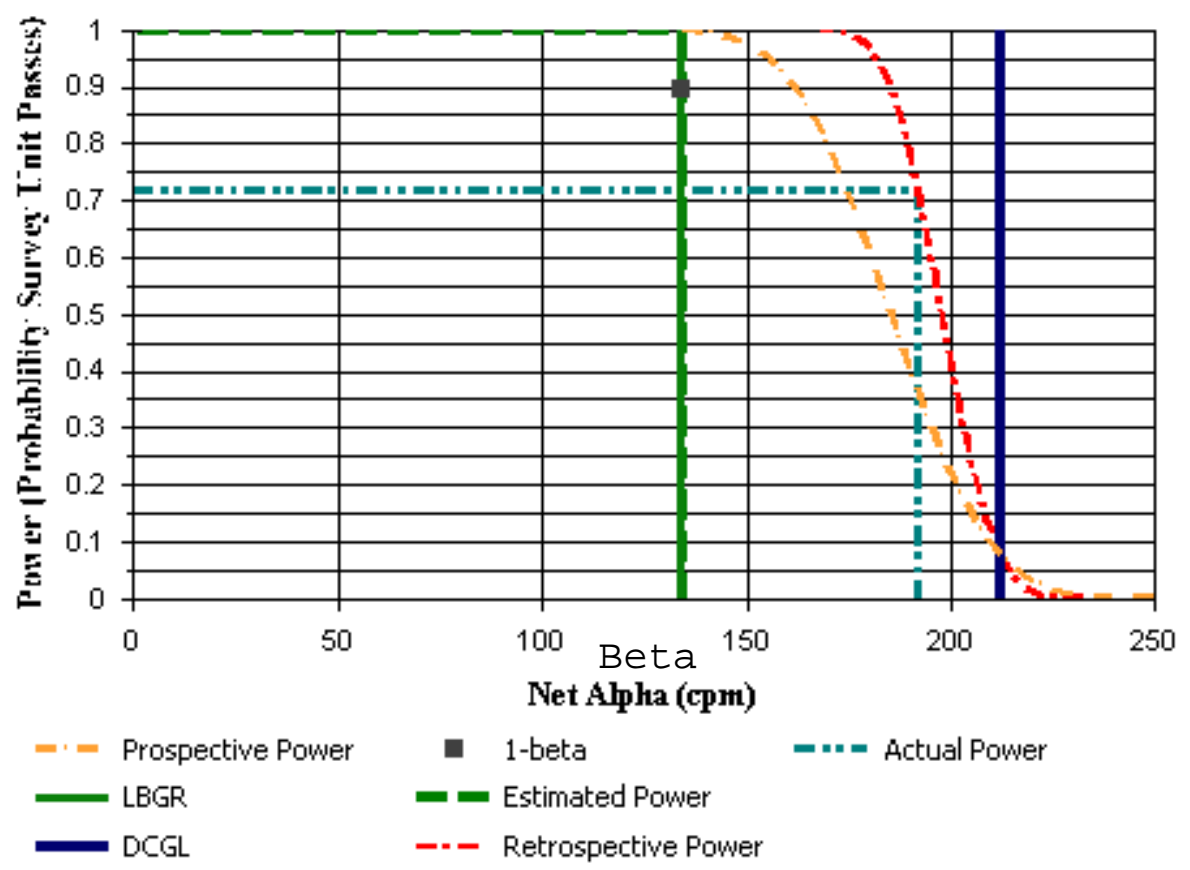




\section{DQA Building Surface Report}

\section{Survey Unit Data}

NOTE: $\quad$ Type $=$ "S" indicates survey measurement.

Type $=$ "R" indicates reference measurement.

\section{Measurement}

$14.1,32.3$

$17.2,32.3$

$20.3,32.3$

$23.3,32.3$

$12.5,35.0$

$15.6,35.0$

$18.7,35.0$

$21.8,35.0$

$24.9,35.0$

$14.1,37.6$

$17.2,37.6$

$20.3,37.6$

$23.3,37.6$

$12.5,40.3$

$15.6,40.3$

$18.7,40.3$

$21.8,40.3$

$24.9,40.3$

\section{Material}

Metal

Metal

Metal

Metal

Metal

Metal

Metal

Metal

Metal

Metal

Metal

Metal

Metal

Metal

Metal

Metal

Metal

Metal
Beta Gross Alpha (cpm)

$\begin{array}{cr}\text { Type } & \text { Gross Alpha } \\ \text { S } & 244\end{array}$

271

261

289

372

200

292

282

275

290

253

280

293

269

301

279

261

284

\section{Basic Statistical Quantities Summary}

\begin{tabular}{lccc} 
Statistic & Survey Unit & Background & DQO Results \\
\hline Sample Number & 18 & N/A & N=18 \\
Mean $\left(\mathrm{dpm} / 100 \mathrm{~cm}^{2}\right)$ & 188.31 & N/A & 134 \\
Median $\left(\mathrm{dpm} / 100 \mathrm{~cm}^{2}\right)$ & 191.99 & $\mathrm{~N} / \mathrm{A}$ & $\mathrm{N} / \mathrm{A}$ \\
Std Dev $\left(\mathrm{dpm} / 100 \mathrm{~cm}^{2}\right)$ & 62.65 & $\mathrm{~N} / \mathrm{A}$ & 61.9 \\
High Value $\left(\mathrm{dpm} / 100 \mathrm{~cm}^{2}\right)$ & 366.78 & $\mathrm{~N} / \mathrm{A}$ & $\mathrm{N} / \mathrm{A}$ \\
Low Value $\left(\mathrm{dpm} / 100 \mathrm{~cm}^{2}\right)$ & 41.76 & $\mathrm{~N} / \mathrm{A}$ & $\mathrm{N} / \mathrm{A}$
\end{tabular}




\section{DQA Building Surface Report}

\section{Assessment Summary}

Site:

Planner(s):

Survey Unit Name:

Report Number:

Survey Unit Measurements:

Reference Area Measurements:

Test Performed:

Judgmental Areas:

Assessment Conclusion:
Hammond Depot FSS Planner

Vitkus

C1 SU24 through 32 BIdg 200E Ceiling C1 SU26

4

18

0

Sign

Test Result:

Not Performed

0

EMC Result:

Not Performed

Reject Null Hypothesis (Survey Unit PASSES)

\section{Retrospective Power Curve}

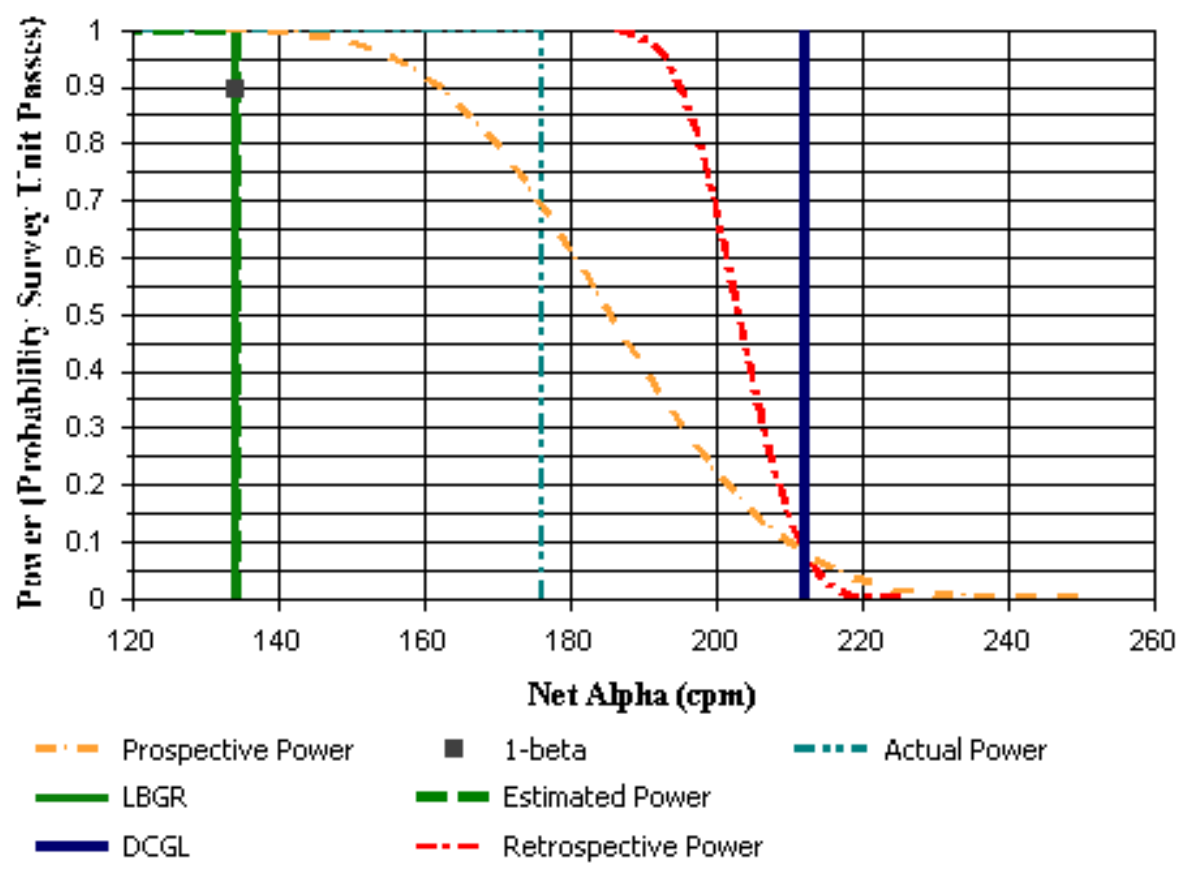




\section{DQA Building Surface Report}

\section{Survey Unit Data}

NOTE: $\quad$ Type $=$ "S" indicates survey measurement.

Type $=$ "R" indicates reference measurement.

\begin{tabular}{lccc} 
Measurement & Material & Type & Gross Alpha (cpm) \\
\hline $26.6,31.0$ & Metal & S & 279 \\
$29.7,31.0$ & Metal & S & 306 \\
$32.8,31.0$ & Metal & S & 251 \\
$35.8,31.0$ & Metal & S & 270 \\
$25.0,33.7$ & Metal & S & 264 \\
$28.1,33.7$ & Metal & S & 283 \\
$31.2,33.7$ & Metal & S & 272 \\
$34.3,33.7$ & Metal & S & 297 \\
$37.4,33.7$ & Metal & S & 260 \\
$26.6,36.4$ & Metal & S & 275 \\
$29.7,36.4$ & Metal & S & 300 \\
$32.8,36.4$ & Metal & S & 249 \\
$35.8,36.4$ & Metal & S & 248 \\
$25.0,39.0$ & Metal & S & 235 \\
$28.1,39.0$ & Metal & S & 276 \\
$31.2,39.0$ & Metal & S & 249 \\
$34.3,39.0$ & Metal & S & 289 \\
$37.4,39.0$ & Metal & S & 240
\end{tabular}

\section{Basic Statistical Quantities Summary}

\begin{tabular}{lccc} 
Statistic & Survey Unit & Background & DQO Results \\
\hline Sample Number & 18 & N/A & $\mathrm{N}=18$ \\
Mean $\left(\mathrm{dpm} / 100 \mathrm{~cm}^{2}\right)$ & 172.25 & $\mathrm{~N} / \mathrm{A}$ & 134 \\
Median $\left(\mathrm{dpm} / 100 \mathrm{~cm}^{2}\right)$ & 175.93 & $\mathrm{~N} / \mathrm{A}$ & $\mathrm{N} / \mathrm{A}$ \\
Std Dev $\left(\mathrm{dpm} / 100 \mathrm{~cm}^{2}\right)$ & 39.88 & $\mathrm{~N} / \mathrm{A}$ & 61.9 \\
High Value $\left(\mathrm{dpm} / 100 \mathrm{~cm}^{2}\right)$ & 242.06 & $\mathrm{~N} / \mathrm{A}$ & $\mathrm{N} / \mathrm{A}$ \\
Low Value $\left(\mathrm{dpm} / 100 \mathrm{~cm}^{2}\right)$ & 107.90 & $\mathrm{~N} / \mathrm{A}$ & $\mathrm{N} / \mathrm{A}$
\end{tabular}




\section{DQA Building Surface Report}

\section{Assessment Summary}

Site:

Planner(s):

Survey Unit Name:

Report Number:

Survey Unit Measurements:

Reference Area Measurements:

Test Performed:

Judgmental Areas:

Assessment Conclusion:
Hammond Depot FSS Planner

Vitkus

C1 SU24 through 32 Bldg 200E Ceiling C1 SU27

5

18

0

Sign

Test Result:

Not Performed

0

EMC Result:

Not Performed

Reject Null Hypothesis (Survey Unit PASSES)

\section{Retrospective Power Curve}

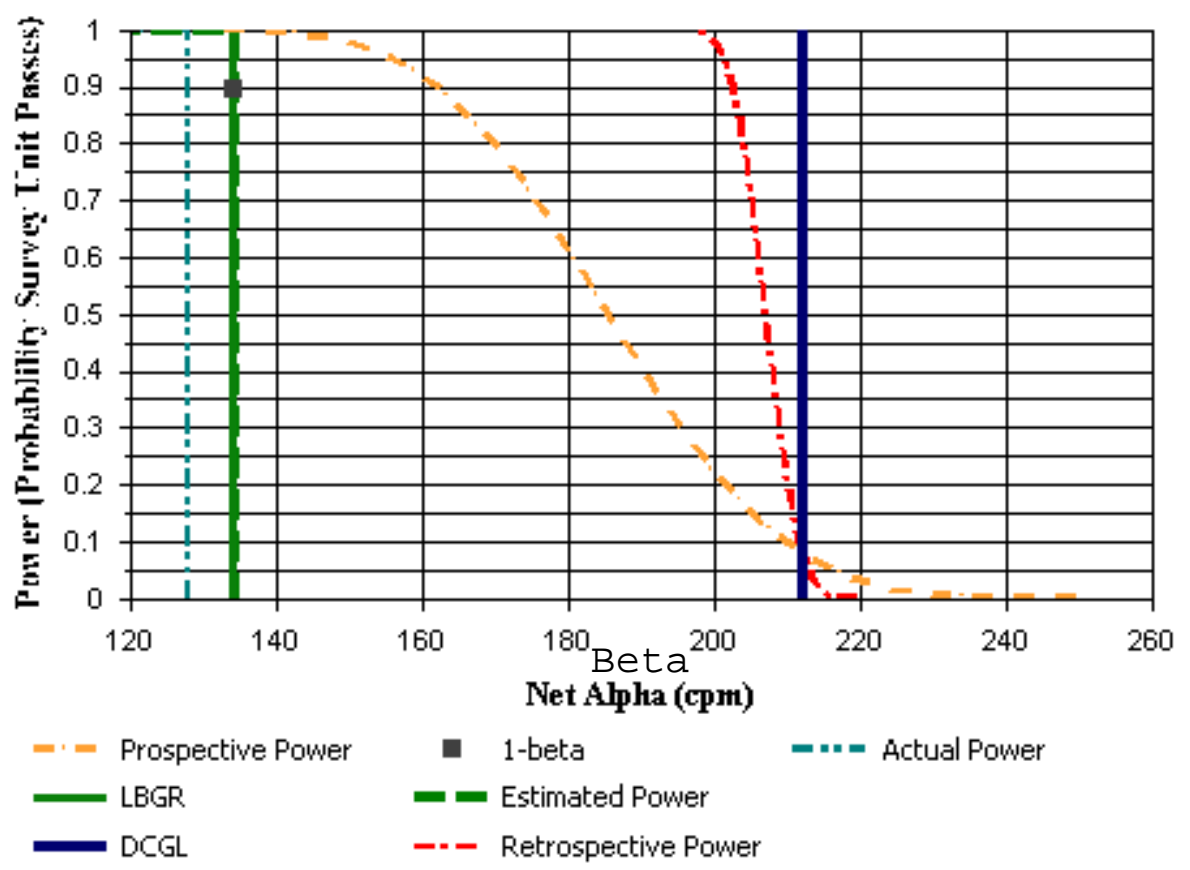




\section{DQA Building Surface Report}

\section{Survey Unit Data}

NOTE: $\quad$ Type $=$ "S" indicates survey measurement.

Type $=$ "R" indicates reference measurement.

\begin{tabular}{|c|c|c|c|}
\hline Measurement & Material & Type & Gross Alpha (cpm) \\
\hline $1.7,42.3$ & Metal & $\mathrm{S}$ & 260 \\
\hline $4.8,42.3$ & Metal & $\mathrm{S}$ & 263 \\
\hline $7.8,42.3$ & Metal & S & 242 \\
\hline $10.9,42.3$ & Metal & $S$ & 244 \\
\hline $0.1,45.0$ & Metal & S & 247 \\
\hline $3.2,45.0$ & Metal & S & 265 \\
\hline $6.3,45.0$ & Metal & S & 239 \\
\hline $9.4,45.0$ & Metal & S & 246 \\
\hline $12.5,45.0$ & Metal & $\mathrm{S}$ & 256 \\
\hline $1.7,47.7$ & Metal & S & 238 \\
\hline $4.8,47.7$ & Metal & S & 283 \\
\hline $7.8,47.7$ & Metal & S & 237 \\
\hline $10.9,47.7$ & Metal & S & 249 \\
\hline $0.1,50.3$ & Metal & S & 240 \\
\hline $3.2,50.3$ & Metal & S & 245 \\
\hline $6.3,50.3$ & Metal & $\mathrm{S}$ & 241 \\
\hline $9.4,50.3$ & Metal & $\mathrm{s}$ & 245 \\
\hline $12.5,50.3$ & Metal & $\mathrm{s}$ & 247 \\
\hline
\end{tabular}

\section{Basic Statistical Quantities Summary}

\begin{tabular}{lccc} 
Statistic & Survey Unit & Background & DQO Results \\
\hline Sample Number & 18 & $\mathrm{~N} / \mathrm{A}$ & $\mathrm{N}=18$ \\
Mean $\left(\mathrm{dpm} / 100 \mathrm{~cm}^{2}\right)$ & 134.88 & $\mathrm{~N} / \mathrm{A}$ & 134 \\
Median $\left(\mathrm{dpm} / 100 \mathrm{~cm}^{2}\right)$ & 127.74 & $\mathrm{~N} / \mathrm{A}$ & $\mathrm{N} / \mathrm{A}$ \\
Std Dev $\left(\mathrm{dpm} / 100 \mathrm{~cm}^{2}\right)$ & 22.50 & $\mathrm{~N} / \mathrm{A}$ & 61.9 \\
High Value $\left(\mathrm{dpm} / 100 \mathrm{~cm}^{2}\right)$ & 198.60 & $\mathrm{~N} / \mathrm{A}$ & $\mathrm{N} / \mathrm{A}$ \\
Low Value $\left(\mathrm{dpm} / 100 \mathrm{~cm}^{2}\right)$ & 111.68 & $\mathrm{~N} / \mathrm{A}$ & $\mathrm{N} / \mathrm{A}$
\end{tabular}




\section{DQA Building Surface Report}

\section{Assessment Summary}

Site:

Planner(s):

Survey Unit Name:

Report Number:

Survey Unit Measurements:

Reference Area Measurements:

Test Performed:

Judgmental Areas:

Assessment Conclusion:
Hammond Depot FSS Planner

Vitkus

C1 SU24 through 32 BIdg 200E Ceiling C1 SU28

7

18

0

Sign

Test Result:

Not Performed

0

EMC Result:

Not Performed

Reject Null Hypothesis (Survey Unit PASSES)

\section{Retrospective Power Curve}

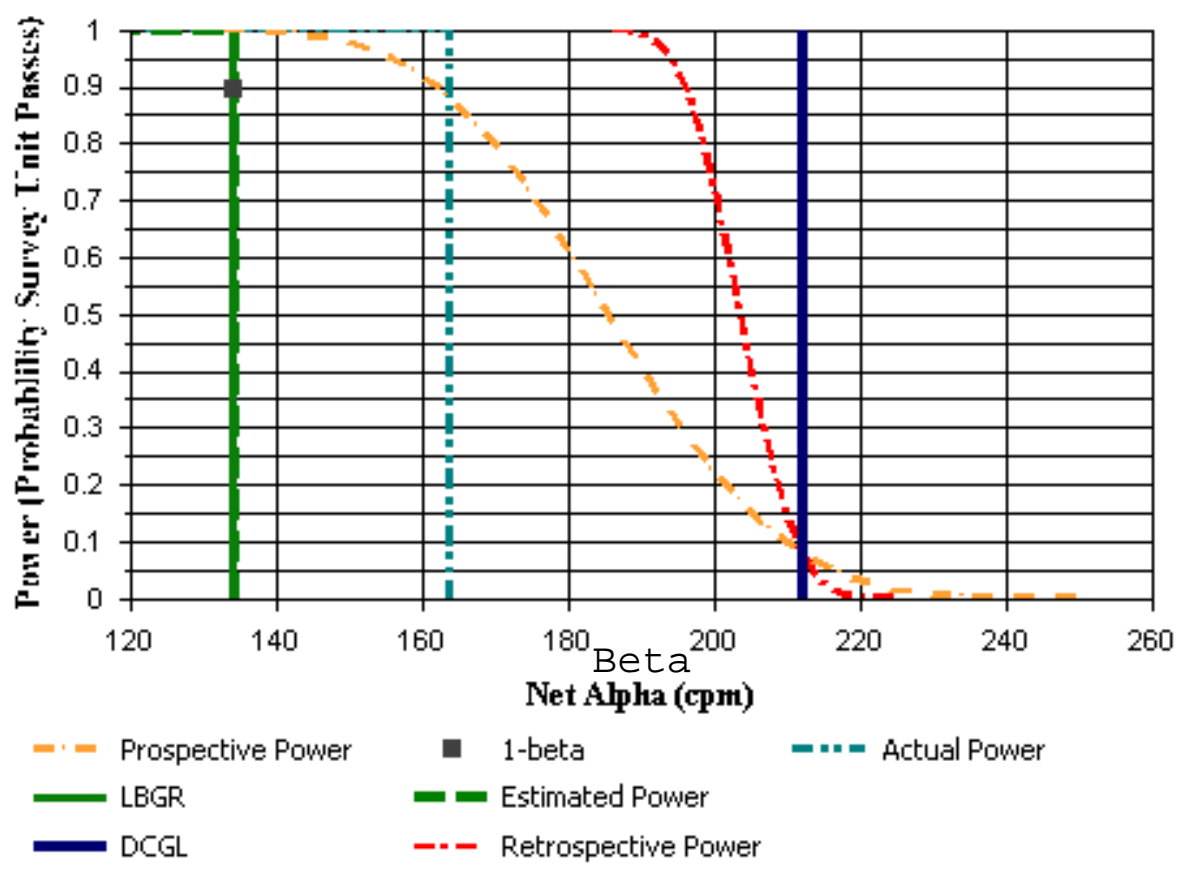




\section{DQA Building Surface Report}

\section{Survey Unit Data}

NOTE: $\quad$ Type $=$ "S" indicates survey measurement.

Type $=$ "R" indicates reference measurement.

\begin{tabular}{lccc} 
Measurement & Material & Type & Gross Alpha (cpm) \\
\hline $14.5,42.2$ & Metal & $\mathrm{S}$ & 249 \\
$17.5,42.2$ & Metal & $\mathrm{S}$ & 250 \\
$20.4,42.2$ & Metal & $\mathrm{S}$ & 248 \\
$23.3,42.2$ & Metal & $\mathrm{S}$ & 252 \\
$13.1,44.8$ & Metal & $\mathrm{S}$ & 288 \\
$16.0,44.8$ & Metal & $\mathrm{S}$ & 239 \\
$18.9,44.8$ & Metal & $\mathrm{S}$ & 264 \\
$21.9,44.8$ & Metal & $\mathrm{S}$ & 238 \\
$24.8,44.8$ & Metal & $\mathrm{S}$ & 277 \\
$14.5,47.3$ & Metal & $\mathrm{S}$ & 265 \\
$17.5,47.3$ & Metal & $\mathrm{S}$ & 313 \\
$20.4,47.3$ & Metal & $\mathrm{S}$ & 281 \\
$23.3,47.3$ & Metal & $\mathrm{S}$ & 280 \\
$13.1,49.9$ & Metal & $\mathrm{S}$ & 267 \\
$16.0,49.9$ & Metal & $\mathrm{S}$ & 264 \\
$18.9,49.9$ & Metal & $\mathrm{S}$ & 286 \\
$21.9,49.9$ & Metal & $\mathrm{S}$ & 247 \\
$24.8,49.9$ & Metal & $\mathrm{S}$ & 279
\end{tabular}

\section{Basic Statistical Quantities Summary}

\begin{tabular}{lccc} 
Statistic & Survey Unit & Background & DQO Results \\
\hline Sample Number & 18 & N/A & N=18 \\
Mean $\left(\mathrm{dpm} / 100 \mathrm{~cm}^{2}\right)$ & 166.37 & $\mathrm{~N} / \mathrm{A}$ & 134 \\
Median $\left(\mathrm{dpm} / 100 \mathrm{~cm}^{2}\right)$ & 163.64 & $\mathrm{~N} / \mathrm{A}$ & $\mathrm{N} / \mathrm{A} / \mathrm{A}$ \\
Std Dev $\left(\mathrm{dpm} / 100 \mathrm{~cm}^{2}\right)$ & 37.66 & $\mathrm{~N} / \mathrm{A}$ & 61.9 \\
High Value $\left(\mathrm{dpm} / 100 \mathrm{~cm}^{2}\right)$ & 255.29 & $\mathrm{~N} / \mathrm{A}$ & $\mathrm{N} / \mathrm{A}$ \\
Low Value $\left(\mathrm{dpm} / 100 \mathrm{~cm}^{2}\right)$ & 113.57 & $\mathrm{~N} / \mathrm{A}$
\end{tabular}




\section{DQA Building Surface Report}

\section{Assessment Summary}

Site:

Planner(s):

Survey Unit Name:

Report Number:

Survey Unit Measurements:

Reference Area Measurements:

Test Performed:

Judgmental Areas:

Assessment Conclusion:
Hammond Depot FSS Planner

Vitkus

C1 SU24 through 32 Bldg 200E Ceiling C1 SU29

10

18

0

Sign

Test Result:

Not Performed

0

EMC Result:

Not Performed

Reject Null Hypothesis (Survey Unit PASSES)

\section{Retrospective Power Curve}

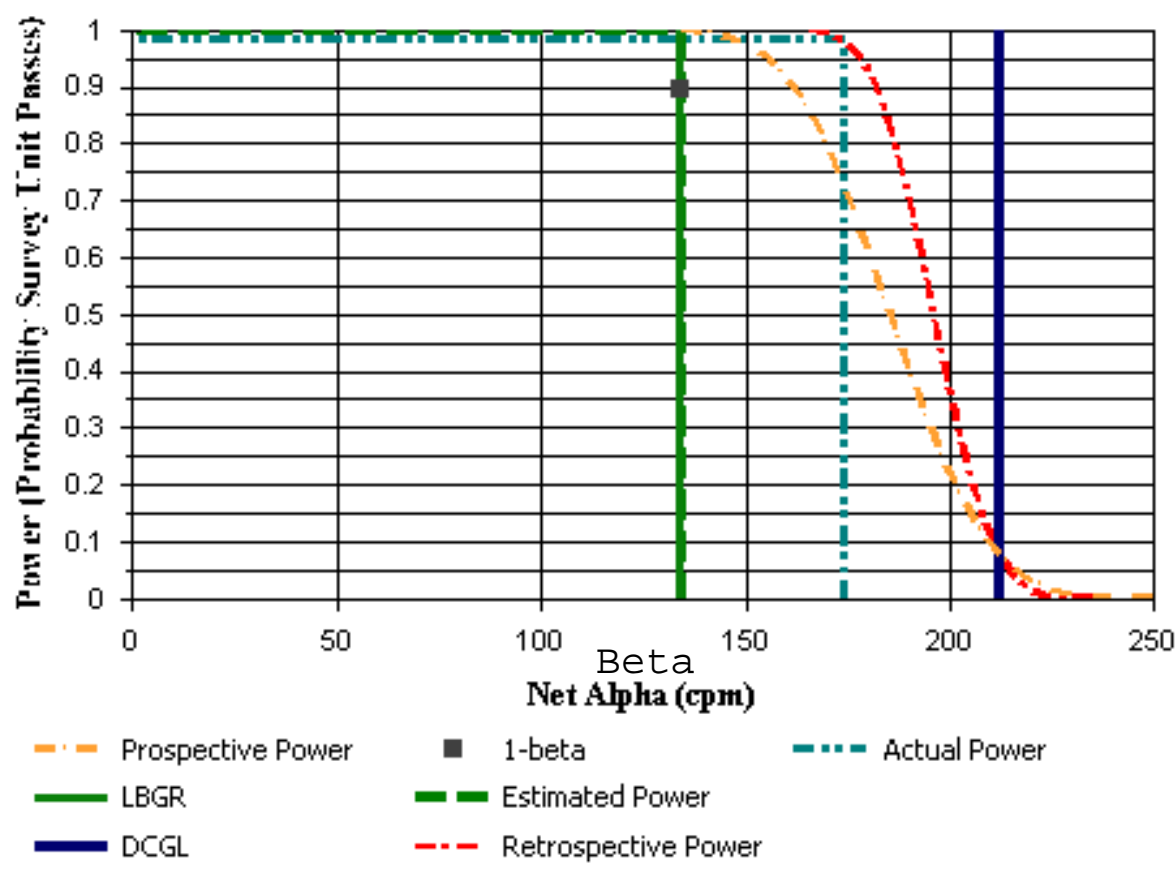




\section{DQA Building Surface Report}

\section{Survey Unit Data}

NOTE: $\quad$ Type $=$ "S" indicates survey measurement.

Type $=$ "R" indicates reference measurement.

\begin{tabular}{lccc} 
Measurement & Material & Type & Gross Alpha (cpm) \\
\hline $25.1,42.4$ & Metal & $\mathrm{S}$ & 268 \\
$28.2,42.4$ & Metal & $\mathrm{S}$ & 294 \\
$31.3,42.4$ & Metal & $\mathrm{S}$ & 272 \\
$34.4,42.4$ & Metal & $\mathrm{S}$ & 275 \\
$37.5,42.4$ & Metal & $\mathrm{S}$ & 290 \\
$26.7,45.1$ & Metal & $\mathrm{S}$ & 260 \\
$29.8,45.1$ & Metal & $\mathrm{S}$ & 365 \\
$32.8,45.1$ & Metal & $\mathrm{S}$ & 350 \\
$35.9,45.1$ & Metal & $\mathrm{S}$ & 216 \\
$25.1,47.8$ & Metal & $\mathrm{S}$ & 321 \\
$28.2,47.8$ & Metal & $\mathrm{S}$ & 247 \\
$31.3,47.8$ & Metal & $\mathrm{S}$ & 266 \\
$34.4,47.8$ & Metal & $\mathrm{S}$ & 242 \\
$37.5,47.8$ & Metal & $\mathrm{S}$ & 287 \\
$26.7,50.4$ & Metal & $\mathrm{S}$ & 266 \\
$29.8,50.4$ & Metal & $\mathrm{S}$ & 283 \\
$32.8,50.4$ & Metal & $\mathrm{S}$ & 255 \\
$35.9,50.4$ & Metal & $\mathrm{S}$ & 246
\end{tabular}

\section{Basic Statistical Quantities Summary}

\begin{tabular}{lccc} 
Statistic & Survey Unit & Background & DQO Results \\
\hline Sample Number & 18 & N/A & $\mathrm{N}=18$ \\
Mean $\left(\mathrm{dpm} / 100 \mathrm{~cm}^{2}\right)$ & 189.05 & $\mathrm{~N} / \mathrm{A}$ & 134 \\
Median $\left(\mathrm{dpm} / 100 \mathrm{~cm}^{2}\right)$ & 174.04 & $\mathrm{~N} / \mathrm{A}$ & $\mathrm{N} / \mathrm{A}$ \\
Std Dev $\left(\mathrm{dpm} / 100 \mathrm{~cm}^{2}\right)$ & 70.29 & $\mathrm{~N} / \mathrm{A}$ & 61.9 \\
High Value $\left(\mathrm{dpm} / 100 \mathrm{~cm}^{2}\right)$ & 353.55 & $\mathrm{~N} / \mathrm{A}$ & $\mathrm{N} / \mathrm{A}$ \\
Low Value $\left(\mathrm{dpm} / 100 \mathrm{~cm}^{2}\right)$ & 72.00 & $\mathrm{~N} / \mathrm{A}$ & $\mathrm{N} / \mathrm{A}$
\end{tabular}




\section{DQA Building Surface Report}

\section{Assessment Summary}

Site:

Planner(s):

Survey Unit Name:

Report Number:

Survey Unit Measurements:

Reference Area Measurements:

Test Performed:

Judgmental Areas:

Assessment Conclusion:
Hammond Depot FSS Planner

Vitkus

C1 SU24 through 32 BIdg 200E Ceiling C1 SU30

8

18

0

Sign

Test Result:

Not Performed

0

EMC Result:

Not Performed

Reject Null Hypothesis (Survey Unit PASSES)

\section{Retrospective Power Curve}

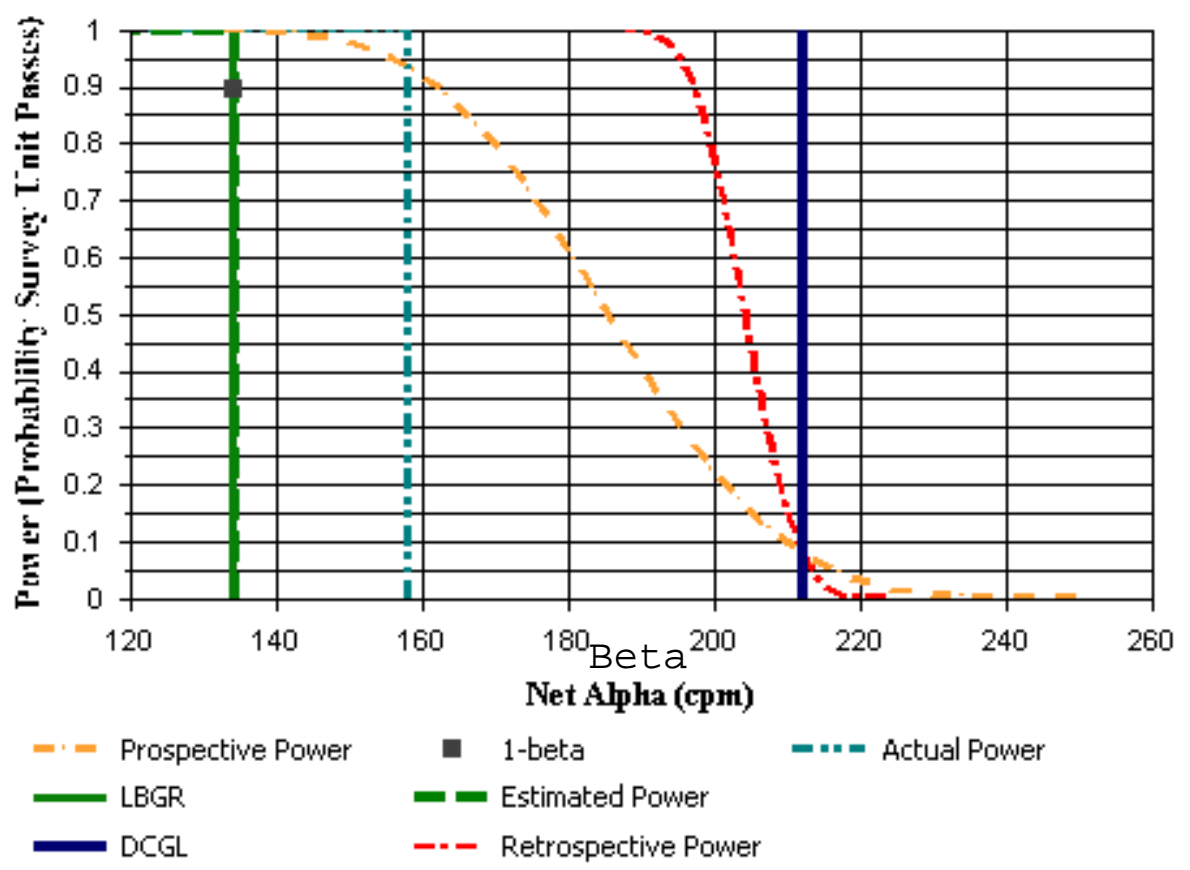




\section{DQA Building Surface Report}

\section{Survey Unit Data}

NOTE: $\quad$ Type $=$ "S" indicates survey measurement.

Type $=$ "R" indicates reference measurement.

\begin{tabular}{lccc} 
Measurement & Material & Type & Gross Alpha (cpm) \\
\hline $1.7,51.5$ & Metal & $\mathrm{S}$ & 236 \\
$4.8,51.5$ & Metal & $\mathrm{S}$ & 229 \\
$7.9,51.5$ & Metal & $\mathrm{S}$ & 242 \\
$10.9,51.5$ & Metal & $\mathrm{S}$ & 253 \\
$0.1,54.2$ & Metal & $\mathrm{S}$ & 262 \\
$3.2,54.2$ & Metal & $\mathrm{S}$ & 274 \\
$6.3,54.2$ & Metal & $\mathrm{S}$ & 293 \\
$9.4,54.2$ & Metal & $\mathrm{S}$ & 290 \\
$12.5,54.2$ & Metal & $\mathrm{S}$ & 236 \\
$1.7,56.9$ & Metal & $\mathrm{S}$ & 275 \\
$4.8,56.9$ & Metal & $\mathrm{S}$ & 261 \\
$7.9,56.9$ & Metal & $\mathrm{S}$ & 242 \\
$10.9,56.9$ & Metal & $\mathrm{S}$ & 257 \\
$0.1,59.6$ & Metal & $\mathrm{S}$ & 262 \\
$3.2,59.6$ & Metal & $\mathrm{S}$ & 263 \\
$6.3,59.6$ & Metal & $\mathrm{S}$ & 280 \\
$9.4,59.6$ & Metal & $\mathrm{S}$ & 263 \\
$12.5,59.6$ & Metal & $\mathrm{S}$ & 249
\end{tabular}

\section{Basic Statistical Quantities Summary}

\begin{tabular}{lccc} 
Statistic & Survey Unit & Background & DQO Results \\
\hline Sample Number & 18 & N/A & $\mathrm{N}=18$ \\
Mean $\left(\mathrm{dpm} / 100 \mathrm{~cm}^{2}\right)$ & 153.78 & $\mathrm{~N} / \mathrm{A}$ & 134 \\
Median $\left(\mathrm{dpm} / 100 \mathrm{~cm}^{2}\right)$ & 157.97 & $\mathrm{~N} / \mathrm{A}$ & $\mathrm{N} / \mathrm{A}$ \\
Std Dev $\left(\mathrm{dpm} / 100 \mathrm{~cm}^{2}\right)$ & 34.81 & $\mathrm{~N} / \mathrm{A}$ & 61.9 \\
High Value $\left(\mathrm{dpm} / 100 \mathrm{~cm}^{2}\right)$ & 217.50 & $\mathrm{~N} / \mathrm{A}$ & $\mathrm{N} / \mathrm{A}$ \\
Low Value $\left(\mathrm{dpm} / 100 \mathrm{~cm}^{2}\right)$ & 96.56 & $\mathrm{~N} / \mathrm{A}$ & $\mathrm{N} / \mathrm{A}$
\end{tabular}




\section{DQA Building Surface Report}

\section{Assessment Summary}

Site:

Planner(s):

Survey Unit Name:

Report Number:

Survey Unit Measurements:

Reference Area Measurements:

Test Performed:

Judgmental Areas:

Assessment Conclusion:
Hammond Depot FSS Planner

Vitkus

C1 SU24 through 32 Bldg 200E Ceiling C1 SU31

9

18

0

Sign

Test Result:

Not Performed

0

EMC Result:

Not Performed

Reject Null Hypothesis (Survey Unit PASSES)

\section{Retrospective Power Curve}

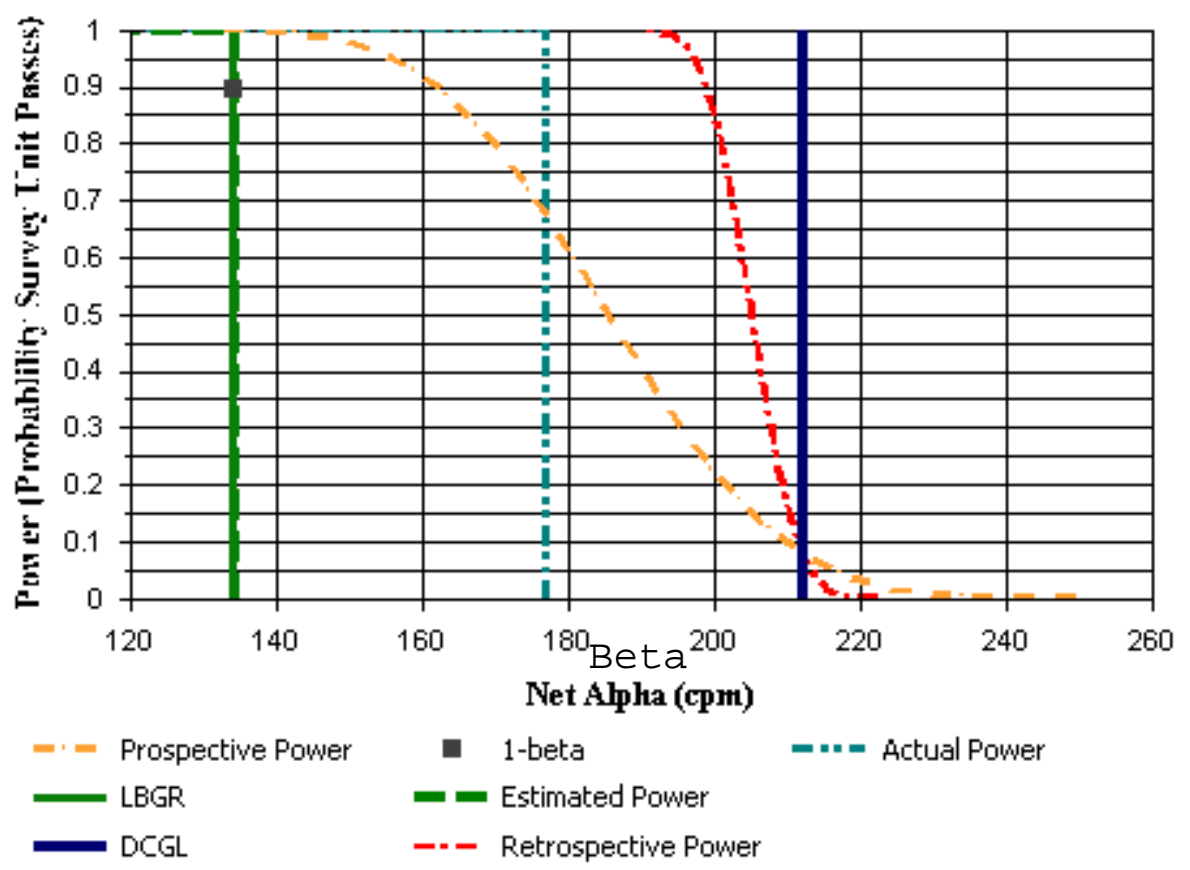




\section{DQA Building Surface Report}

\section{Survey Unit Data}

NOTE: $\quad$ Type $=$ "S" indicates survey measurement.

Type $=$ "R" indicates reference measurement.

\begin{tabular}{lccc} 
Measurement & Material & Type & Gross Alpha (cpm) \\
\hline $14.2,52.4$ & Metal & S & 301 \\
$17.2,52.4$ & Metal & S & 269 \\
$20.3,52.4$ & Metal & S & 294 \\
$23.4,52.4$ & Metal & S & 283 \\
$12.6,55.1$ & Metal & S & 265 \\
$15.7,55.1$ & Metal & S & 269 \\
$18.8,55.1$ & Metal & S & 264 \\
$21.9,55.1$ & Metal & S & 272 \\
$25.0,55.1$ & Metal & S & 268 \\
$14.2,57.8$ & Metal & S & 283 \\
$17.2,57.8$ & Metal & S & 231 \\
$20.3,57.8$ & Metal & S & 278 \\
$23.4,57.8$ & Metal & S & 286 \\
$12.6,60.4$ & Metal & S & 250 \\
$15.7,60.4$ & Metal & S & 271 \\
$18.8,60.4$ & Metal & S & 276 \\
$21.9,60.4$ & Metal & S & 281 \\
$25.0,60.4$ & Metal & S & 253
\end{tabular}

\section{Basic Statistical Quantities Summary}

\begin{tabular}{lccc} 
Statistic & Survey Unit & Background & DQO Results \\
\hline Sample Number & 18 & N/A & N=18 \\
Mean $\left(\mathrm{dpm} / 100 \mathrm{~cm}^{2}\right)$ & 177.61 & $\mathrm{~N} / \mathrm{A}$ & 134 \\
Median $\left(\mathrm{dpm} / 100 \mathrm{~cm}^{2}\right)$ & 176.87 & $\mathrm{~N} / \mathrm{A}$ & $\mathrm{N} / \mathrm{A}$ \\
Std Dev $\left(\mathrm{dpm} / 100 \mathrm{~cm}^{2}\right)$ & 30.98 & $\mathrm{~N} / \mathrm{A}$ & 61.9 \\
High Value $\left(\mathrm{dpm} / 100 \mathrm{~cm}^{2}\right)$ & 232.62 & $\mathrm{~N} / \mathrm{A}$ & $\mathrm{N} / \mathrm{A}$ \\
Low Value $\left(\mathrm{dpm} / 100 \mathrm{~cm}^{2}\right)$ & 100.34 & N/A
\end{tabular}




\section{DQA Building Surface Report}

\section{Assessment Summary}

Site:

Planner(s):

Survey Unit Name:

Report Number:

Survey Unit Measurements:

Reference Area Measurements:

Test Performed:

Judgmental Areas:

Assessment Conclusion:
Hammond Depot FSS Planner

Vitkus

C1 SU24 through 32 Bldg 200E Ceiling

11

18

0

Sign

Test Result:

Not Performed

0

EMC Result:

Not Performed

Reject Null Hypothesis (Survey Unit PASSES)

\section{Retrospective Power Curve}

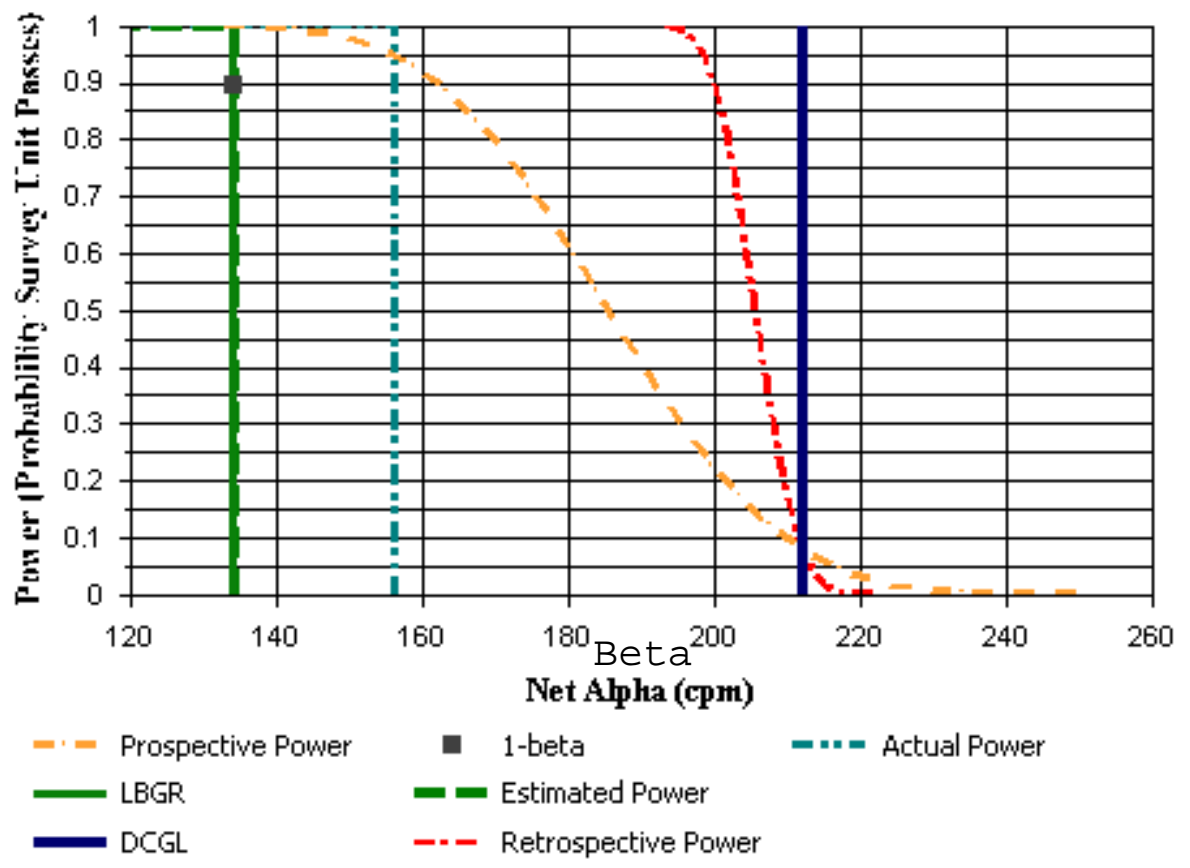




\section{DQA Building Surface Report}

\section{Survey Unit Data}

NOTE: $\quad$ Type $=$ "S" indicates survey measurement.

Type $=$ "R" indicates reference measurement.

\begin{tabular}{lccc} 
Measurement & Material & Type & Gross Alpha (cpm) \\
\hline $26.8,53.1$ & Metal & S & 261 \\
$29.8,53.1$ & Metal & S & 240 \\
$32.7,53.1$ & Metal & S & 255 \\
$35.6,53.1$ & Metal & S & 260 \\
$25.4,55.6$ & Metal & S & 277 \\
$28.3,55.6$ & Metal & S & 280 \\
$31.2,55.6$ & Metal & S & 278 \\
$34.2,55.6$ & Metal & S & 283 \\
$37.1,55.6$ & Metal & S & 275 \\
$26.8,58.2$ & Metal & S & 257 \\
$29.8,58.2$ & Metal & S & 256 \\
$32.7,58.2$ & Metal & S & 270 \\
$35.6,58.2$ & Metal & S & 281 \\
$25.4,60.7$ & Metal & S & 273 \\
$28.3,60.7$ & Metal & S & 251 \\
$31.2,60.7$ & Metal & S & 237 \\
$34.2,60.7$ & Metal & S & 241 \\
$37.1,60.7$ & Metal & S & 250
\end{tabular}

\section{Basic Statistical Quantities Summary}

\begin{tabular}{lccc} 
Statistic & Survey Unit & Background & DQO Results \\
\hline Sample Number & 18 & N/A & $\mathrm{N}=18$ \\
Mean $\left(\mathrm{dpm} / 100 \mathrm{~cm}^{2}\right)$ & 159.86 & $\mathrm{~N} / \mathrm{A}$ & 134 \\
Median $\left(\mathrm{dpm} / 100 \mathrm{~cm}^{2}\right)$ & 156.08 & $\mathrm{~N} / \mathrm{A}$ & $\mathrm{N} / \mathrm{A}$ \\
Std Dev $\left(\mathrm{dpm} / 100 \mathrm{~cm}^{2}\right)$ & 28.57 & $\mathrm{~N} / \mathrm{A}$ & 61.9 \\
High Value $\left(\mathrm{dpm} / 100 \mathrm{~cm}^{2}\right)$ & 198.60 & $\mathrm{~N} / \mathrm{A}$ & $\mathrm{N} / \mathrm{A}$ \\
Low Value $\left(\mathrm{dpm} / 100 \mathrm{~cm}^{2}\right)$ & 111.68 & $\mathrm{~N} / \mathrm{A}$ & $\mathrm{N} / \mathrm{A}$
\end{tabular}




\section{DQA Building Surface Report}

\section{Assessment Summary}

Site:

Planner(s):

Survey Unit Name:

Report Number:

Survey Unit Measurements:

Reference Area Measurements:

Test Performed:

Judgmental Areas:

Assessment Conclusion:
Hammond Depot FSS Planner

Vitkus

C1 SU33 and 34 Bldg 200E columns C1 SU33 Columns 1-20

1

14

0

Sign

Test Result:

Not Performed

0

EMC Result:

Not Performed

Reject Null Hypothesis (Survey Unit PASSES)

\section{Retrospective Power Curve}

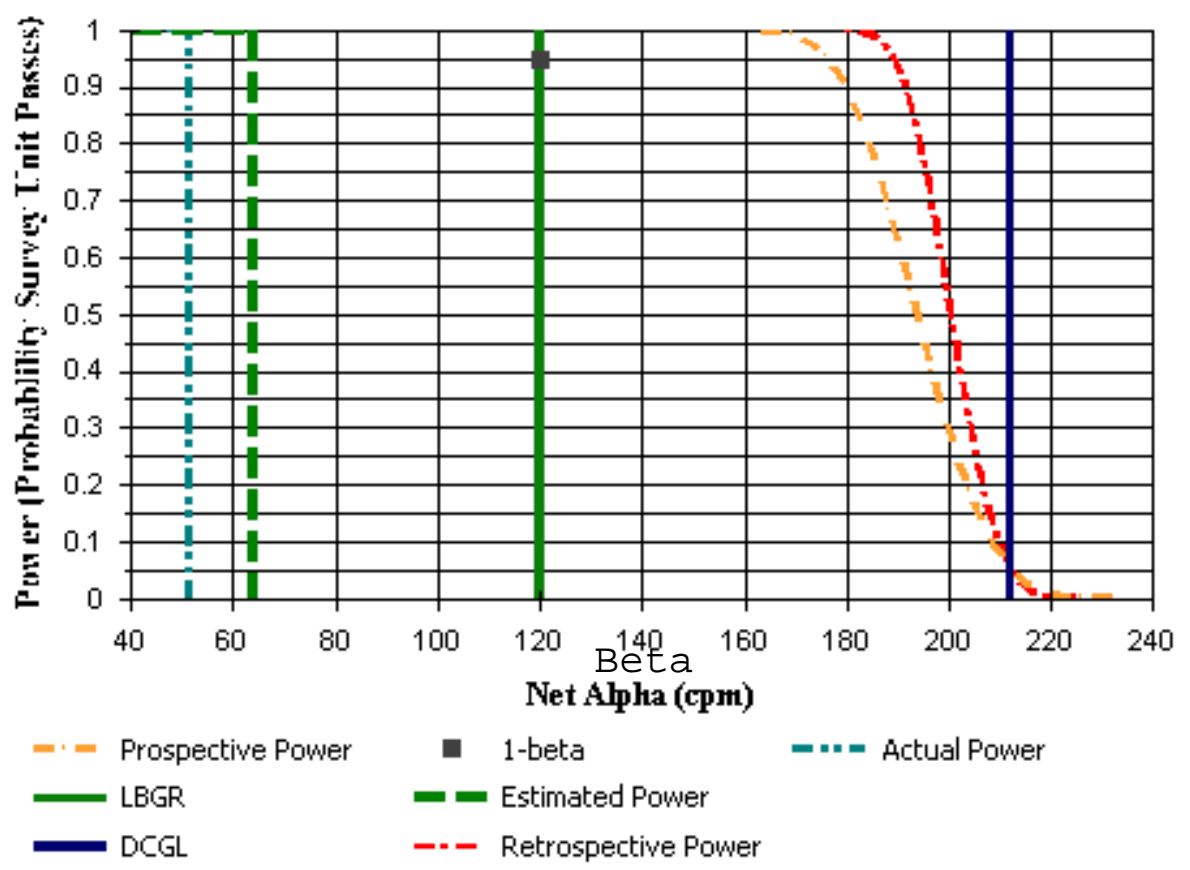




\section{DQA Building Surface Report}

\section{Survey Unit Data}

NOTE: $\quad$ Type $=$ "S" indicates survey measurement.

Type $=$ "R" indicates reference measurement.

Measurement

Material

Beta

Column 1, S. Face /2

Column 2, N. Face /2

Column 5, W. Face /4

Column 6, N. Face 14

Column 7, E. Face $/ 1$

Column 8, S. Face /1

Column 9, S. Face $/ 0$

Column 10, N. Face /

Column 11, E. Face /

Column 12, S. Face /

Column 13, S. Face /

Column 14, W. Face I

Column 19, E. Face /

Metal

Metal

Metal

Metal

Metal

Metal

Metal

Metal

Metal

Metal

Metal

Metal

Metal

Column 20, S. Face I

Metal

Type Gross Alpha (cpm)

\section{Basic Statistical Quantities Summary}

Statistic

Sample Number

Mean $\left(\mathrm{dpm} / 100 \mathrm{~cm}^{2}\right)$

Median (dpm/100 cm²)

Std Dev (dpm/100 $\left.\mathrm{cm}^{2}\right)$

High Value $\left(\mathrm{dpm} / 100 \mathrm{~cm}^{2}\right)$

Low Value $\left(\mathrm{dpm} / 100 \mathrm{~cm}^{2}\right)$
Survey Unit

14
59.31
51.21
38.94
160.81
22.86

14

22.86
Background

DQO Results

$\begin{array}{ll}\text { N/A } & \mathrm{N}=14 \\ \text { N/A } & 64.1 \\ \text { N/A } & \text { N/A } \\ \text { N/A } & 32.3 \\ \text { N/A } & \text { N/A } \\ \text { N/A } & \text { N/A }\end{array}$




\section{DQA Building Surface Report}

\section{Assessment Summary}

Site:

Planner(s):

Survey Unit Name:

Report Number:

Survey Unit Measurements:

Reference Area Measurements:

Test Performed:

Judgmental Areas:

Assessment Conclusion:
Hammond Depot FSS Planner

Vitkus

C1 SU33 and 34 Bldg 200E columns C1 SU34 columns 21-36

2

10

0

Sign

Test Result: $\quad$ Not Performed

0

EMC Result: $\quad$ Not Performed

2 hot spots found and

Reject Null Hypothesis (Survey Unit PASSES) deconned.

\section{Retrospective Power Curve}

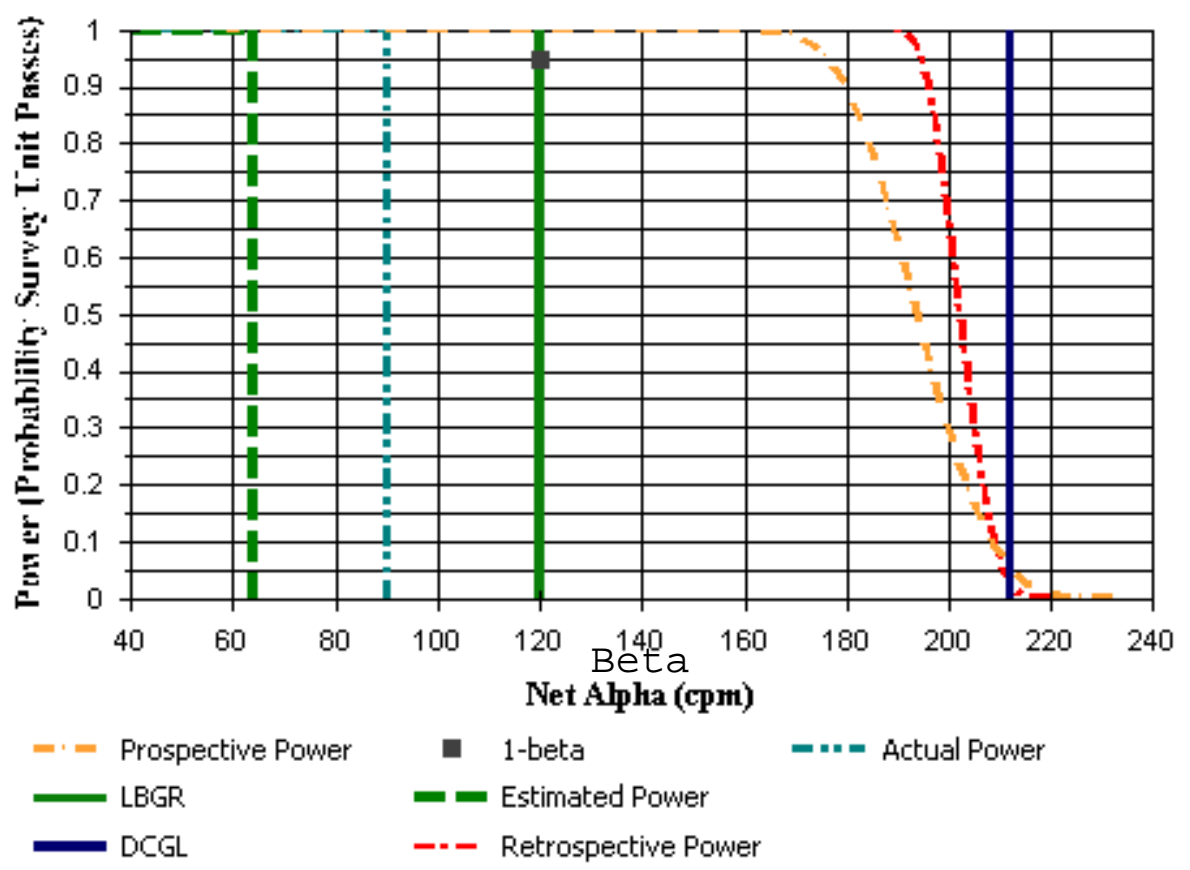




\section{DQA Building Surface Report}

\section{Survey Unit Data}

NOTE: $\quad$ Type $=$ "S" indicates survey measurement.

Type = "R" indicates reference measurement.

\begin{tabular}{lccc} 
Measurement & Material & Type & Gross Alpha (cpm) \\
\hline Column 23, E. Face / & Metal & S & 228 \\
Column 24, S. Face / & Metal & S & 223 \\
Column 25, W. Face / & Metal & S & 240 \\
Column 26, N. Face / & Metal & S & 232 \\
Column 27, E. Face I & Metal & S & 217 \\
Column 28, S. Face / & Metal & S & 219 \\
Column 29, W. Face / & Metal & S & 230 \\
Column 30, N. Face / & Metal & S & 200 \\
Column 35, E. Face I & Metal & S & 236 \\
Column 36, S. Face / & Metal & S & 216
\end{tabular}

\section{Basic Statistical Quantities Summary}

$\begin{array}{lccc}\text { Statistic } & \text { Survey Unit } & \text { Background } & \text { DQO Results } \\ \text { Sample Number } & 10 & \text { N/A } & \text { N=14 } \\ \text { Mean }\left(\mathrm{dpm} / 100 \mathrm{~cm}^{2}\right) & 87.30 & \mathrm{~N} / \mathrm{A} & 64.1 \\ \text { Median }\left(\mathrm{dpm} / 100 \mathrm{~cm}^{2}\right) & 89.95 & \mathrm{~N} / \mathrm{A} & \mathrm{N} / \mathrm{A} \\ \text { Std Dev }\left(\mathrm{dpm} / 100 \mathrm{~cm}^{2}\right) & 22.10 & \mathrm{~N} / \mathrm{A} / \mathrm{A} & 32.3 \\ \text { High Value }\left(\mathrm{dpm} / 100 \mathrm{~cm}^{2}\right) & 117.35 & \mathrm{~N} / \mathrm{A} & \mathrm{N} / \mathrm{A} \\ \text { Low Value }\left(\mathrm{dpm} / 100 \mathrm{~cm}^{2}\right) & 41.76 & \mathrm{~N} / \mathrm{A}\end{array}$




\section{DQA Building Surface Report}

\section{Assessment Summary}

Site:

Planner(s):

Survey Unit Name:

Report Number:

Survey Unit Measurements:

Reference Area Measurements:

Test Performed:

Judgmental Areas:

Assessment Conclusion:
Hammond Depot FSS Planner

Vitkus

C1 SU41 Bldg 200E NW closet Rev 1

1

19

0

Sign

Test Result:

Not Performed

0

EMC Result:

Not Performed

\section{Retrospective Power Curve}

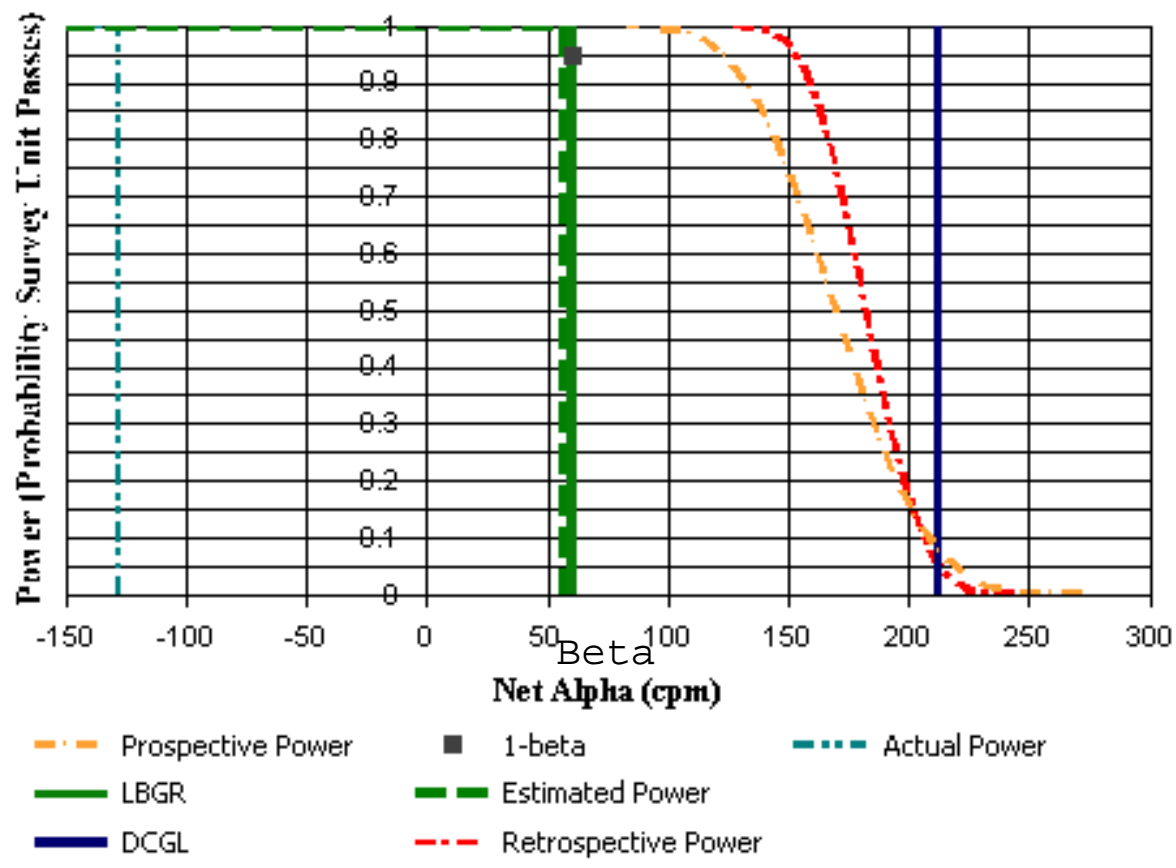




\section{DQA Building Surface Report}

\section{Survey Unit Data}

NOTE: $\quad$ Type = "S" indicates survey measurement.

Type $=$ "R" indicates reference measurement.

\begin{tabular}{lccc} 
Measurement & Material & Type & Gross Alpha (cpm) \\
\hline $0.3,0.2$ & Concrete & $\mathrm{S}$ & 259 \\
$0.7,1.0$ & Concrete & $\mathrm{S}$ & 328 \\
$0.3,0.1,2.5$ & Concrete & $\mathrm{S}$ & 235 \\
$0.8,0.8,2.5$ & Concrete & $\mathrm{S}$ & 269 \\
$0.8,0.0,0.1$ & Concrete & $\mathrm{S}$ & 234 \\
$1.6,0.9,0.1$ & Concrete & $\mathrm{S}$ & 250 \\
$0.3,2.5,0.1$ & Concrete & $\mathrm{S}$ & 411 \\
$0.3,0.0,0.8$ & Concrete & $\mathrm{S}$ & 234 \\
$1.6,0.4,0.8$ & Concrete & $\mathrm{S}$ & 236 \\
$0.7,2.6,0.8$ & Concrete & $\mathrm{S}$ & 403 \\
$0.0,1.0,0.8$ & Concrete & $\mathrm{S}$ & 299 \\
$0.8,0.0,1.6$ & Concrete & $\mathrm{S}$ & 238 \\
$1.6,0.9,1.6$ & Concrete & $\mathrm{S}$ & 284 \\
$0.3,2.5,1.6$ & Concrete & $\mathrm{S}$ & 403 \\
$0.3,0.0,2.4$ & Concrete & $\mathrm{S}$ & 242 \\
$1.6,0.4,2.2$ & Concrete & $\mathrm{S}$ & 252 \\
$0.7,2.6,2.2$ & Concrete & $\mathrm{S}$ & 355 \\
$0.0,1.0,2.0$ & Concrete & $\mathrm{S}$ & 306 \\
$0.0,0.7,1.5$ & Metal & $\mathrm{S}$ & 178
\end{tabular}

\section{Basic Statistical Quantities Summary}

Statistic

Sample Number

Mean $\left(\mathrm{dpm} / 100 \mathrm{~cm}^{2}\right)$

Median (dpm/100 cm²)

Std Dev (dpm/100 cm²)

High Value $\left(\mathrm{dpm} / 100 \mathrm{~cm}^{2}\right)$

Low Value $\left(\mathrm{dpm} / 100 \mathrm{~cm}^{2}\right)$
Survey Unit

19

$-81.80$

$-127.93$

117.72

140.40

$-194.07$
Background

DQO Results

$\begin{array}{ll}\text { N/A } & \mathrm{N}=18 \\ \text { N/A } & 57.1 \\ \text { N/A } & \text { N/A } \\ \text { N/A } & 98.2 \\ \text { N/A } & \text { N/A } \\ \text { N/A } & \text { N/A }\end{array}$




\section{DQA Building Surface Report}

\section{Assessment Summary}

Site:

Planner(s):

Survey Unit Name:

Report Number:

Survey Unit Measurements:

Reference Area Measurements:

Test Performed:

Judgmental Areas:

Assessment Conclusion:
Hammond Depot FSS Planner

Vitkus

C1 SU43 and 44 Building 100E C1 SU43

1

15

0

Sign

Test Result:

Not Performed

0

EMC Result:

Not Performed

\section{Reject Null Hypothesis (Survey Unit PASSES)}

\section{Retrospective Power Curve}

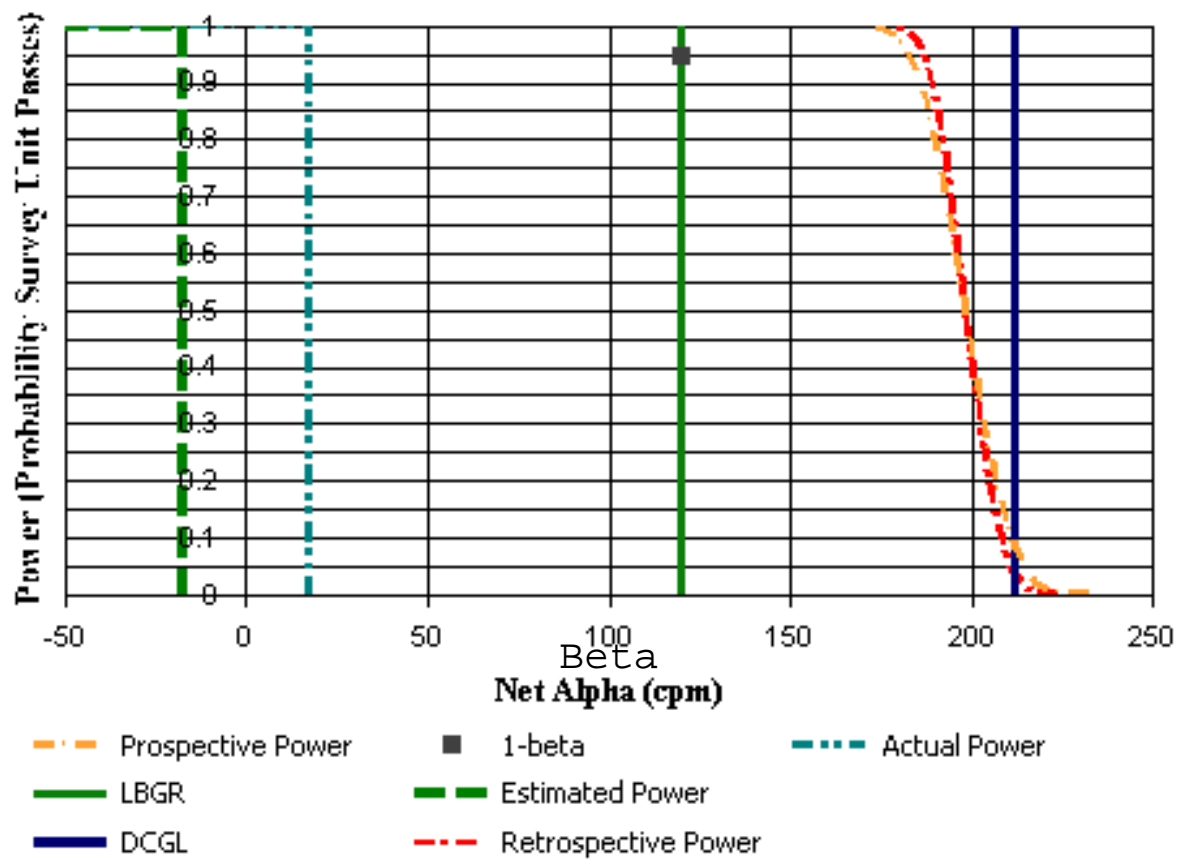




\section{DQA Building Surface Report}

\section{Survey Unit Data}

NOTE: $\quad$ Type = "S" indicates survey measurement.

Type $=$ "R" indicates reference measurement.

\begin{tabular}{llcc} 
Measurement & Material & Type & Gross Alpha (cpm) \\
\hline $7.8,91.6$ & Concrete & $\mathrm{S}$ & 346 \\
$7.8,99.2$ & Concrete & $\mathrm{S}$ & 383 \\
$10.0,87.8$ & Concrete & $\mathrm{S}$ & 341 \\
$10.0,95.4$ & Concrete & $\mathrm{S}$ & 376 \\
$10.0,103.0$ & Concrete & $\mathrm{S}$ & 372 \\
$12.2,91.6$ & Concrete & $\mathrm{S}$ & 341 \\
$12.2,99.2$ & Concrete & $\mathrm{S}$ & 339 \\
$14.4,87.8$ & Concrete & $\mathrm{S}$ & 322 \\
$14.4,95.4$ & Concrete & $\mathrm{S}$ & 349 \\
$14.4,103.0$ & Concrete & $\mathrm{S}$ & 396 \\
$16.5,91.6$ & Concrete & $\mathrm{S}$ & 332 \\
$16.5,99.2$ & Concrete & $\mathrm{S}$ & 374 \\
$18.7,87.8$ & Concrete & $\mathrm{S}$ & 336 \\
$18.7,95.4$ & Concrete & $\mathrm{S}$ & 331 \\
$18.7,103.0$ & Concrete & $\mathrm{S}$ & 354
\end{tabular}

\section{Basic Statistical Quantities Summary}

\begin{tabular}{lccc} 
Statistic & Survey Unit & Background & DQO Results \\
\hline Sample Number & 15 & $\mathrm{~N} / \mathrm{A}$ & $\mathrm{N}=13$ \\
Mean $\left(\mathrm{dpm} / 100 \mathrm{~cm}^{2}\right)$ & 30.42 & $\mathrm{~N} / \mathrm{A}$ & -17.7 \\
Median $\left(\mathrm{dpm} / 100 \mathrm{~cm}^{2}\right)$ & 17.57 & $\mathrm{~N} / \mathrm{A}$ & $\mathrm{N} / \mathrm{A}$ \\
Std Dev $\left(\mathrm{dpm} / 100 \mathrm{~cm}^{2}\right)$ & 41.66 & $\mathrm{~N} / \mathrm{A} / \mathrm{A}$ & 27.7 \\
High Value $\left(\mathrm{dpm} / 100 \mathrm{~cm}^{2}\right)$ & 112.06 & $\mathrm{~N} / \mathrm{A}$ & $\mathrm{N} / \mathrm{A}$ \\
Low Value $\left(\mathrm{dpm} / 100 \mathrm{~cm}^{2}\right)$ & -27.78 & $\mathrm{~N} / \mathrm{A}$
\end{tabular}




\section{DQA Building Surface Report}

\section{Assessment Summary}

Site:

Planner(s):

Survey Unit Name:

Report Number:

Survey Unit Measurements:

Reference Area Measurements:

Test Performed:

Judgmental Areas:

Assessment Conclusion:
Hammond Depot FSS Planner

Vitkus

C1 SU43 and 44 Building 100E C1 SU44

2

15

0

Sign

Test Result:

Not Performed

0

EMC Result:

Not Performed

Reject Null Hypothesis (Survey Unit PASSES)

\section{Retrospective Power Curve}

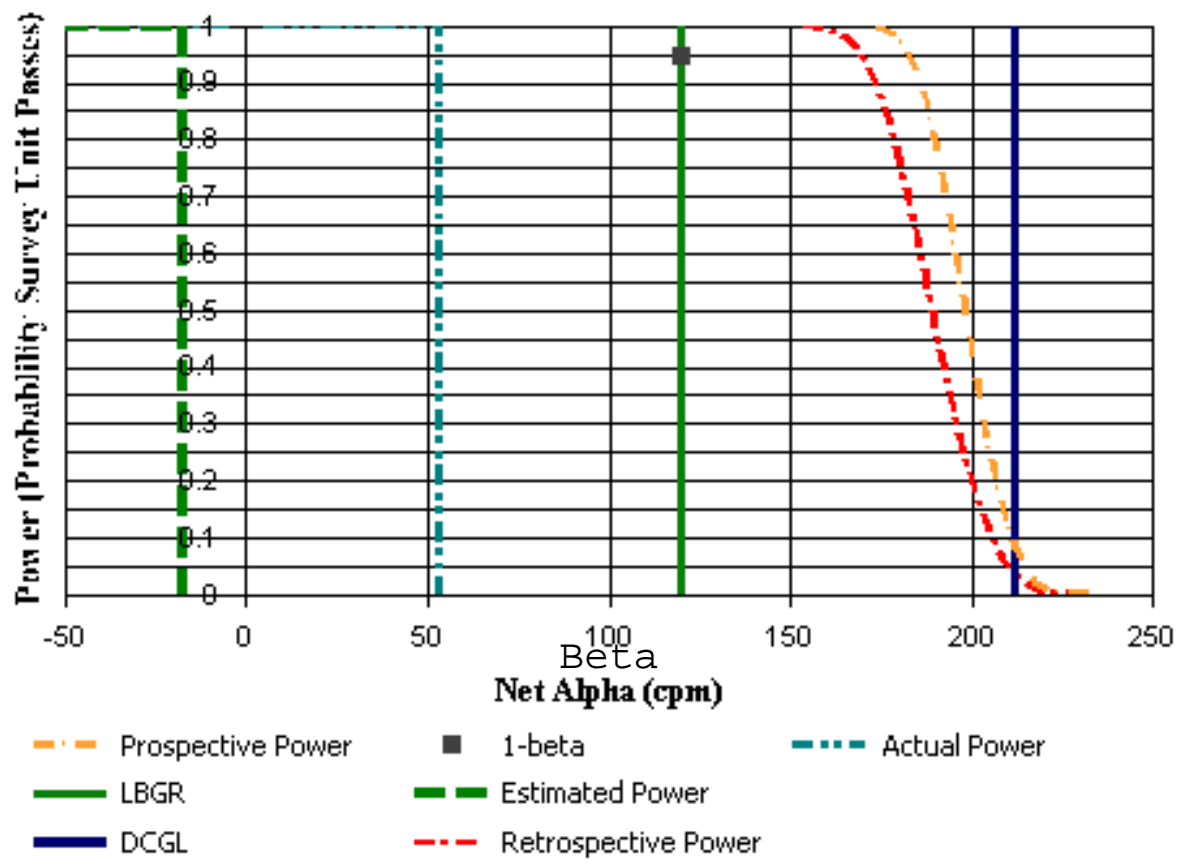




\section{DQA Building Surface Report}

\section{Survey Unit Data}

NOTE: $\quad$ Type = "S" indicates survey measurement.

Type $=$ "R" indicates reference measurement.

\begin{tabular}{llcc} 
Measurement & Material & Type & Gross Alpha (cpm) \\
\hline $19.4,90.4$ & Concrete & S & 368 \\
$19.4,98.1$ & Concrete & S & 340 \\
$21.7,86.5$ & Concrete & S & 324 \\
$21.7,94.3$ & Concrete & S & 334 \\
$21.7,102.0$ & Concrete & S & 372 \\
$23.9,90.4$ & Concrete & S & 359 \\
$23.9,98.1$ & Concrete & S & 365 \\
$26.1,86.5$ & Concrete & S & 432 \\
$26.1,94.3$ & Concrete & S & 341 \\
$26.1,102.0$ & Concrete & S & 449 \\
$28.4,90.4$ & Concrete & S & 369 \\
$28.4,98.1$ & Concrete & S & 408 \\
$30.6,86.5$ & Concrete & S & 389 \\
$30.6,94.3$ & Concrete & S & 350 \\
$30.6,102.0$ & Concrete & S & 331
\end{tabular}

\section{Basic Statistical Quantities Summary}

\begin{tabular}{lccc} 
Statistic & Survey Unit & Background & DQO Results \\
\hline Sample Number & 15 & N/A & N $=13$ \\
Mean $\left(\mathrm{dpm} / 100 \mathrm{~cm}^{2}\right)$ & 60.53 & N/A & -17.7 \\
Median $\left(\mathrm{dpm} / 100 \mathrm{~cm}^{2}\right)$ & 53.48 & N/A $/ A$ & 27.7 \\
Std Dev $\left(\mathrm{dpm} / 100 \mathrm{~cm}^{2}\right)$ & 69.79 & N/A & N/A \\
High Value $\left(\mathrm{dpm} / 100 \mathrm{~cm}^{2}\right)$ & 212.21 & N/A & N/A
\end{tabular}




\section{DQA Building Surface Report}

\section{Assessment Summary}

Site:

Planner(s):

Survey Unit Name:

Report Number:

Survey Unit Measurements:

Reference Area Measurements:

Test Performed:

Judgmental Areas:

Assessment Conclusion:
Hammond Depot FSS Planner

Vitkus

C1 SU43 and 44 Building 100E

C1 SU59

Bays

13 and 14

(added)

3

15

0

Sign

Test Result:

Not Performed

0

EMC Result:

Not Performed

\section{Retrospective Power Curve}

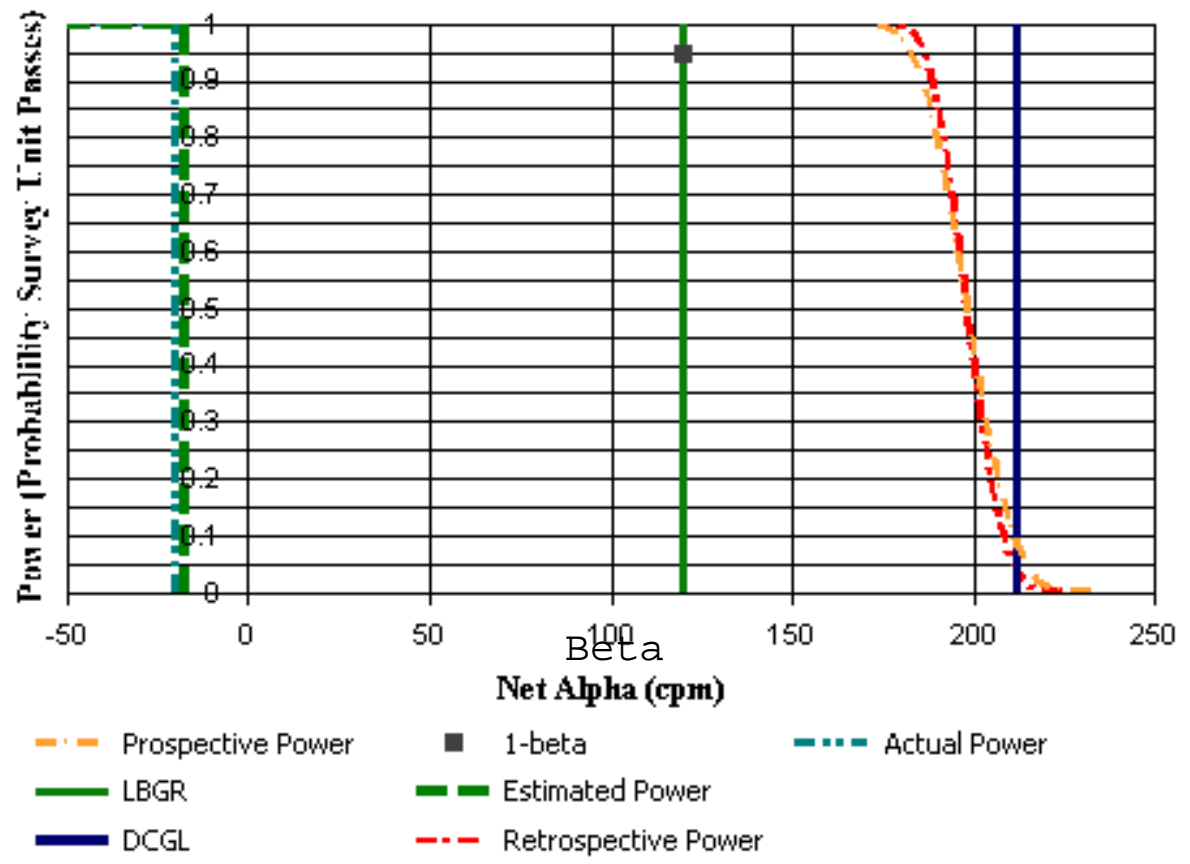




\section{DQA Building Surface Report}

\section{Survey Unit Data}

NOTE: $\quad$ Type = "S" indicates survey measurement.

Type $=$ "R" indicates reference measurement.

\begin{tabular}{llcc} 
Measurement & Material & Type & Gross Alpha (cpm) \\
\hline $15.3,73.2$ & Concrete & $\mathrm{S}$ & 341 \\
$17.9,73.2$ & Concrete & $\mathrm{S}$ & 323 \\
$21.4,73.2$ & Concrete & $\mathrm{S}$ & 271 \\
$16.5,75.7$ & Concrete & $\mathrm{S}$ & 315 \\
$19.9,75.7$ & Concrete & $\mathrm{S}$ & 357 \\
$21.4,75.7$ & Concrete & $\mathrm{S}$ & 324 \\
$15.3,78.2$ & Concrete & $\mathrm{S}$ & 326 \\
$17.9,78.2$ & Concrete & $\mathrm{S}$ & 320 \\
$21.4,78.2$ & Concrete & $\mathrm{S}$ & 334 \\
$16.5,80.7$ & Concrete & $\mathrm{S}$ & 303 \\
$19.9,80.7$ & Concrete & $\mathrm{S}$ & 310 \\
$21.4,80.7$ & Concrete & $\mathrm{S}$ & 329 \\
$15.3,83.2$ & Concrete & $\mathrm{S}$ & 343 \\
$19.5,83.2$ & Concrete & $\mathrm{S}$ & 365 \\
$22.4,83.2$ & Concrete & $\mathrm{S}$ & 329
\end{tabular}

\section{Basic Statistical Quantities Summary}

\begin{tabular}{lccc} 
Statistic & Survey Unit & Background & DQO Results \\
\hline Sample Number & 15 & N/A & N=13 \\
Mean $\left(\mathrm{dpm} / 100 \mathrm{~cm}^{2}\right)$ & -20.22 & $\mathrm{~N} / \mathrm{A}$ & -17.7 \\
Median $\left(\mathrm{dpm} / 100 \mathrm{~cm}^{2}\right)$ & -20.22 & $\mathrm{~N} / \mathrm{A}$ & $\mathrm{N} / \mathrm{A}$ \\
Std Dev $\left(\mathrm{dpm} / 100 \mathrm{~cm}^{2}\right)$ & 42.43 & $\mathrm{~N} / \mathrm{A}$ & 27.7 \\
High Value $\left(\mathrm{dpm} / 100 \mathrm{~cm}^{2}\right)$ & 53.48 & $\mathrm{~N} / \mathrm{A}$ & $\mathrm{N} / \mathrm{A}$ \\
Low Value $\left(\mathrm{dpm} / 100 \mathrm{~cm}^{2}\right)$ & -124.15 & $\mathrm{~N} / \mathrm{A}$ & $\mathrm{N} / \mathrm{A}$
\end{tabular}




\section{DQA Building Surface Report}

\section{Assessment Summary}

Site:

Planner(s):

Survey Unit Name:

Report Number:

Survey Unit Measurements:

Reference Area Measurements:

Test Performed:

Judgmental Areas:

Assessment Conclusion:
Hammond Depot FSS Planner

Vitkus

C2 SU35 and 36 Bldg 200E Ceiling Rev. 1 C2 SU35

1

17

0

Sign

Test Result:

Not Performed

0

EMC Result:

Not Performed

Reject Null Hypothesis (Survey Unit PASSES)

\section{Retrospective Power Curve}

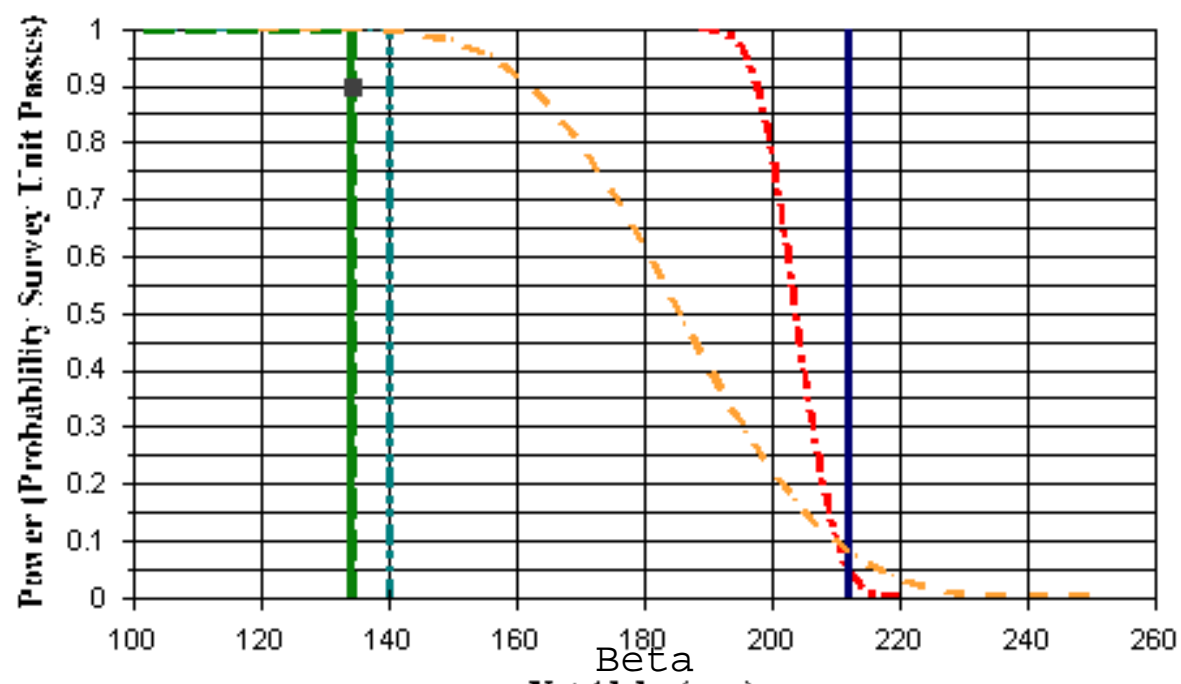

Net Alpha (cpm)

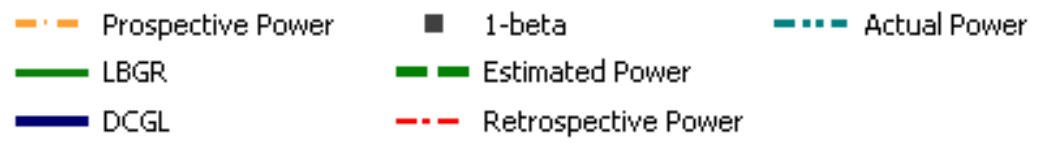




\section{DQA Building Surface Report}

\section{Survey Unit Data}

NOTE: $\quad$ Type $=$ "S" indicates survey measurement.

Type $=$ "R" indicates reference measurement.

\begin{tabular}{|c|c|c|c|}
\hline Measurement & Material & Type & Gross Alpha (cpm) \\
\hline $2.4,3.4$ & Metal & $\mathrm{S}$ & 245 \\
\hline $9.1,3.4$ & Metal & $\mathrm{S}$ & 281 \\
\hline $15.7,3.4$ & Metal & S & 276 \\
\hline $22.4,3.4$ & Metal & S & 250 \\
\hline $29.0,3.4$ & Metal & $S$ & 244 \\
\hline $35.7,3.4$ & Metal & $\mathrm{S}$ & 252 \\
\hline $5.7,9.1$ & Metal & S & 248 \\
\hline $12.4,9.1$ & Metal & $\mathrm{S}$ & 237 \\
\hline $19.1,9.1$ & Metal & S & 278 \\
\hline $25.7,9.1$ & Metal & $\mathrm{S}$ & 237 \\
\hline $32.4,9.1$ & Metal & S & 252 \\
\hline $2.4,14.9$ & Metal & $\mathrm{S}$ & 229 \\
\hline $9.1,14.9$ & Metal & $\mathrm{S}$ & 268 \\
\hline $15.7,14.9$ & Metal & S & 254 \\
\hline $22.4,14.9$ & Metal & S & 270 \\
\hline $29.0,14.9$ & Metal & S & 267 \\
\hline $35.7,14.9$ & Metal & $\mathrm{S}$ & 264 \\
\hline
\end{tabular}

\section{Basic Statistical Quantities Summary}

Statistic

Sample Number

Mean $\left(\mathrm{dpm} / 100 \mathrm{~cm}^{2}\right)$

Median (dpm/100 $\left.\mathrm{cm}^{2}\right)$

Std Dev (dpm/100 $\left.\mathrm{cm}^{2}\right)$

High Value $\left(\mathrm{dpm} / 100 \mathrm{~cm}^{2}\right)$

Low Value $\left(\mathrm{dpm} / 100 \mathrm{~cm}^{2}\right)$
Survey Unit

17
147.58
140.02
29.45
194.82
96.56

Background

$\begin{array}{ll}\text { N/A } & N=18 \\ \text { N/A } & 134 \\ \text { N/A } & \text { N/A } \\ \text { N/A } & 61.9 \\ \text { N/A } & \text { N/A } \\ \text { N/A } & \text { N/A }\end{array}$




\section{DQA Building Surface Report}

\section{Assessment Summary}

Site:

Planner(s):

Survey Unit Name:

Report Number:

Survey Unit Measurements:

Reference Area Measurements:

Test Performed:

Judgmental Areas:

Assessment Conclusion:
Hammond Depot FSS Planner

Vitkus

C2 SU35 and 36 Bldg 200E Ceiling Rev. 1

2

17

0

Sign

Test Result:

Not Performed

0

EMC Result:

Not Performed

\section{Retrospective Power Curve}

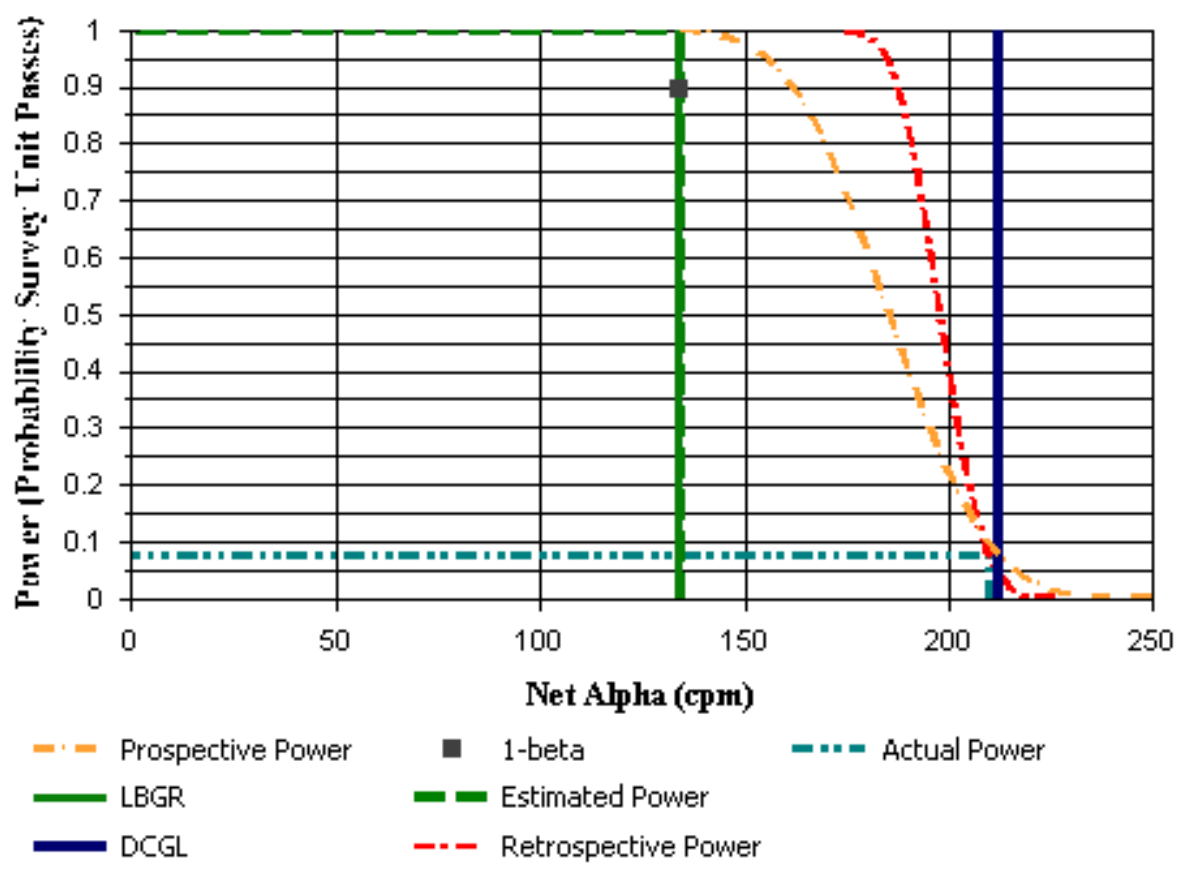




\section{DQA Building Surface Report}

\section{Survey Unit Data}

NOTE: $\quad$ Type $=$ "S" indicates survey measurement.

Type $=$ "R" indicates reference measurement.

\begin{tabular}{lccc} 
Measurement & Material & Type & Gross Alpha (cpm) \\
\hline $2.5,18.4$ & Metal & $\mathrm{S}$ & 296 \\
$9.2,18.4$ & Metal & $\mathrm{S}$ & 291 \\
$15.8,18.4$ & Metal & $\mathrm{S}$ & 301 \\
$22.5,18.4$ & Metal & $\mathrm{S}$ & 298 \\
$29.1,18.4$ & Metal & $\mathrm{S}$ & 297 \\
$35.8,18.4$ & Metal & $\mathrm{S}$ & 276 \\
$5.9,24.1$ & Metal & $\mathrm{S}$ & 263 \\
$12.5,24.1$ & Metal & $\mathrm{S}$ & 312 \\
$19.2,24.1$ & Metal & $\mathrm{S}$ & 290 \\
$25.8,24.1$ & Metal & $\mathrm{S}$ & 263 \\
$32.5,24.1$ & Metal & $\mathrm{S}$ & 289 \\
$2.5,29.9$ & Metal & $\mathrm{S}$ & 269 \\
$9.2,29.9$ & Metal & $\mathrm{S}$ & 230 \\
$15.8,29.9$ & Metal & $\mathrm{S}$ & 219 \\
$22.5,29.9$ & Metal & $\mathrm{S}$ & 297 \\
$29.1,29.9$ & Metal & $\mathrm{S}$ & 257 \\
$35.8,29.9$ & Metal & $\mathrm{S}$ & 250
\end{tabular}

\section{Basic Statistical Quantities Summary}

\begin{tabular}{|c|c|c|c|}
\hline Statistic & Survey Unit & Background & DQO Results \\
\hline Sample Number & 17 & $N / A$ & $\mathrm{~N}=18$ \\
\hline Mean $\left(\mathrm{dpm} / 100 \mathrm{~cm}^{2}\right)$ & 186.04 & N/A & 134 \\
\hline Median (dpm/100 cm²) & 209.94 & N/A & $\mathrm{N} / \mathrm{A}$ \\
\hline Std Dev (dpm/100 cm²) & 49.69 & $N / A$ & 61.9 \\
\hline High Value $\left(\mathrm{dpm} / 100 \mathrm{~cm}^{2}\right)$ & 253.40 & N/A & N/A \\
\hline Low Value $\left(\mathrm{dpm} / 100 \mathrm{~cm}^{2}\right)$ & 77.66 & $N / A$ & $N / A$ \\
\hline
\end{tabular}




\section{DQA Building Surface Report}

\section{Assessment Summary}

Site:

Planner(s):

Survey Unit Name:

Report Number:

Survey Unit Measurements:

Reference Area Measurements:

Test Performed:

Judgmental Areas:

Assessment Conclusion:
Hammond Depot FSS Planner

Vitkus

Class 2 SUs Scoping Rev. 1 C2 SU37 Building 200E, North 1

22

0

Sign

Test Result: $\quad$ Not Performed

0

EMC Result:

Not Performed

Reject Null Hypothesis (Survey Unit PASSES)

\section{Retrospective Power Curve}

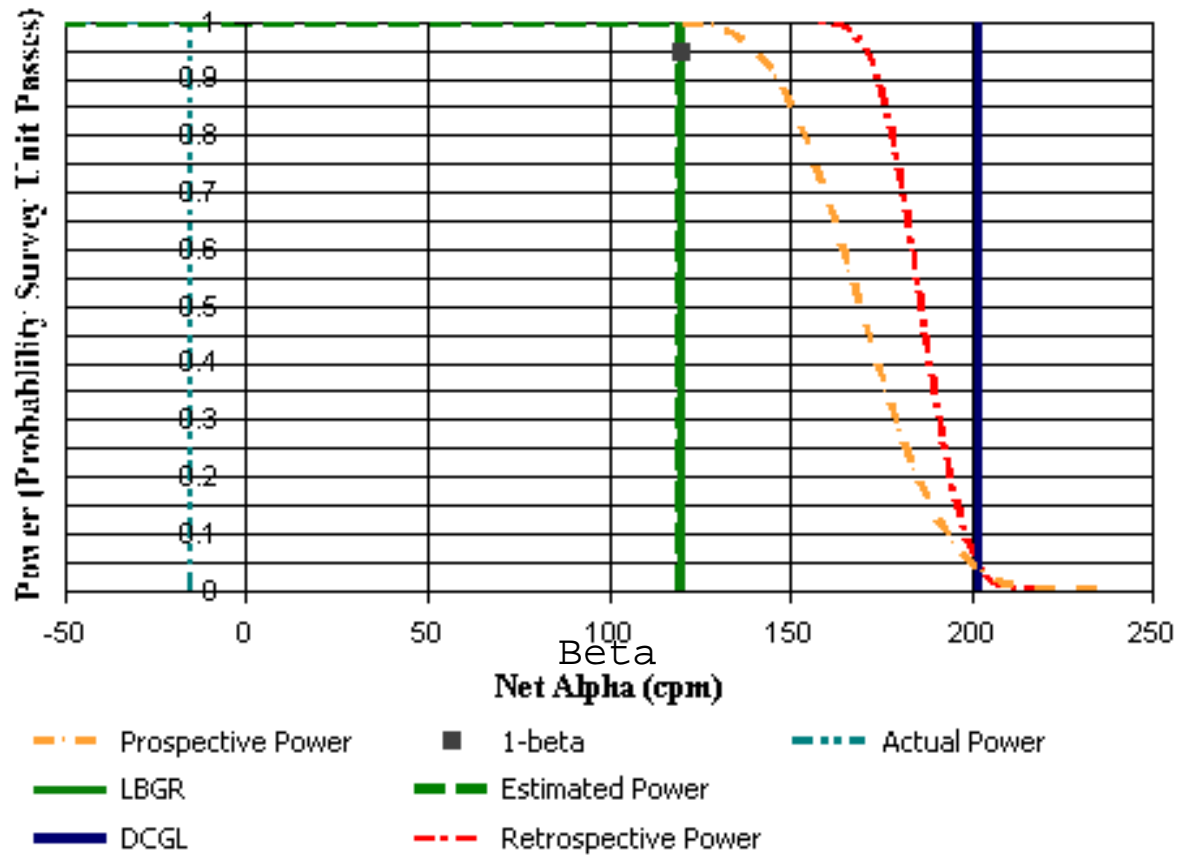




\section{DQA Building Surface Report}

\section{Survey Unit Data}

NOTE: $\quad$ Type = "S" indicates survey measurement.

Type $=$ "R" indicates reference measurement.

\begin{tabular}{|c|c|c|c|}
\hline Measurement & Material & Type & Gross Alpha (cpm) \\
\hline$\overline{46}$ & Concrete & $\mathrm{S}$ & 296 \\
\hline 47 & Concrete & $\mathrm{S}$ & 327 \\
\hline 48 & Concrete & S & 304 \\
\hline 49 & Concrete & S & 329 \\
\hline 50 & Concrete & $S$ & 337 \\
\hline 52 & Concrete & $\mathrm{S}$ & 299 \\
\hline 53 & Concrete & $\mathrm{S}$ & 324 \\
\hline 54 & Concrete & $\mathrm{S}$ & 334 \\
\hline 55 & Concrete & S & 336 \\
\hline 58 & Concrete & S & 286 \\
\hline 59 & Concrete & S & 347 \\
\hline 60 & Concrete & $\mathrm{S}$ & 291 \\
\hline 61 & Concrete & S & 307 \\
\hline 62 & Concrete & S & 309 \\
\hline 63 & Concrete & S & 369 \\
\hline 64 & Concrete & S & 292 \\
\hline 65 & Concrete & $\mathrm{S}$ & 289 \\
\hline 66 & Concrete & $\mathrm{S}$ & 365 \\
\hline 67 & Concrete & S & 434 \\
\hline 68 & Concrete & S & 327 \\
\hline 70 & Concrete & S & 332 \\
\hline 71 & Concrete & $\mathrm{s}$ & 349 \\
\hline
\end{tabular}

\section{Basic Statistical Quantities Summary}

Statistic

Sample Number

Mean $\left(\mathrm{dpm} / 100 \mathrm{~cm}^{2}\right)$

Median (dpm/100 $\left.\mathrm{cm}^{2}\right)$

Std Dev (dpm/100 $\left.\mathrm{cm}^{2}\right)$

High Value $\left(\mathrm{dpm} / 100 \mathrm{~cm}^{2}\right)$

Low Value $\left(\mathrm{dpm} / 100 \mathrm{~cm}^{2}\right)$
Survey Unit

22
-16.27
-15.28
67.70
197.02
-96.63

Background

$\begin{array}{ll}\text { N/A } & N=20 \\ \text { N/A } & 120 \\ \text { N/A } & \text { N/A } \\ \text { N/A } & 62.6 \\ \text { N/A } & \text { N/A } \\ \text { N/A } & \text { N/A }\end{array}$




\section{DQA Building Surface Report}

\section{Assessment Summary}

Site:

Planner(s):

Survey Unit Name:

Report Number:

Survey Unit Measurements:

Reference Area Measurements:

Test Performed:

Judgmental Areas:

Assessment Conclusion:
Hammond Depot FSS Planner

Vitkus

Class 2 SUs Scoping Rev. 1 C2 SU38 Building 200E, N, Bays 16-20

3

24

0

Sign

Test Result:

Not Performed

0

EMC Result:

Not Performed

Reject Null Hypothesis (Survey Unit PASSES)

\section{Retrospective Power Curve}

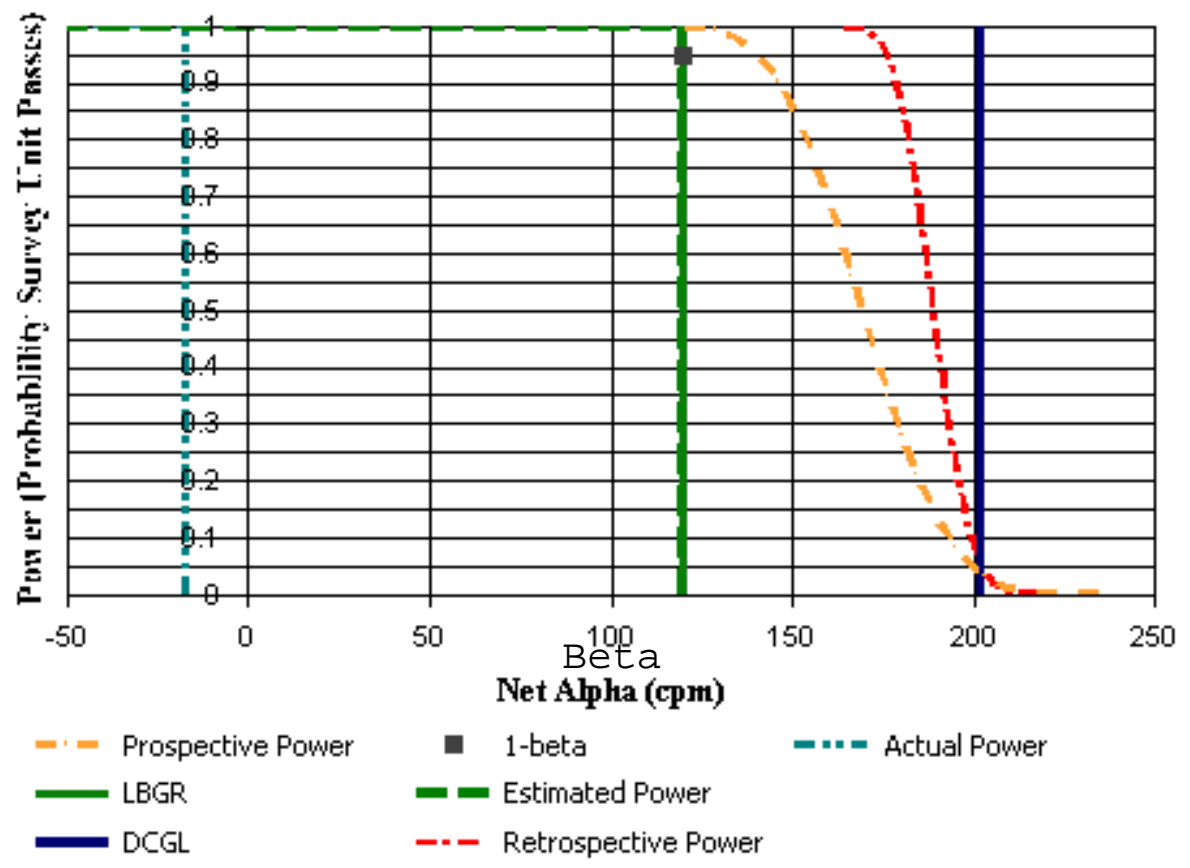




\section{DQA Building Surface Report}

\section{Survey Unit Data}

NOTE: $\quad$ Type = "S" indicates survey measurement.

Type $=$ "R" indicates reference measurement.

\begin{tabular}{llcc} 
Measurement & Material & Type & Gross Alpha (cpm) \\
\hline 21 & Concrete & $\mathrm{S}$ & 348 \\
22 & Concrete & $\mathrm{S}$ & 337 \\
23 & Concrete & $\mathrm{S}$ & 329 \\
25 & Concrete & $\mathrm{S}$ & 439 \\
26 & Concrete & $\mathrm{S}$ & 346 \\
27 & Concrete & $\mathrm{S}$ & 327 \\
28 & Concrete & $\mathrm{S}$ & 312 \\
29 & Concrete & $\mathrm{S}$ & 322 \\
30 & Concrete & $\mathrm{S}$ & 319 \\
31 & Concrete & $\mathrm{S}$ & 354 \\
32 & Concrete & $\mathrm{S}$ & 351 \\
33 & Concrete & $\mathrm{S}$ & 333 \\
34 & Concrete & $\mathrm{S}$ & 356 \\
35 & Concrete & $\mathrm{S}$ & 315 \\
36 & Concrete & $\mathrm{S}$ & 294 \\
37 & Concrete & $\mathrm{S}$ & 290 \\
38 & Concrete & $\mathrm{S}$ & 371 \\
39 & Concrete & $\mathrm{S}$ & 332 \\
40 & Concrete & $\mathrm{S}$ & 325 \\
41 & Concrete & $\mathrm{S}$ & 316 \\
42 & Concrete & $\mathrm{S}$ & 311 \\
43 & Concrete & $\mathrm{S}$ & 291 \\
44 & Concrete & $\mathrm{S}$ & 325 \\
45 & Concrete & $\mathrm{S}$ & 323
\end{tabular}

\section{Basic Statistical Quantities Summary}

Statistic

Sample Number

Mean $\left(\mathrm{dpm} / 100 \mathrm{~cm}{ }^{2}\right)$

Median (dpm/100 $\left.\mathrm{cm}^{2}\right)$

Std Dev (dpm/100 $\left.\mathrm{cm}^{2}\right)$

High Value $\left(\mathrm{dpm} / 100 \mathrm{~cm}^{2}\right)$

Low Value $\left(\mathrm{dpm} / 100 \mathrm{~cm}^{2}\right)$
Survey Unit

24

$-5.52$

$-17.26$

60.84

206.94

$-88.69$
Background

DQO Results

$\begin{array}{ll}\text { N/A } & N=20 \\ \text { N/A } & 120 \\ \text { N/A } & \text { N/A } \\ \text { N/A } & 62.6 \\ \text { N/A } & \text { N/A } \\ \text { N/A } & \text { N/A }\end{array}$




\section{DQA Building Surface Report}

\section{Assessment Summary}

Site:

Planner(s):

Survey Unit Name:

Report Number:

Survey Unit Measurements:

Reference Area Measurements:

Test Performed:

Judgmental Areas:

Assessment Conclusion:
Hammond Depot FSS Planner

Vitkus

Class 2 SUs Scoping Rev. 1 C2 SU42 Building 200E Locker Room

4

21

0

Sign

Test Result:

Not Performed

0

EMC Result:

Not Performed

\section{Retrospective Power Curve}

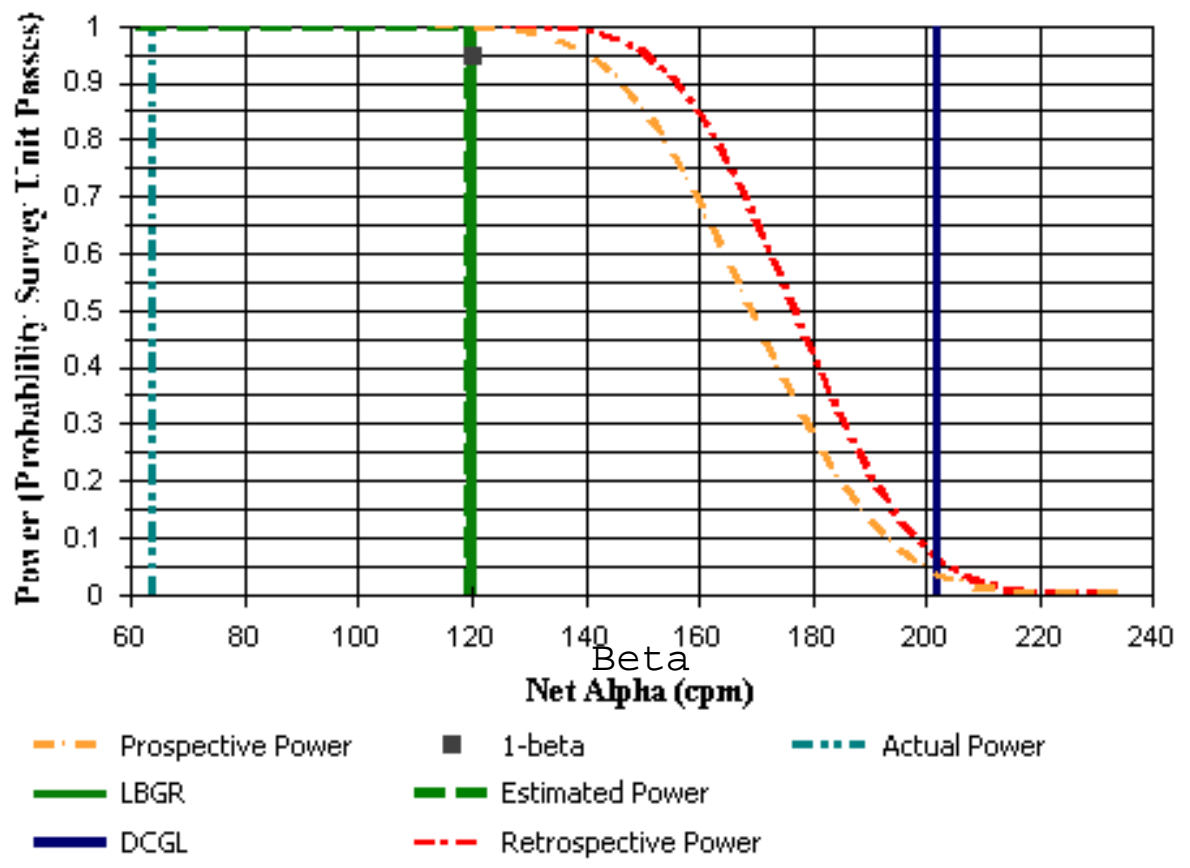




\section{DQA Building Surface Report}

\section{Survey Unit Data}

NOTE: $\quad$ Type $=$ "S" indicates survey measurement.

Type $=$ "R" indicates reference measurement.

\begin{tabular}{|c|c|c|c|}
\hline Measurement & Material & Type & Gross Alpha (cpm) \\
\hline$\overline{51 C}$ & Concrete & $\mathrm{S}$ & 362 \\
\hline $52 \mathrm{C}$ & Concrete & $\mathrm{s}$ & 409 \\
\hline $53 C$ & Concrete & S & 376 \\
\hline $54 \mathrm{C}$ & Concrete & S & 390 \\
\hline $55 \mathrm{C}$ & Concrete & $S$ & 367 \\
\hline $56 C$ & Concrete & $\mathrm{s}$ & 378 \\
\hline $57 \mathrm{C}$ & Concrete & S & 360 \\
\hline $58 \mathrm{C}$ & Concrete & $\mathrm{S}$ & 355 \\
\hline $59 C$ & Concrete & S & 371 \\
\hline $60 C$ & Concrete & S & 380 \\
\hline $61 C$ & Concrete & S & 403 \\
\hline $62 C$ & Concrete & S & 385 \\
\hline $63 C$ & Concrete & $\mathrm{s}$ & 396 \\
\hline $66 \mathrm{C}$ & Unpainted Cinder Block & $\mathrm{s}$ & 438 \\
\hline $67 \mathrm{C}$ & Unpainted Cinder Block & $\mathrm{s}$ & 331 \\
\hline $68 C$ & Unpainted Cinder Block & S & 305 \\
\hline $69 C$ & Unpainted Cinder Block & S & 322 \\
\hline $70 \mathrm{C}$ & Unpainted Cinder Block & $\mathrm{s}$ & 326 \\
\hline $71 \mathrm{C}$ & Unpainted Cinder Block & $\mathrm{s}$ & 330 \\
\hline $72 \mathrm{C}$ & Unpainted Cinder Block & $\mathrm{s}$ & 410 \\
\hline $73 C$ & Unpainted Cinder Block & S & 340 \\
\hline
\end{tabular}

\section{Basic Statistical Quantities Summary}

Statistic

Sample Number

Mean $\left(\mathrm{dpm} / 100 \mathrm{~cm}{ }^{2}\right)$

Median (dpm/100 cm²)

Std Dev (dpm/100 cm²)

High Value $\left(\mathrm{dpm} / 100 \mathrm{~cm}^{2}\right)$

Low Value $\left(\mathrm{dpm} / 100 \mathrm{~cm}^{2}\right)$
Survey Unit

21

12.90

63.89

116.30

147.42

$-200.00$
Background

DQO Results

$\mathrm{N} / \mathrm{A} \quad \mathrm{N}=20$

N/A

120

N/A

N/A

N/A

62.6

N/A

N/A

N/A

N/A 


\section{DQA Building Surface Report}

\section{Assessment Summary}

Site:

Planner(s):

Survey Unit Name:

Report Number:

Survey Unit Measurements:

Reference Area Measurements:

Test Performed:

Judgmental Areas:

Assessment Conclusion:
Hammond Depot FSS Planner

Vitkus

Class 2 SUs C2 SU45 Building 100E bays 15-20 floor

5

15

0

Sign

Test Result: $\quad$ Not Performed

0

EMC Result:

Not Performed

\section{Reject Null Hypothesis (Survey Unit PASSES)}

\section{Retrospective Power Curve}

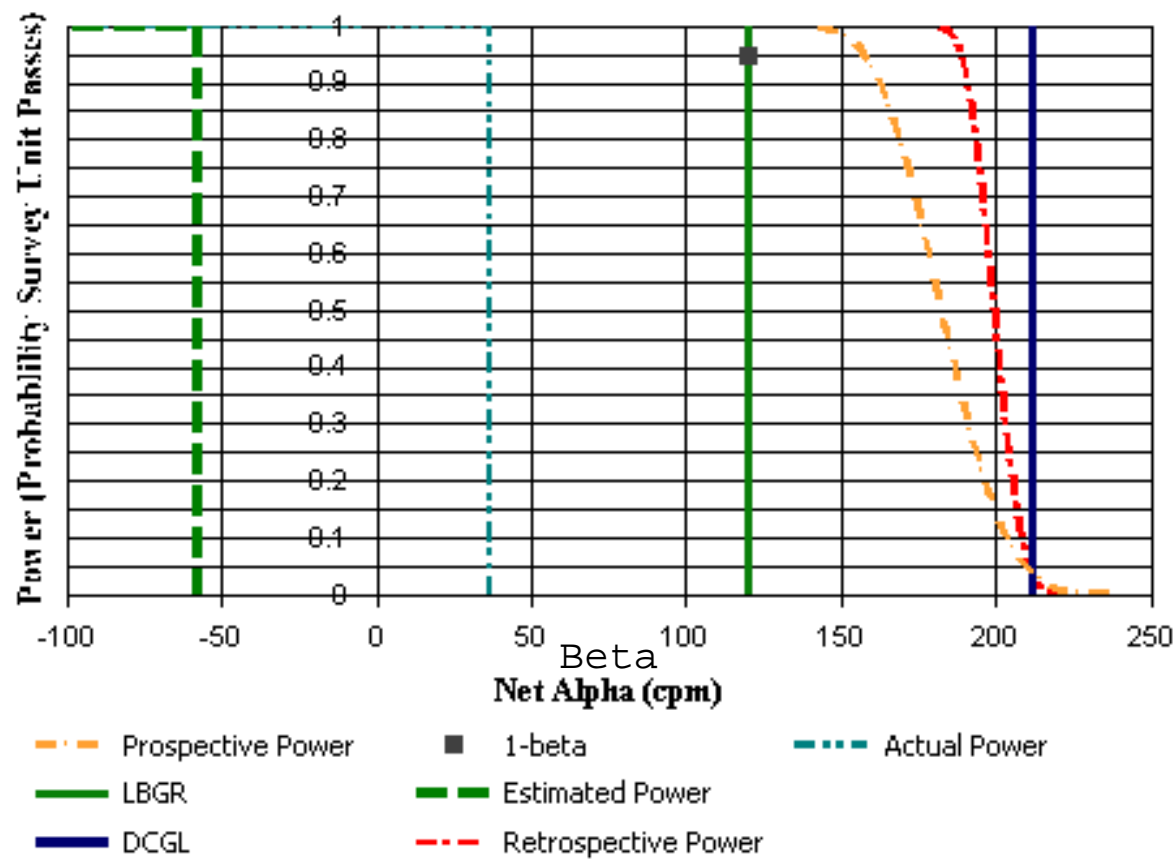




\section{DQA Building Surface Report}

\section{Survey Unit Data}

NOTE: $\quad$ Type = "S" indicates survey measurement.

Type $=$ "R" indicates reference measurement.

\begin{tabular}{llcc} 
Measurement & Material & Type & Gross Alpha (cpm) \\
\hline $2.5,87.2$ & Concrete & $\mathrm{S}$ & 350 \\
$37.3,87.2$ & Concrete & $\mathrm{S}$ & 370 \\
$6.8,94.7$ & Concrete & $\mathrm{S}$ & 376 \\
$32.9,94.7$ & Concrete & $\mathrm{S}$ & 358 \\
$2.5,102.2$ & Concrete & $\mathrm{S}$ & 356 \\
$37.3,102.2$ & Concrete & $\mathrm{S}$ & 348 \\
$6.8,109.8$ & Concrete & $\mathrm{S}$ & 327 \\
$15.5,109.8$ & Concrete & $\mathrm{S}$ & 339 \\
$24.2,109.8$ & Concrete & $\mathrm{S}$ & 348 \\
$32.9,109.8$ & Concrete & $\mathrm{S}$ & 377 \\
$2.5,117.3$ & Concrete & $\mathrm{S}$ & 369 \\
$12.3,117.3$ & Concrete & $\mathrm{S}$ & 310 \\
$19.9,117.3$ & Concrete & $\mathrm{S}$ & 343 \\
$28.6,117.3$ & Concrete & $\mathrm{S}$ & 386 \\
$36.0,117.3$ & Concrete & $\mathrm{S}$ & 361
\end{tabular}

\section{Basic Statistical Quantities Summary}

\begin{tabular}{lccc} 
Statistic & Survey Unit & Background & DQO Results \\
\hline Sample Number & 15 & N/A & N=15 \\
Mean $\left(\mathrm{dpm} / 100 \mathrm{~cm}^{2}\right)$ & 33.70 & $\mathrm{~N} / \mathrm{A}$ & -57.9 \\
Median $\left(\mathrm{dpm} / 100 \mathrm{~cm}^{2}\right)$ & 36.47 & $\mathrm{~N} / \mathrm{A}$ & $\mathrm{N} / \mathrm{A}$ \\
Std Dev $\left(\mathrm{dpm} / 100 \mathrm{~cm}^{2}\right)$ & 38.07 & $\mathrm{~N} / \mathrm{A}$ & 47.4 \\
High Value $\left(\mathrm{dpm} / 100 \mathrm{~cm}^{2}\right)$ & 93.16 & $\mathrm{~N} / \mathrm{A}$ & $\mathrm{N} / \mathrm{A}$ \\
Low Value $\left(\mathrm{dpm} / 100 \mathrm{~cm}^{2}\right)$ & -50.45 & $\mathrm{~N} / \mathrm{A}$ & $\mathrm{N} / \mathrm{A}$
\end{tabular}




\section{DQA Building Surface Report}

\section{Assessment Summary}

Site:

Planner(s):

Survey Unit Name:

Report Number:

Survey Unit Measurements:

Reference Area Measurements:

Test Performed:

Judgmental Areas:

Assessment Conclusion:
Hammond Depot FSS Planner

Vitkus

Class 2 SUs C2 SU46 Building 100E, bays 15-20 walls

3

15

0

Sign

Test Result:

Not Performed

0

EMC Result:

Not Performed

\section{Reject Null Hypothesis (Survey Unit PASSES)}

\section{Retrospective Power Curve}

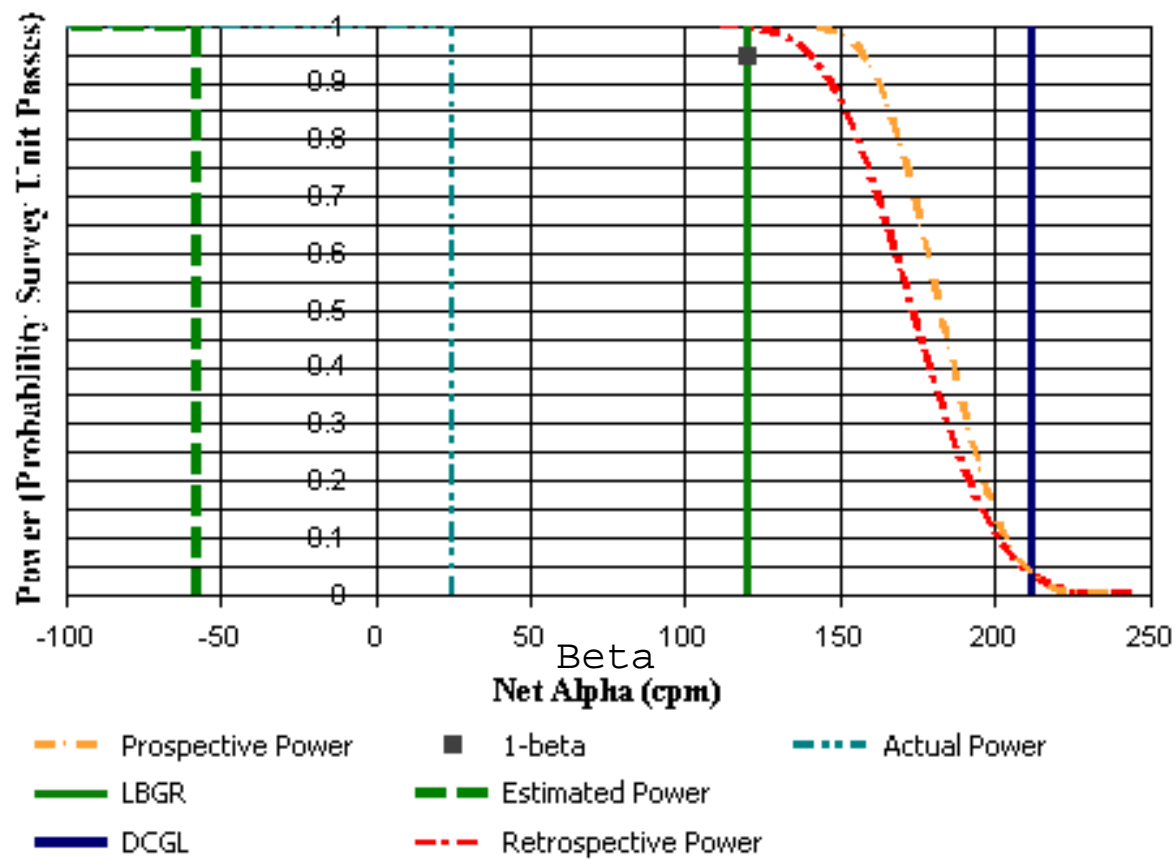




\section{DQA Building Surface Report}

\section{Survey Unit Data}

NOTE: $\quad$ Type $=$ "S" indicates survey measurement.

Type = "R" indicates reference measurement.

Measurement

85.5, 3.1 N. Wall

96.4, 3.1 $\mathrm{W}$. Wall

107.3, 3.1 /W. Wall

$118.2,3.1 / \mathrm{W}$. Wall

$0.1,1.6 / \mathrm{N}$. Wall

$6.3,1.6 / \mathrm{N}$. Wall

$12.6,1.6 / \mathrm{N}$. Wall

$18.9,1.6 / \mathrm{N}$. Wall

25.2, $1.6 / \mathrm{N}$. Wall

$31.5,1.6 / \mathrm{N}$. Wall

$37.8,1.6 / \mathrm{N}$. Wall

36.5, 2.5 /E. Wall

25.6, $2.5 / E$. Wall

14.7, 2.5 /E. Wall

3.8, 2.5/E. Wall
Material

Unpainted Cinder Block

Metal

Unpainted Cinder Block

Unpainted Cinder Block

Unpainted Cinder Block

Unpainted Cinder Block Metal Metal

Unpainted Cinder Block Unpainted Cinder Block

Unpainted Cinder Block Metal

Unpainted Cinder Block

Unpainted Cinder Block
Beta

Type Gross Alpha (cpm)

188

513

202

434

250

447

524

191

194

408

466

454

186

471

477

\section{Basic Statistical Quantities Summary}

$\begin{array}{lccc}\text { Statistic } & \text { Survey Unit } & \text { Background } & \text { DQO Results } \\ \text { Sample Number } & 15 & \text { N/A } & \text { N=15 } \\ \text { Mean }\left(\mathrm{dpm} / 100 \mathrm{~cm}^{2}\right) & 4.85 & \text { N/A N/A } & -57.9 \\ \text { Median }\left(\mathrm{dpm} / 100 \mathrm{~cm}^{2}\right) & 24.75 & \text { N/A } & \text { N/A } \\ \text { Std Dev }\left(\mathrm{dpm} / 100 \mathrm{~cm}^{2}\right) & 117.13 & \text { N/A } & 47.4 \\ \text { High Value }\left(\mathrm{dpm} / 100 \mathrm{~cm}^{2}\right) & 144.18 & \text { N/A } & \text { N/A } \\ \text { Low Value }\left(\mathrm{dpm} / 100 \mathrm{~cm}^{2}\right) & -373.58 & \text { N/A }\end{array}$




\section{DQA Building Surface Report}

\section{Assessment Summary}

Site:

Planner(s):

Survey Unit Name:

Report Number:

Survey Unit Measurements:

Reference Area Measurements:

Test Performed:

Judgmental Areas:

Assessment Conclusion:
Hammond Depot FSS Planner

Vitkus

Class 2 SUs Scoping Rev. 1 C2 SU49 Building 100W, Bays 10-17

2

19

0

Sign

Test Result:

Not Performed

0

EMC Result:

Not Performed

\section{Retrospective Power Curve}

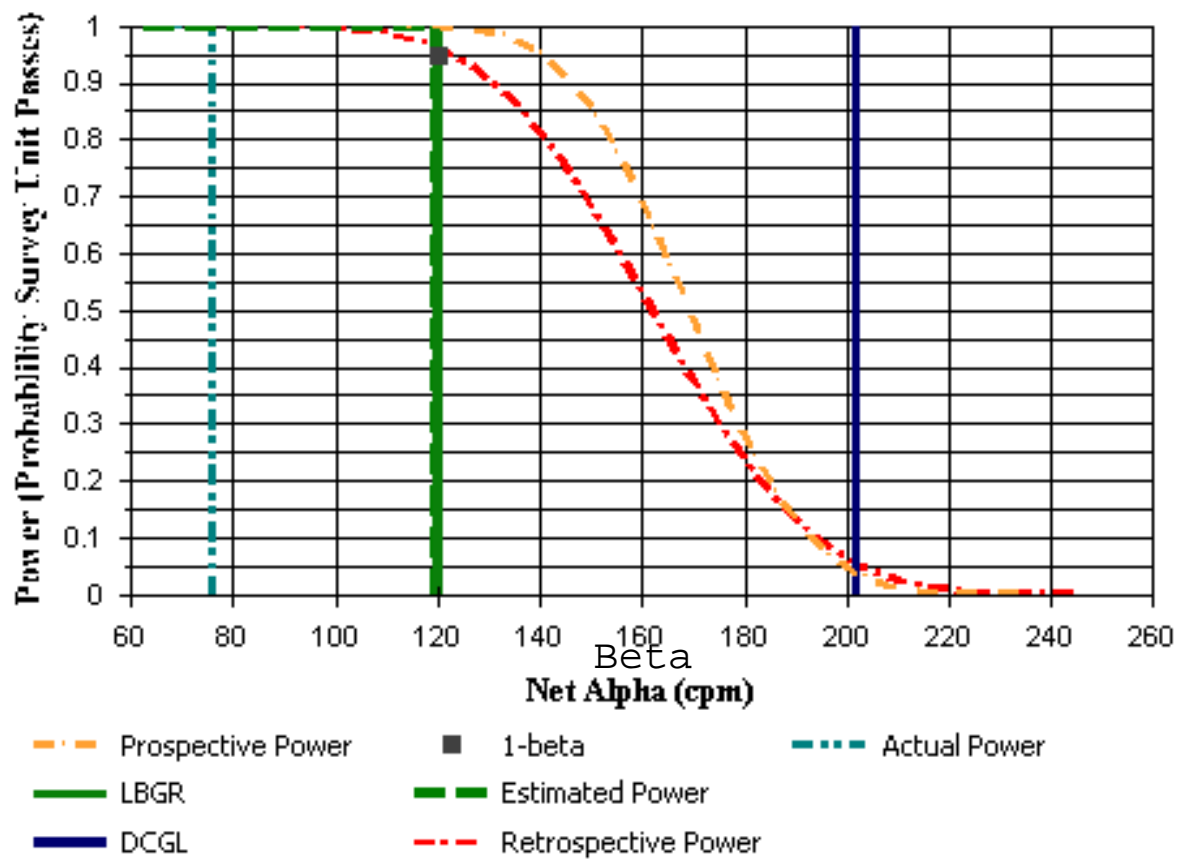




\section{DQA Building Surface Report}

\section{Survey Unit Data}

NOTE: $\quad$ Type $=$ "S" indicates survey measurement.

Type $=$ "R" indicates reference measurement.

\begin{tabular}{llcc} 
Measurement & Material & Type & Gross Alpha (cpm) \\
\hline A54 & Concrete & S & 488 \\
A55 & Concrete & S & 346 \\
A56 & Concrete & S & 489 \\
A57 & Concrete & S & 362 \\
A58 & Concrete & S & 373 \\
A59 & Concrete & S & 465 \\
A60 & Concrete & S & 350 \\
A61 & Concrete & S & 379 \\
A62 & Concrete & S & 409 \\
A63 & Concrete & S & 363 \\
A65 & Concrete & S & 410 \\
A66 & Concrete & S & 431 \\
A83 & Concrete & S & 524 \\
A84 & Concrete & S & 466 \\
A90 & Concrete & S & 243 \\
A91 & Concrete & S & 300 \\
A92 & Concrete & S & 250 \\
A93 & Concrete & S & 277 \\
A94 & Concrete & S & 297
\end{tabular}

\section{Basic Statistical Quantities Summary}

Statistic

Sample Number

Mean $\left(\mathrm{dpm} / 100 \mathrm{~cm}^{2}\right)$

Median (dpm/100 cm²)

Std Dev (dpm/100 cm²)

High Value $\left(\mathrm{dpm} / 100 \mathrm{~cm}^{2}\right)$

Low Value $\left(\mathrm{dpm} / 100 \mathrm{~cm}^{2}\right)$
Survey Unit

19
90.09
75.99
165.69
375.60
-181.94

Background

DQO Results

$\begin{array}{lc}\text { N/A } & N=20 \\ N / A & 120 \\ N / A & N / A \\ N / A & 62.6 \\ N / A & N / A \\ N / A & N / A\end{array}$




\section{DQA Building Surface Report}

\section{Assessment Summary}

Site:

Planner(s):

Survey Unit Name:

Report Number:

Survey Unit Measurements:

Reference Area Measurements:

Test Performed:

Judgmental Areas:

Assessment Conclusion:
Hammond Depot FSS Planner

Vitkus

Class 2 SUs Scoping Rev. 1 C2 SU50 Building 100W, Bays 1-10

5

14

0

Sign

Test Result:

Not Performed

0

EMC Result:

Not Performed

\section{Retrospective Power Curve}

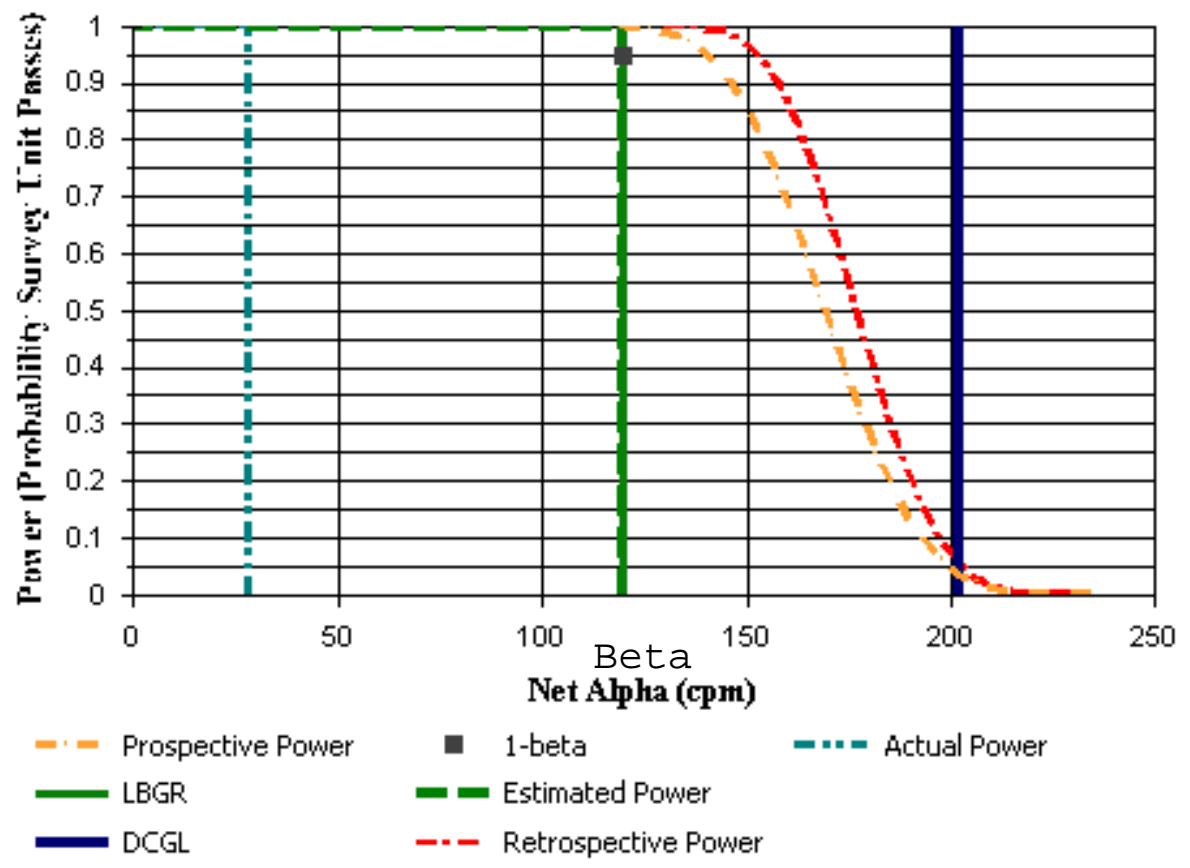




\section{DQA Building Surface Report}

\section{Survey Unit Data}

NOTE: $\quad$ Type = "S" indicates survey measurement.

Type $=$ "R" indicates reference measurement.

\begin{tabular}{lccc} 
Measurement & Material & Type & Gross Alpha (cpm) \\
\hline A40 & Concrete & S & 284 \\
A41 & Unpainted Cinder Block & S & 451 \\
A42 & Concrete & S & 334 \\
A43 & Concrete & S & 298 \\
A44 & Concrete & S & 338 \\
A45 & Concrete & S & 342 \\
A46 & Unpainted Cinder Block & S & 505 \\
A47 & Concrete & S & 353 \\
A48 & Concrete & S & 362 \\
A49 & Concrete & S & 345 \\
A50 & Concrete & S & 390 \\
A51 & Concrete & S & 391 \\
A52 & Unpainted Cinder Block & S & 509 \\
A53 & Concrete & S & 338
\end{tabular}

\section{Basic Statistical Quantities Summary}

Statistic

Sample Number

Mean $\left(\mathrm{dpm} / 100 \mathrm{~cm}{ }^{2}\right)$

Median (dpm/100 cm²)

Std Dev (dpm/100 $\left.\mathrm{cm}^{2}\right)$

High Value $\left(\mathrm{dpm} / 100 \mathrm{~cm}^{2}\right)$

Low Value $\left(\mathrm{dpm} / 100 \mathrm{~cm}^{2}\right)$
Survey Unit

14
48.31
28.37
88.12
204.76
-100.60

Background

N/A

N/A

N/A

N/A

N/A

N/A
DQO Results

$\mathrm{N}=20$

120

N/A

62.6

N/A

N/A 


\section{DQA Building Surface Report}

\section{Assessment Summary}

Site:

Planner(s):

Survey Unit Name:

Report Number:

Survey Unit Measurements:

Reference Area Measurements:

Test Performed:

Judgmental Areas:

Assessment Conclusion:
Hammond Depot FSS Planner

Vitkus

Class 2 SUs Scoping Rev. 1 C2 SU51 Building 100W, Bays 11-20

6

14

0

Sign

Test Result:

Not Performed

0

EMC Result:

Not Performed

\section{Reject Null Hypothesis (Survey Unit PASSES)}

\section{Retrospective Power Curve}

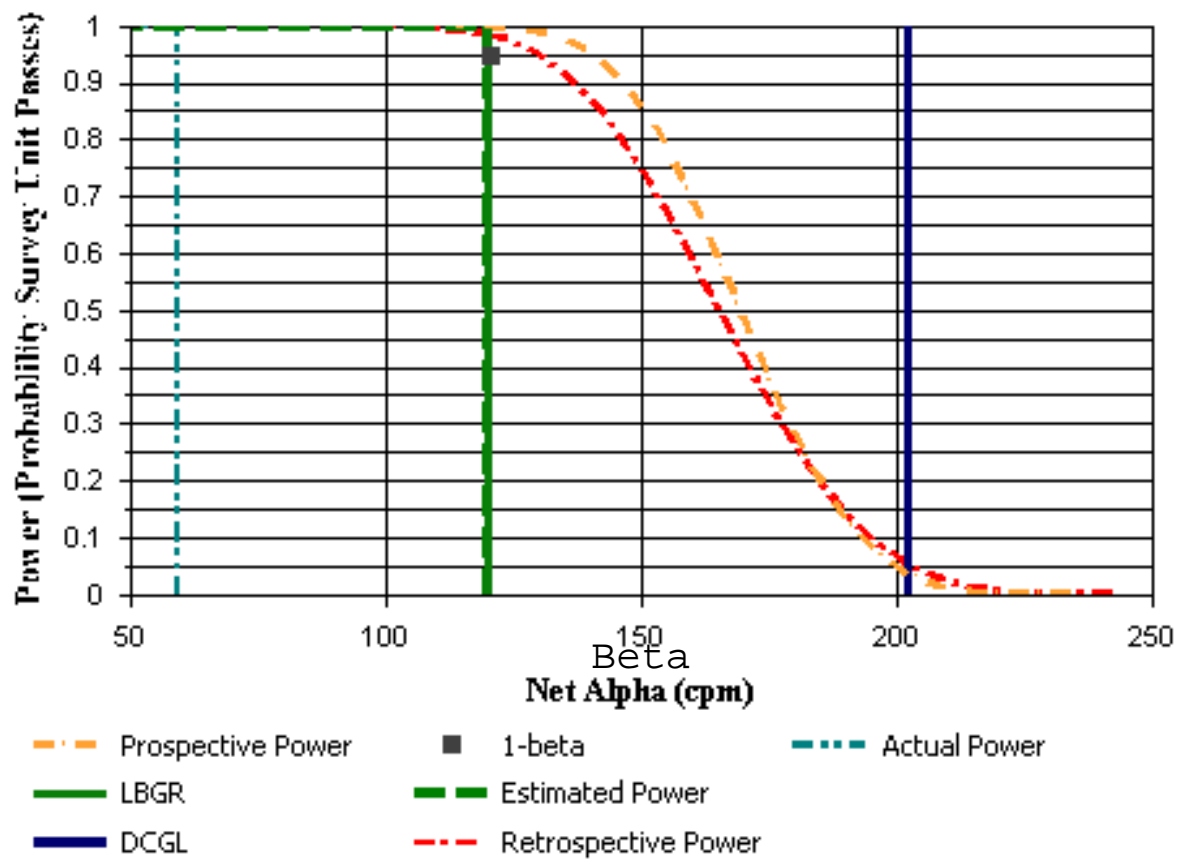




\section{DQA Building Surface Report}

\section{Survey Unit Data}

NOTE: Type $=$ "S" indicates survey measurement.

Type $=$ "R" indicates reference measurement.

\begin{tabular}{lccc} 
Measurement & Material & Type & Gross Alpha (cpm) \\
\hline A68 & Unpainted Cinder Block & S & 533 \\
A69 & Concrete & S & 398 \\
A70 & Concrete & S & 307 \\
A71 & Concrete & S & 329 \\
A72 & Concrete & S & 351 \\
A73 & Concrete & S & 373 \\
A74 & Concrete & S & 425 \\
A75 & Concrete & S & 395 \\
A76 & Unpainted Cinder Block & S & 484 \\
A77 & Concrete & S & 362 \\
A78 & Concrete & S & 339 \\
A79 & Concrete & S & 367 \\
A80 & Concrete & S & 344 \\
A81 & Unpainted Cinder Block & S & 256
\end{tabular}

\section{Basic Statistical Quantities Summary}

Statistic

Sample Number

Mean $\left(\mathrm{dpm} / 100 \mathrm{~cm}{ }^{2}\right)$

Median (dpm/100 cm²)

Std Dev (dpm/100 cm²)

High Value $\left(\mathrm{dpm} / 100 \mathrm{~cm}^{2}\right)$

Low Value $\left(\mathrm{dpm} / 100 \mathrm{~cm}^{2}\right)$
Survey Unit

14

51.57

59.13

129.64

252.38

$-297.22$
Background

DQO Results

$\begin{array}{ll}\text { N/A } & \mathrm{N}=20 \\ \text { N/A } & 120 \\ \text { N/A } & \text { N/A } \\ \text { N/A } & 62.6 \\ \text { N/A } & N / A \\ \text { N/A } & N / A\end{array}$




\section{DQA Building Surface Report}

\section{Assessment Summary}

Site:

Planner(s):

Survey Unit Name:

Report Number:

Survey Unit Measurements:

Reference Area Measurements:

Test Performed:

Judgmental Areas:

Assessment Conclusion:
Hammond Depot FSS Planner

Vitkus

C2 SU56 through 58 Bldg 200E walls C2 SU56 south and southwest walls

1

21

0

Sign

Test Result:

Not Performed

0

EMC Result:

Not Performed

\section{Retrospective Power Curve}

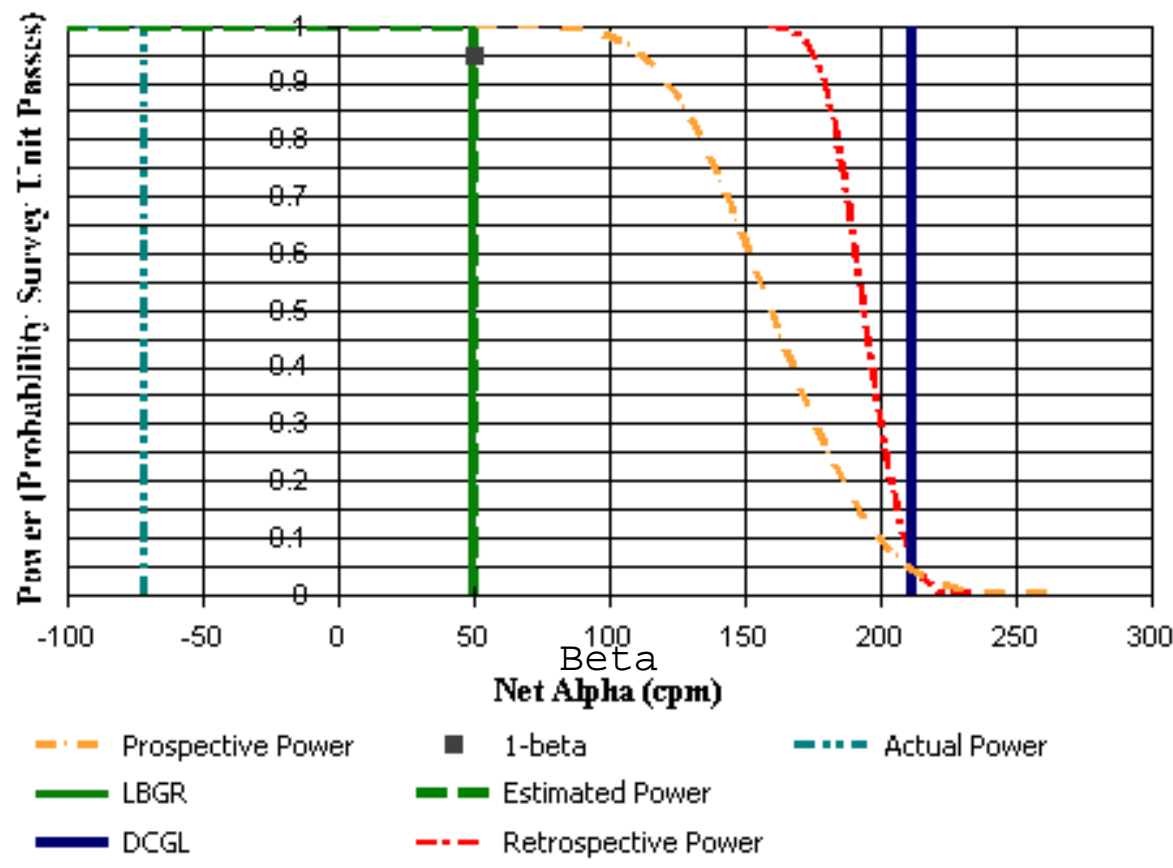




\section{DQA Building Surface Report}

\section{Survey Unit Data}

NOTE: $\quad$ Type = "S" indicates survey measurement.

Type $=$ "R" indicates reference measurement.

Measurement

$1.9,0.2$

$4.4,4.5$

$7.0,0.2$

$9.5,4.5$

$12.1,0.2$

$14.6,4.5$

$17.2,0.2$

$36.1,4.5$

$33.5,0.2$

$31.0,4.5$

$28.4,0.2$

$25.9,4.5$

$23.3,0.2$

$20.8,4.5$

$18.2,0.2$

$15.7,4.5$

$13.1,0.2$

$10.6,4.5$

$8.0,0.2$

$5.5,4.5$

$2.9,0.2$

\section{Material}

Concrete

Metal

Concrete

Metal

Concrete

Metal

Metal

Unpainted Cinder Block Concrete

Unpainted Cinder Block

Unpainted Cinder Block

Unpainted Cinder Block Concrete

Unpainted Cinder Block Metal

Unpainted Cinder Block

Concrete

Metal

Concrete

Concrete

Concrete
Beta

Type Gross Alpha (cpm)

$\begin{array}{ll}\mathrm{S} & 299 \\ \mathrm{~S} & 188\end{array}$

$\mathrm{S} \quad 305$

$\mathrm{S} \quad 184$

S 294

S 209

$\mathrm{S} \quad 210$

$\mathrm{S} \quad 448$

S 288

$\mathrm{S} \quad 403$

$\mathrm{S} \quad 421$

$\mathrm{S} \quad 364$

S 271

S 426

S 244

S 397

S 269

$S \quad 190$

S 273

S 243

S 292

\section{Basic Statistical Quantities Summary}

Statistic

Sample Number

Mean $\left(\mathrm{dpm} / 100 \mathrm{~cm}^{2}\right)$

Median (dpm/100 $\left.\mathrm{cm}^{2}\right)$

Std Dev (dpm/100 cm²)

High Value $\left(\mathrm{dpm} / 100 \mathrm{~cm}^{2}\right)$

Low Value $\left(\mathrm{dpm} / 100 \mathrm{~cm}^{2}\right)$
Survey Unit

21

$-50.92$

$-71.24$

78.50

124.91

$-177.06$
Background

DQO Results

$\begin{array}{ll}\text { N/A } & \mathrm{N}=17 \\ \text { N/A } & 50.1 \\ \text { N/A } & \text { N/A } \\ \text { N/A } & 96.4 \\ \text { N/A } & \text { N/A } \\ \text { N/A } & \text { N/A }\end{array}$




\section{DQA Building Surface Report}

\section{Assessment Summary}

Site:

Planner(s):

Survey Unit Name:

Report Number:

Survey Unit Measurements:

Reference Area Measurements:

Test Performed:

Judgmental Areas:

Assessment Conclusion:
Hammond Depot FSS Planner

Vitkus

C2 SU56 through 58 Bldg 200E walls C2 SU57

3

24

0

Sign

Test Result: Pass

0

EMC Result:

Not Performed

Reject Null Hypothesis (Survey Unit PASSES)

\section{Retrospective Power Curve}

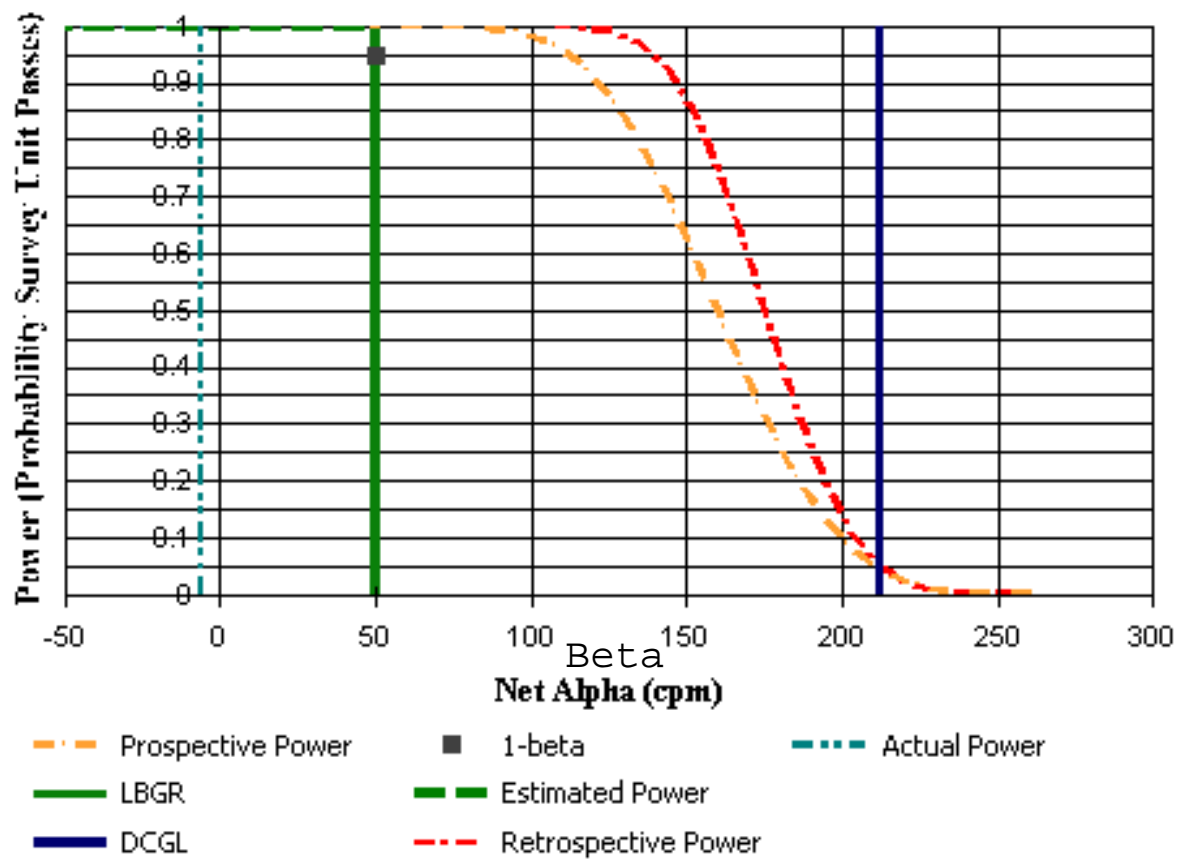




\section{DQA Building Surface Report}

\section{Survey Unit Data}

NOTE: $\quad$ Type $=$ "S" indicates survey measurement.

Type = "R" indicates reference measurement.

\begin{tabular}{lccc} 
Measurement & Material & Type & Gross Alpha (cpm) \\
\hline $33.2,4.5$ & Unpainted Cinder Block & $\mathrm{S}$ & 421 \\
$35.6,0.2$ & Concrete & $\mathrm{S}$ & 599 \\
$38.1,4.5$ & Metal & $\mathrm{S}$ & 240 \\
$40.6,0.2$ & Concrete & $\mathrm{S}$ & 308 \\
$43.1,4.5$ & Unpainted Cinder Block & $\mathrm{S}$ & 427 \\
$45.6,0.2$ & Concrete & $\mathrm{S}$ & 402 \\
$48.1,4.5$ & Metal & $\mathrm{S}$ & 174 \\
$50.6,0.2$ & Metal & $\mathrm{S}$ & 217 \\
$53.1,4.5$ & Metal & $\mathrm{S}$ & 179 \\
$55.6,0.2$ & Concrete & $\mathrm{S}$ & 306 \\
$58.1,4.5$ & Metal & $\mathrm{S}$ & 172 \\
$60.5,0.2$ & Concrete & $\mathrm{S}$ & 289 \\
$1.0,4.5$ & Unpainted Cinder Block & $\mathrm{S}$ & 254 \\
$3.5,0.2$ & Unpainted Cinder Block & $\mathrm{S}$ & 294 \\
$6.0,4.5$ & Unpainted Cinder Block & $\mathrm{S}$ & 349 \\
$8.5,0.2$ & Unpainted Cinder Block & $\mathrm{S}$ & 481 \\
$11.0,4.5$ & Unpainted Cinder Block & $\mathrm{S}$ & 434 \\
$13.5,0.2$ & Unpainted Cinder Block & $\mathrm{S}$ & 477 \\
$16.0,4.5$ & Unpainted Cinder Block & $\mathrm{S}$ & 445 \\
$18.5,0.2$ & Unpainted Cinder Block & $\mathrm{S}$ & 527 \\
$21.0,4.5$ & Unpainted Cinder Block & $\mathrm{S}$ & 464 \\
$23.5,0.2$ & Unpainted Cinder Block & $\mathrm{S}$ & 431 \\
$25.9,4.5$ & Unpainted Cinder Block & $\mathrm{S}$ & 461 \\
$28.4,0.2$ & Unpainted Cinder Block & $\mathrm{S}$ & 519
\end{tabular}

\section{Basic Statistical Quantities Summary}

Statistic

Sample Number

Mean $\left(\mathrm{dpm} / 100 \mathrm{~cm}{ }^{2}\right)$

Median (dpm/100 $\left.\mathrm{cm}^{2}\right)$

Std Dev (dpm/100 $\left.\mathrm{cm}^{2}\right)$

High Value $\left(\mathrm{dpm} / 100 \mathrm{~cm}^{2}\right)$

Low Value $\left(\mathrm{dpm} / 100 \mathrm{~cm}^{2}\right)$
Survey Unit

24

2.30

$-6.24$

161.88

495.65

$-366.02$
Background

DQO Results

$\mathrm{N}=17$

50.1

N/A

N/A

96.4

N/A

N/A

N/A

N/A 


\section{DQA Building Surface Report}

\section{Statistical Test Summary}

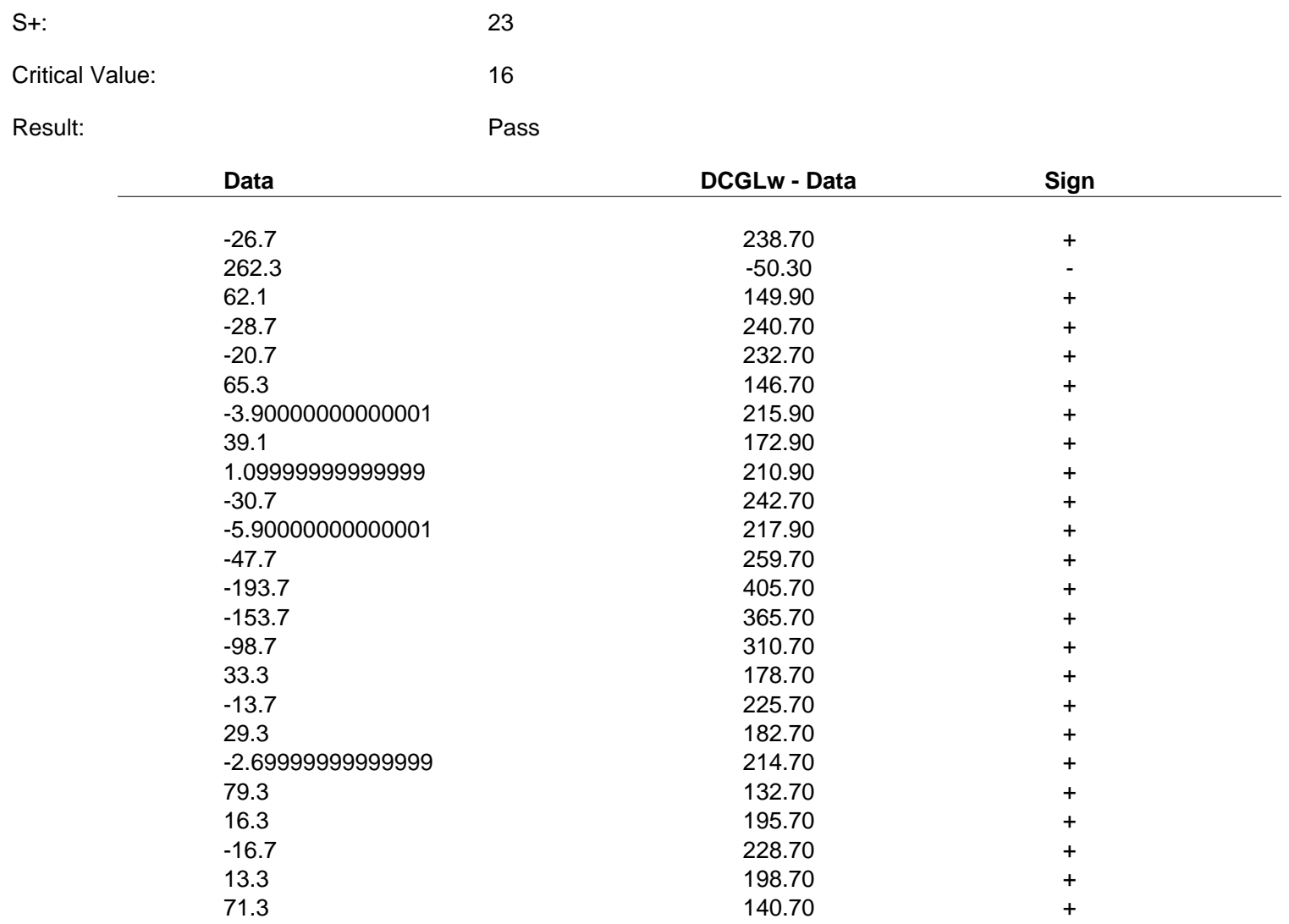




\section{DQA Building Surface Report}

\section{Assessment Summary}

Site:

Planner(s):

Survey Unit Name:

Report Number:

Survey Unit Measurements:

Reference Area Measurements:

Test Performed:

Judgmental Areas:

Assessment Conclusion:
Hammond Depot FSS Planner

Vitkus

C2 SU56 through 58 Bldg 200E walls C2 SU58 East Wall

2

17

0

Sign

Test Result: $\quad$ Not Performed

0

EMC Result:

Not Performed

Reject Null Hypothesis (Survey Unit PASSES)

\section{Retrospective Power Curve}

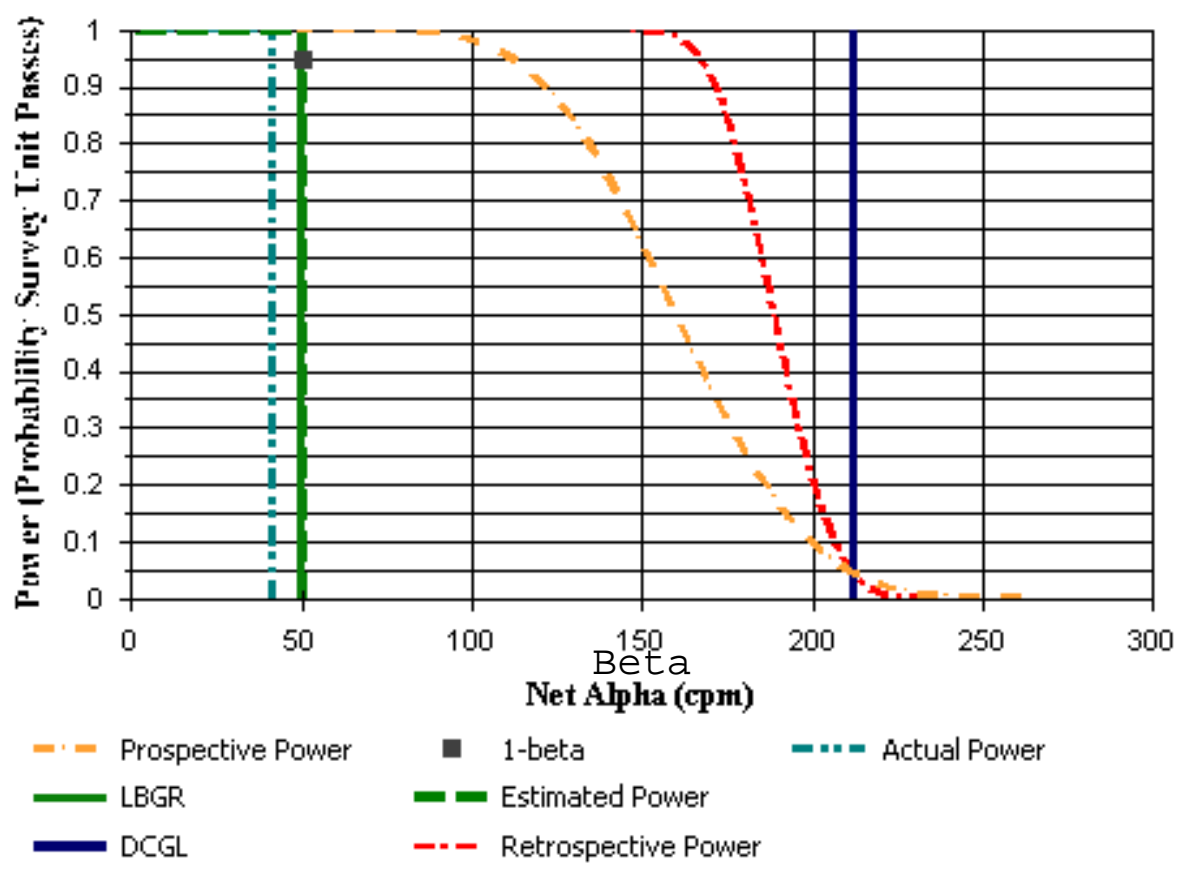




\section{DQA Building Surface Report}

\section{Survey Unit Data}

NOTE: $\quad$ Type = "S" indicates survey measurement.

Type = "R" indicates reference measurement.

Measurement

61.0, 3.6

$57.2,1.4$

$53.4,3.6$

$49.6,1.4$

$45.9,3.6$

$42.1,1.4$

$38.3,3.6$

$34.5,1.4$

$30.7,3.6$

$26.9,1.4$

$23.2,3.6$

$19.4,1.4$

15.6, 3.6

$11.8,1.4$

8.0, 3.6

$4.3,1.4$

$0.5,3.6$

\section{Material}

Unpainted Cinder Block

Unpainted Cinder Block

Unpainted Cinder Block

Unpainted Cinder Block

Unpainted Cinder Block

Unpainted Cinder Block

Unpainted Cinder Block Metal

Unpainted Cinder Block

Unpainted Cinder Block Metal

Unpainted Cinder Block Metal

Unpainted Cinder Block Unpainted Cinder Block

Unpainted Cinder Block

Unpainted Cinder Block
Beta

Type Gross Alpha (cpm)

$\begin{array}{ll}\mathrm{S} & 453 \\ \mathrm{~S} & 539\end{array}$

$\mathrm{S} \quad 415$

$\mathrm{S} \quad 482$

S 519

$\mathrm{S} \quad 515$

$S \quad 436$

$S \quad 200$

S 476

$\mathrm{S} \quad 511$

S $\quad 190$

S $\quad 564$

S 197

S 532

$\mathrm{S} \quad 411$

$\mathrm{S} \quad 465$

S 461

\section{Basic Statistical Quantities Summary}

\section{Statistic}

Sample Number

Mean $\left(\mathrm{dpm} / 100 \mathrm{~cm}^{2}\right)$

Median $\left(\mathrm{dpm} / 100 \mathrm{~cm}^{2}\right)$

Std Dev (dpm/100 cm²)

High Value $\left(\mathrm{dpm} / 100 \mathrm{~cm}^{2}\right)$

Low Value $\left(\mathrm{dpm} / 100 \mathrm{~cm}^{2}\right)$
Survey Unit

17
62.75
41.76
81.66
219.77
-69.35

Background

$\begin{array}{ll}\text { N/A } & \mathrm{N}=17 \\ \text { N/A } & 50.1 \\ \text { N/A } & \text { N/A } \\ \text { N/A } & 96.4 \\ \text { N/A } & \text { N/A } \\ \text { N/A } & \text { N/A }\end{array}$

DQO Results

$N=17$

50.1

N/A

96.4

N/A

N/A 


\section{DQA Building Surface Report}

\section{Assessment Summary}

Site:

Planner(s):

Survey Unit Name:

Report Number:

Survey Unit Measurements:

Reference Area Measurements:

Test Performed:

Judgmental Areas:

Assessment Conclusion:
Hammond Depot FSS Planner

Vitkus

Class 2 SUs C2 SU60 Building 100E Bay 13, 14 west floor

2

15

0

Sign

Test Result: $\quad$ Not Performed

0

EMC Result:

Not Performed

Reject Null Hypothesis (Survey Unit PASSES)

\section{Retrospective Power Curve}

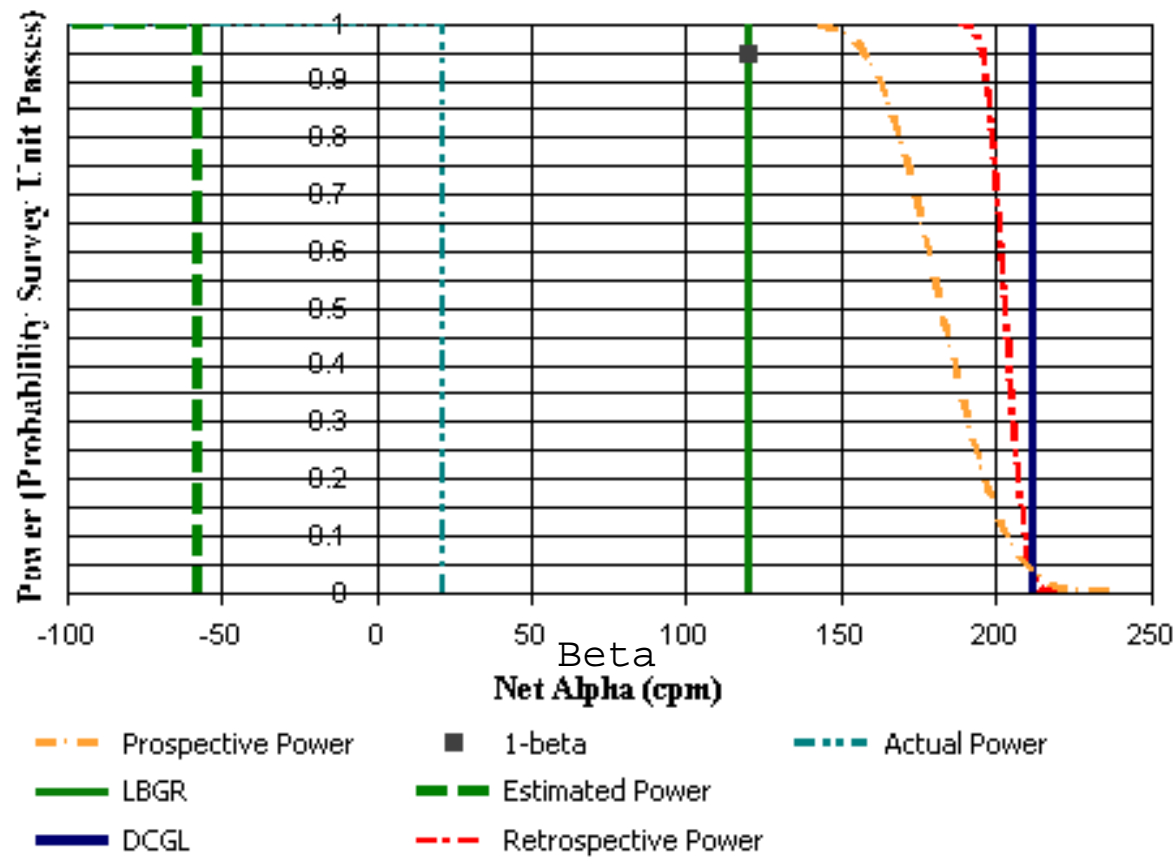




\section{DQA Building Surface Report}

\section{Survey Unit Data}

NOTE: $\quad$ Type = "S" indicates survey measurement.

Type $=$ "R" indicates reference measurement.

\begin{tabular}{llcc} 
Measurement & Material & Type & $\begin{array}{r}\text { Beta } \\
\text { Gross Alpha (cpm) }\end{array}$ \\
\hline $9.0,74.1$ & Concrete & $\mathrm{S}$ & 361 \\
$11.9,74.1$ & Concrete & $\mathrm{S}$ & 344 \\
$14.8,74.1$ & Concrete & $\mathrm{S}$ & 353 \\
$7.6,76.6$ & Concrete & $\mathrm{S}$ & 330 \\
$10.5,76.6$ & Concrete & $\mathrm{S}$ & 351 \\
$13.4,76.6$ & Concrete & $\mathrm{S}$ & 363 \\
$9.0,79.1$ & Concrete & $\mathrm{S}$ & 349 \\
$11.9,79.1$ & Concrete & $\mathrm{S}$ & 316 \\
$14.8,79.1$ & Concrete & $\mathrm{S}$ & 332 \\
$7.6,81.6$ & Concrete & $\mathrm{S}$ & 348 \\
$10.5,81.6$ & Concrete & $\mathrm{S}$ & 316 \\
$13.4,81.6$ & Concrete & $\mathrm{S}$ & 333 \\
$9.0,84.1$ & Concrete & $\mathrm{S}$ & 337 \\
$11.9,84.1$ & Concrete & $\mathrm{S}$ & 350 \\
$14.8,84.1$ & Concrete & $\mathrm{S}$ & 349
\end{tabular}

\section{Basic Statistical Quantities Summary}

\begin{tabular}{lccc} 
Statistic & Survey Unit & Background & DQO Results \\
\hline Sample Number & 15 & N/A & N=15 \\
Mean $\left(\mathrm{dpm} / 100 \mathrm{~cm}^{2}\right)$ & 10.27 & $\mathrm{~N} / \mathrm{A}$ & -57.9 \\
Median $\left(\mathrm{dpm} / 100 \mathrm{~cm}^{2}\right)$ & 21.35 & $\mathrm{~N} / \mathrm{A}$ & $\mathrm{N} / \mathrm{A}$ \\
Std Dev $\left(\mathrm{dpm} / 100 \mathrm{~cm}^{2}\right)$ & 27.23 & $\mathrm{~N} / \mathrm{A}$ & 47.4 \\
High Value $\left(\mathrm{dpm} / 100 \mathrm{~cm}^{2}\right)$ & 49.70 & $\mathrm{~N} / \mathrm{A}$ & $\mathrm{N} / \mathrm{A}$ \\
Low Value $\left(\mathrm{dpm} / 100 \mathrm{~cm}^{2}\right)$ & -39.12 & $\mathrm{~N} / \mathrm{A}$ & $\mathrm{N} / \mathrm{A}$
\end{tabular}




\section{DQA Building Surface Report}

\section{Assessment Summary}

Site:

Planner(s):

Survey Unit Name:

Report Number:

Survey Unit Measurements:

Reference Area Measurements:

Test Performed:

Judgmental Areas:

Assessment Conclusion:
Hammond Depot FSS Planner

Vitkus

Class 2 SUs Scoping C2 SU61 Building 100E bay 13, 14 east

2 floor

14

0

Sign

Test Result:

Not Performed

0

EMC Result:

Not Performed

\section{Retrospective Power Curve}

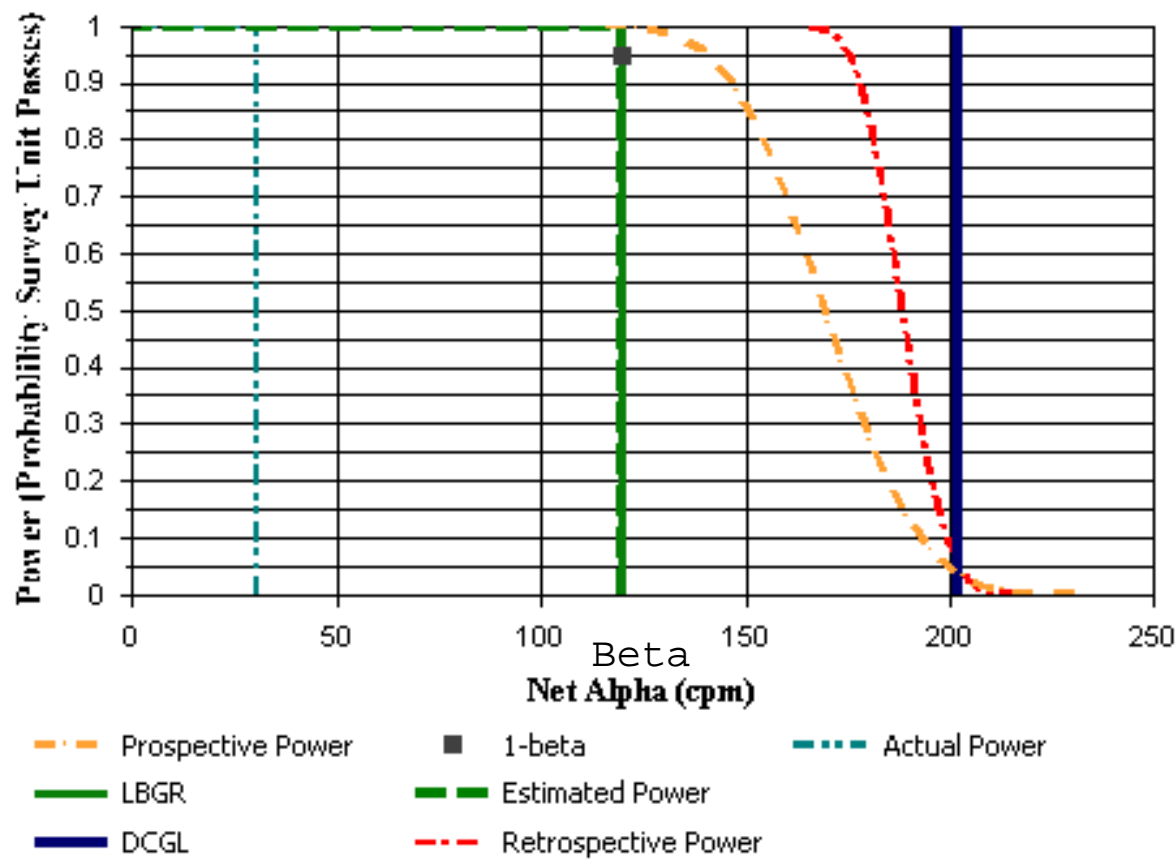




\section{DQA Building Surface Report}

\section{Survey Unit Data}

NOTE: $\quad$ Type = "S" indicates survey measurement.

Type $=$ "R" indicates reference measurement.

\begin{tabular}{llcc} 
Measurement & Material & Type & Gross Alpha (cpm) \\
\hline $24.4,79.6$ & Concrete & S & 360 \\
$26.3,79.6$ & Concrete & S & 365 \\
$28.3,79.6$ & Concrete & S & 312 \\
$23.4,81.3$ & Concrete & S & 380 \\
$25.3,81.3$ & Concrete & S & 389 \\
$27.3,81.3$ & Concrete & S & 390 \\
$29.3,81.3$ & Concrete & S & 333 \\
$24.4,83.0$ & Concrete & S & 372 \\
$26.3,83.0$ & Concrete & S & 345 \\
$28.3,83.0$ & Concrete & S & 344 \\
$23.4,84.7$ & Concrete & S & 321 \\
$25.3,84.7$ & Concrete & S & 343 \\
$27.3,84.7$ & Concrete & S & 336 \\
$29.3,84.7$ & Concrete & S & 355
\end{tabular}

\section{Basic Statistical Quantities Summary}

Statistic

Sample Number

Mean $\left(\mathrm{dpm} / 100 \mathrm{~cm}^{2}\right)$

Median (dpm/100 cm²)

Std Dev (dpm/100 $\left.\mathrm{cm}^{2}\right)$

High Value $\left(\mathrm{dpm} / 100 \mathrm{~cm}^{2}\right)$

Low Value $\left(\mathrm{dpm} / 100 \mathrm{~cm}^{2}\right)$
Survey Unit

14
36.73
30.36
47.94
109.72
-45.04

Background

N/A

N/A

N/A

N/A

N/A

N/A
DQO Results

$\mathrm{N}=25$

120

N/A

69.7

N/A

N/A 


\section{DQA Building Surface Report}

\section{Assessment Summary}

Site:

Planner(s):

Survey Unit Name:

Report Number:

Survey Unit Measurements:

Reference Area Measurements:

Test Performed:

Judgmental Areas:

Assessment Conclusion:
Hammond Depot FSS Planner

Vitkus

Class 3 SUs C3 SU39 Building 200E North, walls

4

15

0

Sign

Test Result: $\quad$ Not Performed

0

EMC Result:

Not Performed

Reject Null Hypothesis (Survey Unit PASSES)

\section{Retrospective Power Curve}

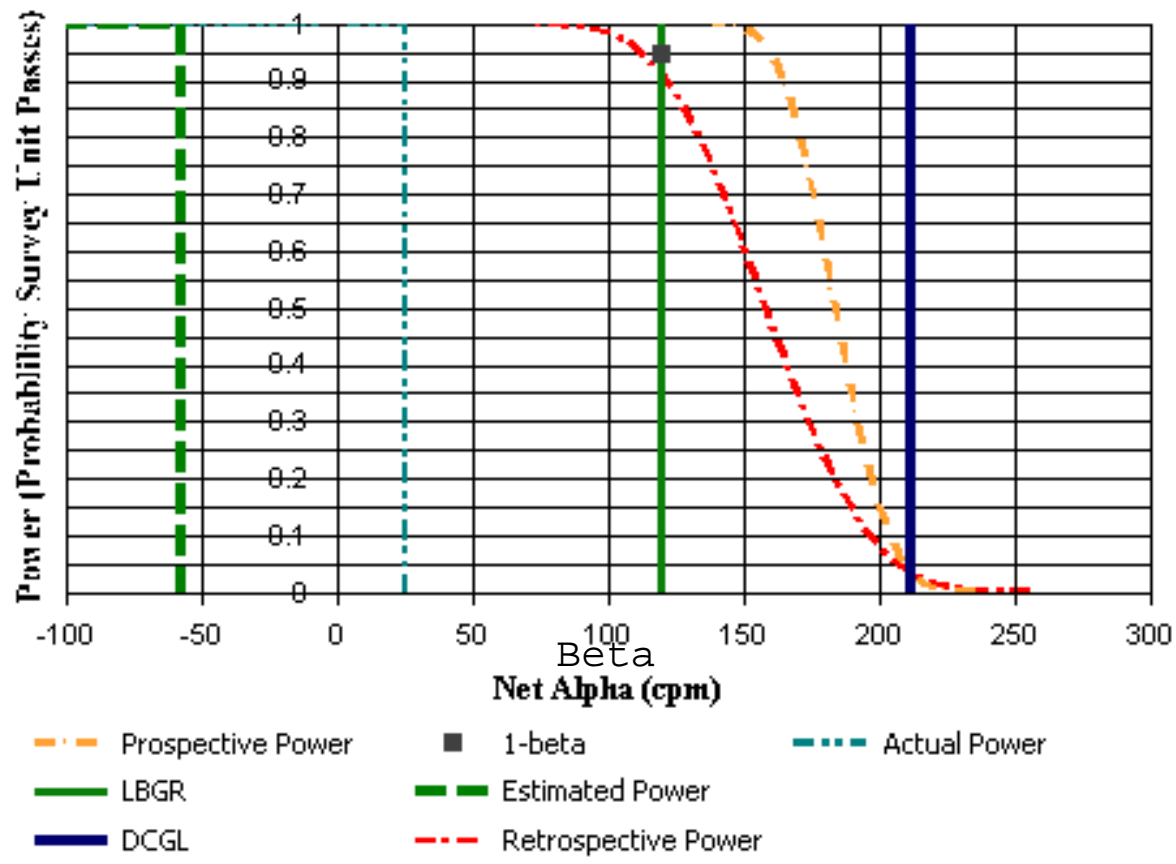




\section{DQA Building Surface Report}

\section{Survey Unit Data}

NOTE: $\quad$ Type = "S" indicates survey measurement.

Type = "R" indicates reference measurement.

Measurement

$0,38.1,2.4$

$0,5.1,3.4$

$37.8,27.7,0.4$

$37.8,17.5,1.5$

$0,54.8,0.9$

$37.8,46.3,2$

$37.8,4.6,3.1$

$14.9,61,3.5$

$37.8,53.6,1.9$

$0,40.3,3.9$

$11.9,61,0.2$

$0,27.2,0.3$

$37.8,46.3,1.3$

$11.4,0,2.9$

$14,0,1.8$

\section{Material}

Unpainted Cinder Block

Unpainted Cinder Block Metal

Unpainted Cinder Block

Unpainted Cinder Block Metal

Unpainted Cinder Block

Unpainted Cinder Block

Unpainted Cinder Block

Unpainted Cinder Block

Unpainted Cinder Block

Metal

Metal

Unpainted Cinder Block

Unpainted Cinder Block
Type- Beta

$\begin{array}{ll}\text { S } & 523 \\ \text { S } & 217 \\ \text { S } & 198 \\ \text { S } & 472 \\ \text { S } & 461 \\ \text { S } & 165 \\ \text { S } & 424 \\ \text { S } & 364 \\ \text { S } & 453 \\ \text { S } & 530 \\ \text { S } & 287 \\ \text { S } & 210 \\ \text { S } & 181 \\ \text { S } & 504 \\ \text { S } & 482\end{array}$

\section{Basic Statistical Quantities Summary}

\begin{tabular}{lccc} 
Statistic & Survey Unit & Background & DQO Results \\
\hline Sample Number & 15 & $\mathrm{~N} / \mathrm{A}$ & $\mathrm{N}=15$ \\
Mean $\left(\mathrm{dpm} / 100 \mathrm{~cm}^{2}\right)$ & -20.82 & $\mathrm{~N} / \mathrm{A}$ & -57.9 \\
Median $\left(\mathrm{dpm} / 100 \mathrm{~cm}^{2}\right)$ & 25.13 & $\mathrm{~N} / \mathrm{A}$ & $\mathrm{N} / \mathrm{A}$ \\
Std Dev $\left(\mathrm{dpm} / 100 \mathrm{~cm}^{2}\right)$ & 162.86 & $\mathrm{~N} / \mathrm{A}$ & 45.2 \\
High Value $\left(\mathrm{dpm} / 100 \mathrm{~cm}^{2}\right)$ & 155.52 & $\mathrm{~N} / \mathrm{A}$ & $\mathrm{N} / \mathrm{A}$ \\
Low Value $\left(\mathrm{dpm} / 100 \mathrm{~cm}^{2}\right)$ & -435.94 & $\mathrm{~N} / \mathrm{A}$ & $\mathrm{N} / \mathrm{A}$
\end{tabular}




\section{DQA Building Surface Report}

\section{Assessment Summary}

Site:

Planner(s):

Survey Unit Name:

Report Number:

Survey Unit Measurements:

Reference Area Measurements:

Test Performed:

Judgmental Areas:

Assessment Conclusion:
Hammond Depot FSS Planner

Vitkus

Class 3 SUs scoping C3 SU40 Building 200E, N, US

1

10

0

Sign

Test Result:

Not Performed

0

EMC Result:

Not Performed

Reject Null Hypothesis (Survey Unit PASSES)

\section{Retrospective Power Curve}

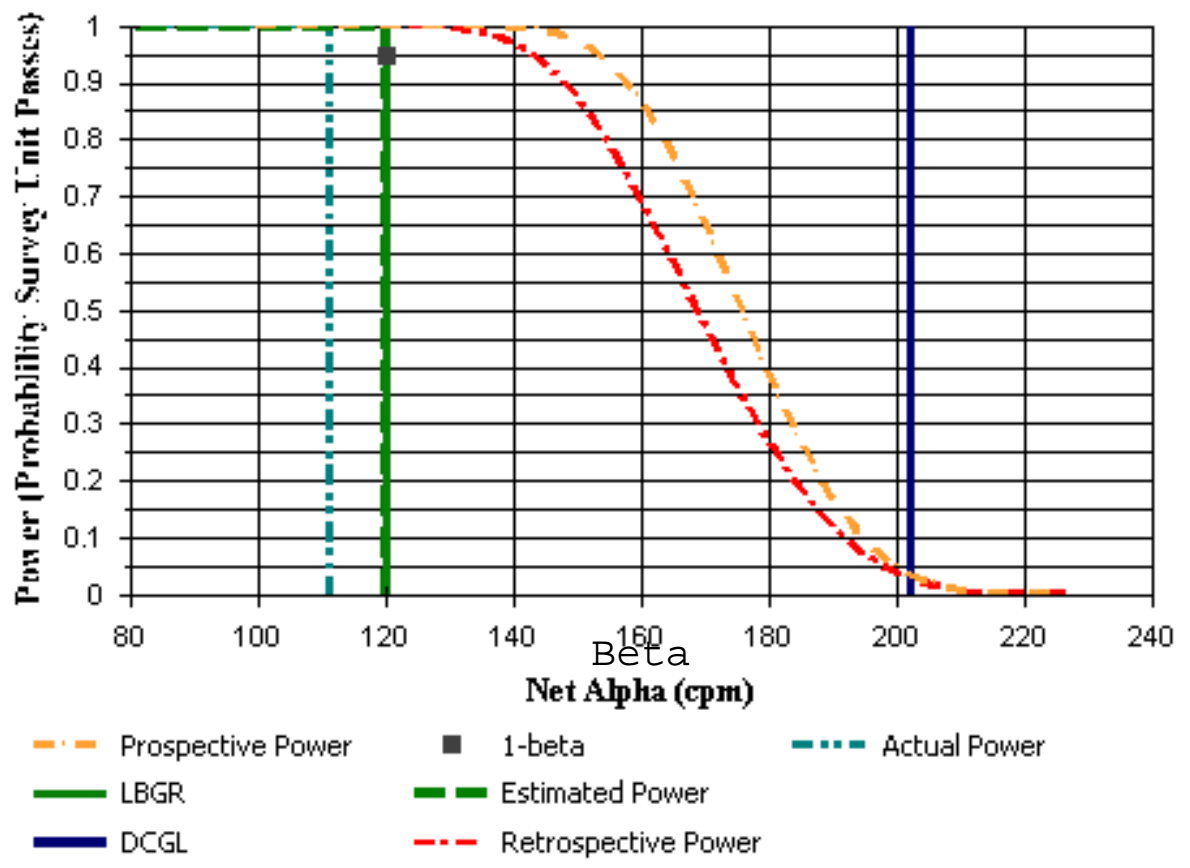




\section{DQA Building Surface Report}

\section{Survey Unit Data}

NOTE: $\quad$ Type $=$ "S" indicates survey measurement.

Type $=$ "R" indicates reference measurement.

\begin{tabular}{lccc} 
Measurement & Material & Type & Gross Alpha (cpm) \\
\hline 72 & Concrete & S & 361 \\
73 & Metal & S & 199 \\
74 & Metal & S & 249 \\
75 & Metal & S & 218 \\
76 & Metal & S & 211 \\
77 & Metal & S & 110 \\
78 & Metal & S & 224 \\
79 & Metal & S & 230 \\
80 & Unpainted Cinder Block & S & 461 \\
81 & Metal & S & 246
\end{tabular}

\section{Basic Statistical Quantities Summary}

\begin{tabular}{|c|c|c|c|}
\hline Statistic & Survey Unit & Background & DQO Results \\
\hline Sample Number & 10 & $\mathrm{~N} / \mathrm{A}$ & $\mathrm{N}=15$ \\
\hline Mean $\left(\mathrm{dpm} / 100 \mathrm{~cm}^{2}\right)$ & 94.86 & N/A & 120 \\
\hline Median (dpm/100 cm²) & 111.01 & $\mathrm{~N} / \mathrm{A}$ & $\mathrm{N} / \mathrm{A}$ \\
\hline Std Dev (dpm/100 cm²) & 78.63 & $\mathrm{~N} / \mathrm{A}$ & 42.4 \\
\hline High Value $\left(\mathrm{dpm} / 100 \mathrm{~cm}^{2}\right)$ & 174.01 & $\mathrm{~N} / \mathrm{A}$ & $\mathrm{N} / \mathrm{A}$ \\
\hline Low Value $\left(\mathrm{dpm} / 100 \mathrm{~cm}^{2}\right)$ & -101.79 & $N / A$ & $N / A$ \\
\hline
\end{tabular}




\section{DQA Building Surface Report}

\section{Assessment Summary}

Site:

Planner(s):

Survey Unit Name:

Report Number:

Survey Unit Measurements:

Reference Area Measurements:

Test Performed:

Judgmental Areas:

Assessment Conclusion:
Hammond Depot FSS Planner

Vitkus

Class 3 SUs C3 SU47 Building 100E ceiling Bays 15-20

5

12

0

Sign

Test Result:

Not Performed

0

EMC Result:

Not Performed

\section{Reject Null Hypothesis (Survey Unit PASSES)}

\section{Retrospective Power Curve}

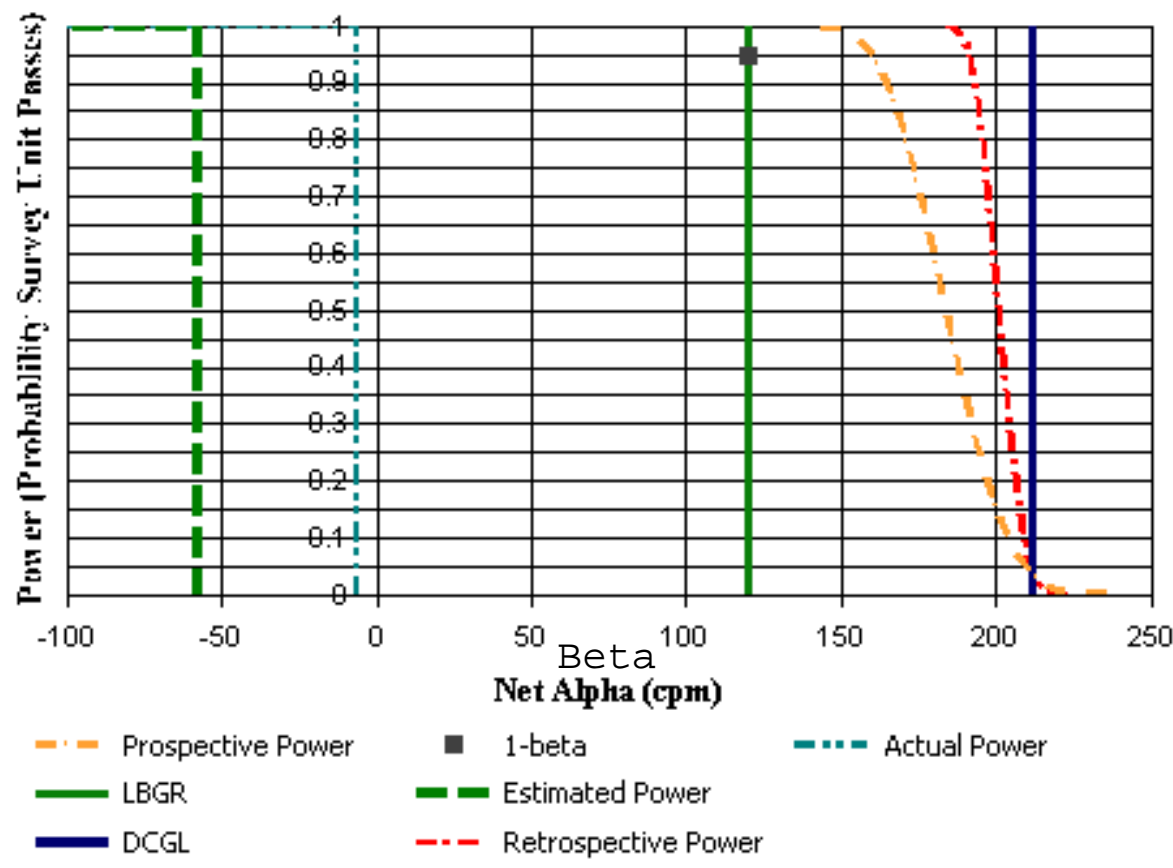




\section{DQA Building Surface Report}

\section{Survey Unit Data}

NOTE: $\quad$ Type = "S" indicates survey measurement.

Type $=$ "R" indicates reference measurement.

\begin{tabular}{lccc} 
Measurement & Material & Type & Gross Alpha (cpm) \\
\hline $20.0,101.5$ & Metal & $\mathrm{S}$ & 198 \\
$34.4,95.2$ & Metal & $\mathrm{S}$ & 174 \\
$5.2,96.9$ & Metal & $\mathrm{S}$ & 184 \\
$38.0,102.9$ & Metal & $\mathrm{S}$ & 175 \\
$29.8,107.4$ & Metal & $\mathrm{S}$ & 171 \\
$27.1,102.3$ & Metal & $\mathrm{S}$ & 172 \\
$3.2,97.7$ & Metal & $\mathrm{S}$ & 158 \\
$36.1,111.7$ & Metal & $\mathrm{S}$ & 159 \\
$35.7,103.9$ & Metal & $\mathrm{S}$ & 196 \\
$21.6,104.1$ & Metal & $\mathrm{S}$ & 176 \\
$17.6,106.2$ & Metal & $\mathrm{S}$ & 196 \\
$15.6,94.1$ & Metal & $\mathrm{S}$ & 145
\end{tabular}

\section{Basic Statistical Quantities Summary}

\begin{tabular}{lccc} 
Statistic & Survey Unit & Background & DQO Results \\
\hline Sample Number & 12 & $\mathrm{~N} / \mathrm{A}$ & $\mathrm{N}=15$ \\
Mean $\left(\mathrm{dpm} / 100 \mathrm{~cm}^{2}\right)$ & -4.85 & $\mathrm{~N} / \mathrm{A}$ & -57.9 \\
Median $\left(\mathrm{dpm} / 100 \mathrm{~cm}^{2}\right)$ & -6.42 & $\mathrm{~N} / \mathrm{A}$ & $\mathrm{N} / \mathrm{A}$ \\
Std Dev $\left(\mathrm{dpm} / 100 \mathrm{~cm}^{2}\right)$ & 31.01 & $\mathrm{~N} / \mathrm{A}$ & 45.2 \\
High Value $\left(\mathrm{dpm} / 100 \mathrm{~cm}^{2}\right)$ & 37.98 & $\mathrm{~N} / \mathrm{A}$ & $\mathrm{N} / \mathrm{A}$ \\
Low Value $\left(\mathrm{dpm} / 100 \mathrm{~cm}^{2}\right)$ & -62.17 & $\mathrm{~N} / \mathrm{A}$ & $\mathrm{N} / \mathrm{A}$
\end{tabular}




\section{DQA Building Surface Report}

\section{Assessment Summary}

Site:

Planner(s):

Survey Unit Name:

Report Number:

Survey Unit Measurements:

Reference Area Measurements:

Test Performed:

Judgmental Areas:

Assessment Conclusion:
Hammond Depot FSS Planner

Vitkus

Class 3 SUs scoping C3 SU48 Building 100E, Bays 1-14

3

9

0

Sign

Test Result: $\quad$ Not Performed

0

EMC Result:

Not Performed

\section{Retrospective Power Curve}

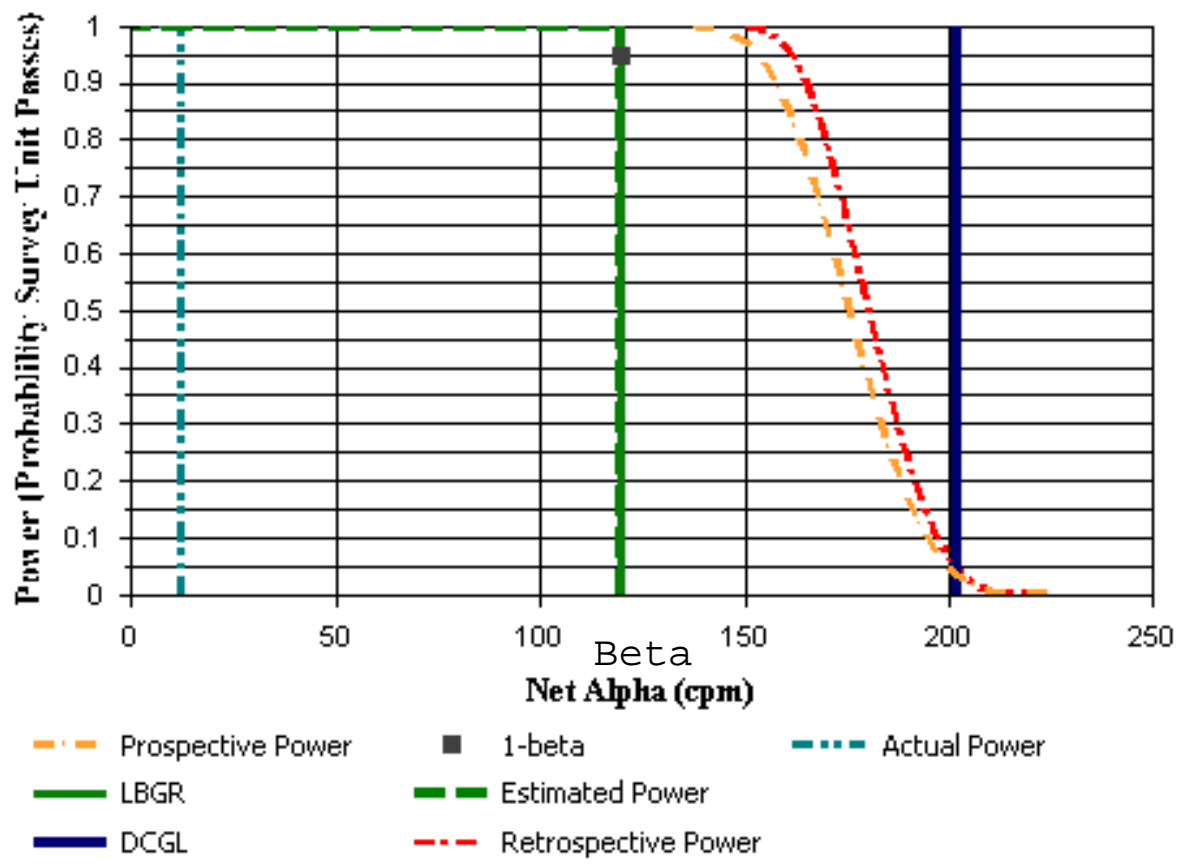




\section{DQA Building Surface Report}

\section{Survey Unit Data}

NOTE: $\quad$ Type = "S" indicates survey measurement.

Type $=$ "R" indicates reference measurement.

\begin{tabular}{lccc} 
Measurement & Material & Type & Gross Alpha (cpm) \\
\hline 8 & Concrete & S & 366 \\
9 & Concrete & S & 386 \\
10 & Concrete & S & 313 \\
11 & Concrete & S & 306 \\
12 & Concrete & S & 341 \\
13 & Concrete & S & 338 \\
14 & Concrete & S & 320 \\
18 & Metal & S & 202 \\
19 & Metal & S & 183
\end{tabular}

\section{Basic Statistical Quantities Summary}

Statistic

Sample Number

Mean $\left(\mathrm{dpm} / 100 \mathrm{~cm}^{2}\right)$

Median (dpm/100 cm²)

Std Dev (dpm/100 cm²)

High Value $\left(\mathrm{dpm} / 100 \mathrm{~cm}^{2}\right)$

Low Value $\left(\mathrm{dpm} / 100 \mathrm{~cm}^{2}\right)$
Survey Unit

9

19.73

12.50

56.16

101.79

$-56.94$
Background

N/A

N/A

N/A

N/A

N/A

N/A
DQO Results

$\mathrm{N}=15$

120

N/A

42.4

N/A

N/A 


\section{DQA Building Surface Report}

\section{Assessment Summary}

Site:

Planner(s):

Survey Unit Name:

Report Number:

Survey Unit Measurements:

Reference Area Measurements:

Test Performed:

Judgmental Areas:

Assessment Conclusion:
Hammond Depot FSS Planner

Vitkus

Class 3 SUs scoping C3 SU52 Building 100W US

2

21

0

Sign

Test Result:

Not Performed

0

EMC Result:

Not Performed

Reject Null Hypothesis (Survey Unit PASSES)

\section{Retrospective Power Curve}

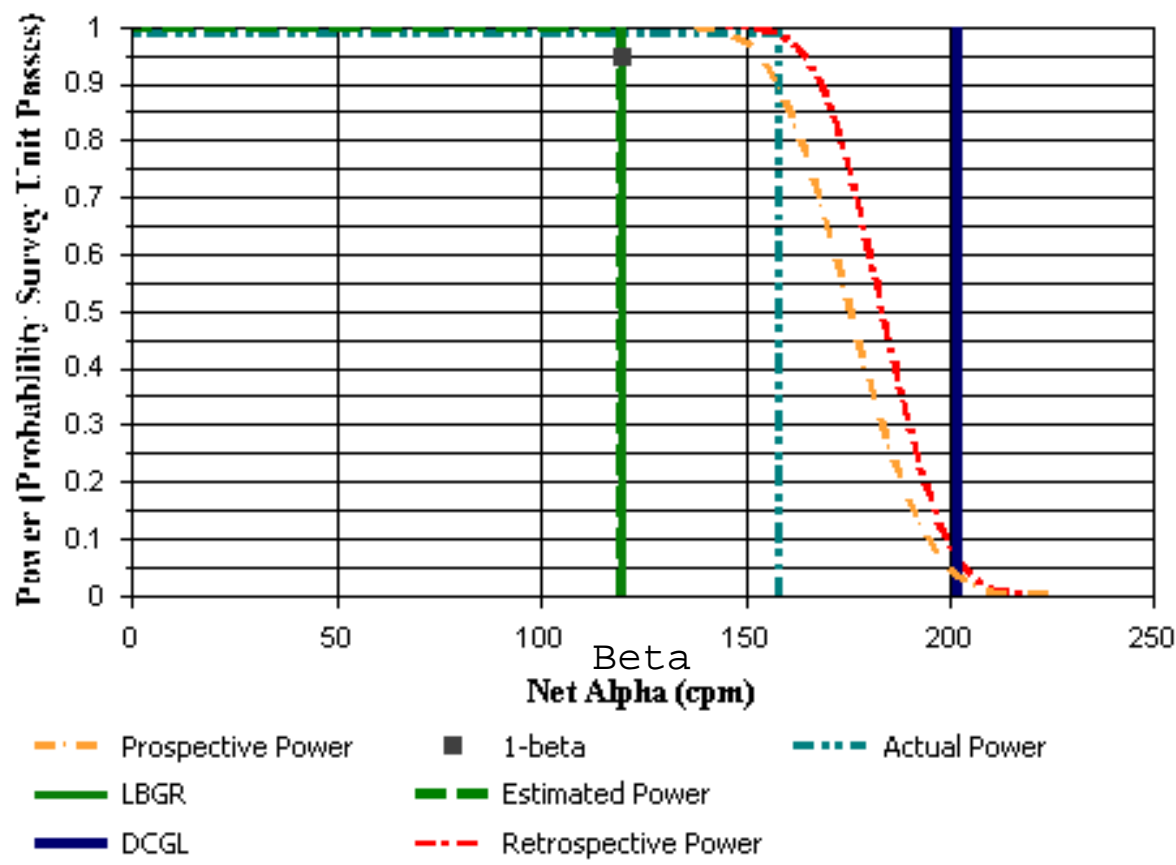




\section{DQA Building Surface Report}

\section{Survey Unit Data}

NOTE: $\quad$ Type $=$ "S" indicates survey measurement.

Type $=$ "R" indicates reference measurement.

\begin{tabular}{lccc} 
Measurement & Material & Type & $\begin{array}{c}\text { Beta } \\
\text { Gross Alpha (cpm) }\end{array}$ \\
\hline A85 & Metal & S & 161 \\
A86 & Metal & S & 235 \\
A87 & Metal & S & 169 \\
A88 & Metal & S & 241 \\
A89 & Metal & S & 224 \\
A90 & Metal & S & 243 \\
A91 & Metal & S & 300 \\
A92 & Metal & S & 250 \\
A93 & Metal & S & 277 \\
A94 & Metal & S & 297 \\
A95 & Metal & S & 282 \\
A96 & Metal & S & 198 \\
A97 & Metal & S & 187 \\
A98 & Setal & S & 210 \\
A99 & Metal & S & 251 \\
A100 & Metal & S & 333 \\
30D & Metal & S & 246 \\
31D & Metal & S & 225 \\
32D & Metal & S & 217 \\
33D & Metal & S & 221 \\
34D & Metal & & 244
\end{tabular}

\section{Basic Statistical Quantities Summary}

Statistic

Sample Number

Mean $\left(\mathrm{dpm} / 100 \mathrm{~cm}{ }^{2}\right)$

Median $\left(\mathrm{dpm} / 100 \mathrm{~cm}^{2}\right)$

Std Dev (dpm/100 cm²)

High Value $\left(\mathrm{dpm} / 100 \mathrm{~cm}^{2}\right)$

Low Value $\left(\mathrm{dpm} / 100 \mathrm{~cm}^{2}\right)$
Survey Unit

21

153.41

158.13

85.37

340.67

$-0.60$
Background

N/A

N/A

N/A

N/A

N/A

N/A
DQO Results

$\mathrm{N}=15$

120

N/A

42.4

N/A

N/A 


\section{DQA Building Surface Report}

\section{Assessment Summary}

Site:

Planner(s):

Survey Unit Name:

Report Number:

Survey Unit Measurements:

Reference Area Measurements:

Test Performed:

Judgmental Areas:

Assessment Conclusion:
Hammond Depot FSS Planner

Vitkus

Class 3 SUs C3 SU53 Garage

3

15

0

Sign

Test Result:

Not Performed

0

EMC Result:

Not Performed

\section{Retrospective Power Curve}

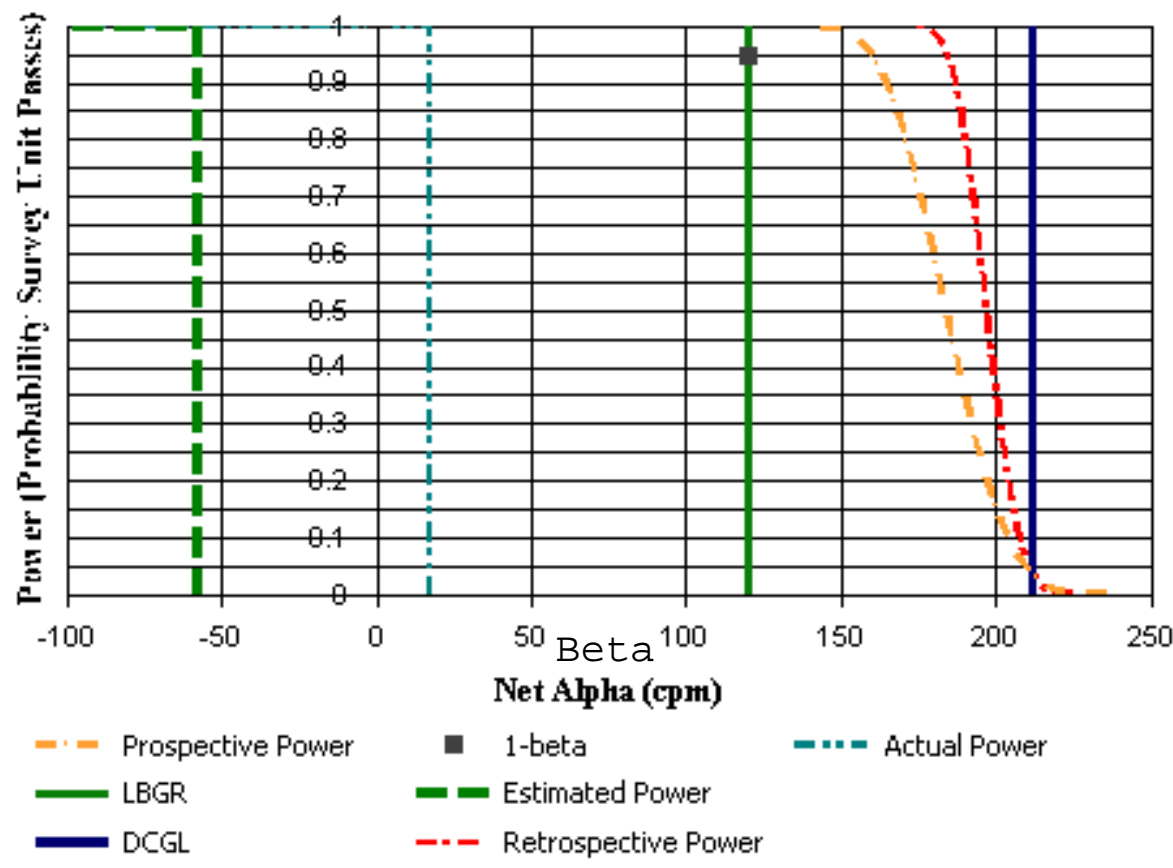




\section{DQA Building Surface Report}

\section{Survey Unit Data}

NOTE: $\quad$ Type = "S" indicates survey measurement.

Type $=$ "R" indicates reference measurement.

\begin{tabular}{llcc} 
Measurement & Material & Type & Gross Alpha (cpm) \\
\hline $19.2,1$ & Concrete & $\mathrm{S}$ & 332 \\
$20.6,4.5$ & Concrete & $\mathrm{S}$ & 395 \\
$16.8,5.1$ & Concrete & $\mathrm{S}$ & 376 \\
$21.4,6.2$ & Concrete & $\mathrm{S}$ & 369 \\
$16.6,9.7$ & Concrete & $\mathrm{S}$ & 343 \\
$9.4,13.9$ & Concrete & $\mathrm{S}$ & 328 \\
$7.2,15$ & Concrete & $\mathrm{S}$ & 381 \\
$3.8,15.7$ & Concrete & $\mathrm{S}$ & 372 \\
$2.8,17.4$ & Concrete & $\mathrm{S}$ & 355 \\
$8.4,19.7$ & Concrete & $\mathrm{S}$ & 323 \\
12,20 & Concrete & $\mathrm{S}$ & 359 \\
$1.9,25$ & Concrete & $\mathrm{S}$ & 330 \\
$18.9,30.3$ & Concrete & $\mathrm{S}$ & 337 \\
$2.1,30.4,1.5$ & Metal & $\mathrm{S}$ & 187 \\
$13.1,30.4 .0 .9$ & Metal & $\mathrm{S}$ & 155
\end{tabular}

\section{Basic Statistical Quantities Summary}

\begin{tabular}{lccc} 
Statistic & Survey Unit & Background & DQO Results \\
\hline Sample Number & 15 & N/A & N=15 \\
Mean $\left(\mathrm{dpm} / 100 \mathrm{~cm}^{2}\right)$ & 26.34 & $\mathrm{~N} / \mathrm{A}$ & -57.9 \\
Median $\left(\mathrm{dpm} / 100 \mathrm{~cm}^{2}\right)$ & 17.20 & $\mathrm{~N} / \mathrm{A}$ & $\mathrm{N} / \mathrm{A}$ \\
Std Dev $\left(\mathrm{dpm} / 100 \mathrm{~cm}^{2}\right)$ & 45.42 & $\mathrm{~N} / \mathrm{A}$ & 45.2 \\
High Value $\left(\mathrm{dpm} / 100 \mathrm{~cm}^{2}\right)$ & 110.17 & $\mathrm{~N} / \mathrm{A}$ & $\mathrm{N} / \mathrm{A}$ \\
Low Value $\left(\mathrm{dpm} / 100 \mathrm{~cm}^{2}\right)$ & -43.27 & $\mathrm{~N} / \mathrm{A}$ & $\mathrm{N} / \mathrm{A}$
\end{tabular}




\section{DQA Building Surface Report}

\section{Assessment Summary}

Site:

Planner(s):

Survey Unit Name:

Report Number:

Survey Unit Measurements:

Reference Area Measurements:

Test Performed:

Judgmental Areas:

Assessment Conclusion:
Hammond Depot FSS Planner

Vitkus

Class 3 SUs C1 SU54 Workshop

2

14

0

Sign

Test Result:

Not Performed

0

EMC Result:

Not Performed

\section{Retrospective Power Curve}

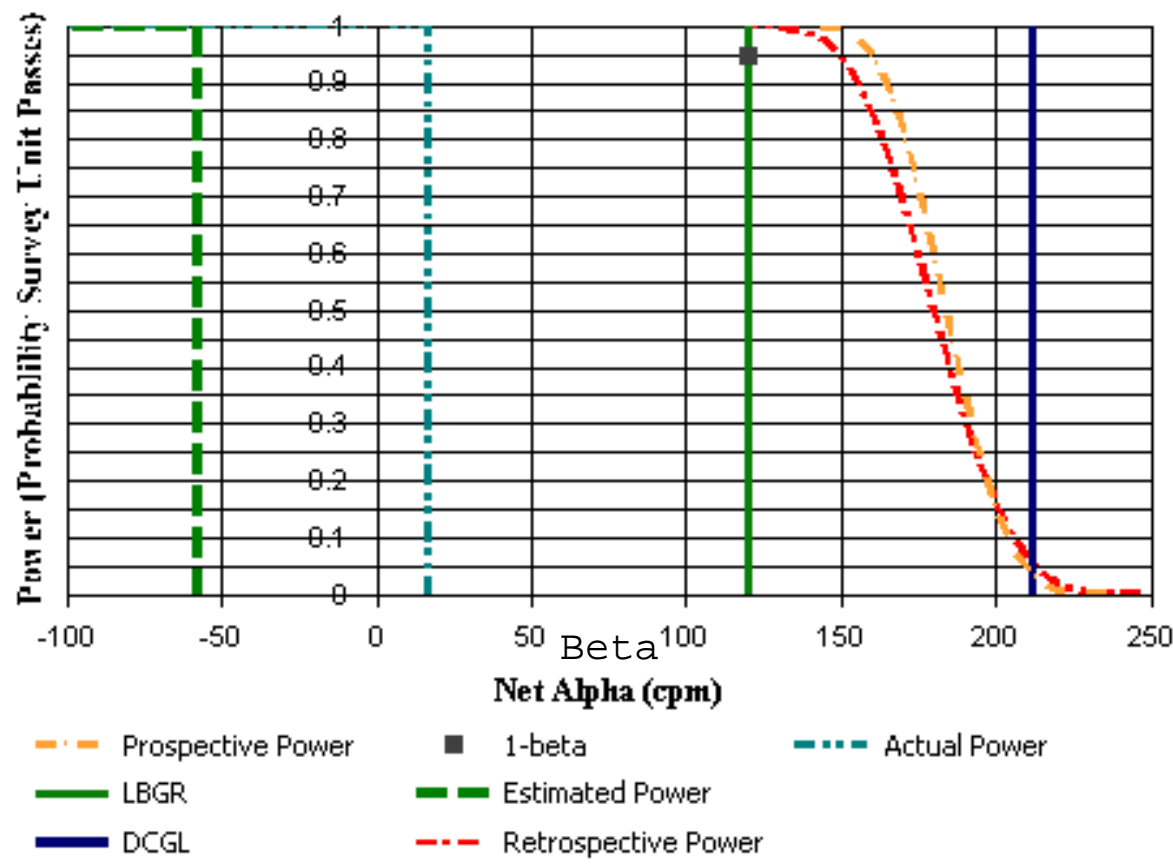




\section{DQA Building Surface Report}

\section{Survey Unit Data}

NOTE: $\quad$ Type = "S" indicates survey measurement.

Type $=$ "R" indicates reference measurement.

Measurement

6.1, 8.6, 0/Floor/WS

7.6, 0.9, 0/Floor/WS

$0.5,13.1$, o/Floor/W

$0.8,3.3,3 / \mathrm{W}$. Wall/

8.6, 7.3, 3/W. Wall/

6.1, 11, 3/Ceiling/O

$0.2,24.5$, 0/Floor/C

$0.2,25.2$, o/Floor/C

$0,24.8,0.9 / \mathrm{W}$. Wall

1.3, 24.6, 0/Floor/C

$0.3,31.8,3 /$ Ceiling

$0.9,35.2,3 /$ Ceiling

3.8, 37.9, 0/Floor/B

$6.4,28.1$, 0/Floor/B
Material

Concrete

Concrete

Concrete

Concrete

Concrete

Metal

Concrete

Concrete

Concrete

Concrete

Concrete

Concrete

Concrete

Concrete
Beta

Type Gross Alpha (cpm)

345

346

384

303

222

285

348

319

300

366

389

411

285

316

\section{Basic Statistical Quantities Summary}

Statistic

Sample Number

Mean $\left(\mathrm{dpm} / 100 \mathrm{~cm}^{2}\right)$

Median (dpm/100 cm²)

Std Dev (dpm/100 $\left.\mathrm{cm}^{2}\right)$

High Value $\left(\mathrm{dpm} / 100 \mathrm{~cm}^{2}\right)$

Low Value $\left(\mathrm{dpm} / 100 \mathrm{~cm}^{2}\right)$
Survey Unit

14
8.64
16.63
107.24
202.38
216.74

Background

N/A

N/A

N/A

N/A

N/A

N/A
DQO Results

$\mathrm{N}=15$

$-57.9$

N/A

45.2

N/A

N/A 


\section{DQA Building Surface Report}

\section{Assessment Summary}

Site:

Planner(s):

Survey Unit Name:

Report Number:

Survey Unit Measurements:

Reference Area Measurements:

Test Performed:

Judgmental Areas:

Assessment Conclusion:
Hammond Depot FSS Planner

Vitkus

Class 3 SUs C3 SU55 Building Exteriors

1

15

0

Sign

Test Result:

Not Performed

0

EMC Result:

Not Performed

Reject Null Hypothesis (Survey Unit PASSES)

\section{Retrospective Power Curve}

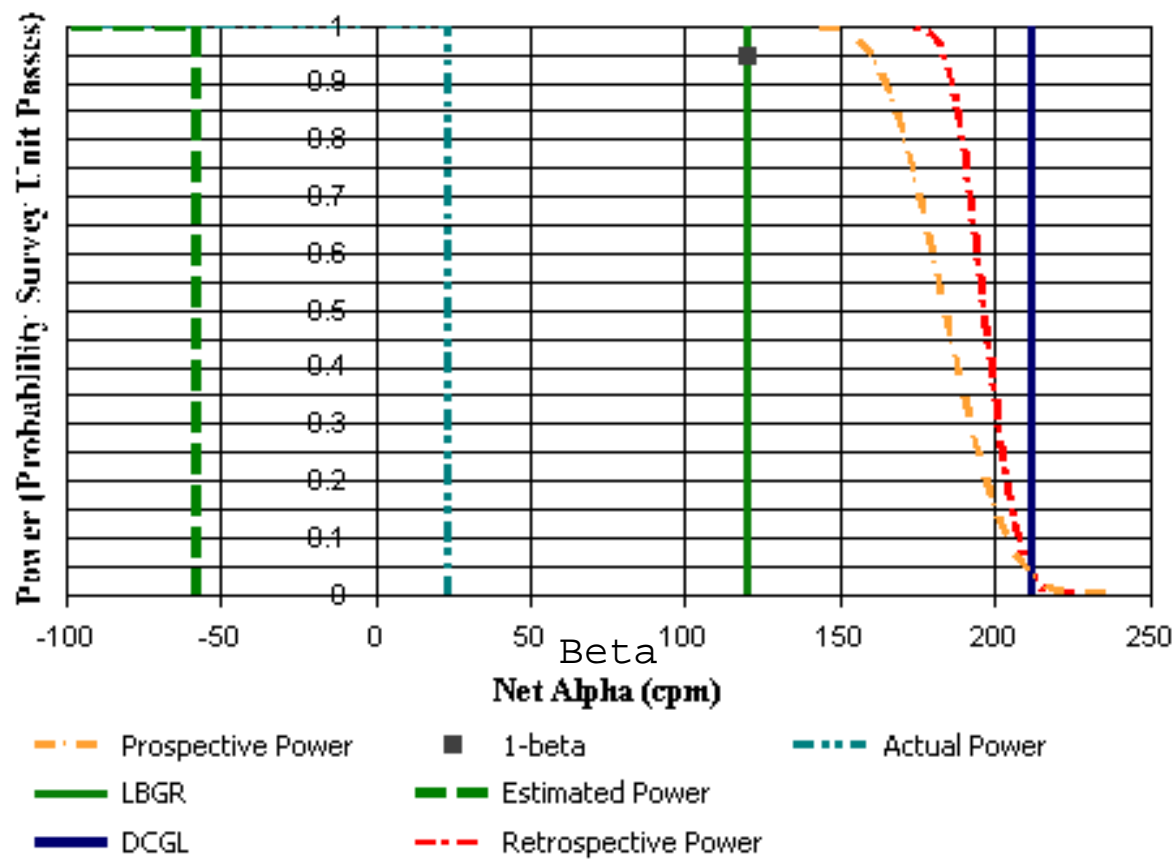




\section{DQA Building Surface Report}

\section{Survey Unit Data}

NOTE: $\quad$ Type $=$ "S" indicates survey measurement.

Type $=$ "R" indicates reference measurement.

\begin{tabular}{lccc} 
Measurement & Material & Type & Gross Alpha (cpm) \\
\hline $75.8,2.7 / 100 W$ West & Concrete & $\mathrm{S}$ & 349 \\
$92.2,2.5 / 100 W$ East & Concrete & $\mathrm{S}$ & 355 \\
$52.8,1.4 / 100$ E West & Concrete & $\mathrm{S}$ & 351 \\
$71.1,2.2 / 100$ E West & Metal & $\mathrm{S}$ & 185 \\
20.9, 2.1/100E North & Metal & $\mathrm{S}$ & 189 \\
$96.9,1.9 / 100$ E East & Concrete & $\mathrm{S}$ & 334 \\
$96.6,0.7 / 200$ E West & Metal & $\mathrm{S}$ & 199 \\
$72.1,2.6 / 200$ E East & Concrete & $\mathrm{S}$ & 361 \\
3.2, 0.1/200E South & Concrete & $\mathrm{S}$ & 342 \\
8.3, 2/Office South & Metal & $\mathrm{S}$ & 206 \\
6, 0.3/Office South & Metal & $\mathrm{S}$ & 252 \\
16, 1.6/Office South & Metal & $\mathrm{S}$ & 181 \\
4.5, 0.6/Guardhouse & Metal & $\mathrm{S}$ & 137 \\
$5.5,2.1 /$ Pumphouse E & Concrete & $\mathrm{S}$ & 347 \\
10.4, 0.6/Garage Eas & Metal & $\mathrm{S}$ & 226
\end{tabular}

\section{Basic Statistical Quantities Summary}

\begin{tabular}{lccc} 
Statistic & Survey Unit & Background & DQO Results \\
\hline Sample Number & 15 & N/A & N=15 \\
Mean $\left(\mathrm{dpm} / 100 \mathrm{~cm}^{2}\right)$ & 29.47 & N/A & -57.9 \\
Median $\left(\mathrm{dpm} / 100 \mathrm{~cm}^{2}\right)$ & 23.24 & N/A $/ A$ \\
Std Dev $\left(\mathrm{dpm} / 100 \mathrm{~cm}^{2}\right)$ & 47.03 & N/A & 45.2 \\
High Value $\left(\mathrm{dpm} / 100 \mathrm{~cm}^{2}\right)$ & 140.02 & N/A & N/A \\
Low Value $\left(\mathrm{dpm} / 100 \mathrm{~cm}^{2}\right)$ & -77.29 & N/A
\end{tabular}


APPENDIX G: LAND SURVEY UNIT DQAs 


\section{DQA Surface Soil Report}

\section{Assessment Summary}

Site:

Planner(s):

Survey Unit Name:

Report Number:

Survey Unit Samples:

Reference Area Samples:

Test Performed:

Judgmental Samples:

Assessment Conclusion:
Hammond Depot FSS Planner

Vitkus

C1 SU1

1

17

17

WRS

Test Result: $\quad$ Pass

0

EMC Result:

Not Performed

Reject Null Hypothesis (Survey Unit PASSES)

\section{Retrospective Power Curve}

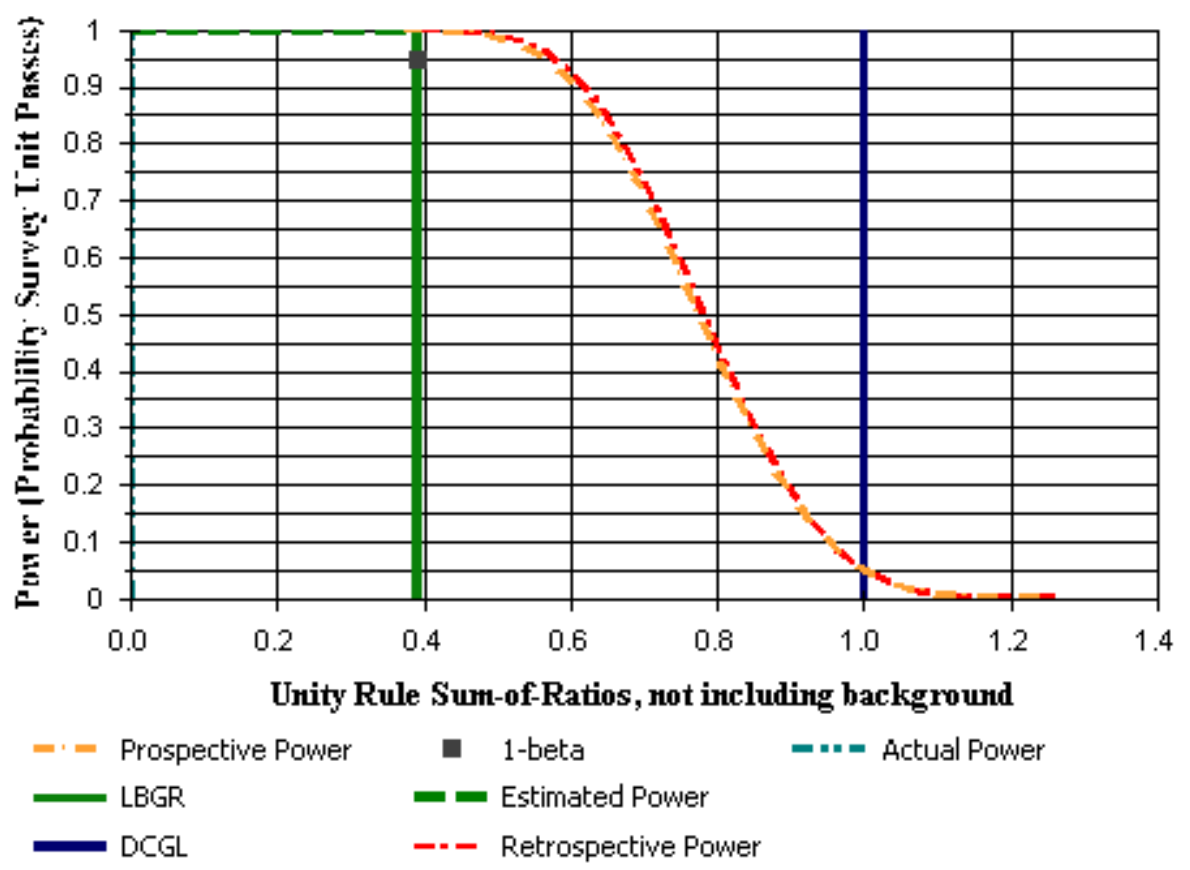




\section{DQA Surface Soil Report}

\section{Survey Unit Data}

NOTE: $\quad$ Type = "S" indicates survey unit sample.

Type = "R" indicates reference area sample.

\begin{tabular}{|c|c|c|c|}
\hline Sample Number & Type & Th-232 (pCi/g) & U-238 (pCi/g) \\
\hline 196 & $\mathrm{~S}$ & 1.17 & 2.16 \\
\hline 197 & $S$ & 1.15 & 2.63 \\
\hline 198 & $S$ & 1.01 & 2.42 \\
\hline 199 & S & 1.15 & 1.54 \\
\hline 200 & $S$ & 1.07 & 2.82 \\
\hline 201 & S & 1.13 & 1.98 \\
\hline 202 & $S$ & 1.2 & 1.37 \\
\hline 203 & $S$ & 0.51 & 0.93 \\
\hline 204 & $S$ & 1.02 & 2.72 \\
\hline 205 & $S$ & 1.22 & 4.09 \\
\hline 206 & S & 0.85 & 2.58 \\
\hline 207 & S & 1.05 & 2.74 \\
\hline 208 & S & 1.24 & 2.8 \\
\hline 209 & S & 1.25 & 1.79 \\
\hline 210 & S & 0.55 & 1.08 \\
\hline 211 & S & 0.88 & 2.48 \\
\hline 212 & S & 1.31 & 3.1 \\
\hline 127 & $\mathrm{R}$ & 1.1 & 2.54 \\
\hline 128 & $\mathrm{R}$ & 0.85 & 2.66 \\
\hline 129 & $\mathrm{R}$ & 0.74 & 2.44 \\
\hline 130 & $\mathrm{R}$ & 1.09 & 2.3 \\
\hline 131 & $\mathrm{R}$ & 0.93 & 2.5 \\
\hline 132 & $\mathrm{R}$ & 0.79 & 1.65 \\
\hline 133 & $\mathrm{R}$ & 0.94 & 2.3 \\
\hline 134 & $\mathrm{R}$ & 0.98 & 2.55 \\
\hline 135 & $\mathrm{R}$ & 0.65 & 2.06 \\
\hline 136 & $\mathrm{R}$ & 0.73 & 2.66 \\
\hline 137 & $\mathrm{R}$ & 1.1 & 2.44 \\
\hline 138 & $\mathrm{R}$ & 1.03 & 2.42 \\
\hline 139 & $\mathrm{R}$ & 1.17 & 2.72 \\
\hline 140 & $\mathrm{R}$ & 1.36 & 3.35 \\
\hline 141 & $\mathrm{R}$ & 0.91 & 1.44 \\
\hline 142 & $\mathrm{R}$ & 1.71 & 3.57 \\
\hline 143 & $\mathrm{R}$ & 0.33 & 0.63 \\
\hline
\end{tabular}




\section{Modified Data (Unity Rule SOR)}

NOTE: $\quad$ Type = "S" indicates survey unit sample.

Type $=$ "R" indicates reference area sample.

\begin{tabular}{lcc} 
Sample Number & Type & Sum-of-Ratios (SOR) \\
\hline 196 & $\mathrm{~S}$ & 1.27 \\
197 & $\mathrm{~S}$ & 1.45 \\
198 & $\mathrm{~S}$ & 1.32 \\
199 & $\mathrm{~S}$ & 1.01 \\
200 & $\mathrm{~S}$ & 1.5 \\
201 & $\mathrm{~S}$ & 1.18 \\
202 & $\mathrm{~S}$ & 0.96 \\
203 & $\mathrm{~S}$ & 0.55 \\
204 & $\mathrm{~S}$ & 1.44 \\
205 & $\mathrm{~S}$ & 2.06 \\
206 & $\mathrm{~S}$ & 1.33 \\
207 & $\mathrm{~S}$ & 1.46 \\
208 & $\mathrm{~S}$ & 1.55 \\
209 & $\mathrm{~S}$ & 1.15 \\
210 & $\mathrm{~S}$ & 0.62 \\
211 & $\mathrm{~S}$ & 1.3 \\
212 & $\mathrm{~S}$ & 1.69 \\
127 & $\mathrm{R}$ & 1.4 \\
128 & $\mathrm{R}$ & 1.36 \\
129 & $\mathrm{R}$ & 1.23 \\
130 & $\mathrm{R}$ & 1.3 \\
131 & $\mathrm{R}$ & 1.32 \\
132 & $\mathrm{R}$ & 0.93 \\
133 & $\mathrm{R}$ & 1.24 \\
134 & $\mathrm{R}$ & 1.36 \\
135 & $\mathrm{R}$ & 1.05 \\
136 & $\mathrm{R}$ & 1.32 \\
137 & $\mathrm{R}$ & 1.36 \\
138 & $\mathrm{R}$ & 1.32 \\
139 & $\mathrm{R}$ & 1.49 \\
140 & $\mathrm{R}$ & 1.81 \\
141 & $\mathrm{R}$ & 0.89 \\
142 & $\mathrm{R}$ & 2.02 \\
143 & $\mathrm{R}$ & 0.37 \\
& &
\end{tabular}




\section{DQA Surface Soil Report}

\section{Basic Statistical Quantities Summary}

\begin{tabular}{lccc} 
Statistic & Survey Unit & Background & DQO Results \\
\hline Sample Number & 17 & 17 & N/2=18 \\
Mean (SOR) & 1.28 & 1.28 & 0.39 \\
Median (SOR) & 1.32 & 1.32 & N/A \\
Std Dev (SOR) & 0.37 & 0.36 & 0.39 \\
High Value (SOR) & 2.06 & 2.02 & N/A \\
Low Value (SOR) & 0.55 & 0.37 & N/A
\end{tabular}

\section{Statistical Test Summary}

$\begin{array}{ll}\text { Sum of Ranks: } & 595 \\ \text { Sum of Reference Ranks: } & 432 \\ \text { Critical Value: } & 345 \\ \text { Result: } & \text { Pass }\end{array}$

\begin{tabular}{lcccc} 
Data & Type & Adjusted Data & Rank & Reference \\
\hline & & & 11 & 11 \\
0.37 & $\mathrm{R}$ & 1.37 & 18 & 18 \\
0.89 & $\mathrm{R}$ & 1.89 & 19 & 19 \\
0.93 & $\mathrm{R}$ & 1.93 & 20 & 20 \\
1.05 & $\mathrm{R}$ & 2.05 & 22 & 22 \\
1.23 & $\mathrm{R}$ & 2.23 & 23 & 23 \\
1.24 & $\mathrm{R}$ & 2.24 & 24 & 24 \\
1.3 & $\mathrm{R}$ & 2.3 & 26 & 26 \\
1.32 & $\mathrm{R}$ & 2.32 & 26 & 26 \\
1.32 & $\mathrm{R}$ & 2.32 & 26 & 26 \\
1.32 & $\mathrm{R}$ & 2.32 & 29 & 29 \\
1.36 & $\mathrm{R}$ & 2.36 & 29 & 29 \\
1.36 & $\mathrm{R}$ & 2.36 & 29 & 29 \\
1.36 & $\mathrm{R}$ & 2.36 & 31 & 31 \\
1.4 & $\mathrm{R}$ & 2.4 & 32 & 32 \\
1.49 & $\mathrm{R}$ & 2.49 & 33 & 33 \\
1.81 & $\mathrm{R}$ & 2.81 & 34 & 34 \\
2.02 & $\mathrm{R}$ & 3.02 & 1 & 0 \\
0.55 & $\mathrm{~S}$ & 0.55 & 2 & 0 \\
0.62 & $\mathrm{~S}$ & 0.62 & 3 & 0 \\
0.96 & $\mathrm{~S}$ & 0.96 & 4 & 0 \\
1.01 & $\mathrm{~S}$ & 1.01 & 5 & 0 \\
1.15 & $\mathrm{~S}$ & 1.15 & 6 & 0 \\
1.18 & $\mathrm{~S}$ & 1.18 & 8 & 0 \\
1.27 & $\mathrm{~S}$ & 1.27 & 8 & 0 \\
1.3 & $\mathrm{~S}$ & 1.3 & & 0
\end{tabular}




\section{DQA Surface Soil Report}

\section{Statistical Test Summary}

\begin{tabular}{lcccc} 
Data & Type & Adjusted Data & Rank & Reference Rank \\
\hline 1.32 & & 1.32 & 9 & 0 \\
1.33 & S & 1.33 & 10 & 0 \\
1.44 & S & 1.44 & 12 & 0 \\
1.45 & S & 1.45 & 13 & 0 \\
1.46 & S & 1.46 & 14 & 0 \\
1.5 & S & 1.5 & 15 & 0 \\
1.55 & S & 1.55 & 16 & 0 \\
1.69 & S & 1.69 & 17 & 0 \\
2.06 & S & 2.06 & 21 & 0
\end{tabular}




\section{DQA Surface Soil Report}

\section{Assessment Summary}

Site:

Planner(s):

Survey Unit Name:

Report Number:

Survey Unit Samples:

Reference Area Samples:

Test Performed:

Judgmental Samples:

Assessment Conclusion:
Hammond Depot FSS Planner

Vitkus

C1 SU2, 3, 4, and 5 (AOC 1 and 2) C1 SU2

1

17

17

WRS

Test Result: $\quad$ Pass

0

EMC Result:

Not Performed

Reject Null Hypothesis (Survey Unit PASSES)

\section{Retrospective Power Curve}

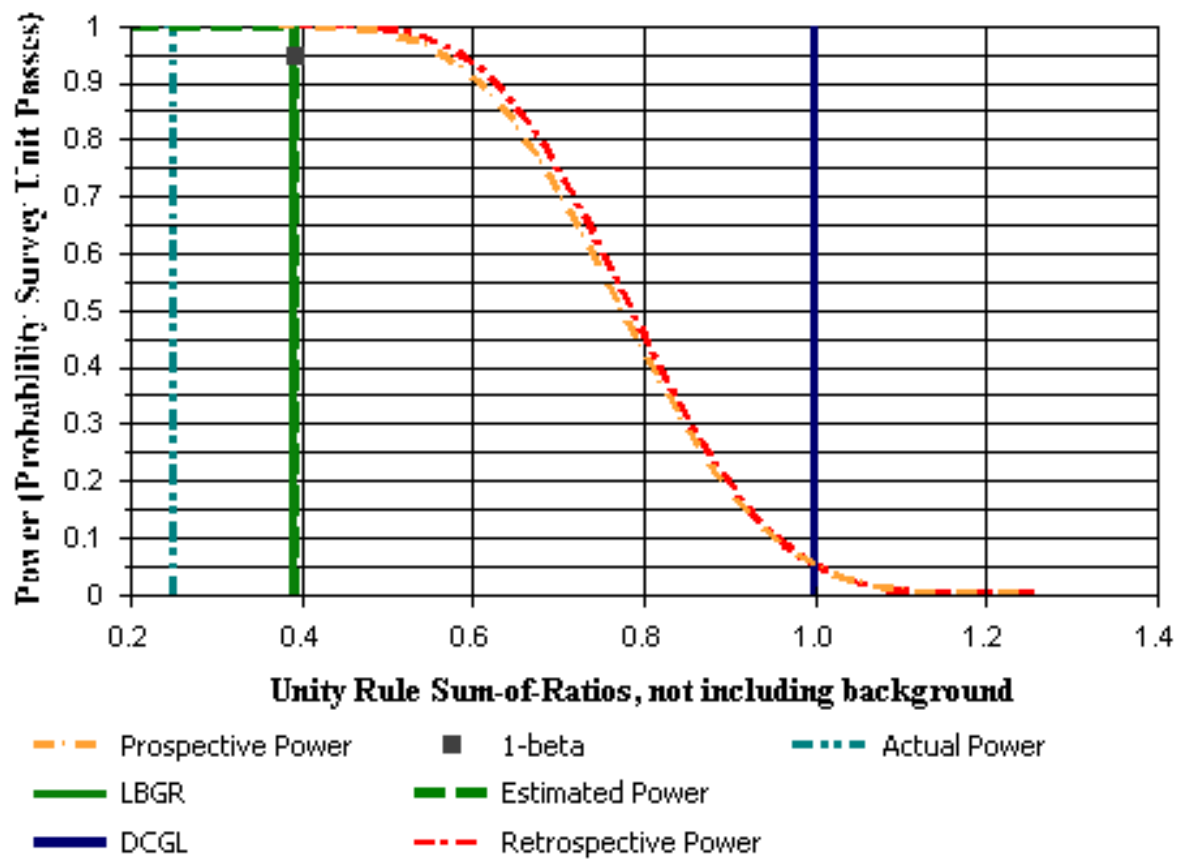




\section{DQA Surface Soil Report}

\section{Survey Unit Data}

NOTE: $\quad$ Type = "S" indicates survey unit sample.

Type = "R" indicates reference area sample.

\begin{tabular}{|c|c|c|c|}
\hline Sample Number & Type & Th-232 (pCi/g) & $\mathrm{U}-238$ (pCi/g) \\
\hline 494 & $\mathrm{~S}$ & 1.19 & 3.15 \\
\hline 495 & $S$ & 1.36 & 2.95 \\
\hline 496 & S & 1.19 & 2.78 \\
\hline 497 & S & 1.43 & 3.14 \\
\hline 498 & S & 0.87 & 1.3 \\
\hline 499 & S & 1.2 & 2.89 \\
\hline 500 & $S$ & 0.8 & 1.8 \\
\hline 501 & $S$ & 1.4 & 3.81 \\
\hline 502 & $S$ & 1.33 & 2.02 \\
\hline 503 & S & 1.14 & 2.59 \\
\hline 504 & S & 1.32 & 3.07 \\
\hline 505 & S & 0.91 & 1.82 \\
\hline 506 & S & 1.13 & 3.02 \\
\hline 507 & $S$ & 1.38 & 3.72 \\
\hline 508 & $S$ & 1.12 & 1.87 \\
\hline 509 & $\mathrm{~S}$ & 1.69 & 2.96 \\
\hline 510 & S & 1.03 & 2.79 \\
\hline 127 & $\mathrm{R}$ & 1.1 & 2.54 \\
\hline 128 & $\mathrm{R}$ & 0.85 & 2.66 \\
\hline 129 & $\mathrm{R}$ & 0.74 & 2.44 \\
\hline 130 & $\mathrm{R}$ & 1.09 & 2.3 \\
\hline 131 & $\mathrm{R}$ & 0.93 & 2.5 \\
\hline 132 & $\mathrm{R}$ & 0.79 & 1.65 \\
\hline 133 & $\mathrm{R}$ & 0.94 & 2.3 \\
\hline 134 & $\mathrm{R}$ & 0.98 & 2.55 \\
\hline 135 & $\mathrm{R}$ & 0.65 & 2.06 \\
\hline 136 & $\mathrm{R}$ & 0.73 & 2.66 \\
\hline 137 & $\mathrm{R}$ & 1.1 & 2.44 \\
\hline 138 & $\mathrm{R}$ & 1.03 & 2.42 \\
\hline 139 & $\mathrm{R}$ & 1.17 & 2.72 \\
\hline 140 & $\mathrm{R}$ & 1.36 & 3.35 \\
\hline 141 & $\mathrm{R}$ & 0.91 & 1.44 \\
\hline 142 & $\mathrm{R}$ & 1.71 & 3.57 \\
\hline 143 & $\mathrm{R}$ & 0.33 & 0.63 \\
\hline
\end{tabular}




\section{Modified Data (Unity Rule SOR)}

NOTE: $\quad$ Type = "S" indicates survey unit sample.

Type $=$ "R" indicates reference area sample.

\begin{tabular}{lcc} 
Sample Number & Type & Sum-of-Ratios (SOR) \\
\hline 494 & $\mathrm{~S}$ & 1.67 \\
495 & $\mathrm{~S}$ & 1.65 \\
496 & $\mathrm{~S}$ & 1.52 \\
497 & $\mathrm{~S}$ & 1.75 \\
498 & $\mathrm{~S}$ & 0.82 \\
499 & $\mathrm{~S}$ & 1.57 \\
500 & $\mathrm{~S}$ & 1 \\
501 & $\mathrm{~S}$ & 2.01 \\
502 & $\mathrm{~S}$ & 1.27 \\
503 & $\mathrm{~S}$ & 1.43 \\
504 & $\mathrm{~S}$ & 1.68 \\
505 & $\mathrm{~S}$ & 1.04 \\
506 & $\mathrm{~S}$ & 1.6 \\
507 & $\mathrm{~S}$ & 1.96 \\
508 & $\mathrm{~S}$ & 1.13 \\
509 & $\mathrm{~S}$ & 1.77 \\
510 & $\mathrm{~S}$ & 1.47 \\
127 & $\mathrm{R}$ & 1.4 \\
128 & $\mathrm{R}$ & 1.36 \\
129 & $\mathrm{R}$ & 1.23 \\
130 & $\mathrm{R}$ & 1.3 \\
131 & $\mathrm{R}$ & 1.32 \\
132 & $\mathrm{R}$ & 0.93 \\
133 & $\mathrm{R}$ & 1.24 \\
134 & $\mathrm{R}$ & 1.36 \\
135 & $\mathrm{R}$ & 1.05 \\
136 & $\mathrm{R}$ & 1.32 \\
137 & $\mathrm{R}$ & 1.36 \\
138 & $\mathrm{R}$ & 1.32 \\
139 & $\mathrm{R}$ & 1.49 \\
140 & $\mathrm{R}$ & 1.81 \\
141 & $\mathrm{R}$ & 0.89 \\
142 & $\mathrm{R}$ & 2.02 \\
143 & $\mathrm{R}$ & 0.37 \\
& &
\end{tabular}




\section{DQA Surface Soil Report}

\section{Basic Statistical Quantities Summary}

\begin{tabular}{lccc} 
Statistic & Survey Unit & Background & DQO Results \\
\hline Sample Number & 17 & 17 & N/2=18 \\
Mean (SOR) & 1.49 & 1.28 & 0.39 \\
Median (SOR) & 1.57 & 1.32 & N/A \\
Std Dev (SOR) & 0.34 & 0.36 & 0.39 \\
High Value (SOR) & 2.01 & 2.02 & N/A \\
Low Value (SOR) & 0.82 & 0.37 & N/A
\end{tabular}

\section{Statistical Test Summary}

$\begin{array}{ll}\text { Sum of Ranks: } & 595 \\ \text { Sum of Reference Ranks: } & 426 \\ \text { Critical Value: } & 345 \\ \text { Result: } & \text { Pass }\end{array}$

$\begin{array}{lll}\text { Data } & \text { Type } & \text { Adjusted Data }\end{array}$

$\begin{array}{llccc}0.37 & \mathrm{R} & 1.37 & 6 & 6 \\ 0.89 & \mathrm{R} & 1.89 & 17 & 17 \\ 0.93 & \mathrm{R} & 1.93 & 18 & 18 \\ 1.05 & \mathrm{R} & 2.05 & 21 & 21 \\ 1.23 & \mathrm{R} & 2.23 & 22 & 22 \\ 1.24 & \mathrm{R} & 2.24 & 23 & 23 \\ 1.3 & \mathrm{R} & 2.3 & 24 & 24 \\ 1.32 & \mathrm{R} & 2.32 & 26 & 26 \\ 1.32 & \mathrm{R} & 2.32 & 26 & 26 \\ 1.32 & \mathrm{R} & 2.32 & 26 & 26 \\ 1.36 & \mathrm{R} & 2.36 & 29 & 29 \\ 1.36 & \mathrm{R} & 2.36 & 29 & 29 \\ 1.36 & \mathrm{R} & 2.36 & 29 & 29 \\ 1.4 & \mathrm{R} & 2.4 & 31 & 31 \\ 1.49 & \mathrm{R} & 2.49 & 32 & 32 \\ 1.81 & \mathrm{R} & 2.81 & 33 & 33 \\ 2.02 & \mathrm{R} & 3.02 & 34 & 34 \\ 0.82 & \mathrm{~S} & 0.82 & 1 & 0 \\ 1 & \mathrm{~S} & 1 & 2 & 0 \\ 1.04 & \mathrm{~S} & 1.04 & 3 & 0 \\ 1.13 & \mathrm{~S} & 1.13 & 4 & 0 \\ 1.27 & \mathrm{~S} & 1.27 & 5 & 7 \\ 1.43 & \mathrm{~S} & 1.43 & 8 & 0 \\ 1.47 & \mathrm{~S} & 1.47 & 9 & 0 \\ 1.52 & \mathrm{~S} & 1.52 & & 0\end{array}$




\section{DQA Surface Soil Report}

\section{Statistical Test Summary}

\begin{tabular}{lcccc} 
Data & Type & Adjusted Data & Rank & Reference Rank \\
\hline 1.57 & & 1.57 & 10 & 0 \\
1.6 & S & 1.6 & 11 & 0 \\
1.65 & S & 1.65 & 12 & 0 \\
1.67 & S & 1.67 & 13 & 0 \\
1.68 & S & 1.68 & 14 & 0 \\
1.75 & S & 1.75 & 15 & 0 \\
1.77 & S & 1.77 & 16 & 0 \\
1.96 & S & 1.96 & 19 & 0 \\
2.01 & S & 2.01 & 20 & 0
\end{tabular}




\section{DQA Building Surface Report}

\section{Assessment Summary}

Site:

Planner(s):

Survey Unit Name:

Report Number:

Survey Unit Measurements:

Reference Area Measurements:

Test Performed:

Judgmental Areas:

Assessment Conclusion:
Hammond Depot FSS Planner

Vitkus

C1 SU2.1 through 5.8 AOC 2 and 3 slag C1 SU2 . 1

1

14

0

Sign

Test Result:

Not Performed

0

EMC Result:

Not Performed

Reject Null Hypothesis (Survey Unit PASSES)

\section{Retrospective Power Curve}

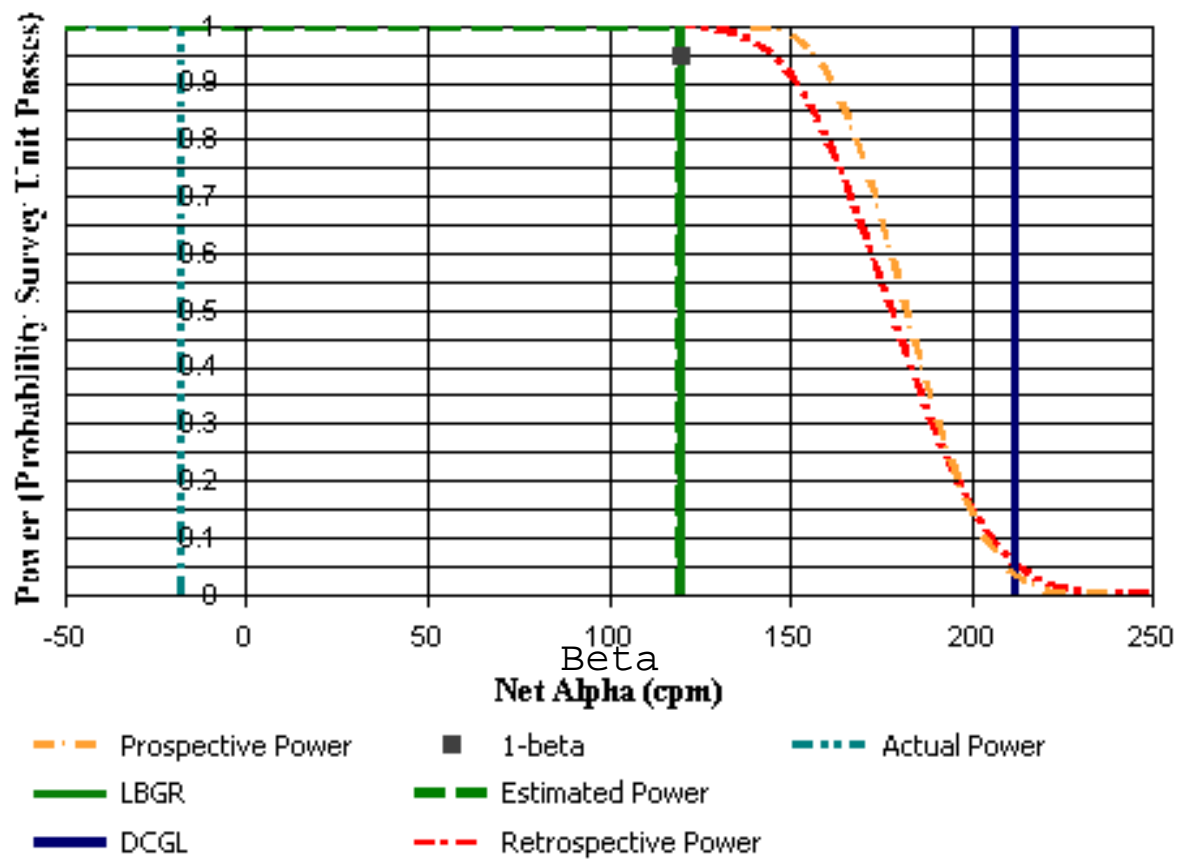




\section{DQA Building Surface Report}

\section{Survey Unit Data}

NOTE: $\quad$ Type = "S" indicates survey measurement.

Type $=$ "R" indicates reference measurement.

\begin{tabular}{lccc} 
Measurement & Material & Type & Gross Alpha (cpm) \\
\hline 4611369,456528 & Slag & S & 479 \\
4611369,456532 & Slag & S & 541 \\
4611369,456536 & Slag & S & 569 \\
4611369,456540 & Slag & S & 481 \\
4611369,456544 & Slag & S & 462 \\
4611372,456527 & Slag & S & 466 \\
4611372,456530 & Slag & S & 498 \\
4611372,456534 & Slag & S & 404 \\
4611372,456538 & Slag & S & 451 \\
4611372,456542 & Slag & S & 408 \\
4611375,456525 & Slag & S & 398 \\
4611375,456528 & Slag & S & 347 \\
4611375,456532 & Slag & $S$ & 405 \\
4611375,456536 & Slag & S & 498
\end{tabular}

\section{Basic Statistical Quantities Summary}

Statistic

Sample Number

Mean $\left(\mathrm{dpm} / 100 \mathrm{~cm}^{2}\right)$

Median (dpm/100 cm²)

Std Dev (dpm/100 $\left.\mathrm{cm}^{2}\right)$

High Value $\left(\mathrm{dpm} / 100 \mathrm{~cm}^{2}\right)$

Low Value $\left(\mathrm{dpm} / 100 \mathrm{~cm}^{2}\right)$
Survey Unit
14
$-29.68$
$-17.67$
114.41
180.74
$-238.76$

Background

DQO Results

$\begin{array}{lc}\text { N/A } & N=15 \\ \text { N/A } & 120 \\ \text { N/A } & N / A \\ \text { N/A } & 48.3 \\ \text { N/A } & \text { N/A } \\ \text { N/A } & \text { N/A }\end{array}$




\section{DQA Building Surface Report}

\section{Assessment Summary}

Site:

Planner(s):

Survey Unit Name:

Report Number:

Survey Unit Measurements:

Reference Area Measurements:

Test Performed:

Judgmental Areas:

Assessment Conclusion:
Hammond Depot FSS Planner

Vitkus

C1 SU2.1 through 5.8 AOC 2 and 3 slag C1 SU2 . 2

2

14

0

Sign

Test Result:

Not Performed

0

EMC Result:

Not Performed

Reject Null Hypothesis (Survey Unit PASSES)

\section{Retrospective Power Curve}

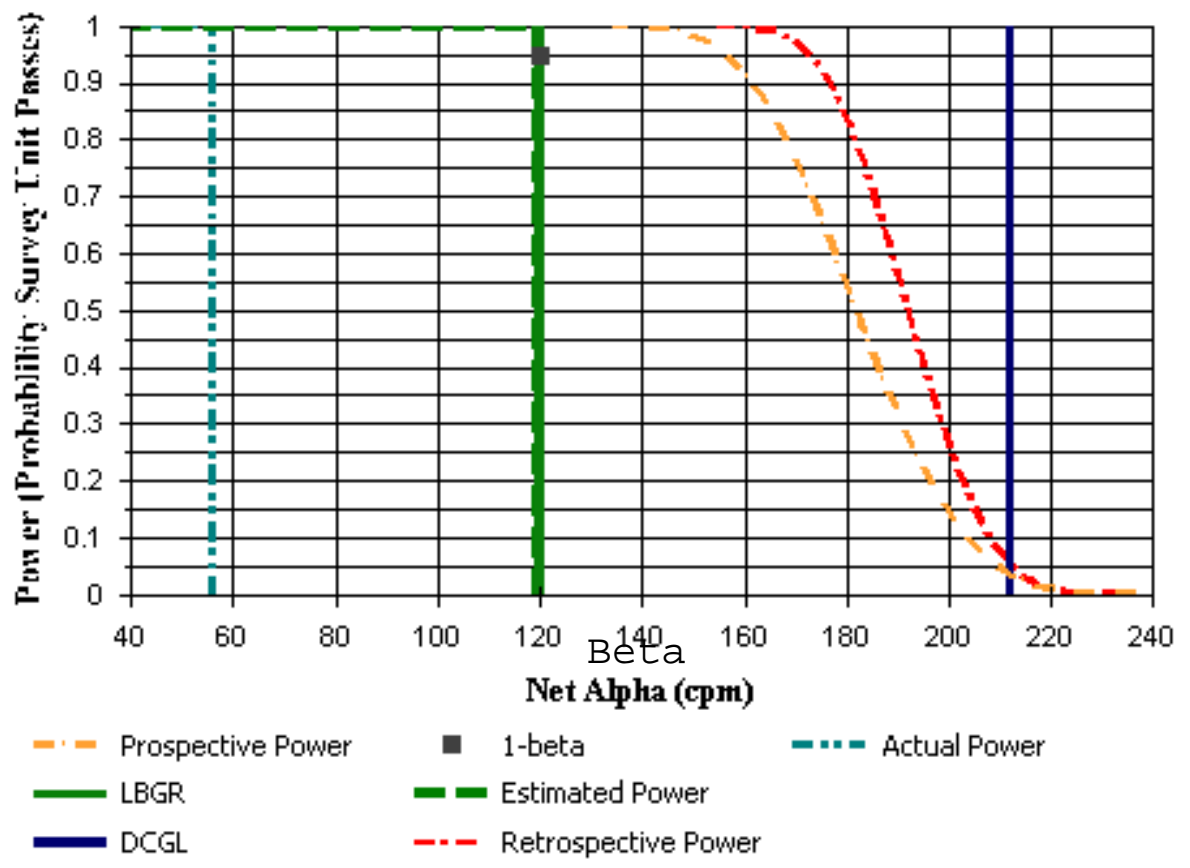




\section{DQA Building Surface Report}

\section{Survey Unit Data}

NOTE: $\quad$ Type $=$ "S" indicates survey measurement.

Type $=$ "R" indicates reference measurement.

\begin{tabular}{lccc} 
Measurement & Material & Type & Gross Alpha (cpm) \\
\hline 4611358,456530 & Slag & S & 525 \\
4611358,456535 & Slag & S & 503 \\
4611358,456539 & Slag & S & 530 \\
4611358,456543 & Slag & S & 552 \\
4611362,456528 & Slag & S & 434 \\
4611362,456533 & Slag & S & 506 \\
4611362,456537 & Slag & S & 487 \\
4611362,456541 & Slag & S & 503 \\
4611362,456545 & Slag & S & 570 \\
4611365,456526 & Slag & S & 459 \\
4611365,456530 & Slag & S & 495 \\
4611365,456535 & Slag & S & 476 \\
4611365,456539 & Slag & $S$ & 485 \\
4611365,456543 & Slag & $S$ & 513
\end{tabular}

\section{Basic Statistical Quantities Summary}

Statistic

Sample Number

Mean $\left(\mathrm{dpm} / 100 \mathrm{~cm}{ }^{2}\right)$

Median (dpm/100 cm²)

Std Dev (dpm/100 $\left.\mathrm{cm}^{2}\right)$

High Value $\left(\mathrm{dpm} / 100 \mathrm{~cm}^{2}\right)$

Low Value $\left(\mathrm{dpm} / 100 \mathrm{~cm}^{2}\right)$
Survey Unit

14
55.49
56.03
66.87
182.63
-74.36

Background

N/A

N/A

N/A

N/A

N/A

N/A
DQO Results

$\mathrm{N}=15$

120

N/A

48.3

N/A

N/A 


\section{DQA Building Surface Report}

\section{Assessment Summary}

Site:

Planner(s):

Survey Unit Name:

Report Number:

Survey Unit Measurements:

Reference Area Measurements:

Test Performed:

Judgmental Areas:

Assessment Conclusion:
Hammond Depot FSS Planner

Vitkus

C1 SU2.1 through 5.8 AOC 2 and 3 slag C1 SU2 . 3

3

14

0

Sign

Test Result:

Not Performed

0

EMC Result:

Not Performed

Reject Null Hypothesis (Survey Unit PASSES)

\section{Retrospective Power Curve}

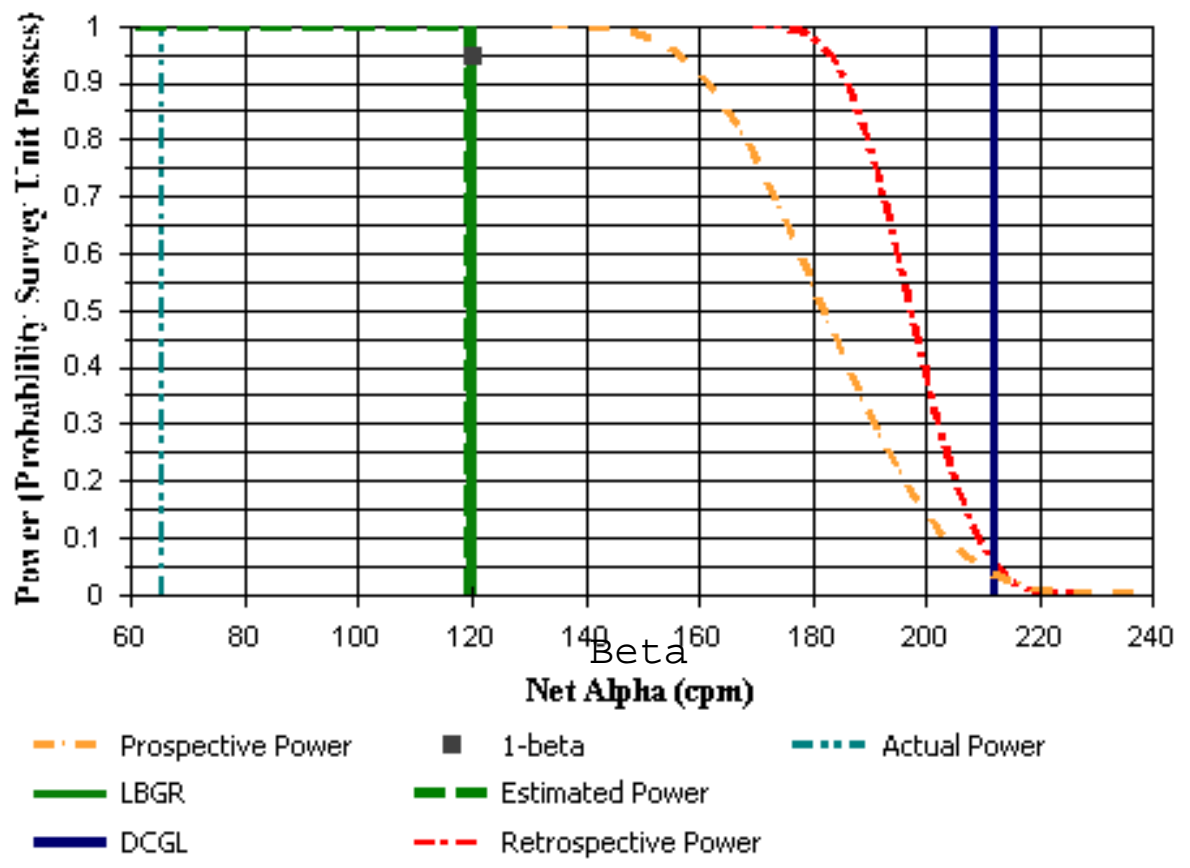




\section{DQA Building Surface Report}

\section{Survey Unit Data}

NOTE: $\quad$ Type $=$ "S" indicates survey measurement.

Type $=$ "R" indicates reference measurement.

\begin{tabular}{lccc} 
Measurement & Material & Type & Gross Alpha (cpm) \\
\hline 4611347,456529 & Slag & S & 539 \\
4611347,456533 & Slag & S & 486 \\
4611347,456537 & Slag & S & 480 \\
4611347,456541 & Slag & S & 478 \\
4611347,456545 & Slag & S & 484 \\
4611350,456531 & Slag & S & 507 \\
4611350,456535 & Slag & S & 534 \\
4611350,456539 & Slag & S & 481 \\
4611350,456543 & Slag & S & 509 \\
4611354,456529 & Slag & S & 505 \\
4611354,456533 & Slag & S & 549 \\
4611354,456537 & Slag & S & 529 \\
4611354,456541 & Slag & $S$ & 518 \\
4611354,456545 & Slag & $S$ & 548
\end{tabular}

\section{Basic Statistical Quantities Summary}

Statistic

Sample Number

Mean $\left(\mathrm{dpm} / 100 \mathrm{~cm}^{2}\right)$

Median (dpm/100 cm²)

Std Dev (dpm/100 $\left.\mathrm{cm}^{2}\right)$

High Value $\left(\mathrm{dpm} / 100 \mathrm{~cm}^{2}\right)$

Low Value $\left(\mathrm{dpm} / 100 \mathrm{~cm}^{2}\right)$
Survey Unit

14
70.20
65.48
49.32
142.95
8.79

Background

N/A

N/A

N/A

N/A

N/A

N/A
DQO Results

$\mathrm{N}=15$

120

N/A

48.3

N/A

N/A 


\section{DQA Building Surface Report}

\section{Assessment Summary}

Site:

Planner(s):

Survey Unit Name:

Report Number:

Survey Unit Measurements:

Reference Area Measurements:

Test Performed:

Judgmental Areas:

Assessment Conclusion:
Hammond Depot FSS Planner

Vitkus

C1 SU2.1 through 5.8 AOC 2 and 3 slag C1 SU2 . 4

4

14

0

Sign

Test Result:

Not Performed

0

EMC Result:

Not Performed

Reject Null Hypothesis (Survey Unit PASSES)

\section{Retrospective Power Curve}

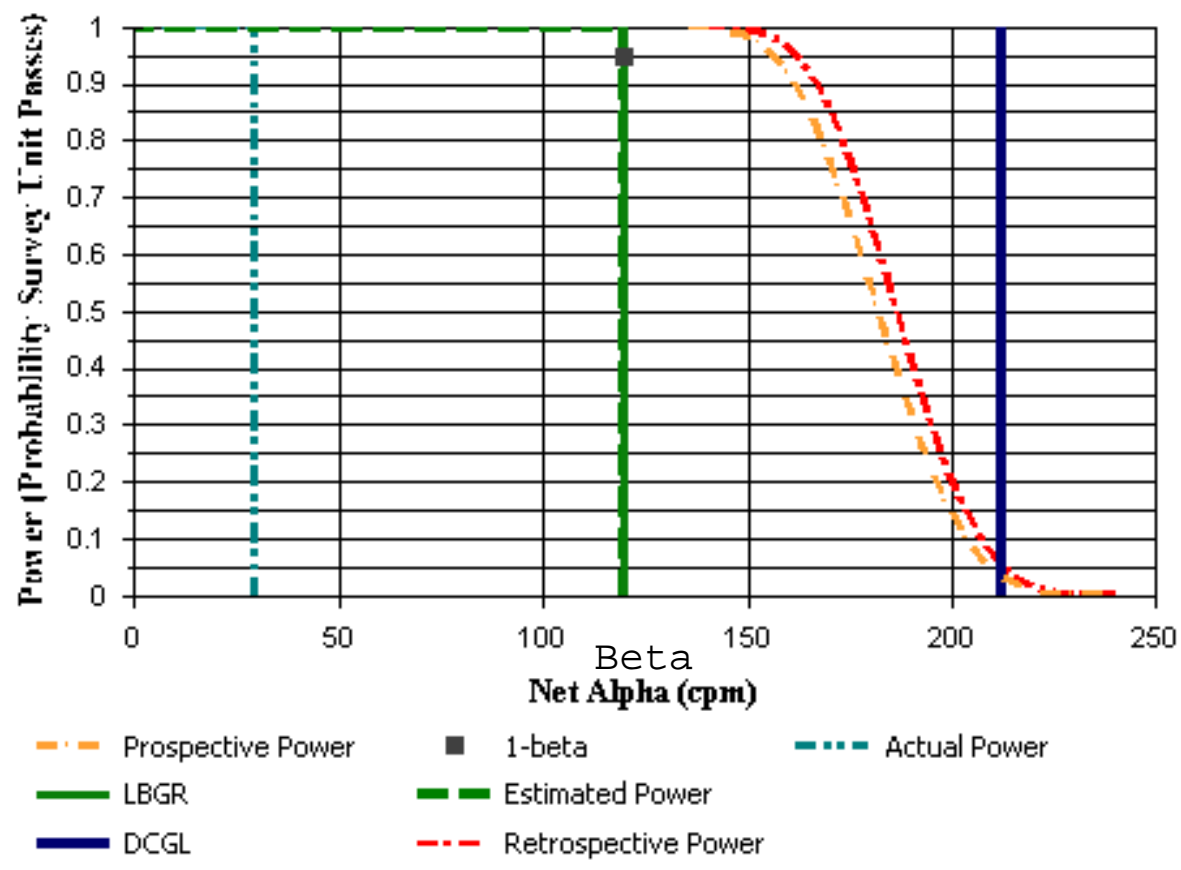




\section{DQA Building Surface Report}

\section{Survey Unit Data}

NOTE: $\quad$ Type = "S" indicates survey measurement.

Type $=$ "R" indicates reference measurement.

\begin{tabular}{lccc} 
Measurement & Material & Type & Gross Alpha (cpm) \\
\hline 4611334,456525 & Slag & S & 498 \\
4611334,456529 & Slag & S & 559 \\
4611334,456532 & Slag & S & 475 \\
4611334,456536 & Slag & S & 431 \\
4611334,456540 & Slag & S & 455 \\
4611334,456544 & Slag & S & 448 \\
4611337,456531 & Slag & S & 589 \\
4611337,456534 & Slag & S & 533 \\
4611337,456538 & Slag & S & 506 \\
4611337,456542 & Slag & S & 480 \\
4611340,456532 & Slag & S & 524 \\
4611340,456536 & Slag & S & 477 \\
4611340,456540 & Slag & S & 457 \\
4611340,456544 & Slag & S & 525
\end{tabular}

\section{Basic Statistical Quantities Summary}

Statistic

Sample Number

Mean $\left(\mathrm{dpm} / 100 \mathrm{~cm}^{2}\right)$

Median (dpm/100 cm²)

Std Dev (dpm/100 $\left.\mathrm{cm}^{2}\right)$

High Value $\left(\mathrm{dpm} / 100 \mathrm{~cm}^{2}\right)$

Low Value $\left(\mathrm{dpm} / 100 \mathrm{~cm}^{2}\right)$
Survey Unit

14
44.56
29.57
85.30
218.54
-80.03

Background

N/A

N/A

N/A

N/A

N/A

N/A
DQO Results

$\mathrm{N}=15$

120

N/A

48.3

N/A

N/A 


\section{DQA Building Surface Report}

\section{Assessment Summary}

Site:

Planner(s):

Survey Unit Name:

Report Number:

Survey Unit Measurements:

Reference Area Measurements:

Test Performed:

Judgmental Areas:

Assessment Conclusion:
Hammond Depot FSS Planner

Vitkus

C1 SU2.1 through 5.8 AOC 2 and 3 slag C1 SU2. 5

18

14

0

Sign

Test Result:

Not Performed

0

EMC Result:

Not Performed

\section{Retrospective Power Curve}

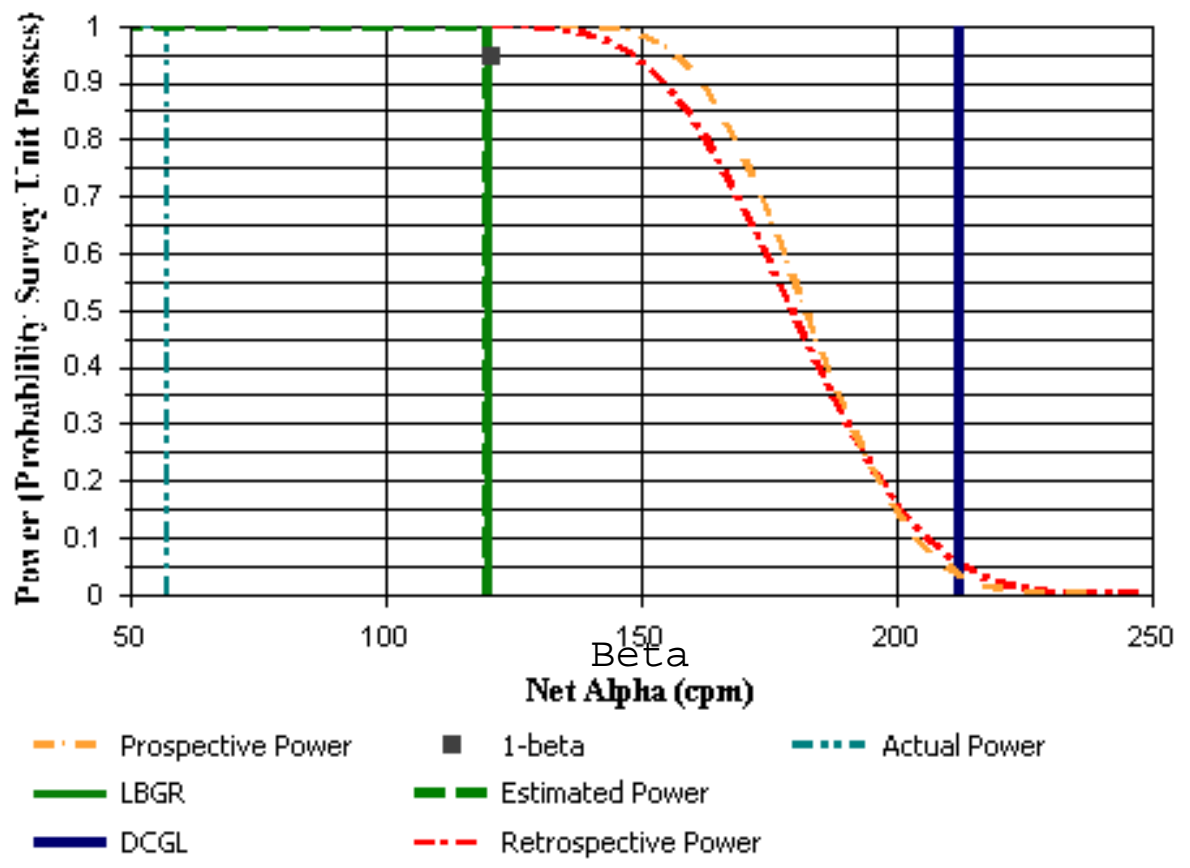




\section{DQA Building Surface Report}

\section{Survey Unit Data}

NOTE: $\quad$ Type = "S" indicates survey measurement.

Type $=$ "R" indicates reference measurement.

\begin{tabular}{lccc} 
Measurement & Material & Type & Gross Alpha (cpm) \\
\hline 4611334,456519 & Slag & S & 658 \\
4611337,456520 & Slag & S & 550 \\
4611339,456519 & Slag & S & 475 \\
4611339,456522 & Slag & S & 541 \\
4611342,456517 & Slag & S & 513 \\
4611342,456520 & Slag & S & 442 \\
4611344,456516 & Slag & S & 447 \\
4611344,456519 & Slag & S & 530 \\
4611347,456514 & Slag & S & 564 \\
4611347,456517 & Slag & S & 482 \\
4611349,456516 & Slag & S & 539 \\
4611352,456514 & Slag & S & 490 \\
4611352,456517 & Slag & $S$ & 450 \\
4611355,456516 & Slag & $S$ & 494
\end{tabular}

\section{Basic Statistical Quantities Summary}

Statistic

Sample Number

Mean $\left(\mathrm{dpm} / 100 \mathrm{~cm}^{2}\right)$

Median (dpm/100 cm²)

Std Dev (dpm/100 $\left.\mathrm{cm}^{2}\right)$

High Value $\left(\mathrm{dpm} / 100 \mathrm{~cm}^{2}\right)$

Low Value $\left(\mathrm{dpm} / 100 \mathrm{~cm}^{2}\right)$
Survey Unit

14
73.98
56.97
109.18
348.92
-59.24

Background

N/A

N/A

N/A

N/A

N/A

N/A
DQO Results

$\mathrm{N}=15$

120

N/A

48.3

N/A

N/A 


\section{DQA Building Surface Report}

\section{Assessment Summary}

Site:

Planner(s):

Survey Unit Name:

Report Number:

Survey Unit Measurements:

Reference Area Measurements:

Test Performed:

Judgmental Areas:

Assessment Conclusion:
Hammond Depot FSS Planner

Vitkus

C1 SU2.1 through 5.8 AOC 2 and 3 slag C1 SU2 . 6

19

14

0

Sign

Test Result:

Not Performed

0

EMC Result:

Not Performed

Reject Null Hypothesis (Survey Unit PASSES)

\section{Retrospective Power Curve}

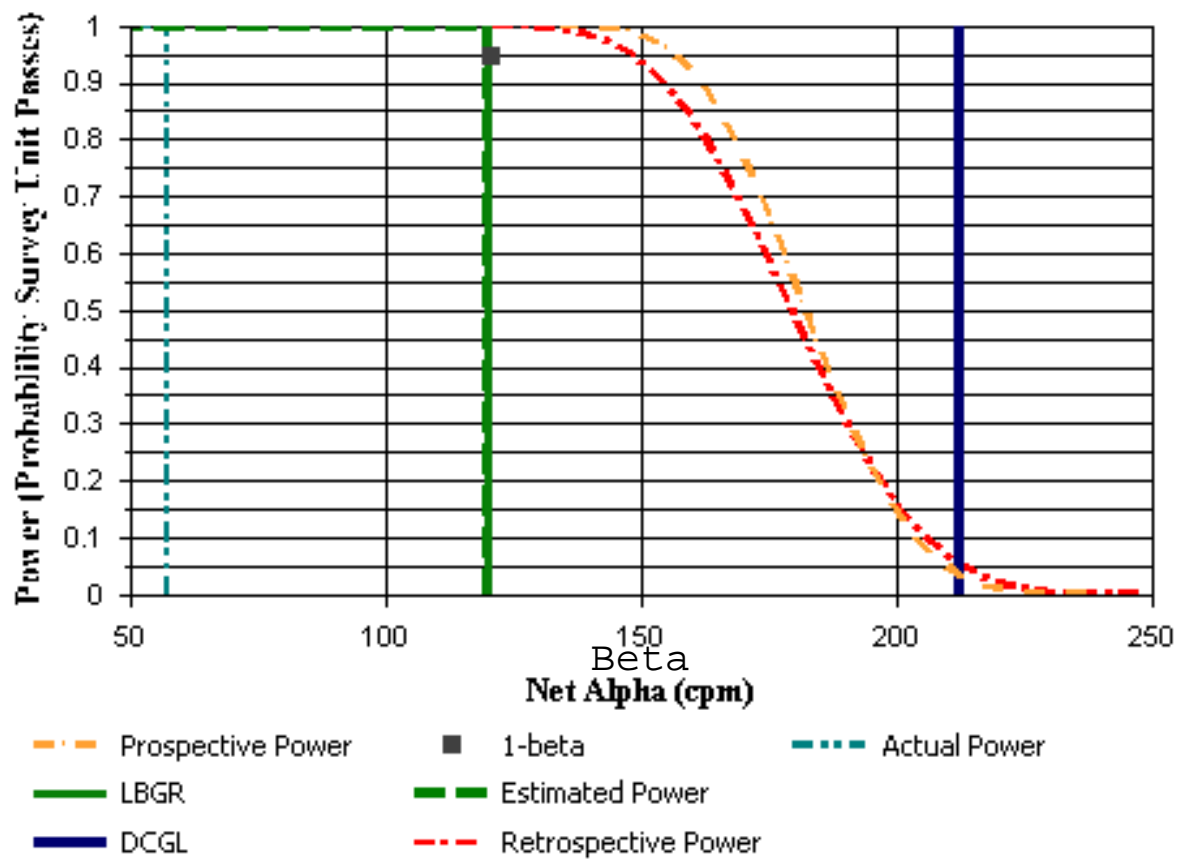




\section{DQA Building Surface Report}

\section{Survey Unit Data}

NOTE: $\quad$ Type = "S" indicates survey measurement.

Type $=$ "R" indicates reference measurement.

\begin{tabular}{lccc} 
Measurement & Material & Type & Gross Alpha (cpm) \\
\hline 4611334,456519 & Slag & S & 658 \\
4611337,456520 & Slag & S & 550 \\
4611339,456519 & Slag & S & 475 \\
4611339,456522 & Slag & S & 541 \\
4611342,456517 & Slag & S & 513 \\
4611342,456520 & Slag & S & 442 \\
4611344,456516 & Slag & S & 447 \\
4611344,456519 & Slag & S & 530 \\
4611347,456514 & Slag & S & 564 \\
4611347,456517 & Slag & S & 482 \\
4611349,456516 & Slag & $S$ & 539 \\
4611352,456514 & Slag & $S$ & 490 \\
4611352,456517 & Slag & $S$ & 450 \\
4611355,456516 & Slag & $S$ & 494
\end{tabular}

\section{Basic Statistical Quantities Summary}

Statistic

Sample Number

Mean $\left(\mathrm{dpm} / 100 \mathrm{~cm}^{2}\right)$

Median (dpm/100 cm²)

Std Dev (dpm/100 $\left.\mathrm{cm}^{2}\right)$

High Value $\left(\mathrm{dpm} / 100 \mathrm{~cm}^{2}\right)$

Low Value $\left(\mathrm{dpm} / 100 \mathrm{~cm}^{2}\right)$
Survey Unit

14
73.98
56.97
109.18
348.92
-59.24

Background

N/A

N/A

N/A

N/A

N/A

N/A
DQO Results

$\mathrm{N}=15$

120

N/A

48.3

N/A

N/A 


\section{DQA Building Surface Report}

\section{Assessment Summary}

Site:

Planner(s):

Survey Unit Name:

Report Number:

Survey Unit Measurements:

Reference Area Measurements:

Test Performed:

Judgmental Areas:

Assessment Conclusion:
Hammond Depot FSS Planner

Vitkus

C1 SU2.1 through 5.8 AOC 2 and 3 slag C1 $\quad$ SU2. 7

20

14

0

Sign

Test Result:

Not Performed

0

EMC Result:

Not Performed

Reject Null Hypothesis (Survey Unit PASSES)

\section{Retrospective Power Curve}

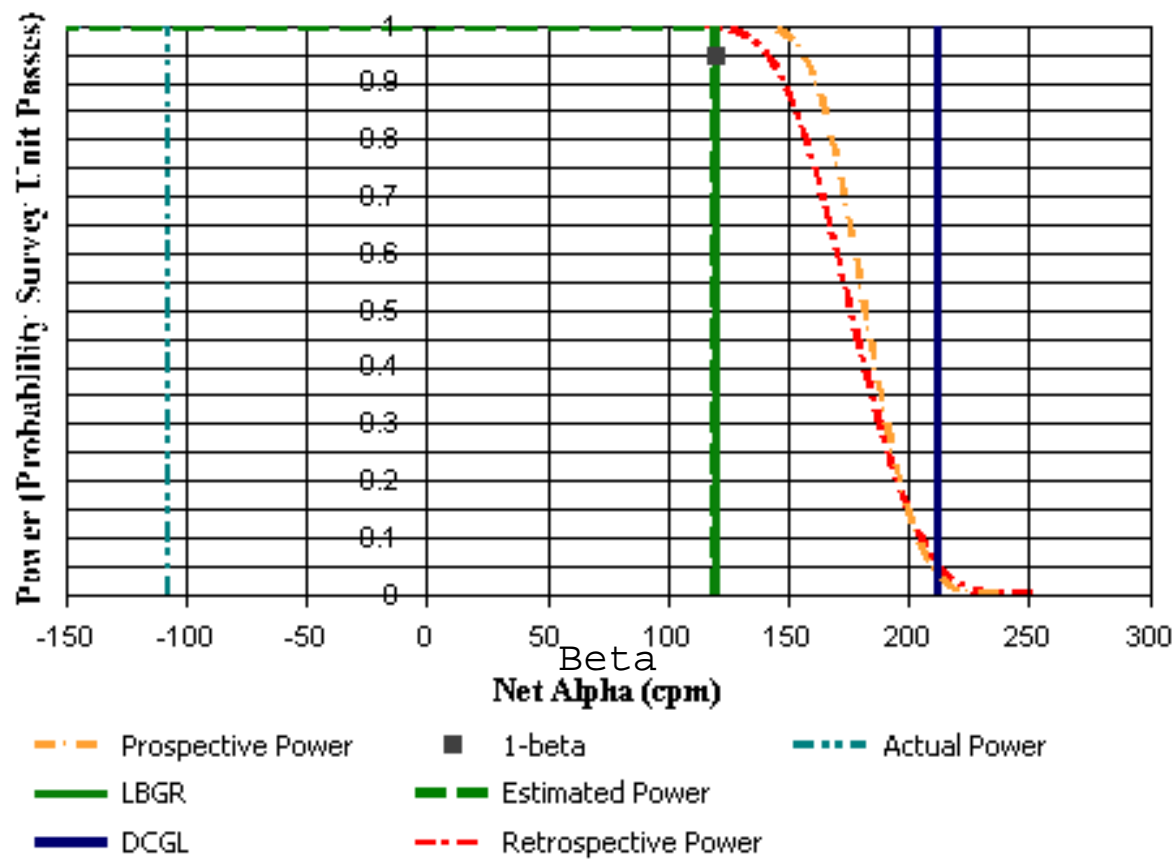




\section{DQA Building Surface Report}

\section{Survey Unit Data}

NOTE: $\quad$ Type = "S" indicates survey measurement.

Type $=$ "R" indicates reference measurement.

\begin{tabular}{lccc} 
Measurement & Material & Type & Gross Alpha (cpm) \\
\hline 4611328,456505 & Slag & S & 409 \\
4611328,456509 & Slag & S & 424 \\
4611331,456503 & Slag & S & 426 \\
4611331,456507 & Slag & S & 543 \\
4611331,456510 & Slag & S & 358 \\
4611331,456514 & Slag & S & 430 \\
4611331,456517 & Slag & S & 445 \\
4611334,456505 & Slag & S & 362 \\
4611334,456509 & Slag & S & 365 \\
4611334,456512 & Slag & S & 390 \\
4611334,456516 & Slag & S & 554 \\
4611337,456510 & Slag & S & 343 \\
4611337,456514 & Slag & $S$ & 408 \\
4611340,456516 & Slag & S & 464
\end{tabular}

\section{Basic Statistical Quantities Summary}

Statistic

Sample Number

Mean $\left(\mathrm{dpm} / 100 \mathrm{~cm}^{2}\right)$

Median (dpm/100 cm²)

Std Dev (dpm/100 $\left.\mathrm{cm}^{2}\right)$

High Value $\left(\mathrm{dpm} / 100 \mathrm{~cm}^{2}\right)$

Low Value $\left(\mathrm{dpm} / 100 \mathrm{~cm}^{2}\right)$
Survey Unit

14
-95.28
-107.43
120.66
152.40
-246.32

Background

N/A

N/A

N/A

N/A

N/A

N/A
DQO Results

$\mathrm{N}=15$

120

N/A

48.3

N/A

N/A 


\section{DQA Surface Soil Report}

\section{Assessment Summary}

Site:

Planner(s):

Survey Unit Name:

Report Number:

Survey Unit Samples:

Reference Area Samples:

Test Performed:

Judgmental Samples:

Assessment Conclusion:
Hammond Depot FSS Planner

Vitkus

C1 SU2, 3, 4, and 5 (AOC 1 and 2) C1 SU3

2

14

17

WRS

Test Result: $\quad$ Pass

0

EMC Result:

Not Performed

Reject Null Hypothesis (Survey Unit PASSES)

\section{Retrospective Power Curve}

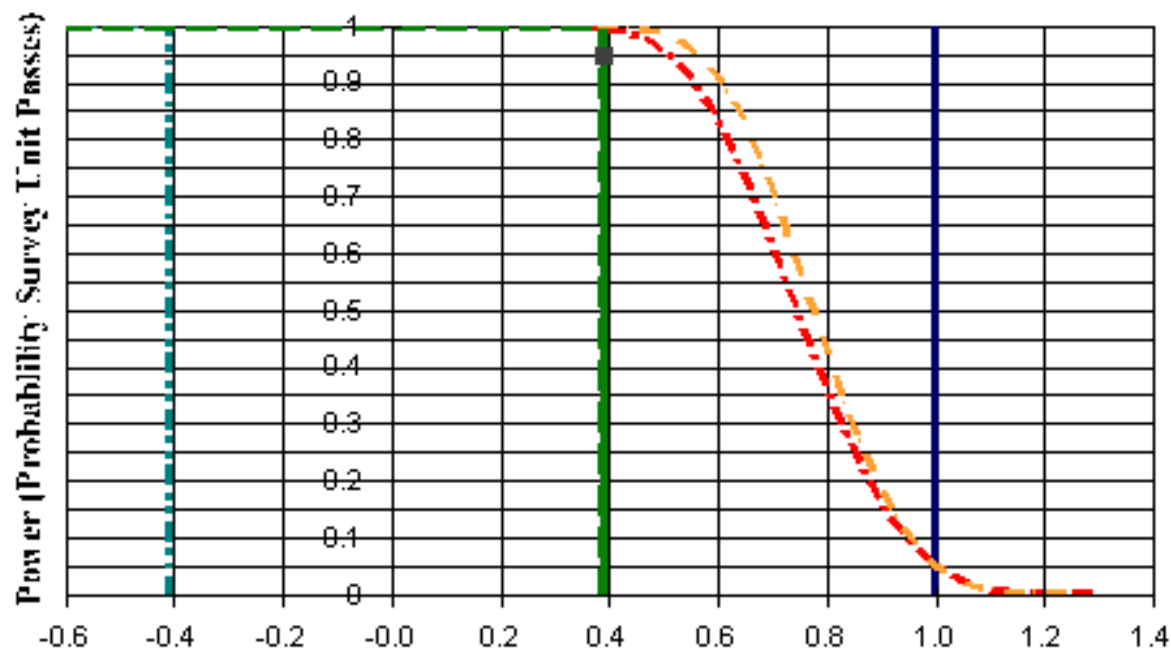

Unity Rule Sum-of-Ratios, not including background

$-\cdot-$ Prospective Power
LBGR
DCGL

- 1-beta

-". Actual Power

- Estimated Power

-- - Retrospective Power 


\section{DQA Surface Soil Report}

\section{Survey Unit Data}

NOTE: $\quad$ Type = "S" indicates survey unit sample.

Type = "R" indicates reference area sample.

\begin{tabular}{|c|c|c|c|}
\hline Sample Number & Type & Th-232 (pCi/g) & $\mathrm{U}-238$ (pCi/g) \\
\hline 512 & $\mathrm{~S}$ & 0.44 & 1.32 \\
\hline 513 & S & 1.34 & 2.94 \\
\hline 514 & S & 0.76 & 1.17 \\
\hline 515 & S & 0.47 & 0.91 \\
\hline 516 & $S$ & 1.34 & 2.38 \\
\hline 517 & $S$ & 0.58 & 1.08 \\
\hline 518 & $S$ & 1.48 & 1.82 \\
\hline 519 & $S$ & 1.05 & 2.74 \\
\hline 520 & S & 0.7 & 1.04 \\
\hline 522 & S & 0.96 & 1.72 \\
\hline 523 & S & 1.62 & 2.25 \\
\hline 524 & $S$ & 0.94 & 1.67 \\
\hline 525 & S & 0.47 & 0.48 \\
\hline 527 & S & 0.85 & 1.35 \\
\hline 127 & $\mathrm{R}$ & 1.1 & 2.54 \\
\hline 128 & $\mathrm{R}$ & 0.85 & 2.66 \\
\hline 129 & $\mathrm{R}$ & 0.74 & 2.44 \\
\hline 130 & $\mathrm{R}$ & 1.09 & 2.3 \\
\hline 131 & $\mathrm{R}$ & 0.93 & 2.5 \\
\hline 132 & $\mathrm{R}$ & 0.79 & 1.65 \\
\hline 133 & $\mathrm{R}$ & 0.94 & 2.3 \\
\hline 134 & $\mathrm{R}$ & 0.98 & 2.55 \\
\hline 135 & $\mathrm{R}$ & 0.65 & 2.06 \\
\hline 136 & $\mathrm{R}$ & 0.73 & 2.66 \\
\hline 137 & $\mathrm{R}$ & 1.1 & 2.44 \\
\hline 138 & $\mathrm{R}$ & 1.03 & 2.42 \\
\hline 139 & $\mathrm{R}$ & 1.17 & 2.72 \\
\hline 140 & $\mathrm{R}$ & 1.36 & 3.35 \\
\hline 141 & $\mathrm{R}$ & 0.91 & 1.44 \\
\hline 142 & $\mathrm{R}$ & 1.71 & 3.57 \\
\hline 143 & $\mathrm{R}$ & 0.33 & 0.63 \\
\hline
\end{tabular}




\section{Modified Data (Unity Rule SOR)}

NOTE: $\quad$ Type = "S" indicates survey unit sample.

Type $=$ "R" indicates reference area sample.

\begin{tabular}{lcc} 
Sample Number & Type & Sum-of-Ratios (SOR) \\
\hline 512 & $\mathrm{~S}$ & 0.68 \\
513 & $\mathrm{~S}$ & 1.64 \\
514 & $\mathrm{~S}$ & 0.73 \\
515 & $\mathrm{~S}$ & 0.53 \\
516 & $\mathrm{~S}$ & 1.41 \\
517 & $\mathrm{~S}$ & 0.63 \\
518 & $\mathrm{~S}$ & 1.24 \\
519 & $\mathrm{~S}$ & 1.46 \\
520 & $\mathrm{~S}$ & 0.66 \\
522 & $\mathrm{~S}$ & 1.02 \\
523 & $\mathrm{~S}$ & 1.46 \\
524 & $\mathrm{~S}$ & 0.99 \\
525 & $\mathrm{~S}$ & 0.35 \\
527 & $\mathrm{~S}$ & 0.83 \\
127 & $\mathrm{R}$ & 1.4 \\
128 & $\mathrm{R}$ & 1.36 \\
129 & $\mathrm{R}$ & 1.23 \\
130 & $\mathrm{R}$ & 1.3 \\
131 & $\mathrm{R}$ & 1.32 \\
132 & $\mathrm{R}$ & 0.93 \\
133 & $\mathrm{R}$ & 1.24 \\
134 & $\mathrm{R}$ & 1.36 \\
135 & $\mathrm{R}$ & 1.05 \\
136 & $\mathrm{R}$ & 1.32 \\
137 & $\mathrm{R}$ & 1.36 \\
138 & $\mathrm{R}$ & 1.32 \\
139 & $\mathrm{R}$ & 1.49 \\
140 & $\mathrm{R}$ & 1.81 \\
141 & $\mathrm{R}$ & 0.89 \\
142 & $\mathrm{R}$ & 2.02 \\
143 & $\mathrm{R}$ & 0.37
\end{tabular}




\section{DQA Surface Soil Report}

\section{Basic Statistical Quantities Summary}

\begin{tabular}{lccc} 
Statistic & Survey Unit & Background & DQO Results \\
\hline Sample Number & 14 & 17 & N/2=18 \\
Mean (SOR) & 0.97 & 1.28 & 0.39 \\
Median (SOR) & 0.91 & 1.32 & N/A \\
Std Dev (SOR) & 0.41 & 0.36 & 0.39 \\
High Value (SOR) & 1.64 & 2.02 & N/A \\
Low Value (SOR) & 0.35 & 0.37 & N/A
\end{tabular}

\section{Statistical Test Summary}

$\begin{array}{ll}\text { Sum of Ranks: } & 496 \\ \text { Sum of Reference Ranks: } & 387 \\ \text { Critical Value: } & 313 \\ \text { Result: } & \text { Pass }\end{array}$

$\begin{array}{lll}\text { Data } & \text { Type } & \text { Adjusted Data }\end{array}$

$\begin{array}{lll}0.37 & \mathrm{R} & 1.37\end{array}$

0.89

0.93

1.05

1.23

1.24

1.3

1.32

1.32

1.32

1.36

1.36

1.36

1.4

1.49

1.81

2.02

0.35

0.53

0.63

0.66

0.68

0.73

0.83

0.99

$R$
$R$
$R$
$R$
$R$
$R$
$R$
$R$
$R$
$R$
$R$
$R$
$R$
$R$
$R$
$R$
$R$
$R$
$S$
$S$
$S$
$S$
$S$
$S$
$S$
$S$




\section{DQA Surface Soil Report}

\section{Statistical Test Summary}

\begin{tabular}{lcccc} 
Data & Type & Adjusted Data & Rank & Reference Rank \\
\hline 1.02 & & 1.02 & 9 & 0 \\
1.24 & S & 1.24 & 10 & 0 \\
1.41 & S & 1.41 & 12 & 0 \\
1.46 & S & 1.46 & 13.5 & 0 \\
1.46 & S & 1.46 & 13.5 & 0 \\
1.64 & S & 1.64 & 15 & 0
\end{tabular}




\section{DQA Building Surface Report}

\section{Assessment Summary}

Site:

Planner(s):

Survey Unit Name:

Report Number:

Survey Unit Measurements:

Reference Area Measurements:

Test Performed:

Judgmental Areas:

Assessment Conclusion:
Hammond Depot FSS Planner

Vitkus

C1 SU2.1 through 5.8 AOC 2 and 3 slag C1 SU3.1

5

14

0

Sign

Test Result:

Not Performed

0

EMC Result:

Not Performed

\section{Reject Null Hypothesis (Survey Unit PASSES)}

\section{Retrospective Power Curve}

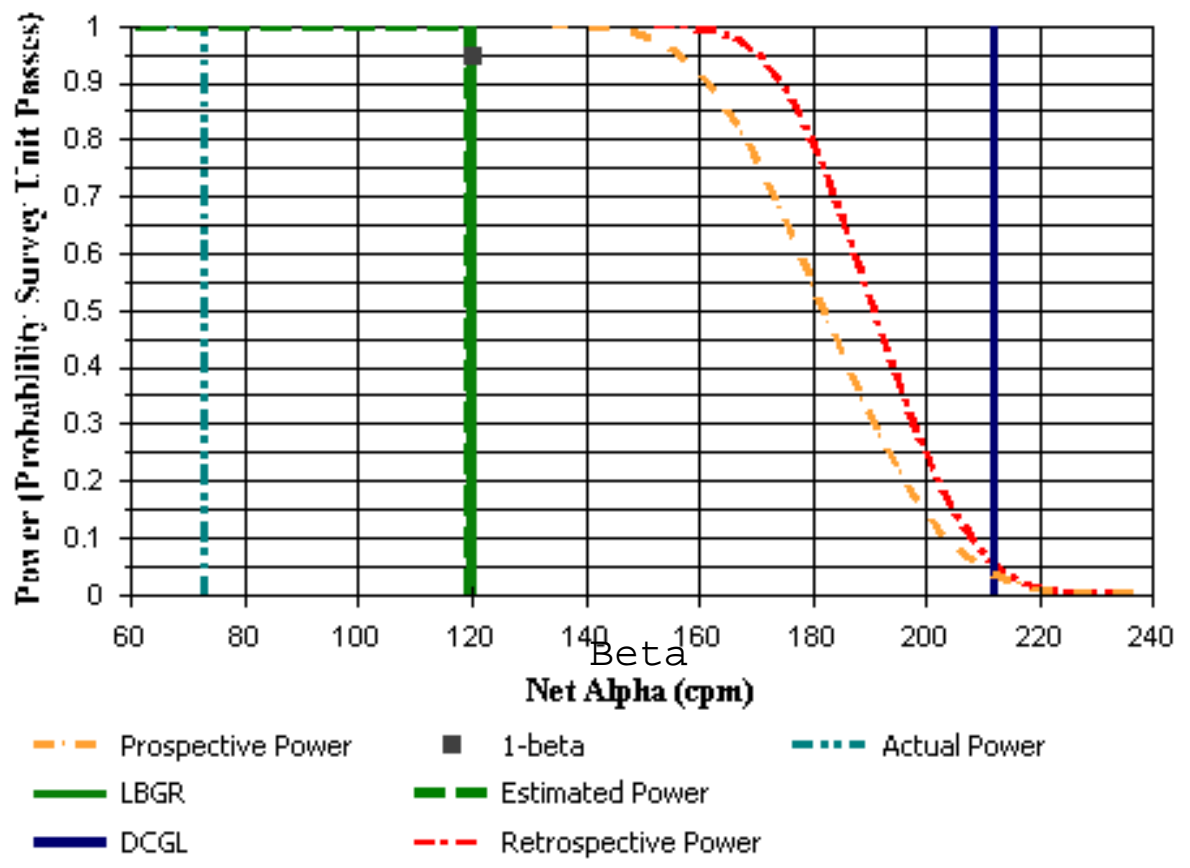




\section{DQA Building Surface Report}

\section{Survey Unit Data}

NOTE: Type $=$ "S" indicates survey measurement.

Type $=$ "R" indicates reference measurement.

\begin{tabular}{lccc} 
Measurement & Material & Type & Gross Alpha (cpm) \\
\hline 4611321,456503 & Slag & S & 450 \\
4611321,456507 & Slag & S & 589 \\
4611321,456511 & Slag & S & 511 \\
4611321,456513 & Slag & S & 565 \\
4611321,456520 & Slag & S & 463 \\
4611324,456505 & Slag & S & 518 \\
4611324,456509 & Slag & S & 511 \\
4611324,456514 & Slag & S & 509 \\
4611324,456518 & Slag & S & 534 \\
4611324,456522 & Slag & S & 539 \\
4611328,456511 & Slag & S & 507 \\
4611328,456516 & Slag & S & 479 \\
4611328,456520 & Slag & $S$ & 549 \\
4611332,456522 & Slag & S & 513
\end{tabular}

\section{Basic Statistical Quantities Summary}

Statistic

Sample Number

Mean $\left(\mathrm{dpm} / 100 \mathrm{~cm}^{2}\right)$

Median (dpm/100 $\left.\mathrm{cm}^{2}\right)$

Std Dev (dpm/100 cm²)

High Value $\left(\mathrm{dpm} / 100 \mathrm{~cm}^{2}\right)$

Low Value $\left(\mathrm{dpm} / 100 \mathrm{~cm}^{2}\right)$
Survey Unit

14
82.35
73.03
71.07
218.54
-44.12

14

$-44.12$
Background

DQO Results

$\begin{array}{ll}\text { N/A } & N=15 \\ \text { N/A } & 120 \\ \text { N/A } & \text { N/A } \\ \text { N/A } & 48.3 \\ \text { N/A } & \text { N/A } \\ \text { N/A } & \text { N/A }\end{array}$




\section{DQA Building Surface Report}

\section{Assessment Summary}

Site:

Planner(s):

Survey Unit Name:

Report Number:

Survey Unit Measurements:

Reference Area Measurements:

Test Performed:

Judgmental Areas:

Assessment Conclusion:
Hammond Depot FSS Planner

Vitkus

C1 SU2.1 through 5.8 AOC 2 and 3 slag C1 SU3 . 2

22

12

0

Sign

Test Result:

Not Performed

0

EMC Result:

Not Performed

Reject Null Hypothesis (Survey Unit PASSES)

\section{Retrospective Power Curve}

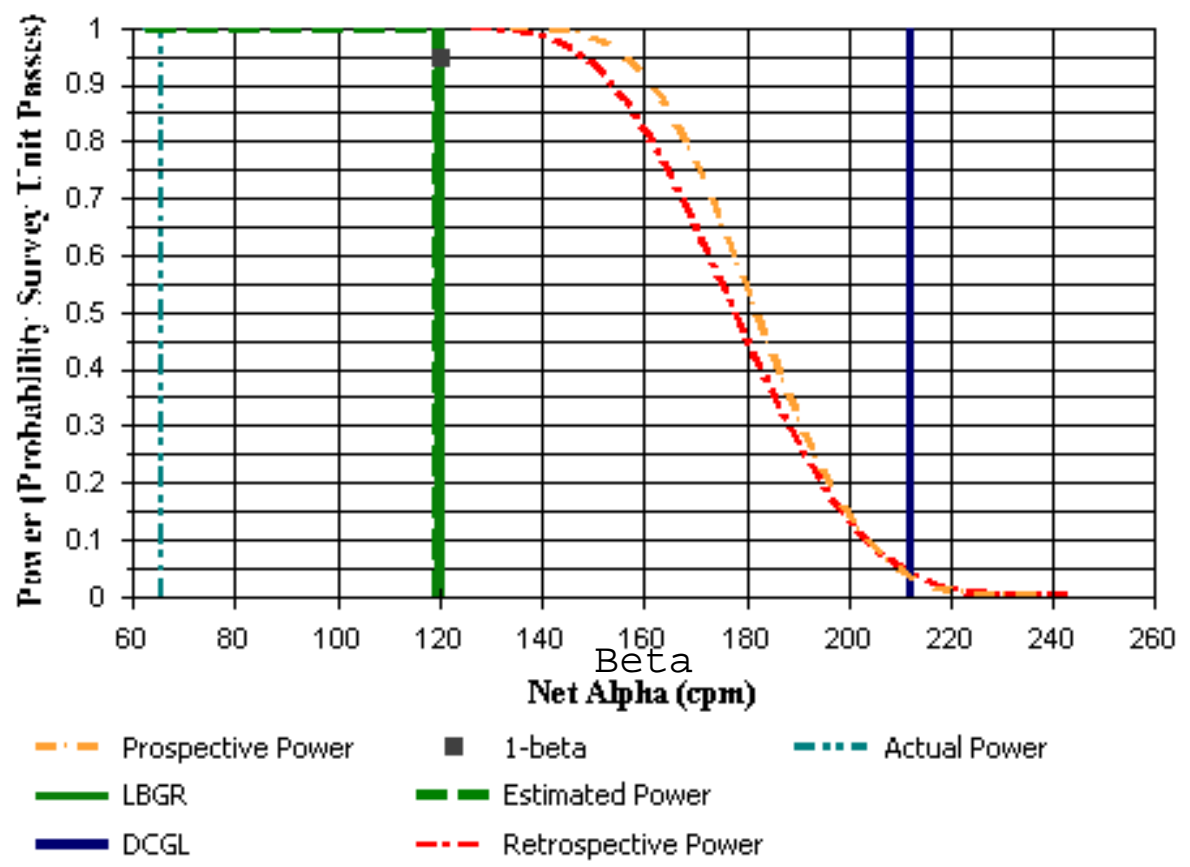




\section{DQA Building Surface Report}

\section{Survey Unit Data}

NOTE: $\quad$ Type $=$ "S" indicates survey measurement.

Type $=$ "R" indicates reference measurement.

\begin{tabular}{lccc} 
Measurement & Material & Type & Gross Alpha (cpm) \\
\hline 4611324,456523 & Slag & S & 500 \\
4611324,456527 & Slag & S & 588 \\
4611324,456531 & Slag & S & 570 \\
4611327,456525 & Slag & S & 610 \\
4611327,456529 & Slag & S & 504 \\
4611327,456533 & Slag & S & 512 \\
4611327,456541 & Slag & S & 490 \\
4611331,456523 & Slag & S & 518 \\
4611331,456527 & Slag & S & 491 \\
4611331,456531 & Slag & S & 540 \\
4611331,456535 & Slag & S & 469 \\
4611331,456539 & Slag & $S$ & 432
\end{tabular}

\section{Basic Statistical Quantities Summary}

Statistic

Sample Number

Mean $\left(\mathrm{dpm} / 100 \mathrm{~cm}^{2}\right)$

Median (dpm/100 $\left.\mathrm{cm}^{2}\right)$

Std Dev (dpm/100 $\left.\mathrm{cm}^{2}\right)$

High Value $\left(\mathrm{dpm} / 100 \mathrm{~cm}^{2}\right)$

Low Value $\left(\mathrm{dpm} / 100 \mathrm{~cm}^{2}\right)$
Survey Unit

12
85.63
65.48
96.04
258.22
-78.14

Background

N/A

N/A

N/A

N/A

N/A

N/A
DQO Results

$\mathrm{N}=15$

120

N/A

48.3

N/A

N/A 


\section{DQA Building Surface Report}

\section{Assessment Summary}

Site:

Planner(s):

Survey Unit Name:

Report Number:

Survey Unit Measurements:

Reference Area Measurements:

Test Performed:

Judgmental Areas:

Assessment Conclusion:
Hammond Depot FSS Planner

Vitkus

C1 SU2.1 through 5.8 AOC 2 and 3 slag C1 SU3. 3

6

14

0

Sign

Test Result:

Not Performed

0

EMC Result:

Not Performed

Reject Null Hypothesis (Survey Unit PASSES)

\section{Retrospective Power Curve}

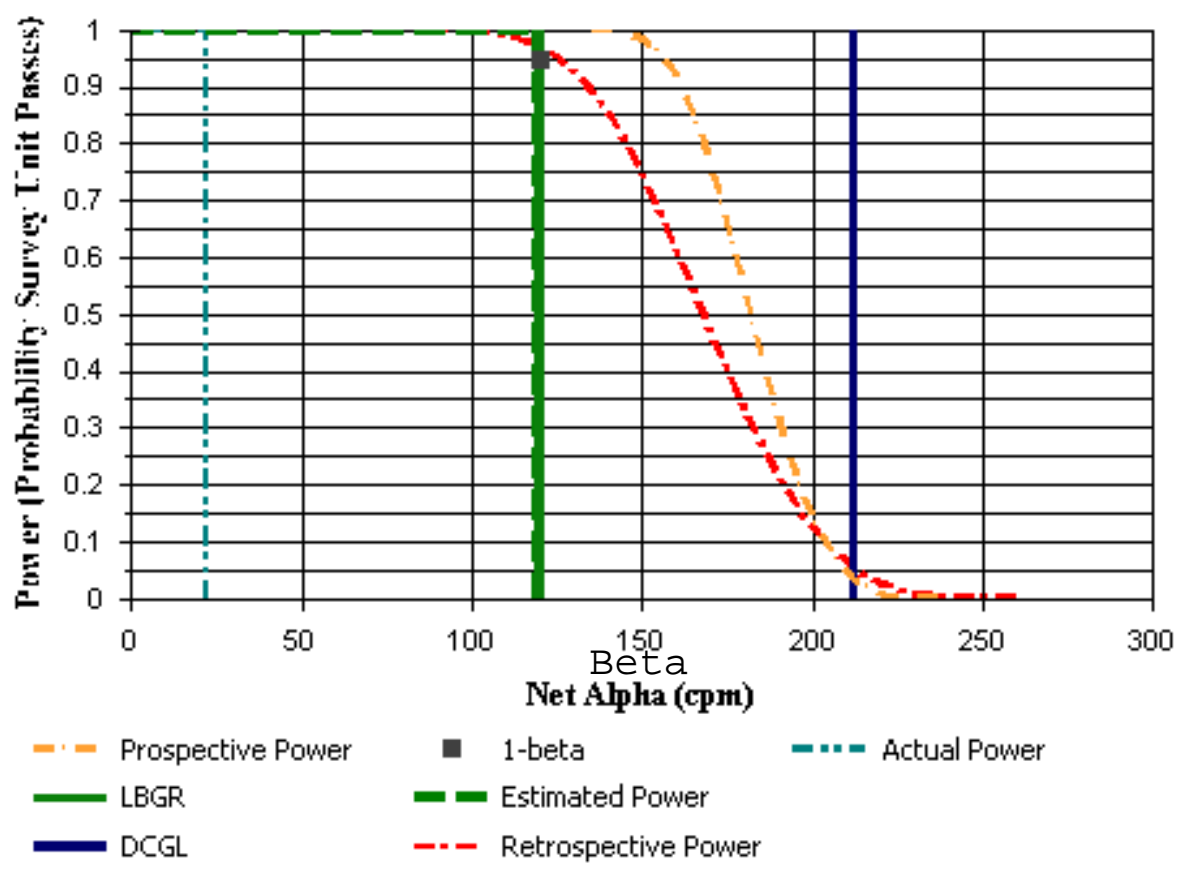




\section{DQA Building Surface Report}

\section{Survey Unit Data}

NOTE: Type = "S" indicates survey measurement.

Type $=$ "R" indicates reference measurement.

\begin{tabular}{lccc} 
Measurement & Material & Type & Gross Alpha (cpm) \\
\hline 4611310,456502 & Slag & S & 585 \\
4611310,456507 & Slag & S & 481 \\
4611310,456512 & Slag & S & 489 \\
4611310,456516 & Slag & S & 385 \\
4611310,456521 & Slag & S & 498 \\
4611314,456504 & Slag & S & 563 \\
4611314,456509 & Slag & S & 393 \\
4611314,456514 & Slag & S & 476 \\
4611314,456519 & Slag & S & 368 \\
4611318,456502 & Slag & S & 633 \\
4611318,456507 & Slag & S & 547 \\
4611318,456512 & Slag & S & 466 \\
4611318,456516 & Slag & S & 447 \\
4611318,456521 & Slag & S & 542
\end{tabular}

\section{Basic Statistical Quantities Summary}

Statistic

Sample Number

Mean $\left(\mathrm{dpm} / 100 \mathrm{~cm}^{2}\right)$

Median (dpm/100 cm²)

Std Dev (dpm/100 cm²)

High Value $\left(\mathrm{dpm} / 100 \mathrm{~cm}^{2}\right)$

Low Value $\left(\mathrm{dpm} / 100 \mathrm{~cm}^{2}\right)$
Survey Unit

14
33.22
22.01
147.15
301.68
199.07

Background

N/A

N/A

N/A

N/A

N/A

N/A
DQO Results

$\mathrm{N}=15$

120

N/A

48.3

N/A

N/A 


\section{DQA Building Surface Report}

\section{Assessment Summary}

Site:

Planner(s):

Survey Unit Name:

Report Number:

Survey Unit Measurements:

Reference Area Measurements:

Test Performed:

Judgmental Areas:

Assessment Conclusion:
Hammond Depot FSS Planner

Vitkus

C1 SU2.1 through 5.8 AOC 2 and 3 slag C1 SU3 . 4

7

11

0

Sign

Test Result:

Not Performed

0

EMC Result:

Not Performed

Reject Null Hypothesis (Survey Unit PASSES)

\section{Retrospective Power Curve}

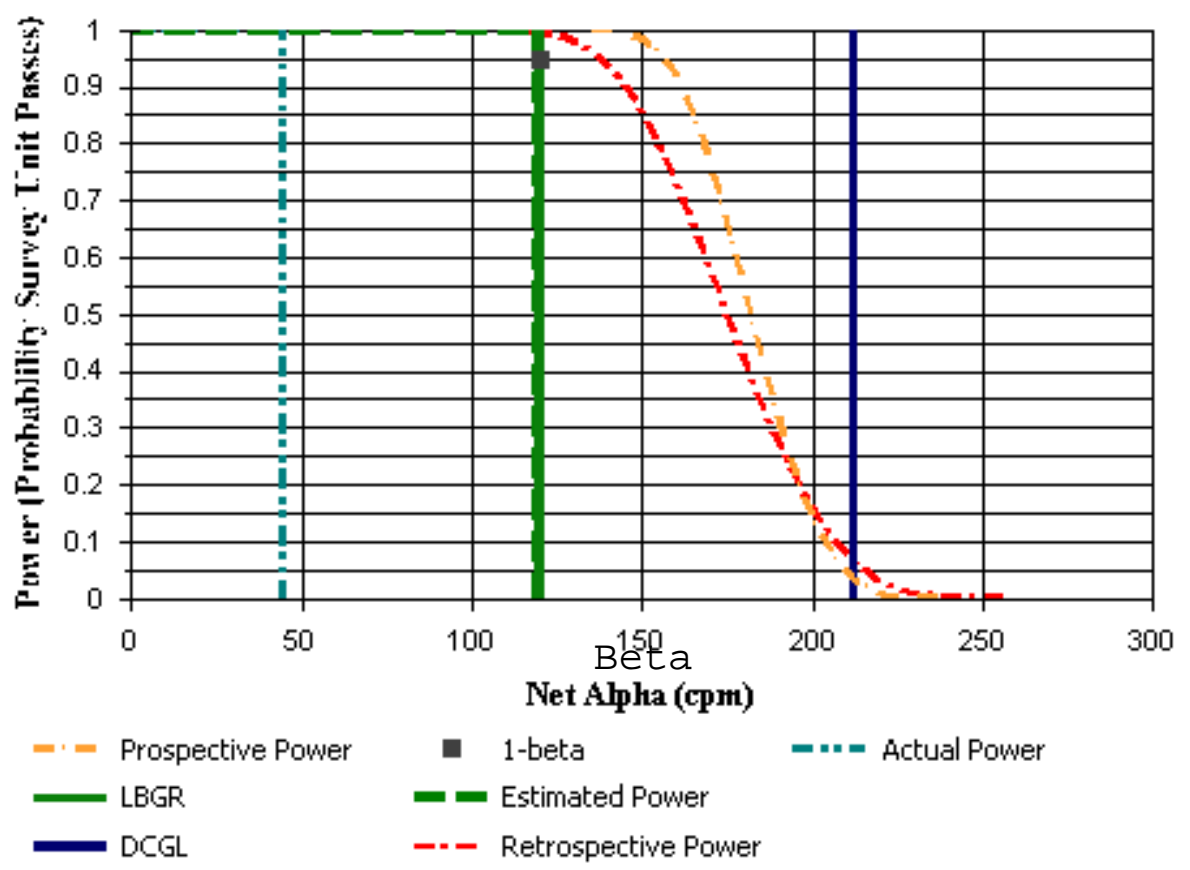




\section{DQA Building Surface Report}

\section{Survey Unit Data}

NOTE: $\quad$ Type = "S" indicates survey measurement.

Type = "R" indicates reference measurement.

\begin{tabular}{lccc} 
Measurement & Material & Type & Gross Alpha (cpm) \\
\hline 4611309,456523 & Slag & S & 440 \\
4611309,456527 & Slag & S & 368 \\
4611313,456532 & Slag & S & 519 \\
4611313,456527 & Slag & S & 497 \\
4611313,456530 & Slag & S & 480 \\
4611313,456534 & Slag & S & 514 \\
4611316,456525 & Slag & S & 390 \\
4611316,456527 & Slag & S & 489 \\
4611316,456532 & Slag & S & 513 \\
4611320,456525 & Slag & S & 541 \\
4611320,456530 & Slag & S & 568
\end{tabular}

\section{Basic Statistical Quantities Summary}

\begin{tabular}{lccc} 
Statistic & Survey Unit & Background & DQO Results \\
\hline Sample Number & 11 & N/A & N=15 \\
Mean $\left(\mathrm{dpm} / 100 \mathrm{~cm}^{2}\right)$ & 19.27 & $\mathrm{~N} / \mathrm{A}$ & 120 \\
Median $\left(\mathrm{dpm} / 100 \mathrm{~cm}^{2}\right)$ & 44.69 & $\mathrm{~N} / \mathrm{A}$ & $\mathrm{N} / \mathrm{A}$ \\
Std Dev $\left(\mathrm{dpm} / 100 \mathrm{~cm}^{2}\right)$ & 116.04 & $\mathrm{~N} / \mathrm{A}$ & 48.3 \\
High Value $\left(\mathrm{dpm} / 100 \mathrm{~cm}^{2}\right)$ & 178.85 & $\mathrm{~N} / \mathrm{A}$ & $\mathrm{N} / \mathrm{A}$ \\
Low Value $\left(\mathrm{dpm} / 100 \mathrm{~cm}^{2}\right)$ & -199.07 & $\mathrm{~N} / \mathrm{A}$ & $\mathrm{N} / \mathrm{A}$
\end{tabular}




\section{DQA Building Surface Report}

\section{Assessment Summary}

Site:

Planner(s):

Survey Unit Name:

Report Number:

Survey Unit Measurements:

Reference Area Measurements:

Test Performed:

Judgmental Areas:

Assessment Conclusion:
Hammond Depot FSS Planner

Vitkus

C1 SU2.1 through 5.8 AOC 2 and 3 slag C1 SU3. 5

8

14

0

Sign

Test Result:

Not Performed

0

EMC Result:

Not Performed

Reject Null Hypothesis (Survey Unit PASSES)

\section{Retrospective Power Curve}

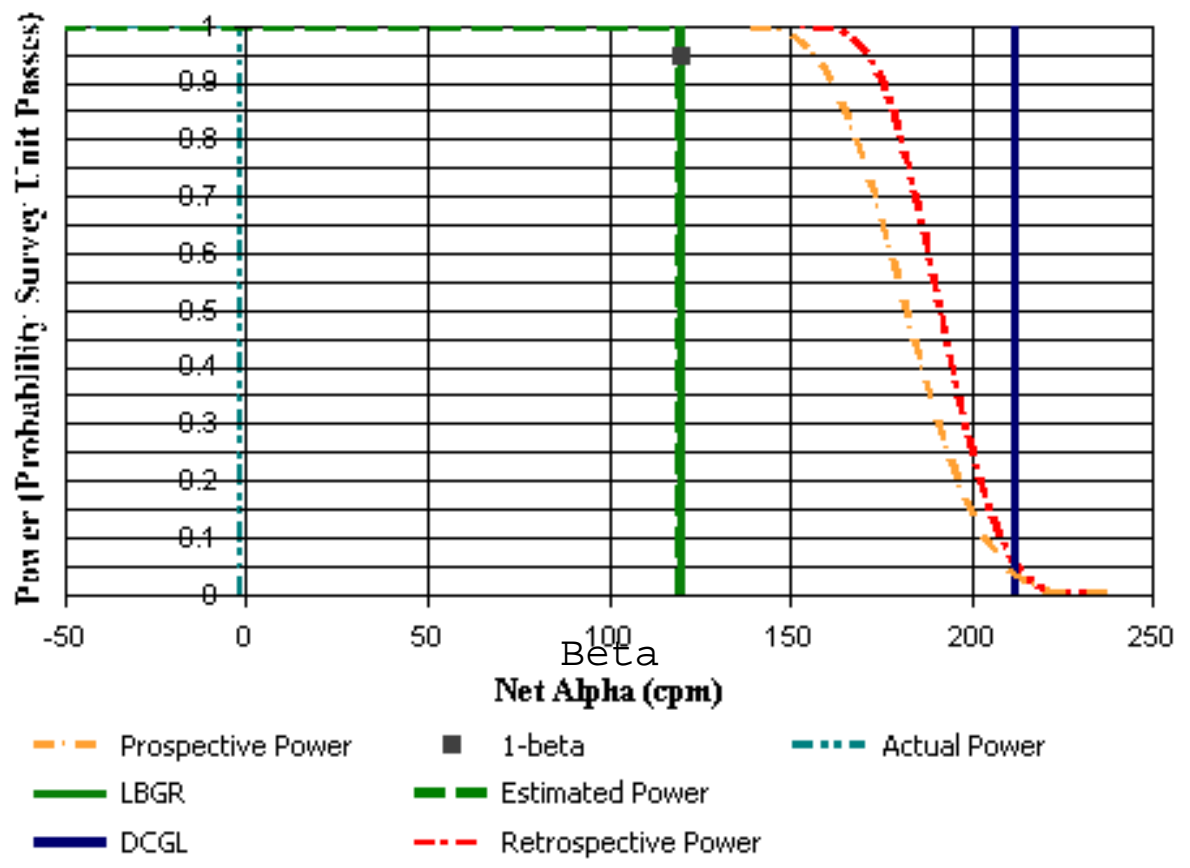




\section{DQA Building Surface Report}

\section{Survey Unit Data}

NOTE: Type $=$ "S" indicates survey measurement.

Type $=$ "R" indicates reference measurement.

\begin{tabular}{lccc} 
Measurement & Material & Type & Gross Alpha (cpm) \\
\hline 4611301,456506 & Slag & S & 479 \\
4611301,456510 & Slag & S & 517 \\
4611301,456514 & Slag & S & 424 \\
4611301,456518 & Slag & S & 409 \\
4611304,456504 & Slag & S & 501 \\
4611304,456508 & Slag & S & 441 \\
4611304,456512 & Slag & S & 458 \\
4611304,456516 & Slag & S & 466 \\
4611304,456520 & Slag & S & 423 \\
4611307,456502 & Slag & S & 523 \\
4611307,456506 & Slag & S & 506 \\
4611307,456510 & Slag & S & 480 \\
4611307,456514 & Slag & S & 464 \\
4611307,456518 & Slag & S & 502
\end{tabular}

\section{Basic Statistical Quantities Summary}

Statistic

Sample Number

Mean $\left(\mathrm{dpm} / 100 \mathrm{~cm}^{2}\right)$

Median (dpm/100 cm²)

Std Dev (dpm/100 cm²)

High Value $\left(\mathrm{dpm} / 100 \mathrm{~cm}^{2}\right)$

Low Value $\left(\mathrm{dpm} / 100 \mathrm{~cm}^{2}\right)$
Survey Unit

14
-4.58
-1.61
69.43
93.82
-121.60

Background

N/A

N/A

N/A

N/A

N/A

N/A

$N / A$
$N / A$
$N / A$
$N / A$
$N / A$
$N / A$

DQO Results

$\mathrm{N}=15$

120

N/A

48.3

N/A

N/A 


\section{DQA Building Surface Report}

\section{Assessment Summary}

Site:

Planner(s):

Survey Unit Name:

Report Number:

Survey Unit Measurements:

Reference Area Measurements:

Test Performed:

Judgmental Areas:

Assessment Conclusion:
Hammond Depot FSS Planner

Vitkus

C1 SU2.1 through 5.8 AOC 2 and 3 slag C1 SU3. 6

9

14

0

Sign

Test Result:

Not Performed

0

EMC Result:

Not Performed

Reject Null Hypothesis (Survey Unit PASSES)

\section{Retrospective Power Curve}

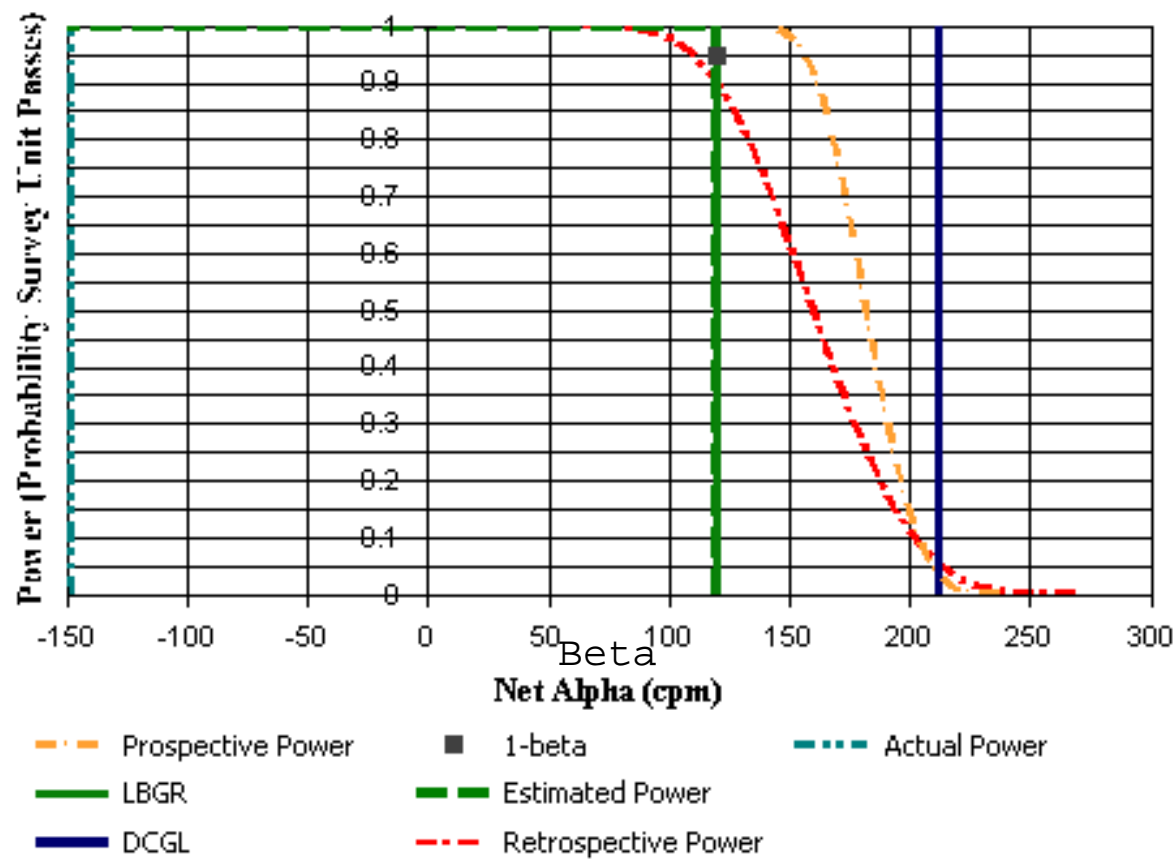




\section{DQA Building Surface Report}

\section{Survey Unit Data}

NOTE: Type = "S" indicates survey measurement.

Type $=$ "R" indicates reference measurement.

\begin{tabular}{lccc} 
Measurement & Material & Type & Gross Alpha (cpm) \\
\hline 4611298,456522 & Slag & S & 375 \\
4611298,456526 & Slag & S & 391 \\
4611298,456531 & Slag & S & 392 \\
4611298,456535 & Slag & S & 495 \\
4611298,456539 & Slag & S & 654 \\
4611301,456524 & Slag & S & 375 \\
4611301,456529 & Slag & S & 398 \\
4611301,456533 & Slag & S & 618 \\
4611301,456537 & Slag & S & 457 \\
4611305,456522 & Slag & S & 373 \\
4611305,456526 & Slag & S & 368 \\
4611305,456531 & Slag & S & 376 \\
4611305,456535 & Slag & S & 421 \\
4611305,456539 & Slag & S & 411
\end{tabular}

\section{Basic Statistical Quantities Summary}

Statistic

Sample Number

Mean $\left(\mathrm{dpm} / 100 \mathrm{~cm}^{2}\right)$

Median (dpm/100 cm²)

Std Dev (dpm/100 cm²)

High Value $\left(\mathrm{dpm} / 100 \mathrm{~cm}^{2}\right)$

Low Value $\left(\mathrm{dpm} / 100 \mathrm{~cm}^{2}\right)$
Survey Unit

14
-70.58
-148.05
174.19
341.36
-199.07

Background

N/A

N/A

N/A

N/A

N/A

N/A

N/A
N/A
N/A
N/A
N/A
N/A

DQO Results

$\mathrm{N}=15$

120

N/A

48.3

N/A

N/A 


\section{DQA Building Surface Report}

\section{Assessment Summary}

Site:

Planner(s):

Survey Unit Name:

Report Number:

Survey Unit Measurements:

Reference Area Measurements:

Test Performed:

Judgmental Areas:

Assessment Conclusion:
Hammond Depot FSS Planner

Vitkus

C1 SU2.1 through 5.8 AOC 2 and 3 slag $\quad$ C1 $\quad$ SU3. 7

21

14

0

Sign

Test Result:

Not Performed

0

EMC Result:

Not Performed

Reject Null Hypothesis (Survey Unit PASSES)

\section{Retrospective Power Curve}

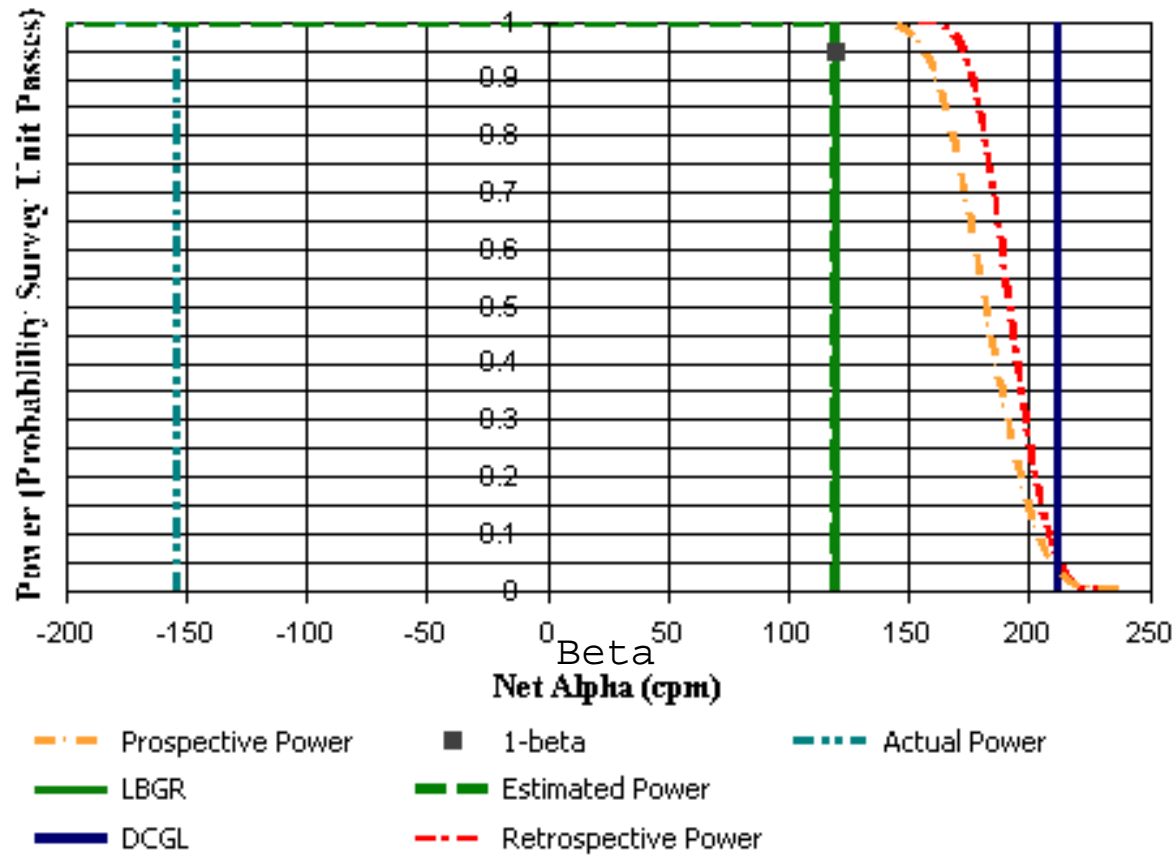




\section{DQA Building Surface Report}

\section{Survey Unit Data}

NOTE: $\quad$ Type = "S" indicates survey measurement.

Type $=$ "R" indicates reference measurement.

\begin{tabular}{lccc} 
Measurement & Material & Type & Gross Alpha (cpm) \\
\hline 4611290,456505 & Slag & S & 376 \\
4611290,456510 & Slag & S & 367 \\
4611290,456514 & Slag & S & 361 \\
4611290,456518 & Slag & S & 417 \\
4611290,456522 & Slag & S & 376 \\
4611294,456507 & Slag & S & 432 \\
4611294,456512 & Slag & S & 385 \\
4611294,456516 & Slag & S & 474 \\
4611294,456520 & Slag & S & 454 \\
4611297,456505 & Slag & S & 420 \\
4611297,456510 & Slag & S & 399 \\
4611297,456514 & Slag & S & 374 \\
4611297,456518 & Slag & $S$ & 359 \\
4611297,456522 & Slag & $S$ & 409
\end{tabular}

\section{Basic Statistical Quantities Summary}

Statistic

Sample Number

Mean $\left(\mathrm{dpm} / 100 \mathrm{~cm}^{2}\right)$

Median (dpm/100 cm²)

Std Dev (dpm/100 $\left.\mathrm{cm}^{2}\right)$

High Value $\left(\mathrm{dpm} / 100 \mathrm{~cm}^{2}\right)$

Low Value $\left(\mathrm{dpm} / 100 \mathrm{~cm}^{2}\right)$
Survey Unit

14
138.20
153.72
67.38
1.23
-216.08

Background

N/A

N/A

N/A

N/A

N/A

N/A
DQO Results

$\mathrm{N}=15$

120

N/A

48.3

N/A

N/A 


\section{DQA Surface Soil Report}

\section{Assessment Summary}

Site:

Planner(s):

Survey Unit Name:

Report Number:

Survey Unit Samples:

Reference Area Samples:

Test Performed:

Judgmental Samples:

Assessment Conclusion:
Hammond Depot FSS Planner

Vitkus

C1 SU2, 3, 4, and 5 (AOC 1 and 2) C1 SU4

3

16

17

WRS

Test Result: $\quad$ Pass

0

EMC Result:

Not Performed

Reject Null Hypothesis (Survey Unit PASSES)

\section{Retrospective Power Curve}

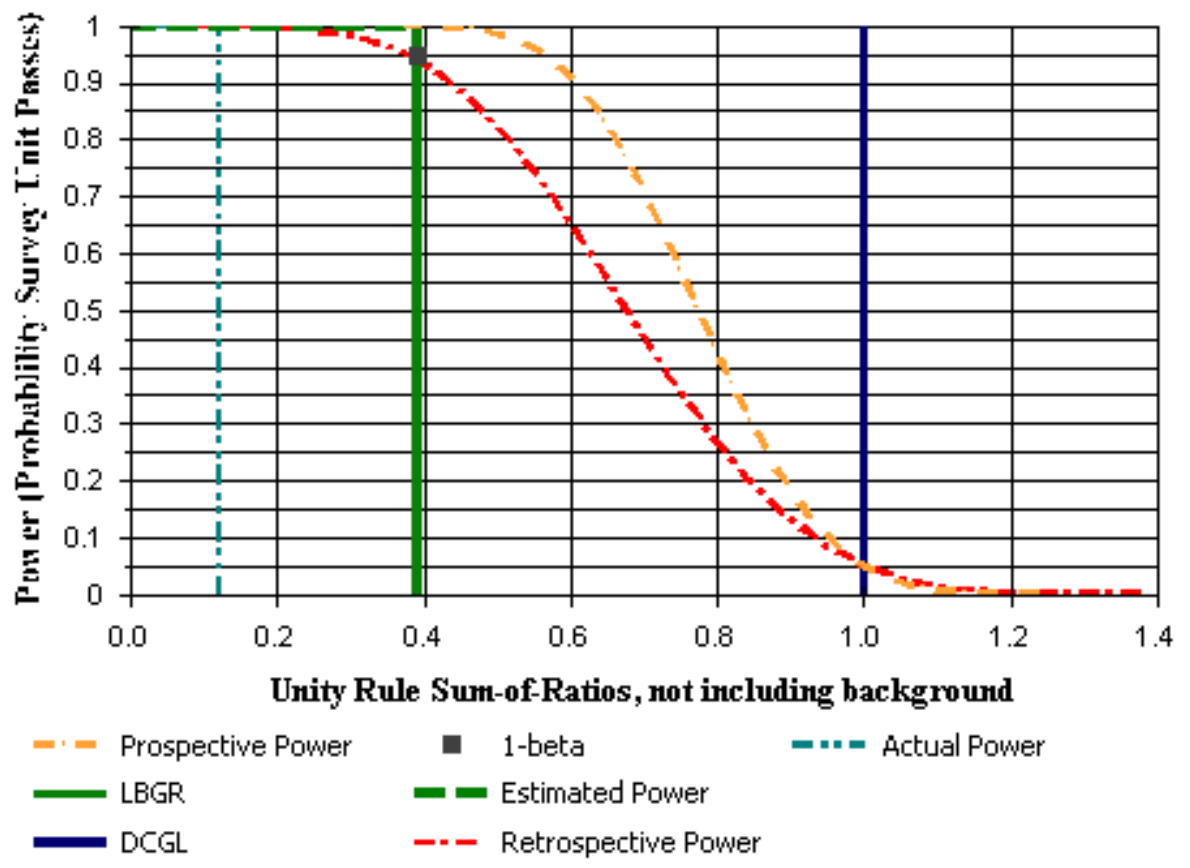




\section{DQA Surface Soil Report}

\section{Survey Unit Data}

NOTE: $\quad$ Type = "S" indicates survey unit sample.

Type = "R" indicates reference area sample.

\begin{tabular}{|c|c|c|c|}
\hline Sample Number & Type & Th-232 (pCi/g) & $\mathrm{U}-238$ (pCi/g) \\
\hline 529 & $\mathrm{~S}$ & 1.88 & 3.33 \\
\hline 530 & $S$ & 1.11 & 1.96 \\
\hline 531 & S & 2.47 & 3.9 ballast \\
\hline 532 & S & 1.08 & 3.36 \\
\hline 533 & S & 1.44 & 3.55 \\
\hline 534 & $S$ & 0.99 & 1.66 \\
\hline 535 & $S$ & 2.05 & 2.29 \\
\hline 536 & $S$ & 0.85 & 1.96 \\
\hline 537 & S & 3.02 & 2.56 \\
\hline 538 & S & 1.97 & 2.1 \\
\hline 539 & S & 0.88 & 1.54 \\
\hline 540 & S & 1.26 & 1.48 \\
\hline 541 & S & 1.45 & 2.27 \\
\hline 542 & S & 0.64 & 1.2 \\
\hline 543 & S & 1.06 & 2.76 \\
\hline 544 & S & 0.41 & 0.81 \\
\hline 127 & $\mathrm{R}$ & 1.1 & 2.54 \\
\hline 128 & $\mathrm{R}$ & 0.85 & 2.66 \\
\hline 129 & $\mathrm{R}$ & 0.74 & 2.44 \\
\hline 130 & $\mathrm{R}$ & 1.09 & 2.3 \\
\hline 131 & $\mathrm{R}$ & 0.93 & 2.5 \\
\hline 132 & $\mathrm{R}$ & 0.79 & 1.65 \\
\hline 133 & $\mathrm{R}$ & 0.94 & 2.3 \\
\hline 134 & $\mathrm{R}$ & 0.98 & 2.55 \\
\hline 135 & $\mathrm{R}$ & 0.65 & 2.06 \\
\hline 136 & $\mathrm{R}$ & 0.73 & 2.66 \\
\hline 137 & $\mathrm{R}$ & 1.1 & 2.44 \\
\hline 138 & $\mathrm{R}$ & 1.03 & 2.42 \\
\hline 139 & $\mathrm{R}$ & 1.17 & 2.72 \\
\hline 140 & $\mathrm{R}$ & 1.36 & 3.35 \\
\hline 141 & $\mathrm{R}$ & 0.91 & 1.44 \\
\hline 142 & $\mathrm{R}$ & 1.71 & 3.57 \\
\hline 143 & $\mathrm{R}$ & 0.33 & 0.63 \\
\hline
\end{tabular}




\section{Modified Data (Unity Rule SOR)}

NOTE: $\quad$ Type = "S" indicates survey unit sample.

Type $=$ "R" indicates reference area sample.

\begin{tabular}{|c|c|c|}
\hline Sample Number & Type & Sum-of-Ratios (SOR) \\
\hline 529 & $\mathrm{~S}$ & 1.98 \\
\hline 530 & $\mathrm{~S}$ & 1.17 \\
\hline 531 & $S$ & 2.41 \\
\hline 532 & S & 1.72 \\
\hline 533 & S & 1.92 \\
\hline 534 & $S$ & 1.01 \\
\hline 535 & S & 1.62 \\
\hline 536 & S & 1.08 \\
\hline 537 & $\mathrm{~S}$ & 2.07 \\
\hline 538 & $S$ & 1.52 \\
\hline 539 & $S$ & 0.92 \\
\hline 540 & S & 1.03 \\
\hline 541 & S & 1.41 \\
\hline 542 & $S$ & 0.7 \\
\hline 543 & $S$ & 1.47 \\
\hline 544 & S & 0.47 \\
\hline 127 & $\mathrm{R}$ & 1.4 \\
\hline 128 & $\mathrm{R}$ & 1.36 \\
\hline 129 & $\mathrm{R}$ & 1.23 \\
\hline 130 & $\mathrm{R}$ & 1.3 \\
\hline 131 & $\mathrm{R}$ & 1.32 \\
\hline 132 & $\mathrm{R}$ & 0.93 \\
\hline 133 & $\mathrm{R}$ & 1.24 \\
\hline 134 & $\mathrm{R}$ & 1.36 \\
\hline 135 & $\mathrm{R}$ & 1.05 \\
\hline 136 & $\mathrm{R}$ & 1.32 \\
\hline 137 & $\mathrm{R}$ & 1.36 \\
\hline 138 & $\mathrm{R}$ & 1.32 \\
\hline 139 & $\mathrm{R}$ & 1.49 \\
\hline 140 & $\mathrm{R}$ & 1.81 \\
\hline 141 & $\mathrm{R}$ & 0.89 \\
\hline 142 & $\mathrm{R}$ & 2.02 \\
\hline 143 & $\mathrm{R}$ & 0.37 \\
\hline
\end{tabular}




\section{DQA Surface Soil Report}

\section{Basic Statistical Quantities Summary}

\begin{tabular}{lccc} 
Statistic & Survey Unit & Background & DQO Results \\
\hline Sample Number & 16 & 17 & N/2=18 \\
Mean (SOR) & 1.41 & 1.28 & 0.39 \\
Median (SOR) & 1.44 & 1.32 & N/A \\
Std Dev (SOR) & 0.53 & 0.36 & 0.39 \\
High Value (SOR) & 2.41 & 2.02 & N/A \\
Low Value (SOR) & 0.47 & 0.37 & N/A
\end{tabular}

\section{Statistical Test Summary}

$\begin{array}{ll}\text { Sum of Ranks: } & 561 \\ \text { Sum of Reference Ranks: } & 397 \\ \text { Critical Value: } & 335 \\ \text { Result: } & \text { Pass }\end{array}$

Data $\quad$ Type $\quad$ Adjusted Data $\quad$ Rank

0.37

0.89

0.93

1.05

1.23

1.24

1.3

1.32

1.32

1.32

1.36

1.36

1.36

1.4

1.49

1.81

2.02

0.47

0.7

0.92

1.01

1.03

1.08

1.17

1.41
561

397

35

Pass

$\begin{array}{ll}\mathrm{R} & 1.37\end{array}$

1.89

1.93

2.05

2.23

2.24

2.3

2.32

2.32

2.32

2.36

2.36

2.36

2.4

2.49

2.81

3.02

0.47

0.7

0.92

1.01

1.03

1.08

1.17

1.41
Rank

Reference Rank

$\begin{array}{cc}8 & 8 \\ 14 & 14 \\ 16 & 16 \\ 18 & 18 \\ 20 & 20 \\ 21 & 21 \\ 22 & 22 \\ 24 & 24 \\ 24 & 24 \\ 24 & 24 \\ 27 & 27 \\ 27 & 27 \\ 27 & 27 \\ 29 & 29 \\ 31 & 31 \\ 32 & 32 \\ 33 & 33 \\ 1 & 0 \\ 2 & 0 \\ 3 & 0 \\ 4 & 0 \\ 5 & 0 \\ 6 & 0 \\ 7 & 0 \\ 9 & 0\end{array}$




\section{DQA Surface Soil Report}

\section{Statistical Test Summary}

\begin{tabular}{lcccc} 
Data & Type & Adjusted Data & Rank & Reference Rank \\
\hline 1.47 & S & 1.47 & 10 & 0 \\
1.52 & S & 1.52 & 11 & 0 \\
1.62 & S & 1.62 & 12 & 0 \\
1.72 & S & 1.72 & 13 & 0 \\
1.92 & S & 1.92 & 15 & 0 \\
1.98 & S & 1.98 & 17 & 0 \\
2.07 & S & 2.07 & 19 & 0 \\
2.41 & S & 2.41 & 30 & 0
\end{tabular}




\section{DQA Surface Soil Report}

\section{Assessment Summary}

Site:

Planner(s):

Survey Unit Name:

Report Number:

Survey Unit Samples:

Reference Area Samples:

Test Performed:

Judgmental Samples:

Assessment Conclusion:
Hammond Depot FSS Planner

Vitkus

C1 SU2, 3, 4, and 5 (AOC 1 and 2) C1 SU5

4

17

17

WRS

Test Result: $\quad$ Pass

0

EMC Result: $\quad$ Not Performed

Reject Null Hypothesis (Survey Unit PASSES)

\section{Retrospective Power Curve}

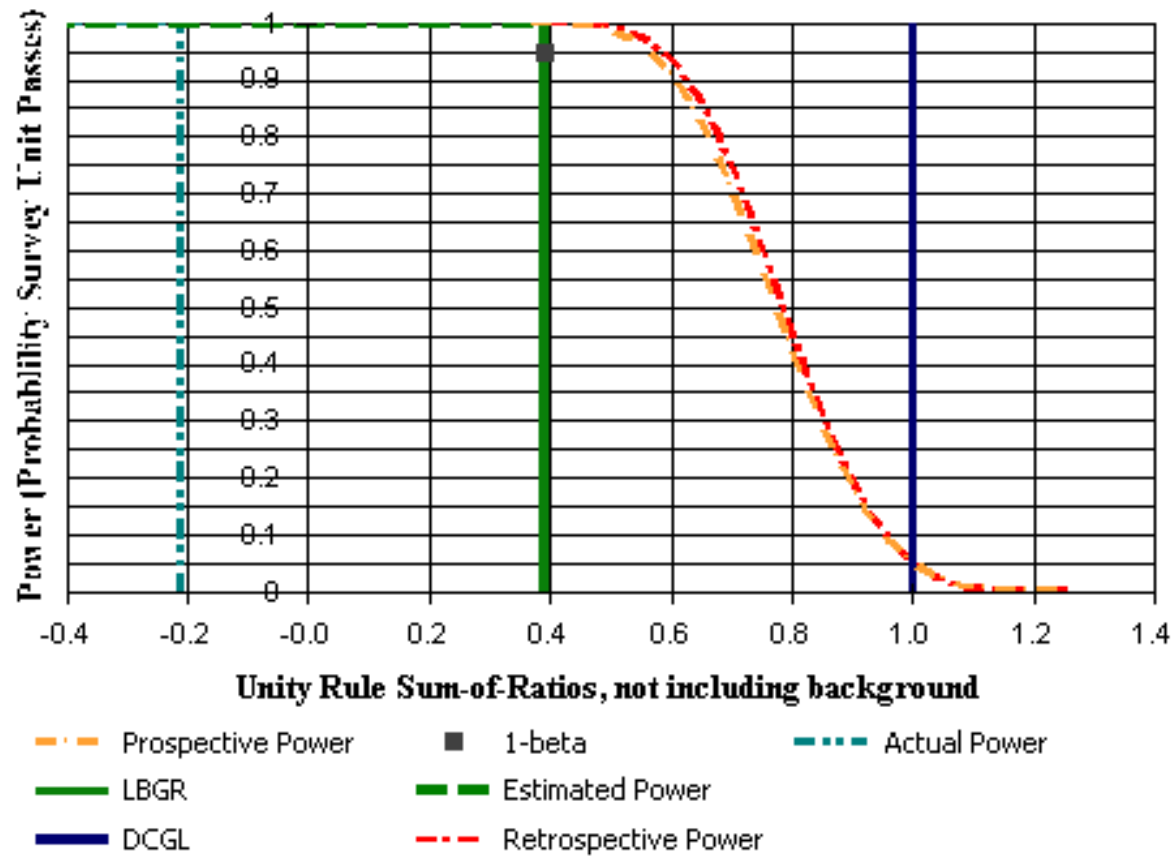




\section{DQA Surface Soil Report}

\section{Survey Unit Data}

NOTE: $\quad$ Type = "S" indicates survey unit sample.

Type = "R" indicates reference area sample.

\begin{tabular}{|c|c|c|c|}
\hline Sample Number & Type & Th-232 (pCi/g) & U-238 (pCi/g) \\
\hline$\overline{545}$ & $\mathrm{~S}$ & 1.06 & 2.04 \\
\hline 546 & S & 1 & 1.56 \\
\hline 547 & S & 1.06 & 1.87 \\
\hline 548 & S & 1.24 & 1.46 \\
\hline 549 & $\mathrm{~S}$ & 1.01 & 1.09 \\
\hline 550 & S & 1.42 & 2.06 \\
\hline 551 & S & 1.12 & 1.11 \\
\hline 552 & $S$ & 1.28 & 1.48 \\
\hline 553 & $S$ & 1.42 & 1.98 \\
\hline 554 & $S$ & 1.32 & 1.61 \\
\hline 555 & S & 1.48 & 1.51 \\
\hline 556 & S & 1.22 & 1.6 \\
\hline 557 & S & 1.47 & 2.21 \\
\hline 558 & S & 1.4 & 1.58 \\
\hline 559 & $S$ & 1.13 & 1.54 \\
\hline 560 & $\mathrm{~S}$ & 1.68 & 1.85 \\
\hline 561 & S & 1.52 & 1.65 \\
\hline 127 & $\mathrm{R}$ & 1.1 & 2.54 \\
\hline 128 & $\mathrm{R}$ & 0.85 & 2.66 \\
\hline 129 & $\mathrm{R}$ & 0.74 & 2.44 \\
\hline 130 & $\mathrm{R}$ & 1.09 & 2.3 \\
\hline 131 & $\mathrm{R}$ & 0.93 & 2.5 \\
\hline 132 & $\mathrm{R}$ & 0.79 & 1.65 \\
\hline 133 & $\mathrm{R}$ & 0.94 & 2.3 \\
\hline 134 & $\mathrm{R}$ & 0.98 & 2.55 \\
\hline 135 & $\mathrm{R}$ & 0.65 & 2.06 \\
\hline 136 & $\mathrm{R}$ & 0.73 & 2.66 \\
\hline 137 & $\mathrm{R}$ & 1.1 & 2.44 \\
\hline 138 & $\mathrm{R}$ & 1.03 & 2.42 \\
\hline 139 & $\mathrm{R}$ & 1.17 & 2.72 \\
\hline 140 & $\mathrm{R}$ & 1.36 & 3.35 \\
\hline 141 & $\mathrm{R}$ & 0.91 & 1.44 \\
\hline 142 & $\mathrm{R}$ & 1.71 & 3.57 \\
\hline 143 & $\mathrm{R}$ & 0.33 & 0.63 \\
\hline
\end{tabular}




\section{Modified Data (Unity Rule SOR)}

NOTE: $\quad$ Type = "S" indicates survey unit sample.

Type $=$ "R" indicates reference area sample.

\begin{tabular}{lcc} 
Sample Number & Type & Sum-of-Ratios (SOR) \\
\hline 545 & $\mathrm{~S}$ & 1.18 \\
546 & $\mathrm{~S}$ & 0.97 \\
547 & $\mathrm{~S}$ & 1.11 \\
548 & $\mathrm{~S}$ & 1.01 \\
549 & $\mathrm{~S}$ & 0.78 \\
550 & $\mathrm{~S}$ & 1.31 \\
551 & $\mathrm{~S}$ & 0.83 \\
552 & $\mathrm{~S}$ & 1.03 \\
553 & $\mathrm{~S}$ & 1.28 \\
554 & $\mathrm{~S}$ & 1.1 \\
555 & $\mathrm{~S}$ & 1.11 \\
556 & $\mathrm{~S}$ & 1.06 \\
557 & $\mathrm{~S}$ & 1.39 \\
558 & $\mathrm{~S}$ & 1.11 \\
559 & $\mathrm{~S}$ & 1.01 \\
560 & $\mathrm{~S}$ & 1.32 \\
561 & $\mathrm{~S}$ & 1.18 \\
127 & $\mathrm{R}$ & 1.4 \\
128 & $\mathrm{R}$ & 1.36 \\
129 & $\mathrm{R}$ & 1.23 \\
130 & $\mathrm{R}$ & 1.3 \\
131 & $\mathrm{R}$ & 1.32 \\
132 & $\mathrm{R}$ & 0.93 \\
133 & $\mathrm{R}$ & 1.24 \\
134 & $\mathrm{R}$ & 1.36 \\
135 & $\mathrm{R}$ & 1.05 \\
136 & $\mathrm{R}$ & 1.32 \\
137 & $\mathrm{R}$ & 1.36 \\
138 & $\mathrm{R}$ & 1.32 \\
139 & $\mathrm{R}$ & 1.49 \\
140 & $\mathrm{R}$ & 1.81 \\
141 & $\mathrm{R}$ & 0.89 \\
142 & $\mathrm{R}$ & 2.02 \\
143 & $\mathrm{R}$ & 0.37 \\
& &
\end{tabular}




\section{DQA Surface Soil Report}

\section{Basic Statistical Quantities Summary}

\begin{tabular}{lccc} 
Statistic & Survey Unit & Background & DQO Results \\
\hline Sample Number & 17 & 17 & N/2=18 \\
Mean (SOR) & 1.10 & 1.28 & 0.39 \\
Median (SOR) & 1.11 & 1.32 & N/A \\
Std Dev (SOR) & 0.17 & 0.36 & 0.39 \\
High Value (SOR) & 1.39 & 2.02 & N/A \\
Low Value (SOR) & 0.78 & 0.37 & N/A
\end{tabular}

\section{Statistical Test Summary}

$\begin{array}{lc}\text { Sum of Ranks: } & 595 \\ \text { Sum of Reference Ranks: } & 441 \\ \text { Critical Value: } & 345 \\ \text { Result: } & \text { Pass }\end{array}$

\begin{tabular}{|c|c|c|}
\hline Data & Type & Adjusted Data \\
\hline
\end{tabular}

0.37

0.89

0.93

1.05

1.23

1.24

1.3

1.32

1.32

1.32

1.36

1.36

1.36

1.4

1.49

1.81

2.02

0.78

0.83

0.97

1.01

1.01

1.03

1.06

1.1
595

345

Pass

1.37

1.89

1.93

2.05

2.23

2.24

2.3

2.32

2.32

2.32

2.36

2.36

2.36

2.4

2.49

2.81

3.02

0.78

0.83

0.97

1.01

1.01

1.03

1.06

1.1

17

17

19
20

21

22

23

24

26

26

26

29

29

29

31
32

32
33

33
34
1

1

2
3

4.5

4.5

6

7

8
Reference Rank

17
19
20
21
22
23
24
26
26
26
29
29
29
31
32
33
34
0
0
0
0
0
0
0
0
0 


\section{DQA Surface Soil Report}

Statistical Test Summary

\begin{tabular}{lcccc} 
Data & Type & Adjusted Data & Rank & Reference Rank \\
\hline 1.11 & & 1.11 & 10 & 0 \\
1.11 & S & 1.11 & 10 & 0 \\
1.11 & S & 1.11 & 10 & 0 \\
1.18 & S & 1.18 & 12.5 & 0 \\
1.18 & S & 1.18 & 12.5 & 0 \\
1.28 & S & 1.28 & 14 & 0 \\
1.31 & S & 1.31 & 15 & 0 \\
1.32 & S & 1.32 & 16 & 0 \\
1.39 & S & 1.39 & 18 & 0
\end{tabular}




\section{DQA Building Surface Report}

\section{Assessment Summary}

Site:

Planner(s):

Survey Unit Name:

Report Number:

Survey Unit Measurements:

Reference Area Measurements:

Test Performed:

Judgmental Areas:

Assessment Conclusion:
Hammond Depot FSS Planner

Vitkus

C1 SU2.1 through 5.8 AOC 2 and 3 slag C1 SU5.1

10

14

0

Sign

Test Result:

Not Performed

0

EMC Result:

Not Performed

Reject Null Hypothesis (Survey Unit PASSES)

\section{Retrospective Power Curve}

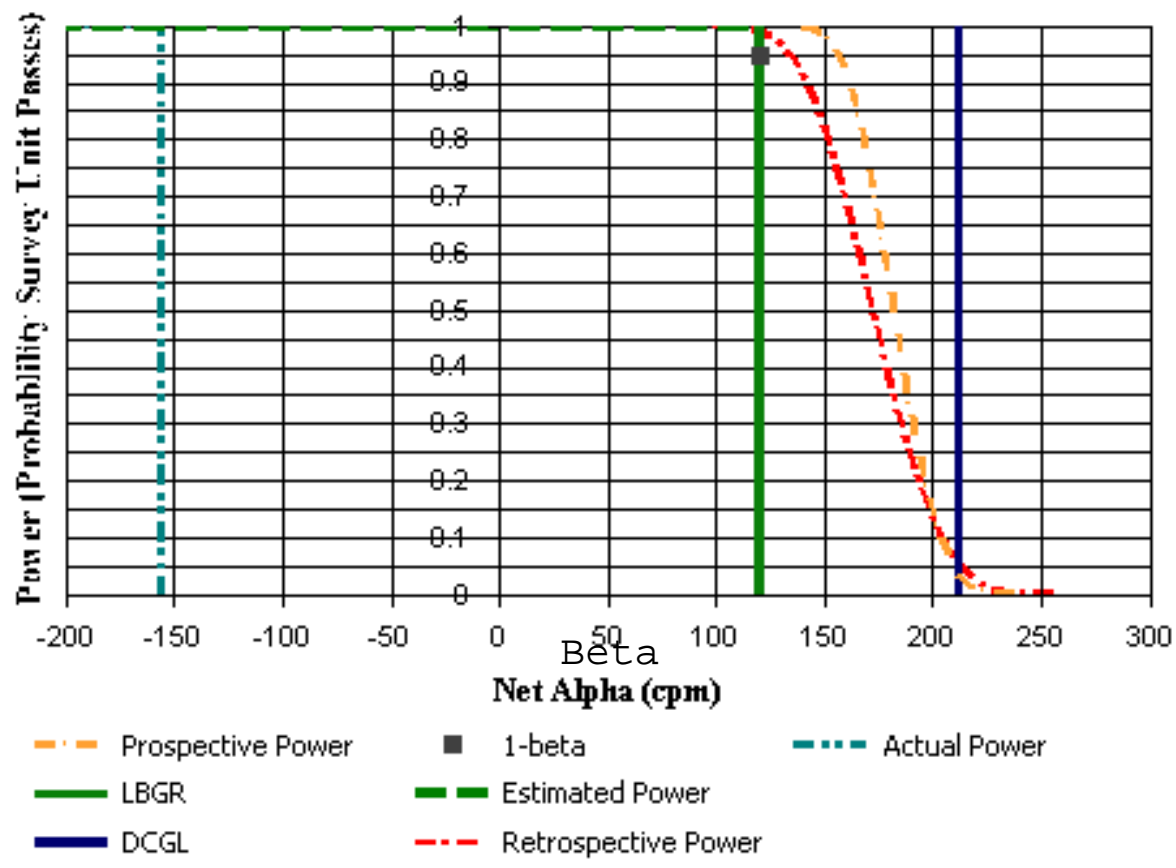




\section{DQA Building Surface Report}

\section{Survey Unit Data}

NOTE: $\quad$ Type $=$ "S" indicates survey measurement.

Type $=$ "R" indicates reference measurement.

\begin{tabular}{lccc} 
Measurement & Material & Type & Gross Alpha (cpm) \\
\hline 4611287,456524 & Slag & S & 373 \\
4611287,456528 & Slag & S & 350 \\
4611287,456532 & Slag & S & 366 \\
4611287,456536 & Slag & S & 503 \\
4611287,456540 & Slag & S & 464 \\
4611290,456526 & Slag & S & 436 \\
4611290,456530 & Slag & S & 357 \\
4611290,456534 & Slag & S & 402 \\
4611290,456538 & Slag & S & 551 \\
4611294,456524 & Slag & S & 344 \\
4611294,456528 & Slag & $S$ & 348 \\
4611294,456532 & Slag & $S$ & 380 \\
4611294,456536 & Slag & $S$ & 437 \\
4611294,456540 & Slag & $S$ & 524
\end{tabular}

\section{Basic Statistical Quantities Summary}

Statistic

Sample Number

Mean $\left(\mathrm{dpm} / 100 \mathrm{~cm}^{2}\right)$

Median (dpm/100 cm²)

Std Dev (dpm/100 $\left.\mathrm{cm}^{2}\right)$

High Value $\left(\mathrm{dpm} / 100 \mathrm{~cm}^{2}\right)$

Low Value $\left(\mathrm{dpm} / 100 \mathrm{~cm}^{2}\right)$
Survey Unit

14
-106.89
-155.61
132.67
146.73
-244.43

Background

N/A

N/A

N/A

N/A

N/A

N/A
DQO Results

$\mathrm{N}=15$

120

N/A

48.3

N/A

N/A 


\section{DQA Building Surface Report}

\section{Assessment Summary}

Site:

Planner(s):

Survey Unit Name:

Report Number:

Survey Unit Measurements:

Reference Area Measurements:

Test Performed:

Judgmental Areas:

Assessment Conclusion:
Hammond Depot FSS Planner

Vitkus

C1 SU2.1 through 5.8 AOC 2 and 3 slag C1 SU5. 2

11

14

0

Sign

Test Result:

Not Performed

0

EMC Result:

Not Performed

\section{Retrospective Power Curve}

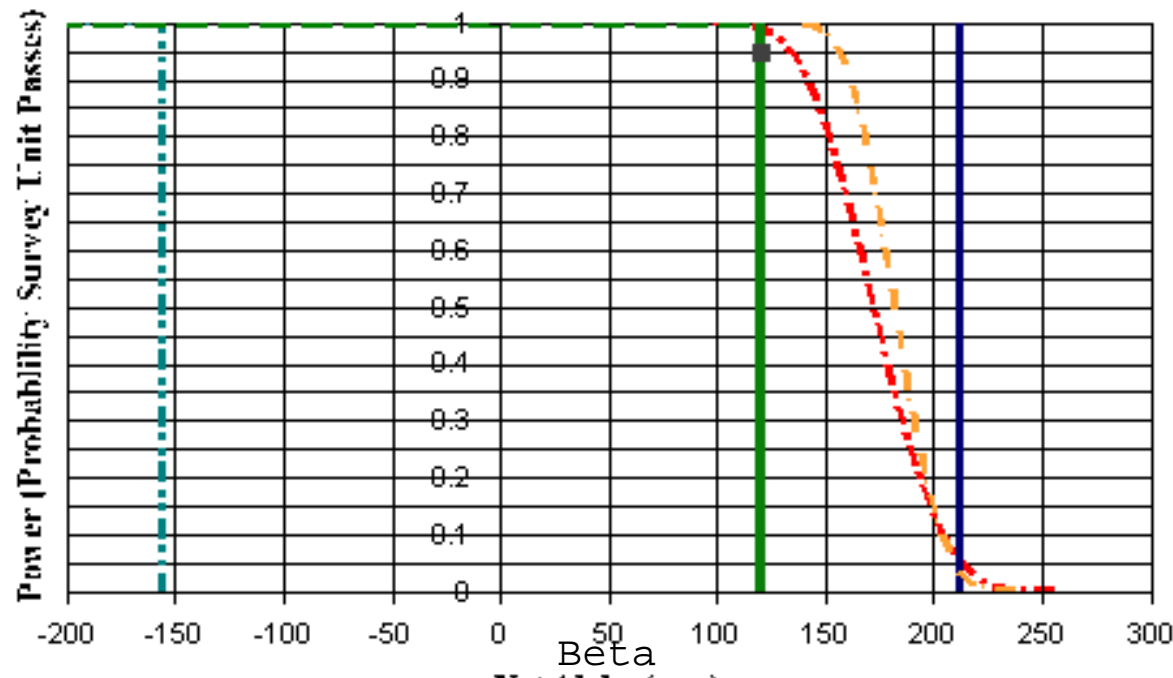

Net Alpha (cpm)

$\begin{array}{lc}- \text { - Prospective Power } & \text { 1-beta } \\ \text { LBGR } & =- \text { Estimated Power } \\ \text { DCGL } & --- \text { Retrospective Power }\end{array}$




\section{DQA Building Surface Report}

\section{Survey Unit Data}

NOTE: $\quad$ Type = "S" indicates survey measurement.

Type $=$ "R" indicates reference measurement.

\begin{tabular}{lccc} 
Measurement & Material & Type & Gross Alpha (cpm) \\
\hline 4611287,456524 & Slag & S & 373 \\
4611287,456528 & Slag & S & 350 \\
4611287,456532 & Slag & S & 366 \\
4611287,456536 & Slag & S & 503 \\
4611287,456540 & Slag & S & 464 \\
4611290,456526 & Slag & S & 436 \\
4611290,456530 & Slag & S & 357 \\
4611290,456534 & Slag & S & 402 \\
4611290,456538 & Slag & S & 551 \\
4611294,456524 & Slag & S & 344 \\
4611294,456528 & Slag & S & 348 \\
4611294,456532 & Slag & S & 380 \\
4611294,456536 & Slag & $S$ & 437 \\
4611294,456540 & Slag & $S$ & 524
\end{tabular}

\section{Basic Statistical Quantities Summary}

Statistic

Sample Number

Mean $\left(\mathrm{dpm} / 100 \mathrm{~cm}^{2}\right)$

Median (dpm/100 cm²)

Std Dev (dpm/100 $\left.\mathrm{cm}^{2}\right)$

High Value $\left(\mathrm{dpm} / 100 \mathrm{~cm}^{2}\right)$

Low Value $\left(\mathrm{dpm} / 100 \mathrm{~cm}^{2}\right)$
Survey Unit

14
-106.89
-155.61
132.67
146.73
-244.43

Background

N/A

N/A

N/A

N/A

N/A

N/A
DQO Results

$\mathrm{N}=15$

120

N/A

48.3

N/A

N/A 


\section{DQA Building Surface Report}

\section{Assessment Summary}

Site:

Planner(s):

Survey Unit Name:

Report Number:

Survey Unit Measurements:

Reference Area Measurements:

Test Performed:

Judgmental Areas:

Assessment Conclusion:
Hammond Depot FSS Planner

Vitkus

C1 SU2.1 through 5.8 AOC 2 and 3 slag C1 SU5. 3

12

14

0

Sign

Test Result:

Not Performed

0

EMC Result:

Not Performed

Reject Null Hypothesis (Survey Unit PASSES)

\section{Retrospective Power Curve}

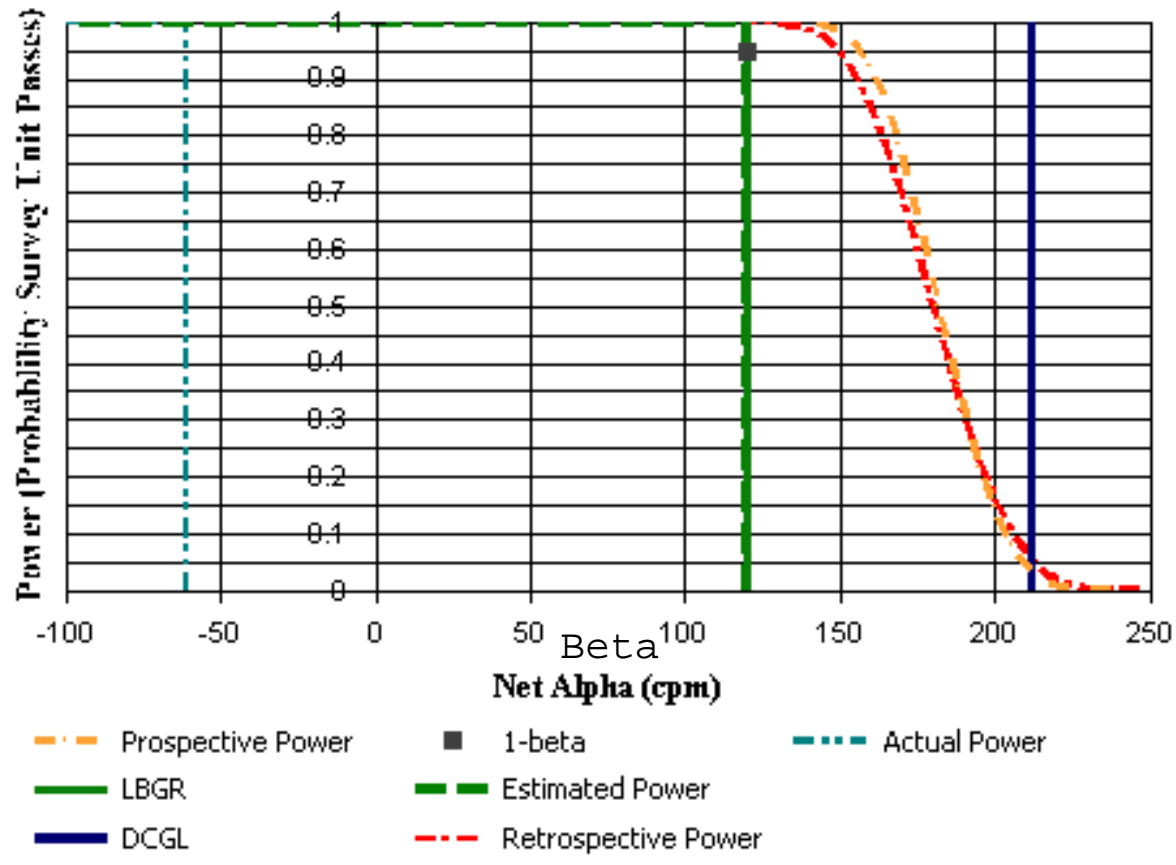




\section{DQA Building Surface Report}

\section{Survey Unit Data}

NOTE: $\quad$ Type = "S" indicates survey measurement.

Type $=$ "R" indicates reference measurement.

\begin{tabular}{lccc} 
Measurement & Material & Type & Gross Alpha (cpm) \\
\hline 4611277,456525 & Slag & S & 344 \\
4611277,456530 & Slag & S & 523 \\
4611277,456535 & Slag & S & 489 \\
4611277,456540 & Slag & S & 386 \\
4611277,456545 & Slag & S & 455 \\
4611281,456523 & Slag & S & 391 \\
4611281,456528 & Slag & S & 408 \\
4611281,456533 & Slag & S & 502 \\
4611281,456538 & Slag & S & 439 \\
4611281,456543 & Slag & S & 511 \\
4611286,456525 & Slag & S & 362 \\
4611286,456530 & Slag & S & 399 \\
4611286,456535 & Slag & $S$ & 449 \\
4611286,456540 & Slag & $S$ & 443
\end{tabular}

\section{Basic Statistical Quantities Summary}

Statistic

Sample Number

Mean $\left(\mathrm{dpm} / 100 \mathrm{~cm}^{2}\right)$

Median (dpm/100 cm²)

Std Dev (dpm/100 $\left.\mathrm{cm}^{2}\right)$

High Value $\left(\mathrm{dpm} / 100 \mathrm{~cm}^{2}\right)$

Low Value $\left(\mathrm{dpm} / 100 \mathrm{~cm}^{2}\right)$
Survey Unit

14
-70.98
-61.13
106.85
93.82
-244.43

Background

N/A

N/A

N/A

N/A

N/A

N/A
DQO Results

$\mathrm{N}=15$

120

N/A

48.3

N/A

N/A 


\section{DQA Building Surface Report}

\section{Assessment Summary}

Site:

Planner(s):

Survey Unit Name:

Report Number:

Survey Unit Measurements:

Reference Area Measurements:

Test Performed:

Judgmental Areas:

Assessment Conclusion:
Hammond Depot FSS Planner

Vitkus

C1 SU2.1 through 5.8 AOC 2 and 3 slag C1 SU5. 4

13

14

0

Sign

Test Result:

Not Performed

0

EMC Result:

Not Performed

Reject Null Hypothesis (Survey Unit PASSES)

\section{Retrospective Power Curve}

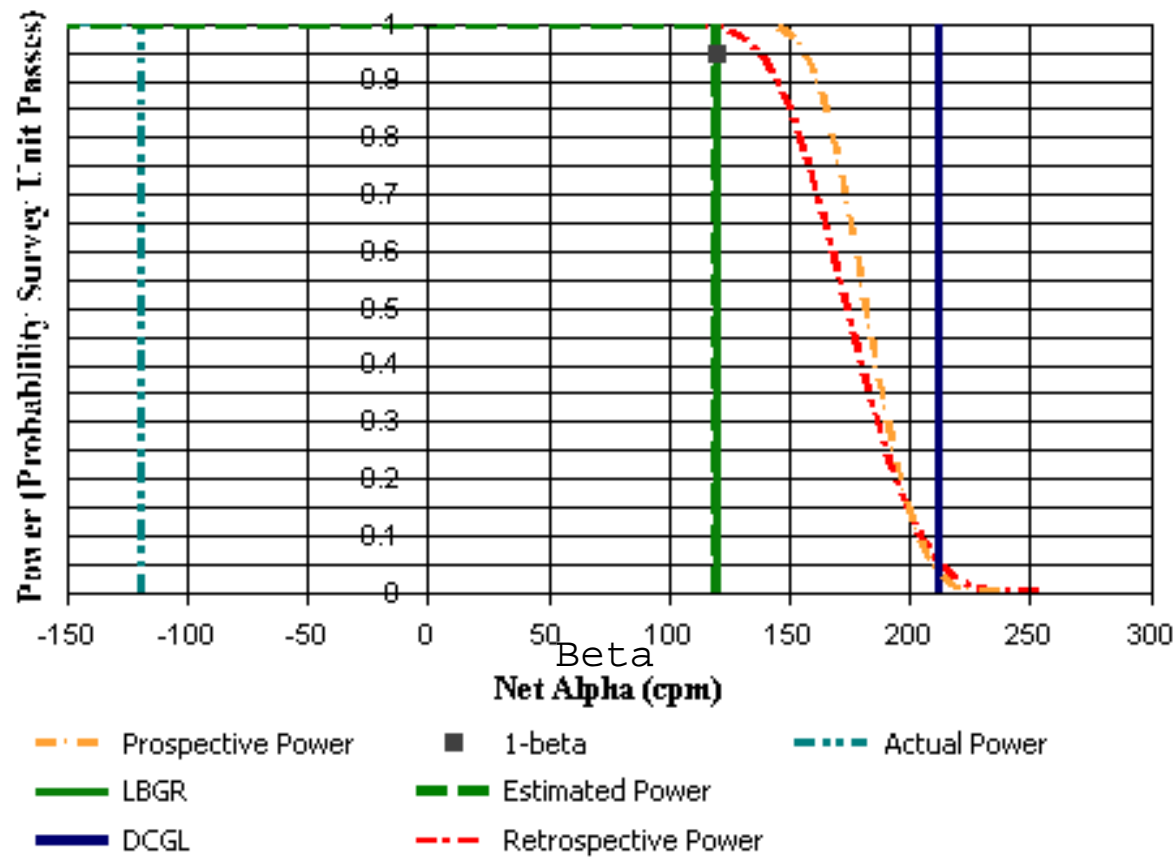




\section{DQA Building Surface Report}

\section{Survey Unit Data}

NOTE: $\quad$ Type = "S" indicates survey measurement.

Type $=$ "R" indicates reference measurement.

\begin{tabular}{lccc} 
Measurement & Material & Type & Gross Alpha (cpm) \\
\hline 4611250,456521 & Slag & S & 359 \\
4611253,456519 & Slag & S & 592 \\
4611257,456521 & Slag & S & 520 \\
4611260,456519 & Slag & S & 415 \\
4611264,456517 & Slag & S & 393 \\
4611264,456521 & Slag & S & 418 \\
4611267,456515 & Slag & S & 402 \\
4611267,456519 & Slag & S & 444 \\
4611271,456513 & Slag & S & 447 \\
4611271,456517 & Slag & S & 370 \\
4611271,456521 & Slag & S & 330 \\
4611274,456511 & Slag & S & 406 \\
4611274,456515 & Slag & $S$ & 391 \\
4611274,456519 & Slag & S & 463
\end{tabular}

\section{Basic Statistical Quantities Summary}

Statistic

Sample Number

Mean $\left(\mathrm{dpm} / 100 \mathrm{~cm}^{2}\right)$

Median (dpm/100 cm²)

Std Dev (dpm/100 $\left.\mathrm{cm}^{2}\right)$

High Value $\left(\mathrm{dpm} / 100 \mathrm{~cm}^{2}\right)$

Low Value $\left(\mathrm{dpm} / 100 \mathrm{~cm}^{2}\right)$
Survey Unit

14
-91.36
-118.76
127.12
224.21
-270.88

Background

N/A

N/A

N/A

N/A

N/A

N/A
DQO Results

$\mathrm{N}=15$

120

N/A

48.3

N/A

N/A 


\section{DQA Building Surface Report}

\section{Assessment Summary}

Site:

Planner(s):

Survey Unit Name:

Report Number:

Survey Unit Measurements:

Reference Area Measurements:

Test Performed:

Judgmental Areas:

Assessment Conclusion:
Hammond Depot FSS Planner

Vitkus

C1 SU2.1 through 5.8 AOC 2 and 3 slag C1 SU5. 5

14

19

0

Sign

Test Result:

Not Performed

0

EMC Result:

Not Performed

Reject Null Hypothesis (Survey Unit PASSES)

\section{Retrospective Power Curve}

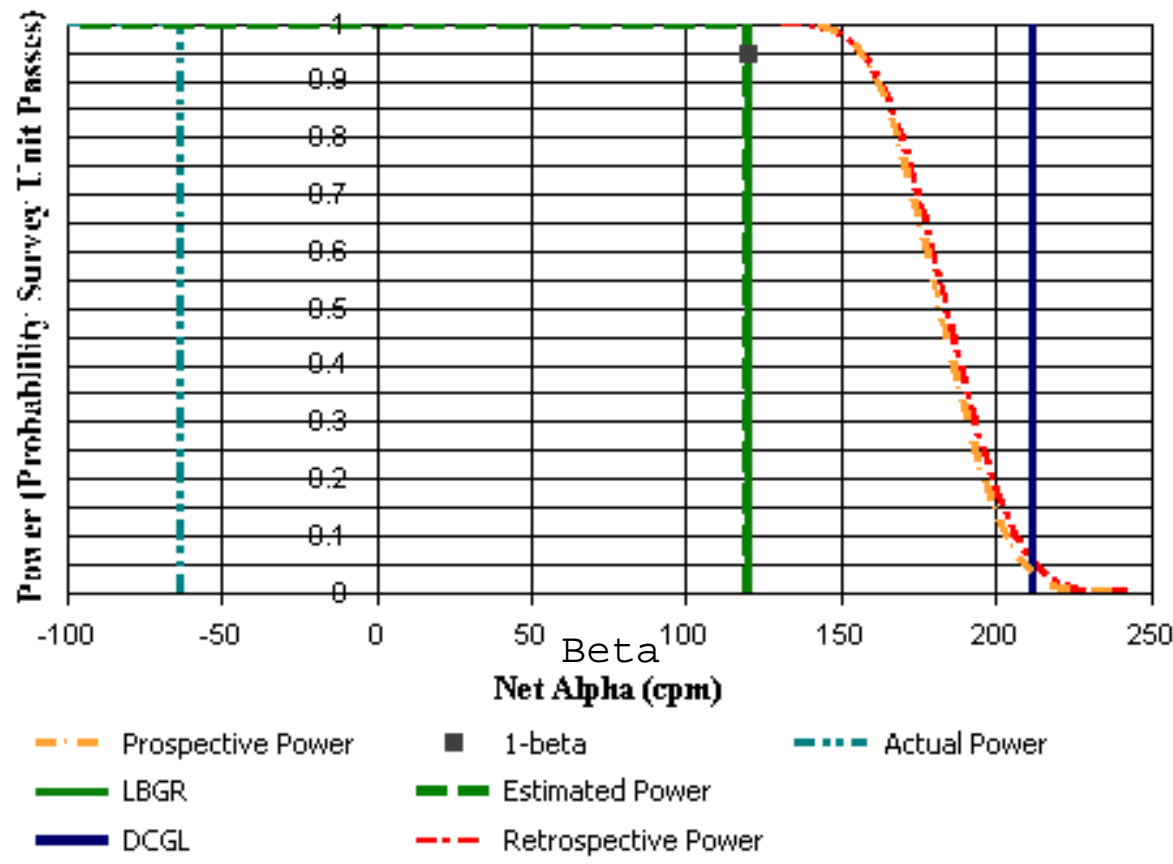




\section{DQA Building Surface Report}

\section{Survey Unit Data}

NOTE: Type $=$ "S" indicates survey measurement.

Type $=$ "R" indicates reference measurement.

\begin{tabular}{lccc} 
Measurement & Material & Type & Gross Alpha (cpm) \\
\hline 4611268,456523 & Slag & S & 388 \\
4611268,456527 & Slag & S & 434 \\
4611268,456532 & Slag & S & 458 \\
4611268,456536 & Slag & S & 563 \\
4611268,456540 & Slag & S & 447 \\
4611268,456545 & Slag & S & 396 \\
4611268,456549 & Slag & S & 380 \\
4611272,456525 & Slag & S & 358 \\
4611272,456529 & Slag & S & 440 \\
4611272,456534 & Slag & S & 524 \\
4611272,456538 & Slag & S & 427 \\
4611272,456542 & Slag & S & 477 \\
4611272,456547 & Slag & S & 462 \\
4611276,456523 & Slag & S & 412 \\
4611276,456527 & Slag & S & 387 \\
4611276,456532 & Slag & S & 509 \\
4611276,456536 & Slag & S & 558 \\
4611276,456540 & Slag & S & 440 \\
4611276,456545 & Slag & S & 471
\end{tabular}

\section{Basic Statistical Quantities Summary}

Statistic

Sample Number

Mean $\left(\mathrm{dpm} / 100 \mathrm{~cm}^{2}\right)$

Median (dpm/100 cm²)

Std Dev (dpm/100 cm²)

High Value $\left(\mathrm{dpm} / 100 \mathrm{~cm}^{2}\right)$

Low Value $\left(\mathrm{dpm} / 100 \mathrm{~cm}^{2}\right)$
Survey Unit

19
-46.01
-63.02
110.26
169.41
217.97

Background

$\begin{array}{lc}\text { N/A } & \mathrm{N}=15 \\ \text { N/A } & 120 \\ \text { N/A } & \text { N/A } \\ \text { N/A } & 48.3 \\ \text { N/A } & \text { N/A } \\ \text { N/A } & \text { N/A }\end{array}$




\section{DQA Building Surface Report}

\section{Assessment Summary}

Site:

Planner(s):

Survey Unit Name:

Report Number:

Survey Unit Measurements:

Reference Area Measurements:

Test Performed:

Judgmental Areas:

Assessment Conclusion:
Hammond Depot FSS Planner

Vitkus

C1 SU2.1 through 5.8 AOC 2 and 3 slag C1 SU5. 6

15

14

0

Sign

Test Result:

Not Performed

0

EMC Result:

Not Performed

Reject Null Hypothesis (Survey Unit PASSES)

\section{Retrospective Power Curve}

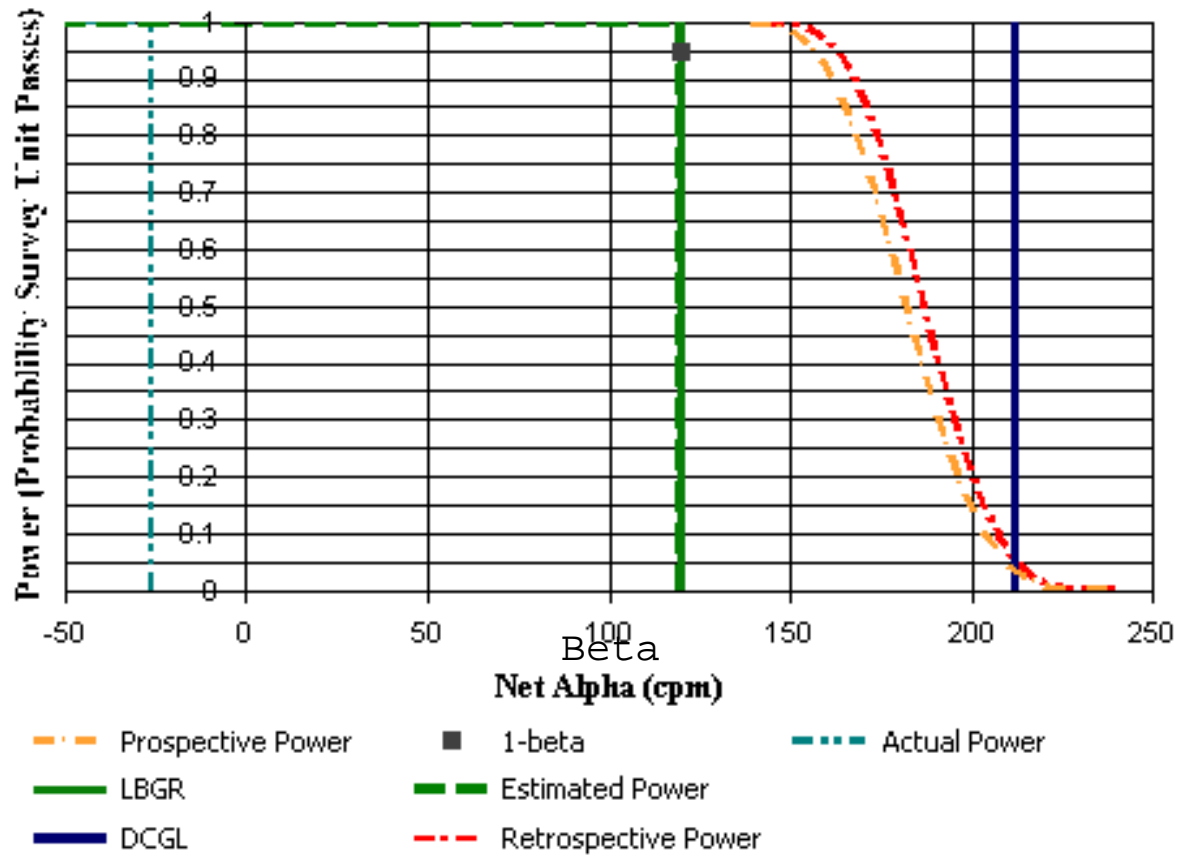




\section{DQA Building Surface Report}

\section{Survey Unit Data}

NOTE: $\quad$ Type = "S" indicates survey measurement.

Type $=$ "R" indicates reference measurement.

\begin{tabular}{lccc} 
Measurement & Material & Type & Gross Alpha (cpm) \\
\hline 4611251,456525 & Slag & S & 448 \\
4611251,456530 & Slag & S & 352 \\
4611255,456523 & Slag & S & 449 \\
4611255,456528 & Slag & S & 458 \\
4611255,456532 & Slag & S & 471 \\
4611259,456525 & Slag & S & 476 \\
4611259,456530 & Slag & S & 461 \\
4611259,456534 & Slag & S & 463 \\
4611263,456523 & Slag & S & 517 \\
4611263,456528 & Slag & S & 389 \\
4611263,456531 & Slag & S & 399 \\
4611267,456525 & Slag & S & 479 \\
4611267,456530 & Slag & $S$ & 447 \\
4611267,456534 & Slag & S & 505
\end{tabular}

\section{Basic Statistical Quantities Summary}

Statistic

Sample Number

Mean $\left(\mathrm{dpm} / 100 \mathrm{~cm}^{2}\right)$

Median (dpm/100 cm²)

Std Dev (dpm/100 $\left.\mathrm{cm}^{2}\right)$

High Value $\left(\mathrm{dpm} / 100 \mathrm{~cm}^{2}\right)$

Low Value $\left(\mathrm{dpm} / 100 \mathrm{~cm}^{2}\right)$
Survey Unit

14
-42.23
-26.17
84.10
82.48
-229.31

Background

N/A

N/A

N/A

N/A

N/A

N/A
DQO Results
$\mathrm{N}=15$
120
N/A
48.3
N/A
N/A 


\section{DQA Building Surface Report}

\section{Assessment Summary}

Site:

Planner(s):

Survey Unit Name:

Report Number:

Survey Unit Measurements:

Reference Area Measurements:

Test Performed:

Judgmental Areas:

Assessment Conclusion:
Hammond Depot FSS Planner

Vitkus

C1 SU2.1 through 5.8 AOC 2 and 3 slag C1 SU5. 7

16

14

0

Sign

Test Result:

Not Performed

0

EMC Result:

Not Performed

Reject Null Hypothesis (Survey Unit PASSES)

\section{Retrospective Power Curve}

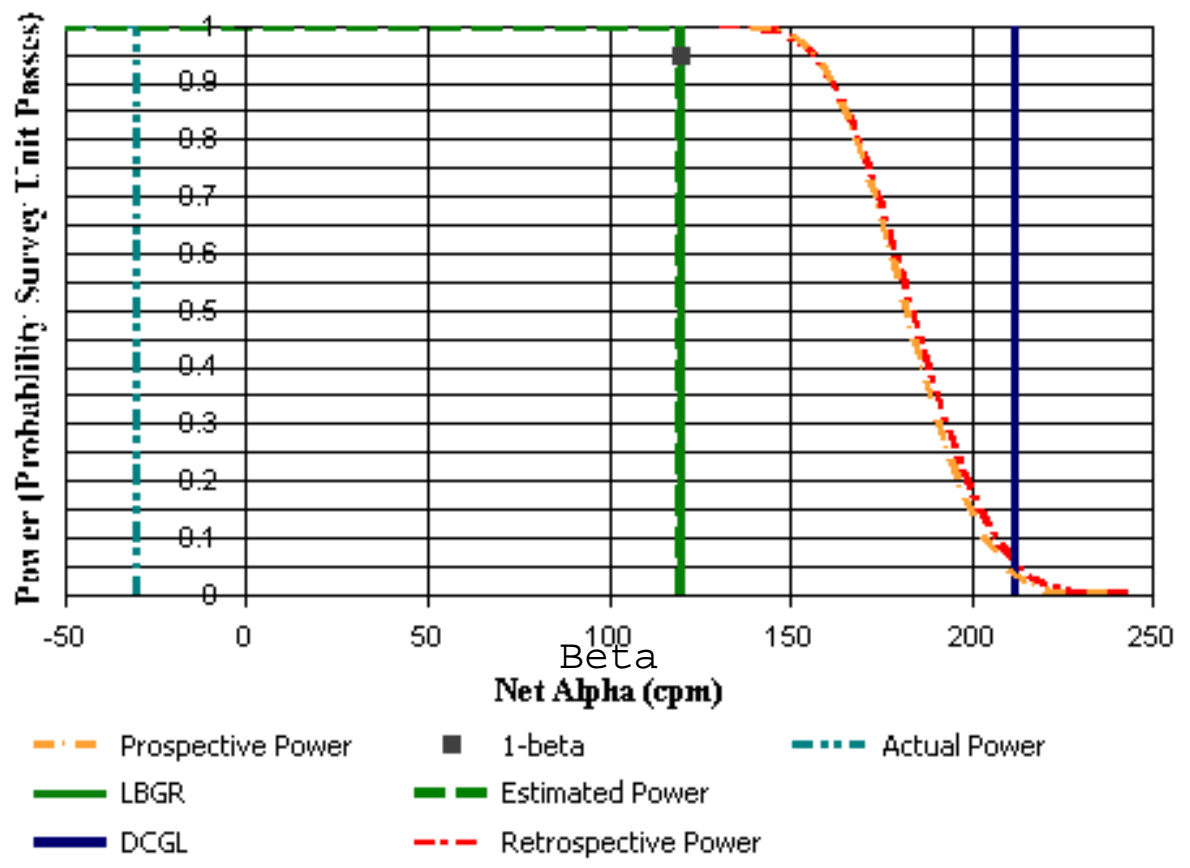




\section{DQA Building Surface Report}

\section{Survey Unit Data}

NOTE: $\quad$ Type = "S" indicates survey measurement.

Type $=$ "R" indicates reference measurement.

\begin{tabular}{lccc} 
Measurement & Material & Type & Gross Alpha (cpm) \\
\hline 4611259,456537 & Slag & S & 481 \\
4611259,456541 & Slag & S & 480 \\
4611259,456544 & Slag & S & 455 \\
4611259,456548 & Slag & S & 438 \\
4611259,456552 & Slag & S & 471 \\
4611262,456535 & Slag & S & 461 \\
4611262,456539 & Slag & S & 377 \\
4611262,456543 & Slag & S & 376 \\
4611262,456546 & Slag & S & 411 \\
4611262,456550 & Slag & S & 460 \\
4611266,456537 & Slag & S & 538 \\
4611266,456541 & Slag & $S$ & 381 \\
4611266,456544 & Slag & $S$ & 368 \\
4611266,456548 & Slag & S & 464
\end{tabular}

\section{Basic Statistical Quantities Summary}

Statistic

Sample Number

Mean $\left(\mathrm{dpm} / 100 \mathrm{~cm}^{2}\right)$

Median (dpm/100 cm²)

Std Dev (dpm/100 $\left.\mathrm{cm}^{2}\right)$

High Value $\left(\mathrm{dpm} / 100 \mathrm{~cm}^{2}\right)$

Low Value $\left(\mathrm{dpm} / 100 \mathrm{~cm}^{2}\right)$
Survey Unit

14
-62.88
-29.95
95.30
122.17
-199.07

Background

N/A

N/A

N/A

N/A

N/A

N/A

N/A
N/A
N/A
$N / A$
$N / A$
$N / A$

DQO Results

$\mathrm{N}=15$

120

N/A

48.3

N/A

N/A 


\section{DQA Building Surface Report}

\section{Assessment Summary}

Site:

Planner(s):

Survey Unit Name:

Report Number:

Survey Unit Measurements:

Reference Area Measurements:

Test Performed:

Judgmental Areas:

Assessment Conclusion:
Hammond Depot FSS Planner

Vitkus

C1 SU2.1 through 5.8 AOC 2 and 3 slag C1 SU5. 8

17

14

0

Sign

Test Result:

Not Performed

0

EMC Result:

Not Performed

Reject Null Hypothesis (Survey Unit PASSES)

\section{Retrospective Power Curve}

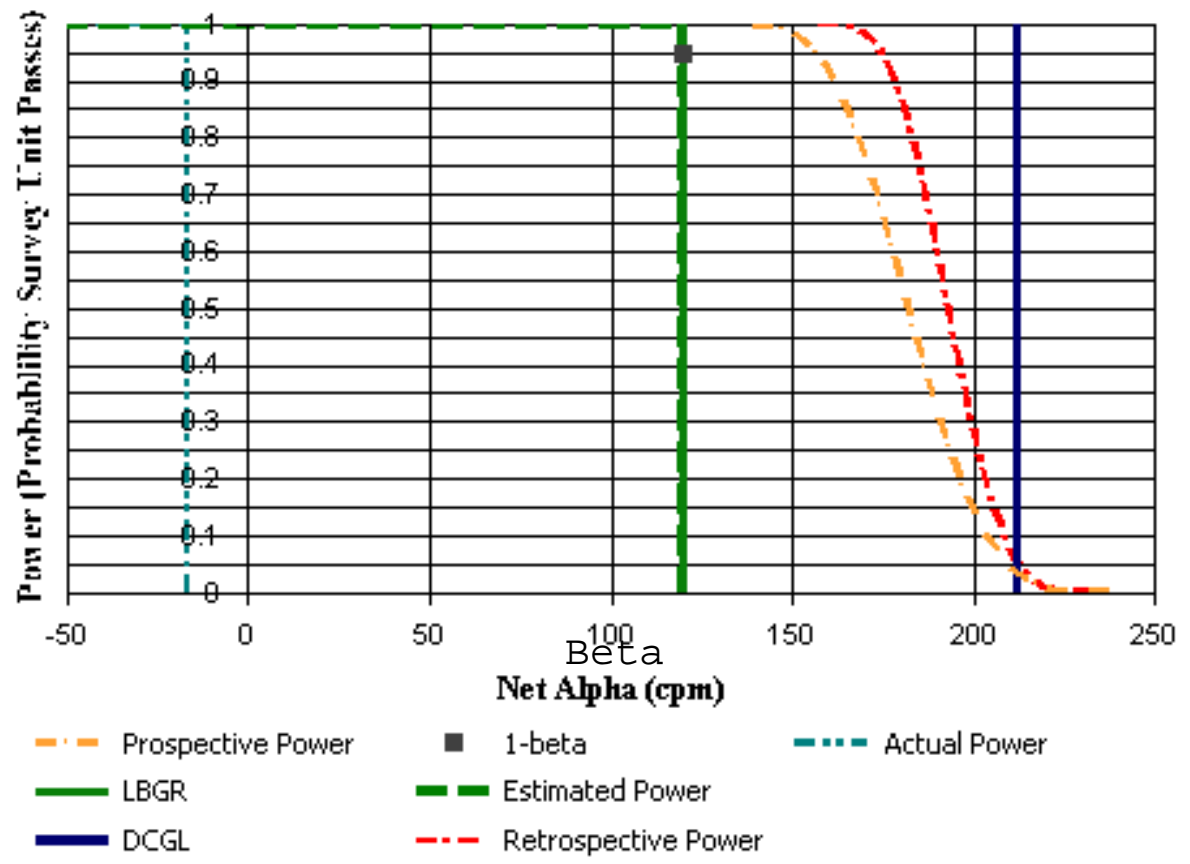




\section{DQA Building Surface Report}

\section{Survey Unit Data}

NOTE: $\quad$ Type = "S" indicates survey measurement.

Type $=$ "R" indicates reference measurement.

\begin{tabular}{lccc} 
Measurement & Material & Type & Gross Alpha (cpm) \\
\hline 4611251,456544 & Slag & S & 446 \\
4611251,456547 & Slag & S & 472 \\
4611251,456551 & Slag & S & 436 \\
4611251,456553 & Slag & S & 513 \\
4611254,456538 & Slag & S & 517 \\
4611254,456542 & Slag & S & 438 \\
4611254,456545 & Slag & S & 459 \\
4611254,456549 & Slag & S & 452 \\
4611254,456552 & Slag & S & 448 \\
4611257,456537 & Slag & S & 472 \\
4611257,456540 & Slag & S & 482 \\
4611257,456544 & Slag & S & 470 \\
4611257,456547 & Slag & $S$ & 399 \\
4611257,456551 & Slag & S & 517
\end{tabular}

\section{Basic Statistical Quantities Summary}

Statistic

Sample Number

Mean $\left(\mathrm{dpm} / 100 \mathrm{~cm}{ }^{2}\right)$

Median (dpm/100 cm²)

Std Dev (dpm/100 $\left.\mathrm{cm}^{2}\right)$

High Value $\left(\mathrm{dpm} / 100 \mathrm{~cm}^{2}\right)$

Low Value $\left(\mathrm{dpm} / 100 \mathrm{~cm}^{2}\right)$
Survey Unit

14
-14.29
-16.72
63.97
82.48
-140.50

Background

N/A

N/A

N/A

N/A

N/A

N/A

N/A
N/A
N/A
N/A
N/A
N/A

DQO Results

$\mathrm{N}=15$

120

N/A

48.3

N/A

N/A 


\section{DQA Surface Soil Report}

\section{Assessment Summary}

Site:

Planner(s):

Survey Unit Name:

Report Number:

Survey Unit Samples:

Reference Area Samples:

Test Performed:

Judgmental Samples:

Assessment Conclusion:
Hammond Depot FSS Planner

Vitkus

C1 SU6 and $7 \quad$ C1 SU6

1

18

17

WRS

Test Result: $\quad$ Pass

0

EMC Result: Not Performed

Reject Null Hypothesis (Survey Unit PASSES)

\section{Retrospective Power Curve}

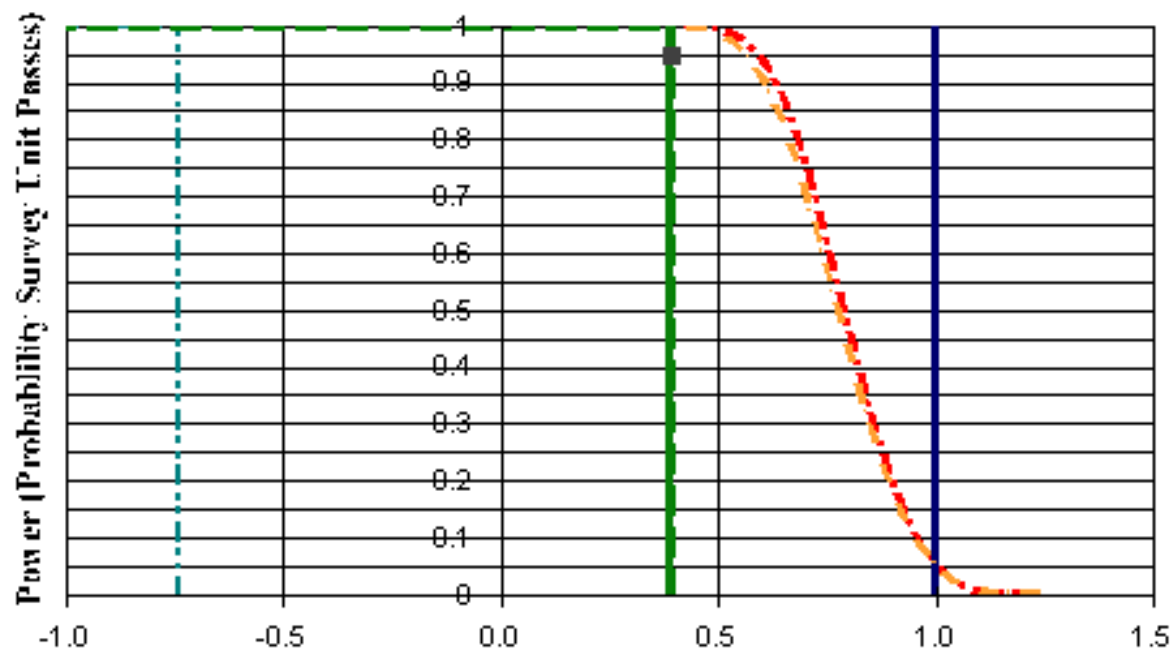

Unity Rule Sum-of-Ratios, not including background

$\begin{array}{lc}-\cdot-\text { Prospective Power } & \text { 1-beta } \\ \text { LBGR } & - \text { Estimated Power } \\ \text { DCGL } & --- \text { Retrospective Power }\end{array}$




\section{DQA Surface Soil Report}

\section{Survey Unit Data}

NOTE: $\quad$ Type = "S" indicates survey unit sample.

Type = "R" indicates reference area sample.

\begin{tabular}{|c|c|c|c|}
\hline Sample Number & Type & Th-232 (pCi/g) & U-238 (pCi/g) \\
\hline$\overline{438}$ & $\mathrm{~S}$ & 0.56 & 0.75 \\
\hline 439 & $S$ & 0.53 & 0.64 \\
\hline 440 & S & 0.65 & 1.26 \\
\hline 441 & S & 0.89 & 1.42 \\
\hline 442 & $\mathrm{~S}$ & 0.38 & 0.94 \\
\hline 443 & $S$ & 0.48 & 0.81 \\
\hline 444 & $S$ & 0.65 & 0.82 \\
\hline 445 & $S$ & 0.58 & 1.4 \\
\hline 446 & S & 0.57 & 1.41 \\
\hline 447 & S & 0.47 & 0.75 \\
\hline 448 & S & 0.48 & 0.97 \\
\hline 449 & S & 0.51 & 0.77 \\
\hline 450 & S & 0.77 & 1.77 \\
\hline 451 & S & 0.67 & 0.92 \\
\hline 452 & S & 0.64 & 1.02 \\
\hline 453 & S & 0.74 & 0.67 \\
\hline 454 & S & 0.49 & 1.35 \\
\hline 455 & $S$ & 1.29 & 2.29 \\
\hline 127 & $\mathrm{R}$ & 1.1 & 2.54 \\
\hline 128 & $\mathrm{R}$ & 0.85 & 2.66 \\
\hline 129 & $\mathrm{R}$ & 0.74 & 2.44 \\
\hline 130 & $\mathrm{R}$ & 1.09 & 2.3 \\
\hline 131 & $\mathrm{R}$ & 0.93 & 2.5 \\
\hline 132 & $\mathrm{R}$ & 0.79 & 1.65 \\
\hline 133 & $\mathrm{R}$ & 0.94 & 2.3 \\
\hline 134 & $\mathrm{R}$ & 0.98 & 2.55 \\
\hline 135 & $\mathrm{R}$ & 0.65 & 2.06 \\
\hline 136 & $\mathrm{R}$ & 0.73 & 2.66 \\
\hline 137 & $\mathrm{R}$ & 1.1 & 2.44 \\
\hline 138 & $\mathrm{R}$ & 1.03 & 2.42 \\
\hline 139 & $\mathrm{R}$ & 1.17 & 2.72 \\
\hline 140 & $\mathrm{R}$ & 1.36 & 3.35 \\
\hline 141 & $\mathrm{R}$ & 0.91 & 1.44 \\
\hline 142 & $\mathrm{R}$ & 1.71 & 3.57 \\
\hline 143 & $\mathrm{R}$ & 0.33 & 0.63 \\
\hline
\end{tabular}




\section{Modified Data (Unity Rule SOR)}

NOTE: $\quad$ Type = "S" indicates survey unit sample.

Type $=$ "R" indicates reference area sample.

\begin{tabular}{|c|c|c|}
\hline Sample Number & Type & Sum-of-Ratios (SOR) \\
\hline 438 & $\mathrm{~S}$ & 0.49 \\
\hline 439 & $S$ & 0.44 \\
\hline 440 & $\mathrm{~S}$ & 0.73 \\
\hline 441 & $\mathrm{~S}$ & 0.87 \\
\hline 442 & $\mathrm{~S}$ & 0.51 \\
\hline 443 & $S$ & 0.49 \\
\hline 444 & $S$ & 0.55 \\
\hline 445 & $S$ & 0.76 \\
\hline 446 & S & 0.76 \\
\hline 447 & $S$ & 0.46 \\
\hline 448 & $\mathrm{~S}$ & 0.55 \\
\hline 449 & $\mathrm{~S}$ & 0.48 \\
\hline 450 & $\mathrm{~S}$ & 0.97 \\
\hline 451 & $S$ & 0.6 \\
\hline 452 & $S$ & 0.63 \\
\hline 453 & $\mathrm{~S}$ & 0.52 \\
\hline 454 & $\mathrm{~S}$ & 0.71 \\
\hline 455 & $\mathrm{~S}$ & 1.36 \\
\hline 127 & $\mathrm{R}$ & 1.4 \\
\hline 128 & $\mathrm{R}$ & 1.36 \\
\hline 129 & $\mathrm{R}$ & 1.23 \\
\hline 130 & $\mathrm{R}$ & 1.3 \\
\hline 131 & $\mathrm{R}$ & 1.32 \\
\hline 132 & $\mathrm{R}$ & 0.93 \\
\hline 133 & $\mathrm{R}$ & 1.24 \\
\hline 134 & $\mathrm{R}$ & 1.36 \\
\hline 135 & $\mathrm{R}$ & 1.05 \\
\hline 136 & $\mathrm{R}$ & 1.32 \\
\hline 137 & $\mathrm{R}$ & 1.36 \\
\hline 138 & $\mathrm{R}$ & 1.32 \\
\hline 139 & $\mathrm{R}$ & 1.49 \\
\hline 140 & $\mathrm{R}$ & 1.81 \\
\hline 141 & $\mathrm{R}$ & 0.89 \\
\hline 142 & $\mathrm{R}$ & 2.02 \\
\hline 143 & $\mathrm{R}$ & 0.37 \\
\hline
\end{tabular}




\section{DQA Surface Soil Report}

\section{Basic Statistical Quantities Summary}

\begin{tabular}{lccc} 
Statistic & Survey Unit & Background & DQO Results \\
\hline Sample Number & 18 & 17 & N/2=18 \\
Mean (SOR) & 0.66 & 1.28 & 0.39 \\
Median (SOR) & 0.58 & 1.32 & N/A \\
Std Dev (SOR) & 0.23 & 0.36 & 0.39 \\
High Value (SOR) & 1.36 & 2.02 & N/A \\
Low Value (SOR) & 0.44 & 0.37 & N/A
\end{tabular}

\section{Statistical Test Summary}

$\begin{array}{ll}\text { Sum of Ranks: } & 630 \\ \text { Sum of Reference Ranks: } & 459 \\ \text { Critical Value: } & 356 \\ \text { Result: } & \text { Pass }\end{array}$

$\begin{array}{lll}\text { Data } & \text { Type } & \text { Adjusted Data }\end{array}$

Reference Rank

0.37

0.89

0.93

1.05

1.23

1.24

1.3

1.32

1.32

1.32

1.36

1.36

1.36

1.4

1.49

1.81

2.02

0.44

0.46

0.48

0.49

0.49

0.51

0.52

0.55
459

56

Pass

$\begin{array}{ll}\mathrm{R} & 1.37\end{array}$

1.89

1.93

2.05

2.23

2.24

2.3

2.32

2.32

2.32

2.36

2.36

2.36

2.4

2.49

2.81

3.02

0.44

0.46

0.48

0.49

0.49

0.51

0.52

0.55
19

20

21

22

23

24

25

27

27

27

30

30

30

32

33

34

35

1

2

3

4.5

4.5

6

7

8.5
19

20

21

22

23

24

25

27

27

27

30

30

30

32

33

34

35

0

0

0

0

0

0

0 


\section{DQA Surface Soil Report}

Statistical Test Summary

\begin{tabular}{|c|c|c|c|c|}
\hline Data & Type & Adjusted Data & Rank & Reference Rank \\
\hline 0.55 & $\mathrm{~S}$ & 0.55 & 8.5 & 0 \\
\hline 0.6 & $\mathrm{~S}$ & 0.6 & 10 & 0 \\
\hline 0.63 & $\mathrm{~S}$ & 0.63 & 11 & 0 \\
\hline 0.71 & $\mathrm{~S}$ & 0.71 & 12 & 0 \\
\hline 0.73 & $\mathrm{~S}$ & 0.73 & 13 & 0 \\
\hline 0.76 & $\mathrm{~S}$ & 0.76 & 14.5 & 0 \\
\hline 0.76 & $\mathrm{~S}$ & 0.76 & 14.5 & 0 \\
\hline 0.87 & $\mathrm{~S}$ & 0.87 & 16 & 0 \\
\hline 0.97 & $\mathrm{~S}$ & 0.97 & 17 & 0 \\
\hline 1.36 & $\mathrm{~S}$ & 1.36 & 18 & 0 \\
\hline
\end{tabular}




\section{DQA Surface Soil Report}

\section{Assessment Summary}

Site:

Planner(s):

Survey Unit Name:

Report Number:

Survey Unit Samples:

Reference Area Samples:

Test Performed:

Judgmental Samples:

Assessment Conclusion:
Hammond Depot FSS Planner

Vitkus

C1 SU6 and $7 \quad$ C1 $\quad$ SU7

2

17

17

WRS

Test Result: $\quad$ Pass

0

EMC Result:

Not Performed

Reject Null Hypothesis (Survey Unit PASSES)

\section{Retrospective Power Curve}

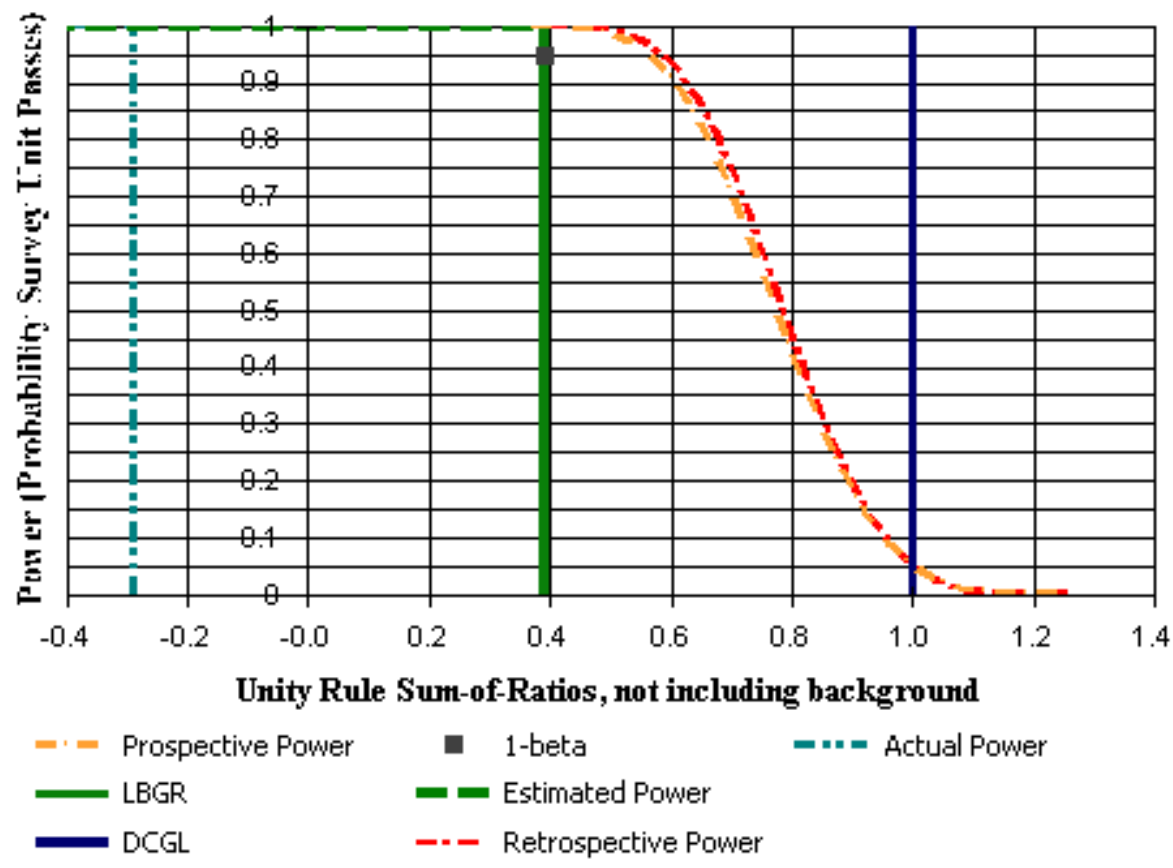




\section{DQA Surface Soil Report}

\section{Survey Unit Data}

NOTE: $\quad$ Type = "S" indicates survey unit sample.

Type = "R" indicates reference area sample.

\begin{tabular}{|c|c|c|c|}
\hline Sample Number & Type & Th-232 (pCi/g) & U-238 (pCi/g) \\
\hline 456 & $\mathrm{~S}$ & 0.98 & 1.9 \\
\hline 457 & $S$ & 0.83 & 1.54 \\
\hline 458 & $S$ & 0.88 & 1.82 \\
\hline 459 & S & 0.72 & 1.46 \\
\hline 460 & $S$ & 1.36 & 1.84 \\
\hline 461 & $S$ & 0.75 & 1.75 \\
\hline 462 & $S$ & 1 & 1.84 \\
\hline 463 & $S$ & 0.57 & 0.87 \\
\hline 464 & $S$ & 0.97 & 1.77 \\
\hline 465 & $S$ & 0.73 & 1.38 \\
\hline 466 & S & 0.91 & 2.19 \\
\hline 467 & S & 0.95 & 2.54 \\
\hline 468 & S & 1.01 & 1.5 \\
\hline 469 & S & 0.76 & 1.21 \\
\hline 470 & S & 2.06 & 2.42 \\
\hline 471 & S & 1.32 & 2.58 \\
\hline 472 & S & 0.32 & 0.72 \\
\hline 127 & $\mathrm{R}$ & 1.1 & 2.54 \\
\hline 128 & $\mathrm{R}$ & 0.85 & 2.66 \\
\hline 129 & $\mathrm{R}$ & 0.74 & 2.44 \\
\hline 130 & $\mathrm{R}$ & 1.09 & 2.3 \\
\hline 131 & $\mathrm{R}$ & 0.93 & 2.5 \\
\hline 132 & $\mathrm{R}$ & 0.79 & 1.65 \\
\hline 133 & $\mathrm{R}$ & 0.94 & 2.3 \\
\hline 134 & $\mathrm{R}$ & 0.98 & 2.55 \\
\hline 135 & $\mathrm{R}$ & 0.65 & 2.06 \\
\hline 136 & $\mathrm{R}$ & 0.73 & 2.66 \\
\hline 137 & $\mathrm{R}$ & 1.1 & 2.44 \\
\hline 138 & $\mathrm{R}$ & 1.03 & 2.42 \\
\hline 139 & $\mathrm{R}$ & 1.17 & 2.72 \\
\hline 140 & $\mathrm{R}$ & 1.36 & 3.35 \\
\hline 141 & $\mathrm{R}$ & 0.91 & 1.44 \\
\hline 142 & $\mathrm{R}$ & 1.71 & 3.57 \\
\hline 143 & $\mathrm{R}$ & 0.33 & 0.63 \\
\hline
\end{tabular}




\section{Modified Data (Unity Rule SOR)}

NOTE: $\quad$ Type = "S" indicates survey unit sample.

Type $=$ "R" indicates reference area sample.

\begin{tabular}{lcc} 
Sample Number & Type & Sum-of-Ratios (SOR) \\
\hline 456 & $\mathrm{~S}$ & 1.1 \\
457 & $\mathrm{~S}$ & 0.9 \\
458 & $\mathrm{~S}$ & 1.03 \\
459 & $\mathrm{~S}$ & 0.83 \\
460 & $\mathrm{~S}$ & 1.2 \\
461 & $\mathrm{~S}$ & 0.96 \\
462 & $\mathrm{~S}$ & 1.08 \\
463 & $\mathrm{~S}$ & 0.54 \\
464 & $\mathrm{~S}$ & 1.04 \\
465 & $\mathrm{~S}$ & 0.8 \\
466 & $\mathrm{~S}$ & 1.19 \\
467 & $\mathrm{~S}$ & 1.34 \\
468 & $\mathrm{~S}$ & 0.95 \\
469 & $\mathrm{~S}$ & 0.75 \\
470 & $\mathrm{~S}$ & 1.68 \\
471 & $\mathrm{~S}$ & 1.49 \\
472 & $\mathrm{~S}$ & 0.4 \\
127 & $\mathrm{R}$ & 1.4 \\
128 & $\mathrm{R}$ & 1.36 \\
129 & $\mathrm{R}$ & 1.23 \\
130 & $\mathrm{R}$ & 1.3 \\
131 & $\mathrm{R}$ & 1.32 \\
132 & $\mathrm{R}$ & 0.93 \\
133 & $\mathrm{R}$ & 1.24 \\
134 & $\mathrm{R}$ & 1.36 \\
135 & $\mathrm{R}$ & 1.05 \\
136 & $\mathrm{R}$ & 1.32 \\
137 & $\mathrm{R}$ & 1.36 \\
138 & $\mathrm{R}$ & 1.32 \\
139 & $\mathrm{R}$ & 1.49 \\
140 & $\mathrm{R}$ & 1.81 \\
141 & $\mathrm{R}$ & 0.89 \\
142 & $\mathrm{R}$ & 2.02 \\
143 & $\mathrm{R}$ & 0.37 \\
& &
\end{tabular}




\section{DQA Surface Soil Report}

\section{Basic Statistical Quantities Summary}

\begin{tabular}{lccc} 
Statistic & Survey Unit & Background & DQO Results \\
\hline Sample Number & 17 & 17 & N/2=18 \\
Mean (SOR) & 1.02 & 1.28 & 0.39 \\
Median (SOR) & 1.03 & 1.32 & N/A \\
Std Dev (SOR) & 0.32 & 0.36 & 0.39 \\
High Value (SOR) & 1.68 & 2.02 & N/A \\
Low Value (SOR) & 0.40 & 0.37 & N/A
\end{tabular}

\section{Statistical Test Summary}

$\begin{array}{ll}\text { Sum of Ranks: } & 595 \\ \text { Sum of Reference Ranks: } & 440 \\ \text { Critical Value: } & 345 \\ \text { Result: } & \text { Pass }\end{array}$

\begin{tabular}{lll} 
Data & Type & Adjusted Data \\
\hline
\end{tabular}

0.37

0.89

0.93

1.05

1.23

1.24

1.3

1.32

1.32

1.32

1.36

1.36

1.36

1.4

1.49

1.81

2.02

0.4

0.54

0.75

0.8

0.83

0.9

0.95

0.96
595

Pass

$\begin{array}{ll}\mathrm{R} & 1.37\end{array}$

1.89

1.93

2.05

2.23

2.24

2.3

2.32

2.32

2.32

2.36

2.36

2.36

2.4

2.49

2.81

3.02

0.4

0.54

0.75

0.8

0.83

0.9

0.95

0.96
Rank

Reference Rank

$\begin{array}{cc}16 & 16 \\ 19 & 19 \\ 20 & 20 \\ 21 & 21 \\ 22 & 22 \\ 23 & 23 \\ 24 & 24 \\ 26 & 26 \\ 26 & 26 \\ 26 & 26 \\ 29 & 29 \\ 29 & 29 \\ 29 & 29 \\ 31 & 31 \\ 32 & 32 \\ 33 & 33 \\ 34 & 34 \\ 1 & 0 \\ 2 & 0 \\ 3 & 0 \\ 4 & 0 \\ 5 & 0 \\ 6 & 0 \\ 7 & 0 \\ 8 & 0\end{array}$




\section{DQA Surface Soil Report}

\section{Statistical Test Summary}

\begin{tabular}{lcccc} 
Data & Type & Adjusted Data & Rank & Reference Rank \\
\hline 1.03 & & 1.03 & 9 & 0 \\
1.04 & S & 1.04 & 10 & 0 \\
1.08 & S & 1.08 & 11 & 0 \\
1.1 & S & 1.1 & 12 & 0 \\
1.19 & S & 1.19 & 13 & 0 \\
1.2 & S & 1.2 & 14 & 0 \\
1.34 & S & 1.34 & 15 & 0 \\
1.49 & S & 1.49 & 17 & 0 \\
1.68 & S & 1.68 & 18 & 0
\end{tabular}




\section{DQA Surface Soil Report}

\section{Assessment Summary}

Site:

Planner(s):

Survey Unit Name:

Report Number:

Survey Unit Samples:

Reference Area Samples:

Test Performed:

Judgmental Samples:

Assessment Conclusion:
Hammond Depot FSS Planner

Vitkus

C2 SU1 through $12 \quad$ C2 SU1

1

14

17

WRS

Test Result: $\quad$ Pass

0

EMC Result:

Not Performed

Reject Null Hypothesis (Survey Unit PASSES)

\section{Retrospective Power Curve}

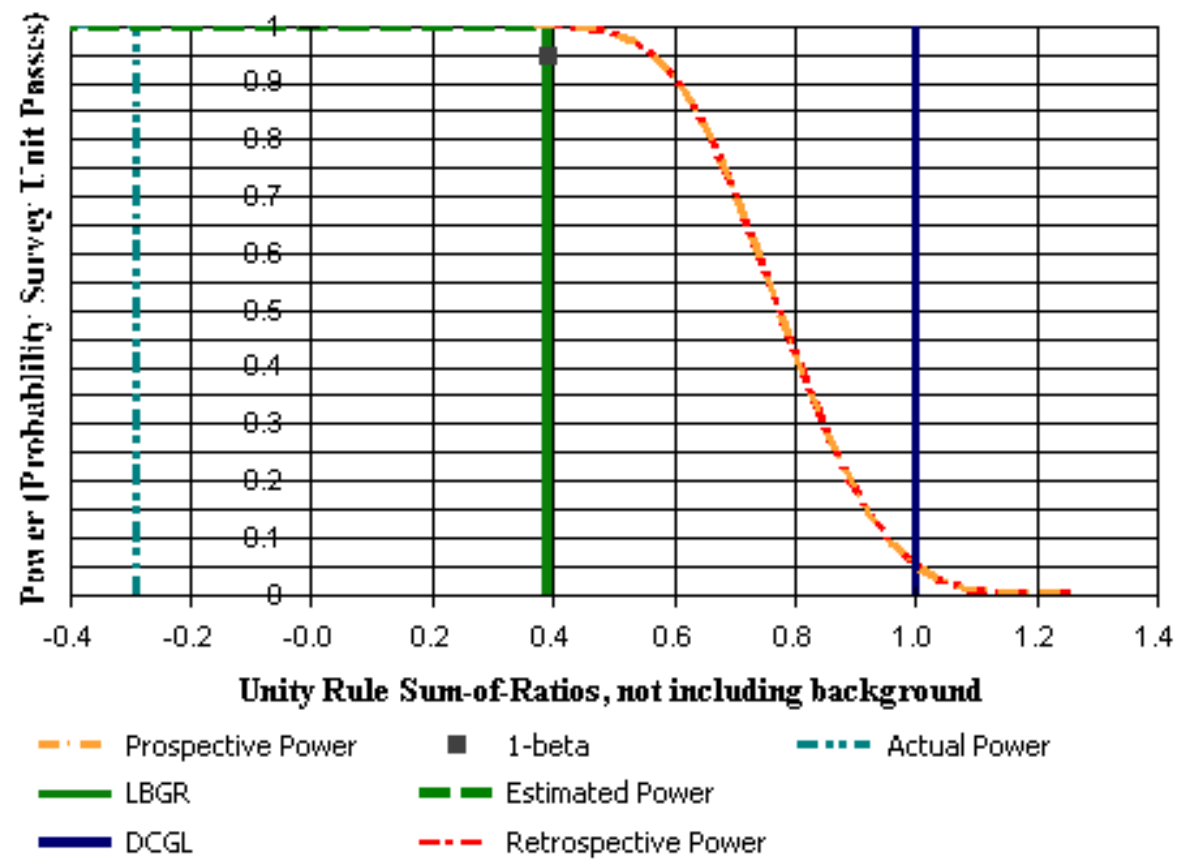




\section{DQA Surface Soil Report}

\section{Survey Unit Data}

NOTE: $\quad$ Type = "S" indicates survey unit sample.

Type = "R" indicates reference area sample.

\begin{tabular}{|c|c|c|c|}
\hline Sample Number & Type & Th-232 (pCi/g) & $\mathrm{U}-238(\mathrm{pCi} / \mathrm{g})$ \\
\hline$\overline{251}$ & $\mathrm{~S}$ & 1.08 & 2.4 \\
\hline 253 & $S$ & 0.75 & 1.33 \\
\hline 254 & $S$ & 1.19 & 1.38 \\
\hline 255 & S & 1.15 & 3.18 \\
\hline 256 & $S$ & 0.6 & 1.24 \\
\hline 258 & S & 0.93 & 1.95 \\
\hline 259 & $S$ & 0.54 & 0.94 \\
\hline 261 & $S$ & 0.98 & 2.34 \\
\hline 262 & $S$ & 0.77 & 1.62 \\
\hline 263 & $S$ & 0.93 & 2.01 \\
\hline 264 & S & 0.8 & 1.03 \\
\hline 265 & S & 1.03 & 1.86 \\
\hline 266 & S & 0.59 & 1.4 \\
\hline 267 & S & 1.17 & 2.35 \\
\hline 127 & $\mathrm{R}$ & 1.1 & 2.54 \\
\hline 128 & $\mathrm{R}$ & 0.85 & 2.66 \\
\hline 129 & $\mathrm{R}$ & 0.74 & 2.44 \\
\hline 130 & $\mathrm{R}$ & 1.09 & 2.3 \\
\hline 131 & $\mathrm{R}$ & 0.93 & 2.5 \\
\hline 132 & $\mathrm{R}$ & 0.79 & 1.65 \\
\hline 133 & $\mathrm{R}$ & 0.94 & 2.3 \\
\hline 134 & $\mathrm{R}$ & 0.98 & 2.55 \\
\hline 135 & $\mathrm{R}$ & 0.65 & 2.06 \\
\hline 136 & $\mathrm{R}$ & 0.73 & 2.66 \\
\hline 137 & $\mathrm{R}$ & 1.1 & 2.44 \\
\hline 138 & $\mathrm{R}$ & 1.03 & 2.42 \\
\hline 139 & $\mathrm{R}$ & 1.17 & 2.72 \\
\hline 140 & $\mathrm{R}$ & 1.36 & 3.35 \\
\hline 141 & $\mathrm{R}$ & 0.91 & 1.44 \\
\hline 142 & $\mathrm{R}$ & 1.71 & 3.57 \\
\hline 143 & $\mathrm{R}$ & 0.33 & 0.63 \\
\hline
\end{tabular}




\section{Modified Data (Unity Rule SOR)}

NOTE: $\quad$ Type = "S" indicates survey unit sample.

Type $=$ "R" indicates reference area sample.

\begin{tabular}{lcc} 
Sample Number & Type & Sum-of-Ratios (SOR) \\
\hline 251 & $\mathrm{~S}$ & 1.33 \\
253 & $\mathrm{~S}$ & 0.79 \\
254 & $\mathrm{~S}$ & 0.96 \\
255 & $\mathrm{~S}$ & 1.67 \\
256 & $\mathrm{~S}$ & 0.7 \\
258 & $\mathrm{~S}$ & 1.1 \\
259 & $\mathrm{~S}$ & 0.56 \\
261 & $\mathrm{~S}$ & 1.27 \\
262 & $\mathrm{~S}$ & 0.91 \\
263 & $\mathrm{~S}$ & 1.12 \\
264 & $\mathrm{~S}$ & 0.69 \\
265 & $\mathrm{~S}$ & 1.1 \\
266 & $\mathrm{~S}$ & 0.76 \\
267 & $\mathrm{~S}$ & 1.34 \\
127 & $\mathrm{R}$ & 1.4 \\
128 & $\mathrm{R}$ & 1.36 \\
129 & $\mathrm{R}$ & 1.23 \\
130 & $\mathrm{R}$ & 1.3 \\
131 & $\mathrm{R}$ & 1.32 \\
132 & $\mathrm{R}$ & 0.93 \\
133 & $\mathrm{R}$ & 1.24 \\
134 & $\mathrm{R}$ & 1.36 \\
135 & $\mathrm{R}$ & 1.05 \\
136 & $\mathrm{R}$ & 1.32 \\
137 & $\mathrm{R}$ & 1.36 \\
138 & $\mathrm{R}$ & 1.32 \\
139 & $\mathrm{R}$ & 1.49 \\
140 & $\mathrm{R}$ & 1.81 \\
141 & $\mathrm{R}$ & 0.89 \\
142 & $\mathrm{R}$ & 2.02 \\
143 & $\mathrm{R}$ & 0.37
\end{tabular}




\section{DQA Surface Soil Report}

\section{Basic Statistical Quantities Summary}

\begin{tabular}{lccc} 
Statistic & Survey Unit & Background & DQO Results \\
\hline Sample Number & 14 & 17 & N/2=18 \\
Mean (SOR) & 1.02 & 1.28 & 0.39 \\
Median (SOR) & 1.03 & 1.32 & N/A \\
Std Dev (SOR) & 0.31 & 0.36 & 0.39 \\
High Value (SOR) & 1.67 & 2.02 & N/A \\
Low Value (SOR) & 0.56 & 0.37 & N/A
\end{tabular}

\section{Statistical Test Summary}

$\begin{array}{ll}\text { Sum of Ranks: } & 496 \\ \text { Sum of Reference Ranks: } & 390 \\ \text { Critical Value: } & 313 \\ \text { Result: } & \text { Pass }\end{array}$

$\begin{array}{lll}\text { Data } & \text { Type } & \text { Adjusted Data }\end{array}$

$0.37-\mathrm{R} \quad 1.37 \quad 14$

0.89

0.93

1.05

1.23

1.24

1.3

1.32

1.32

1.32

1.36

1.36

1.36

1.4

1.49

1.81

2.02

0.56

0.69

0.7

0.76

0.79

0.91

0.96

1.1
390

1.89

1.93

2.05

2.23

2.24

2.3

2.32

2.32

2.32

2.36

2.36

2.36

2.4

2.49

2.81

3.02

0.56

0.69

0.7

0.76

0.79

0.91

0.96

1.1
Rank

Reference Rank

$\begin{array}{cc}14 & 14 \\ 16 & 16 \\ 17 & 17 \\ 18 & 18 \\ 19 & 19 \\ 20 & 20 \\ 21 & 21 \\ 23 & 23 \\ 23 & 23 \\ 23 & 23 \\ 26 & 26 \\ 26 & 26 \\ 26 & 26 \\ 28 & 28 \\ 29 & 29 \\ 30 & 30 \\ 31 & 31 \\ 1 & 0 \\ 2 & 0 \\ 3 & 0 \\ 4 & 0 \\ 5 & 0 \\ 6 & 0 \\ 7 & 0 \\ 8.5 & 0\end{array}$




\section{DQA Surface Soil Report}

\section{Statistical Test Summary}

\begin{tabular}{lcccc} 
Data & Type & Adjusted Data & Rank & Reference Rank \\
\hline 1.1 & & 1.1 & 8.5 & 0 \\
1.12 & S & 1.12 & 10 & 0 \\
1.27 & S & 1.27 & 11 & 0 \\
1.33 & S & 1.33 & 12 & 0 \\
1.34 & S & 1.34 & 13 & 0 \\
1.67 & S & 1.67 & 15 & 0
\end{tabular}




\section{DQA Surface Soil Report}

\section{Assessment Summary}

Site:

Planner(s):

Survey Unit Name:

Report Number:

Survey Unit Samples:

Reference Area Samples:

Test Performed:

Judgmental Samples:

Assessment Conclusion:
Hammond Depot FSS Planner

Vitkus

C2 SU1 through $12 \quad$ C2 SU2

2

14

17

WRS

0

Test Result: $\quad$ Pass

EMC Result: Not Performed

Reject Null Hypothesis (Survey Unit PASSES)

\section{Retrospective Power Curve}

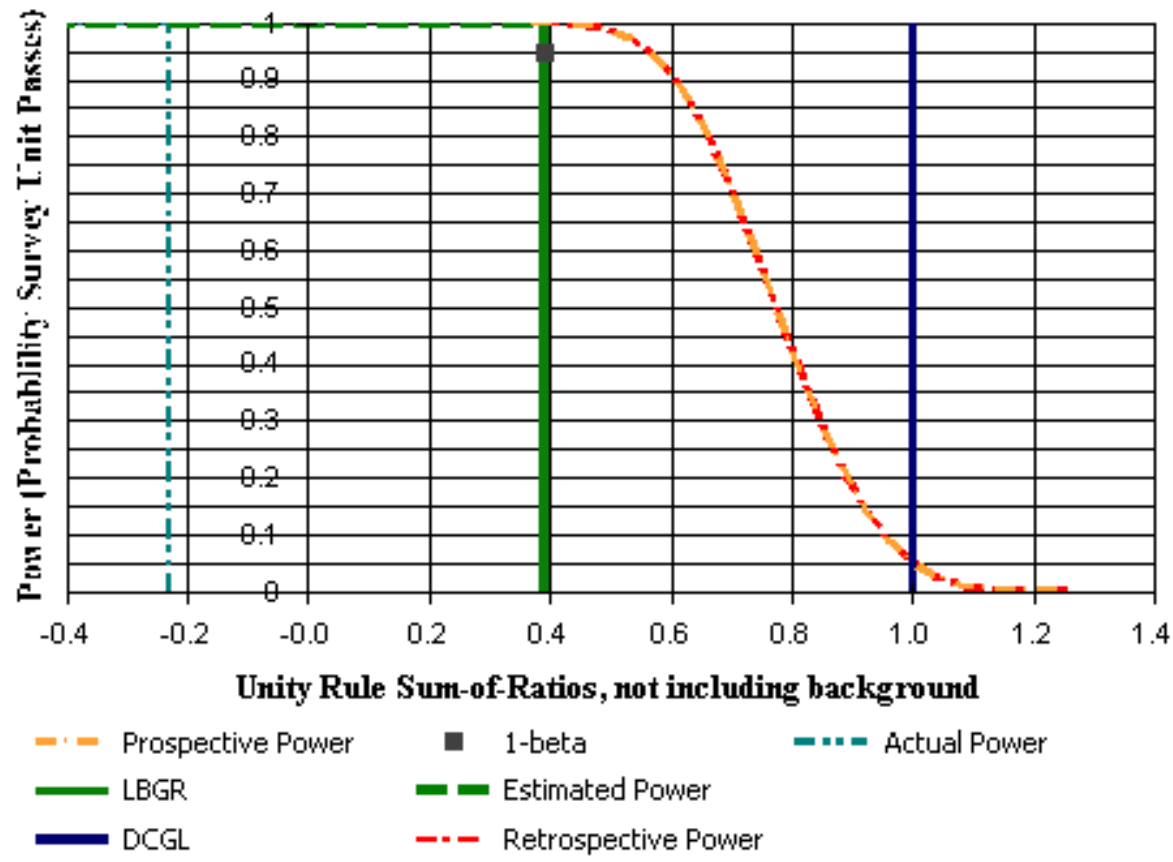




\section{DQA Surface Soil Report}

\section{Survey Unit Data}

NOTE: $\quad$ Type = "S" indicates survey unit sample.

Type = "R" indicates reference area sample.

\begin{tabular}{|c|c|c|c|}
\hline Sample Number & Type & Th-232 (pCi/g) & $\mathrm{U}-238$ (pCi/g) \\
\hline 269 & $\mathrm{~S}$ & 0.77 & 1.47 \\
\hline 270 & S & 0.87 & 1.81 \\
\hline 271 & S & 0.73 & 1.01 \\
\hline 272 & $\mathrm{~S}$ & 1.42 & 1.75 \\
\hline 273 & $S$ & 0.78 & 1.55 \\
\hline 274 & S & 0.94 & 2.1 \\
\hline 276 & $S$ & 0.96 & 2.46 \\
\hline 277 & $S$ & 1.3 & 2 \\
\hline 278 & S & 0.57 & 0.74 \\
\hline 279 & S & 1.05 & 1.62 \\
\hline 280 & S & 1.19 & 2.24 \\
\hline 281 & $S$ & 0.81 & 2.17 \\
\hline 283 & $S$ & 1.08 & 1.58 \\
\hline 284 & S & 1.03 & 2.04 \\
\hline 127 & $\mathrm{R}$ & 1.1 & 2.54 \\
\hline 128 & $\mathrm{R}$ & 0.85 & 2.66 \\
\hline 129 & $\mathrm{R}$ & 0.74 & 2.44 \\
\hline 130 & $\mathrm{R}$ & 1.09 & 2.3 \\
\hline 131 & $\mathrm{R}$ & 0.93 & 2.5 \\
\hline 132 & $\mathrm{R}$ & 0.79 & 1.65 \\
\hline 133 & $\mathrm{R}$ & 0.94 & 2.3 \\
\hline 134 & $\mathrm{R}$ & 0.98 & 2.55 \\
\hline 135 & $\mathrm{R}$ & 0.65 & 2.06 \\
\hline 136 & $\mathrm{R}$ & 0.73 & 2.66 \\
\hline 137 & $\mathrm{R}$ & 1.1 & 2.44 \\
\hline 138 & $\mathrm{R}$ & 1.03 & 2.42 \\
\hline 139 & $\mathrm{R}$ & 1.17 & 2.72 \\
\hline 140 & $\mathrm{R}$ & 1.36 & 3.35 \\
\hline 141 & $\mathrm{R}$ & 0.91 & 1.44 \\
\hline 142 & $\mathrm{R}$ & 1.71 & 3.57 \\
\hline 143 & $\mathrm{R}$ & 0.33 & 0.63 \\
\hline
\end{tabular}




\section{DQA Surface Soil Report}

\section{Modified Data (Unity Rule SOR)}

NOTE: $\quad$ Type = "S" indicates survey unit sample.

Type = "R" indicates reference area sample.

\begin{tabular}{lcc} 
Sample Number & Type & Sum-of-Ratios (SOR) \\
\hline 269 & S & 0.85 \\
270 & S & 1.02 \\
271 & S & 0.66 \\
272 & S & 1.19 \\
273 & S & 0.89 \\
274 & S & 1.16 \\
276 & S & 1.32 \\
277 & S & 1.25 \\
278 & S & 0.49 \\
279 & S & 1.01 \\
280 & S & 1.31 \\
281 & S & 1.15 \\
283 & S & 1 \\
284 & S & 1.17 \\
127 & R & 1.4 \\
128 & R & 1.36 \\
129 & R & 1.23 \\
130 & R & 1.3 \\
131 & R & 1.32 \\
132 & R & 0.93 \\
133 & R & 1.24 \\
134 & R & 1.36 \\
135 & R & 1.05 \\
136 & R & 1.32 \\
137 & $\mathrm{R}$ & 1.36 \\
138 & $\mathrm{R}$ & 1.32 \\
139 & $\mathrm{R}$ & 1.49 \\
140 & $\mathrm{R}$ & 1.81 \\
141 & $\mathrm{R}$ & 0.89 \\
142 & $\mathrm{R}$ & 2.02 \\
143 & $\mathrm{R}$ & 0.37
\end{tabular}




\section{DQA Surface Soil Report}

\section{Basic Statistical Quantities Summary}

\begin{tabular}{lccc} 
Statistic & Survey Unit & Background & DQO Results \\
\hline Sample Number & 14 & 17 & N/2=18 \\
Mean (SOR) & 1.03 & 1.28 & 0.39 \\
Median (SOR) & 1.09 & 1.32 & N/A \\
Std Dev (SOR) & 0.24 & 0.36 & 0.39 \\
High Value (SOR) & 1.32 & 2.02 & N/A \\
Low Value (SOR) & 0.49 & 0.37 & N/A
\end{tabular}

\section{Statistical Test Summary}

$\begin{array}{ll}\text { Sum of Ranks: } & 496 \\ \text { Sum of Reference Ranks: } & 391 \\ \text { Critical Value: } & 313 \\ \text { Result: } & \text { Pass }\end{array}$

$\begin{array}{lll}\text { Data } & \text { Type } & \text { Adjusted Data }\end{array}$

$0.37-\mathrm{R} \quad 1.37$

0.89

0.93

1.05

1.23

1.24

1.3

1.32

1.32

1.32

1.36

1.36

1.36

1.4

1.49

1.81

2.02

0.49

0.66

0.85

0.89

1

1.01

1.02

1.15 


\section{DQA Surface Soil Report}

Statistical Test Summary

\begin{tabular}{lcccc} 
Data & Type & Adjusted Data & Rank & Reference Rank \\
\hline 1.16 & & 1.16 & 9 & 0 \\
1.17 & S & 1.17 & 10 & 0 \\
1.19 & S & 1.19 & 11 & 0 \\
1.25 & S & 1.25 & 12 & 0 \\
1.31 & S & 1.31 & 13 & 0 \\
1.32 & S & 1.32 & 14 & 0
\end{tabular}




\section{DQA Surface Soil Report}

\section{Assessment Summary}

Site:

Planner(s):

Survey Unit Name:

Report Number:

Survey Unit Samples:

Reference Area Samples:

Test Performed:

Judgmental Samples:

Assessment Conclusion:
Hammond Depot FSS Planner

Vitkus

C2 SU1 through 12 C2 SU3

3

14

17

WRS

Test Result: $\quad$ Pass

0

EMC Result: Not Performed

Reject Null Hypothesis (Survey Unit PASSES)

\section{Retrospective Power Curve}

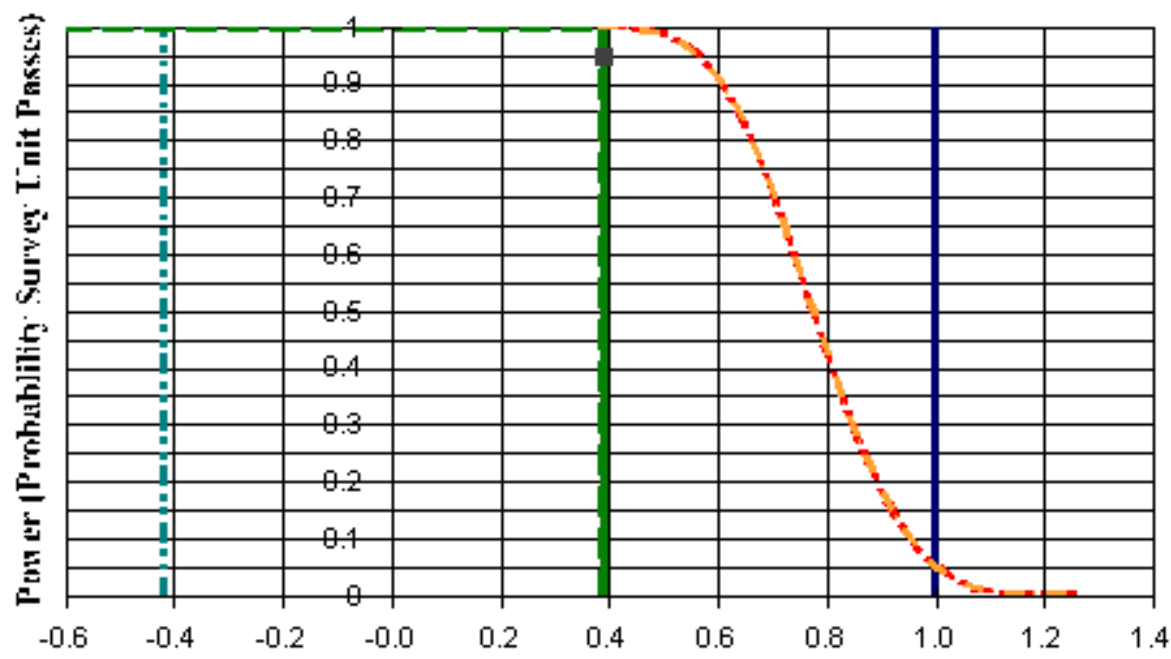

Unity Rule Sum-of-Ratios, not including background

$-\cdot=$ Prospective Power
LBGR
DCGL

- 1-beta

- Estimated Power

-- - Retrospective Power 


\section{DQA Surface Soil Report}

\section{Survey Unit Data}

NOTE: $\quad$ Type = "S" indicates survey unit sample.

Type = "R" indicates reference area sample.

\begin{tabular}{|c|c|c|c|}
\hline Sample Number & Type & Th-232 (pCi/g) & $\mathrm{U}-238$ (pCi/g) \\
\hline 285 & $\mathrm{~S}$ & 0.69 & 2.06 \\
\hline 286 & S & 0.44 & 1.12 \\
\hline 287 & S & 0.69 & 1.8 \\
\hline 288 & S & 0.75 & 1.33 \\
\hline 290 & $S$ & 0.99 & 1.6 \\
\hline 291 & S & 1.16 & 3.45 \\
\hline 292 & $S$ & 0.62 & 1.7 \\
\hline 293 & $S$ & 0.65 & 1.68 \\
\hline 294 & S & 0.75 & 1.2 \\
\hline 295 & S & 0.58 & 1.47 \\
\hline 296 & S & 0.84 & 2.06 \\
\hline 297 & S & 0.48 & 1.52 \\
\hline 298 & $S$ & 0.96 & 1.8 \\
\hline 301 & S & 0.77 & 1.36 \\
\hline 127 & $\mathrm{R}$ & 1.1 & 2.54 \\
\hline 128 & $\mathrm{R}$ & 0.85 & 2.66 \\
\hline 129 & $\mathrm{R}$ & 0.74 & 2.44 \\
\hline 130 & $\mathrm{R}$ & 1.09 & 2.3 \\
\hline 131 & $\mathrm{R}$ & 0.93 & 2.5 \\
\hline 132 & $\mathrm{R}$ & 0.79 & 1.65 \\
\hline 133 & $\mathrm{R}$ & 0.94 & 2.3 \\
\hline 134 & $\mathrm{R}$ & 0.98 & 2.55 \\
\hline 135 & $\mathrm{R}$ & 0.65 & 2.06 \\
\hline 136 & $\mathrm{R}$ & 0.73 & 2.66 \\
\hline 137 & $\mathrm{R}$ & 1.1 & 2.44 \\
\hline 138 & $\mathrm{R}$ & 1.03 & 2.42 \\
\hline 139 & $\mathrm{R}$ & 1.17 & 2.72 \\
\hline 140 & $\mathrm{R}$ & 1.36 & 3.35 \\
\hline 141 & $\mathrm{R}$ & 0.91 & 1.44 \\
\hline 142 & $\mathrm{R}$ & 1.71 & 3.57 \\
\hline 143 & $\mathrm{R}$ & 0.33 & 0.63 \\
\hline
\end{tabular}




\section{DQA Surface Soil Report}

\section{Modified Data (Unity Rule SOR)}

NOTE: $\quad$ Type = "S" indicates survey unit sample.

Type = "R" indicates reference area sample.

\begin{tabular}{lcc} 
Sample Number & Type & Sum-of-Ratios (SOR) \\
\hline 285 & S & 1.06 \\
286 & S & 0.6 \\
287 & S & 0.96 \\
288 & S & 0.79 \\
290 & S & 0.98 \\
291 & S & 1.78 \\
292 & S & 0.89 \\
293 & S & 0.9 \\
294 & S & 0.74 \\
295 & S & 0.79 \\
296 & S & 1.11 \\
297 & S & 0.77 \\
298 & S & 1.05 \\
301 & S & 0.81 \\
127 & R & 1.4 \\
128 & R & 1.36 \\
129 & R & 1.23 \\
130 & R & 1.3 \\
131 & R & 1.32 \\
132 & R & 0.93 \\
133 & R & 1.24 \\
134 & R & 1.36 \\
135 & R & 1.05 \\
136 & R & 1.32 \\
137 & $\mathrm{R}$ & 1.36 \\
138 & $\mathrm{R}$ & 1.32 \\
139 & $\mathrm{R}$ & 1.49 \\
140 & $\mathrm{R}$ & 1.81 \\
141 & $\mathrm{R}$ & 0.89 \\
142 & $\mathrm{R}$ & 2.02 \\
143 & $\mathrm{R}$ & 0.37
\end{tabular}




\section{DQA Surface Soil Report}

\section{Basic Statistical Quantities Summary}

\begin{tabular}{lccc} 
Statistic & Survey Unit & Background & DQO Results \\
\hline Sample Number & 14 & 17 & N/2=18 \\
Mean (SOR) & 0.95 & 1.28 & 0.39 \\
Median (SOR) & 0.90 & 1.32 & N/A \\
Std Dev (SOR) & 0.28 & 0.36 & 0.39 \\
High Value (SOR) & 1.78 & 2.02 & N/A \\
Low Value (SOR) & 0.60 & 0.37 & N/A
\end{tabular}

\section{Statistical Test Summary}

$\begin{array}{ll}\text { Sum of Ranks: } & 496 \\ \text { Sum of Reference Ranks: } & 390 \\ \text { Critical Value: } & 313 \\ \text { Result: } & \text { Pass }\end{array}$

$\begin{array}{lll}\text { Data } & \text { Type } & \text { Adjusted Data }\end{array}$

$0.37-\mathrm{R} \quad 1.37 \quad 14$

0.89

0.93

1.05

1.23

1.24

1.3

1.32

1.32

1.32

1.36

1.36

1.36

1.4

1.49

1.81

2.02

0.6

0.74

0.77

0.79

0.79

0.81

0.89

0.9
390

13

Pass

1.89

1.93

2.05

2.23

2.24

2.3

2.32

2.32

2.32

2.36

2.36

2.36

2.4

2.49

2.81

3.02

0.6

0.74

0.77

0.79

0.79

0.81

0.89

0.9
Rank

Reference Rank

$\begin{array}{cc}14 & 14 \\ 16 & 16 \\ 17 & 17 \\ 18 & 18 \\ 19 & 19 \\ 20 & 20 \\ 21 & 21 \\ 23 & 23 \\ 23 & 23 \\ 23 & 23 \\ 26 & 26 \\ 26 & 26 \\ 26 & 26 \\ 28 & 28 \\ 29 & 29 \\ 30 & 30 \\ 31 & 31 \\ 1 & 0 \\ 2 & 0 \\ 3 & 0 \\ 4.5 & 0 \\ 4.5 & 0 \\ 6 & 0 \\ 7 & 0 \\ 8 & 0\end{array}$




\section{DQA Surface Soil Report}

Statistical Test Summary

\begin{tabular}{lcccc} 
Data & Type & Adjusted Data & Rank & Reference Rank \\
\hline 0.96 & & & & 0 \\
0.98 & S & 0.96 & 10 & 0 \\
1.05 & S & 0.98 & 11 & 0 \\
1.06 & S & 1.05 & 12 & 0 \\
1.11 & S & 1.06 & 13 & 0 \\
1.78 & S & 1.11 & 15 & 0
\end{tabular}




\section{DQA Surface Soil Report}

\section{Assessment Summary}

Site:

Planner(s):

Survey Unit Name:

Report Number:

Survey Unit Samples:

Reference Area Samples:

Test Performed:

Judgmental Samples:

Assessment Conclusion:
Hammond Depot FSS Planner

Vitkus

C2 SU1 through $12 \quad$ C2 SU4

5

16

17

WRS

Test Result: $\quad$ Pass

0

EMC Result:

Not Performed

Reject Null Hypothesis (Survey Unit PASSES)

\section{Retrospective Power Curve}

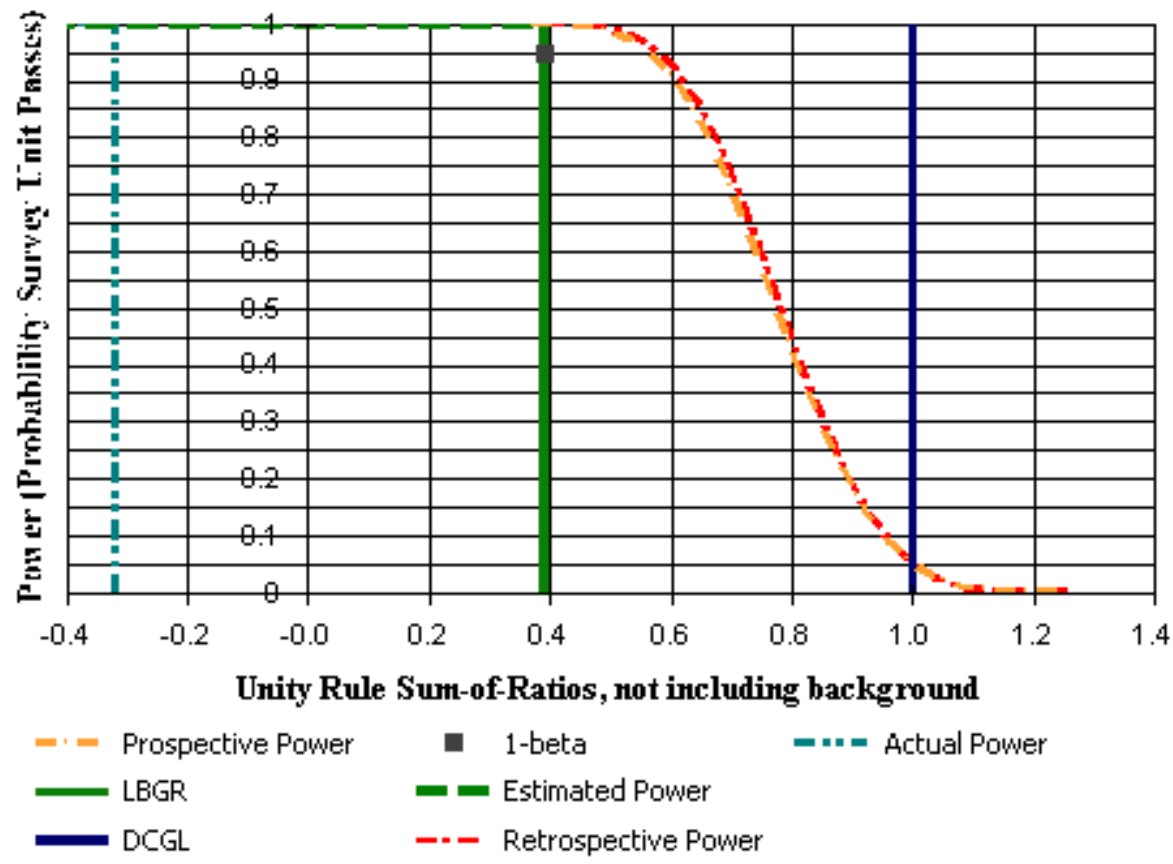




\section{DQA Surface Soil Report}

\section{Survey Unit Data}

NOTE: $\quad$ Type = "S" indicates survey unit sample.

Type = "R" indicates reference area sample.

\begin{tabular}{|c|c|c|c|}
\hline Sample Number & Type & Th-232 (pCi/g) & U-238 (pCi/g) \\
\hline 302 & $\mathrm{~S}$ & 0.86 & 1.2 \\
\hline 303 & $S$ & 0.94 & 1.92 \\
\hline 304 & S & 0.88 & 2.8 \\
\hline 305 & S & 0.89 & 1.81 \\
\hline 306 & S & 0.84 & 1.58 \\
\hline 307 & $S$ & 0.68 & 2.35 \\
\hline 308 & $S$ & 1.18 & 1.72 \\
\hline 309 & $S$ & 0.96 & 1.47 \\
\hline 310 & $S$ & 0.78 & 2.15 \\
\hline 311 & S & 0.88 & 2.48 \\
\hline 312 & S & 0.83 & 2 \\
\hline 313 & S & 0.81 & 1.65 \\
\hline 315 & S & 0.28 & 0.57 \\
\hline 316 & S & 0.71 & 1.79 \\
\hline 317 & S & 1.01 & 1.53 \\
\hline 318 & S & 1.08 & 1.35 \\
\hline 127 & $\mathrm{R}$ & 1.1 & 2.54 \\
\hline 128 & $\mathrm{R}$ & 0.85 & 2.66 \\
\hline 129 & $\mathrm{R}$ & 0.74 & 2.44 \\
\hline 130 & $\mathrm{R}$ & 1.09 & 2.3 \\
\hline 131 & $\mathrm{R}$ & 0.93 & 2.5 \\
\hline 132 & $\mathrm{R}$ & 0.79 & 1.65 \\
\hline 133 & $\mathrm{R}$ & 0.94 & 2.3 \\
\hline 134 & $\mathrm{R}$ & 0.98 & 2.55 \\
\hline 135 & $\mathrm{R}$ & 0.65 & 2.06 \\
\hline 136 & $\mathrm{R}$ & 0.73 & 2.66 \\
\hline 137 & $\mathrm{R}$ & 1.1 & 2.44 \\
\hline 138 & $\mathrm{R}$ & 1.03 & 2.42 \\
\hline 139 & $\mathrm{R}$ & 1.17 & 2.72 \\
\hline 140 & $\mathrm{R}$ & 1.36 & 3.35 \\
\hline 141 & $\mathrm{R}$ & 0.91 & 1.44 \\
\hline 142 & $\mathrm{R}$ & 1.71 & 3.57 \\
\hline 143 & $\mathrm{R}$ & 0.33 & 0.63 \\
\hline
\end{tabular}




\section{DQA Surface Soil Report}

\section{Modified Data (Unity Rule SOR)}

NOTE: $\quad$ Type = "S" indicates survey unit sample.

Type = "R" indicates reference area sample.

\begin{tabular}{lcc} 
Sample Number & Type & Sum-of-Ratios (SOR) \\
\hline 302 & S & 0.78 \\
303 & S & 1.09 \\
304 & S & 1.42 \\
305 & S & 1.03 \\
306 & S & 0.92 \\
307 & S & 1.17 \\
308 & S & 1.09 \\
309 & S & 0.92 \\
310 & S & 1.13 \\
311 & S & 1.3 \\
312 & S & 1.09 \\
313 & S & 0.94 \\
315 & S & 0.32 \\
316 & S & 0.96 \\
317 & S & 0.96 \\
318 & S & 0.91 \\
127 & R & 1.4 \\
128 & R & 1.36 \\
129 & R & 1.23 \\
130 & R & 1.3 \\
131 & R & 1.32 \\
132 & R & 0.93 \\
133 & $\mathrm{R}$ & 1.24 \\
134 & $\mathrm{R}$ & 1.36 \\
135 & $\mathrm{R}$ & 1.05 \\
136 & $\mathrm{R}$ & 1.32 \\
137 & $\mathrm{R}$ & 1.36 \\
138 & $\mathrm{R}$ & 1.32 \\
139 & $\mathrm{R}$ & 1.49 \\
140 & $\mathrm{R}$ & 1.81 \\
141 & $\mathrm{R}$ & 0.89 \\
142 & $\mathrm{R}$ & 2.02 \\
143 & $\mathrm{R}$ & 0.37 \\
& &
\end{tabular}




\section{DQA Surface Soil Report}

\section{Basic Statistical Quantities Summary}

\begin{tabular}{lccc} 
Statistic & Survey Unit & Background & DQO Results \\
\hline Sample Number & 16 & 17 & N/2=18 \\
Mean (SOR) & 1.00 & 1.28 & 0.39 \\
Median (SOR) & 1.00 & 1.32 & N/A \\
Std Dev (SOR) & 0.24 & 0.36 & 0.39 \\
High Value (SOR) & 1.42 & 2.02 & N/A \\
Low Value (SOR) & 0.32 & 0.37 & N/A
\end{tabular}

\section{Statistical Test Summary}

$\begin{array}{ll}\text { Sum of Ranks: } & 561 \\ \text { Sum of Reference Ranks: } & 424 \\ \text { Critical Value: } & 335 \\ \text { Result: } & \text { Pass }\end{array}$

\begin{tabular}{lcccc} 
Data & Type & Adjusted Data & Rank & Reference \\
\hline & & & 16 & 16 \\
0.37 & $\mathrm{R}$ & 1.37 & 18 & 18 \\
0.89 & $\mathrm{R}$ & 1.89 & 19 & 19 \\
0.93 & $\mathrm{R}$ & 1.93 & 20 & 20 \\
1.05 & $\mathrm{R}$ & 2.05 & 21 & 21 \\
1.23 & $\mathrm{R}$ & 2.23 & 22 & 22 \\
1.24 & $\mathrm{R}$ & 2.24 & 23 & 23 \\
1.3 & $\mathrm{R}$ & 2.3 & 25 & 25 \\
1.32 & $\mathrm{R}$ & 2.32 & 25 & 25 \\
1.32 & $\mathrm{R}$ & 2.32 & 25 & 25 \\
1.32 & $\mathrm{R}$ & 2.32 & 28 & 28 \\
1.36 & $\mathrm{R}$ & 2.36 & 28 & 28 \\
1.36 & $\mathrm{R}$ & 2.36 & 28 & 28 \\
1.36 & $\mathrm{R}$ & 2.36 & 30 & 30 \\
1.4 & $\mathrm{R}$ & 2.4 & 31 & 31 \\
1.49 & $\mathrm{R}$ & 2.49 & 32 & 32 \\
1.81 & $\mathrm{R}$ & 2.81 & 33 & 33 \\
2.02 & $\mathrm{R}$ & 3.02 & 1 & 0 \\
0.32 & $\mathrm{~S}$ & 0.32 & 2 & 0 \\
0.78 & $\mathrm{~S}$ & 0.78 & 3 & 0 \\
0.91 & $\mathrm{~S}$ & 0.91 & 4.5 & 0 \\
0.92 & $\mathrm{~S}$ & 0.92 & 4.5 & 0 \\
0.92 & $\mathrm{~S}$ & 0.92 & 7.5 & 0 \\
0.94 & $\mathrm{~S}$ & 0.94 & 7.5 & 0 \\
0.96 & $\mathrm{~S}$ & 0.96 & & 0 \\
0.96 & $\mathrm{~S}$ & 0.96 & & 0
\end{tabular}




\section{DQA Surface Soil Report}

\section{Statistical Test Summary}

\begin{tabular}{lcccc} 
Data & Type & Adjusted Data & Rank & Reference Rank \\
\hline 1.03 & & & & 0 \\
1.09 & S & 1.03 & 11 & 0 \\
1.09 & S & 1.09 & 11 & 0 \\
1.09 & S & 1.09 & 11 & 0 \\
1.13 & S & 1.09 & 13 & 0 \\
1.17 & S & 1.13 & 14 & 0 \\
1.3 & S & 1.17 & 15 & 0 \\
1.42 & S & 1.3 & 17 & 0
\end{tabular}




\section{DQA Surface Soil Report}

\section{Assessment Summary}

Site:

Planner(s):

Survey Unit Name:

Report Number:

Survey Unit Samples:

Reference Area Samples:

Test Performed:

Judgmental Samples:

Assessment Conclusion:
Hammond Depot FSS Planner

Vitkus

C2 SU1 through 12 C2 SU5

6

13

17

WRS

Test Result: $\quad$ Pass

0

EMC Result:

Not Performed

Reject Null Hypothesis (Survey Unit PASSES)

\section{Retrospective Power Curve}

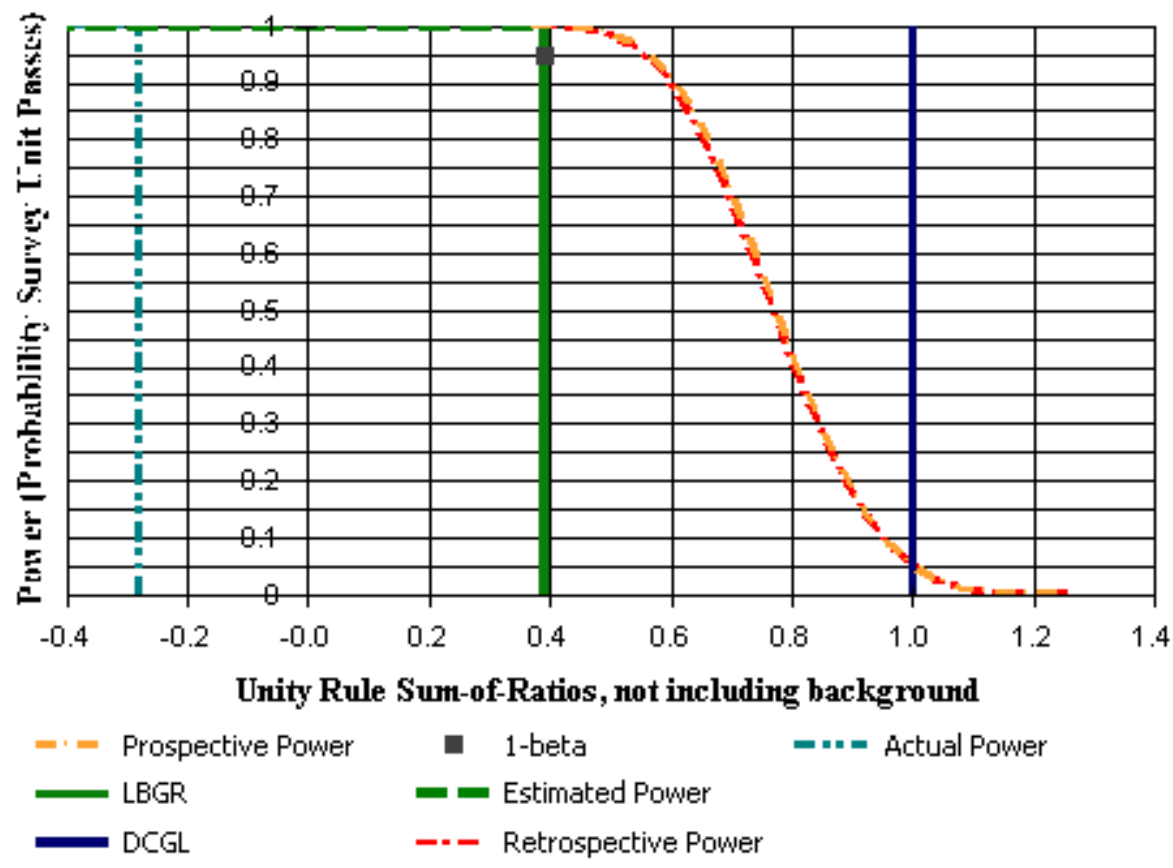




\section{DQA Surface Soil Report}

\section{Survey Unit Data}

NOTE: $\quad$ Type = "S" indicates survey unit sample.

Type = "R" indicates reference area sample.

\begin{tabular}{|c|c|c|c|}
\hline Sample Number & Type & Th-232 (pCi/g) & $\mathrm{U}-238$ (pCi/g) \\
\hline 319 & $\mathrm{~S}$ & 0.82 & 2 \\
\hline 320 & $S$ & 0.63 & 1.31 \\
\hline 324 & S & 0.55 & 0.56 \\
\hline 325 & S & 0.99 & 2.85 \\
\hline 326 & S & 0.64 & 1.43 \\
\hline 327 & S & 0.85 & 2.1 \\
\hline 328 & $S$ & 0.42 & 0.45 \\
\hline 329 & $S$ & 0.96 & 2.01 \\
\hline 331 & S & 0.57 & 0.78 \\
\hline 332 & S & 0.7 & 1.37 \\
\hline 333 & S & 1.02 & 2.43 \\
\hline 334 & S & 0.82 & 2.05 \\
\hline 335 & S & 0.73 & 1.97 \\
\hline 127 & $\mathrm{R}$ & 1.1 & 2.54 \\
\hline 128 & $\mathrm{R}$ & 0.85 & 2.66 \\
\hline 129 & $\mathrm{R}$ & 0.74 & 2.44 \\
\hline 130 & $\mathrm{R}$ & 1.09 & 2.3 \\
\hline 131 & $\mathrm{R}$ & 0.93 & 2.5 \\
\hline 132 & $\mathrm{R}$ & 0.79 & 1.65 \\
\hline 133 & $\mathrm{R}$ & 0.94 & 2.3 \\
\hline 134 & $\mathrm{R}$ & 0.98 & 2.55 \\
\hline 135 & $\mathrm{R}$ & 0.65 & 2.06 \\
\hline 136 & $\mathrm{R}$ & 0.73 & 2.66 \\
\hline 137 & $\mathrm{R}$ & 1.1 & 2.44 \\
\hline 138 & $\mathrm{R}$ & 1.03 & 2.42 \\
\hline 139 & $\mathrm{R}$ & 1.17 & 2.72 \\
\hline 140 & $\mathrm{R}$ & 1.36 & 3.35 \\
\hline 141 & $\mathrm{R}$ & 0.91 & 1.44 \\
\hline 142 & $\mathrm{R}$ & 1.71 & 3.57 \\
\hline 143 & $\mathrm{R}$ & 0.33 & 0.63 \\
\hline
\end{tabular}




\section{DQA Surface Soil Report}

\section{Modified Data (Unity Rule SOR)}

NOTE: $\quad$ Type = "S" indicates survey unit sample.

Type = "R" indicates reference area sample.

\begin{tabular}{|c|c|c|}
\hline Sample Number & Type & Sum-of-Ratios (SOR) \\
\hline 319 & $\mathrm{~S}$ & 1.08 \\
\hline 320 & $S$ & 0.74 \\
\hline 324 & $\mathrm{~S}$ & 0.41 \\
\hline 325 & $\mathrm{~S}$ & 1.48 \\
\hline 326 & $S$ & 0.79 \\
\hline 327 & $S$ & 1.13 \\
\hline 328 & $S$ & 0.32 \\
\hline 329 & $\mathrm{~S}$ & 1.14 \\
\hline 331 & $\mathrm{~S}$ & 0.51 \\
\hline 332 & $\mathrm{~S}$ & 0.79 \\
\hline 333 & $\mathrm{~S}$ & 1.32 \\
\hline 334 & $S$ & 1.1 \\
\hline 335 & $S$ & 1.04 \\
\hline 127 & $\mathrm{R}$ & 1.4 \\
\hline 128 & $\mathrm{R}$ & 1.36 \\
\hline 129 & $\mathrm{R}$ & 1.23 \\
\hline 130 & $\mathrm{R}$ & 1.3 \\
\hline 131 & $\mathrm{R}$ & 1.32 \\
\hline 132 & $\mathrm{R}$ & 0.93 \\
\hline 133 & $\mathrm{R}$ & 1.24 \\
\hline 134 & $\mathrm{R}$ & 1.36 \\
\hline 135 & $\mathrm{R}$ & 1.05 \\
\hline 136 & $\mathrm{R}$ & 1.32 \\
\hline 137 & $\mathrm{R}$ & 1.36 \\
\hline 138 & $\mathrm{R}$ & 1.32 \\
\hline 139 & $\mathrm{R}$ & 1.49 \\
\hline 140 & $\mathrm{R}$ & 1.81 \\
\hline 141 & $\mathrm{R}$ & 0.89 \\
\hline 142 & $\mathrm{R}$ & 2.02 \\
\hline 143 & $\mathrm{R}$ & 0.37 \\
\hline
\end{tabular}




\section{DQA Surface Soil Report}

\section{Basic Statistical Quantities Summary}

\begin{tabular}{lccc} 
Statistic & Survey Unit & Background & DQO Results \\
\hline Sample Number & 13 & 17 & N/2=18 \\
Mean (SOR) & 0.91 & 1.28 & 0.39 \\
Median (SOR) & 1.04 & 1.32 & N/A \\
Std Dev (SOR) & 0.35 & 0.36 & 0.39 \\
High Value (SOR) & 1.48 & 2.02 & N/A \\
Low Value (SOR) & 0.32 & 0.37 & N/A
\end{tabular}

\section{Statistical Test Summary}

$\begin{array}{ll}\text { Sum of Ranks: } & 465 \\ \text { Sum of Reference Ranks: } & 373 \\ \text { Critical Value: } & 303 \\ \text { Result: } & \text { Pass }\end{array}$

$\begin{array}{lll}\text { Data } & \text { Type } & \text { Adjusted Data }\end{array}$

Reference Rank

0.37

0.89

0.93

1.05

1.23

1.24

1.3

1.32

1.32

1.32

1.36

1.36

1.36

1.4

1.49

1.81

2.02

0.32

0.41

0.51

0.74

0.79

0.79

1.04

1.08

$\begin{array}{lc}\mathrm{R} & 1.37 \\ \mathrm{R} & 1.89 \\ \mathrm{R} & 1.93 \\ \mathrm{R} & 2.05 \\ \mathrm{R} & 2.23 \\ \mathrm{R} & 2.24 \\ \mathrm{R} & 2.3 \\ \mathrm{R} & 2.32 \\ \mathrm{R} & 2.32 \\ \mathrm{R} & 2.32 \\ \mathrm{R} & 2.36 \\ \mathrm{R} & 2.36 \\ \mathrm{R} & 2.36 \\ \mathrm{R} & 2.4 \\ \mathrm{R} & 2.49 \\ \mathrm{R} & 2.81 \\ \mathrm{R} & 3.02 \\ \mathrm{~S} & 0.32 \\ \mathrm{~S} & 0.41 \\ \mathrm{~S} & 0.51 \\ \mathrm{~S} & 0.74 \\ \mathrm{~S} & 0.79 \\ \mathrm{~S} & 0.79 \\ \mathrm{~S} & 1.04 \\ \mathrm{~S} & 1.08\end{array}$

1.37

1.89

2.05

2.23

2.24

2.32

2.32

2.32

2.36

2.36

2.4

2.49

3.02

0.32

0.41

0.51

0.74

0.79

1.08

\section{3}

15

16

17

18

19

20

22

22

22
25
25

25

25

27

28

29

30

1

2

3

4
5.5

5.5

7
13
15
16
17
18
19
20
22
22
22
25
25
25
27
28
29
30
0
0
0
0
0
0
0
0 


\section{DQA Surface Soil Report}

\section{Statistical Test Summary}

\begin{tabular}{lcccc} 
Data & Type & Adjusted Data & Rank & Reference Rank \\
\hline 1.1 & & & 9 & 0 \\
1.13 & S & 1.1 & 10 & 0 \\
1.14 & S & 1.13 & 11 & 0 \\
1.32 & S & 1.14 & 12 & 0 \\
1.48 & S & 1.32 & 14 & 0
\end{tabular}




\section{DQA Surface Soil Report}

\section{Assessment Summary}

Site:

Planner(s):

Survey Unit Name:

Report Number:

Survey Unit Samples:

Reference Area Samples:

Test Performed:

Judgmental Samples:

Assessment Conclusion:
Hammond Depot FSS Planner

Vitkus

C2 SU1 through $12 \quad$ C2 SU6

4

13

17

WRS

Test Result: $\quad$ Pass

0

EMC Result:

Not Performed

Reject Null Hypothesis (Survey Unit PASSES)

\section{Retrospective Power Curve}

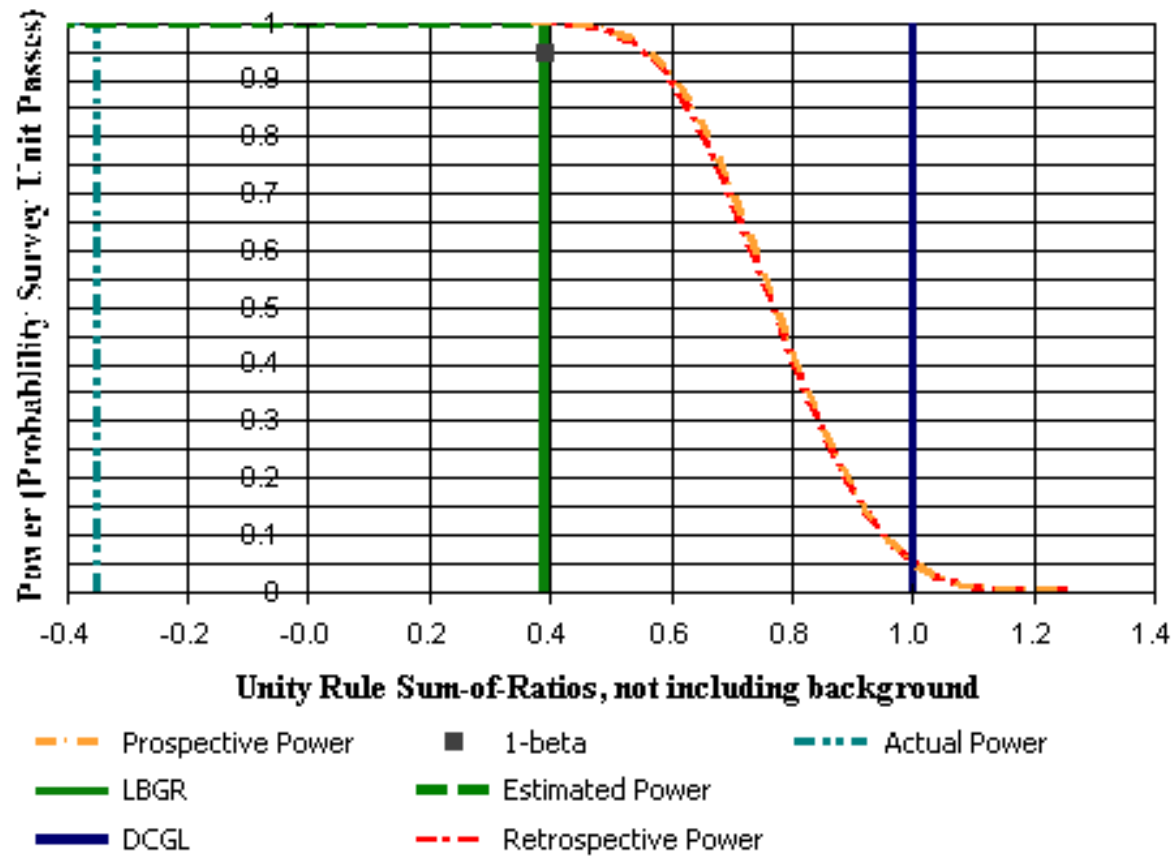




\section{DQA Surface Soil Report}

\section{Survey Unit Data}

NOTE: $\quad$ Type = "S" indicates survey unit sample.

Type = "R" indicates reference area sample.

\begin{tabular}{lccc} 
Sample Number & Type & Th-232 (pCi/g) & U-238 (pCi/g) \\
\hline 336 & $\mathrm{~S}$ & 0.42 & 0.77 \\
339 & $\mathrm{~S}$ & 0.57 & 1.34 \\
341 & $\mathrm{~S}$ & 0.58 & 1.41 \\
342 & $\mathrm{~S}$ & 1.42 & 1.73 \\
343 & $\mathrm{~S}$ & 0.87 & 2.59 \\
344 & $\mathrm{~S}$ & 0.86 & 1.69 \\
345 & $\mathrm{~S}$ & 0.65 & 1.7 \\
347 & $\mathrm{~S}$ & 0.66 & 1.35 \\
348 & $\mathrm{~S}$ & 0.95 & 2.58 \\
349 & $\mathrm{~S}$ & 0.73 & 1.64 \\
350 & $\mathrm{~S}$ & 1.06 & 1.51 \\
351 & $\mathrm{~S}$ & 0.9 & 2.16 \\
352 & $\mathrm{~S}$ & 0.99 & 1.9 \\
127 & $\mathrm{R}$ & 1.1 & 2.54 \\
128 & $\mathrm{R}$ & 0.85 & 2.66 \\
129 & $\mathrm{R}$ & 0.74 & 2.44 \\
130 & $\mathrm{R}$ & 1.09 & 2.3 \\
131 & $\mathrm{R}$ & 0.93 & 2.5 \\
132 & $\mathrm{R}$ & 0.79 & 1.65 \\
133 & $\mathrm{R}$ & 0.94 & 2.3 \\
134 & $\mathrm{R}$ & 0.98 & 2.55 \\
135 & $\mathrm{R}$ & 0.65 & 2.06 \\
136 & $\mathrm{R}$ & 0.73 & 2.66 \\
137 & $\mathrm{R}$ & 1.1 & 2.44 \\
138 & $\mathrm{R}$ & 1.03 & 2.42 \\
139 & $\mathrm{R}$ & 1.17 & 2.72 \\
140 & $\mathrm{R}$ & 1.36 & 3.35 \\
141 & $\mathrm{R}$ & 0.91 & 1.44 \\
142 & $\mathrm{R}$ & 1.71 & 3.57 \\
143 & $\mathrm{R}$ & 0.33 & 0.63 \\
& & &
\end{tabular}




\section{Modified Data (Unity Rule SOR)}

NOTE: $\quad$ Type = "S" indicates survey unit sample.

Type $=$ "R" indicates reference area sample.

\begin{tabular}{lcc} 
Sample Number & Type & Sum-of-Ratios (SOR) \\
\hline 336 & $\mathrm{~S}$ & 0.45 \\
339 & $\mathrm{~S}$ & 0.73 \\
341 & $\mathrm{~S}$ & 0.76 \\
342 & $\mathrm{~S}$ & 1.18 \\
343 & $\mathrm{~S}$ & 1.34 \\
344 & $\mathrm{~S}$ & 0.97 \\
345 & $\mathrm{~S}$ & 0.9 \\
347 & $\mathrm{~S}$ & 0.77 \\
348 & $\mathrm{~S}$ & 1.36 \\
349 & $\mathrm{~S}$ & 0.91 \\
350 & $\mathrm{~S}$ & 0.97 \\
351 & $\mathrm{~S}$ & 1.17 \\
352 & $\mathrm{~S}$ & 1.1 \\
127 & $\mathrm{R}$ & 1.4 \\
128 & $\mathrm{R}$ & 1.36 \\
129 & $\mathrm{R}$ & 1.23 \\
130 & $\mathrm{R}$ & 1.3 \\
131 & $\mathrm{R}$ & 1.32 \\
132 & $\mathrm{R}$ & 0.93 \\
133 & $\mathrm{R}$ & 1.24 \\
134 & $\mathrm{R}$ & 1.36 \\
135 & $\mathrm{R}$ & 1.05 \\
136 & $\mathrm{R}$ & 1.32 \\
137 & $\mathrm{R}$ & 1.36 \\
138 & $\mathrm{R}$ & 1.32 \\
139 & $\mathrm{R}$ & 1.49 \\
140 & $\mathrm{R}$ & 1.81 \\
141 & $\mathrm{R}$ & 0.89 \\
142 & $\mathrm{R}$ & 2.02 \\
143 & $\mathrm{R}$ & 0.37
\end{tabular}




\section{DQA Surface Soil Report}

\section{Basic Statistical Quantities Summary}

\begin{tabular}{lccc} 
Statistic & Survey Unit & Background & DQO Results \\
\hline Sample Number & 13 & 17 & N/2=18 \\
Mean (SOR) & 0.97 & 1.28 & 0.39 \\
Median (SOR) & 0.97 & 1.32 & N/A \\
Std Dev (SOR) & 0.26 & 0.36 & 0.39 \\
High Value (SOR) & 1.36 & 2.02 & N/A \\
Low Value (SOR) & 0.45 & 0.37 & N/A
\end{tabular}

\section{Statistical Test Summary}

$\begin{array}{ll}\text { Sum of Ranks: } & 465 \\ \text { Sum of Reference Ranks: } & 374 \\ \text { Critical Value: } & 303 \\ \text { Result: } & \text { Pass }\end{array}$

$\begin{array}{lll}\text { Data } & \text { Type } & \text { Adjusted Data }\end{array}$

Reference Rank

0.37

0.89

0.93

1.05

1.23

1.24

1.3

1.32

1.32

1.32

1.36

1.36

1.36

1.4

1.49

1.81

2.02

0.45

0.73

0.76

0.77

0.9

0.91

0.97

0.97

$\begin{array}{lc}\mathrm{R} & 1.37 \\ \mathrm{R} & 1.89 \\ \mathrm{R} & 1.93 \\ \mathrm{R} & 2.05 \\ \mathrm{R} & 2.23 \\ \mathrm{R} & 2.24 \\ \mathrm{R} & 2.3 \\ \mathrm{R} & 2.32 \\ \mathrm{R} & 2.32 \\ \mathrm{R} & 2.32 \\ \mathrm{R} & 2.36 \\ \mathrm{R} & 2.36 \\ \mathrm{R} & 2.36 \\ \mathrm{R} & 2.4 \\ \mathrm{R} & 2.49 \\ \mathrm{R} & 2.81 \\ \mathrm{R} & 3.02 \\ \mathrm{~S} & 0.45 \\ \mathrm{~S} & 0.73 \\ \mathrm{~S} & 0.76 \\ \mathrm{~S} & 0.77 \\ \mathrm{~S} & 0.9 \\ \mathrm{~S} & 0.91 \\ \mathrm{~S} & 0.97 \\ \mathrm{~S} & 0.97\end{array}$

1.37

189

2.05

2.23

2.24

2.32

2.32

2.32

2.36

2.36

2.4

2.49

3.02

0.45

0.73

0.76

0.77

0.91

0.97

0.97

$\begin{array}{cc}14 & 14 \\ 15 & 15 \\ 16 & 16 \\ 17 & 17 \\ 18 & 18 \\ 19 & 19 \\ 20 & 20 \\ 22 & 22 \\ 22 & 22 \\ 22 & 22 \\ 25 & 25 \\ 25 & 25 \\ 25 & 25 \\ 27 & 27 \\ 28 & 28 \\ 29 & 29 \\ 30 & 30 \\ 1 & 0 \\ 2 & 0 \\ 3 & 0 \\ 4 & 0 \\ 5 & 0 \\ 6 & 0 \\ 7.5 & 0 \\ 7.5 & 0\end{array}$




\section{DQA Surface Soil Report}

\section{Statistical Test Summary}

\begin{tabular}{lcccc} 
Data & Type & Adjusted Data & Rank & Reference Rank \\
\hline 1.1 & & & 9 & 0 \\
1.17 & S & 1.1 & 10 & 0 \\
1.18 & S & 1.17 & 11 & 0 \\
1.34 & S & 1.18 & 12 & 0 \\
1.36 & S & 1.34 & 13 & 0
\end{tabular}




\section{DQA Surface Soil Report}

\section{Assessment Summary}

Site:

Planner(s):

Survey Unit Name:

Report Number:

Survey Unit Samples:

Reference Area Samples:

Test Performed:

Judgmental Samples:

Assessment Conclusion:
Hammond Depot FSS Planner

Vitkus

C2 SU1 through $12 \quad$ C2 SU7

11

14

17

WRS

Test Result: $\quad$ Pass

0

EMC Result: Not Performed

Reject Null Hypothesis (Survey Unit PASSES)

\section{Retrospective Power Curve}

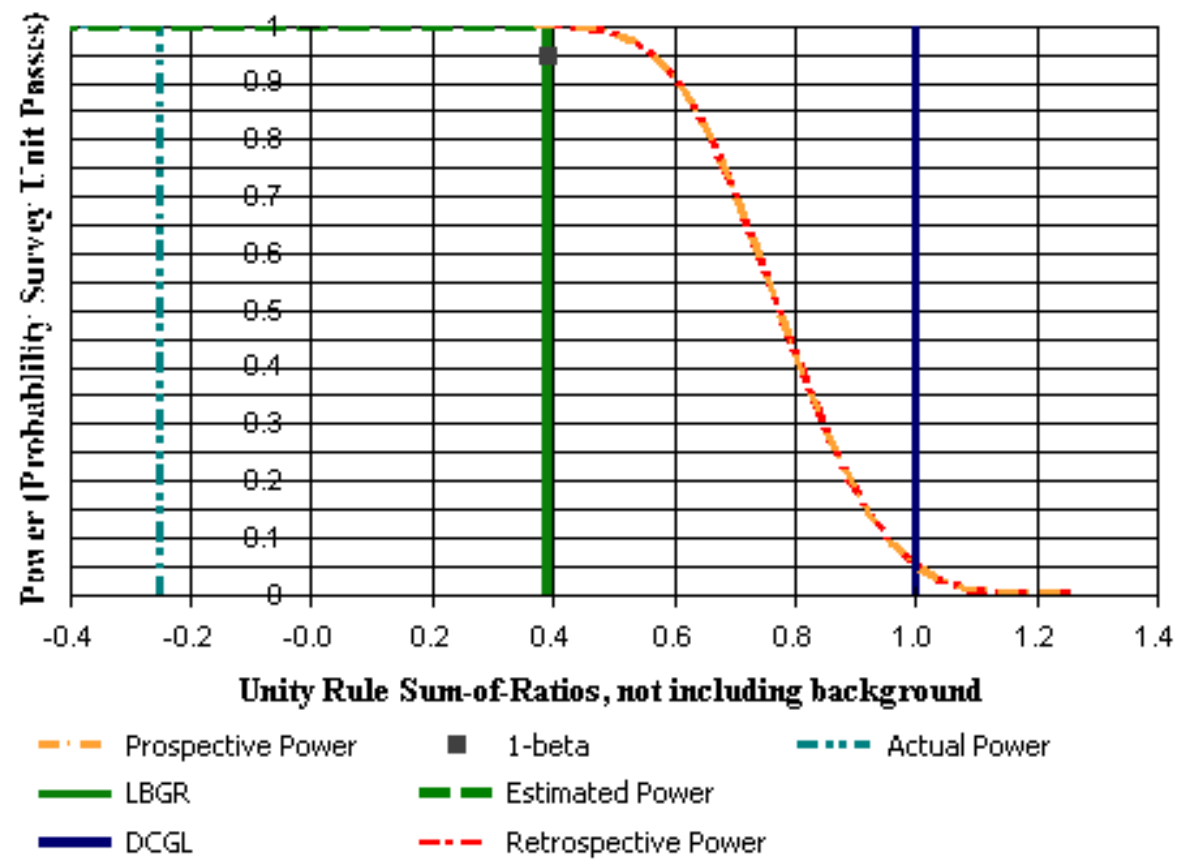




\section{DQA Surface Soil Report}

\section{Survey Unit Data}

NOTE: $\quad$ Type = "S" indicates survey unit sample.

Type = "R" indicates reference area sample.

\begin{tabular}{|c|c|c|c|}
\hline Sample Number & Type & Th-232 (pCi/g) & $\mathrm{U}-238$ (pCi/g) \\
\hline 353 & $\mathrm{~S}$ & 0.67 & 1.58 \\
\hline 356 & S & 0.84 & 1.14 \\
\hline 357 & S & 1.23 & 2.16 \\
\hline 359 & S & 1 & 1.78 \\
\hline 360 & $S$ & 1.05 & 1.96 \\
\hline 361 & S & 1.11 & 3.07 \\
\hline 362 & $S$ & 0.45 & 0.93 \\
\hline 363 & $S$ & 1.09 & 2.28 \\
\hline 364 & S & 0.98 & 2.26 \\
\hline 365 & S & 0.94 & 1.89 \\
\hline 366 & S & 0.96 & 3.35 \\
\hline 367 & $S$ & 0.78 & 1.28 \\
\hline 368 & $S$ & 0.75 & 1.75 \\
\hline 369 & S & 1.03 & 1.72 \\
\hline 127 & $\mathrm{R}$ & 1.1 & 2.54 \\
\hline 128 & $\mathrm{R}$ & 0.85 & 2.66 \\
\hline 129 & $\mathrm{R}$ & 0.74 & 2.44 \\
\hline 130 & $\mathrm{R}$ & 1.09 & 2.3 \\
\hline 131 & $\mathrm{R}$ & 0.93 & 2.5 \\
\hline 132 & $\mathrm{R}$ & 0.79 & 1.65 \\
\hline 133 & $\mathrm{R}$ & 0.94 & 2.3 \\
\hline 134 & $\mathrm{R}$ & 0.98 & 2.55 \\
\hline 135 & $\mathrm{R}$ & 0.65 & 2.06 \\
\hline 136 & $\mathrm{R}$ & 0.73 & 2.66 \\
\hline 137 & $\mathrm{R}$ & 1.1 & 2.44 \\
\hline 138 & $\mathrm{R}$ & 1.03 & 2.42 \\
\hline 139 & $\mathrm{R}$ & 1.17 & 2.72 \\
\hline 140 & $\mathrm{R}$ & 1.36 & 3.35 \\
\hline 141 & $\mathrm{R}$ & 0.91 & 1.44 \\
\hline 142 & $\mathrm{R}$ & 1.71 & 3.57 \\
\hline 143 & $\mathrm{R}$ & 0.33 & 0.63 \\
\hline
\end{tabular}




\section{DQA Surface Soil Report}

\section{Modified Data (Unity Rule SOR)}

NOTE: $\quad$ Type = "S" indicates survey unit sample.

Type = "R" indicates reference area sample.

\begin{tabular}{lcc} 
Sample Number & Type & Sum-of-Ratios (SOR) \\
\hline 353 & S & 0.86 \\
356 & S & 0.75 \\
357 & S & 1.29 \\
359 & S & 1.06 \\
360 & S & 1.15 \\
361 & S & 1.61 \\
362 & S & 0.53 \\
363 & S & 1.29 \\
364 & S & 1.24 \\
365 & S & 1.08 \\
366 & S & 1.67 \\
367 & S & 0.78 \\
368 & S & 0.96 \\
369 & S & 1.04 \\
127 & R & 1.4 \\
128 & R & 1.36 \\
129 & R & 1.23 \\
130 & R & 1.3 \\
131 & R & 1.32 \\
132 & R & 0.93 \\
133 & R & 1.24 \\
134 & R & 1.36 \\
135 & $\mathrm{R}$ & 1.05 \\
136 & $\mathrm{R}$ & 1.32 \\
137 & $\mathrm{R}$ & 1.36 \\
138 & $\mathrm{R}$ & 1.32 \\
139 & $\mathrm{R}$ & 1.49 \\
140 & $\mathrm{R}$ & 1.81 \\
141 & $\mathrm{R}$ & 0.89 \\
142 & $\mathrm{R}$ & 2.02 \\
143 & $\mathrm{R}$ & 0.37
\end{tabular}




\section{DQA Surface Soil Report}

\section{Basic Statistical Quantities Summary}

\begin{tabular}{lccc} 
Statistic & Survey Unit & Background & DQO Results \\
\hline Sample Number & 14 & 17 & N/2=18 \\
Mean (SOR) & 1.09 & 1.28 & 0.39 \\
Median (SOR) & 1.07 & 1.32 & N/A \\
Std Dev (SOR) & 0.32 & 0.36 & 0.39 \\
High Value (SOR) & 1.67 & 2.02 & N/A \\
Low Value (SOR) & 0.53 & 0.37 & N/A
\end{tabular}

\section{Statistical Test Summary}

$\begin{array}{ll}\text { Sum of Ranks: } & 496 \\ \text { Sum of Reference Ranks: } & 389 \\ \text { Critical Value: } & 313 \\ \text { Result: } & \text { Pass }\end{array}$

$\begin{array}{lll}\text { Data } & \text { Type } & \text { Adjusted Data }\end{array}$

$\begin{array}{lcc}0.37 & \mathrm{R} & 1.37 \\ 0.89 & \mathrm{R} & 1.89 \\ 0.93 & \mathrm{R} & 1.93 \\ 1.05 & \mathrm{R} & 2.05 \\ 1.23 & \mathrm{R} & 2.23 \\ 1.24 & \mathrm{R} & 2.24 \\ 1.3 & \mathrm{R} & 2.3 \\ 1.32 & \mathrm{R} & 2.32 \\ 1.32 & \mathrm{R} & 2.32 \\ 1.32 & \mathrm{R} & 2.32 \\ 1.36 & \mathrm{R} & 2.36 \\ 1.36 & \mathrm{R} & 2.36 \\ 1.36 & \mathrm{R} & 2.36 \\ 1.4 & \mathrm{R} & 2.4 \\ 1.49 & \mathrm{R} & 2.49 \\ 1.81 & \mathrm{R} & 2.81 \\ 2.02 & \mathrm{R} & 3.02 \\ 0.53 & \mathrm{~S} & 0.53 \\ 0.75 & \mathrm{~S} & 0.75 \\ 0.78 & \mathrm{~S} & 0.78 \\ 0.86 & \mathrm{~S} & 0.86 \\ 0.96 & \mathrm{~S} & 0.96 \\ 1.04 & \mathrm{~S} & 1.04 \\ 1.06 & \mathrm{~S} & 1.06 \\ 1.08 & \mathrm{~S} & 1.08\end{array}$




\section{DQA Surface Soil Report}

\section{Statistical Test Summary}

\begin{tabular}{lcccc} 
Data & Type & Adjusted Data & Rank & Reference Rank \\
\hline 1.15 & & & & 9 \\
1.24 & S & 1.15 & 10 & 0 \\
1.29 & S & 1.24 & 11.5 & 0 \\
1.29 & S & 1.29 & 11.5 & 0 \\
1.61 & S & 1.29 & 14 & 0 \\
1.67 & S & 1.61 & 15 & 0 \\
\end{tabular}




\section{DQA Surface Soil Report}

\section{Assessment Summary}

Site:

Planner(s):

Survey Unit Name:

Report Number:

Survey Unit Samples:

Reference Area Samples:

Test Performed:

Judgmental Samples:

Assessment Conclusion:
Hammond Depot FSS Planner

Vitkus

C2 SU1 through $12 \quad$ C2 SU8

7

14

17

WRS

Test Result: $\quad$ Pass

0

EMC Result:

Not Performed

Reject Null Hypothesis (Survey Unit PASSES)

\section{Retrospective Power Curve}

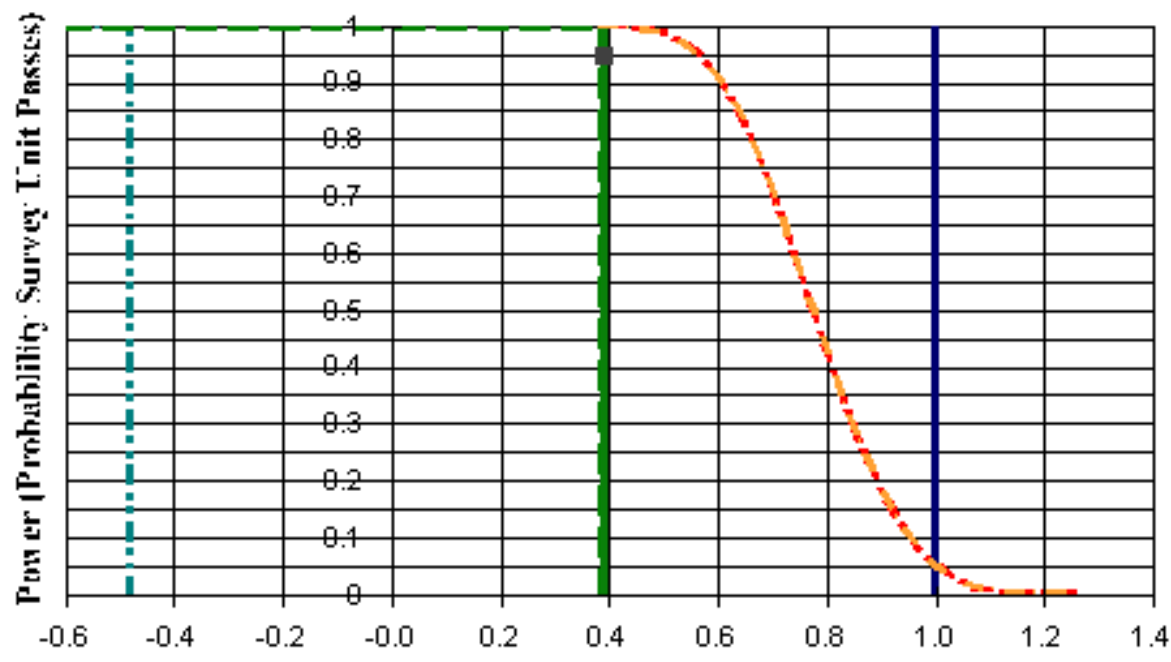

Unity Rule Sum-of-Ratios, not including background

$-\cdot=$ Prospective Power
LBGR
DCGL

- 1-beta

-". Actual Power

- Estimated Power

-- - Retrospective Power 


\section{DQA Surface Soil Report}

\section{Survey Unit Data}

NOTE: $\quad$ Type = "S" indicates survey unit sample.

Type = "R" indicates reference area sample.

\begin{tabular}{|c|c|c|c|}
\hline Sample Number & Type & Th-232 (pCi/g) & $\mathrm{U}-238$ (pCi/g) \\
\hline 370 & $\mathrm{~S}$ & 0.76 & 1.54 \\
\hline 371 & S & 0.53 & 0.98 \\
\hline 372 & S & 0.45 & 0.78 \\
\hline 373 & $S$ & 0.98 & 1.5 \\
\hline 374 & $S$ & 0.88 & 1.27 \\
\hline 376 & S & 0.86 & 2.13 \\
\hline 377 & $S$ & 0.48 & 0.49 \\
\hline 379 & $S$ & 0.98 & 1.52 \\
\hline 380 & S & 0.69 & 1.31 \\
\hline 381 & S & 0.56 & 1.17 \\
\hline 382 & S & 1.31 & 1.39 \\
\hline 384 & $S$ & 0.69 & 1.24 \\
\hline 385 & S & 0.79 & 1.85 \\
\hline 386 & S & 0.58 & 1.64 \\
\hline 127 & $\mathrm{R}$ & 1.1 & 2.54 \\
\hline 128 & $\mathrm{R}$ & 0.85 & 2.66 \\
\hline 129 & $\mathrm{R}$ & 0.74 & 2.44 \\
\hline 130 & $\mathrm{R}$ & 1.09 & 2.3 \\
\hline 131 & $\mathrm{R}$ & 0.93 & 2.5 \\
\hline 132 & $\mathrm{R}$ & 0.79 & 1.65 \\
\hline 133 & $\mathrm{R}$ & 0.94 & 2.3 \\
\hline 134 & $\mathrm{R}$ & 0.98 & 2.55 \\
\hline 135 & $\mathrm{R}$ & 0.65 & 2.06 \\
\hline 136 & $\mathrm{R}$ & 0.73 & 2.66 \\
\hline 137 & $\mathrm{R}$ & 1.1 & 2.44 \\
\hline 138 & $\mathrm{R}$ & 1.03 & 2.42 \\
\hline 139 & $\mathrm{R}$ & 1.17 & 2.72 \\
\hline 140 & $\mathrm{R}$ & 1.36 & 3.35 \\
\hline 141 & $\mathrm{R}$ & 0.91 & 1.44 \\
\hline 142 & $\mathrm{R}$ & 1.71 & 3.57 \\
\hline 143 & $\mathrm{R}$ & 0.33 & 0.63 \\
\hline
\end{tabular}




\section{Modified Data (Unity Rule SOR)}

NOTE: $\quad$ Type = "S" indicates survey unit sample.

Type $=$ "R" indicates reference area sample.

\begin{tabular}{lcc} 
Sample Number & Type & Sum-of-Ratios (SOR) \\
\hline 370 & $\mathrm{~S}$ & 0.88 \\
371 & $\mathrm{~S}$ & 0.57 \\
372 & $\mathrm{~S}$ & 0.47 \\
373 & $\mathrm{~S}$ & 0.94 \\
374 & $\mathrm{~S}$ & 0.81 \\
376 & $\mathrm{~S}$ & 1.15 \\
377 & $\mathrm{~S}$ & 0.36 \\
379 & $\mathrm{~S}$ & 0.95 \\
380 & $\mathrm{~S}$ & 0.76 \\
381 & $\mathrm{~S}$ & 0.66 \\
382 & $\mathrm{~S}$ & 1.01 \\
384 & $\mathrm{~S}$ & 0.73 \\
385 & $\mathrm{~S}$ & 1.01 \\
386 & $\mathrm{~S}$ & 0.86 \\
127 & $\mathrm{R}$ & 1.4 \\
128 & $\mathrm{R}$ & 1.36 \\
129 & $\mathrm{R}$ & 1.23 \\
130 & $\mathrm{R}$ & 1.3 \\
131 & $\mathrm{R}$ & 1.32 \\
132 & $\mathrm{R}$ & 0.93 \\
133 & $\mathrm{R}$ & 1.24 \\
134 & $\mathrm{R}$ & 1.36 \\
135 & $\mathrm{R}$ & 1.05 \\
136 & $\mathrm{R}$ & 1.32 \\
137 & $\mathrm{R}$ & 1.36 \\
138 & $\mathrm{R}$ & 1.32 \\
139 & $\mathrm{R}$ & 1.49 \\
140 & $\mathrm{R}$ & 1.81 \\
141 & $\mathrm{R}$ & 0.89 \\
142 & $\mathrm{R}$ & 2.02 \\
143 & $\mathrm{R}$ & 0.37
\end{tabular}




\section{DQA Surface Soil Report}

\section{Basic Statistical Quantities Summary}

\begin{tabular}{lccc} 
Statistic & Survey Unit & Background & DQO Results \\
\hline Sample Number & 14 & 17 & N/2=18 \\
Mean (SOR) & 0.80 & 1.28 & 0.39 \\
Median (SOR) & 0.84 & 1.32 & N/A \\
Std Dev (SOR) & 0.22 & 0.36 & 0.39 \\
High Value (SOR) & 1.15 & 2.02 & N/A \\
Low Value (SOR) & 0.36 & 0.37 & N/A
\end{tabular}

\section{Statistical Test Summary}

$\begin{array}{ll}\text { Sum of Ranks: } & 496 \\ \text { Sum of Reference Ranks: } & 391 \\ \text { Critical Value: } & 313 \\ \text { Result: } & \text { Pass }\end{array}$

$\begin{array}{lll}\text { Data } & \text { Type } & \text { Adjusted Data }\end{array}$

0.37

0.89

0.93

1.05

1.23

1.24

1.3

1.32

1.32

1.32

1.36

1.36

1.36

1.4

1.49

1.81

2.02

0.36

0.47

0.57

0.66

0.73

0.76

0.81

0.86
496

Pass

1.37

1.89

1.93

2.05

2.23

2.24

2.3

2.32

2.32

2.32

2.36

2.36

2.36

2.4

2.49

2.81

3.02

0.36

0.47

0.57

0.66

0.73

0.76

0.81

0.86
Rank

Reference Rank

$\begin{array}{cc}15 & 15 \\ 16 & 16 \\ 17 & 17 \\ 18 & 18 \\ 19 & 19 \\ 20 & 20 \\ 21 & 21 \\ 23 & 23 \\ 23 & 23 \\ 23 & 23 \\ 26 & 26 \\ 26 & 26 \\ 26 & 26 \\ 28 & 28 \\ 29 & 29 \\ 30 & 30 \\ 31 & 31 \\ 1 & 0 \\ 2 & 0 \\ 3 & 0 \\ 4 & 0 \\ 5 & 0 \\ 6 & 0 \\ 7 & 0 \\ 8 & 0\end{array}$




\section{DQA Surface Soil Report}

Statistical Test Summary

\begin{tabular}{lcccc} 
Data & Type & Adjusted Data & Rank & Reference Rank \\
\hline & & & & 9 \\
0.88 & S & 0.88 & 10 & 0 \\
0.94 & S & 0.94 & 11 & 0 \\
0.95 & S & 0.95 & 12.5 & 0 \\
1.01 & S & 1.01 & 12.5 & 0 \\
1.01 & S & 1.01 & 14 & 0 \\
1.15 & S & 1.15 & & 0
\end{tabular}




\section{DQA Surface Soil Report}

\section{Assessment Summary}

Site:

Planner(s):

Survey Unit Name:

Report Number:

Survey Unit Samples:

Reference Area Samples:

Test Performed:

Judgmental Samples:

Assessment Conclusion:
Hammond Depot FSS Planner

Vitkus

C2 SU1 through $12 \quad$ C2 SU9

8

16

17

WRS

Test Result: $\quad$ Pass

0

EMC Result:

Not Performed

Reject Null Hypothesis (Survey Unit PASSES)

\section{Retrospective Power Curve}

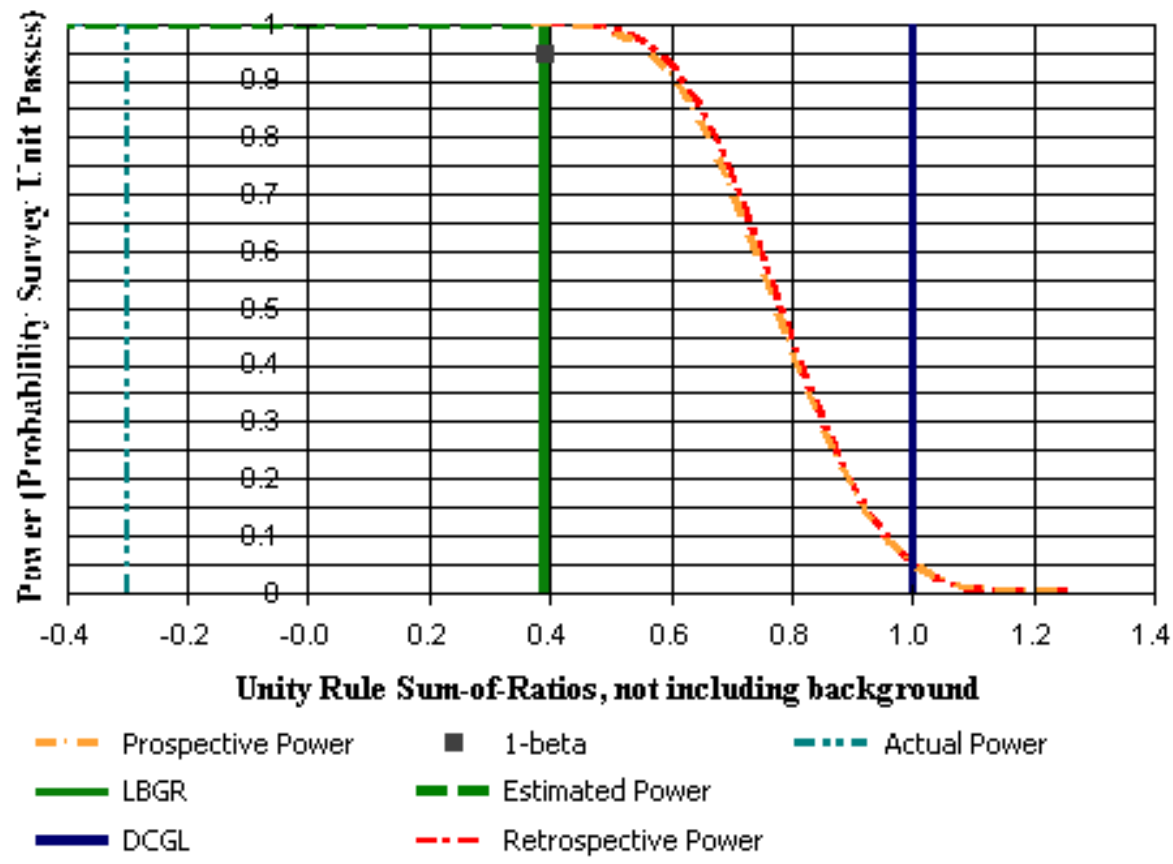




\section{DQA Surface Soil Report}

\section{Survey Unit Data}

NOTE: $\quad$ Type = "S" indicates survey unit sample.

Type = "R" indicates reference area sample.

\begin{tabular}{|c|c|c|c|}
\hline Sample Number & Type & Th-232 (pCi/g) & U-238 (pCi/g) \\
\hline 387 & $\mathrm{~S}$ & 0.79 & 1.61 \\
\hline 388 & $S$ & 1.02 & 1.93 \\
\hline 389 & S & 0.8 & 1.54 \\
\hline 390 & S & 0.56 & 0.84 \\
\hline 391 & S & 1.02 & 2.1 \\
\hline 392 & S & 0.97 & 1.14 \\
\hline 393 & $S$ & 0.91 & 2.55 \\
\hline 394 & $S$ & 0.63 & 1.08 \\
\hline 395 & S & 0.99 & 2.17 \\
\hline 396 & S & 0.8 & 1.9 \\
\hline 397 & S & 0.94 & 2.34 \\
\hline 399 & S & 1 & 1.76 \\
\hline 400 & S & 0.97 & 1.39 \\
\hline 401 & S & 0.86 & 1.93 \\
\hline 402 & S & 0.82 & 1.78 \\
\hline 403 & S & 0.65 & 0.98 \\
\hline 127 & $\mathrm{R}$ & 1.1 & 2.54 \\
\hline 128 & $\mathrm{R}$ & 0.85 & 2.66 \\
\hline 129 & $\mathrm{R}$ & 0.74 & 2.44 \\
\hline 130 & $\mathrm{R}$ & 1.09 & 2.3 \\
\hline 131 & $\mathrm{R}$ & 0.93 & 2.5 \\
\hline 132 & $\mathrm{R}$ & 0.79 & 1.65 \\
\hline 133 & $\mathrm{R}$ & 0.94 & 2.3 \\
\hline 134 & $\mathrm{R}$ & 0.98 & 2.55 \\
\hline 135 & $\mathrm{R}$ & 0.65 & 2.06 \\
\hline 136 & $\mathrm{R}$ & 0.73 & 2.66 \\
\hline 137 & $\mathrm{R}$ & 1.1 & 2.44 \\
\hline 138 & $\mathrm{R}$ & 1.03 & 2.42 \\
\hline 139 & $\mathrm{R}$ & 1.17 & 2.72 \\
\hline 140 & $\mathrm{R}$ & 1.36 & 3.35 \\
\hline 141 & $\mathrm{R}$ & 0.91 & 1.44 \\
\hline 142 & $\mathrm{R}$ & 1.71 & 3.57 \\
\hline 143 & $\mathrm{R}$ & 0.33 & 0.63 \\
\hline
\end{tabular}




\section{Modified Data (Unity Rule SOR)}

NOTE: $\quad$ Type = "S" indicates survey unit sample.

Type $=$ "R" indicates reference area sample.

\begin{tabular}{lcc} 
Sample Number & Type & Sum-of-Ratios (SOR) \\
\hline 387 & $\mathrm{~S}$ & 0.92 \\
388 & $\mathrm{~S}$ & 1.12 \\
389 & $\mathrm{~S}$ & 0.89 \\
390 & $\mathrm{~S}$ & 0.53 \\
391 & $\mathrm{~S}$ & 1.19 \\
392 & $\mathrm{~S}$ & 0.79 \\
393 & $\mathrm{~S}$ & 1.33 \\
394 & $\mathrm{~S}$ & 0.65 \\
395 & $\mathrm{~S}$ & 1.21 \\
396 & $\mathrm{~S}$ & 1.04 \\
397 & $\mathrm{~S}$ & 1.26 \\
399 & $\mathrm{~S}$ & 1.05 \\
400 & $\mathrm{~S}$ & 0.89 \\
401 & $\mathrm{~S}$ & 1.07 \\
402 & $\mathrm{~S}$ & 0.99 \\
403 & $\mathrm{~S}$ & 0.62 \\
127 & $\mathrm{R}$ & 1.4 \\
128 & $\mathrm{R}$ & 1.36 \\
129 & $\mathrm{R}$ & 1.23 \\
130 & $\mathrm{R}$ & 1.3 \\
131 & $\mathrm{R}$ & 1.32 \\
132 & $\mathrm{R}$ & 0.93 \\
133 & $\mathrm{R}$ & 1.24 \\
134 & $\mathrm{R}$ & 1.36 \\
135 & $\mathrm{R}$ & 1.05 \\
136 & $\mathrm{R}$ & 1.32 \\
137 & $\mathrm{R}$ & 1.36 \\
138 & $\mathrm{R}$ & 1.32 \\
139 & $\mathrm{R}$ & 1.49 \\
140 & $\mathrm{R}$ & 1.81 \\
141 & $\mathrm{R}$ & 0.89 \\
142 & $\mathrm{R}$ & 2.02 \\
143 & $\mathrm{R}$ & 0.37 \\
& &
\end{tabular}




\section{DQA Surface Soil Report}

\section{Basic Statistical Quantities Summary}

\begin{tabular}{lccc} 
Statistic & Survey Unit & Background & DQO Results \\
\hline Sample Number & 16 & 17 & N/2=18 \\
Mean (SOR) & 0.97 & 1.28 & 0.39 \\
Median (SOR) & 1.02 & 1.32 & N/A \\
Std Dev (SOR) & 0.24 & 0.36 & 0.39 \\
High Value (SOR) & 1.33 & 2.02 & N/A \\
Low Value (SOR) & 0.53 & 0.37 & N/A
\end{tabular}

\section{Statistical Test Summary}

$\begin{array}{ll}\text { Sum of Ranks: } & 561 \\ \text { Sum of Reference Ranks: } & 425 \\ \text { Critical Value: } & 335 \\ \text { Result: } & \text { Pass }\end{array}$

$\begin{array}{lll}\text { Data } & \text { Type } & \text { Adjusted Data }\end{array}$

$\begin{array}{llll}0.37 & \mathrm{R} & 1.37 & 17\end{array}$

0.89

0.93

1.05

1.23

1.24

1.3

1.32

1.32

1.32

1.36

1.36

1.36

1.4

1.49

1.81

2.02

0.53

0.62

0.65

0.79

0.89

0.89

0.92

0.99
425

35

1.89

1.93

2.05

2.23

2.24

2.3

2.32

2.32

2.32

2.36

2.36

2.36

2.4

2.49

2.81

3.02

0.53

0.62

0.65

0.79

0.89

0.89

0.92

0.99
Rank

Reference Rank

$\begin{array}{cc}17 & 17 \\ 18 & 18 \\ 19 & 19 \\ 20 & 20 \\ 21 & 21 \\ 22 & 22 \\ 23 & 23 \\ 25 & 25 \\ 25 & 25 \\ 25 & 25 \\ 28 & 28 \\ 28 & 28 \\ 28 & 28 \\ 30 & 30 \\ 31 & 31 \\ 32 & 32 \\ 33 & 33 \\ 1 & 0 \\ 2 & 0 \\ 3 & 0 \\ 4 & 0 \\ 5.5 & 0 \\ 5.5 & 0 \\ 7 & 0 \\ 8 & 0\end{array}$




\section{DQA Surface Soil Report}

\section{Statistical Test Summary}

\begin{tabular}{lcccc} 
Data & Type & Adjusted Data & Rank & Reference Rank \\
\hline 1.04 & & & & 0 \\
1.05 & S & 1.04 & 10 & 0 \\
1.07 & S & 1.05 & 11 & 0 \\
1.12 & S & 1.07 & 12 & 0 \\
1.19 & S & 1.12 & 13 & 0 \\
1.21 & S & 1.19 & 14 & 0 \\
1.26 & S & 1.21 & 15 & 0 \\
1.33 & S & 1.26 & 16 & 0
\end{tabular}




\section{DQA Surface Soil Report}

\section{Assessment Summary}

Site:

Planner(s):

Survey Unit Name:

Report Number:

Survey Unit Samples:

Reference Area Samples:

Test Performed:

Judgmental Samples:

Assessment Conclusion:
Hammond Depot FSS Planner

Vitkus

C2 SU1 through $12 \quad$ C2 SU10

9

14

17

WRS

Test Result: $\quad$ Pass

0

EMC Result:

Not Performed

\section{Reject Null Hypothesis (Survey Unit PASSES)}

\section{Retrospective Power Curve}

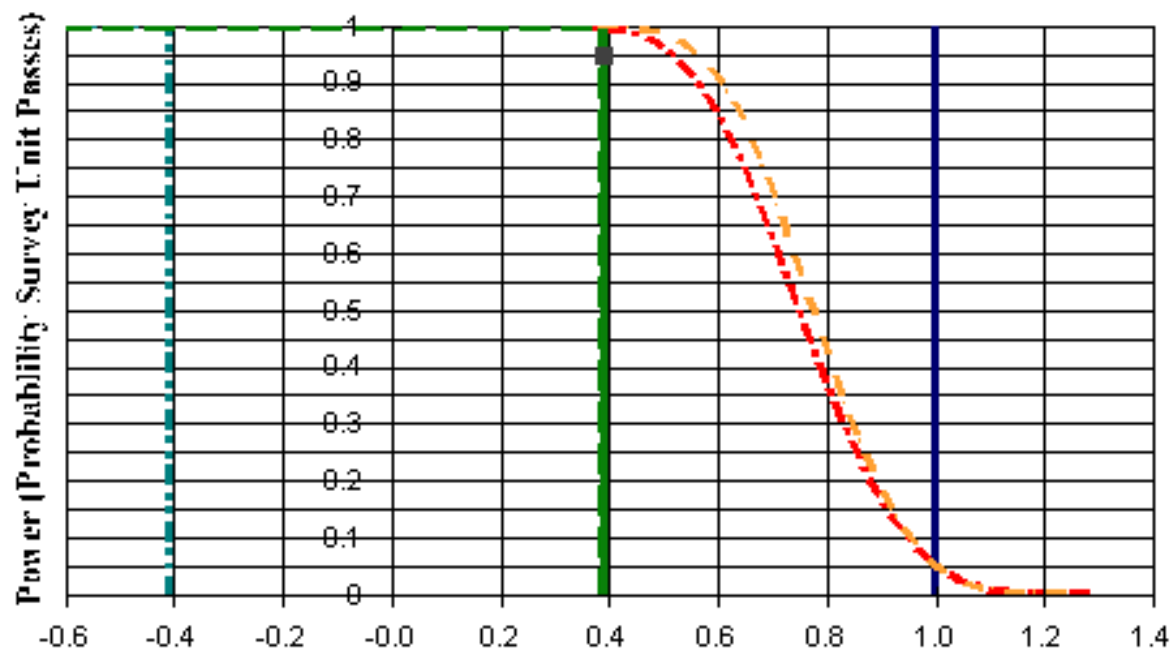

Unity Rule Sum-of-Ratios, not including background

$-\cdot=$ Prospective Power
LBGR
DCGL

- 1-beta

- Estimated Power

-- - Retrospective Power 


\section{DQA Surface Soil Report}

\section{Survey Unit Data}

NOTE: $\quad$ Type = "S" indicates survey unit sample.

Type = "R" indicates reference area sample.

\begin{tabular}{|c|c|c|c|}
\hline Sample Number & Type & Th-232 (pCi/g) & $\mathrm{U}-238$ (pCi/g) \\
\hline 405 & $\mathrm{~S}$ & 0.33 & 0.87 \\
\hline 407 & S & 1.09 & 2.22 \\
\hline 408 & S & 0.56 & 1.14 \\
\hline 409 & S & 0.49 & 0.82 \\
\hline 410 & $S$ & 0.59 & 1.1 \\
\hline 411 & $S$ & 0.33 & 0.43 \\
\hline 412 & $S$ & 1.13 & 2.62 \\
\hline 414 & $S$ & 0.97 & 2.35 \\
\hline 415 & S & 0.71 & 1.13 \\
\hline 416 & S & 0.99 & 1.92 \\
\hline 417 & S & 0.81 & 2.68 \\
\hline 418 & $S$ & 0.61 & 1.11 \\
\hline 419 & S & 1.01 & 2.63 \\
\hline 420 & S & 0.74 & 2.29 \\
\hline 127 & $\mathrm{R}$ & 1.1 & 2.54 \\
\hline 128 & $\mathrm{R}$ & 0.85 & 2.66 \\
\hline 129 & $\mathrm{R}$ & 0.74 & 2.44 \\
\hline 130 & $\mathrm{R}$ & 1.09 & 2.3 \\
\hline 131 & $\mathrm{R}$ & 0.93 & 2.5 \\
\hline 132 & $\mathrm{R}$ & 0.79 & 1.65 \\
\hline 133 & $\mathrm{R}$ & 0.94 & 2.3 \\
\hline 134 & $\mathrm{R}$ & 0.98 & 2.55 \\
\hline 135 & $\mathrm{R}$ & 0.65 & 2.06 \\
\hline 136 & $\mathrm{R}$ & 0.73 & 2.66 \\
\hline 137 & $\mathrm{R}$ & 1.1 & 2.44 \\
\hline 138 & $\mathrm{R}$ & 1.03 & 2.42 \\
\hline 139 & $\mathrm{R}$ & 1.17 & 2.72 \\
\hline 140 & $\mathrm{R}$ & 1.36 & 3.35 \\
\hline 141 & $\mathrm{R}$ & 0.91 & 1.44 \\
\hline 142 & $\mathrm{R}$ & 1.71 & 3.57 \\
\hline 143 & $\mathrm{R}$ & 0.33 & 0.63 \\
\hline
\end{tabular}




\section{DQA Surface Soil Report}

\section{Modified Data (Unity Rule SOR)}

NOTE: $\quad$ Type = "S" indicates survey unit sample.

Type = "R" indicates reference area sample.

\begin{tabular}{lcc} 
Sample Number & Type & Sum-of-Ratios (SOR) \\
\hline 405 & S & 0.46 \\
407 & S & 1.26 \\
408 & S & 0.65 \\
409 & S & 0.5 \\
410 & S & 0.64 \\
411 & S & 0.29 \\
412 & S & 1.44 \\
414 & S & 1.27 \\
415 & S & 0.7 \\
416 & S & 1.11 \\
417 & S & 1.35 \\
418 & S & 0.65 \\
419 & S & 1.4 \\
420 & S & 1.17 \\
127 & R & 1.4 \\
128 & R & 1.36 \\
129 & R & 1.23 \\
130 & R & 1.3 \\
131 & R & 1.32 \\
132 & R & 0.93 \\
133 & R & 1.24 \\
134 & R & 1.36 \\
135 & R & 1.05 \\
136 & R & 1.32 \\
137 & $\mathrm{R}$ & 1.36 \\
138 & $\mathrm{R}$ & 1.32 \\
139 & $\mathrm{R}$ & 1.49 \\
140 & $\mathrm{R}$ & 1.81 \\
141 & $\mathrm{R}$ & 0.89 \\
142 & $\mathrm{R}$ & 2.02 \\
143 & $\mathrm{R}$ & 0.37
\end{tabular}




\section{DQA Surface Soil Report}

\section{Basic Statistical Quantities Summary}

\begin{tabular}{lccc} 
Statistic & Survey Unit & Background & DQO Results \\
\hline Sample Number & 14 & 17 & N/2=18 \\
Mean (SOR) & 0.92 & 1.28 & 0.39 \\
Median (SOR) & 0.91 & 1.32 & N/A \\
Std Dev (SOR) & 0.40 & 0.36 & 0.39 \\
High Value (SOR) & 1.44 & 2.02 & N/A \\
Low Value (SOR) & 0.29 & 0.37 & N/A
\end{tabular}

\section{Statistical Test Summary}

$\begin{array}{ll}\text { Sum of Ranks: } & 496 \\ \text { Sum of Reference Ranks: } & 389 \\ \text { Critical Value: } & 313 \\ \text { Result: } & \text { Pass }\end{array}$

Data Type Adjusted Data $\quad$ Rank

0.37

0.89

0.93

1.05

1.23

1.24

1.3

1.32

1.32

1.32

1.36

1.36

1.36

1.4

1.49

1.81

2.02

0.29

0.46

0.5

0.64

0.65

0.65

0.7

1.11
496

Pass

1.37

1.89

1.93

2.05

2.23

2.24

2.3

2.32

2.32

2.32

2.36

2.36

2.36

2.4

2.49

2.81

3.02

0.29

0.46

0.5

0.64

0.65

0.65

0.7

1.11
Rank

Reference Rank

$\begin{array}{cc}13 & 13 \\ 16 & 16 \\ 17 & 17 \\ 18 & 18 \\ 19 & 19 \\ 20 & 20 \\ 21 & 21 \\ 23 & 23 \\ 23 & 23 \\ 23 & 23 \\ 26 & 26 \\ 26 & 26 \\ 26 & 26 \\ 28 & 28 \\ 29 & 29 \\ 30 & 30 \\ 31 & 31 \\ 1 & 0 \\ 2 & 0 \\ 3 & 0 \\ 4 & 0 \\ 5.5 & 0 \\ 5.5 & 0 \\ 7 & 0 \\ 8 & 0\end{array}$




\section{DQA Surface Soil Report}

\section{Statistical Test Summary}

\begin{tabular}{lcccc} 
Data & Type & Adjusted Data & Rank & Reference Rank \\
\hline 1.17 & & 1.17 & 9 & 0 \\
1.26 & S & 1.26 & 10 & 0 \\
1.27 & S & 1.27 & 11 & 0 \\
1.35 & S & 1.35 & 12 & 0 \\
1.4 & S & 1.4 & 14 & 0 \\
1.44 & S & 1.44 & 15 & 0
\end{tabular}




\section{DQA Surface Soil Report}

\section{Assessment Summary}

Site:

Planner(s):

Survey Unit Name:

Report Number:

Survey Unit Samples:

Reference Area Samples:

Test Performed:

Judgmental Samples:

Assessment Conclusion:
Hammond Depot FSS Planner

Vitkus

C2 SU1 through $12 \quad$ C2 SU11

10

17

17

WRS

Test Result: $\quad$ Pass

0

EMC Result:

Not Performed

Reject Null Hypothesis (Survey Unit PASSES)

\section{Retrospective Power Curve}

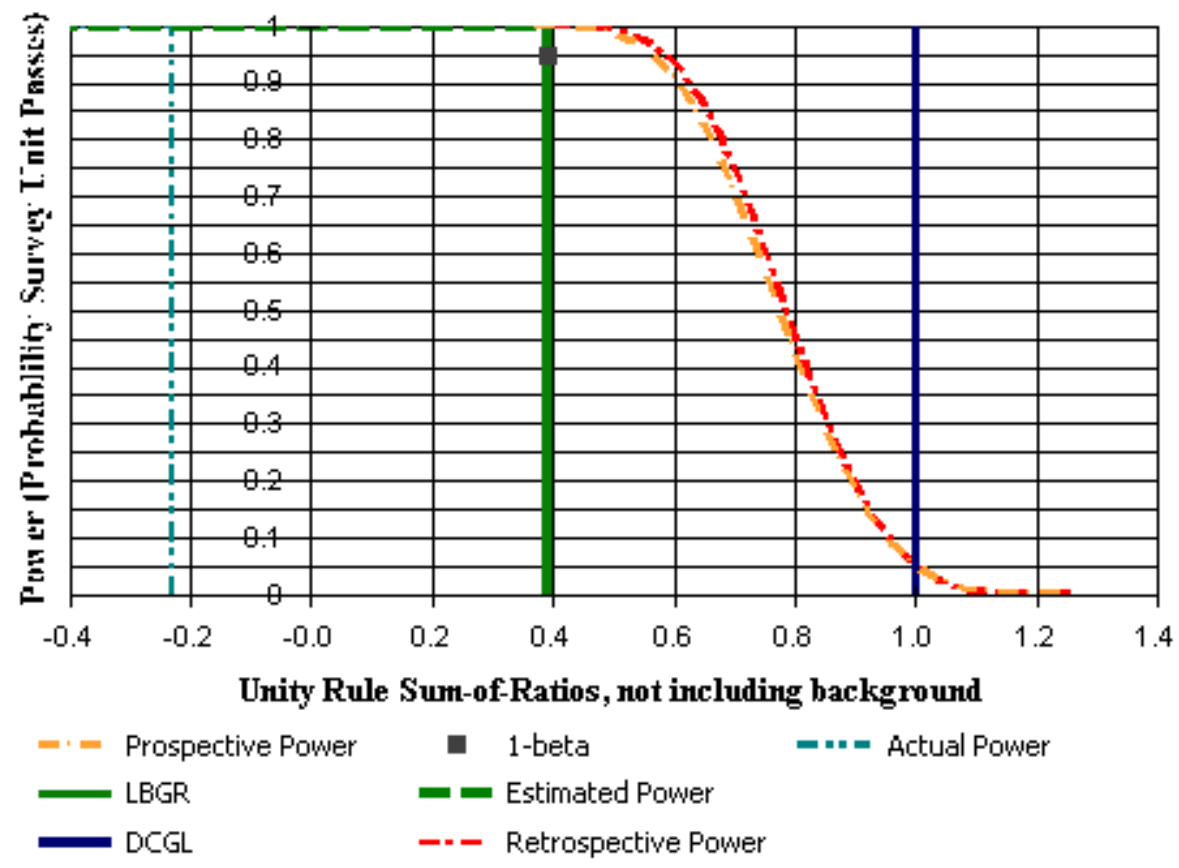




\section{DQA Surface Soil Report}

\section{Survey Unit Data}

NOTE: $\quad$ Type = "S" indicates survey unit sample.

Type = "R" indicates reference area sample.

\begin{tabular}{|c|c|c|c|}
\hline Sample Number & Type & Th-232 (pCi/g) & $\mathrm{U}-238$ (pCi/g) \\
\hline$\overline{421}$ & $\mathrm{~S}$ & 0.98 & 2.15 \\
\hline 422 & $S$ & 1.09 & 2.28 \\
\hline 423 & S & 0.95 & 1.73 \\
\hline 424 & S & 0.68 & 1.72 \\
\hline 425 & S & 1.31 & 1.74 \\
\hline 426 & S & 1.26 & 1.61 \\
\hline 427 & S & 1.14 & 2.01 \\
\hline 428 & $S$ & 0.62 & 1.63 \\
\hline 429 & S & 0.75 & 2.29 \\
\hline 430 & S & 0.82 & 1.67 \\
\hline 431 & S & 1.05 & 1.83 \\
\hline 432 & S & 0.77 & 2.24 \\
\hline 433 & S & 1.01 & 1.76 \\
\hline 434 & S & 0.96 & 2.82 \\
\hline 435 & $S$ & 1.21 & 2.66 \\
\hline 436 & S & 1.02 & 1.33 \\
\hline 437 & S & 0.87 & 1.39 \\
\hline 127 & $\mathrm{R}$ & 1.1 & 2.54 \\
\hline 128 & $\mathrm{R}$ & 0.85 & 2.66 \\
\hline 129 & $\mathrm{R}$ & 0.74 & 2.44 \\
\hline 130 & $\mathrm{R}$ & 1.09 & 2.3 \\
\hline 131 & $\mathrm{R}$ & 0.93 & 2.5 \\
\hline 132 & $\mathrm{R}$ & 0.79 & 1.65 \\
\hline 133 & $\mathrm{R}$ & 0.94 & 2.3 \\
\hline 134 & $\mathrm{R}$ & 0.98 & 2.55 \\
\hline 135 & $\mathrm{R}$ & 0.65 & 2.06 \\
\hline 136 & $\mathrm{R}$ & 0.73 & 2.66 \\
\hline 137 & $\mathrm{R}$ & 1.1 & 2.44 \\
\hline 138 & $\mathrm{R}$ & 1.03 & 2.42 \\
\hline 139 & $\mathrm{R}$ & 1.17 & 2.72 \\
\hline 140 & $\mathrm{R}$ & 1.36 & 3.35 \\
\hline 141 & $\mathrm{R}$ & 0.91 & 1.44 \\
\hline 142 & $\mathrm{R}$ & 1.71 & 3.57 \\
\hline 143 & $\mathrm{R}$ & 0.33 & 0.63 \\
\hline
\end{tabular}




\section{Modified Data (Unity Rule SOR)}

NOTE: $\quad$ Type = "S" indicates survey unit sample.

Type $=$ "R" indicates reference area sample.

\begin{tabular}{lcc} 
Sample Number & Type & Sum-of-Ratios (SOR) \\
\hline 421 & $\mathrm{~S}$ & 1.2 \\
422 & $\mathrm{~S}$ & 1.29 \\
423 & $\mathrm{~S}$ & 1.02 \\
424 & $\mathrm{~S}$ & 0.92 \\
425 & $\mathrm{~S}$ & 1.15 \\
426 & $\mathrm{~S}$ & 1.08 \\
427 & $\mathrm{~S}$ & 1.2 \\
428 & $\mathrm{~S}$ & 0.87 \\
429 & $\mathrm{~S}$ & 1.17 \\
430 & $\mathrm{~S}$ & 0.95 \\
431 & $\mathrm{~S}$ & 1.09 \\
432 & $\mathrm{~S}$ & 1.16 \\
433 & $\mathrm{~S}$ & 1.05 \\
434 & $\mathrm{~S}$ & 1.46 \\
435 & $\mathrm{~S}$ & 1.48 \\
436 & $\mathrm{~S}$ & 0.88 \\
437 & $\mathrm{~S}$ & 0.86 \\
127 & $\mathrm{R}$ & 1.4 \\
128 & $\mathrm{R}$ & 1.36 \\
129 & $\mathrm{R}$ & 1.23 \\
130 & $\mathrm{R}$ & 1.3 \\
131 & $\mathrm{R}$ & 1.32 \\
132 & $\mathrm{R}$ & 0.93 \\
133 & $\mathrm{R}$ & 1.24 \\
134 & $\mathrm{R}$ & 1.36 \\
135 & $\mathrm{R}$ & 1.05 \\
136 & $\mathrm{R}$ & 1.32 \\
137 & $\mathrm{R}$ & 1.36 \\
138 & $\mathrm{R}$ & 1.32 \\
139 & $\mathrm{R}$ & 1.49 \\
140 & $\mathrm{R}$ & 1.81 \\
141 & $\mathrm{R}$ & 0.89 \\
142 & $\mathrm{R}$ & 2.02 \\
143 & $\mathrm{R}$ & 0.37 \\
& &
\end{tabular}




\section{DQA Surface Soil Report}

\section{Basic Statistical Quantities Summary}

\begin{tabular}{lccc} 
Statistic & Survey Unit & Background & DQO Results \\
\hline Sample Number & 17 & 17 & N/2=18 \\
Mean (SOR) & 1.11 & 1.28 & 0.39 \\
Median (SOR) & 1.09 & 1.32 & N/A \\
Std Dev (SOR) & 0.19 & 0.36 & 0.39 \\
High Value (SOR) & 1.48 & 2.02 & N/A \\
Low Value (SOR) & 0.86 & 0.37 & N/A
\end{tabular}

\section{Statistical Test Summary}

$\begin{array}{ll}\text { Sum of Ranks: } & 595 \\ \text { Sum of Reference Ranks: } & 440 \\ \text { Critical Value: } & 345 \\ \text { Result: } & \text { Pass }\end{array}$

$\begin{array}{lll}\text { Data } & \text { Type } & \text { Adjusted Data }\end{array}$

$0.37-\mathrm{R}=1.37-16$

0.89

0.93

1.05

1.23

1.24

1.3

1.32

1.32

1.32

1.36

1.36

1.36

1.4

1.49

1.81

2.02

0.86

0.87

0.88

0.92

0.95

1.02

1.05

1.08
440

45

Pass

1.89

1.93

2.05

2.23

2.24

2.3

2.32

2.32

2.32

2.36

2.36

2.36

2.4

2.49

2.81

3.02

0.86

0.87

0.88

0.92

0.95

1.02

1.05

1.08
Rank

Reference Rank

$\begin{array}{cc}16 & 16 \\ 19 & 19 \\ 20 & 20 \\ 21 & 21 \\ 22 & 22 \\ 23 & 23 \\ 24 & 24 \\ 26 & 26 \\ 26 & 26 \\ 26 & 26 \\ 29 & 29 \\ 29 & 29 \\ 29 & 29 \\ 31 & 31 \\ 32 & 32 \\ 33 & 33 \\ 34 & 34 \\ 1 & 0 \\ 2 & 0 \\ 3 & 0 \\ 4 & 0 \\ 5 & 0 \\ 6 & 0 \\ 7 & 0 \\ 8 & 0\end{array}$




\section{DQA Surface Soil Report}

Statistical Test Summary

\begin{tabular}{lcccc} 
Data & Type & Adjusted Data & Rank & Reference Rank \\
\hline 1.09 & & 1.09 & 9 & 0 \\
1.15 & S & 1.15 & 10 & 0 \\
1.16 & S & 1.16 & 11 & 0 \\
1.17 & S & 1.17 & 12 & 0 \\
1.2 & S & 1.2 & 13.5 & 0 \\
1.2 & S & 1.2 & 13.5 & 0 \\
1.29 & S & 1.29 & 15 & 0 \\
1.46 & S & 1.46 & 17 & 0 \\
1.48 & S & 1.48 & 18 & 0
\end{tabular}




\section{DQA Surface Soil Report}

\section{Assessment Summary}

Site:

Planner(s):

Survey Unit Name:

Report Number:

Survey Unit Samples:

Reference Area Samples:

Test Performed:

Judgmental Samples:

Assessment Conclusion:
Hammond Depot FSS Planner

Vitkus

C2 SU1 through $12 \quad$ C2 SU12

12

16

17

WRS

Test Result: $\quad$ Pass

0

EMC Result:

Not Performed

Reject Null Hypothesis (Survey Unit PASSES)

\section{Retrospective Power Curve}

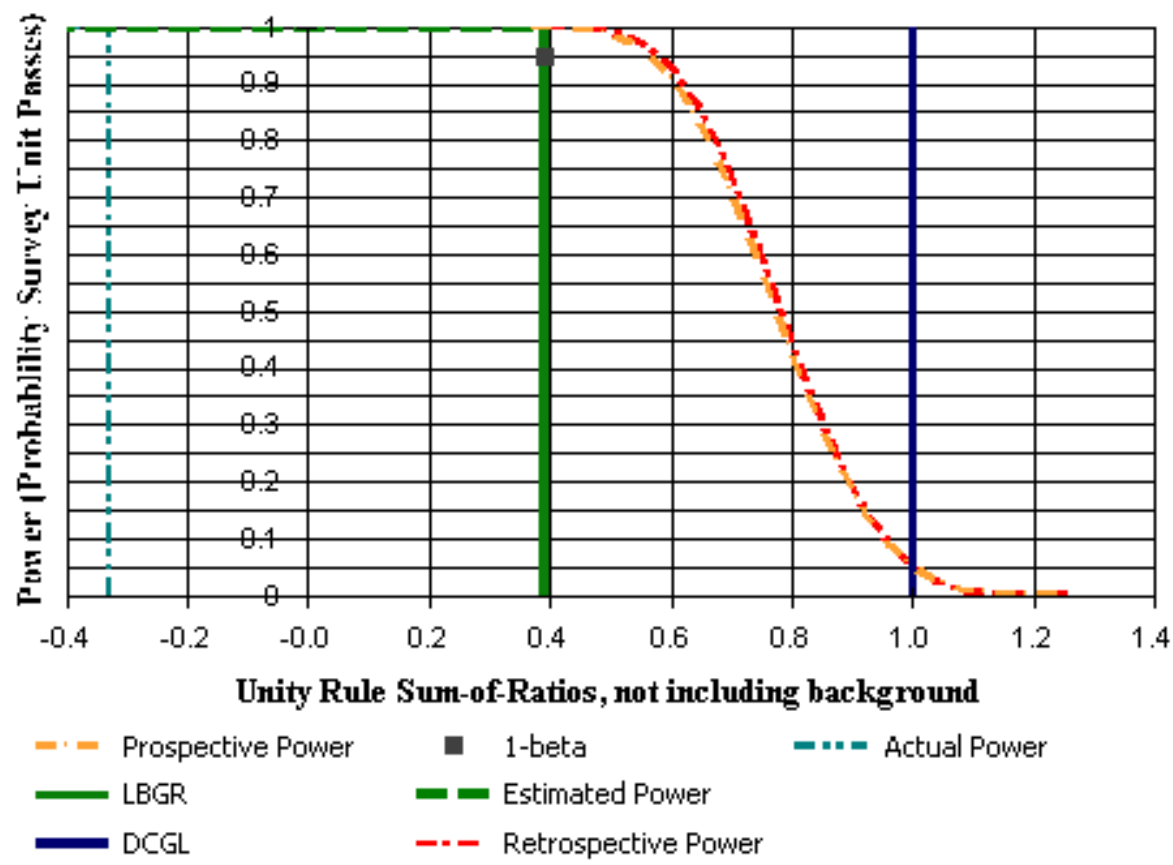




\section{DQA Surface Soil Report}

\section{Survey Unit Data}

NOTE: $\quad$ Type = "S" indicates survey unit sample.

Type = "R" indicates reference area sample.

\begin{tabular}{|c|c|c|c|}
\hline Sample Number & Type & Th-232 (pCi/g) & $\mathrm{U}-238$ (pCi/g) \\
\hline$\overline{478}$ & $\mathrm{~S}$ & 0.71 & 2.06 \\
\hline 479 & $S$ & 0.77 & 2.01 \\
\hline 480 & S & 0.67 & 1.04 \\
\hline 481 & S & 0.91 & 1.54 \\
\hline 482 & S & 0.78 & 1.81 \\
\hline 483 & $S$ & 0.56 & 1.15 \\
\hline 484 & $S$ & 0.76 & 1.4 \\
\hline 485 & $S$ & 0.75 & 1.8 \\
\hline 486 & S & 0.77 & 1.56 \\
\hline 487 & S & 0.9 & 1.93 \\
\hline 488 & S & 0.77 & 1.79 \\
\hline 489 & S & 1.36 & 2.11 \\
\hline 490 & S & 2.31 & 2.74 \\
\hline 491 & S & 1.43 & 2.56 \\
\hline 492 & S & 0.87 & 1.59 \\
\hline 493 & S & 1.5 & 2.34 \\
\hline 127 & $\mathrm{R}$ & 1.1 & 2.54 \\
\hline 128 & $\mathrm{R}$ & 0.85 & 2.66 \\
\hline 129 & $\mathrm{R}$ & 0.74 & 2.44 \\
\hline 130 & $\mathrm{R}$ & 1.09 & 2.3 \\
\hline 131 & $\mathrm{R}$ & 0.93 & 2.5 \\
\hline 132 & $\mathrm{R}$ & 0.79 & 1.65 \\
\hline 133 & $\mathrm{R}$ & 0.94 & 2.3 \\
\hline 134 & $\mathrm{R}$ & 0.98 & 2.55 \\
\hline 135 & $\mathrm{R}$ & 0.65 & 2.06 \\
\hline 136 & $\mathrm{R}$ & 0.73 & 2.66 \\
\hline 137 & $\mathrm{R}$ & 1.1 & 2.44 \\
\hline 138 & $\mathrm{R}$ & 1.03 & 2.42 \\
\hline 139 & $\mathrm{R}$ & 1.17 & 2.72 \\
\hline 140 & $\mathrm{R}$ & 1.36 & 3.35 \\
\hline 141 & $\mathrm{R}$ & 0.91 & 1.44 \\
\hline 142 & $\mathrm{R}$ & 1.71 & 3.57 \\
\hline 143 & $\mathrm{R}$ & 0.33 & 0.63 \\
\hline
\end{tabular}




\section{Modified Data (Unity Rule SOR)}

NOTE: $\quad$ Type = "S" indicates survey unit sample.

Type $=$ "R" indicates reference area sample.

\begin{tabular}{lcc} 
Sample Number & Type & Sum-of-Ratios (SOR) \\
\hline 478 & $\mathrm{~S}$ & 1.07 \\
479 & $\mathrm{~S}$ & 1.07 \\
480 & $\mathrm{~S}$ & 0.65 \\
481 & $\mathrm{~S}$ & 0.93 \\
482 & $\mathrm{~S}$ & 0.99 \\
483 & $\mathrm{~S}$ & 0.65 \\
484 & $\mathrm{~S}$ & 0.82 \\
485 & $\mathrm{~S}$ & 0.98 \\
486 & $\mathrm{~S}$ & 0.89 \\
487 & $\mathrm{~S}$ & 1.08 \\
488 & $\mathrm{~S}$ & 0.98 \\
489 & $\mathrm{~S}$ & 1.31 \\
490 & $\mathrm{~S}$ & 1.89 \\
491 & $\mathrm{~S}$ & 1.52 \\
492 & $\mathrm{~S}$ & 0.94 \\
493 & $\mathrm{~S}$ & 1.45 \\
127 & $\mathrm{R}$ & 1.4 \\
128 & $\mathrm{R}$ & 1.36 \\
129 & $\mathrm{R}$ & 1.23 \\
130 & $\mathrm{R}$ & 1.3 \\
131 & $\mathrm{R}$ & 1.32 \\
132 & $\mathrm{R}$ & 0.93 \\
133 & $\mathrm{R}$ & 1.24 \\
134 & $\mathrm{R}$ & 1.36 \\
135 & $\mathrm{R}$ & 1.05 \\
136 & $\mathrm{R}$ & 1.32 \\
137 & $\mathrm{R}$ & 1.36 \\
138 & $\mathrm{R}$ & 1.32 \\
139 & $\mathrm{R}$ & 1.49 \\
140 & $\mathrm{R}$ & 1.81 \\
141 & $\mathrm{R}$ & 0.89 \\
142 & $\mathrm{R}$ & 2.02 \\
143 & $\mathrm{R}$ & 0.37 \\
& &
\end{tabular}




\section{DQA Surface Soil Report}

\section{Basic Statistical Quantities Summary}

\begin{tabular}{lccc} 
Statistic & Survey Unit & Background & DQO Results \\
\hline Sample Number & 16 & 17 & N/2=18 \\
Mean (SOR) & 1.08 & 1.28 & 0.39 \\
Median (SOR) & 0.99 & 1.32 & N/A \\
Std Dev (SOR) & 0.32 & 0.36 & 0.39 \\
High Value (SOR) & 1.89 & 2.02 & N/A \\
Low Value (SOR) & 0.65 & 0.37 & N/A
\end{tabular}

\section{Statistical Test Summary}

$\begin{array}{ll}\text { Sum of Ranks: } & 561 \\ \text { Sum of Reference Ranks: } & 422 \\ \text { Critical Value: } & 335 \\ \text { Result: } & \text { Pass }\end{array}$

$\begin{array}{lll}\text { Data } & \text { Type } & \text { Adjusted Data }\end{array}$

0.37

0.89

0.93

1.05

1.23

1.24

1.3

1.32

1.32

1.32

1.36

1.36

1.36

1.4

1.49

1.81

2.02

0.65

0.65

0.82

0.89

0.93

0.94

0.98

0.98
561

Pass

$\begin{array}{ll}\mathrm{R} & 1.37\end{array}$

1.89

1.93

2.05

2.23

2.24

2.3

2.32

2.32

2.32

2.36

2.36

2.36

2.4

2.49

2.81

3.02

0.65

0.65

0.82

0.89

0.93

0.94

0.98

0.98
Rank

Reference Rank

$\begin{array}{cc}14 & 14 \\ 18 & 18 \\ 19 & 19 \\ 20 & 20 \\ 21 & 21 \\ 22 & 22 \\ 23 & 23 \\ 25 & 25 \\ 25 & 25 \\ 25 & 25 \\ 28 & 28 \\ 28 & 28 \\ 28 & 28 \\ 30 & 30 \\ 31 & 31 \\ 32 & 32 \\ 33 & 33 \\ 1.5 & 0 \\ 1.5 & 0 \\ 3 & 0 \\ 4 & 0 \\ 5 & 0 \\ 6 & 0 \\ 7.5 & 0 \\ 7.5 & 0\end{array}$




\section{DQA Surface Soil Report}

Statistical Test Summary

\begin{tabular}{lcccc} 
Data & Type & Adjusted Data & Rank & Reference Rank \\
\hline 0.99 & & & & 0 \\
1.07 & S & 0.99 & 10.5 & 0 \\
1.07 & S & 1.07 & 10.5 & 0 \\
1.08 & S & 1.07 & 12 & 0 \\
1.31 & S & 1.08 & 13 & 0 \\
1.45 & S & 1.31 & 15 & 0 \\
1.52 & S & 1.45 & 16 & 0 \\
1.89 & S & 1.52 & 17 & 0
\end{tabular}




\section{DQA Building Surface Report}

\section{Assessment Summary}

Site:

Planner(s):

Survey Unit Name:

Report Number:

Survey Unit Measurements:

Reference Area Measurements:

Test Performed:

Judgmental Areas:

Assessment Conclusion:
Hammond Depot FSS Planner

Vitkus

C2 SU12 Debris Pile DM Rev. 1

1

20

0

Sign

Test Result: Pass

0

EMC Result:

Not Performed

Reject Null Hypothesis (Survey Unit PASSES)

\section{Retrospective Power Curve}

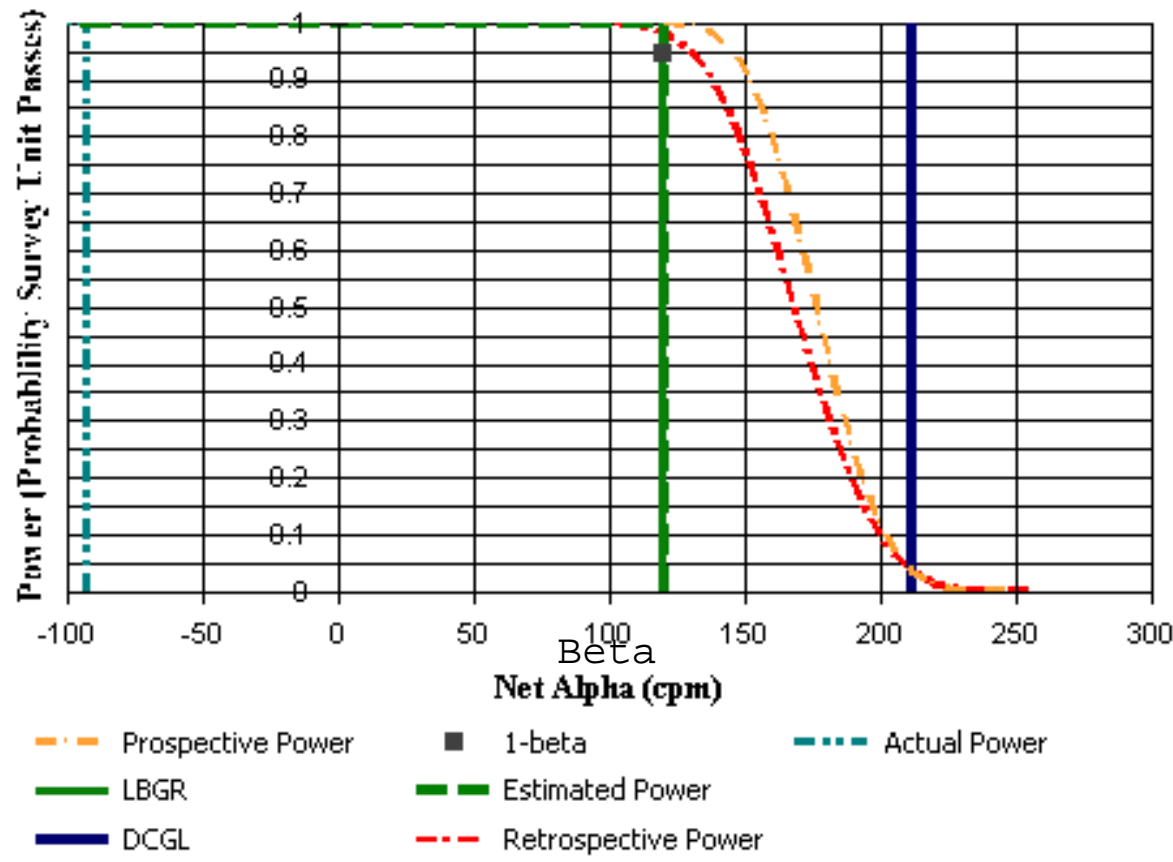




\section{DQA Building Surface Report}

\section{Survey Unit Data}

NOTE: $\quad$ Type = "S" indicates survey measurement.

Type = "R" indicates reference measurement.

\begin{tabular}{lccc} 
Measurement & Material & Type & Gross Alpha (cpm) \\
\hline 456512,4611391 & Concrete & S & 378 \\
456510,4611395 & Unpainted Cinder Block & S & 303 \\
456507,4611399 & Slag & S & 454 \\
456505,4611404 & Concrete & S & 319 \\
456515,4611404 & Slag & S & 495 \\
456502,4611408 & Slag & S & 410 \\
456512,4611408 & Small Brick & S & 619 \\
456500,4611412 & Unpainted Cinder Block & S & 313 \\
456510,4611412 & Slag & S & 399 \\
456510,4611421 & Slag & S & 405 \\
456522,4611425 & Unpainted Cinder Block & S & 318 \\
456527,4611425 & Slag & S & 365 \\
456520,4611430 & Slag & S & 455 \\
456525,4611430 & Small Brick & S & 793 Location investigated, piece of fire brick \\
456525,4611438 & Slag & S & 369 with background higher due to natural U/Th \\
456530,4611447 & Slag & S & 381 \\
456522,4611452 & Small Brick & S & 574 \\
456527,4611452 & Slag & S & 439 \\
456525,4611456 & Slag & S & 365 \\
456530,4611456 & & 470
\end{tabular}

\section{Basic Statistical Quantities Summary}

\begin{tabular}{|c|c|c|c|}
\hline Statistic & Survey Unit & Background & DQO Results \\
\hline Sample Number & 20 & $\mathrm{~N} / \mathrm{A}$ & $N=20$ \\
\hline Mean $\left(\mathrm{dpm} / 100 \mathrm{~cm}^{2}\right)$ & -73.51 & N/A & 120 \\
\hline Median $\left(\mathrm{dpm} / 100 \mathrm{~cm}^{2}\right)$ & -92.31 & $\mathrm{~N} / \mathrm{A}$ & $\mathrm{N} / \mathrm{A}$ \\
\hline Std Dev (dpm/100 cm²) & 156.70 & $\mathrm{~N} / \mathrm{A}$ & 67.3 \\
\hline High Value $\left(\mathrm{dpm} / 100 \mathrm{~cm}^{2}\right)$ & 408.16 & $\mathrm{~N} / \mathrm{A}$ & $\mathrm{N} / \mathrm{A}$ \\
\hline Low Value $\left(\mathrm{dpm} / 100 \mathrm{~cm}^{2}\right)$ & -273.43 & $\mathrm{~N} / \mathrm{A}$ & $\mathrm{N} / \mathrm{A}$ \\
\hline
\end{tabular}




\section{DQA Building Surface Report}

\section{Statistical Test Summary}

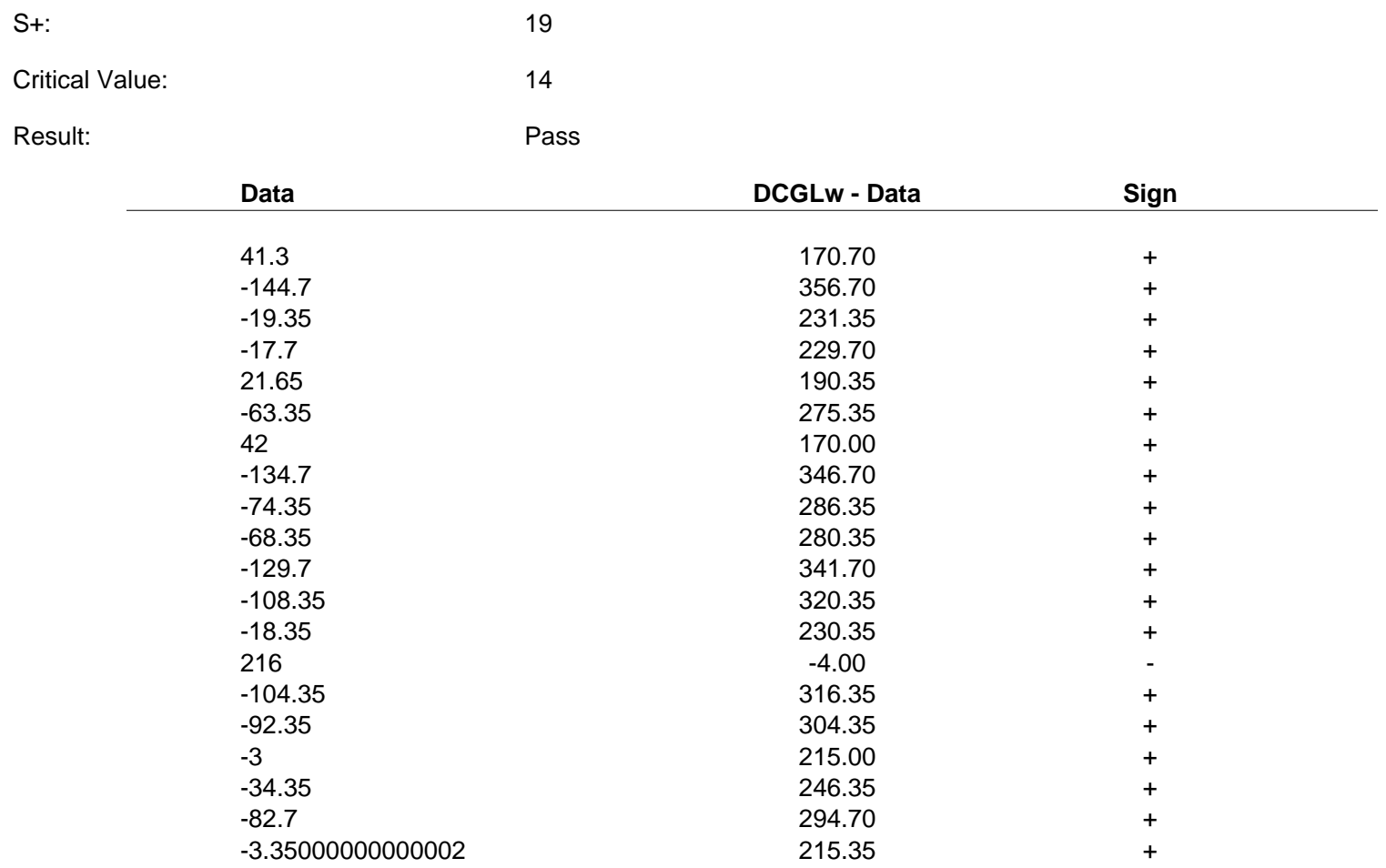




\section{DQA Surface Soil Report}

\section{Assessment Summary}

Site:

Planner(s):

Survey Unit Name:

Report Number:

Survey Unit Samples:

Reference Area Samples:

Test Performed:

Judgmental Samples:

Assessment Conclusion:
Hammond Depot FSS Planner

Vitkus

C3 SU1 through 5 C3 SU1

1

13

17

WRS

Test Result: $\quad$ Pass

0

EMC Result:

Not Performed

Reject Null Hypothesis (Survey Unit PASSES)

\section{Retrospective Power Curve}

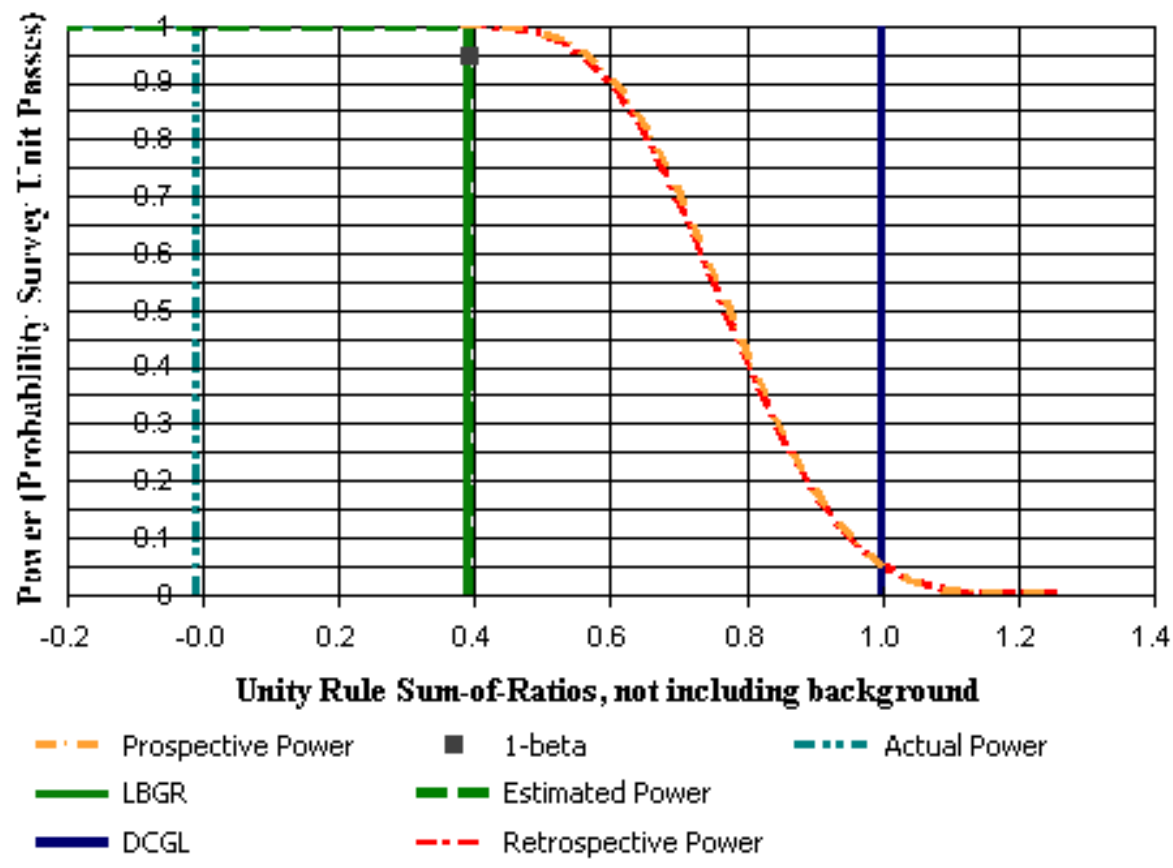




\section{DQA Surface Soil Report}

\section{Survey Unit Data}

NOTE: $\quad$ Type = "S" indicates survey unit sample.

Type = "R" indicates reference area sample.

\begin{tabular}{lccc} 
Sample Number & Type & Th-232 (pCi/g) & U-238 (pCi/g) \\
\hline 144 & S & 0.77 & 2.3 \\
145 & S & 0.95 & 2.6 \\
147 & S & 0.94 & 2.46 \\
148 & S & 1.1 & 2.9 \\
149 & S & 0.9 & 2.4 \\
151 & S & 0.67 & 2.68 \\
153 & S & 1.02 & 2.66 \\
154 & S & 1.1 & 2.81 \\
155 & S & 0.8 & 1.96 \\
156 & S & 1.43 & 1.84 \\
157 & S & 0.88 & 2.85 \\
158 & S & 0.89 & 1.4 \\
160 & S & 1.13 & 3.31 \\
127 & $\mathrm{R}$ & 1.1 & 2.54 \\
128 & $\mathrm{R}$ & 0.85 & 2.66 \\
129 & $\mathrm{R}$ & 0.74 & 2.44 \\
130 & $\mathrm{R}$ & 1.09 & 2.3 \\
131 & $\mathrm{R}$ & 0.93 & 2.5 \\
132 & $\mathrm{R}$ & 0.79 & 1.65 \\
133 & $\mathrm{R}$ & 0.94 & 2.3 \\
134 & $\mathrm{R}$ & 0.98 & 2.55 \\
135 & $\mathrm{R}$ & 0.65 & 2.06 \\
136 & $\mathrm{R}$ & 0.73 & 2.66 \\
137 & $\mathrm{R}$ & 1.1 & 2.44 \\
138 & $\mathrm{R}$ & 1.03 & 2.42 \\
139 & $\mathrm{R}$ & 1.17 & 2.72 \\
140 & $\mathrm{R}$ & 1.36 & 3.35 \\
141 & $\mathrm{R}$ & 0.91 & 1.44 \\
142 & $\mathrm{R}$ & 1.71 & 3.57 \\
143 & $\mathrm{R}$ & 0.33 & 0.63 \\
& & &
\end{tabular}




\section{DQA Surface Soil Report}

\section{Modified Data (Unity Rule SOR)}

NOTE: $\quad$ Type = "S" indicates survey unit sample.

Type $=$ "R" indicates reference area sample.

\begin{tabular}{lcc} 
Sample Number & Type & Sum-of-Ratios (SOR) \\
\hline 144 & $\mathrm{~S}$ & 1.19 \\
145 & $\mathrm{~S}$ & 1.37 \\
147 & $\mathrm{~S}$ & 1.31 \\
148 & $\mathrm{~S}$ & 1.54 \\
149 & $\mathrm{~S}$ & 1.27 \\
151 & $\mathrm{~S}$ & 1.3 \\
153 & $\mathrm{~S}$ & 1.42 \\
154 & $\mathrm{~S}$ & 1.5 \\
155 & $\mathrm{~S}$ & 1.06 \\
156 & $\mathrm{~S}$ & 1.23 \\
157 & $\mathrm{~S}$ & 1.44 \\
158 & $\mathrm{~S}$ & 0.87 \\
160 & $\mathrm{~S}$ & 1.71 \\
127 & $\mathrm{R}$ & 1.4 \\
128 & $\mathrm{R}$ & 1.36 \\
129 & $\mathrm{R}$ & 1.23 \\
130 & $\mathrm{R}$ & 1.3 \\
131 & $\mathrm{R}$ & 1.32 \\
132 & $\mathrm{R}$ & 0.93 \\
133 & $\mathrm{R}$ & 1.24 \\
134 & $\mathrm{R}$ & 1.36 \\
135 & $\mathrm{R}$ & 1.05 \\
136 & $\mathrm{R}$ & 1.32 \\
137 & $\mathrm{R}$ & 1.36 \\
138 & $\mathrm{R}$ & 1.32 \\
139 & $\mathrm{R}$ & 1.49 \\
140 & $\mathrm{R}$ & 1.81 \\
141 & $\mathrm{R}$ & 0.89 \\
142 & $\mathrm{R}$ & 2.02 \\
143 & $\mathrm{R}$ & 0.37
\end{tabular}




\section{DQA Surface Soil Report}

\section{Basic Statistical Quantities Summary}

\begin{tabular}{lccc} 
Statistic & Survey Unit & Background & DQO Results \\
\hline Sample Number & 13 & 17 & N/2=18 \\
Mean (SOR) & 1.32 & 1.28 & 0.39 \\
Median (SOR) & 1.31 & 1.32 & $\mathrm{~N} / \mathrm{A}$ \\
Std Dev (SOR) & 0.22 & 0.36 & 0.39 \\
High Value (SOR) & 1.71 & 2.02 & $\mathrm{~N} / \mathrm{A}$ \\
Low Value (SOR) & 0.87 & 0.37 & $\mathrm{~N} / \mathrm{A}$
\end{tabular}

\section{Statistical Test Summary}

$\begin{array}{ll}\text { Sum of Ranks: } & 465 \\ \text { Sum of Reference Ranks: } & 368 \\ \text { Critical Value: } & 303 \\ \text { Result: } & \text { Pass }\end{array}$

$\begin{array}{llll}\text { Data } & \text { Type } & \text { Adjusted Data } & \text { Ran }\end{array}$

$\begin{array}{lll}0.37 & \mathrm{R} & 1.37\end{array}$

0.89

0.93

1.05

1.23

1.24

1.3

1.32

1.32

1.32

1.36

1.36

1.36

1.4

1.49

1.81

2.02

0.87

1.06

1.19

1.23

1.27

1.3

1.31

1.37

1.37

1.89

1.93

2.05

2.23

2.24

2.3

2.32

2.32

2.32

2.36

2.36

2.36

2.4

2.49

2.81

3.02

0.87

1.06

1.19

1.23

1.27

1.3

1.31

1.37

Rank

Reference Rank

$8 \quad 8$

$15 \quad 15$

$16 \quad 16$

$\begin{array}{ll}17 & 17\end{array}$

$\begin{array}{ll}18 & 18\end{array}$

$19 \quad 19$

$20-20$

$22 \quad 22$

$22 \quad 22$

$22 \quad 22$

$25 \quad 25$

$25-25$

25
25

$27 \quad 27$

$\begin{array}{ll}28 & 28\end{array}$

$29 \quad 29$

$30 \quad 30$

1 - 0

$\begin{array}{ll}1 & 0 \\ 3 & 0\end{array}$

4 0

$\begin{array}{ll}4 & 0 \\ 5 & 0\end{array}$

$\begin{array}{ll}6 & 0\end{array}$

$\begin{array}{ll}7 & 0 \\ 9 & 0\end{array}$




\section{DQA Surface Soil Report}

\section{Statistical Test Summary}

\begin{tabular}{lcccc} 
Data & Type & Adjusted Data & Rank & Reference Rank \\
\hline 1.42 & & & 10 & 0 \\
1.44 & S & 1.42 & 11 & 0 \\
1.5 & S & 1.44 & 12 & 0 \\
1.54 & S & 1.5 & 13 & 0 \\
1.71 & S & 1.54 & 14 & 0
\end{tabular}




\section{DQA Surface Soil Report}

\section{Assessment Summary}

Site:

Planner(s):

Survey Unit Name:

Report Number:

Survey Unit Samples:

Reference Area Samples:

Test Performed:

Judgmental Samples:

Assessment Conclusion:
Hammond Depot FSS Planner

Vitkus

C3 SU1 through 5 C3 SU2

2

17

17

WRS

Test Result: $\quad$ Pass

0

EMC Result:

Not Performed

Reject Null Hypothesis (Survey Unit PASSES)

\section{Retrospective Power Curve}

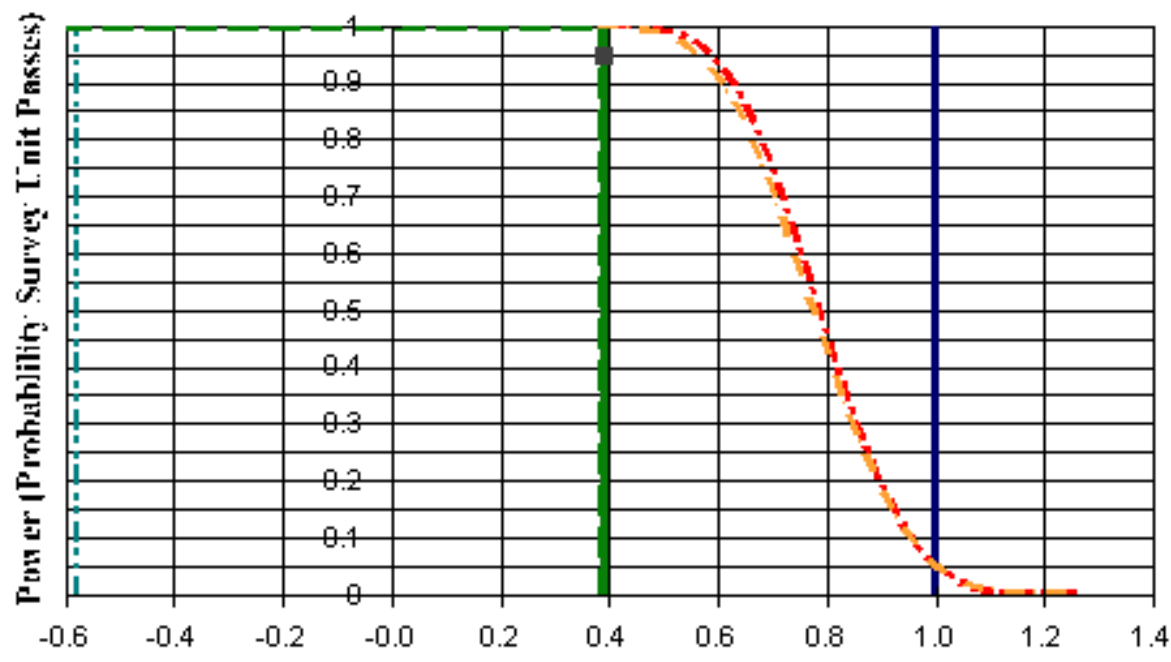

Unity Rule Sum-of-Ratios, not including background

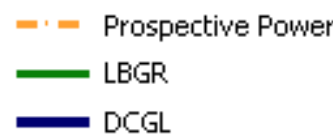

1-beta

n " - Actual Power

- Estimated Power

-- - Retrospective Power 


\section{DQA Surface Soil Report}

\section{Survey Unit Data}

NOTE: $\quad$ Type = "S" indicates survey unit sample.

Type = "R" indicates reference area sample.

\begin{tabular}{|c|c|c|c|}
\hline Sample Number & Type & Th-232 (pCi/g) & U-238 (pCi/g) \\
\hline 161 & $\mathrm{~S}$ & 0.82 & 1.6 \\
\hline 163 & $S$ & 0.47 & 1.34 \\
\hline 164 & $S$ & 0.61 & 1.2 \\
\hline 165 & S & 0.64 & 1.39 \\
\hline 166 & $S$ & 0.68 & 1.6 \\
\hline 167 & S & 0.7 & 1.08 \\
\hline 168 & $S$ & 0.5 & 0.66 \\
\hline 169 & $S$ & 0.81 & 1.19 \\
\hline 170 & $S$ & 0.72 & 1.83 \\
\hline 171 & $S$ & 0.77 & 1.06 \\
\hline 172 & S & 0.58 & 0.99 \\
\hline 173 & S & 0.77 & 1.19 \\
\hline 174 & S & 0.7 & 1.46 \\
\hline 175 & S & 0.6 & 0.86 \\
\hline 176 & S & 0.29 & 0.62 \\
\hline 177 & S & 0.86 & 1.54 \\
\hline 178 & S & 0.99 & 2.16 \\
\hline 127 & $\mathrm{R}$ & 1.1 & 2.54 \\
\hline 128 & $\mathrm{R}$ & 0.85 & 2.66 \\
\hline 129 & $\mathrm{R}$ & 0.74 & 2.44 \\
\hline 130 & $\mathrm{R}$ & 1.09 & 2.3 \\
\hline 131 & $\mathrm{R}$ & 0.93 & 2.5 \\
\hline 132 & $\mathrm{R}$ & 0.79 & 1.65 \\
\hline 133 & $\mathrm{R}$ & 0.94 & 2.3 \\
\hline 134 & $\mathrm{R}$ & 0.98 & 2.55 \\
\hline 135 & $\mathrm{R}$ & 0.65 & 2.06 \\
\hline 136 & $\mathrm{R}$ & 0.73 & 2.66 \\
\hline 137 & $\mathrm{R}$ & 1.1 & 2.44 \\
\hline 138 & $\mathrm{R}$ & 1.03 & 2.42 \\
\hline 139 & $\mathrm{R}$ & 1.17 & 2.72 \\
\hline 140 & $\mathrm{R}$ & 1.36 & 3.35 \\
\hline 141 & $\mathrm{R}$ & 0.91 & 1.44 \\
\hline 142 & $\mathrm{R}$ & 1.71 & 3.57 \\
\hline 143 & $\mathrm{R}$ & 0.33 & 0.63 \\
\hline
\end{tabular}




\section{Modified Data (Unity Rule SOR)}

NOTE: $\quad$ Type = "S" indicates survey unit sample.

Type $=$ "R" indicates reference area sample.

\begin{tabular}{lcc} 
Sample Number & Type & Sum-of-Ratios (SOR) \\
\hline 161 & $\mathrm{~S}$ & 0.92 \\
163 & $\mathrm{~S}$ & 0.7 \\
164 & $\mathrm{~S}$ & 0.69 \\
165 & $\mathrm{~S}$ & 0.78 \\
166 & $\mathrm{~S}$ & 0.87 \\
167 & $\mathrm{~S}$ & 0.67 \\
168 & $\mathrm{~S}$ & 0.44 \\
169 & $\mathrm{~S}$ & 0.76 \\
170 & $\mathrm{~S}$ & 0.98 \\
171 & $\mathrm{~S}$ & 0.69 \\
172 & $\mathrm{~S}$ & 0.6 \\
173 & $\mathrm{~S}$ & 0.74 \\
174 & $\mathrm{~S}$ & 0.83 \\
175 & $\mathrm{~S}$ & 0.55 \\
176 & $\mathrm{~S}$ & 0.35 \\
177 & $\mathrm{~S}$ & 0.91 \\
178 & $\mathrm{~S}$ & 1.21 \\
127 & $\mathrm{R}$ & 1.4 \\
128 & $\mathrm{R}$ & 1.36 \\
129 & $\mathrm{R}$ & 1.23 \\
130 & $\mathrm{R}$ & 1.3 \\
131 & $\mathrm{R}$ & 1.32 \\
132 & $\mathrm{R}$ & 0.93 \\
133 & $\mathrm{R}$ & 1.24 \\
134 & $\mathrm{R}$ & 1.36 \\
135 & $\mathrm{R}$ & 1.05 \\
136 & $\mathrm{R}$ & 1.32 \\
137 & $\mathrm{R}$ & 1.36 \\
138 & $\mathrm{R}$ & 1.32 \\
139 & $\mathrm{R}$ & 1.49 \\
140 & $\mathrm{R}$ & 1.81 \\
141 & $\mathrm{R}$ & 0.89 \\
142 & $\mathrm{R}$ & 2.02 \\
143 & $\mathrm{R}$ & 0.37 \\
& &
\end{tabular}




\section{DQA Surface Soil Report}

\section{Basic Statistical Quantities Summary}

\begin{tabular}{lccc} 
Statistic & Survey Unit & Background & DQO Results \\
\hline Sample Number & 17 & 17 & N/2=18 \\
Mean (SOR) & 0.75 & 1.28 & 0.39 \\
Median (SOR) & 0.74 & 1.32 & N/A \\
Std Dev (SOR) & 0.21 & 0.36 & 0.39 \\
High Value (SOR) & 1.21 & 2.02 & N/A \\
Low Value (SOR) & 0.35 & 0.37 & N/A
\end{tabular}

\section{Statistical Test Summary}

$\begin{array}{ll}\text { Sum of Ranks: } & 595 \\ \text { Sum of Reference Ranks: } & 442 \\ \text { Critical Value: } & 345 \\ \text { Result: } & \text { Pass }\end{array}$

\begin{tabular}{lcccc} 
Data & Type & Adjusted Data & Rank & Reference \\
\hline & & & 18 & 18 \\
0.37 & $\mathrm{R}$ & 1.37 & 19 & 19 \\
0.89 & $\mathrm{R}$ & 1.89 & 20 & 20 \\
0.93 & $\mathrm{R}$ & 1.93 & 21 & 21 \\
1.05 & $\mathrm{R}$ & 2.05 & 22 & 22 \\
1.23 & $\mathrm{R}$ & 2.23 & 23 & 23 \\
1.24 & $\mathrm{R}$ & 2.24 & 24 & 24 \\
1.3 & $\mathrm{R}$ & 2.3 & 26 & 26 \\
1.32 & $\mathrm{R}$ & 2.32 & 26 & 26 \\
1.32 & $\mathrm{R}$ & 2.32 & 26 & 26 \\
1.32 & $\mathrm{R}$ & 2.32 & 29 & 29 \\
1.36 & $\mathrm{R}$ & 2.36 & 29 & 29 \\
1.36 & $\mathrm{R}$ & 2.36 & 29 & 29 \\
1.36 & $\mathrm{R}$ & 2.36 & 31 & 31 \\
1.4 & $\mathrm{R}$ & 2.4 & 32 & 32 \\
1.49 & $\mathrm{R}$ & 2.49 & 33 & 33 \\
1.81 & $\mathrm{R}$ & 2.81 & 34 & 34 \\
2.02 & $\mathrm{R}$ & 3.02 & 1 & 0 \\
0.35 & $\mathrm{~S}$ & 0.35 & 2 & 0 \\
0.44 & $\mathrm{~S}$ & 0.44 & 3 & 0 \\
0.55 & $\mathrm{~S}$ & 0.55 & 4 & 0 \\
0.6 & $\mathrm{~S}$ & 0.6 & 5 & 0 \\
0.67 & $\mathrm{~S}$ & 0.67 & 6.5 & 0 \\
0.69 & $\mathrm{~S}$ & 0.69 & 8 & 0 \\
0.69 & $\mathrm{~S}$ & 0.69 & & 0 \\
0.7 & $\mathrm{~S}$ & 0.7 & & 0
\end{tabular}




\section{DQA Surface Soil Report}

Statistical Test Summary

\begin{tabular}{lcccc} 
Data & Type & Adjusted Data & Rank & Reference Rank \\
\hline 0.74 & & & & 0 \\
0.76 & S & 0.74 & 10 & 0 \\
0.78 & S & 0.76 & 11 & 0 \\
0.83 & S & 0.78 & 12 & 0 \\
0.87 & S & 0.83 & 13 & 0 \\
0.91 & S & 0.87 & 14 & 0 \\
0.92 & S & 0.91 & 15 & 0 \\
0.98 & S & 0.92 & 16 & 0 \\
1.21 & S & 0.98 & 17 & 0
\end{tabular}




\section{DQA Surface Soil Report}

\section{Assessment Summary}

Site:

Planner(s):

Survey Unit Name:

Report Number:

Survey Unit Samples:

Reference Area Samples:

Test Performed:

Judgmental Samples:

Assessment Conclusion:
Hammond Depot FSS Planner

Vitkus

C3 SU1 through 5 C3 SU3

3

17

17

WRS

0

Test Result: $\quad$ Pass

EMC Result: Not Performed

Reject Null Hypothesis (Survey Unit PASSES)

\section{Retrospective Power Curve}

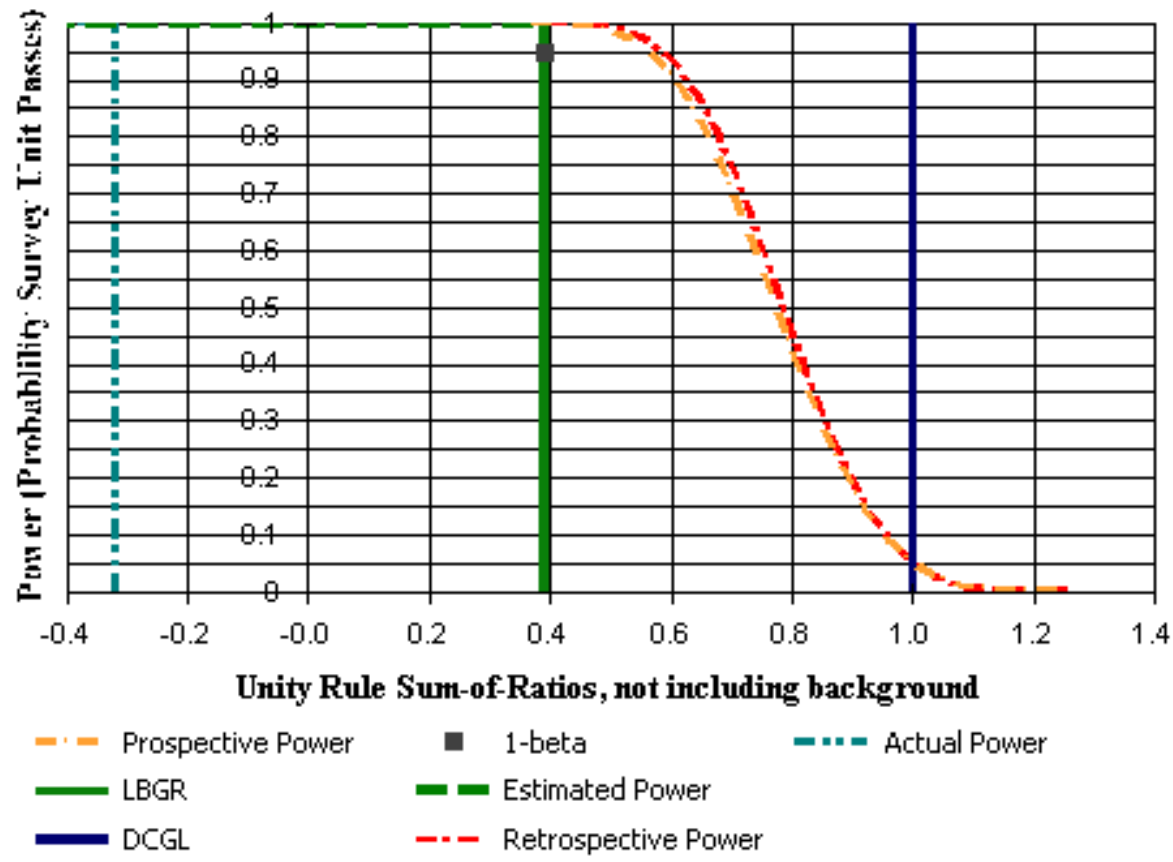




\section{DQA Surface Soil Report}

\section{Survey Unit Data}

NOTE: $\quad$ Type = "S" indicates survey unit sample.

Type = "R" indicates reference area sample.

\begin{tabular}{|c|c|c|c|}
\hline Sample Number & Type & Th-232 (pCi/g) & U-238 (pCi/g) \\
\hline$\overline{179}$ & $\mathrm{~S}$ & 0.88 & 1.74 \\
\hline 180 & S & 0.44 & 1.37 \\
\hline 181 & S & 0.89 & 1.53 \\
\hline 182 & S & 1.06 & 2.78 \\
\hline 183 & $S$ & 1.05 & 1.6 \\
\hline 184 & S & 0.66 & 1.59 \\
\hline 185 & $S$ & 0.36 & 0.69 \\
\hline 186 & $S$ & 0.87 & 1.37 \\
\hline 187 & S & 0.82 & 1.82 \\
\hline 188 & S & 1.15 & 2.43 \\
\hline 189 & S & 0.88 & 1.18 \\
\hline 190 & S & 1.2 & 2.24 \\
\hline 191 & $S$ & 0.76 & 2.22 \\
\hline 192 & S & 0.86 & 2.31 \\
\hline 193 & S & 0.74 & 2.39 \\
\hline 194 & S & 1.02 & 1.82 \\
\hline 195 & S & 0.39 & 0.97 \\
\hline 127 & $\mathrm{R}$ & 1.1 & 2.54 \\
\hline 128 & $\mathrm{R}$ & 0.85 & 2.66 \\
\hline 129 & $\mathrm{R}$ & 0.74 & 2.44 \\
\hline 130 & $\mathrm{R}$ & 1.09 & 2.3 \\
\hline 131 & $\mathrm{R}$ & 0.93 & 2.5 \\
\hline 132 & $\mathrm{R}$ & 0.79 & 1.65 \\
\hline 133 & $\mathrm{R}$ & 0.94 & 2.3 \\
\hline 134 & $\mathrm{R}$ & 0.98 & 2.55 \\
\hline 135 & $\mathrm{R}$ & 0.65 & 2.06 \\
\hline 136 & $\mathrm{R}$ & 0.73 & 2.66 \\
\hline 137 & $\mathrm{R}$ & 1.1 & 2.44 \\
\hline 138 & $\mathrm{R}$ & 1.03 & 2.42 \\
\hline 139 & $\mathrm{R}$ & 1.17 & 2.72 \\
\hline 140 & $\mathrm{R}$ & 1.36 & 3.35 \\
\hline 141 & $\mathrm{R}$ & 0.91 & 1.44 \\
\hline 142 & $\mathrm{R}$ & 1.71 & 3.57 \\
\hline 143 & $\mathrm{R}$ & 0.33 & 0.63 \\
\hline
\end{tabular}




\section{Modified Data (Unity Rule SOR)}

NOTE: $\quad$ Type = "S" indicates survey unit sample.

Type $=$ "R" indicates reference area sample.

\begin{tabular}{lcc} 
Sample Number & Type & Sum-of-Ratios (SOR) \\
\hline 179 & $\mathrm{~S}$ & 1 \\
180 & $\mathrm{~S}$ & 0.7 \\
181 & $\mathrm{~S}$ & 0.92 \\
182 & $\mathrm{~S}$ & 1.48 \\
183 & $\mathrm{~S}$ & 1 \\
184 & $\mathrm{~S}$ & 0.86 \\
185 & $\mathrm{~S}$ & 0.4 \\
186 & $\mathrm{~S}$ & 0.85 \\
187 & $\mathrm{~S}$ & 1.01 \\
188 & $\mathrm{~S}$ & 1.37 \\
189 & $\mathrm{~S}$ & 0.78 \\
190 & $\mathrm{~S}$ & 1.31 \\
191 & $\mathrm{~S}$ & 1.15 \\
192 & $\mathrm{~S}$ & 1.22 \\
193 & $\mathrm{~S}$ & 1.21 \\
194 & $\mathrm{~S}$ & 1.08 \\
195 & $\mathrm{~S}$ & 0.52 \\
127 & $\mathrm{R}$ & 1.4 \\
128 & $\mathrm{R}$ & 1.36 \\
129 & $\mathrm{R}$ & 1.23 \\
130 & $\mathrm{R}$ & 1.3 \\
131 & $\mathrm{R}$ & 1.32 \\
132 & $\mathrm{R}$ & 0.93 \\
133 & $\mathrm{R}$ & 1.24 \\
134 & $\mathrm{R}$ & 1.36 \\
135 & $\mathrm{R}$ & 1.05 \\
136 & $\mathrm{R}$ & 1.32 \\
137 & $\mathrm{R}$ & 1.36 \\
138 & $\mathrm{R}$ & 1.32 \\
139 & $\mathrm{R}$ & 1.49 \\
140 & $\mathrm{R}$ & 1.81 \\
141 & $\mathrm{R}$ & 0.89 \\
142 & $\mathrm{R}$ & 2.02 \\
143 & $\mathrm{R}$ & 0.37 \\
& &
\end{tabular}




\section{DQA Surface Soil Report}

\section{Basic Statistical Quantities Summary}

\begin{tabular}{lccc} 
Statistic & Survey Unit & Background & DQO Results \\
\hline Sample Number & 17 & 17 & N/2=18 \\
Mean (SOR) & 0.99 & 1.28 & 0.39 \\
Median (SOR) & 1.00 & 1.32 & N/A \\
Std Dev (SOR) & 0.29 & 0.36 & 0.39 \\
High Value (SOR) & 1.48 & 2.02 & N/A \\
Low Value (SOR) & 0.40 & 0.37 & N/A
\end{tabular}

\section{Statistical Test Summary}

$\begin{array}{ll}\text { Sum of Ranks: } & 595 \\ \text { Sum of Reference Ranks: } & 440 \\ \text { Critical Value: } & 345 \\ \text { Result: } & \text { Pass }\end{array}$

\begin{tabular}{lcccc} 
Data & Type & Adjusted Data & Rank & Reference \\
\hline & & & & 16 \\
0.37 & $\mathrm{R}$ & 1.37 & 19 & 19 \\
0.89 & $\mathrm{R}$ & 1.89 & 20 & 20 \\
0.93 & $\mathrm{R}$ & 1.93 & 21 & 21 \\
1.05 & $\mathrm{R}$ & 2.05 & 22 & 22 \\
1.23 & $\mathrm{R}$ & 2.23 & 23 & 23 \\
1.24 & $\mathrm{R}$ & 2.24 & 24 & 24 \\
1.3 & $\mathrm{R}$ & 2.3 & 26 & 26 \\
1.32 & $\mathrm{R}$ & 2.32 & 26 & 26 \\
1.32 & $\mathrm{R}$ & 2.32 & 26 & 26 \\
1.32 & $\mathrm{R}$ & 2.32 & 29 & 29 \\
1.36 & $\mathrm{R}$ & 2.36 & 29 & 29 \\
1.36 & $\mathrm{R}$ & 2.36 & 29 & 29 \\
1.36 & $\mathrm{R}$ & 2.36 & 31 & 31 \\
1.4 & $\mathrm{R}$ & 2.4 & 32 & 32 \\
1.49 & $\mathrm{R}$ & 2.49 & 33 & 33 \\
1.81 & $\mathrm{R}$ & 2.81 & 34 & 34 \\
2.02 & $\mathrm{R}$ & 3.02 & 1 & 0 \\
0.4 & $\mathrm{~S}$ & 0.4 & 2 & 0 \\
0.52 & $\mathrm{~S}$ & 0.52 & 3 & 0 \\
0.7 & $\mathrm{~S}$ & 0.7 & 4 & 0 \\
0.78 & $\mathrm{~S}$ & 0.78 & 5 & 0 \\
0.85 & $\mathrm{~S}$ & 0.85 & 6 & 7 \\
0.86 & $\mathrm{~S}$ & 0.86 & 8.5 & 0 \\
0.92 & $\mathrm{~S}$ & 0.92 & 1 & 0 \\
1 & $\mathrm{~S}$ & & & 0
\end{tabular}




\section{DQA Surface Soil Report}

Statistical Test Summary

\begin{tabular}{lcccc} 
Data & Type & Adjusted Data & Rank & Reference \\
\hline 1 & & 1 & 8.5 & 0 \\
1.01 & S & 1.01 & 10 & 0 \\
1.08 & S & 1.08 & 11 & 0 \\
1.15 & S & 1.15 & 12 & 0 \\
1.21 & S & 1.21 & 13 & 0 \\
1.22 & S & 1.22 & 14 & 0 \\
1.31 & S & 1.31 & 15 & 0 \\
1.37 & S & 1.37 & 17 & 0 \\
1.48 & S & 1.48 & 18 & 0
\end{tabular}




\section{DQA Surface Soil Report}

\section{Assessment Summary}

Site:

Planner(s):

Survey Unit Name:

Report Number:

Survey Unit Samples:

Reference Area Samples:

Test Performed:

Judgmental Samples:

Assessment Conclusion:
Hammond Depot FSS Planner

Vitkus

C3 SU1 through 5 C3 SU4

4

17

17

WRS

Test Result: $\quad$ Pass

0

EMC Result:

Not Performed

Reject Null Hypothesis (Survey Unit PASSES)

\section{Retrospective Power Curve}

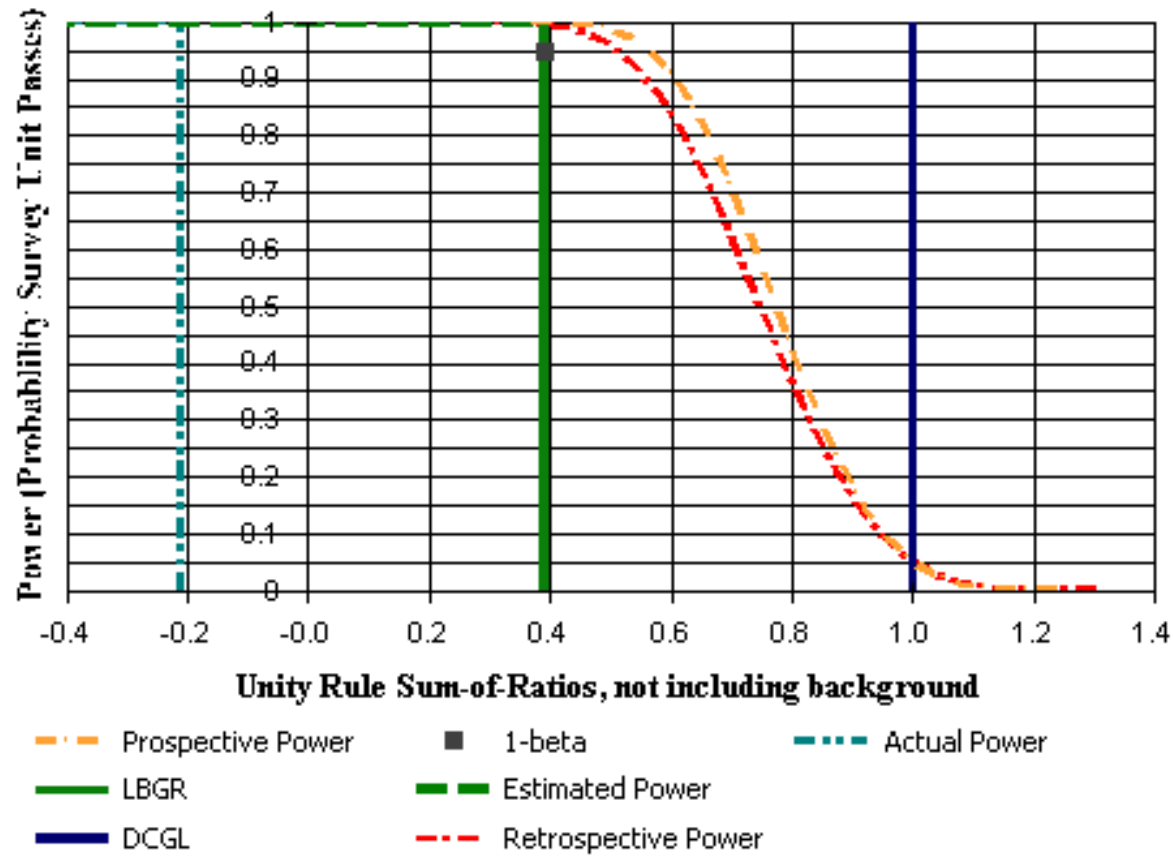




\section{DQA Surface Soil Report}

\section{Survey Unit Data}

NOTE: $\quad$ Type = "S" indicates survey unit sample.

Type = "R" indicates reference area sample.

\begin{tabular}{|c|c|c|c|}
\hline Sample Number & Type & Th-232 (pCi/g) & $\mathrm{U}-238$ (pCi/g) \\
\hline$\overline{215}$ & $\mathrm{~S}$ & 1.4 & 3.7 \\
\hline 216 & S & 1.66 & 2.63 \\
\hline 217 & S & 1.19 & 1.88 \\
\hline 218 & S & 1.01 & 1.24 \\
\hline 219 & S & 1.53 & 2.3 \\
\hline 220 & S & 1.55 & 2.16 \\
\hline 221 & $S$ & 1.38 & 2.34 \\
\hline 222 & $S$ & 1.5 & 2.31 \\
\hline 223 & $S$ & 0.9 & 2.01 \\
\hline 224 & $S$ & 1.04 & 1.72 \\
\hline 225 & S & 1.44 & 2.3 \\
\hline 226 & S & 1.24 & 1.65 \\
\hline 227 & S & 1.09 & 1.39 \\
\hline 228 & S & 0.96 & 1.94 \\
\hline 229 & $S$ & 0.47 & 0.6 \\
\hline 230 & S & 0.41 & 0.74 \\
\hline 231 & S & 0.53 & 0.85 \\
\hline 127 & $\mathrm{R}$ & 1.1 & 2.54 \\
\hline 128 & $\mathrm{R}$ & 0.85 & 2.66 \\
\hline 129 & $\mathrm{R}$ & 0.74 & 2.44 \\
\hline 130 & $\mathrm{R}$ & 1.09 & 2.3 \\
\hline 131 & $\mathrm{R}$ & 0.93 & 2.5 \\
\hline 132 & $\mathrm{R}$ & 0.79 & 1.65 \\
\hline 133 & $\mathrm{R}$ & 0.94 & 2.3 \\
\hline 134 & $\mathrm{R}$ & 0.98 & 2.55 \\
\hline 135 & $\mathrm{R}$ & 0.65 & 2.06 \\
\hline 136 & $\mathrm{R}$ & 0.73 & 2.66 \\
\hline 137 & $\mathrm{R}$ & 1.1 & 2.44 \\
\hline 138 & $\mathrm{R}$ & 1.03 & 2.42 \\
\hline 139 & $\mathrm{R}$ & 1.17 & 2.72 \\
\hline 140 & $\mathrm{R}$ & 1.36 & 3.35 \\
\hline 141 & $\mathrm{R}$ & 0.91 & 1.44 \\
\hline 142 & $\mathrm{R}$ & 1.71 & 3.57 \\
\hline 143 & $\mathrm{R}$ & 0.33 & 0.63 \\
\hline
\end{tabular}




\section{Modified Data (Unity Rule SOR)}

NOTE: $\quad$ Type = "S" indicates survey unit sample.

Type $=$ "R" indicates reference area sample.

\begin{tabular}{lcc} 
Sample Number & Type & Sum-of-Ratios (SOR) \\
\hline 215 & S & 1.96 \\
216 & S & 1.62 \\
217 & S & 1.16 \\
218 & S & 0.84 \\
219 & S & 1.45 \\
220 & S & 1.4 \\
221 & S & 1.41 \\
222 & S & 1.44 \\
223 & S & 1.11 \\
224 & S & 1.05 \\
225 & S & 1.42 \\
226 & S & 1.09 \\
227 & S & 0.93 \\
228 & S & 1.11 \\
229 & S & 0.4 \\
230 & S & 0.44 \\
231 & S & 0.52 \\
127 & R & 1.4 \\
128 & R & 1.36 \\
129 & R & 1.23 \\
130 & R & 1.3 \\
131 & R & 1.32 \\
132 & R & 0.93 \\
133 & R & 1.24 \\
134 & R & 1.36 \\
135 & R & 1.05 \\
136 & $\mathrm{R}$ & 1.32 \\
137 & $\mathrm{R}$ & 1.36 \\
138 & $\mathrm{R}$ & 1.32 \\
139 & $\mathrm{R}$ & 1.49 \\
140 & $\mathrm{R}$ & 1.81 \\
141 & $\mathrm{R}$ & 0.89 \\
142 & $\mathrm{R}$ & 2.02 \\
143 & $\mathrm{R}$ & 0.37 \\
& &
\end{tabular}




\section{DQA Surface Soil Report}

\section{Basic Statistical Quantities Summary}

\begin{tabular}{lccc} 
Statistic & Survey Unit & Background & DQO Results \\
\hline Sample Number & 17 & 17 & N/2=18 \\
Mean (SOR) & 1.14 & 1.28 & 0.39 \\
Median (SOR) & 1.11 & 1.32 & N/A \\
Std Dev (SOR) & 0.42 & 0.36 & 0.39 \\
High Value (SOR) & 1.96 & 2.02 & N/A \\
Low Value (SOR) & 0.40 & 0.37 & N/A
\end{tabular}

\section{Statistical Test Summary}

$\begin{array}{ll}\text { Sum of Ranks: } & 595 \\ \text { Sum of Reference Ranks: } & 433 \\ \text { Critical Value: } & 345 \\ \text { Result: } & \text { Pass }\end{array}$

$\begin{array}{lll}\text { Data } & \text { Type } & \text { Adjusted Data }\end{array}$

$\begin{array}{lll}0.37 & \mathrm{R} & 1.37\end{array}$

0.89

0.93

1.05

1.23

1.24

1.3

1.32

1.32

1.32

1.36

1.36

1.36

1.4

1.49

1.81

2.02

0.4

0.44

0.52

0.84

0.93

1.05

1.09

1.11
433

45

Pass

1.93

2.05

2.23

2.24

2.3

2.32

2.32

2.32

2.36

2.36

2.36

2.4

2.49

2.81

3.02

0.4

0.44

0.52

0.84

0.93

1.05

1.09

1.11
Rank

Reference Rank

$\begin{array}{cc}11 & 11 \\ 18 & 18 \\ 19 & 19 \\ 21 & 21 \\ 22 & 22 \\ 23 & 23 \\ 24 & 24 \\ 26 & 26 \\ 26 & 26 \\ 26 & 26 \\ 29 & 29 \\ 29 & 29 \\ 29 & 29 \\ 31 & 31 \\ 32 & 32 \\ 33 & 33 \\ 34 & 34 \\ 1 & 0 \\ 2 & 0 \\ 3 & 0 \\ 4 & 0 \\ 5 & 0 \\ 6 & 0 \\ 7 & 0 \\ 8.5 & 0\end{array}$




\section{DQA Surface Soil Report}

Statistical Test Summary

\begin{tabular}{lcccc} 
Data & Type & Adjusted Data & Rank & Reference Rank \\
\hline 1.11 & & 1.11 & 8.5 & 0 \\
1.16 & S & 1.16 & 10 & 0 \\
1.4 & S & 1.4 & 12 & 0 \\
1.41 & S & 1.41 & 13 & 0 \\
1.42 & S & 1.42 & 14 & 0 \\
1.44 & S & 1.44 & 15 & 0 \\
1.45 & S & 1.45 & 16 & 0 \\
1.62 & S & 1.62 & 17 & 0 \\
1.96 & S & 1.96 & 20 & 0
\end{tabular}




\section{DQA Surface Soil Report}

\section{Assessment Summary}

Site:

Planner(s):

Survey Unit Name:

Report Number:

Survey Unit Samples:

Reference Area Samples:

Test Performed:

Judgmental Samples:

Assessment Conclusion:
Hammond Depot FSS Planner

Vitkus

C3 SU1 through 5 C3 SU5

5

17

17

WRS

Test Result: $\quad$ Pass

0

EMC Result:

Not Performed

Reject Null Hypothesis (Survey Unit PASSES)

\section{Retrospective Power Curve}

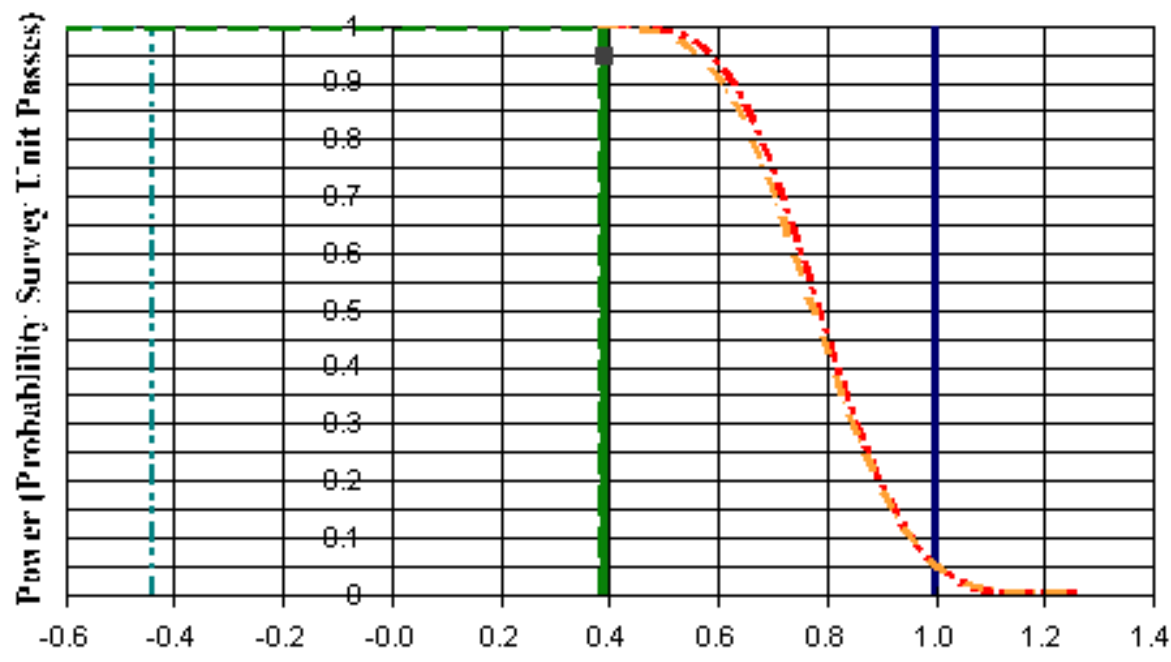

Unity Rule Sum-of-Ratios, not including background

$-\cdot=$ Prospective Power
LBGR
DCGL

- 1-beta

- Estimated Power

-- - Retrospective Power 


\section{DQA Surface Soil Report}

\section{Survey Unit Data}

NOTE: $\quad$ Type = "S" indicates survey unit sample.

Type = "R" indicates reference area sample.

\begin{tabular}{|c|c|c|c|}
\hline Sample Number & Type & Th-232 (pCi/g) & U-238 (pCi/g) \\
\hline 233 & $\mathrm{~S}$ & 1.21 & 1.15 \\
\hline 234 & $S$ & 1.18 & 1.72 \\
\hline 235 & $S$ & 0.99 & 1.6 \\
\hline 236 & S & 1.45 & 1.94 \\
\hline 237 & $S$ & 0.38 & 0.6 \\
\hline 238 & S & 0.99 & 1.67 \\
\hline 239 & $S$ & 0.36 & 0.63 \\
\hline 240 & $S$ & 0.87 & 1.55 \\
\hline 241 & $S$ & 1.35 & 2.42 \\
\hline 242 & $S$ & 0.63 & 0.84 \\
\hline 243 & S & 0.88 & 1.43 \\
\hline 244 & S & 0.75 & 0.91 \\
\hline 245 & S & 0.59 & 1.14 \\
\hline 246 & S & 0.76 & 1.62 \\
\hline 247 & S & 0.38 & 0.65 \\
\hline 248 & S & 0.64 & 1.48 \\
\hline 249 & S & 0.58 & 1.25 \\
\hline 127 & $\mathrm{R}$ & 1.1 & 2.54 \\
\hline 128 & $\mathrm{R}$ & 0.85 & 2.66 \\
\hline 129 & $\mathrm{R}$ & 0.74 & 2.44 \\
\hline 130 & $\mathrm{R}$ & 1.09 & 2.3 \\
\hline 131 & $\mathrm{R}$ & 0.93 & 2.5 \\
\hline 132 & $\mathrm{R}$ & 0.79 & 1.65 \\
\hline 133 & $\mathrm{R}$ & 0.94 & 2.3 \\
\hline 134 & $\mathrm{R}$ & 0.98 & 2.55 \\
\hline 135 & $\mathrm{R}$ & 0.65 & 2.06 \\
\hline 136 & $\mathrm{R}$ & 0.73 & 2.66 \\
\hline 137 & $\mathrm{R}$ & 1.1 & 2.44 \\
\hline 138 & $\mathrm{R}$ & 1.03 & 2.42 \\
\hline 139 & $\mathrm{R}$ & 1.17 & 2.72 \\
\hline 140 & $\mathrm{R}$ & 1.36 & 3.35 \\
\hline 141 & $\mathrm{R}$ & 0.91 & 1.44 \\
\hline 142 & $\mathrm{R}$ & 1.71 & 3.57 \\
\hline 143 & $\mathrm{R}$ & 0.33 & 0.63 \\
\hline
\end{tabular}




\section{Modified Data (Unity Rule SOR)}

NOTE: $\quad$ Type = "S" indicates survey unit sample.

Type $=$ "R" indicates reference area sample.

\begin{tabular}{lcc} 
Sample Number & Type & Sum-of-Ratios (SOR) \\
\hline 233 & S & 0.88 \\
234 & S & 1.09 \\
235 & S & 0.98 \\
236 & S & 1.28 \\
237 & S & 0.37 \\
238 & S & 1.01 \\
239 & S & 0.38 \\
240 & S & 0.92 \\
241 & S & 1.43 \\
242 & S & 0.55 \\
243 & S & 0.88 \\
244 & S & 0.62 \\
245 & S & 0.66 \\
246 & S & 0.91 \\
247 & S & 0.39 \\
248 & S & 0.81 \\
249 & S & 0.7 \\
127 & R & 1.4 \\
128 & R & 1.36 \\
129 & R & 1.23 \\
130 & R & 1.3 \\
131 & R & 1.32 \\
132 & R & 0.93 \\
133 & R & 1.24 \\
134 & $\mathrm{R}$ & 1.36 \\
135 & $\mathrm{R}$ & 1.05 \\
136 & $\mathrm{R}$ & 1.32 \\
137 & $\mathrm{R}$ & 1.36 \\
138 & $\mathrm{R}$ & 1.32 \\
139 & $\mathrm{R}$ & 1.49 \\
140 & $\mathrm{R}$ & 1.81 \\
141 & $\mathrm{R}$ & 0.89 \\
142 & $\mathrm{R}$ & 2.02 \\
143 & $\mathrm{R}$ & 0.37 \\
& &
\end{tabular}




\section{DQA Surface Soil Report}

\section{Basic Statistical Quantities Summary}

\begin{tabular}{lccc} 
Statistic & Survey Unit & Background & DQO Results \\
\hline Sample Number & 17 & 17 & N/2=18 \\
Mean (SOR) & 0.82 & 1.28 & 0.39 \\
Median (SOR) & 0.88 & 1.32 & N/A \\
Std Dev (SOR) & 0.30 & 0.36 & 0.39 \\
High Value (SOR) & 1.43 & 2.02 & N/A \\
Low Value (SOR) & 0.37 & 0.37 & N/A
\end{tabular}

\section{Statistical Test Summary}

$\begin{array}{lc}\text { Sum of Ranks: } & 595 \\ \text { Sum of Reference Ranks: } & 441 \\ \text { Critical Value: } & 345 \\ \text { Result: } & \text { Pass }\end{array}$

$\begin{array}{lll}\text { Data } & \text { Type } & \text { Adjusted Data }\end{array}$

\begin{tabular}{|c|c|c|c|c|}
\hline 0.37 & $\mathrm{R}$ & 1.37 & 17 & 17 \\
\hline 0.89 & $\mathrm{R}$ & 1.89 & 19 & 19 \\
\hline 0.93 & $\mathrm{R}$ & 1.93 & 20 & 20 \\
\hline 1.05 & $\mathrm{R}$ & 2.05 & 21 & 2 \\
\hline 1.23 & $\mathrm{R}$ & 2.23 & 22 & 2 \\
\hline 1.24 & $\mathrm{R}$ & 2.24 & 23 & \\
\hline 1.3 & $\mathrm{R}$ & 2.3 & 24 & \\
\hline 1.32 & $\mathrm{R}$ & 2.32 & 26 & \\
\hline 1.32 & $\mathrm{R}$ & 2.32 & 26 & \\
\hline 1.32 & $\mathrm{R}$ & 2.32 & 26 & \\
\hline 1.36 & $\mathrm{R}$ & 2.36 & 29 & 9 \\
\hline 1.36 & $\mathrm{R}$ & 2.36 & 29 & \\
\hline 1.36 & $\mathrm{R}$ & 2.36 & 29 & \\
\hline 1.4 & $\mathrm{R}$ & 2.4 & 31 & 31 \\
\hline 1.49 & $\mathrm{R}$ & 2.49 & 32 & \\
\hline 1.81 & $\mathrm{R}$ & 2.81 & 33 & \\
\hline 2.02 & $\mathrm{R}$ & 3.02 & 34 & \\
\hline 0.37 & $\mathrm{~S}$ & 0.37 & 1 & \\
\hline 0.38 & $\mathrm{~S}$ & 0.38 & 2 & \\
\hline 0.39 & $\mathrm{~S}$ & 0.39 & 3 & \\
\hline 0.55 & $S$ & 0.55 & 4 & C \\
\hline 0.62 & $S$ & 0.62 & 5 & \\
\hline 0.66 & $\mathrm{~S}$ & 0.66 & 6 & \\
\hline 0.7 & $\mathrm{~S}$ & 0.7 & 7 & c \\
\hline 0.81 & $\mathrm{~S}$ & 0.81 & 8 & \\
\hline
\end{tabular}




\section{DQA Surface Soil Report}

Statistical Test Summary

\begin{tabular}{|c|c|c|c|c|}
\hline Data & Type & Adjusted Data & Rank & Reference Rank \\
\hline 0.88 & $\mathrm{~S}$ & 0.88 & 9.5 & 0 \\
\hline 0.88 & $\mathrm{~S}$ & 0.88 & 9.5 & 0 \\
\hline 0.91 & $\mathrm{~S}$ & 0.91 & 11 & 0 \\
\hline 0.92 & $\mathrm{~S}$ & 0.92 & 12 & 0 \\
\hline 0.98 & $\mathrm{~S}$ & 0.98 & 13 & 0 \\
\hline 1.01 & $\mathrm{~S}$ & 1.01 & 14 & 0 \\
\hline 1.09 & $\mathrm{~S}$ & 1.09 & 15 & 0 \\
\hline 1.28 & $S$ & 1.28 & 16 & 0 \\
\hline 1.43 & $\mathrm{~S}$ & 1.43 & 18 & 0 \\
\hline
\end{tabular}

